\title{
The Impact of HBO1 in Ovarian Cancer
}

\section{Quintela Vazquez, Marcos}

How to cite:

Quintela Vazquez, Marcos (2018) The Impact of HBO1 in Ovarian Cancer. Doctoral thesis, Swansea University. http://cronfa.swan.ac.uk/Record/cronfa49209

Use policy:

This item is brought to you by Swansea University. Any person downloading material is agreeing to abide by the terms of the repository licence: copies of full text items may be used or reproduced in any format or medium, without prior permission for personal research or study, educational or non-commercial purposes only. The copyright for any work remains with the original author unless otherwise specified. The full-text must not be sold in any format or medium without the formal permission of the copyright holder. Permission for multiple reproductions should be obtained from the original author.

Authors are personally responsible for adhering to copyright and publisher restrictions when uploading content to the repository.

Please link to the metadata record in the Swansea University repository, Cronfa (link given in the citation reference above.)

http://www.swansea.ac.uk/library/researchsupport/ris-support/ 


\section{Prifysgol Abertawe}

Swansea University

\section{The Impact of HBO1 in Ovarian Cancer}

Marcos Quintela Vazquez, MSc

Submitted to Swansea University in fulfilment of the requirements for the Degree of Doctor of Philosophy.

2018 


\section{Summary}

New approaches to improve current detection and treatment of ovarian cancer (OC), now the fifth leading cause of cancer death among women in the United Kingdom, are increasingly needed. Current cytotoxic chemotherapy and targeted therapies have failed to significantly impact overall patient survival and drugs targeting epigenetic readers, writers and erasers are receiving much attention as potential sources of novel OC therapies. The identification of target molecules and/or epigenetic regulators with key roles in the OC phenotype and progression could facilitate the development of new effective treatment strategies.

The main purpose of this study was to assess the role of the 'histone acetyltransferase binding to ORC-1' (HBO1), an epigenetic regulator that plays important roles in diverse molecular processes including DNA replication and transcription. Previous studies found HBO1 overexpressed in $\mathrm{OC}$ tissues and, although the molecular basis of its role is still unclear, it may represent a novel target involved in the modulation of the OC phenotype.

To better understand the roles of HBO1 in OC, we first profiled its expression in cellular models and found it to be overexpressed compared to a non-cancerous cell model. Further analysis of the HBO1 downstream regulatory pathways through the use of two different silencing methodologies confirmed that HBO1 is involved in the regulation of important cancer associated pathways and processes, as well as individual oncogenes and tumour suppressor genes. Analysis of the biomechanical properties of OC cells using Atomic Force Microscopy outlined a role for HBO1 in the establishment of an aggressive cancerous phenotype, as the membranes of HBO1-overexpressing cells were significantly more elastic than their HBO1depleted counterparts. Overall, the results of this study suggest dynamic roles for HBO1, which ought to be studied further to determine whether this molecule could be considered an attractive target for the future development of OC therapies. 


\section{Declarations and Statements}

\section{DECLARATION}

This work has not previously been accepted in substance for any degree and is not being concurrently submitted in candidature for any degree.

Signed

Date

\section{STATEMENT 1}

This thesis is the result of my own investigations, except where otherwise stated.

Other sources are acknowledged by footnotes giving explicit references. A bibliography is appended.

Signed

Date

\section{STATEMENT 2}

I hereby give consent for my thesis, if accepted, to be available for photocopying and for inter-library loan, and for the title and summary to be made available to outside organisations.

Signed

Date 


\section{List of Contents}

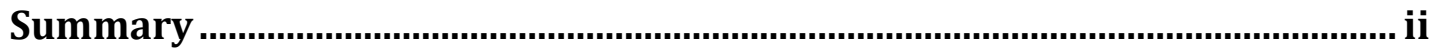

Declarations and Statements ..........................................................................ii

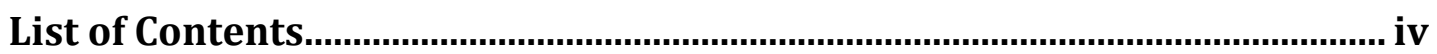

Acknowledgements .............................................................................................viii

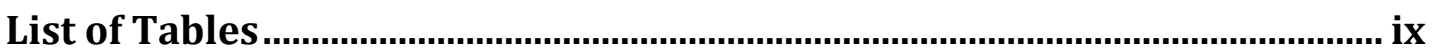

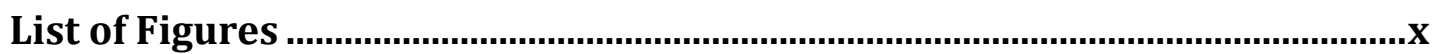

List of Abbreviations ......................................................................................

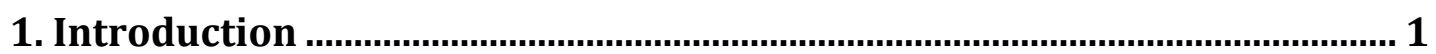

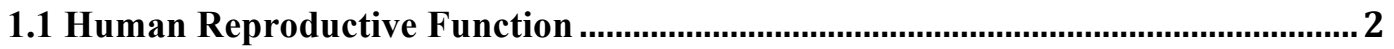

1.1.1 Female reproductive system................................................................................................ 2

1.1.2 Female hormonal system................................................................................................4

1.1.3 Formation of female gametes: Oogenesis ...........................................................................

1.2. Onset of Cancer and Tumour Progression ............................................................. 8

1.2.1 Carcinogenesis .............................................................................................................................

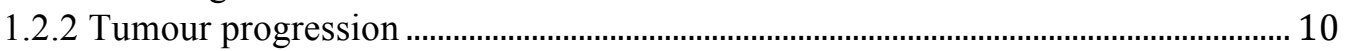

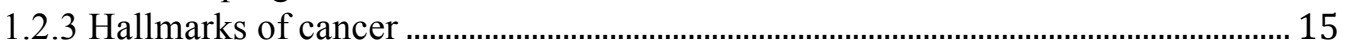

1.2.4 Tumour heterogeneity and microenvironment .............................................................. 17

1.3 Cancer in the Ovaries: Ovarian Cancer ................................................................. 17

1.3.1 Origins of ovarian carcinomas.................................................................................. 18

1.3.2 Disease progression..........................................................................................................2 20

1.3.3 Epidemiology and clinical presentation ...................................................................2.

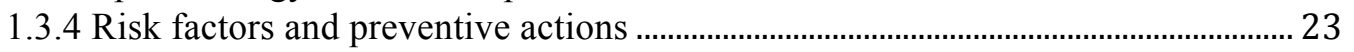

1.3.5 Diagnostic evaluation and staging............................................................................2. 24

1.3.6 Histological classification ..........................................................................................26

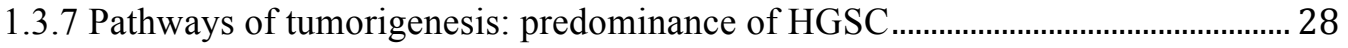

1.4 Ovarian Cancer Treatment ...................................................................................... 33

1.4.1 First line treatment for advanced ovarian cancer..............................................................33

1.4.2 Ovarian cancer recurrence...................................................................................................35

1.4.3 Targeting cancer angiogenesis.......................................................................................... 36

1.4.4 Targeting cancer 'BRCAness' ………………………………………………………….....37

1.4.5 Alternative ovarian cancer treatment strategies ..........................................................39

1.5 Epigenetic Regulatory Mechanisms in OC Progression ...................................... 41

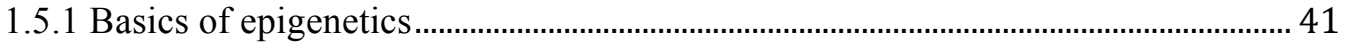

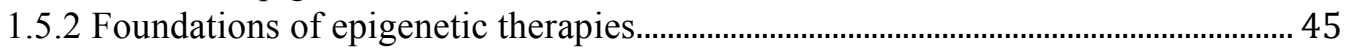

1.5.3 Epigenetic therapies for the treatment of ovarian cancer............................................ 47

1.5.3 Targeting histone acetyltransferases...........................................................................50

1.6 Histone Acetyltransferase Binding to ORC1 - HBO1............................................ 54

1.6.1 Structure and functions of the $\mathrm{N}$-terminus domain.....................................................5 54

1.6.2 Structure and functions of the C-terminus domain ............................................................56

1.6.3 HBO1 cell localization .......................................................................................................57

1.6.4 Roles of HBO1 in DNA replication..........................................................................5 57

1.6.5 Roles of HBO1 in transcription.....................................................................................6

$1.6 .7 \mathrm{HBO} 1$ at the crossroads........................................................................................ 65

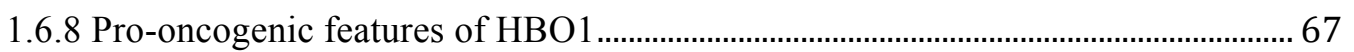

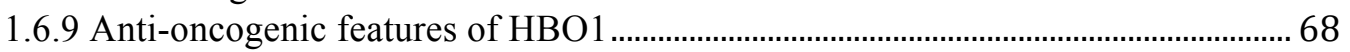

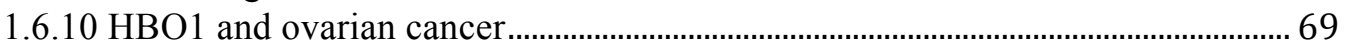

1.7 Research Aims ........................................................................................................... 70 


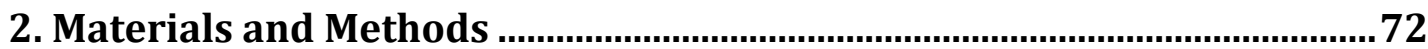

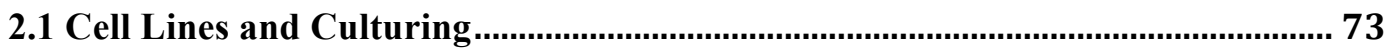

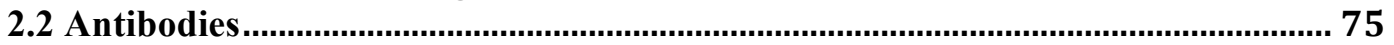

2.3 Two Step quantitative Real Time-Polymerase Chain Reaction ............................. 76

2.3.1 Extraction of RNA and synthesis of complementary DNA ..................................... 76

2.3.2 Design and characterization of primers ……………..................................................... 76

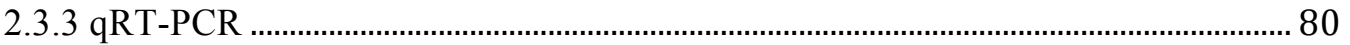

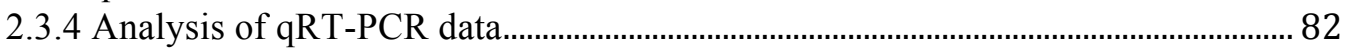

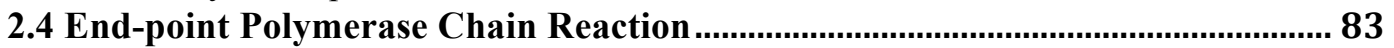

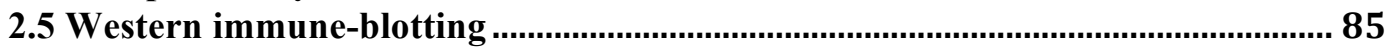

2.5.1 Protein extraction and quantification..................................................................... 85

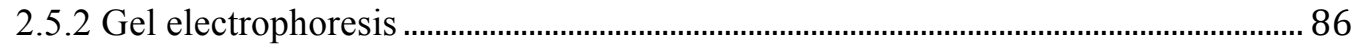

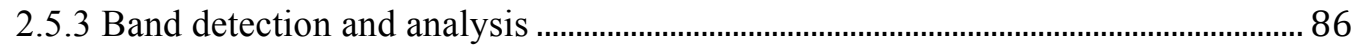

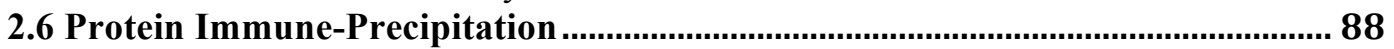

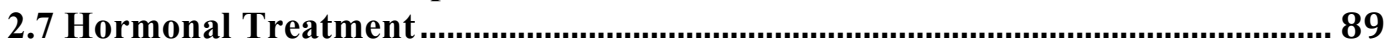

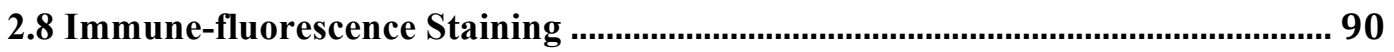

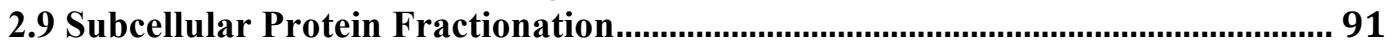

2.10 Chromatin Immune-precipitation coupled to qRT-PCR .................................... 91

2.10.1 Chromatrap ${ }^{\circledR}$ and EZ-Magna ChIP protocols....................................................... 93

2.10.2 Non-commercial ChIP protocol.................................................................................. 94

2.10.3 Design and characterization of ChIP-qRT-PCR primers ........................................ 97

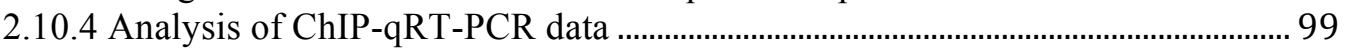

2.11 Structured Query Language Relational Database ..................................................99

2.12 ChIP combined with Sequencing .........................................................................100

2.12.1 Preparation of samples and acquisition of data ..................................................100

2.12.2 Analysis of ChIP-Seq raw data ..........................................................................101

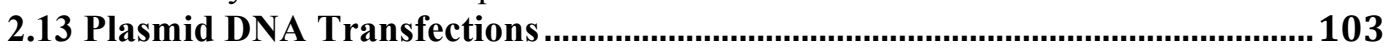

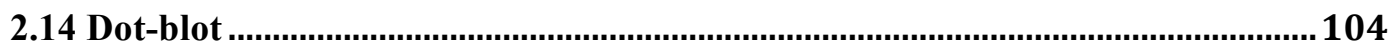

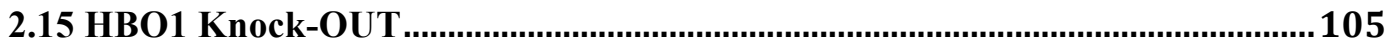

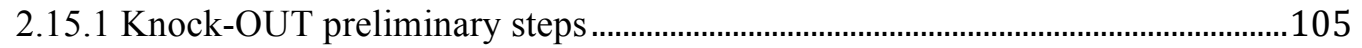

2.15.2 Knock-OUT and T7EI surveyor nuclease assay ……...........................................109

2.16 Small interfering RNA Knockdown .............................................................111

2.17 Lentiviral transduction of short hairpin RNAs ..............................................113

2.17.1 Characteristics and structure of selected molecules..............................................113

2.17.2 Optimization of the lentiviral transduction .........................................................114

2.17.3 Transduction of shRNAs targeting HBO1 …….................................................115

2.18 Design and construction of an HBO1-expressing vector ..................................116

2.18.1 Plasmid design .........................................................................................................116

2.18.2 Plasmid construction..................................................................................................117

2.18.3 Plasmid isolation........................................................................................................118

2.18.4 Directed-site mutation of the HBO1-expression plasmid .....................................118

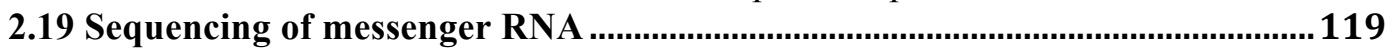

2.19.1 Sample collection and sequencing ……….......................................................119

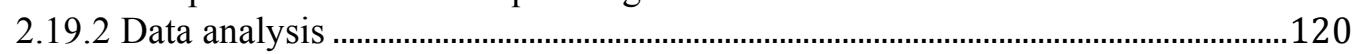

2.20 Gene Expression Profiling - Microarrays.........................................................121

2.20.1 Synthesis of complementary RNA.......................................................................121

2.20.2 BeadChip hybridization, scan and analysis..........................................................122

2.21 Pathway Analysis of Gene Expression Profiles....................................................123

2.21.2 Gene Set Enrichment Analysis.............................................................................123

2.21.2 Ingenuity Pathway Analysis......................................................................................124

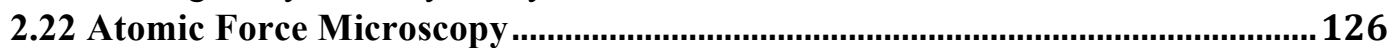

2.23 Scratch/Wound-healing and Cell Invasion Assays.............................................127

2.24 Enzyme-Linked Immune-Sorbent Assays.......................................................128

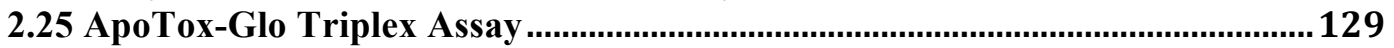


3. Expression and Regulation of HBO1 in Ovarian Cancer ........................ 131

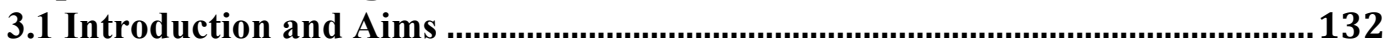

3.2 Ovarian Cancer Cell Line Model Systems ……...................................................133

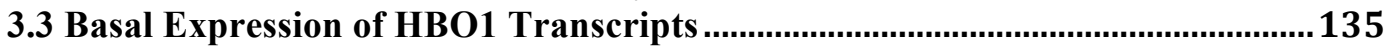

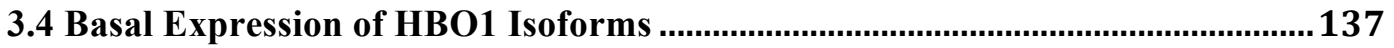

3.4.1 Structure and characteristics of the HBO1 isoforms ...............................................137

3.4.2 Differential expression of HBO1 isoforms in OC cell lines ...................................138

3.5 Basal Expression of HBO1 Protein in OC ............................................................140

3.5.1 Selection of antibodies targeting HBO1 ……........................................................140

4.5.3 Differential expression of HBO1 protein in OC cell lines .......................................143

3.6 Analysis of Potential Sources of HBO1 De-regulation in OC ...............................144

3.7 HBO1 Genome Wide Localization................................................................147

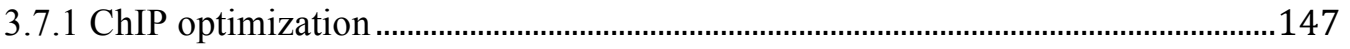

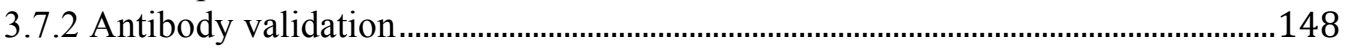

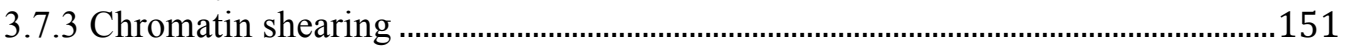

3.7.4 ChIP-qRT-PCR enrichment..............................................................................152

3.7.5 Potential HBO1 targets for ChIP-qRT-PCR enrichment .......................................155

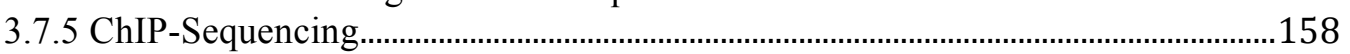

3.7.6 Further ChIP-qRT-PCR optimization ................................................................162

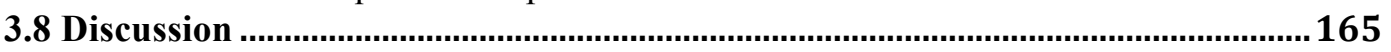

4. Optimization of Genetic Tools for the Study of HBO1 Function ............. 169

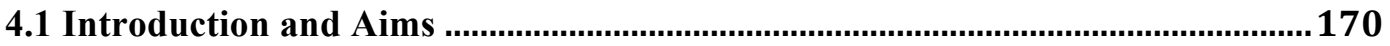

4.2 Generation of an HBO1 Knock-OUT Cell Line.................................................172

4.2.1 Cas9 transfection into UWB1.289 cells ..................................................................172

4.2.2 CRISPR RNA transfection and confirmation of mutational events......................176

4.3 Transduction of shRNAs to Constitutively Knockdown HBO1 ...........................181

4.3 Delivery of siRNAs to Transiently Knockdown HBO1 …..................................183

4.3.1 Optimization of the siRNA delivery to OC cells....................................................183

4.3.2 Delivery of siRNAs to OC cell lines .......................................................................186

4.3.3 Validation of siRNA efficiency.............................................................................187

4.5 Gene Expressing Vector: a Tool to Up-regulate HBO1 Levels.............................188

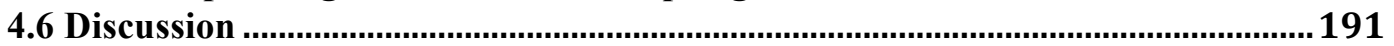

\section{Functional HBO1 Evaluation using a Stable RNAi-based Knockdown}

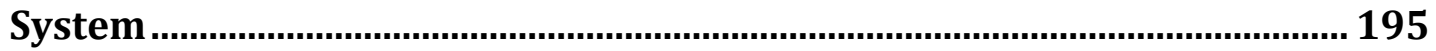

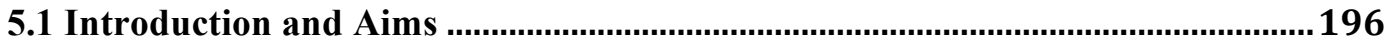

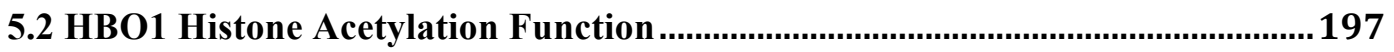

5.3 Gene Expression Profiling of Transduced UWB1.289 Cells ...............................199

5.3 Pathway Analysis of the Stable HBO1 Knockdown Profile ..................................201

5.4 In vitro Evaluation of HBO1-predicted Biological Functions ...............................202

5.4.1 Assessment of HBO1 suggested role in cell proliferation......................................203

5.4.2 Assessment of HBO1 suggested involvement in cell motility................................204

5.4.3 Assessment of HBO1 suggested involvement in cell organization........................205

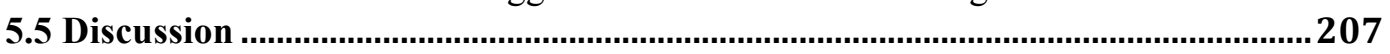




\section{Functional HBO1 Evaluation using a Transient RNAi-based Knockdown}

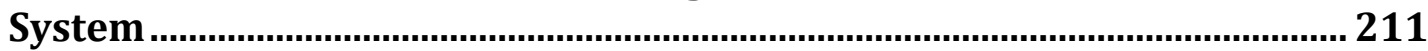

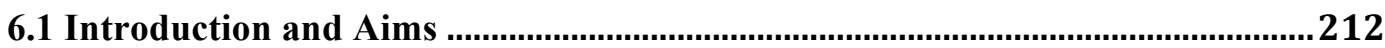

6.2 HBO1 Histone Acetylation Function in OC Cells..............................................213

6.3 Microarray Analysis of Transient HBO1 Knockdown Profiles .........................214

6.2.1 Gene expression profiling of OVCAR-3 cells..........................................................214

6.2.2 Gene expression profiling of SKOV-3 cells .............................................................218

6.2.3 Gene expression profiling of UWB1.289 cells .....................................................22

6.2.4 Study of HBO1 responsive genes in multiple OC cell lines....................................222

6.3 Functional Analysis of Transient HBO1 Knockdown Profiles .............................226

6.3.1 Ingenuity Pathway Analysis of HBO1 knockdown profiles....................................228

6.3.2 Gene Set Enrichment Analysis of HBO1 knockdown profiles.................................231

6.3.3 Validation of HBO1 KD inflammatory responses ...................................................233

6.4 In vitro Evaluation of HBO1-predicted Biological Functions ...............................234

6.4.1 Assessment of HBO1 suggested role in cell proliferation.......................................235

6.4.2 Assessment of HBO1 predicted involvement in cell motility ................................236

6.4.3 Assessment of HBO1 predicted involvement in cellular invasion ........................237

6.4.4 Assessment of HBO1 predicted involvement in cell viability ...............................239

6.4.5 Assessment of $\mathrm{HBO} 1$ predicted involvement in inflammatory responses...........241

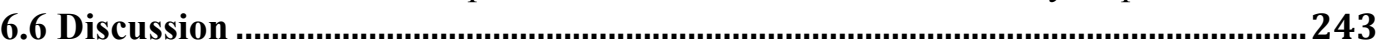

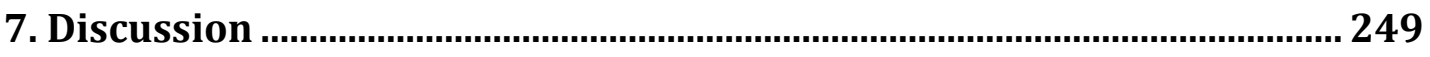

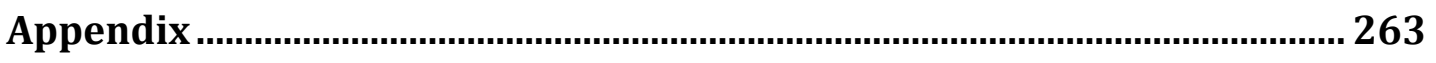

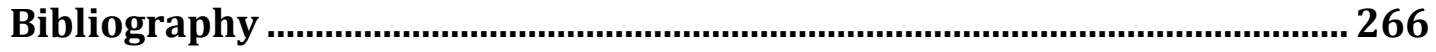




\section{Acknowledgements}

I wish to express my sincere thanks to my supervisor, Professor R. Steven Conlan, for giving me the opportunity to take on this $\mathrm{PhD}$, for his advice, supervision and patience over the course of my research. Likewise, I would like to thank Dr. Lewis Francis and Dr. Deyarina Gonzalez for their invaluable support and assistance.

My sincere gratitude goes to Dr. Paul Webb and Dr. Douglas Sieglaff, as well as the whole group at the Houston Methodist Research Institute, for their assistance, guidance, expertise and encouragement extended to me.

I also would like to take this opportunity to record my thanks to all the members of the $\mathrm{CNH}$ in Swansea University, who welcomed me and helped me in each step of the way.

Me gustaría también dar las gracias de manera especial a la doctora Belen Pan Castillo, sin ella no hubiera llegado hasta aquí... literalmente.

A Beth, mi corazón

A mis padres, por todo 


\section{List of Tables}

TABLE 1. 1 LIST OF EUKARYOTIC DNA DAMAGE REPAIR PATHWAYS ................................................ 14

TABLE 1. 2 SUMMARY TABLE OF THE 'HALLMARKS OF CANCER' .................................................... 16

TABLE 1. 3 SUMMARY OF THE INTERNATIONAL STAGING SYSTEM FOR OVARIAN CARCINOMAS .............25

TABLE 1. 4 CLINICAL FEATURES OF THE FIVE MOST COMMON SUB-TYPES OF EOC ….........................27

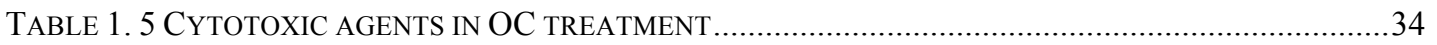

TABLE 1. 6 CLINICAL EXPERIENCE WITH EPIGENETIC THERAPIES IN OC .........................................49

TABLE 1. 7 LIST OF PREVIOUS HBO1 KNOCKDOWN EXPERIMENTS …….............................................66

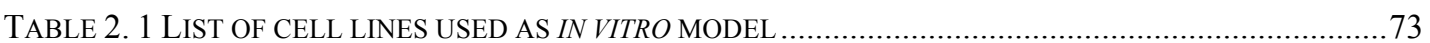

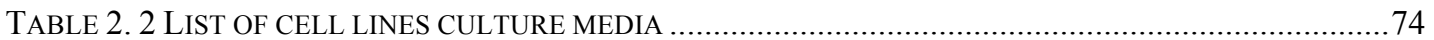

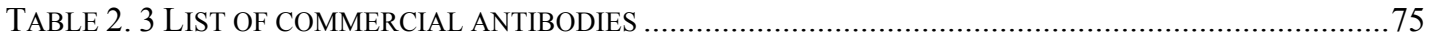

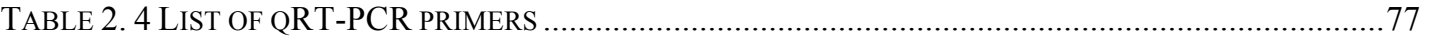

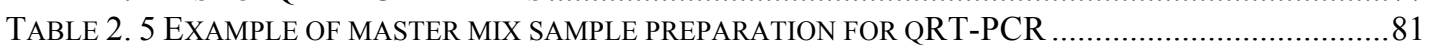

TABLE 2. 6 AMPLIFICATION STEPS USED IN QRT-PCR EXPERIMENTS .................................................82

TABLE 2. 7 EXAMPLE OF FOLD-CHANGE CALCULATION FOLLOWING THE $\Delta \Delta C_{\mathrm{T}}$ METHOD ....................83

TABLE 2. 8 BASIC COMPONENTS AND AMPLIFICATION STEPS USED IN END-POINT PCR ........................84

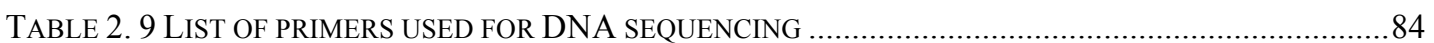

TABLE 2. 10 EXAMPLE OF RELATIVE DENSITY CALCULATIONS IN WB ANALYSIS ...............................87

TABLE 2. 11 EXAMPLE OF 'PERCENTAGE OF CELL LOCALIZATION' CALCULATIONS.............................91

TABLE 2. 12 RECIPES FOR THE NON-COMMERCIAL CHIP PROTOCOL ...................................................95

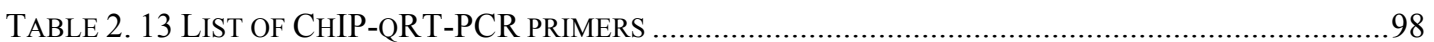

TABLE 2. 14 EXAMPLE OF 'PERCENTAGE OF INPUT' ENRICHMENT CALCULATIONS ................................99

TABLE 2. 15 LIST OF REAGENTS USED FOR PLASMID DNA TRANSFECTION ...................................... 104

TABLE 2. 16 LIST OF CRISPR RNA SEQUENCES AND HBO1-KNOCKOUT PRIMERS ............................107

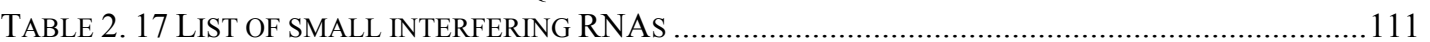

TABLE 2. 18 LIST OF SIRNA TRANSFECTION CONDITIONS FOR OC CELL LINES .................................112

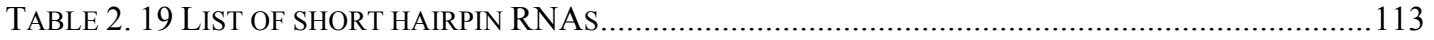

TABLE 2. 20 LIST OF PRIMERS USED TO CONSTRUCT AN HBO1-EXPRESSION PLASMID ........................117

TABLE 2. 21 EXAMPLE OF RELATIVE CELL CALCULATIONS FOR APOTOX-GLOTM ASSAYS ..................130

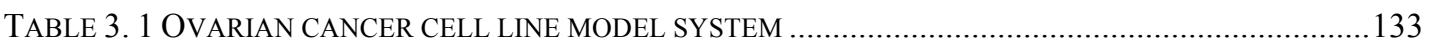

TABLE 3. 2 STRUCTURAL COMPOSITION OF THE FIVE HBO1 ISOFORMS .............................................137

TABLE 3.3 RELEVANT HBO1-RELATED DATASETS INTEGRATED INTO THE SQL DATABASE ..............155

TABLE 3. 4 LIST OF POTENTIAL HBO1 TARGET GENES DERIVED FROM SQL CORRELATIONS ...............156

TABLE 3. 5 LIST OF POTENTIAL HBO1 TARGET GENES DERIVED FROM CHIP-SEQ.............................160

TABLE 4. 1 AdVANTAGES AND DISADVANTAGES OF KD AND KO AVAILABLE TECHNIQUES ...............171

TABLE 4. 2 LIST OF POSSIBLE OUTCOMES OF THE CRISPR-CAS9 GENOME EDITING ...........................192

TABLE 5. 1 LIST OF ALTERED TRANSCRIPTS AFTER CONSTITUTIVE KD OF HBO1 ..............................200

TABLE 5. 2 LIST OF PREDICTED IPA ALTERATIONS FOLLOWING STABLE HBO1 KD ...........................2202

TABLE 6. 1 LIST OF ALTERED GENES AFTER TRANSIENT HBO1 KD IN OVCAR-3...........................215

TABLE 6. 2 LIST OF GENE VALIDATION TARGETS FOR THE OVCAR-3 EXPRESSION PROFILE ...............216

TABLE 6. 3 LIST OF ALTERED GENES AFTER TRANSIENT HBO1 KD IN SKOV-3 ..............................218

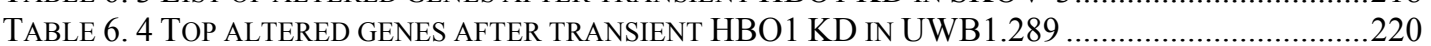

TABLE 6. 5 SUMMARY OF GENE EXPRESSION CHANGES CAUSED BY TRANSIENT HBO1 KD .................222

TABLE 6. 6 COMPARISON OF GENE EXPRESSION CHANGES BETWEEN OC CELL LINES .........................226

TABLE 6. 7 LIST OF COMMON ALTERED IPA FUNCTIONS AFTER TRANSIENT HBO1 KD .....................229

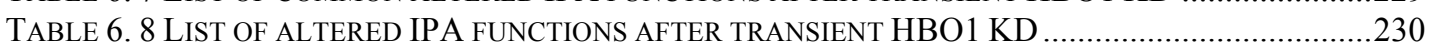

TABLE 6. 9 LIST OF COMMON ALTERED GENE-SETS FOLLOWING TRANSIENT HBO1 KD ...................231

TABLE 6. 10 LIST OF GSEA ACTIVATED GENE-SETS AFTER TRANSIENT HBO1 KD ..........................233

TABLE 7. 1 LIST OF COMMON ALTERED IPA FUNCTIONS AFTER HBO1 KD .....................................259 


\section{List of Figures}

FIGURE 1. 1 SCHEMATIC REPRESENTATION OF THE HUMAN FEMALE INTERNAL GENITALIA.....................

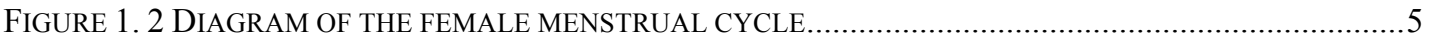

FIGURE 1. 3 SCHEMATIC REPRESENTATION OF FOLLICLE DEVELOPMENT IN THE OVARY .........................7

FIGURE 1. 4 DIAGRAM SUMMARIZING CELL CARCINOGENESIS AND TUMOUR PROGRESSION ...................11

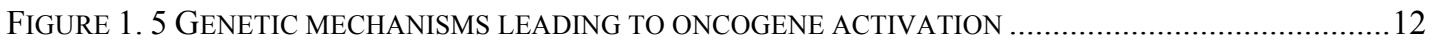

FIGURE 1. 6 EFFECT OF GENETIC MODIFICATIONS ON TUMOUR SUPPRESSOR GENES ...........................13

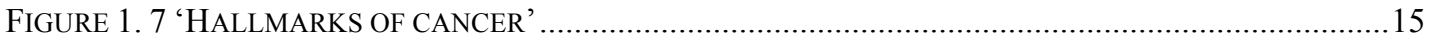

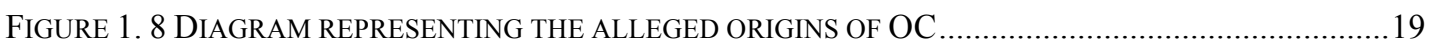

FIGURE 1.9 SCHEMATIC DEPICTION OF THE MOST FREQUENT OC METASTASIS SITES...........................20

FIGURE 1. 10 COMPARISON OF DEATH-TO-INCIDENCE RATES FOR COMMON FEMALE CANCERS.............22

FIGURE 1. 11 REPRESENTATIVE EXAMPLES OF HGSC HISTOLOGICAL EXAMINATIONS .........................29

FIGURE 1. 12 SCHEMATIC REPRESENTATION OF ALTERED SIGNALLING PATHWAYS IN HGSC .................31

FIGURE 1. 13 MODELS OF INITIATION AND PROGRESSION OF HGSC ....................................................3

FIGURE 1. 14 SENSITIVITY TO PLATINUM CHEMOTHERAPY OF HGSC .................................................35

FIGURE 1. 15 SYNTHETIC LETHALITY COUPLING $B R C A$ DEFICIENCY AND PARP INHIBITION .................38

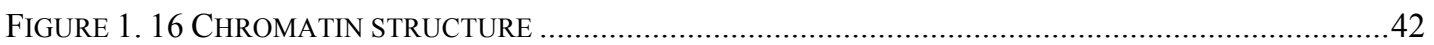

FIGURE 1. 17 EPIGENETIC REGULATION OF GENE EXPRESSION ........................................................43

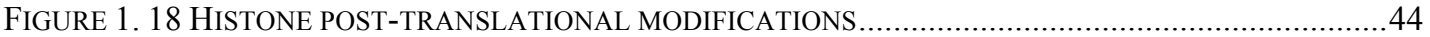

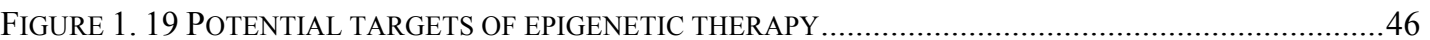

FIGURE 1.20 POTENTIAL TARGETS OF EPIGENETIC THERAPY IN OC …............................................4

FIGURE 1.21 STRUCTURE OF A CANONICAL PROTEIN MULTI-SUBUNIT COMPLEX ...............................51

FIGURE 1.22 SCHEMATIC REPRESENTATION OF THE STRUCTURE OF HUMAN PROTEIN HBO1 ..............54

FIGURE 1. 23 REGULATION OF DNA REPLICATION LICENSING BY HBO1 …......................................58

FIGURE 1.24 MODEL FOR HBO1 ASSEMBLY IN ALTERNATE MULTI-PROTEIN COMPLEXES ....................61

FIGURE 1. 25 SCHEMATIC REPRESENTATION OF THE STRUCTURE AND INTERACTIONS OF HUMAN

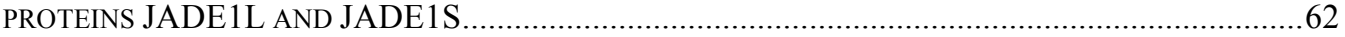

FIGURE 2. 1 MAIN CHARACTERISTICS OF CHIP AND CHIP-QRT-PCR PRIMER DESIGN .........................92

FIGURE 2. 2 EXAMPLE OF SQL RELATIONAL DATABASE CORRELATIONS........................................... 100

FIGURE 2. 3 WORKFLOW DIAGRAM OF THE CHIP-SEQUENCING ANALYSIS PIPELINE .........................102

FIGURE 2. 4 EXAMPLE OF CHANCE ANALYSIS ON CHIP-SEQUENCING DATA.................................103

FIGURE 2. 5 SELECTION OF CRISPR RNAS TARGETING THE HBO1 LOCUS........................................106

FIGURE 2. 6 METHODOLOGY TO TRANSIENTLY EXPRESS CAS9 IN OC CELLS .....................................108

FIGURE 2. 7 ANALYSIS OF CRISPR MUTAGENESIS EFFICIENCY USING THE T7EI ASSAY ....................110

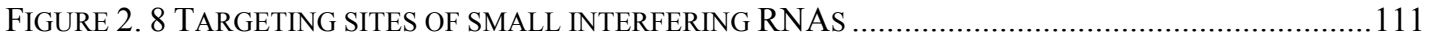

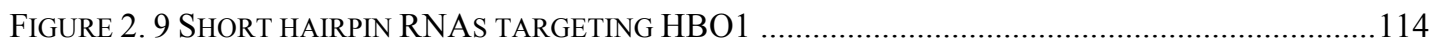

FIGURE 2. 10 DESIGN AND CONSTRUCTION OF AN HBO1-EXPRESSING PLASMID ................................116

FIGURE 2. 11 GENERATION OF AN HBO1-MUTANT VIA SITE-DIRECTED MUTAGENESIS .......................119

FIGURE 2. 12 WORKFLOW DIAGRAM OF THE RNASEQ ANALYSIS PIPELINE ...................................120

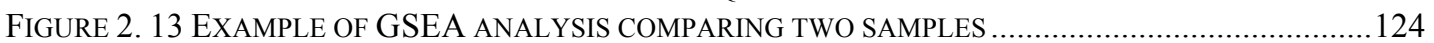

FIGURE 2. 14 EXAMPLE OF AN IPA 'DOWNSTREAM EFFECTS' OUTCOME ............................................125

FIGURE 2. 15 EXAMPLE OF RELATIVE AREA CALCULATIONS FOR WOUND-HEALING ASSAYS ...............127

FIGURE 3. 1 COMPARATIVE ANALYSIS OF HBO1 TRANSCRIPT EXPRESSION ......................................136

FIGURE 3. 2 SCHEMATIC DEPICTION OF THE HUMAN HBO1 PROTEIN VARIANTS.................................138

FIGURE 3. 3 COMPARATIVE ANALYSIS OF HBO1 ISOFORM EXPRESSION ............................................139

FIGURE 3. 4 WESTERN BLOT ANALYSES OF HBO1 ANTIBODIES PERFORMANCE ...............................141

FIGURE 3. 5 ANALYSIS OF HBO1 UBIQUITINATION STATE VIA IP USING THE G-2 ANTIBODY ...............142

FIGURE 3. 6 COMPARATIVE ANALYSIS OF HBO1 PROTEIN EXPRESSION ...........................................143

FIGURE 3. 7 COMPARATIVE ANALYSIS OF NUCLEAR RECEPTOR EXPRESSION LEVELS ............................ 145

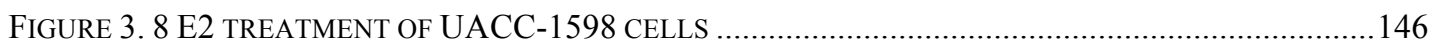

FIGURE 3. 9 HBO1 IP AND ING4 CO-IP USING AB70 ANTIBODY .................................................. 149

FIGURE 3. 10 HBO1 IMMUNE-FLUORESCENCE STAINING …........................................................... 149

FIGURE 3. 11 SUBCELLULAR PROTEIN LOCALIZATION OF HBO1 IN UWB1.289 CELLS .........................150

FIGURE 3. 12 SONICATION EFFICIENCY OF UWB1.289 CROSS-LINKED CHROMATIN SAMPLES..............152

FIGURE 3. 13 ENRICHMENT OF POTENTIAL HBO1 TARGETS: ORIGINS OF REPLICATION ......................153

FIGURE 3. 14 IGV ANALYSIS OF ALLEGED HBO1-TARGETED ORIGINS OF REPLICATION.....................154 
FIGURE 3. 15 SQL ISOLATION OF POTENTIAL HBO1 TARGETS ................................................... 156

FIGURE 3. 16 ENRICHMENT OF POTENTIAL HBO1 TARGETS: SQL-DERIVED GENES ............................157

FIGURE 3. 17 GRAPHICAL REPRESENTATION OF ACTIVE MOTIF ${ }^{\circledR}$ CHIP-SEQ RESULTS ..........................159

FIGURE 3. 18 IGV ANALYSIS OF POTENTIAL HBO1 TARGETS DERIVED FROM CHIP-SEQ ....................161

FIGURE 3. 19 ENRICHMENT OF POTENTIAL HBO1-TARGETS: AM-DERIVED GENES ..............................162

FIGURE 3. 20 COMPARATIVE ANALYSIS OF HBO1-COMPLEX COMPONENTS ........................................ 164

FIGURE 4. 1 UWB1.289 TRANSFECTION EFFICIENCY TEST ............................................................... 173

FIGURE 4. 2 TRANSFECTION OF A CAS9-EXPRESSING PLASMID IN UWB1.289 AND HELA....................174

FIGURE 4. 3 UWB1.289 ELECTROPORATION EFFICIENCY TEST ...................................................... 175

FIGURE 4. 4 ELECTROPORATION OF A CAS9-EXPRESSION PLASMID IN UWB1.289 CELLS....................176

FIGURE 4. 5 T7EI SURVEYOR ASSAY OF CAS9-ELECTROPORATED UWB1.289 CELLS ..........................177

FIGURE 4. 6 ANALYSIS OF POTENTIAL UWB1.289 KNOCK-OUT INDIVIDUAL COLONIES.....................178

FIGURE 4. 7 SEQUENCING ANALYSIS OF THE TARGETED HBO1 LOCUS ...........................................179

FIGURE 4. 8 LENTIVIRAL TRANSDUCTION OF SHRNAS INTO UWB1.289 CELLS ................................181

FIGURE 4. 9 HBO1 MRNA RELATIVE LEVELS IN TRANSDUCED UWB1.289 CELLS............................182

FIGURE 4. 10 HBO1 PROTEIN RELATIVE LEVELS IN TRANSDUCED UWB1.289 CELLS ........................182

FIGURE 4. 11 HBO1 MRNA RELATIVE LEVELS FOLLOWING SIRNA KD IN OVCAR-3 ......................184

FIGURE 4. 12 HBO1 PROTEIN RELATIVE LEVELS FOLLOWING SIRNA KD IN OVCAR-3 ....................185

FIGURE 4. 13 TIME-COURSE ANALYSIS OF HBO1 LEVELS FOLLOWING SIRNA KD ...........................185

FIGURE 4. 14 HBO1 RELATIVE LEVELS FOLLOWING SIRNA KD IN OC CELL LINES ...........................186

FIGURE 4. 15 VALIDATION OF HBO1 KD USING INDIVIDUAL SIRNAS ............................................187

FIGURE 4. 16 TRANSFECTION OF AN HBO1-EXPRESSING VECTOR INTO OVCAR-3 CELLS ..................189

FIGURE 4. 17 ECTOPIC EXPRESSION OF HBO1 IN SHRNA-TRANSDUCED UWB1.289 CELLS ...............190

FIGURE 5. 1 HiSTONE ACETYLATION PATTERNS AFTER STABLE HBO1 KD ........................................197

FigURE 5. 2 HiSTONE ACETYLATION RELATIVE LEVELS AFTER STABLE HBO1 KD ...........................198

FIGURE 5. 3 HISTONE ACETYLATION PATTERNS LINKED TO HBO1 ECTOPIC EXPRESSION....................199

FIGURE 5. 4 VALIDATION OF GENE EXPRESSION CHANGES CAUSED BY STABLE HBO1 KD ..................201

FIGURE 5. 5 STUDY OF CELL PROLIFERATION AFTER CONSTITUTIVE HBO1 KD .................................2203

FIGURE 5. 6 EXAMPLE OF SCRATCH ASSAY WITH STABLE HBO1 KD UWB1.289 CELLS....................204

FIGURE 5. 7 STUDY OF CELL ORGANIZATION AFTER STABLE HBO1 KD IN UWB1.289 CELLS ............206

FIGURE 6. 1 HISTONE ACETYLATION PATTERNS AFTER TRANSIENT HBO1 KD.................................213

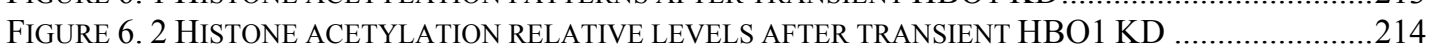

FIGURE 6. 3 VALIDATION OF THE HBO1 KD OVCAR-3 GENE EXPRESSION PROFILE ..........................216

FIGURE 6. 4 TIME-COURSE ANALYSIS OF THE TRANSIENT HBO1 KD EFFECT IN OVCAR-3 ...............217

FIGURE 6. 5 VALIDATION OF THE HBO1 KD SKOV-3 GENE EXPRESSION PROFILE ...........................2219

FIGURE 6. 6 VALIDATION OF THE HBO1 KD UWB1.289 GENE EXPRESSION PROFILE ..............................221

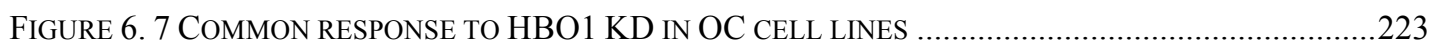

FIGURE 6. 8 VALIDATION OF COMMON GENE EXPRESSION CHANGES CAUSED BY HBO1 KD..............224

Figure 6. 9 GENE RELATIVE LEVELS AFTER HBO1 KD IN UACC-1598 AND TOV-112D ................225

FIGURE 6. 10 ALTERED EXPRESSION OF KEY CANCER GENES CAUSED BY HBO1 KD ..........................22

FIGURE 6. 11 IPA CANONICAL PATHWAYS PREDICTION AFTER HBO1 KD IN UWB1 289 ..................228

FIGURE 6. 12 IPA DOWNSTREAM EFFECTS PREDICTION AFTER HBO1 KD IN UWB1.289 _................229

FigurE 6. 13 EXAMPLE OF GENE-SET ENRICHMENT AFTER HBO1 KD IN OVCAR-3 .......................232

FIGURE 6. 14 VALIDATION OF IFN-RELATED GENE CHANGES IN OVCAR-3 CELLS ..............................2. 234

FIGURE 6. 15 STUDY OF CELL PROLIFERATION AFTER HBO1 KD IN OC CELLS ..................................235

FIGURE 6. 16 EXAMPLE OF WOUND-HEALING ASSAY AFTER HBO1 KD IN OVCAR-3 ......................236

FIGURE 6. 17 STUDY OF CELL INVASIVENESS AFTER HBO1 KD IN OC CELLS ..................................2238

FIGURE 6. 18 STUDY OF CELL VIABILITY, CYTOTOXICITY AND APOPTOSIS AFTER HBO1 KD ...............240

FIGURE 6. 19 IFN PRODUCTION AND SECRETION AFTER HBO1 KD IN OC CELLS..............................242

FIGURE 7. 1 SCHEMATIC DEPICTION OF POTENTIAL HBO1 PROTEIN PTMS ....................................253 


\section{List of Abbreviations}

\begin{tabular}{ll} 
A & \\
\hline Acetyl-CoA & Acetyl-coenzyme A \\
AFM & Atomic force microscopy \\
AM & Active Motif \\
ANOVA & Analysis of variance \\
AR & Androgen receptor \\
ATP & Adenosine triphosphate
\end{tabular}

$\mathrm{B}$

\begin{tabular}{ll}
\hline BRCA1/2 & Breast cancer susceptibility gene/DNA repair associated 1/2 \\
BrdU & 5'-Bromo-2'-deoxyuridine \\
BRPF1-3 & Bromodomain and PHD finger containing protein 1-3 \\
BSA & Bovine serum albumin \\
BST2 & Bone marrow stromal cell antigen 2
\end{tabular}

$\mathrm{C}$

$\begin{array}{ll}\text { CA-125/MUC16 } & \text { Cancer antigen 125/Mucin 16 } \\ \text { Cas9 } & \text { CRISPR-associated protein 9 } \\ \text { CCC } & \text { Clear cell carcinoma } \\ \text { CCNE1 } & \text { Cyclin E1 } \\ \text { CD46 } & \text { CD46 molecule/antigen } \\ \text { CDK } & \text { Cyclin-dependent kinase } \\ \text { CDKN1A/p21 } & \text { Cyclin-dependent kinase inhibitor 1A } \\ \text { cDNA } & \text { Complementary DNA } \\ \text { Cdt1 } & \text { Chromatin licensing and DNA replication factor 1 } \\ \text { ChIP } & \text { Chromatin immune-precipitation } \\ \text { ChIP-Seq } & \text { ChIP combined with sequencing } \\ \text { CIC } & \text { Cortical inclusion cyst } \\ \text { CMV } & \text { Cytomegalovirus } \\ \text { CpG } & \text { Cytosine-guanine di-nucleotide } \\ \text { CRISPR } & \text { Clustered regularly interspaced short palindromic repeats } \\ \text { cRNA } & \text { Complementary RNA } \\ \text { cRNA } & \text { CRISPR RNA } \\ \text { CTD } & \text { Carboxyl-terminus domain }\end{array}$

D

\begin{tabular}{ll}
\hline DAPI & 4', 6'-Diamidine-2'-phenylindole dihydrochloride \\
DB & Dot-blot \\
DNA & Deoxyribonucleic acid \\
DNMT & DNA methyl-transferase \\
dNTP & Deoxy-nucleotide triphosphate \\
DSB & Double-strand break
\end{tabular}

E

\begin{tabular}{ll}
\hline E. coli & Escherichia coli \\
E2 & Estradiol \\
EC & Endometrioid carcinoma \\
EDTA & Ethylenediaminetetraacetic acid \\
ELISA & Enzyme-linked immune-sorbent assay \\
EMT & Epithelial-mesenchymal transition \\
EOC & Epithelial ovarian cancer \\
ERBB2/HER2 & Epidermal growth factor receptor 2 \\
ERA/ & Estrogen receptor A/alpha \\
ERB/ $/ \boldsymbol{l}$ & Estrogen receptor B/beta
\end{tabular}




$\begin{array}{ll}\text { FC } & \text { Fold-change } \\ \text { FBS } & \text { Foetal calf/bovine serum } \\ \text { FDR } & \text { False discovery rate } \\ \text { FGFR4 } & \text { Fibroblast growth factor receptor } 4 \\ \text { FTE } & \text { Fallopian tube epithelium }\end{array}$

G

$\begin{array}{ll}\text { GAPDH } & \text { Glyceraldehyde-3-phosphate dehydrogenase } \\ \text { GCN5/KAT2A } & \text { Lysine acetyltransferase 2A } \\ \text { gDNA } & \text { Genomic DNA } \\ \text { GFP } & \text { Green fluorescent protein } \\ \text { GS } & \text { GSEA Gene-set } \\ \text { GSEA } & \text { Gene set enrichment analysis }\end{array}$

$\mathrm{H}$

$\begin{array}{ll}\text { H1, 2A/B, 3, 4 } & \text { Histone 1, 2A/B, 3, 4 } \\ \text { H2AFY } & \text { H2A histone family member Y } \\ \text { HAT } & \text { Histone acetyltransferase } \\ \text { HBO1/KAT7/MYST2 } & \text { Histone acetyltransferase binding to ORC-1 } \\ \text { HDAC } & \text { Histone deacetylase } \\ \text { HGSC } & \text { High-grade serous carcinoma } \\ \text { HOSEpiC } & \text { Human ovarian surface epithelial cells } \\ \text { HPC } & \text { High performance computing } \\ \text { HPRT1 } & \text { Hypoxanthine phosphoribosyltransferase 1 } \\ \text { HR } & \text { Homologous recombination } \\ \text { HRR } & \text { Homologous recombination repair }\end{array}$

I

Immune-fluorescence

$\begin{array}{ll}\text { IgG } & \text { Interferon alpha, beta } \\ \text { Immunoglobulin } G\end{array}$

IGR Intergenic region

IGV Integrative genomics viewer

INDEL Insertions and/or deletions

ING4/5 Inhibitor of growth family member 4/5

IP Immune-precipitation

ISG15 Interferon stimulated protein 15

$\mathrm{J}$

JADE1-3 JADE family PHD finger 1-3

K

$\begin{array}{ll}\text { KD } & \text { Knockdown } \\ \text { KO } & \text { Knockout }\end{array}$

$\mathrm{L}$

$\begin{array}{ll}\text { LAT/KAT } & \text { Lysine acetyltransferase } \\ \text { LGSC } & \text { Low-grade serous carcinoma }\end{array}$

$\mathrm{M}$

MC

MCM1-7

Mek-1

MEV

MLH1

MOF/KAT8

Mucinous carcinoma

Minichromosome maintenance complex component 1-7

Mitogen-activated protein kinase 1

Multiple experiment viewer

MutL homolog 1

Males absent on the first 
MOI

MORF/KAT6B

MOZ/KAT6A

mRNA

MSH2
Multiplicity of infection

Lysine acetyltransferase 6B

Lysine acetyltransferase 6A

Messenger RNA

MutS homolog 2

\begin{tabular}{l} 
N \\
\hline NCBI \\
NEOC \\
NF-KB/ $\boldsymbol{~} \beta$ \\
NHEJ \\
NLS \\
NR \\
NTD
\end{tabular}

$\mathrm{O}$

$\begin{array}{ll}\text { O/N } & \text { Over-night } \\ \text { OC } & \text { Ovarian cancer } \\ \text { ORC } & \text { Origin recognition complex } \\ \text { ORF } & \text { Open reading frame } \\ \text { OSE } & \text { Ovarian surface epithelium }\end{array}$

$\mathrm{P}$

p300/KAT3B

PAM

PARP

PBS

PCAF/KAT2B

PCR

PHD

PI

PI3K

PIC

Plk1

PMS2

PR

Pre-RC

PTM

PVDF
National centre for biotechnology information

Non-epithelial ovarian cancer

Nuclear factor kappa beta

Non-homologous end joining

Nuclear localization signal

Nuclear receptor

Amino-terminus domain

E1A binding protein $\mathrm{p} 300$

Proto-spacer adjacent motif

Poly-ADP ribose polymerase

Phosphate buffered saline solution

p300/CBP-associated factor

Polymerase chain reaction

Plant homeodomain

Percentage of input

Phosphatidylinositol 3-kinase

Protease inhibitor cocktail

Polo-kinase 1

Mismatch repair PMS1 homolog 2

Progesterone receptor

Pre-replication complex

Post-translational modification

Polyvinylidine difluoride

Q qRT-PCR

Quantitative real time-PCR

$\mathrm{R}$

\section{RA}

RD

RIPA

RNA

RNAi

RNAse

RNAseq

RPL5

RR

RT
Relative area

Relative density

Radio-immune-precipitation assay

Ribonucleic acid

RNA interference

Ribonuclease

RNA sequencing

Ribosomal protein L5

Response rate

Room temperature 


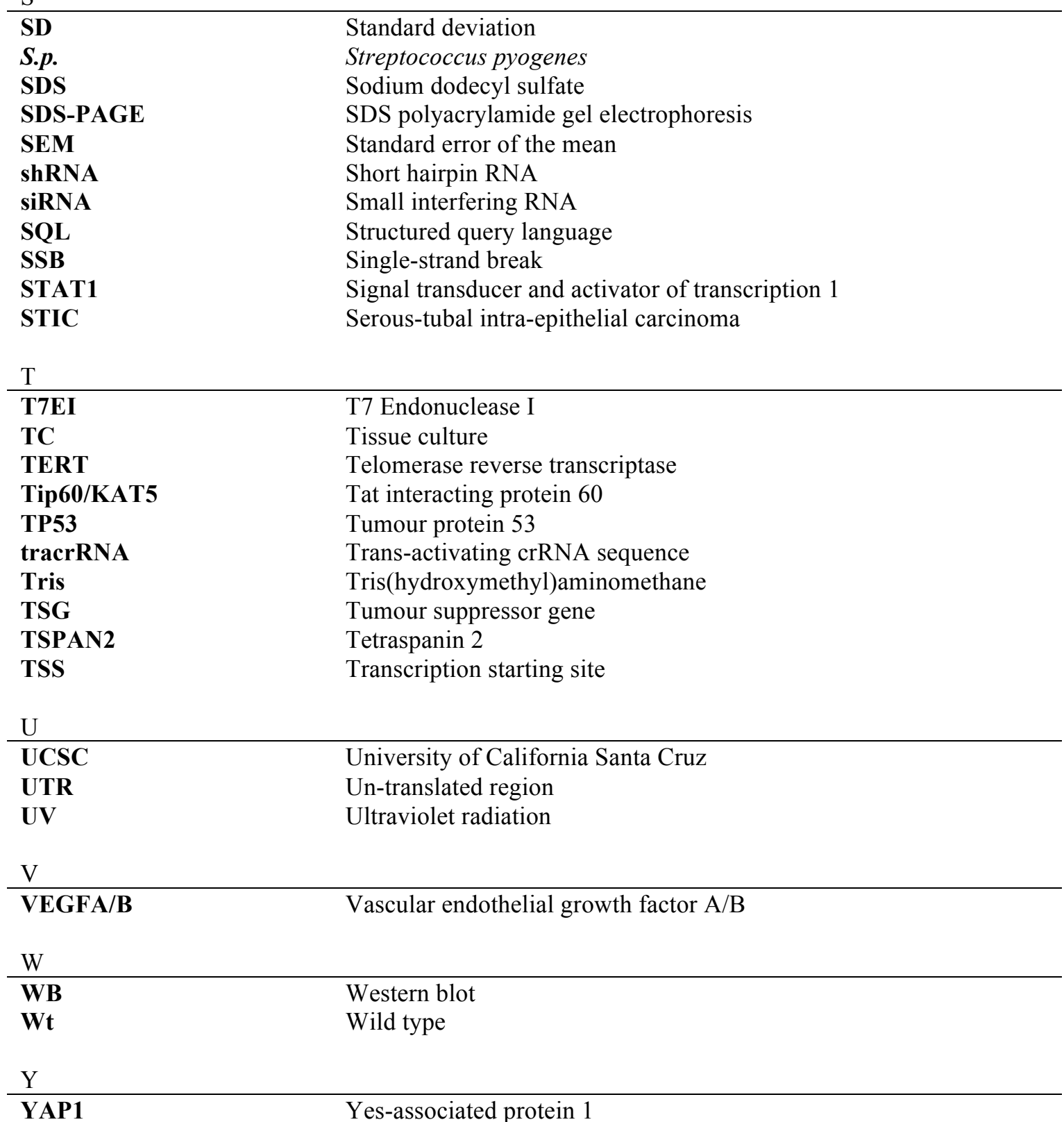

YAP1

Yes-associated protein 1

The following typographical conventions are used in this work:

- Gene, mRNA and cDNA names are written in Italics, but only those consistently used are included in the list of abbreviations. They are not written in Italics when incorporated in titles, figures, tables or lists.

- When corresponding, protein names contain Greek alphabet letters (e.g. ER $\alpha$ ), whereas gene, mRNA and cDNA names contain Latin alphabet letters (e.g. ERA). 


\section{Introduction}




\subsection{Human Reproductive Function}

Normal reproductive function is essential for the production of healthy offspring and therefore, survival of the species (Johnson \& Everitt 2000). Unlike asexual reproduction, where progeny arise from a single organism, sexual reproduction is based on the combination of two morphologically distinct reproductive cells denominated gametes (Crow 1994). The male gametes are the spermatozoa (spermatozoon in singular) and the female gametes are the ova (ovum), both haploid germ cells that fuse during fertilization to conceive a zygote containing genetic material from the two progenitors (Piñon Jr 2002). Sexual reproduction and the merging of parental chromosomes provide the necessary biological variation to adapt in a changing environment (Johnson \& Everitt 2000).

The biological process that leads to the generation and maturation of reproductive cells or haploid gametes is known as gametogenesis (Johnson \& Everitt 2000) or oogenesis in females (Gilbert 2000) and spermatogenesis in males (Cheng \& Mruk 2010). These biological processes take place in the reproductive/sex glands or gonads: testes in males and ovaries in females (Piñon Jr 2002). Both sexes are able to synthesize and secrete steroid/sex hormones such as androgens, estrogens and progesterone, which play vital roles both in the development and function of the reproductive organs and in sexual behaviour (Finlayson 2007).

Unlike the continuous sperm production of the male, the maturation of the female gamete followed by its release from the ovary (ovulation) is cyclical (Fathalla 2015). During each ovarian/menstrual cycle, one gamete becomes fully mature and is expelled from the ovary in order to encounter the male gamete and merge (Johnson \& Everitt 2000).

\subsubsection{Female reproductive system}

The female reproductive system is comprised of external and internal sex organs, commonly termed female external or internal genitalia respectively (Impey \& Child 2012). Whereas the female external genitalia have protective and stimulatory functions, the internal counterparts are involved in producing, nourishing and transporting functional female gametes, amongst other functions (Martini et al. 
2012). The female internal genitalia localize to the pelvis, the lower part of the abdomen, and is surrounded by pelvic bones and muscles (Marieb \& Hoehn 2015). These organs are also enclosed within an extensive mesentery known as the broad ligament of the uterus (Oats \& Abraham 2016). It includes two fallopian tubes, two female gonads, uterus, cervix and vagina (Impey \& Child 2012) (Fig. 1.1).

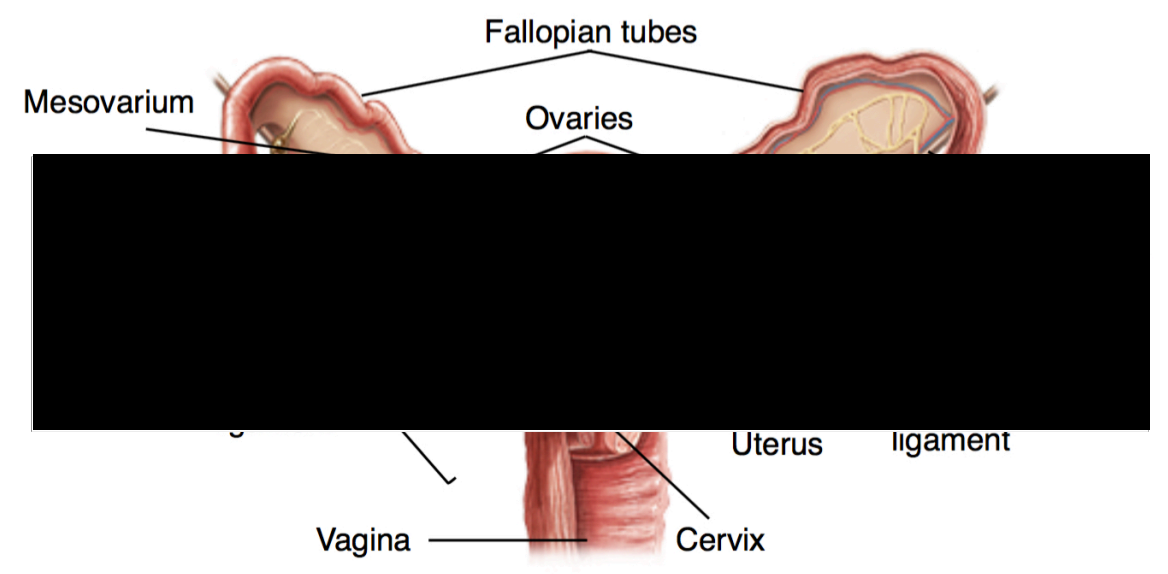

Figure 1. 1 Schematic representation of the human female internal genitalia The image illustrates the main structures of the human female internal genitalia. Source: Adapted from an online source (Kenhub.com 2017).

The two fallopian tubes, also called uterine tubes, oviducts or salpinges, each measure about $10 \mathrm{~cm}$ and extend laterally from the uterus, lying between the folds of the broad ligament (Jenkins et al. 2008) (Fig. 1.1). They receive the female gamete when it leaves the ovary, provide a route for sperm to reach it and transport fertilized ova from the ovaries to the uterus (Marieb \& Hoehn 2015). To achieve this goal, one end of each fallopian tube is attached to the uterus and the other is close but not directly attached to the ovary, and therefore empties into the pelvic cavity (Oats \& Abraham 2016). On the latter, the uterine tubes end in a fringe of long, finger like projections lined with ciliated epithelium called fimbria (Jenkins et al. 2008). The mucosal lining of the salpinges consists of fallopian tube epithelium (FTE) cells and connective tissue (Martini et al. 2012).

The paired ovaries are small, lumpy, almond-shaped organs/glands that flank the uterus and are confined in the upper pelvic cavity (Widmaier et al. 2015) (Fig. 1.1). 
A typical ovary is about $5 \mathrm{~cm}$ long, $2.5 \mathrm{~cm}$ wide and $8 \mathrm{~cm}$ thick, weighs $6-8 \mathrm{~g}$, displays either pink or yellowish colouration and has a nodular consistency (Martini et al. 2012). One on each side of the uterus, ovaries are held into position in the peritoneal cavity by a series of ligaments (Jenkins et al. 2008). The ovarian ligament extends from the uterus, near the attachment of the fallopian tube, to the medial surface of the ovaries (Marieb \& Hoehn 2015). The suspensory ligament anchors the ovaries laterally to the pelvic wall and contains the major blood vessels of the ovaries: the ovarian artery and the ovarian vein (Martini et al. 2012). Lastly, the mesovarium, a thickened double-layered fold of mesentery that forms part of the broad ligament of the uterus, supports and stabilizes the position of the ovaries (Jenkins et al. 2008) (Fig. 1.1).

A simple layer of epithelial cells called ovarian surface epithelium (OSE) covers each ovary externally (Auersperg et al. 2001). Immediately below the OSE, a layer of dense, fibrous, irregular connective tissue called tunica albuginea surrounds the interior tissues (stroma) of the ovary, which are divided into a superficial cortex and a deeper medulla (Martini et al. 2012). The ovarian cortex contains dense irregular connective tissue surrounding sac-like structures called ovarian follicles, which harbour the future gametes (Cambridge University Press 1995). Conversely, the medulla comprises more loosely arranged connective tissue and contains the largest blood vessels, lymphatic vessels and nerves (Oats \& Abraham 2016).

\subsubsection{Female hormonal system}

To ensure the optimal regulation of the menstrual cycle, the female reproductive system is under hormonal control, which involves interplay between different endocrine glands: hypothalamus, anterior pituitary and the ovaries (Finlayson 2007). Various hormones are secreted at drastically disparate rates during different parts of the female monthly sexual cycle or period (Hall 2016) (Fig. 1.2). 
Introduction

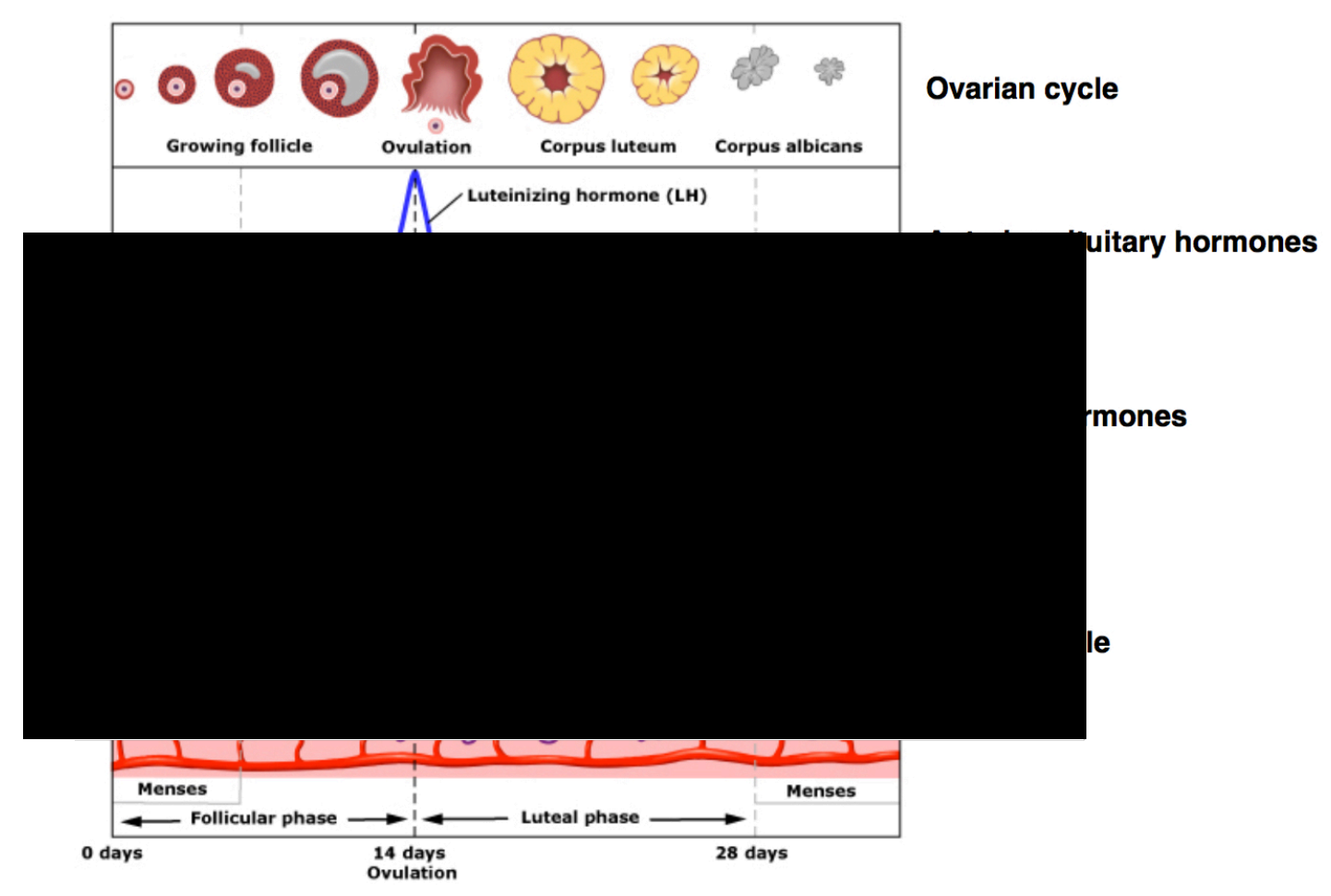

Figure 1. 2 Diagram of the female menstrual cycle

Diagrammatic representation of the menstrual cycle phases (ovarian and uterine), alongside with changes that occur in the anterior pituitary and ovarian hormones. The duration of the menstrual cycle is represented at the bottom of the panel. Source: Adapted from an online source (SportsMD 2015).

The gonadotropin-releasing hormone $(\mathrm{GnRH})$ is secreted by the hypothalamus and stimulates the synthesis and secretion of follicle-stimulating hormone (FSH) and luteinizing hormone (LH) at the anterior pituitary gland (Piñon Jr 2002). Its secretion is very low during childhood due to the negative feedback exerted by circulating steroid hormones (Jones \& Lopez 2013). However, beginning with puberty and during a woman's reproductive years, pulses of gonadotropin-releasing hormone are critical for the successful maturation of ova and completion of menstrual cycles (Finlayson 2007). Finally, the anterior pituitary sex hormones, follicle-stimulating hormone and luteinizing hormone, target the ovaries and stimulate the secretion of estrogens and progesterone (Johnson \& Everitt 2000).

An extremely complex interplay between these hormones regulates the female period, inducing cyclical changes in the ovaries, the fallopian tubes and the uterus (Donnez et al. 1985; Hall 2016) (Fig. 1.2). When the menstrual cycle ceases (age 45- 
55), the concomitant hormonal production concludes too, resulting in important hormonal imbalances (Marieb \& Hoehn 2015). In an effort to palliate physical discomfort and to prevent skeletal and cardiovascular complications associated with the fall in hormone levels, physicians often prescribe hormonal replacement therapies (Jane et al. 2012).

\subsubsection{Formation of female gametes: Oogenesis}

During early embryonic development, primordial diploid germ cells migrate to the outer surface of the ovary and become oogonia or primordial ova (Witschi 1948). These undifferentiated primordial germ cells proliferate rapidly via mitosis and collect around them layers of epithelial cells to form structures called primordial follicles (Cambridge University Press 1995). Nonetheless, most primordial follicles degenerate in a process known as follicular atresia and only a few develop into primary follicles, containing primary oocytes/germ cells (Johnson \& Everitt 2000).

Approximately 1-2 million primary follicles, each containing one primary oocyte (a female's presumed lifetime supply) are distributed in the cortex of immature ovaries at birth (Zuckerman 1951). However, certain studies claim to have demonstrated the existence of proliferative ovarian germline stem cells in the ovaries of adult mice (Johnson et al. 2004; Zou et al. 2009), and more recently, that ovarian germline stem cells were isolated and characterized in the ovaries of postnatal mice and reproductive-age women (White et al. 2012). Nowadays, the field of reproductive biology is still divided between the supporters of this new neo-oogenesis theory (Pacchiarotti et al. 2010) and its opponents (Zhang et al. 2012).

Over time, beginning in late foetal development and until the supply of primordial follicles is depleted (menopause), quiescent primordial follicles are recruited into a growing pool of primary follicles (Cambridge University Press 1995). All recruited primordial follicles undergo follicular atresia until hormone activation, starting in puberty, rescues a small portion from that fate. By then, an endowment of perhaps 200,000-400,000 primary oocytes remain in the ovaries (Hall 2016). From this moment on, primordial follicles are continuously activated and enter a growth pattern (folliculogenesis) that will finally lead to ovulation (Johnson \& Everitt 2000). 


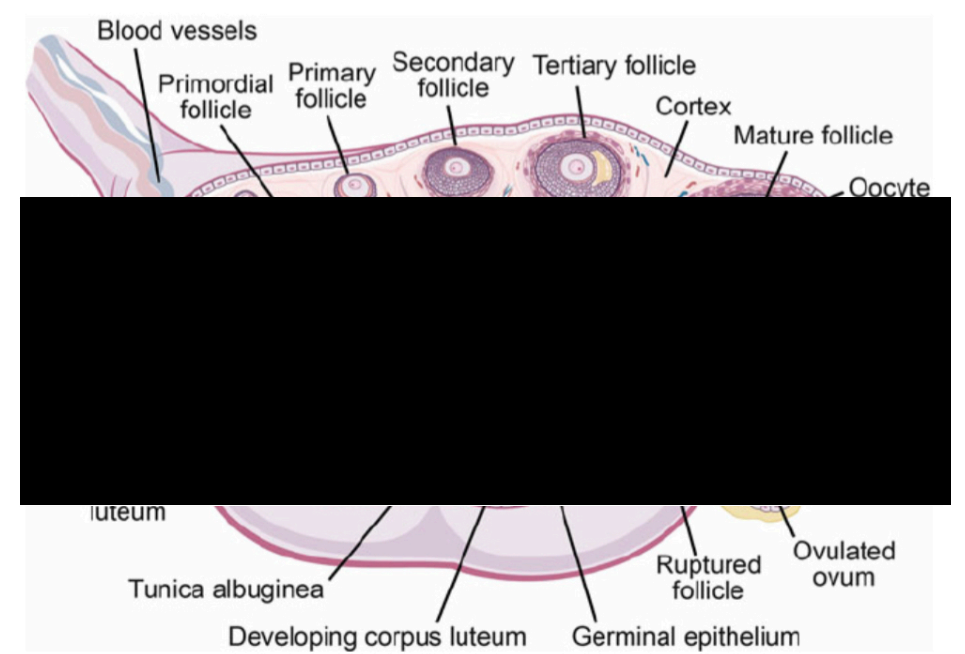

Figure 1. 3 Schematic representation of follicle development in the ovary

Throughout a women's life until menopause, quiescent primordial follicles undergo folliculogenesis, whose aim is to develop the structure containing the female gametes until they are released from the ovary. Source: 'Female and male gametogenesis' (Desai et al. 2013).

Throughout the process of folliculogenesis, illustrated in Figure 1.3, primordial follicles evolve progressively over different stages: primary/pre-antral, secondary/antral and mature/graafian follicles. Alongside follicle development, primary oocytes evolve into secondary oocytes (Hall 2016).

Even though 10-25 pre-antral follicles are recruited at the beginning of each menstrual cycle, only one -the dominant follicle- fully matures (Armstrong \& Webb 1997). By the $14^{\text {th }}$ day of the menstrual cycle, the dominant follicle creates a prominent bulge in the surface of the ovary and eventually releases the secondary oocyte into the pelvic cavity (Murdoch et al. 2010). After the mature follicle discharges the secondary oocyte, it collapses and transforms into the corpus luteum, an endocrine structure that manufactures different hormones (Marieb \& Hoehn 2015). Around the time of ovulation, the beating cilia on the fimbria create currents in the peritoneal fluid that sweep the ovum into the fallopian tube as it emerges onto the ovarian surface (Widmaier et al. 2015).

If a spermatozoon fertilizes the secondary oocyte on its way to the uterus, a zygote is formed. Conversely, if there is no fertilization, both the secondary oocyte and the 
corpus luteum degenerate leading to menstruation and the beginning of a new ovarian cycle (Hall 2016). Approximately 400-500 primordial follicles develop enough to expel their ova throughout the reproductive life of a healthy woman (Widmaier et al. 2015). This incessant rupture-repair leads to scarred and pitted ovarian surfaces, with irregular contours and invaginations (Auersperg et al. 2001; Martini et al. 2012).

Even though usually only one ovum is expelled every menstrual cycle, there are always many follicles at different stages of growth in an adult's female ovary (Marieb \& Hoehn 2015) (Fig. 1.3). Continuous ovarian proliferative activity, together with constant rupture-repair associated with ovulation and the stringent hormone control pose a significant risk of malfunctioning. This will impact on a woman's ability to reproduce successfully but can also increase the risk of other health problems such as polycystic ovary syndrome and ovarian cancer (OC) (Hennessy et al. 2009; Sirmans \& Pate 2013).

\subsection{Onset of Cancer and Tumour Progression}

The cells that are part of the female reproductive system, as any other in the human body, might experience environmental or internal alterations that disturb cell homeostasis (e.g. genetic mutations), eliciting abnormal attributes such as sustained proliferation, which may alter adjacent tissue structures (Cooper 2000; Weiderpass \& Labrèche 2012). For instance, ovarian surface cells, which are in constant hustle due to hormonal and/or cytokine cues and membrane remodelling have a high risk of suffering homeostatic disturbances (Mendelsohn et al. 2015).

Between a fully normal, well-differentiated tissue and an abnormal un-differentiated tissue (lost all tissue-specific traits) lies a broad spectrum of intermediate appearance growths, which are collectively denominated neoplasms or tumours (Mendelsohn et al. 2015). Tumours that grow locally without invading adjacent tissues are classified as benign; they represent the great majority of primary tumours arising in humans and are generally harmless to their host (Knowles \& Selby 2005). On the contrary, tumours that invade underlying tissue, spread throughout the body and establish new 
colonies or metastasise, are classified as malignant (Weinberg 2014). Although clinical oncologists and surgeons often reserve the word 'cancer' for these metastatic tumours, in contemporary cancer research it is commonly used to include all types of abnormal growths.

\subsubsection{Carcinogenesis}

The risk of uncontrolled cell proliferation, and the formation of tumours as a consequence, is substantial throughout the lives of humans (Weinberg 2014). In addition to innate malfunctioning, 'age' is the biggest risk factor for developing cancer, external agents (carcinogens) can cause or contribute to carcinogenesis, the initiation of a tumour (Knowles \& Selby 2005). Carcinogens act at the molecular level, damaging the structure of deoxyribonucleic acid (DNA) strands and generating errors that can lead to mutations during DNA synthesis (Weston \& Harris 2003). Interestingly, a dividing cell is more susceptible to carcinogens and thus mutations are more frequent in tissues with increased cellular activity (Baba \& Catoi 2007). Exposure to chemical carcinogens such as ultraviolet radiation (UV) or certain elements contained within tobacco, alcohol or food are the main recognised aetiology for cancer in the western world (Thun et al. 2002; Boffetta et al. 2006; Loeb \& Harris 2008; Khandekar et al. 2011).

Tumour viruses like the human papillomavirus also possess the ability to induce carcinogenesis, forcing infected cells to thrive and proliferate uncontrollably rather than kill them (Moore \& Chang 2010). For instance, human papilloma virus infection is linked with several cancers affecting the female genitalia (e.g. vagina, cervix), as well as others like head and neck cancer (Bansal et al. 2016). Other factors that either contribute directly to or pose an important risk of carcinogenesis include hereditary syndromes, chronic inflammation and hormonal cues (Shacter \& Weitzman 2002; Banerjee 2011; Kulkarni \& Carley 2016).

Hereditary cancer syndromes entail the inheritance of genetic mutations in one or more genes, determine the tendency of an individual to develop certain cancers and may also cause the early onset of these cancers. The most common hereditary cancer syndromes are the 'hereditary breast and ovarian cancer syndrome' (HBOC) and the 
'hereditary non-polyposis colorectal cancer' (HNPCC, also known as 'Lynch syndrome') (Kulkarni \& Carley 2016). A localized chronic inflammation involves the accumulation of large numbers of inflammatory mediators, which contribute to carcinogenesis by inducing pro-tumorigenic internal (DNA mutations) and environmental alterations in the surrounding cells (Shacter \& Weitzman 2002). Similarly, carcinogenic events might be potentiated or promoted by unbalanced hormones (Banerjee 2011).

\subsubsection{Tumour progression}

The formation of a tumour is an intricate multi-step process that usually takes decades. Once the affected cell has acquired a functionally advantageous genetic or epigenetic modification, it will proliferate more effectively than its neighbours and generate large clonal populations (Nowell 1976; Baxter et al. 2014). As years go by, tumour cells acquire more advantageous alterations and evolve progressively into un-differentiated malignant states (Mendelsohn et al. 2015). By the time of detection, tumours are described and classified upon grade and stage, depending on how far they have progressed in the tumorigenesis process. Tumour grading (I-IV) describes the abnormality of tumour cells and tissues observed under a microscope, where the highest 'abnormality' or un-differentiated state (IV) represents the highest malignancy and aggressiveness (Weinberg 2014). On the other hand, tumour staging (I-IV) determines the extent to which a tumour has developed by local growth (I) or invasive spreading (IV) (Greene \& Sobin 2008).

During the course of tumour progression or tumorigenesis, key changes occur in cancer cells, granting them the ability to survive and proliferate uncontrollably (Clark 1991) (Fig. 1.4). There are two broad classes of genes that play a key role in carcinogenesis and tumour progression: proto-oncogenes and tumour suppressor genes (Weinberg 2014). 


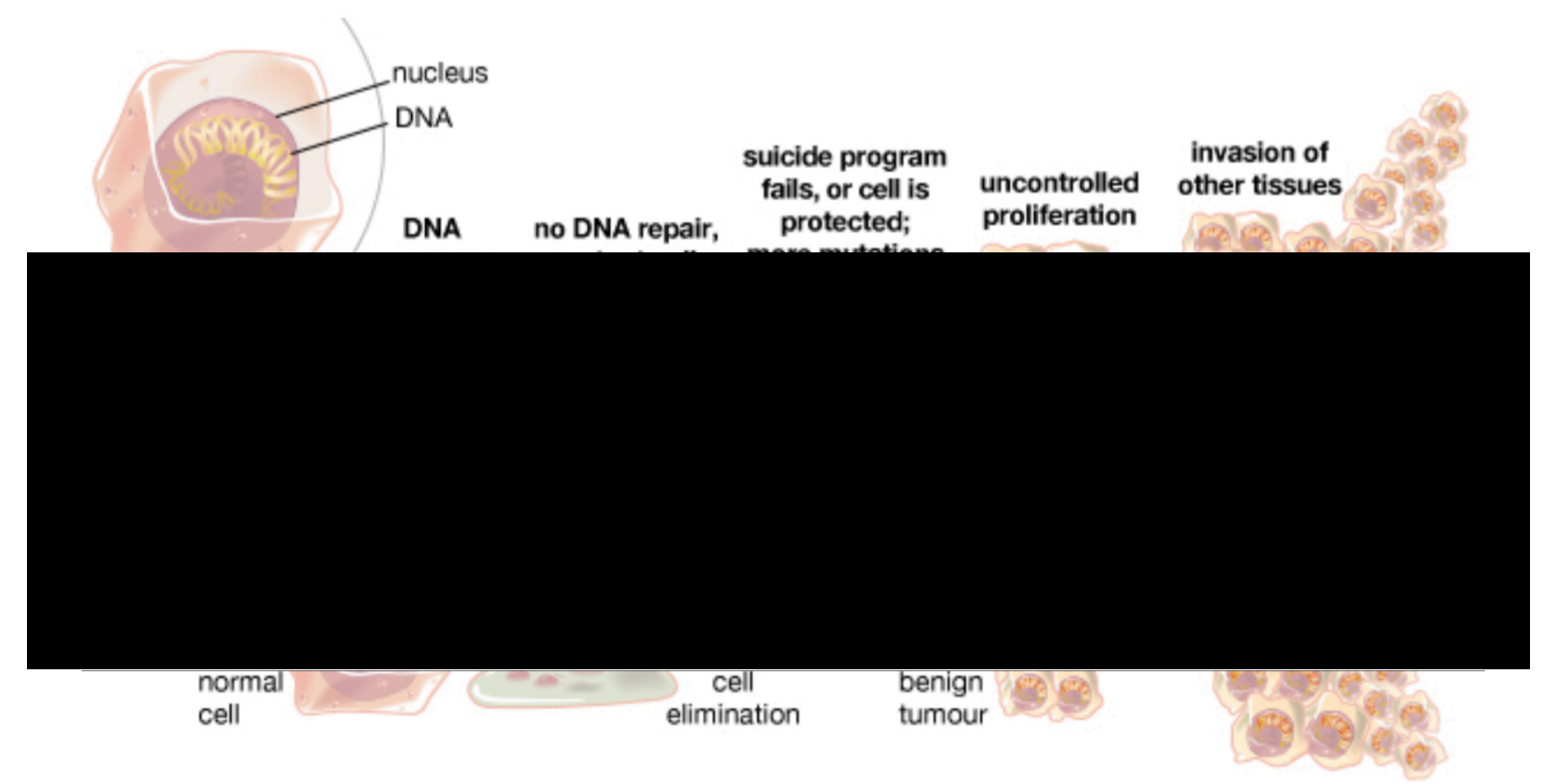

(9) 2014 Encyclopædia Britannica, Inc.

Figure 1. 4 Diagram summarizing cell carcinogenesis and tumour progression DNA repair mechanisms maintain the integrity of DNA, which may acquire mutations during normal replication or prompted by the action of carcinogens. If mutations are accurately repaired, the mutant cell develops into a normal cell once again, but if they are not repaired, the mutant cell survives. The apoptotic machinery is responsible for detecting un-repaired mutant cells and provoke cell death if the mutations are un-reparable. However, if the suicide programme fails or the mutant cells are protected against it, more mutations occur, which could lead to uncontrolled cell proliferation. Depending on the tendency of the mutant cells to spread, tumours are classified into benign or malignant. Source: Encyclopaedia Britannica, Inc (2014).

Proto-oncogenes are critical growth-controlling cellular genes and, as such, are tightly regulated under normal physiological circumstances (Todd \& Wong 1999). As a result of epigenetic disturbances and genetic alterations such as mutations, gene amplifications and chromosome rearrangements, proto-oncogenes no longer respond to restrictive cellular signals (e.g. apoptosis signals), becoming active oncogenes and contributing to cancer progression (Pierotti et al. 2003) (Fig. 1.5). Regardless of the activating mechanism, an oncogene always behaves as a dominant allele in its ability to confer malignant properties on cells (Vogelstein \& Kinzler 2004). 


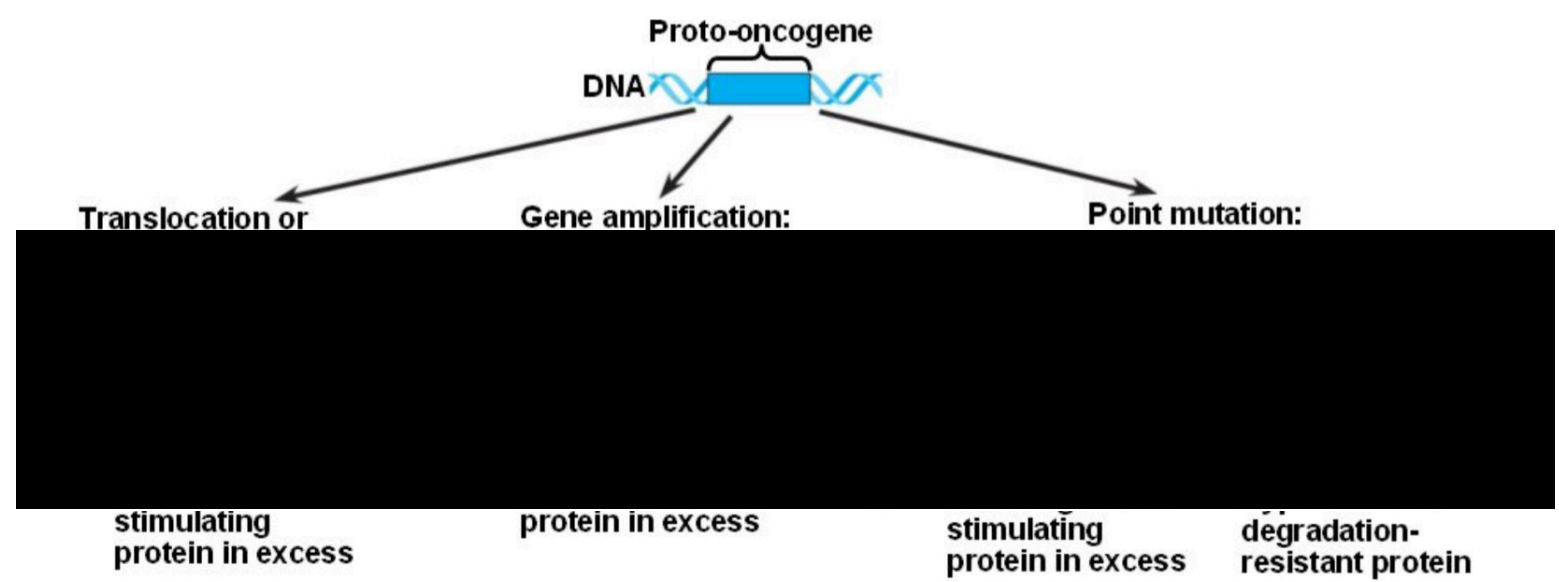

Figure 1. 5 Genetic mechanisms leading to oncogene activation

Increased expression of normal growth-stimulating proteins result from various genetic mechanisms including: (1) chromosomal translocations, that situate the target gene under the regulation of a different promoter, (2) gene amplification and point mutations (insertion, deletion) within (3) the promoter element or (4) the gene body of a target proto-oncogene. Source: 'Biochemistry for Medics" (Chhabra 2013).

The most frequently mutated oncogenes detected in human cancer are members of the RAS signalling family (h-RAS, k-RAS and n-RAS), involved in proliferation, differentiation, survival and other essential cellular mechanisms (Karnoub \& Weinberg 2008). These genes are commonly targeted by single nucleotide/point mutations at conserved sites, eliciting the constitutive activation of Ras proteins (Prior et al. 2012).

Tumour suppressor genes (TSGs) encode proteins that are functionally integrated into pathways that prevent unscheduled cell proliferation, stimulate cell death or trigger the induction of cell cycle arrest (Sherr 2004). Accordingly, TSGs act as negative regulators of cancer progression and hence mutations in these genes lead to pro-oncogenic effects (Berger et al. 2011) (Fig. 1.6). Equivalent to proto-oncogenes, TSGs are commonly inactivated via genetic and epigenetic alterations (Vogelstein \& Kinzler 2004). 


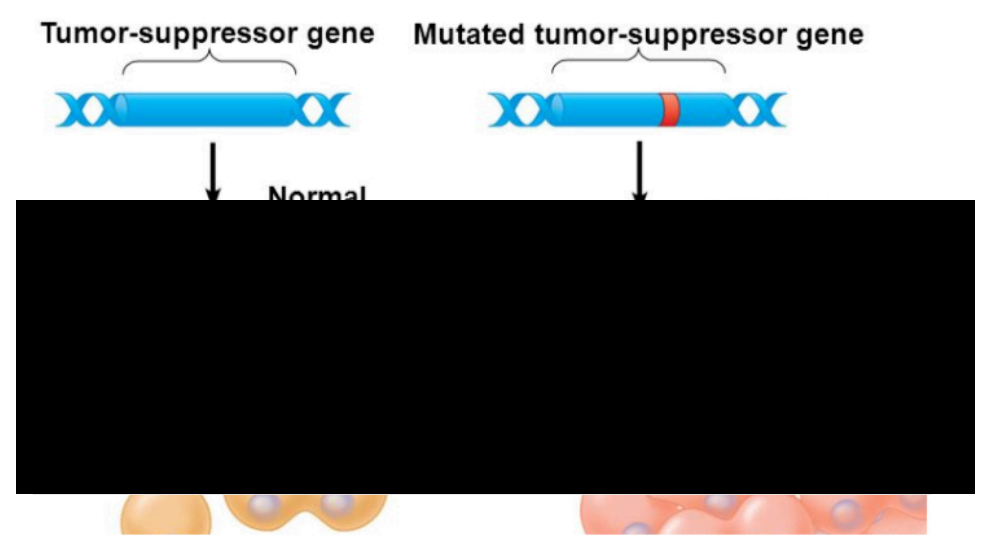

Figure 1. 6 Effect of genetic modifications on tumour suppressor genes

Tumour-suppressor genes are under strict control and code key regulatory proteins. The mutation of a tumour suppressor gene could lead to uncontrolled cell growth, contributing to cancer progression. Source: 'Principles of Cancer Biology' (Kleinsmith 2006).

Cancer-preventive effects of most TSGs normally require the presence of a single functional allele, a genetic property denominated haplo-sufficiency, which means that in order to achieve a successful malignant transformation both gene alleles must be inactivated (Mendelsohn et al. 2015). Usually, the first allele is inactivated via genetic mutation and the second gene copy (and its chromosomal surroundings) is lost through mechanisms that depend on loss of heterozygosity, including mitotic recombination, inappropriate chromosomal segregation or gene conversion (Lodish et al. 2000). This process is commonly observed when genetic mutations affect TSGs in germline cells, which are therefore inherited and responsible for hereditary cancer syndromes (Kulkarni \& Carley 2016). In these cases, the hereditary pattern is dominant, since the inactivation of the remaining allele via loss of heterozygosity suffices to promote tumorigenesis (Weinberg 2014).

On the other hand, certain TSGs like the 'tumour protein tp53' (TP53) only need one inactivated allele to induce tumour progression, a genetic property denominated haplo-insufficiency (Goh et al. 2011). The TP53 TSG encodes a multifunctional transcription factor which can impose cell cycle arrest, cellular quiescence or induce apoptosis when is activated in response to a variety of conditions including DNA damage, oxidative stress, osmotic shock and others (Kastan et al. 1991; Lu \& Lane 1993; Graeber et al. 1994; Kishi et al. 2001; Vousden \& Lu 2002). Accordingly, 
TP53 haplo-insufficiency and the loss of activity that it entails confer a relative advantage to cells, since the complete loss of function would lead to apoptosis or senescence (Berger et al. 2011). For instance, while wild-type (wt) p53 proteins bind DNA as tetramers, mutant p53 cannot bind DNA but still forms hetero-oligomers with wt-p53. Consequently, these complexes show impaired DNA association and transcriptional activity, resulting in loss of p53 function (Goh et al. 2011).

Some TSGs, often called stability genes, encode proteins that are involved in DNA damage response and repair mechanisms, whose function is to keep genetic alterations to a minimum (Friedberg 2003). Deficiencies in DNA repair through inactivation of stability genes lead to higher rates of mutations, genomic instability, altered karyotype and thus tumour progression. Accordingly, stability genes are commonly inactivated in cancers (Jeggo et al. 2015). Most are haplo-sufficient (Weinberg 2014) and some are responsible for hereditary cancer syndromes (e.g. 'mutS homolog 2' or MSH2) (Müller \& Fishel 2002).

Table 1. 1 List of eukaryotic DNA damage repair pathways

\begin{tabular}{|c|l|}
\hline DNA damage & \multicolumn{1}{c|}{ DNA repair pathway } \\
\hline \multirow{2}{*}{ Single strand damage } & Base-excision repair \\
\cline { 2 - 2 } & Nucleotide-excision repair \\
\cline { 2 - 2 } & Mismatch repair \\
\hline \multirow{2}{*}{ Double-strand break } & Homologous recombination repair \\
\cline { 2 - 2 } & Non-homologous end joining \\
\hline
\end{tabular}

There are 450 known genes implicated in DNA damage response and repair (Pearl et al. 2015), divided into distinct pathways responsible for specific types of DNA damage (Ciccia \& Elledge 2010) (Table 1.1). Double-strand breaks (DSBs), the most lethal of DNA insults, are repaired using either the error-proof homologous recombination repair (HRR) or the error-prone non-homologous end joining (NHEJ) (Lieber 2010; Jasin \& Rothstein 2013). Even though it has some limitations, the preferred pathway to repair DSBs is HRR, which involves breast cancer susceptibility genes 1 and $2(B R C A 1 / 2)$ and other nuclear enzymes such as polyADP ribose polymerases ( $P A R P)\left(\mathrm{O}^{\prime}\right.$ Driscoll \& Jeggo 2006). 
$B R C A 1 / 2$ are the most extensively studied DNA repair genes and deficiencies within either of them result in defective HRR and subsequent loss of efficient and effective DNA DSB repair (Gudmundsdottir \& Ashworth 2006). Specifically, BRCA1 interacts with other protein complexes stimulating HRR and repressing NHEJ for the initial processing of DSB ends (Chapman et al. 2012). In turn, BRCA2 acts as a scaffold for additional DSB-related factors on single strand DNA (Fradet-Turcotte et al. 2016).

\subsubsection{Hallmarks of cancer}

Over time, the activation of oncogenes and silencing of TSG/stability genes give tumour cells the ability to acquire certain molecular characteristics, the 'Hallmarks of Cancer', which are probably all necessary to a greater or lesser extent for tumour progression (Hanahan \& Weinberg 2000; Hanahan et al. 2011) (Fig. 1.7 \& Table 1.2).

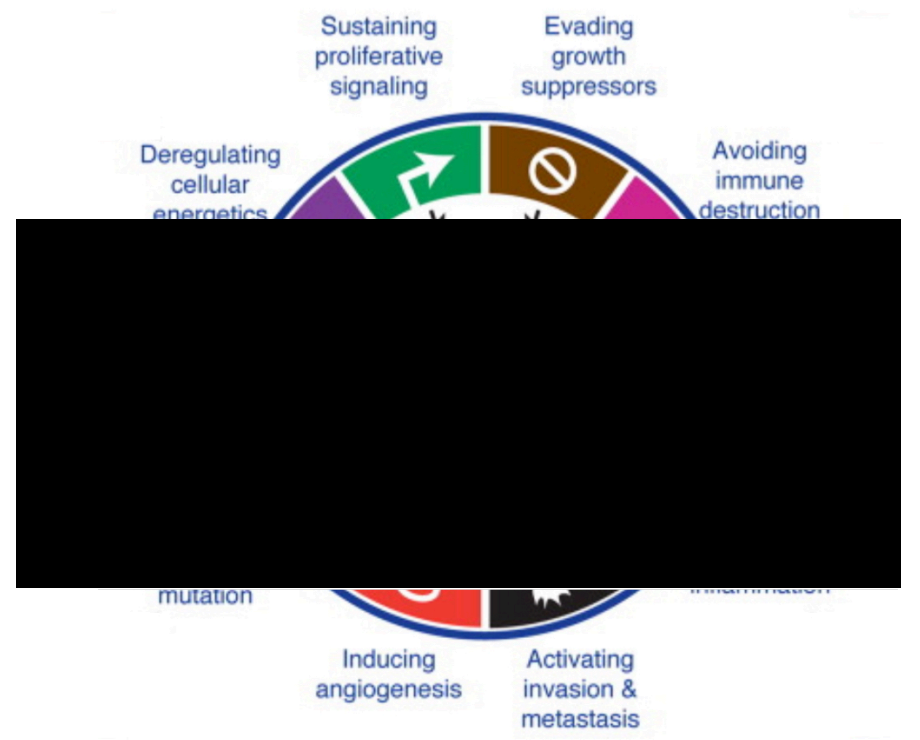

\section{Figure 1. 7 'Hallmarks of cancer'}

The illustration encompasses the six hallmark capabilities originally proposed in 'Hallmarks of cancer' plus two emerging hallmarks related to the avoidance of the immune system and cancer energetics, as well as two enabling characteristics of cancer: genome instability and tumour-promoting inflammation. Source: 'Hallmarks of Cancer: The Next Generation' (Hanahan et al. 2011). 
Table 1. 2 Summary table of the 'Hallmarks of cancer'

\begin{tabular}{|c|c|}
\hline \multicolumn{2}{|r|}{ Hallmarks of Cancer } \\
\hline $\begin{array}{l}\text { Sustained } \\
\text { proliferative } \\
\text { signalling }\end{array}$ & $\begin{array}{l}\text { - The interaction of a growth factor with its specific receptor } \\
\text { activates cellular signalling transduction cascades, ultimately } \\
\text { responsible for cell proliferation and survival. Oncogenic } \\
\text { alterations grant conditions to achieve growth self- } \\
\text { sufficiency. (Bower \& Waxman 2015). } \\
\text { - Recurrent targets: components of cellular signalling cascades, } \\
\text { e.g. tyrosine kinase receptors (Yarden \& Pines 2012). }\end{array}$ \\
\hline $\begin{array}{l}\text { Evading growth } \\
\text { suppressors }\end{array}$ & $\begin{array}{l}\text { - The cell cycle is a precisely programmed series of events that } \\
\text { enables a cell to duplicate its contents and divide. Oncogenic } \\
\text { alterations grant independency from anti-proliferative signals } \\
\text { and cell cycle checkpoints (Barbacid et al. 2005). } \\
\text { - Recurrent targets: cell cycle regulators, e.g. cyclins or cyclin- } \\
\text { dependent kinases (CDK) (Inoue \& Fry 2015). }\end{array}$ \\
\hline $\begin{array}{l}\text { Resisting cell } \\
\text { death }\end{array}$ & $\begin{array}{l}\text { - Apoptosis is a pre-programmed process of cell death, } \\
\text { characterized by a cascade of events that trigger the action of } \\
\text { caspase proteases. Oncogenic alterations inactivate the } \\
\text { apoptotic machinery in order to survive and thrive, e.g. TP53 } \\
\text { mutations (Pop \& Salvesen 2009). }\end{array}$ \\
\hline $\begin{array}{l}\text { Enabling } \\
\text { replicative } \\
\text { immortality }\end{array}$ & $\begin{array}{l}\text { - Telomeres are chromatin structures located at the ends of } \\
\text { chromosomes that are not copied completely in each cycle of } \\
\text { DNA replication, shortening progressively, leading to } \\
\text { genomic instability and natural cell death. Oncogenic } \\
\text { alterations activate telomerase activity, thus enabling cell } \\
\text { death evasion (Aubert \& Lansdorp 2008). }\end{array}$ \\
\hline Angiogenesis & $\begin{array}{l}\text { - Tumour masses recruit new blood vessels to access blood } \\
\text { circulation in order to grow and metastasize. Oncogenic } \\
\text { alterations modify the balance between pro- and anti- } \\
\text { angiogenic molecules in the tumour microenvironment, e.g. } \\
\text { increased presence of vascular endothelial growth factor A } \\
(V E G F A) \text { (pro-angiogenic) (Baeriswyl \& Christofori 2009). }\end{array}$ \\
\hline $\begin{array}{l}\text { Invasion and } \\
\text { metastasis }\end{array}$ & $\begin{array}{l}\text { - Epithelial cancer cells, precursors to over } 90 \% \text { of human } \\
\text { cancers, undergo a drastic morphology conversion } \\
\text { denominated epithelial-mesenchymal transition (EMT) and } \\
\text { acquire invasive properties (Yeung \& Yang 2017). } \\
\text { - This process may be followed by dissemination, invasion and } \\
\text { colonization of new organs or metastasis (Obenauf \& } \\
\text { Massagué 2015). }\end{array}$ \\
\hline $\begin{array}{l}\text { Avoid destruction } \\
\text { by the host } \\
\text { immune system }\end{array}$ & $\begin{array}{l}\text { - Cancer cells try to avoid destruction by the immune system } \\
\text { and promote the generation of an immunosuppressive tumour } \\
\text { microenvironment (Mittal et al. 2014). }\end{array}$ \\
\hline $\begin{array}{l}\text { Deregulate } \\
\text { metabolism }\end{array}$ & $\begin{array}{l}\text { - Cancer cells need to acquire the means to support abnormal } \\
\text { cancer growth by means of triggering severe metabolic re- } \\
\text { programming (Courtnay et al. 2015). }\end{array}$ \\
\hline
\end{tabular}




\subsubsection{Tumour heterogeneity and microenvironment}

Human cancers display substantial intra-tumour heterogeneity in most phenotypic features, such as cellular morphology, gene expression, metabolism and also angiogenic, proliferative, immunogenic and metastatic potential (Fidler \& Hart 1982; Nicolson 1984; Heppner 1984). This heterogeneity is often attributed to intra-tumour clonal diversity, the outcome of a succession of clonal expansions where the rate of genetic change is so extreme that, at some point, genetically divergent clones coexist within a mass (Maley et al. 2004; Merlo et al. 2006; Marusyk \& Polyak 2010). The discovery of cancer stem cells with the ability to self-renew and differentiate into bulk tumour non-stem cells (Dick 2008; O'Brien et al. 2010) led to the assumption that, in cancers that follow a stem cell model, cancer stem cells are the target of mutations and clonal selection (Shackleton et al. 2009).

The last but no less important contribution to tumour intra-heterogeneity comes from the influence of the tumour microenvironment, the cellular environment surrounding the tumour with which it shares a constant and close interaction (Park et al. 2000). The tumour microenvironment is formed by differing concentrations of cells of haematopoietic origin (e.g. macrophages), cells of mesenchymal origin (e.g. fibroblasts) and non-cellular components (extracellular matrix) (Pattabiraman \& Weinberg 2014). As a consequence, cancerous cells within a tumour experience a range of different micro-environmental signals, which translate into a range of phenotypic manifestations (Polyak et al. 2009).

\subsection{Cancer in the Ovaries: Ovarian Cancer}

The five areas of the female reproductive system that harbour major gynaecological cancer malignancies are: uterine cervix, uterine corpus (endometrium), ovaries, vulva and vagina (Siegel et al. 2016). Malignant tumours located at the ovaries can arise from any cell type contained within this structure, including germ, stromal and epithelial cells (National Academy of Medicine 2016). As a consequence, OC is a highly heterogeneous malignancy that encloses a myriad of distinct diseases which happen to share an anatomical location (Karnezis et al. 2016). 
Ovarian cancers are broadly divided into two major types: epithelial ovarian cancers (EOCs) and non-epithelial ovarian cancers (NEOCs), which account for approximately $90 \%$ and $10 \%$ of all malignant OCs respectively (Matulonis et al. 2016). Whereas NEOCs originate from germ and stromal cells within the ovaries, EOCs or ovarian carcinomas were traditionally thought to develop from the single layer of epithelium that covers the ovaries (Bell 2005). However, recent studies suggest that these malignancies actually develop from multiple sites, mostly outside the ovaries (Kurman \& Shih 2010; Nezhat et al. 2015; Temkin et al. 2016).

\subsubsection{Origins of ovarian carcinomas}

The initial lesion that gives rise to ovarian carcinomas has thus far not been identified, and several theories regarding its origin have been put under the spotlight over the years (Cardenas et al. 2016). There are two unique ovarian functional characteristics that undoubtedly partake and/or boost the establishment of carcinomas: the cyclical ovulation process and the ovarian microenvironment (Fathalla 1971; Karnezis et al. 2016).

Every menstrual cycle one ovary releases a single ovum that breaks through the OSE, which consequently has to be fully restored (Murdoch et al. 2010). This rupture generates a local pro-inflammatory microenvironment that may lead to DNA damage, replication errors and eventually the malignant transformation of cells (Murdoch et al. 2001; Richards et al. 2002; Murdoch 2005). Cyclical reparations of OSE and the continuous pro-inflammatory setting also lead to invaginations and the formation of cortical inclusion cysts (CICs) (Auersperg et al. 2001; Mehra et al. 2011) (Fig. 1.8). As a consequence, either via invagination of normal or earlymalignant OSE CICs, local factors (e.g. hormones) are thought to influence ectopic cells to undergo tumorigenesis (Risch 1998; Zheng et al. 2000; Hennessy \& Mills 2006).

Apart from OSE, ectopic fallopian tubal epithelium (FTE) can implant on the site of rupture when ovulation occurs -endosalpingosis-, resulting in the formation of a tubal CIC that may undergo malignant transformation (Prentice et al. 2012) (Fig. 1.8). Furthermore, the pro-inflammatory microenvironment that recruits FTEs is also 
able to recruit serous tubal intra-epithelial carcinomas (STICs) from the distal fimbriated end of the fallopian tube (Lee et al. 2007; Kindelberger et al. 2007) (Fig. 1.8). These STICs fulfil anatomical, phenotypic and biological criteria as precursors of the most common sub-type of EOC, termed high-grade serous carcinoma (Karst, Levanon \& Drapkin 2011; Perets et al. 2013).

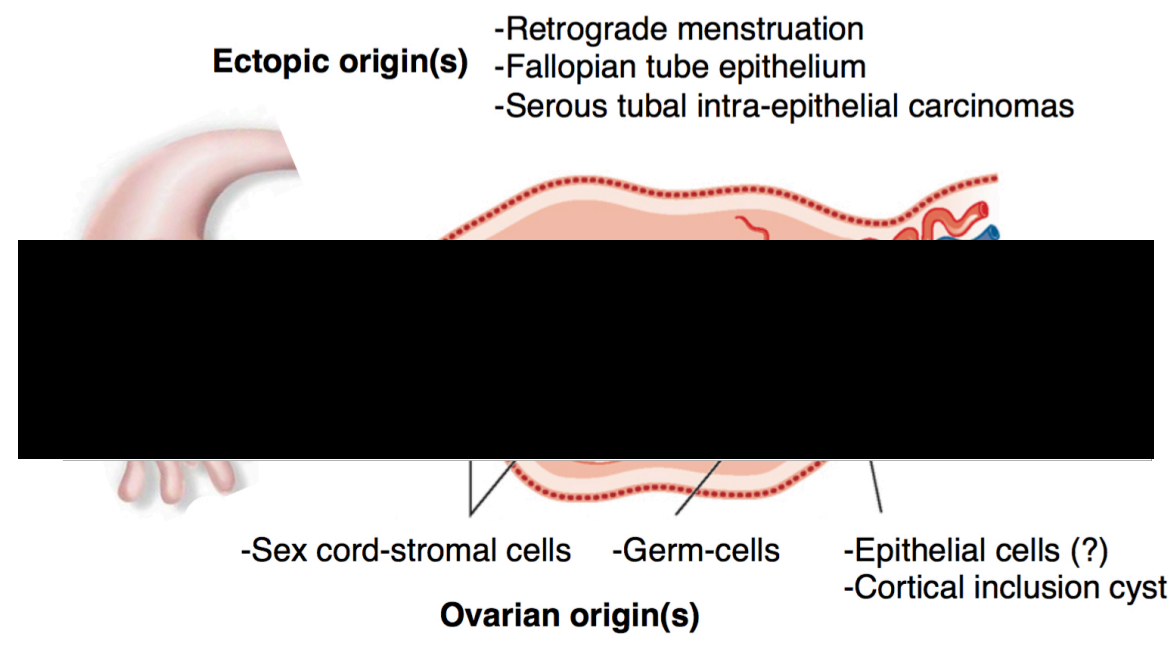

Figure 1. 8 Diagram representing the alleged origins of $\mathrm{OC}$

Ovarian cancer allegedly originates in the three distinct types of cells that are part of the ovary: sex cord-stromal, germ and epithelial cells. Other possible sources of OC initiation include retrograde menstruation and endosalpingosis of fallopian tube epithelium or serous tubal intra-epithelial carcinomas (STICs). Source: Adapted from (Gershenson 2016).

A wide variety of cancers (e.g. breast, lung, gastrointestinal tract) often present dominant ovarian masses, highlighting the idea that the ovary accommodates a favourable environment for ectopic cell growths (Yang-Hartwich et al. 2014). In line with this, the settlement of endometrial cells (endometriosis) via retrograde menstruation is linked with the establishment of other EOC sub-types, endometrioid carcinomas and clear-cell carcinomas (Prowse et al. 2006; Worley et al. 2013) (Fig. 1.8). Additionally, several studies have suggested the involvement of OSE and fimbrial stem cells that normally participate in post-ovulatory wound closure in the transformations that lead to OC (Szotek et al. 2008; Bowen et al. 2009; Paik et al. 2012; Ng \& Barker 2015). 


\subsubsection{Disease progression}

Regardless of the origin of the first cell that gave rise to OC, a tumour mass is formed either inside or adnexal to the ovary (Bower \& Waxman 2015). If the tumour is localized within the organ, the disease progression is slower, as it will need to grow extensively and achieve sufficient size to cause the rupture of the ovary in order to progress. Conversely, tumours that arise from the surface of the ovary can spread quickly before a palpable mass is formed (Mendelsohn et al. 2015).

OC metastasises either by direct extension from the ovarian tumour to neighbouring organs (e.g. fallopian tube, intestine, bladder, colon) or more typically when cancer cells detach from the primary tumour (Lengyel 2010) (Fig. 1.9). Cell detachment and subsequent spread is thought to occur either via transcoelomic passive dissemination or haematogenous metastasis (Yeung et al. 2015).

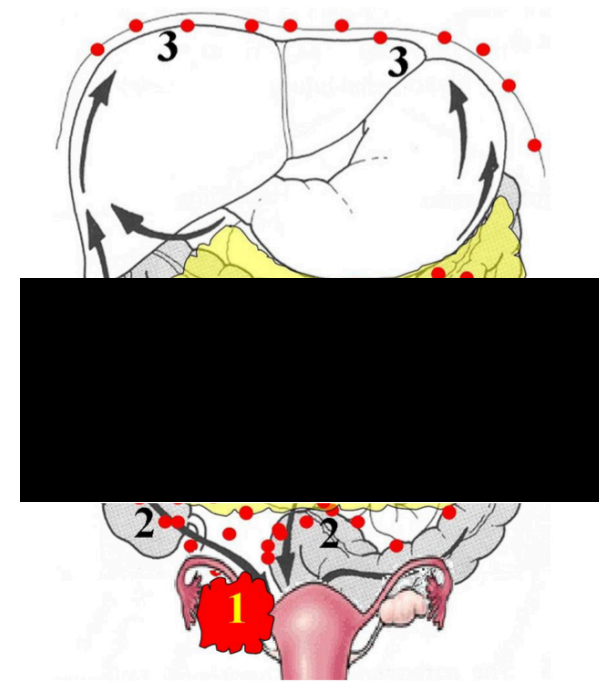

Figure 1.9 Schematic depiction of the most frequent OC metastasis sites

Illustrative representation of the female internal organs localized in the upper/lower abdomen, which constitute the most common sites of OC metastasis. In this case, the image represents a case of cancer originally located in the right ovary with metastases in the abdominal cavity (1), on the intestines (2), against the diaphragm (3) and on the omentum (4). Red dots represent metastatic tumour cell implantations, with the highest density located on the omentum. This image was obtained from Mulier (2009) (Mulier 2009).

The transcoelomic passive dissemination mechanism is the most common route of OC metastasis and involves the circulation of excess peritoneal fluid (ascites fluid) through the body cavities (Tan et al. 2006). The aetiology of this excess fluid is not well understood, but preclinical and clinical observations have demonstrated that the vascular permeability-enhancing factor VEGF is responsible for ascites accumulation (Xu et al. 2000). Currents of ascites fluids are thought to detach OC cells, carry them in the form of multicellular spheroids, and spread them throughout the peritoneal cavity, resulting in tumour nodules in the diaphragm, bowel, 
parenchyma of liver or lung and most notably the omentum, OC primary metastasis site (Fig. 1.9) (Sehouli et al. 2009; Herold \& Cannistra 2013).

Implantation in peritoneal organs involves interaction between the cancer cells and the mesothelium, which covers all organs within the peritoneal cavity (Kenny et al. 2014; Davidowitz et al. 2014). After implantation, the resultant inflammation stimulate mesothelial cells and their associated immune and stromal cells to release cytokines such as interleukin (IL)-1, -6 and -8 , which subsequently enhance tumour angiogenesis and further ascites formation via increased secretion of VEGF by cancer cells, creating a favourable OC microenvironment (Freedman et al. 2004; Stadlmann et al. 2005; Thibault et al. 2014). The predilection for the omentum, a fat pad primarily composed of adipocytes, both for primary and recurrent OC, might be explained by the alleged contribution of those cancer-associated adipocytes to cancer progression (Fig. 1.9) (Dirat et al. 2010). For instance, studies have demonstrated that cancer-associated adipocytes promote cancer cell growth and invasion via cytokine secretion (e.g. interleukins) as well as serving as an energy source (Nieman et al. 2011; Clark et al. 2013).

Ovarian cancer cells can also follow a traditional pattern of haematogenous metastasis via cell epithelial-mesenchymal transition (EMT), the detachment from the ovarian mass and intravasation to the blood circulatory system. Nevertheless, ovarian circulating tumour cells preferentially implant in the omentum and subsequently spread to other peritoneal surfaces (Pradeep et al. 2014).

\subsubsection{Epidemiology and clinical presentation}

Ovarian cancer is relatively uncommon, accounting for only $2.6 \%$ of new women cancer cases in the United States of America (USA) (Siegel et al. 2016) and 2\% in the United Kingdom (UK) (Cancer Statistics UK 2014). Nevertheless, it is the fifth leading cause of cancer death among American and British women, accounting for $5 \%$ of all women cancer deaths in each country. Specifically, 14,240 and 4,128 women died from OC in the USA and the UK respectively, according to the latest estimates made public (Siegel et al. 2016; Cancer Statistics UK 2014). Indeed, although the estimated number of new OC cases each year among British and 
American women is only one-tenth the number of new cases of breast cancer, the death-incidence ratio for $\mathrm{OC}$ is more than three times higher than that for breast cancer (Fig. 1.10).

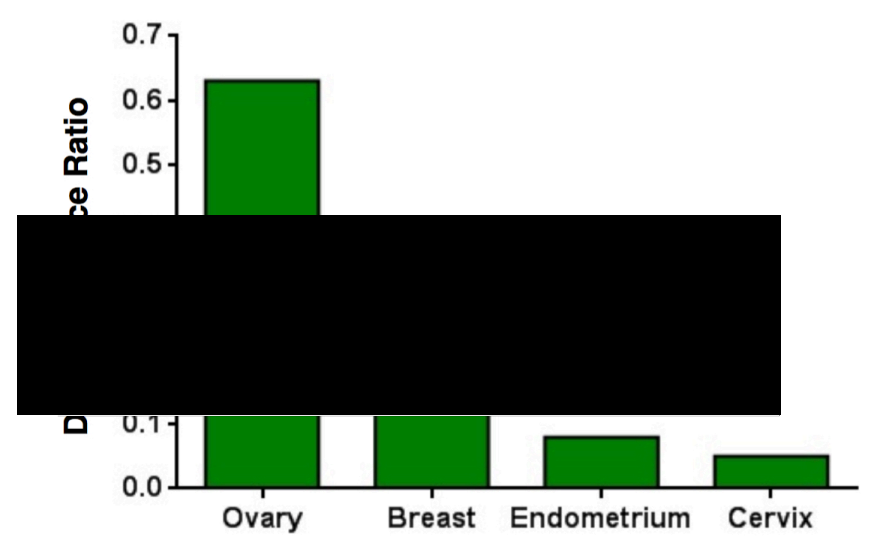

Figure 1. 10 Comparison of death-to-incidence rates for common female cancers Ratio between the death and incidence rates for cancer of the ovary, breast, endometrium and cervix per 100,000 women in the United States, 2008-2012. Source: 'Evolving paradigms...' (National Academy of Medicine 2016).

Most women with OC are diagnosed in later life, with a median age of diagnosis of 63 years in the UK (Cancer Statistics UK 2014) and the lifetime risk for a woman to develop ovarian cancer is approximately 1 in 70 (Mendelsohn et al. 2015). However, roughly two-thirds of women diagnosed with OC present with an aggressive, latestage metastatic disease state (Herold \& Cannistra 2013), thus largely explaining the staggering digits of OC-related deaths (Fig. 1.10). This late diagnosis is mostly due to the neglecting of early symptoms, since studies have shown that $90 \%$ of women with early-stage disease and $100 \%$ of women with late-stage disease reported having at least one symptom (Goff et al. 2004; Lataifeh et al. 2005). Symptoms of OC at initial presentation include abdominal bloating, pelvic and/or abdominal pain, fatigue, difficulty eating and urinary-related symptoms; mostly caused by accumulated ascites fluid (Goff et al. 2000). Because they are general, vague and non-specific, both patients and physicians usually overlook these symptoms or attribute them to other diseases (Goff et al. 2004). Accordingly, diagnosis frequently occurs tardily, when symptoms become apparent and severe as a result of the cancer reaching a late, aggressive and metastatic stage (Goff et al. 2000).

Overall survival rates of OC have increased little in the last 20 to 30 years (Vaughan et al. 2011) and this slight improvement is mostly related to better surgery and new 
chemotherapy options (Bristow et al. 2002). The 5-year survival rate of OC is currently below $46 \%$ and as low as $35-40 \%$ when considering only high-grade serous carcinomas, the deadliest and most common sub-type of disease, accounting for the worst prognosis of all gynaecological cancers (Cancer Research UK 2010; Quaresma et al. 2015). In comparison, other malignancies like breast cancer (80$90 \%)$, endometrial cancer $(70-80 \%)$ or cervical cancer $(60-70 \%)$ have much higher 5-year survival rates (Siegel et al. 2016; Cancer Statistics UK 2014).

\subsubsection{Risk factors and preventive actions}

Several aspects can impact the risk of developing OC, including genetic factors, hormonal treatments and reproductive history. Nonetheless, age represents the key risk factor, since most women who develop OC are diagnosed after menopause, at age 55 or older (Purdie et al. 2003).

- Genetic factors: Approximately $18 \%$ of all OC patients carry germline mutations in genes associated with increased OC risk (Norquist et al. 2016). The most significant risk factors are germline BRCA1/2 mutations, found in $10-15 \%$ of all OC patients (Alsop et al. 2012). Besides BRCA1/2, other germline mutations in genes involved in DNA repair such as the 'MutL homolog 1' (MLH1), MSH2 or the 'PMS1 homolog 2' (PMS2) can also increase the risk of developing OC (Walsh et al. 2011; Pennington \& Swisher 2012).

- Hormonal treatments: Hormone replacement therapy consists in the use of female hormones (estrogen, progesterone) to treat common symptoms of menopause and aging (Hildebrand et al. 2010). In recent years, it has been shown to slightly increase the risk of OC in post-menopausal women, presumably due to carcinogenic effects of excess hormones (Shi et al. 2015). In contrast, the early use of oral contraceptives (also containing estrogen and progesterone) is generally associated with reductions in the risk of developing OC (Havrilesky et al. 2013), probably due to the decline in lifetime ovulation events (Fathalla 2013).

- Reproductive history: Parity (Tourgeman et al. 2002), tubal ligation, unilateral/bilateral salpingectomy (removal of the fallopian tube) and/or oophorectomy (removal of the ovary) reduce the risk of OC (Rice et al. 2014; Gaitskell et al. 2016). Risk-reducing salpingo-oophorectomy, complete removal 
of the ovaries and the fallopian tubes, reduces the risk of malignancy by up to 96\% (Rebbeck et al. 2002; Olivier et al. 2004). Non-hormonal interruption of incessant ovulation could also be a potential approach to reduce the risk of OC (Tung et al. 2005; Fathalla 2016).

\subsubsection{Diagnostic evaluation and staging}

In patients with indicative symptoms, a diagnostic evaluation includes:

- Study of the family history of breast and ovarian cancers. The risk of OC is greatly increased when a family history of breast or ovarian cancer is present due to the possibility of carrying an inherited germline mutation (e.g. BRCA1/2) (Bergfeldt et al. 2002; Edlich et al. 2005).

- Physical examinations such as abdominal/pelvic and recto-vaginal explorations. For instance, a palpable ovary is not a normal finding and raises suspicion of malignancy (Herold \& Cannistra 2013).

- Imaging tests: trans-vaginal/abdominal ultrasonography (Varras 2004), Doppler ultrasound (Shah et al. 2013), computed tomography (Santoso et al. 2014), positron emission tomography (Yoshida et al. 2009) and/or magnetic resonance imaging (Sohaib \& Reznek 2007).

- Blood tests: complete blood count, chemistry profiling and kidney/liver function tests are performed in order to determine a woman's general health status. Specific tests for serum biomarkers such as inhibin A/B (Walentowicz et al. 2014), beta-human chorionic gonadotropin ( $\beta$-hCG) (Lenhard et al. 2012), and mostly cancer antigen 125/mucin 16 (CA-125/MUC16) can indicate the presence or absence of OC (Rustin et al. 2001), since they are commonly overrepresented, to a greater or lesser extent, in the bloodstream of OC patients (Sölétormos et al. 2016). CA-125 is the most important biomarker for OC, and it is useful to assess the response of OC patients to chemotherapy as well as the detection of relapse (Bast 2010). Nevertheless, CA-125 levels are elevated in only 50\% early-stage patients and can also be high in other tumours and normal tissues (Jacobs et al. 1999; Kobayashi et al. 2008). The lack of specificity and sensitivity of current early detection biomarkers makes a screening test for general population impossible, although it should be common practice amongst female at increased 
genetic risk (e.g. carriers of $B R C A 1 / 2$ germline mutations) (Yurkovetsky et al. 2010; Vaughan et al. 2011).

- Paracentesis: In patients with ascites fluid (fluid build-up inside the abdomen), samples of this fluid can be extracted to clarify its origin (Decruze et al. 2010).

If the results from the diagnostic testing, specially trans-vaginal ultrasonography, suggest the presence of a complex tumorigenic mass, the next step is a laparotomy surgery (Sokalska et al. 2009). The surgical process allows to obtain biopsies from the tumour, pelvis and abdomen for histological examination by pathology (tissue grading/staging), as well as remove as much tumour mass as possible (Herold \& Cannistra 2013). Surgical approaches vary if a woman is suspected to have benign, borderline or early-stage tumours (Weber et al. 2011; Falcetta et al. 2016). The staging system for $\mathrm{OC}$ is derived from the International Federation of Gynaecology and Obstetrics or FIGO classification (Table 1.3).

Table 1. 3 Summary of the international staging system for ovarian carcinomas

\begin{tabular}{|c|c|}
\hline Stage I & Limited to ovary or ovaries \\
\hline IA & One ovary, surface involvement or rupture \\
\hline IB & Both ovaries, surface involvement or rupture \\
\hline IC & Malignant ascites \\
\hline Stage II & Pelvic extension \\
\hline IIA & Involvement of the uterus or the fallopian tubes \\
\hline IIB & Involvement of the other pelvic organs (e.g. bladder, rectum) \\
\hline Stage III & Involvement of the upper abdomen or lymph nodes \\
\hline IIIA & Microscopic peritoneal disease outside pelvis \\
\hline IIIB & Macroscopic peritoneal metastases $\leq 2 \mathrm{~cm}$ diameter \\
\hline IIIC & Macroscopic peritoneal metastases $>2 \mathrm{~cm}$ diameter \\
\hline Stage IV & Distant organ involvement \\
\hline IVA & Pleural effusion with positive cytology \\
\hline IVB & Metastases to extra-abdominal sites \\
\hline
\end{tabular}

Herold \& Cannistra (2013) 
If the disease is localized to the ovaries (stage I), the prognosis of OC is excellent, with up to $90 \%$ 5-year survival rates using currently available surgery and chemotherapy. As the disease spreads to the pelvis (II), the peritoneal cavity (III), or outside the abdomen (IV), the prognosis becomes progressively worse, with a 5-year survival of less than $10 \%$ in the last group (Mendelsohn et al. 2015).

\subsubsection{Histological classification}

As well as staging, post-surgical histo-pathological analysis of the cancerous tissue allows tumour grading and the classification of OCs depending on histological differences (Rosen et al. 2009). EOCs or ovarian carcinomas, the most common type of diagnosed malignant OC ( $90 \%)$, are responsible for most OC-related deaths (Seidman et al. 2004; Braicu et al. 2011) and, as OC in general, they are highly heterogeneous themselves (Meinhold-Heerlein \& Hauptmann 2014).

EOCs are usually classified into sub-types or histo-types according to how closely tumour cells resemble the epithelia lining different organs in the female genitourinary tract (Chen et al. 2003). Specifically mucinous, endometrioid/clear cell, transitional and serous carcinomas exhibit morphological features similar to epithelial cells in the endocervix/intestine, endometrium, urinary bladder and fallopian tube respectively. Each histo-type exhibits distinct expression profiles, molecular composition, clinical features, patterns of spread, response to chemotherapy and outcome (Marquez et al. 2005; Gilks \& Prat 2009) (Table 1.4). Consequently, ovarian carcinoma histo-types are considered different diseases (Köbel et al. 2008; Vaughan et al. 2011; Prat 2012b).

Primary mucinous carcinomas (MCs) account for $\sim 3-4 \%$ of all ovarian carcinomas (Seidman et al. 2003). They are usually confined to the ovary, have low response rate to standard chemotherapy (detailed in the next section: 1.4 Ovarian Cancer Treatment), and a generally favourable prognosis (Seidman et al. 2003; Hess et al. 2004) (Table 1.4). The response rate (RR) defines the percentage of patients whose cancer shrinks or disappears after treatment. 
Table 1. 4 Clinical features of the five most common sub-types of EOC

\begin{tabular}{|c|c|c|c|c|}
\cline { 2 - 5 } \multicolumn{1}{c|}{} & Proportion (\%) & Localization $^{1}$ & Chemo-sensitivity $^{2}$ & Prognosis \\
\hline MC & $3-4$ & Confined ovary & Low & Favourable \\
\hline EC & $8-10$ & \multirow{2}{*}{ Confined pelvis } & High & Favourable \\
\cline { 5 - 5 } CCC & $8-10$ & & Low & Intermediate \\
\hline HGSC & $70-78$ & Transcoelomic & High & Poor \\
\cline { 1 - 2 } LGSC & $3-5$ & spread & Intermediate & Intermediate \\
\hline
\end{tabular}

${ }^{1}$ Usual localization or pattern of spread

${ }^{2}$ Chemo-sensitivity: response rate to standard chemotherapy treatment

MC: Primary mucinous carcinoma; EC: Endometrioid carcinoma; CCC: Clear-cell carcinoma; HGSC: High-grade serous carcinoma; LGSC: Low-grade serous carcinoma

Endometrioid carcinomas (ECs) and clear-cell carcinomas (CCCs) represent approximately $10 \%$ of all ovarian carcinomas respectively (Gilks \& Prat 2009). These two subtypes are characterized by a well-defined association with atypical endometriosis (Yoshikawa et al. 2000; Erzen et al. 2001) and pelvic localization without transcoelomic spread. However, while ECs have good RR to standard chemotherapy and a favourable prognosis (Gilks et al. 2008), CCCs do not respond well to chemotherapy and have poor survival rates (Sugiyama et al. 2000; Lee et al. 2011) (Table 1.4).

Serous carcinomas (SCs, also referred to as serous-papillary carcinomas) are divided into two distinct diseases: low-grade serous carcinomas (LGSCs) and high-grade serous carcinomas (HGSCs) (Malpica et al. 2004; Lengyel 2010) (Table 1.4). LGSCs account for 3-5\% of all ovarian carcinomas (Bell 2014). They follow a pattern of transcoloemic spread, have bad RR to standard chemotherapy and intermediate to poor survival rates (Charlie Gourley et al. 2014; Gershenson et al. 2015). HGSCs are the most common type of OC, representing approximately 70$80 \%$ of all ovarian carcinomas (Seidman et al. 2004; Soslow 2008) (Table 1.4). HGSC patients typically present with advanced stage disease ( $80 \%)$, and even though the majority show a great response to standard chemotherapy, almost every patient experience incurable recurrences (Baldwin et al. 2012; Bowtell et al. 2015; Dao et al. 2016). Due to the overwhelming prevalence of HGSCs, from now on 
when referring to historical or large-scale epidemiologic studies of OC readers can reasonably assume that most of the tumours were HGSCs.

Other infrequent carcinomas include transitional carcinomas $(<0.5 \%)$ (Cuatrecasas et al. 2009) and ovarian carcinosarcomas, often termed epithelial-stromal or mixed mesodermal tumours, which display mixed histology and thus can be grouped with either EOC or NEOC (Harris et al. 2003; Makris et al. 2015).

NEOC are a group of uncommon, histologically and clinically distinct tumours, further subdivided into germ-cell tumours and sex cord-stromal cell tumours (Koulouris \& Penson 2009; Boussios et al. 2016). Germ cell tumours originate from the embryonic germ cells (ova/oocytes) of the ovary, occur principally in children or young women aged 10-30 and represent approximately $\sim 3 \%$ of all diagnosed malignant OC cases (Zalel et al. 1996; Tavassoli \& Deville 2003). On the other hand, sex cord-stromal tumours arise from the maturing follicles and stroma of the ovaries, occur over a wide age range and represent approximately $7 \%$ of all malignant OC cases (Tavassoli \& Deville 2003). This group also includes other rare ovarian malignancies such as small cell carcinoma of the ovary, whose histology do not resemble any structure in the human body (Callegaro-Filho et al. 2016).

\subsubsection{Pathways of tumorigenesis: predominance of HGSC}

As well as the origin, the earliest events of OC tumorigenesis and the developmental progression model that follows are still unclear (Bowtell 2010). Evidence suggests that OC can progress, at least, along two different pathways: type I and type II (Shih \& Kurman 2004; Kurman \& Shih 2010).

The type I pathway group includes LGSCs, low-grade MCs, low-grade ECs, lowgrade CCCs and transitional carcinomas (Shih \& Kurman 2004). Type I tumours are highly differentiated (low-grade) and often diagnosed in early stages (Kurman \& Shih 2008). They grow slowly and develop stepwise from well-recognized precursor lesions such as benign neoplasms and borderline tumours (Shih \& Kurman 2005; Patrono et al. 2013). Although every sub-type is defined by specific molecular patterns, the main feature that characterizes type I tumours is the genetic stability 
(low frequency of mutations), typified in the lack of TP53 mutations (Hauptmann et al. 2002; Bauerschlag et al. 2010).

The type II group includes HGSC, high-grade ECs, undifferentiated carcinomas and carcinosarcomas (Shih \& Kurman 2004). Type II tumours are poorly differentiated (high-grade) and often diagnosed in late stages (Kurman \& Shih 2008). They grow aggressively without known or morphologically visible precursor lesions -'de novo development'-, metastasize quickly and thus are associated with high mortality resulting from OC (Kurman \& Shih 2010). Unlike type I, high-grade tumours are usually genetically unstable and more than $80 \%$ carry TP53 mutations (Hauptmann et al. 2002; Bauerschlag et al. 2010). Type II tumours account for approximately $75 \%$ of all EOC and, within this group, HGSC is the most prevalent (Soslow 2008).

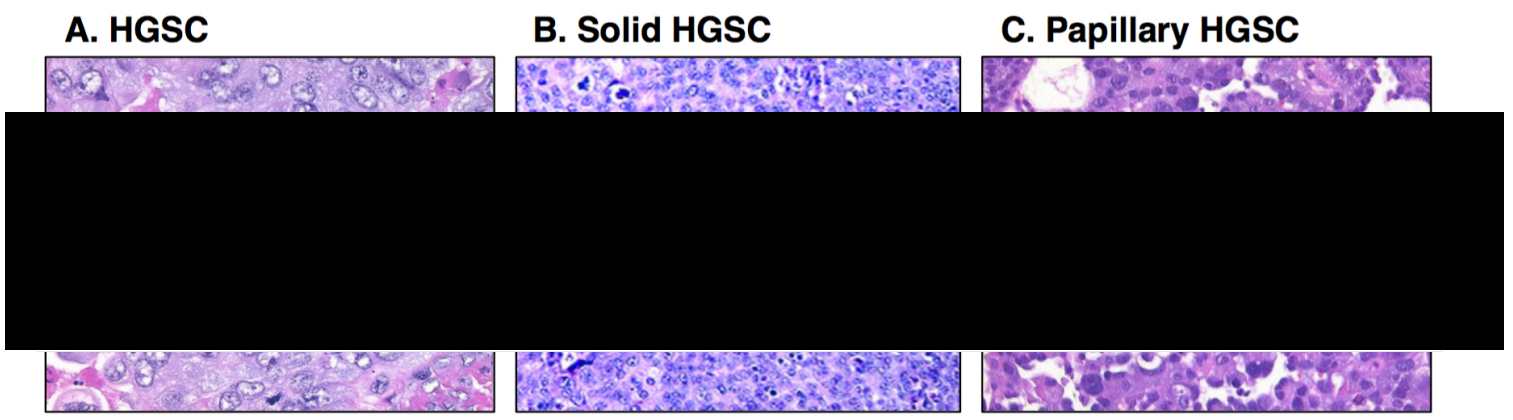

Figure 1. 11 Representative examples of HGSC histological examinations Histological images of: (A) High-grade serous carcinoma (HGSC) sample displaying nuclear atypia, atypical mitotic figures and high mitotic/apoptotic rates (60x). (B) Solid HGSC sample; tumour cells are arranged in solid sheets with no glandular or papillary formations (10x). (C) Papillary HGSC; papillae covered by stratified, highly atypical epithelium (40x). All images were stained using haematoxylin \& eosin. Source: 'High-grade serous carcinoma involving fallopian tube, ovary and peritoneum' (Al-Agha \& Blake Gilks 2011).

HGSCs are characterized by cytological features of high-grade malignancy, including nuclear atypia (high nuclear-to-cytoplasmic ratio, prominent nucleoli, bizarre nuclei), atypical mitotic figures (Bavle 2014), high mitotic/apoptotic rates (Malpica et al. 2004; Elmore 2007) and high proliferative index measured via Ki-67 protein immune-staining (Mahadevappa et al. 2017) (Fig. 1.11 A). Microscopically, 
HGSCs usually show either solid or papillary growth patterns (Fig. 1.11 B, C) (Prat 2012a).

Although diagnosis of HGSC is primarily based on routine cell morphology, certain immune-markers stain positively in the majority of HGSC and allow differentiate it from the rest of sub-types (Köbel et al. 2009). For instance, Ki-67 moderate to high expression levels distinguish between LGSC and HGSC samples. Others include estrogen receptor alpha $(\mathrm{ERA} / \alpha)$ (positive HGSC, negative $\mathrm{CC}$ ), p53 (positive HGSC, negative LGSC and CC) and the 'Wilms tumour protein 1' (WT1) (positive HGSC, negative EC and CC) (Al-Agha \& Blake Gilks 2011).

The mutational spectrum of HGSC is surprisingly simple (Network 2011). Apart from TP53 and BRCA1/2, other significant mutations are infrequent (2-6\%) reflecting the underlying complexity of this disease (Bashashati et al. 2013). TP53 mutations occur in almost $100 \%$ of HGSCs causing the loss of wild-type p53 functions due to either gain of abnormal function of mutant p53, or absent-low mutant p53 protein (Ahmed et al. 2010; Cole et al. 2016). Approximately 50\% of HGSC exhibit $B R C A$ and/or HRR-related defects, establishing a 'BRCAness' patient profile (Rigakos \& Razis 2012; Lord \& Ashworth 2016). These defects include:

- Germline and somatic mutations of BRCA1/2 ( 22\%) (Foster et al. 1996; Hilton et al. 2002).

- Epigenetic silencing of BRCA1 via promoter hyper-methylation (Rae Lynn Baldwin et al. 2000).

- Genetic or epigenetic alterations in genes whose function either affect or are affected by normal $B R C A$ gene function. For instance, amplification of the 'BRCA2-interacting transcriptional repressor' (EMSY) (Brown et al. 2006) or promoter hyper-methylation of the 'Falconi anemia group $\mathrm{F}$ protein' or FANCF (Lim et al. 2008).

- Defects in proteins involved in DNA repair besides $B R C A$. For instance, focal deletion/mutation of the 'tumour suppressor phosphate and tensin homolog' (PTEN) or hyper-methylation of the 'RAD51 paralog C' (RAD51C) (Network 2011). 
HGSCs are characterized by genomic structural variation, an important mechanism to inactivate TSGs (Martins et al. 2014; Patch et al. 2015). Recurrent molecular alterations include de-regulated retinoblastoma protein (RB), phosphatidylinositol 3kinase (PI3K)/RAS and NOTCH signalling pathways (Fig. 1.12 A-C), as well as high level of somatic DNA copy number alterations affecting genes like 'cyclin E1' (CCNE1), MYC and the 'telomerase reverse transcriptase' (TERT) (Gorringe et al. 2007; Etemadmoghadam et al. 2010; Network 2011).

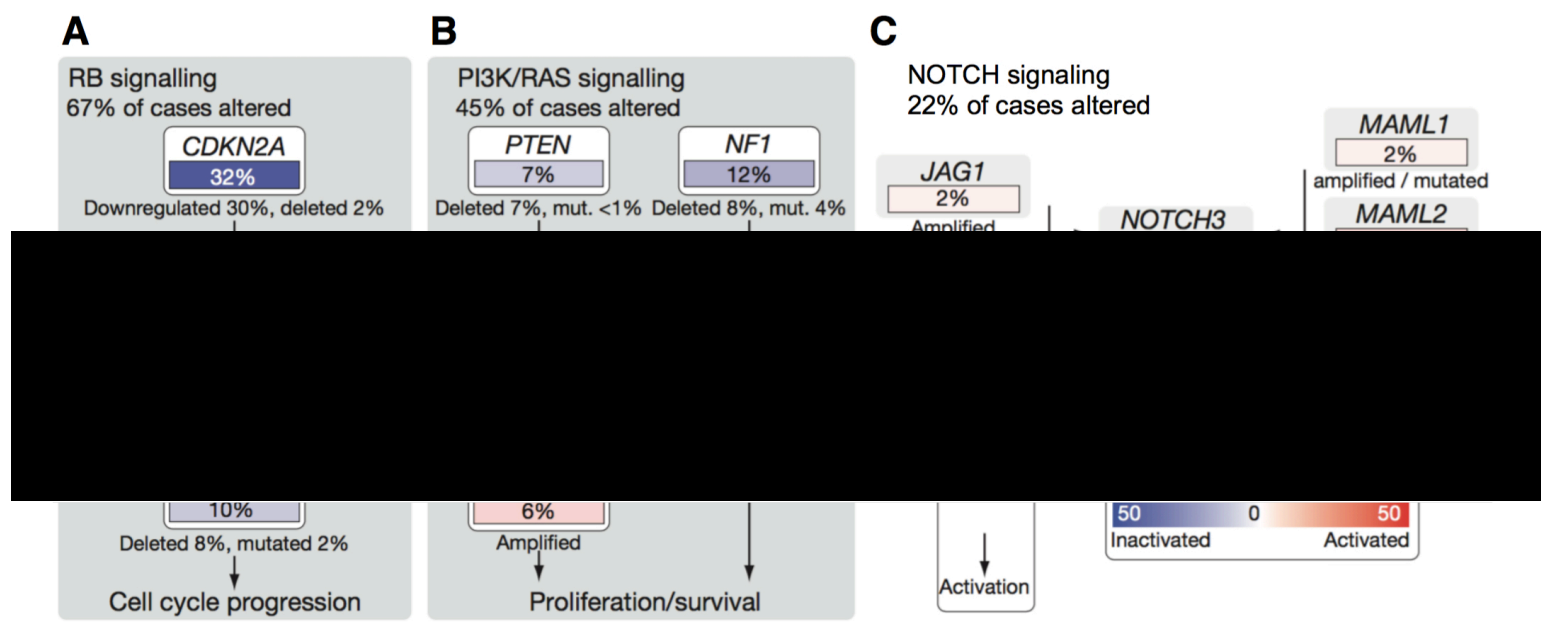

Figure 1. 12 Schematic representation of altered signalling pathways in HGSC (A) Retinoblastoma protein (RB), (B) PI3K/RAS and (C) NOTCH signalling pathways are commonly altered in HGSC. These alterations were defined by somatic mutations, DNA copy number changes (e.g. amplification) or, in some cases, by significant up- or down-regulation relative to expression in diploid tumours. Alteration frequencies are expressed as the percentage of all considered cases in the particular study. Activated genes are represented in red colour whereas inactivated genes are represented in blue. Source: 'Integrated genomic analyses of ovarian carcinoma' (Network 2011). CDKN2A: cyclin dependent kinase inhibitor 2A; $C C N D 1 / 2$ : cyclin D1/2; RB1: RB transcriptional corepressor 1; $N F 1$ : Neurofibromin 1; PIK3CA: PI3K catalytic subunit alpha; $A K T 1 / 2$ : AKT serine/threonine kinase $1 / 2$; $B R A F$ : B-Raf proto-oncogene, serine/threonine kinase; JAG1/2: Jagged $1 / 2$; $M A M L 1 / 2 / 3$ : mastermind like transcriptional coactivator 1/2/3.

An important number of HGSCs are thought to arise from STICs, found in the fallopian tubes of both germline BRCA mutation carriers and sporadic HGSCs ( 60\%) (Przybycin et al. 2010; Karst, Levanon \& Drapkin 2011; Kuhn et al. 2012)

(Fig. 1.8). These carcinomas are in turn thought to progress from normal FTE, characterized by low proliferation rates, normal cell polarity and overexpression of 
p53 protein present in the tubes regardless of BRCA status (George \& Shaw 2014)

(Fig. 1.13 A). With time, FTEs carrying the so-called 'p53 signature' progress to STICs, developing genomic alterations common to those observed in HGSCs, including CCNE1 and TERT amplification and up-regulation of the PI3K pathway (Kuhn et al. 2010; Sehdev et al. 2010; Karst, Levanon, Duraisamy, et al. 2011) (Fig. 1.13). However, and consistent with mouse models, some HGSCs do not show a direct evolution from precursors in the fallopian tube (J. Kim et al. 2015) and their origin is still unclear (Perets et al. 2013; Howitt et al. 2015).

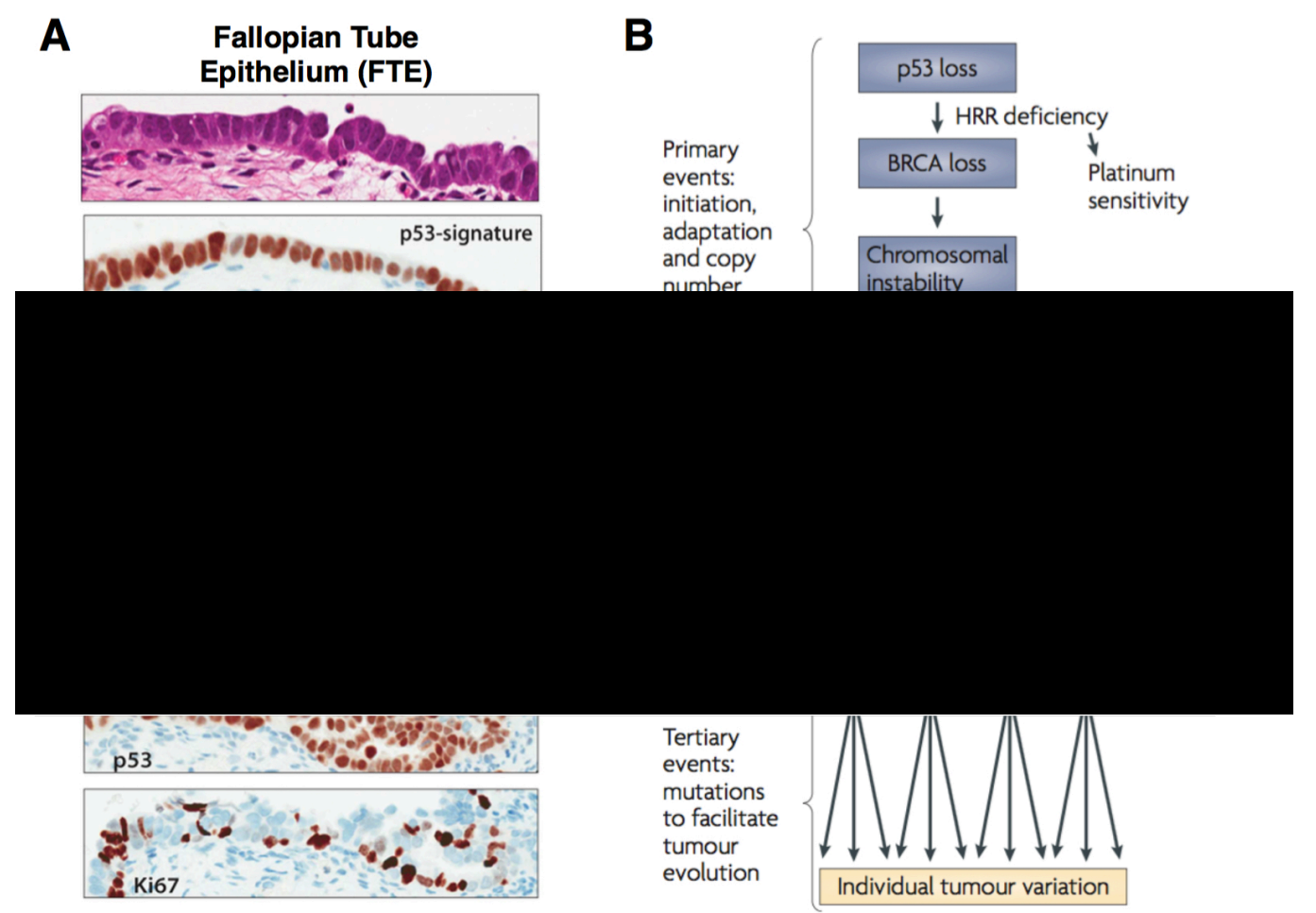

Figure 1. 13 Models of initiation and progression of HGSC

(A) Serous-tubal intraepithelial carcinomas (bottom three panels) are thought to progress from normal fallopian tube epithelium (top three panels). The top panel of both FTE and STIC histology display haematoxylin \& eosin stain. The middle and bottom panels show p53 and Ki67 staining respectively. Source: 'BRCA and early events...' (George \& Shaw 2014). (B) Model outlining the stages of the initiation and progression of HGSC. Loss of TP53 and BRCA, leading to a deficiency in homologous recombination repair, initiate chromosomal instability and widespread copy number changes. These alterations are thought to drive molecular subtype specification $(\mathrm{C} 1, \mathrm{C} 2, \mathrm{C} 4, \mathrm{C} 5)$ resulting in global changes in gene expression. Subsequent mutations provide further advantages for tumour growth but may not be molecular subtype specific. Source: 'The genesis and evolution...' (Bowtell 2010). 
There is currently no accepted model for initiation and progression of HGSC (Bowtell et al. 2015). TP53 mutation and stabilization is an early and invariant event in HGSCs but other than that, little is known of the temporal sequence of other molecular changes or the dynamics of chromosomal instability that drive HGSC progression (Berns \& Bowtell 2012). Bowtell et al (2010) proposed a model for initiation and progression of BRCAness-associated HGSC, where TP53 mutations and deficiencies in HRR pathway lead to chromosomal instability and widespread copy number changes (Bowtell 2010) (Fig. 1.13 B).

Based on different gene expression profiles and distinct clinical outcomes, HGSC has been further categorized into four subtypes: differentiated, immunoreactive, mesenchymal and proliferative; which are thought to account for HGSC histological variations (e.g. solid and/or papillary histology) (Tothill et al. 2008; Helland et al. 2011; Network 2011) (Fig. 1.13 B). However, these molecular subtypes have not yet been integrated into the clinical setting (Bowtell et al. 2015).

\subsection{Ovarian Cancer Treatment}

\subsubsection{First line treatment for advanced ovarian cancer}

The first line standard treatment for advanced stage OC starts with optimal cytoreduction, often via a extensive laparotomy surgery (Herold \& Cannistra 2013). This procedure permits efficient tumour grading and staging, thereby allowing the precise selection of subsequent treatments (Weinberg 2014). In any case, surgery is currently followed by adjuvant intravenous or intraperitoneal (less frequent) chemotherapy with a platinum-based agent combined with anti-microtubule drugs (Piccart et al. 2000; Elit et al. 2007). Specifically, the most widely practiced first-line treatment regimen is six cycles of intravenous platinum (preferably carboplatin) with paclitaxel infusion once every three weeks (Ye et al. 2014). However, based on patient-susceptibility to the side effects of chemotherapy, carboplatin/pegylated liposomal doxorubicin is often used as alternative to standard therapy (Pignata et al. 2011). 
Chemotherapy treatment consists of the use of a drug or, most commonly, a combination of drugs (also known as chemotherapeutic/cytotoxic agents) to stop or slow the growth of cancer cells (Weinberg 2014). Common cytotoxic agents previously or currently used for OC treatment are summarized in Table 1.5.

Table 1. 5 Cytotoxic agents in ovarian cancer treatment

\begin{tabular}{|c|c|c|}
\hline Cytotoxic agent & Mechanism of action & Examples OC treatment \\
\hline $\begin{array}{c}\text { Alkylating } \\
\text { agents }\end{array}$ & $\begin{array}{c}\text { Generation of DNA crosslinks, } \\
\text { leading to cell apoptosis }\end{array}$ & $\begin{array}{c}\text { Cyclophosphamide } \\
\text { (McGuire et al. 2003) }\end{array}$ \\
\hline Antimetabolites & Interference of DNA production & $\begin{array}{c}\text { Gemcitabine, decitabine } \\
\text { (Parker 2009) }\end{array}$ \\
\hline $\begin{array}{c}\text { Intercalating } \\
\text { agents }\end{array}$ & $\begin{array}{c}\text { Inhibition of DNA replication } \\
\text { via DNA strand intercalation }\end{array}$ & $\begin{array}{c}\text { Platinum derivatives such as } \\
\text { carboplatin and cisplatin } \\
\text { (Monneret 2011) }\end{array}$ \\
\hline Spindle poisons & $\begin{array}{c}\text { Disruption of microtubule } \\
\text { function, inhibiting cell division }\end{array}$ & $\begin{array}{c}\text { Taxanes such as paclitaxel } \\
\text { (Wani \& Horwitz 2014) }\end{array}$ \\
\hline $\begin{array}{c}\text { Topoisomerase } \\
\text { inhibitors }\end{array}$ & $\begin{array}{c}\text { Blockage of topoisomerase } \\
\text { DNA arrangements, leading to } \\
\text { cell apoptosis }\end{array}$ & $\begin{array}{c}\text { Doxorubicin, tocotecan } \\
\text { (Nitiss 2009) }\end{array}$ \\
\hline
\end{tabular}

Most cytotoxic agents are thought to preferentially attack high-rate proliferating cancerous cells, although the actual selection mechanism is not quite well understood (Amadori et al. 1997; Weinberg 2014). In fact, proliferation rates are quite low in some chemo-sensitive human cancers and the mechanism of tumour regression is unknown (Mitchison 2012). Nonetheless, major obstacles to successful cures with chemotherapy are the development of intrinsic or acquired mechanisms of drug resistance and a narrow therapeutic index, customarily having little difference between toxic and therapeutic doses (Crawford 2013). In general, the concept of selection for resistance implies that mutations either may pre-exist as small subpopulations within the cancer (intrinsic resistance) or may arise during the course of therapy (acquired resistance) (Lee \& Swanton 2012). The standard of care following completion of platinum-based chemotherapy include physical examination, monitoring CA-125 levels, and imaging follow-up (Salani et al. 2011). 


\subsubsection{Ovarian cancer recurrence}

Approximately $70-80 \%$ of women with advanced OC suffer recurrence of their primary cancer following first-line treatment (Cannistra 2004). In most cases, these tumours acquire resistance to chemotherapy via overexpression of drug transporters (Patch et al. 2015) or restoration of BRCA genes by means of secondary somatic mutations (Norquist et al. 2011), amongst other strategies. Following definitive diagnosis of recurrent $\mathrm{OC}$, several factors determine subsequent treatment options including location of metastases, complications from previous therapies, degree of platinum sensitivity and other patient-related issues (Matulonis et al. 2016) (Fig. 1.14). In general terms, the choice of treatment for recurrent $\mathrm{OC}$ is based on the interval between the completion of the last platinum-based treatment and the detection of the relapse -platinum-free interval (Luvero et al. 2014).

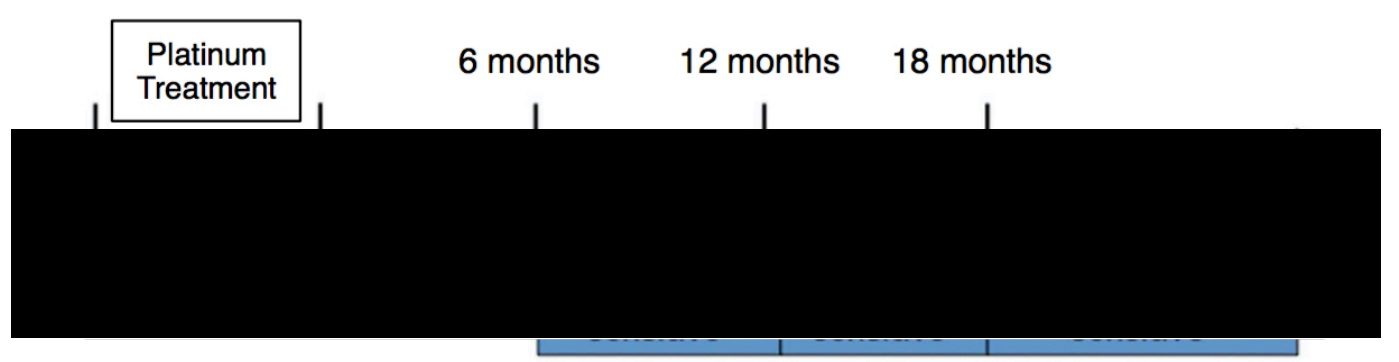

\section{Figure 1. 14 Sensitivity to platinum chemotherapy of HGSC}

High-grade serous carcinoma recurrent tumours are classified into platinum-sensitive (intermediately, highly, extremely highly) or platinum-resistant according to their sensitivity to platinum chemotherapy. The platinum-resistant group include tumours that relapse within 6 months after chemotherapy and also those who do not respond to platinum treatment, also called refractory tumours. Source: 'Redistribution of resistance...' (Yokoyama 2013).

The standard of care for patients with platinum-sensitive recurrent $\mathrm{OC}$ is the reutilization of combinatory platinum-based therapies (Raja et al. 2013). Even though response rates are approximately 50\% (Pfisterer et al. 2006; Pujade-Lauraine et al. 2010), these responses usually last a few months and their efficiency decrease with subsequent platinum use until the tumour is declared platinum resistant (Markman et al. 2004; Hanker et al. 2012). 
Platinum-resistant recurrent $\mathrm{OC}$ includes patients who do not respond or relapse within 6 months after first-line treatment and patients who relapse within 6 months of several lines of treatment for recurrent OC (Mantia-Smaldone et al. 2011) (Fig. 1.14). The standard of care for these patients includes non platinum-based therapies using combinations or single-agent treatments of pegylated liposomal doxorubicin (Monk et al. 2010), paclitaxel (Thigpen et al. 1994), topotecan (ten Bokkel Huinink et al. 1997) and gemcitabine (Mutch et al. 2007). New cytotoxic compounds under clinical trials include microtubule inhibitors epothilones (Rustin et al. 2011) and the thymidylate synthase inhibitor pemetrexed (Vergote et al. 2009). Paclitaxel is considered the standard regimen in platinum-resistant OC, used in combination with a variety of non-chemotherapeutic agents (Rosenberg et al. 2002).

\subsubsection{Targeting cancer angiogenesis}

Chemotherapy remains the backbone of OC treatment, however, novel therapies, both in combination with chemotherapy and alone, have demonstrated significant improvements (Grunewald \& Ledermann 2016). Even though there are several drugs being tested in OC patients, at the moment only two agents are licensed for OC treatment: Bevacizumab and Olaparib ${ }^{\circledR}$, which target tumorigenic angiogenesis and BRCAness respectively (Symeonides \& Gourley 2015).

High-grade serous ovarian carcinomas express moderate to high levels of proangiogenic proteins, particularly VEGFA and VEGFB (Bowtell et al. 2015). Inhibition of VEGFs restores the balance between pro and anti angiogenic factors, negatively influencing tumour growth and decreasing its metastatic potential (Mendelsohn et al. 2015). Bevacizumab is a humanized monoclonal antibody directed against circulating VEGFA (Ribatti 2011). It has been explored as a firstline single agent, in combination with chemotherapy and as maintenance treatment post chemotherapy (Perren et al. 2011; Burger et al. 2011). Furthermore, it has been studied in the setting of second-line treatment of platinum-sensitive (Aghajanian et al. 2015) and platinum-resistant recurrent OC (Pujade-Lauraine et al. 2014). All these studies demonstrated statistically significant increase in progression-free survival, the length of time during and after treatment that a patient lives with cancer without detection of new disease progression (Korn \& Crowley 2013), compared to 
chemotherapy alone. However, there is still no evidence of increase in overall survival, the length of time from the date of diagnosis/start of treatment that patients diagnosed with the disease are still alive (Oliver \& McGuire 2014).

Resistance to bevacizumab emerges in most patients with HGSC who initially responded to the treatment (Bottsford-Miller et al. 2012). Nevertheless, there is a subset of patients that is clearly benefiting from anti-angiogenic therapies, although that effect is being diluted by the lack of predictive biomarkers, which are only now starting to emerge (Lambrechts et al. 2013). In fact, a recently identified transcriptomic signature predicts distinct responses to bevacizumab treatment in the different subtypes of HGSC (C Gourley et al. 2014).

New anti-angiogenic strategies that are currently being studied include: trebananib (inhibitor of angiopoietins 1 and 2) (Monk et al. 2014), aflibercept (inhibitor of VEGF receptors 1 and 2) (Tew et al. 2014) and other multi-target tyrosine kinase inhibitors such as cediranib (Ledermann et al. 2016).

\subsubsection{Targeting cancer 'BRCAness'}

There are 17 members of the PARP nuclear superfamily, of which PARPI and PARP2 have key roles in DNA repair (Amé et al. 1999; Rouleau et al. 2010). PARP1/2, together with the HRR pathway (Table 1.1), are essential in the reparation of collapsed replication forks happening alongside DNA replication (Bryant et al. 2009; Fradet-Turcotte et al. 2016).

Initially, PARP inhibitors were investigated due to their chemo-sensitizing potential, enhancing the cytotoxic effects of chemotherapy (Purnell \& Whish 1980; Ferraris 2010). More recently, they are being studied as single agents due to their ability to selectively kill cells with defects in DNA repair pathways (Drew 2015). PARP inhibition leads to accumulation of single-strand breaks (SSBs), unrepaired replication forks and ultimately DSBs which a cell with functional HRR is able to accurately and efficiently repair (Hoeijmakers 2001). On the contrary, those cells with homologous BRCA1/2 mutations either resolve these DBS via the error-prone NHEJ pathway or not repair them at all, which leads to genomic instability and 
ultimately cell death (Ashworth 2008) (Fig. 1.15). Given the fact that 50\% of HGSC patients display a 'BRCAness' profile, the use of PARP inhibitors has been extendedly studied (Network 2011).

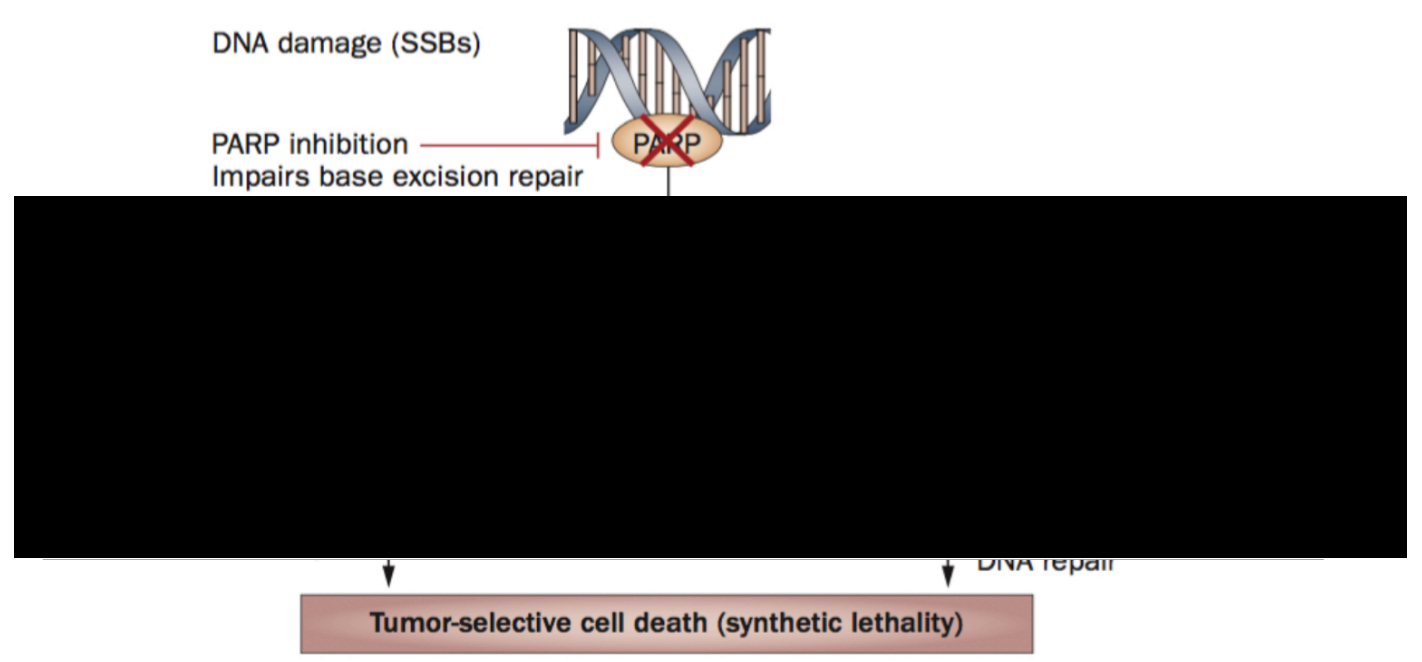

Figure 1. 15 Synthetic lethality coupling $B R C A$ deficiency and PARP inhibition Cellular DNA is frequently subject to damage, although single strand breaks (SSBs) are normally efficiently repaired by base-excision repair (BER). PARP1/2 are key components of BER mechanisms and PARP inhibition leads, therefore, to persistence of SSBs, which might be encountered by a replication fork, collapse, and form double-strand breaks (DSBs). In the absence of BRCA1/2, DSBs are repaired by alternative pathways like the error-prone non-homologous end joining (NHEJ), which result in gross genomic instability and cell death. Source: 'Making the best of PARP inhibitors in ovarian cancer' (Banerjee et al. 2010).

Olaparib ${ }^{\circledR}$ is a small molecule with activity against PARP1 and 2 that has been explored as first-line treatment (Fong et al. 2009) and as maintenance therapy for mutated BRCA1/2 platinum-sensitive recurrent HGSC, rendering significant improvements in patients' progression-free survival (Ledermann et al. 2014; Domchek et al. 2016). Importantly, secondary mutations restoring mutated $B R C A 1 / 2$ predict resistance to chemotherapy and PARP inhibitors (Edwards et al. 2008; Sakai et al. 2008; Norquist et al. 2011). The use of combination therapies, e.g. targeting at the same time 'BRCAness' deficiencies and OC angiogenic potential, in order to improve responses and overcome resistance has yielded significant increase in progression-free survival (Liu et al. 2014). 
Other PARP inhibitors that are currently under investigation in phase III studies include niraparib $^{\circledR}$ (Mirza et al. 2016) or veliparib ${ }^{\circledR}$ (Coleman et al. 2015).

\subsubsection{Alternative ovarian cancer treatment strategies}

Distinctive traits of the OC malignancy have prompted a variety of therapeutic approaches, aside from the inhibition of PARPs and angiogenesis. These procedures include endocrine therapy, immunotherapy, antibody targeted therapies and epigenetic therapies:

- Endocrine therapy is involved in managing cancers whose growth is dependent on hormones, and its aim is to antagonize hormone actions (Abdulkareem \& Zurmi 2012; Simpkins et al. 2013). The most common example of effective endocrine therapy is the treatment of ER $\alpha$-positive breast cancer with tamoxifen, which binds to the receptor blocking the tumorigenic effects of estrogen (Singh \& Kumar 2005).

HGSCs usually express ER $\alpha(60-70 \%)$ and the aromatase enzyme, which is responsible for the biosynthesis of estrogen (Rao \& Slotman 1996; Kitawaki et al. 1996); both molecules previously linked to OC growth stimulation in several OC cell lines (Galtier-Dereure et al. 1992; Langdon et al. 1994). However, clinical trials with anti-estrogenic therapies including tamoxifen and aromatase inhibitors, both in primary and recurrent OC settings, have shown disappointing therapeutic efficacy when compared to ER $\alpha$-positive breast cancer associated responses (Schwartz et al. 1989; Ramirez et al. 2008; Argenta et al. 2009; Simpkins et al. 2013).

- Immunotherapy is the treatment of a disease by supplementing or strengthening the patient's immune response against a tumour (Baxevanis et al. 2009). Accumulated evidence indicates that $\mathrm{OC}$ has therapeutic potential for immunebased therapies via activation of effector immune cells or blockade of immuneinhibitory pathways (Adams \& Benencia 2015; Coukos et al. 2016). Accordingly, several strategies are currently being tested in clinical trials, including cancer vaccines (Kandalaft et al. 2013), immune checkpoint inhibitors (Duraiswamy et al. 2013) and T-cell-activating interleukins (Vlad et al. 2010). 
- Antibody targeted therapies: Similar to bevacizumab, other antibodies specifically designed to target molecules involved in a variety of OC-related processes have been tested, e.g. the folate receptor alpha and the epidermal growth factor receptor 2 (ERBB2, also known as HER2).

The folate cycle has a crucial role in biosynthesis reactions, essential for rapid cell growth (Elnakat \& Ratnam 2006), and the main cellular receptor of folate is commonly overexpressed in HGSC (Kalli et al. 2008). However, monoclonal antibody farletuzumab and folate-conjugated vintafolide targeting folate receptor alpha did not show any significant benefit in phase III trials (Oza et al. 2015; Vergote et al. 2016).

Trans-membrane epidermal growth factor receptors have key roles in cancer and ERBB2 is commonly overexpressed in HGSC (Serrano-Olvera et al. 2006; Teplinsky \& Muggia 2014). Accordingly, breast cancer-approved anti-ERBB2 agents such as herceptin/trastuzumab have been investigated in OC, although none have showed significant improvements in progression-free or overall survival thus far (Bookman et al. 2003; Teplinsky \& Muggia 2015).

- Epigenetic therapies: One of the most exciting areas of biology over the past decade has been the expanding understanding of how epigenetic control influences the patterns of gene expression in cells, a knowledge that has immediate translational implications for targeting epigenetic abnormalities in cancer for therapeutic purposes (Jones \& Baylin 2007). Epigenetic regulation and therapies aimed at $\mathrm{OC}$ are discussed in the next section. 


\subsection{Epigenetic Regulatory Mechanisms in OC Progression}

In principle, 'epigenetics' refers to the somatically heritable differences in gene expression not attributable to intrinsic alterations in the primary sequence of DNA (Kouzarides 2007). Individual cells of different types all have the same genome, but have multiple different 'epigenomes' that define their respective phenotypes (Jones $\&$ Baylin 2002). In the same way, cancers of all types, and even different cell populations within a single patient's cancer, undergo extensive and biologically significant changes in their epigenomes, ending with aberrant activation of oncogenes and inactivation of tumour suppressor genes favouring cancer progression (Allis \& Jenuwein 2016). Furthermore, virtually all tumours harbour mutations in genes that encode proteins that control the epigenome (Azad et al. 2013).

\subsubsection{Basics of epigenetics}

The genetic material of eukaryotic cells is packaged into the nucleus in the form of chromatin fibres, consisting of nucleosomes, RNA and a number of other associated proteins (Kornberg 1974). Each nucleosome is composed of a segment of 147 base pairs of DNA wrapped almost two times around a cylindrical protein core containing two copies of each histone protein: histone 2A (H2A), histone 2B (H2B), histone 3 (H3) and histone 4 (H4) (Kornberg 1974; Richmond et al. 1997) (Fig. 1.16). Histones are small basic proteins of around $14 \mathrm{kDa}$ that contain a high percentage of positively charged amino acids and are the most abundant proteins bound to DNA in eukaryotic cells (Richmond et al. 1997).

DNA packaging organization in a chromatin fibre generates restrictions for all processes involving DNA such as replication, repair, recombination and transcription (Ehrenhofer-Murray 2004). Chromatin structural rearrangements, also known as epigenetic changes, which include DNA methylation, covalent modification of histones, the incorporation of histone variants (Henikoff \& Smith 2015) and noncovalent remodelling by adenosine triphosphate (ATP)-dependent remodelling enzymes (Vignali et al. 2000), allow these constraints to be overcome (EhrenhoferMurray 2004). Additionally, non-coding RNAs also play a role in epigenetic gene regulation (Peschansky \& Wahlestedt 2014). Currently, the most studied and 
recognized cancer-specific epigenetic changes are alterations in: DNA methylation and histone acetylation, a specific covalent post-translational modification of histones (Esteller 2008).

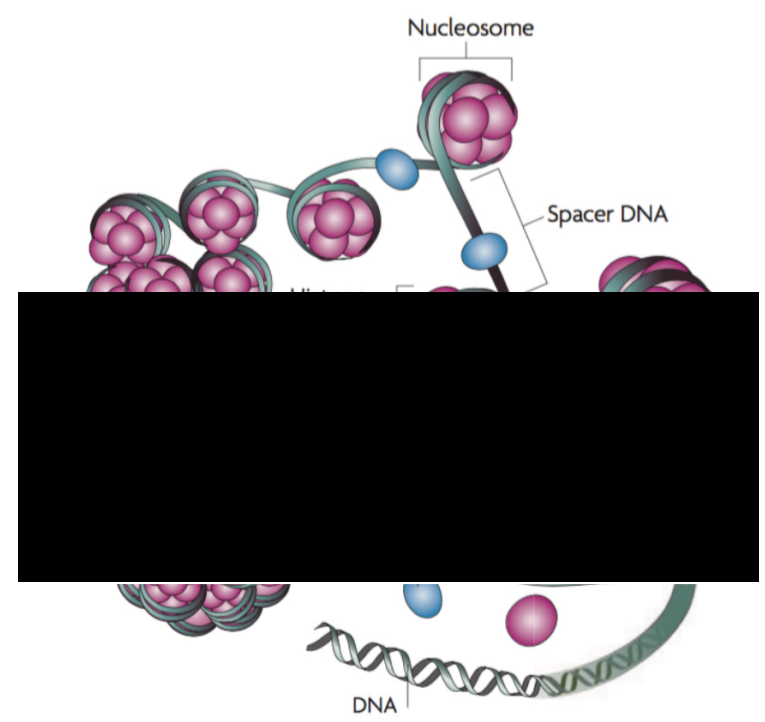

Figure 1. 16 Chromatin structure

This figure illustrates a representation of the structure of a chromatin fibre, whose basic units are the nucleosomes. Each nucleosome is composed of approximately 147 base pairs of DNA wrapped around an octamer of histones (two copies of $\mathrm{H} 2 \mathrm{~A}, \mathrm{H} 2 \mathrm{~B}, \mathrm{H} 3$ and $\mathrm{H} 4$ ). Histone $\mathrm{H} 1$ binds to the DNA that links two nucleosomes. Source: 'Epigenetic regulation in African trypanosomes...' (Figueiredo et al. 2009).

DNA methylation: DNA methylation occurs among cytosine residues in cytosineguanine $(\mathrm{CpG})$ di-nucleotides, which are mostly distributed in $\mathrm{CpG}$-rich regions or 'CpG islands' (Holliday \& Pugh 1975; Bird et al. 1985), and this reaction is catalysed by DNA methyl-transferase (DNMT) enzymes (Bestor \& Ingram 1983). The addition of methyl groups to cytosine molecules alters the activity of DNA sequences; for instance, when located in a gene promoter, DNA methylation typically acts to repress gene transcription (Moore et al. 2013) (Fig. 1.17). Aberrant methylation of ' $\mathrm{CpG}$ islands' has been linked to tumour initiation and progression (Costello et al. 2000). While cancer-related DNA hyper-methylation is commonly associated with silencing of TSG (Feinberg \& Tycko 2004), hypo-methylation is linked to transcriptional activation of oncogenes (Feinberg \& Vogelstein 1983) (Fig. 1.17). 


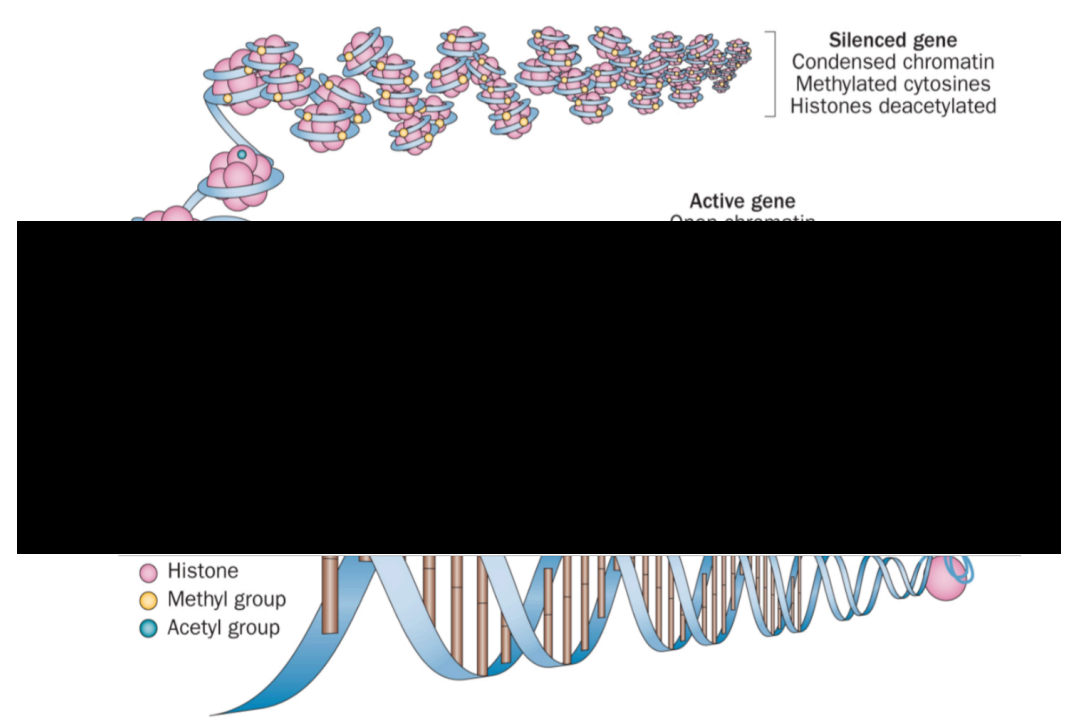

Figure 1. 17 Epigenetic regulation of gene expression

Broadly, gene expression is controlled at promoter regions by a combination of DNA methylation and chromatin configuration. In normal cells, gene expression is commonly silenced by the condensation of chromatin structures via DNA methylation and histone de-acetylation. By contrast, active genes are those characterized by open nucleosome spacing around the transcriptional start site, lack of DNA methylation and enhanced histone acetylation. Source: 'The future of epigenetic therapy in solid tumours...' (Azad et al. 2013).

Covalent modification of histones: The N-terminal tail of histone proteins protrude from the nucleosome core and provide sites for reversible post-translational modifications (PTMs), including acetylation, ubiquitination, methylation, phosphorylation and sumoylation (Tessarz \& Kouzarides 2014) (Fig. 1.18 A). PTMs can influence histone interactions or function as docking sites for other proteins that specifically recognize these modifications (Musselman et al. 2012). A new terminology describes molecules that lay down histone modifications as 'writers', those who remove them as 'erasers' and 'readers' as those who interpret the signals and influence subsequent changes (Dawson et al. 2012) (Fig. 1.18 B). By tightly condensing or loosening up the structure of chromatin, aberrant PTMs can deregulate DNA-accessibility and thus promote oncogene expression or silence TSG/stability genes (Seeber \& van Diest 2012) (Fig. 1.17). 
Introduction

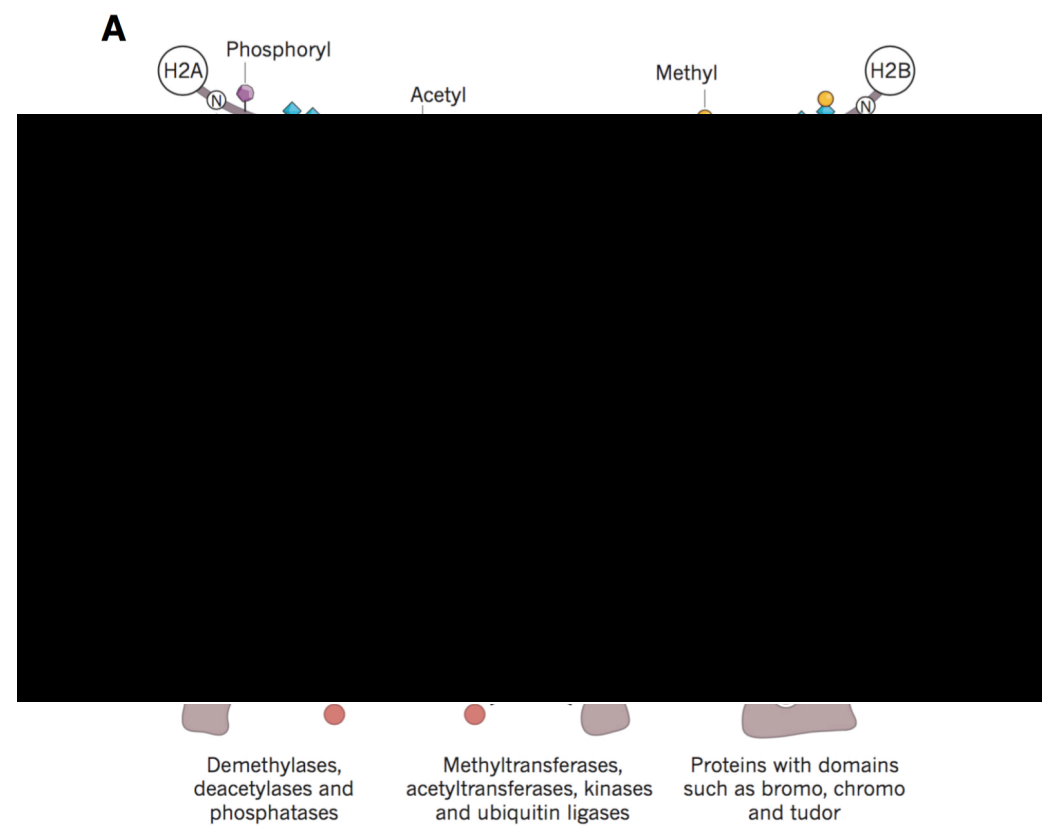

Figure 1. 18 Histone post-translational modifications

(A) The majority of post-translational modifications (PTMs) occur in the N-terminus tail of histones protruded from nucleosome cores. These PTMs include the addition of phosphoryl, acetyl and methyl groups, amongst others. (B) Molecules denominated 'writers' introduce histone marks (e.g. methyltransferases), 'erasers' take them out (e.g. demethylases) and 'readers' can recognize particular forms of histone modification and influence subsequent changes. Source: 'Chromatin proteins and modifications as drug targets' (Helin \& Dhanak 2013).

Histone acetylation: The acetylation reaction is the process of substituting an active hydrogen atom of a protein for an acetyl group $\left(\mathrm{C}_{2} \mathrm{H}_{3} \mathrm{O}\right)$ (Phillips 1963). In cell biology, acetylation is a major regulatory mechanism, conferring reversible novel properties to altered proteins, including changes in enzymatic activity, localization, stability and interaction partners (Drazic et al. 2016).

In the context of chromatin, the addition of an acetyl group to a protruding histone $\mathrm{N}$-terminal tail disrupts the electrostatic interactions between the targeted histone and DNA by neutralizing positively charged lysine residues within histones (Carrozza et al. 2003). This action results in disruption of the nucleosomal structure and prompt unfolding of local DNA, making it more accessible by transcriptional machinery. As a consequence, the acetylation and de-acetylation of core histone proteins regulate gene transcription, replication and DNA repair processes, 
influencing chromatin configuration and providing important platforms or docking sites for the recruitment of proteins and enzyme complexes required for further chromatin modelling (Shahbazian \& Grunstein 2007) (Fig. 1.18 B).

Histone $\mathrm{N}$-terminal tails are acetylated in the $\varepsilon$-amino group of lysine residues, a reaction that relies on acetyl-coenzyme A (acetyl-CoA) and is carried out by lysine acetyltransferases (LATs or KATs) (Gershey et al. 1968; Yang 2004). Owing to the discovery of lysine acetylation as a PTM of histones (Phillips 1963), the enzymes that regulate global acetylation levels are termed histone acetyltransferases (HATs) and histone deacetylases (HDACs) (Kleff et al. 1995; Rundlett et al. 1996; Sadoul et al. 2008). HATs introduce acetyl groups into histones and are commonly associated with an open chromatin structure that facilitates transcription, whereas HDACs remove acetyl residues and consequently are associated with gene repression (Zhang \& Dent 2005).

\subsubsection{Foundations of epigenetic therapies}

Mutations of genes involved in epigenetic mechanisms, such as DNMTs and HDACs, have been previously linked to cancer (Ley et al. 2010; Guglielmelli et al. 2011). These mutations are thought to set up downstream abnormalities in the normal cell epigenome, remodelling it and establishing a completely different 'cancer epigenome', prone to favour tumour progression (Berdasco \& Esteller 2013). However, downstream genes affected by these epigenetic abnormalities are generally wild type for the underlying DNA sequence. Thus, normal function might be restored since, theoretically, reversing these epigenetic abnormalities should be easier than repairing the original upstream somatic mutations. Consequently, the aim of the so-called epigenetic therapies is to reverse key features of abnormal cancer epigenomes (Jones et al. 2016).

Traditional therapies aimed at single gene abnormalities (e.g. OC targeted antibodies) commonly provide non-durable therapeutic responses, largely because the carcinogenic potential of most tumours relies on the existence of multiple signalling abnormalities, e.g. HGSCs (Mendelsohn et al. 2015; Matulonis et al. 2016). Moreover, compensatory events can lead to resistance to the targeted 
inhibition of any single gene-mediated event to which a cancer cell is addicted (Housman et al. 2014). Conversely, epigenetic therapies have the potential ability to simultaneously reverse tumorigenic abnormalities via multiple signalling pathways, mediating cell re-programming to inhibit cancer initiation and progression (Allis \& Jenuwein 2016) (Fig. 1.19).

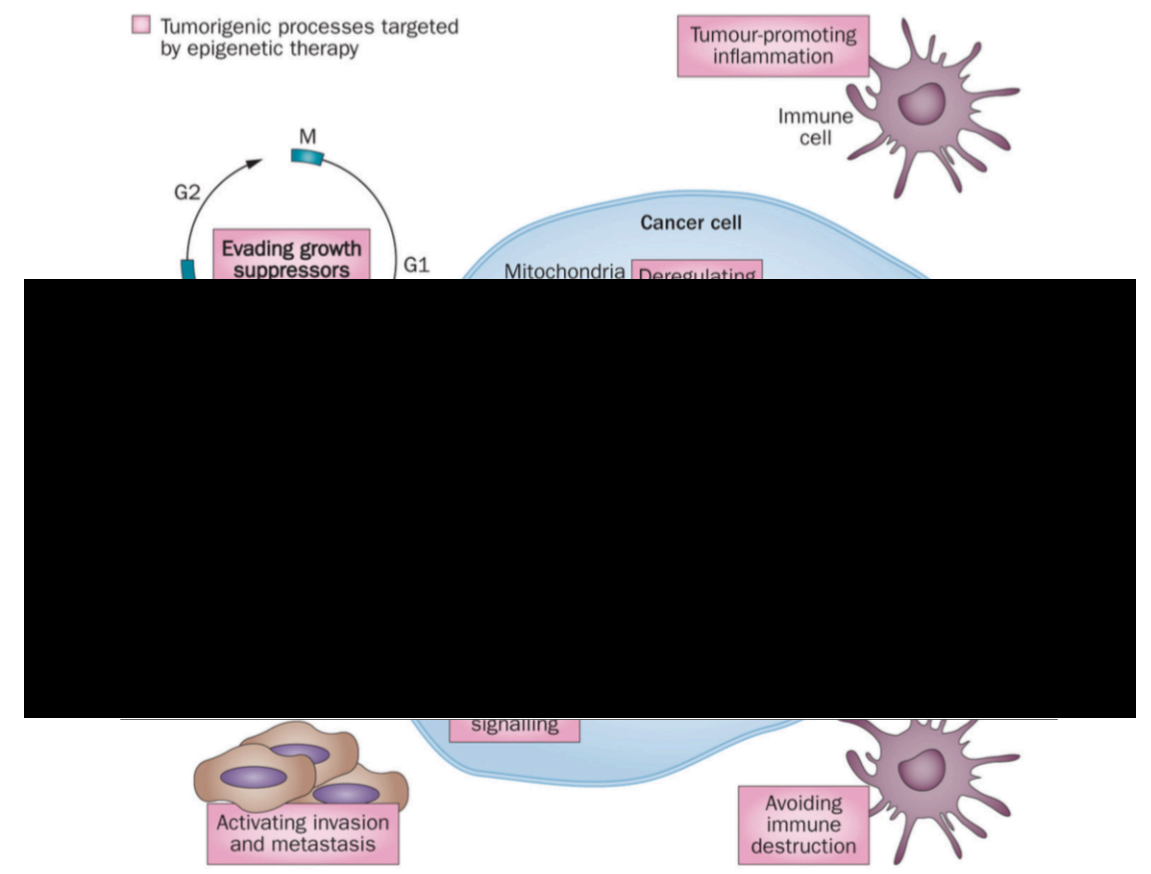

Figure 1. 19 Potential targets of epigenetic therapy

Targeting one signalling pathway or biological function can result in compensatory modulation of other, off-target drivers of tumorigenesis. The epigenetic control is involved in all the hallmarks of tumour initiation and progression (e.g. resistance to cell death, replicative immortality). Therefore, epigenetic therapy offers the ability to concurrently target, and reverse, multiple aberrant signalling pathways as well as the expected compensatory changes. Source: 'The future of epigenetic therapy...' (Azad et al. 2013).

The hypothesis behind this assumption is based on the premises that for every genetic mutation in a given patient's tumour, hundreds of genes have epigenetic abnormalities within the deviant cell signalling pathways that drive tumorigenesis (Herman \& Baylin 2003; Ushijima \& Asada 2010). Therefore, the loss of function or expression of many important candidate genes among the genes scattered throughout the pathways can be critical to the initiation and progression of tumours, as well as to their sensitivity and resistance to chemotherapy (Easwaran et al. 2012). 


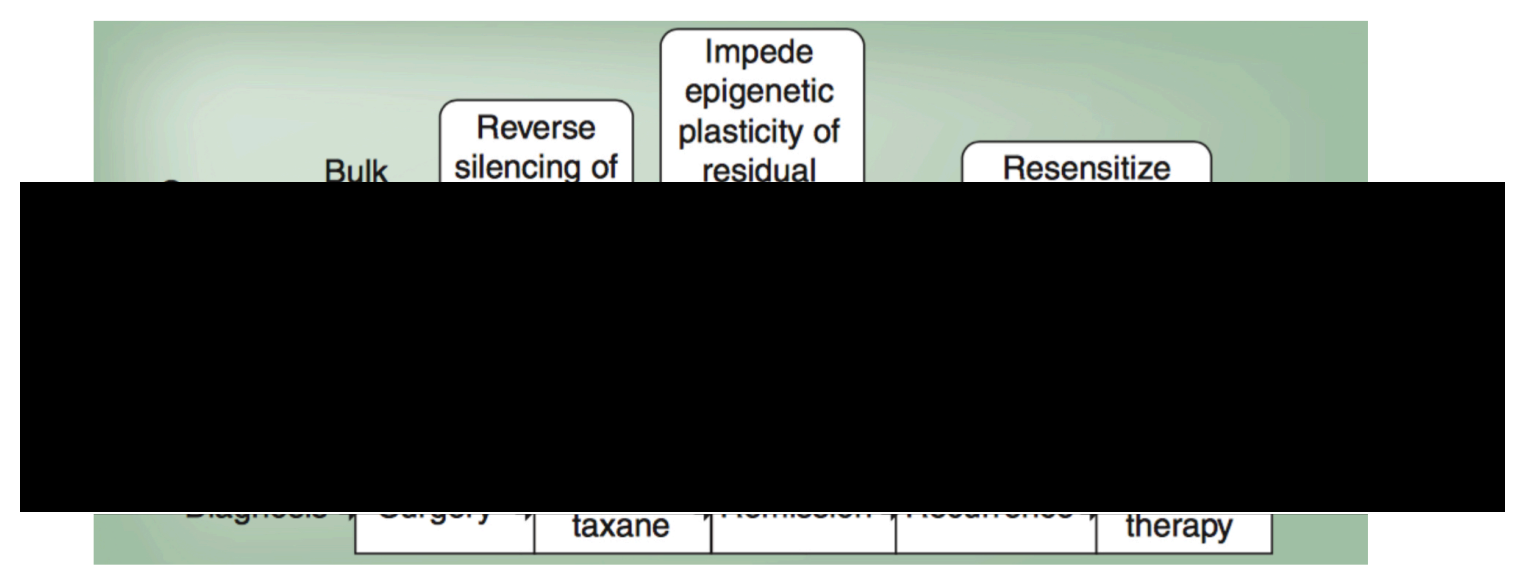

Figure 1. 20 Potential targets of epigenetic therapy in OC

The progress of OC treatment, from diagnosis to recurrence, is depicted at the bottom panels, with the cell population thought to be present at each stage of this process shown within the shaded boxes. Potential opportunities for epigenetic therapies are depicted at the top panels, and include time points prior to treatment with chemotherapy, during remission and after recurrence. Source: 'Targeting the epigenome in ovarian cancer' (Murphy 2012).

A shortcoming potentially applicable to most OC therapies is the inefficient targeting of cancer cells responsible for the disease itself or its recurrence, the ovarian cancer stem cells (Yinu Wang et al. 2014) (Fig. 1.20). Ovarian cancer stem cells have been isolated from established OC cell lines, ascites, primary and metastatic tumours and are hypothesized to be largely responsible for chemo-resistance (Bapat et al. 2005; S. Zhang et al. 2008; Curley et al. 2009). Similar to normal embryonic or tissue stem cells, ovarian cancer stem cells are believed to harbour a significantly altered epigenome, offering a useful arena for epigenetic therapies to develop strategies to target cancer stem-like cells (Balch et al. 2009; Y. Wang et al. 2014).

\subsubsection{Epigenetic therapies for the treatment of ovarian cancer}

Known aberrant epigenetic changes contribute to the inactivation of tumour suppressor genes and the activation of oncogenes in HGSC (Balch et al. 2009). Some altered epigenetic mechanisms include:

- Epigenetic silencing via promoter hyper-methylation of TSGs such as BRCA1 (R L Baldwin et al. 2000), the 'Ras association domain-containing protein 1' 
(RASSF1A) (BonDurant et al. 2011), the 'opioid-binding protein/cell adhesion molecule' (OPCML) (Sellar et al. 2003), MLH1, MSH2 (H. Zhang et al. 2008) or the 'SPARC-like protein 1' (SPARCL1) (Network 2011).

- Epigenetic activation via promoter hypo-methylation of potential oncogenes, including the 'preferentially expressed antigen in melanoma' (PRAME) (Zhang et al. 2016), PARPl (Bi et al. 2013), the 'solute carrier family 6 member 8' (SLC6A8) or nucleolin (NCL) (Shih et al. 2010).

- Histone modifications, such as the promoter hypo-acetylation of the 'deleted in lung and oesophageal cancer protein 1' (DLEC1) TSG (Kwong et al. 2006) or the overexpression of sirtuin 1 (SIRT1) deacetylase (Shuang et al. 2015).

- MicroRNAs: Epigenetic regulation via microRNA expression is significantly deregulated in HGSC (Vilming Elgaaen et al. 2014). Some microRNA act as TSGs and are down-regulated in HGSC (e.g. miR-152) (S. Zhang et al. 2015). Others, like the miR-200 family, are strongly up-regulated and promote tumorigenesis (Chen et al. 2013).

Despite the relatively rudimentary understanding of the HGSC epigenome, these epigenetic aberrations underpin new therapeutic options (Nguyen et al. 2014). DNMT and HDAC inhibitors are the two major classes of epigenetic modulating agents that have been investigated for the treatment of OC, both as single agents and in combination with other therapies (Murphy 2012) (Table 1.6). Nonetheless, early clinical trials soon confirmed the lack of utility of these epigenetic drugs as single agents, similar to clinical findings observed in other diseases (Smith et al. 2017).

Several DNMT inhibitors have been tested in clinical trials of patients with recurrent resistant OC (Fang et al. 2010; Falchook et al. 2013; Odunsi et al. 2014) (Table 1.6). Low doses of 5-aza-2'-deoxycytidine (decitabine) seemed to restore sensitivity to chemotherapy via de-methylation of individual TSGs (e.g. MLH1, RASSF1A) and multiple other important genes involved in key regulatory pathways. Specifically, pre-treatment with decitabine increased the objective response to re-treatment with platinum from $<10 \%$ to $35 \%$ of patients (Matei et al. 2012). The objective response (rate) is defined as the proportion of patients with tumour size reduction of a predefined amount and for a minimum time period. A similar result was partially 
achieved by 5 -AzaC (azacitidine), which increased the objective response to a $22 \%$ of the patients (Fu et al. 2011) (Table 1.6). Larger trials testing de-methylating agents to overcome platinum resistance are currently on-going (Smith et al. 2017). However, while these studies indicate that DNMT inhibitors may improve response to platinum in patients with platinum-resistant $\mathrm{OC}$, they failed to deliver any improvement in platinum-sensitive OC (Glasspool et al. 2014).

Table 1. 6 Clinical experience with epigenetic therapies in OC

\begin{tabular}{|c|c|c|c|c|c|c|c|}
\hline Citation & $\begin{array}{l}1 \text { st } \\
\text { Author }\end{array}$ & Year & $\begin{array}{l}\text { Study } \\
\text { Type }\end{array}$ & Regimen & \# Pts & Population & Findings \\
\hline \multicolumn{8}{|l|}{ HDAC Inhibitors } \\
\hline (Modesitt et al., 2008) & Modesitt & 2008 & Phase 2 & Vorinostat & 27 & Platinum-resistant & 1 PR, 9 SD, only 2 patients had PFS $>6$ months \\
\hline (Mendivil et al., 2013) & Mendivil & 2013 & Phase 2 & $\begin{array}{l}\text { Vorinostat }+ \\
\text { paclitaxel/carbo }\end{array}$ & 18 & Primary therapy & $\begin{array}{l}7 \mathrm{CR}, 2 \mathrm{PR}, 2 \mathrm{SD}, \mathrm{ORR} 50 \% \text {. Terminated early due to } \mathrm{GI} \\
\text { perforation in } 3 \text { patients }\end{array}$ \\
\hline (Matulonis et al., 2015) & Matulonis & 2015 & Phase 1 & $\begin{array}{l}\text { Vorinostat }+ \\
\text { gemcitabine/carbo }\end{array}$ & 15 & $\begin{array}{l}\text { 1st recurrence, } \\
\text { platinum-sensitive }\end{array}$ & $\begin{array}{l}6 \mathrm{PR}, 1 \mathrm{SD} \text {. Terminated early due to hematologic } \\
\text { toxicity }\end{array}$ \\
\hline (Mackay et al., 2010) & Mackay & 2010 & Phase 2 & Belinostat & 32 & $\begin{array}{l}\text { Platinum-resistant EOC } \\
\text { or LMP }\end{array}$ & $\begin{array}{l}\text { LMP: } 1 \text { PR, } 10 \text { SD } \\
\text { EOC: } 9 \text { SD }\end{array}$ \\
\hline (Dizon et al., 2012a) & Dizon & 2012 & Phase 2 & Belinostat + carbo & 27 & Platinum-resistant & ORR $7.4 \%$. Terminated early due to lack of activity \\
\hline (Dizon et al., 2012b) & Dizon & 2012 & $\begin{array}{l}\text { Phase } \\
1 \mathrm{~b} / 2\end{array}$ & $\begin{array}{l}\text { Belinostat }+ \\
\text { paclitaxel/carbo }\end{array}$ & 35 & Recurrent EOC & 3 CR, 12 PR, ORR 43\% \\
\hline \multicolumn{8}{|l|}{ DNMT Inhibitors } \\
\hline (Falchook et al., 2013) & Falchook & 2013 & Phase 1 & $5 A Z A+V P A+$ carbo & $\begin{array}{l}32(10 \\
\mathrm{EOC})\end{array}$ & Platinum-resistant & $\begin{array}{l}3 / 10 \text { EOC patients had minor response or } \mathrm{SD}>4 \\
\text { months }\end{array}$ \\
\hline (Fang et al., 2010) & Fang & 2010 & Phase 1 & Decitabine + carbo & 9 & Platinum-resistant & $1 \mathrm{CR}, 3 \mathrm{SD}>6$ months \\
\hline (Matei et al., 2012) & Matei & 2012 & Phase 2 & Decitabine + carbo & 17 & Platinum-resistant & $1 \mathrm{CR}, 5 \mathrm{PR}, 6 \mathrm{SD} .35 \%$ ORR \\
\hline (Odunsi et al., 2014) & Odunsi & 2014 & Phase 1 & $\begin{array}{l}\text { NY-ESO-1 vaccine }+ \\
\text { decitabine }+ \text { PLD }\end{array}$ & 10 & Recurrent EOC & $5 \mathrm{SD}, 1 \mathrm{PR}$ \\
\hline
\end{tabular}

Carbo $=$ Carboplatin; 5AZA $=$ 5-azacytidine; VPA $=$ Valproic acid, PLD: Pegylated liposomal doxorubicin; LMP: Low malignant potential; $\mathrm{CR}=$ Complete response, absence of all detectable cancer; $\mathrm{PR}=$ Partial response, at least $50 \%$ reduction in measurable tumour; $\mathrm{SD}=$ Stable disease, cancer neither growing not shrinking not metastasizing further; ORR $=$ Objective response rate, proportion of patients with tumour size reduction of a predefined amount and for a minimum time period; PFS = Progression-free survival, length of time during and after the treatment that a patients lives with the disease but it does not get worse; GI = Gastrointestinal. Source: 'Epigenetic therapy for the treatment of...' (Smith et al. 2017).

The experience with HDAC inhibitors has been less encouraging thus far. For instance, SAHA (Vorinostat) has shown recurrent toxicity issues and limited effects in platinum-resistant trial settings (Modesitt et al. 2008; Mendivil et al. 2013; Matulonis et al. 2015) (Table 1.6). Conversely, the effects of Belinostat range from absence of activity to discrete benefits when combined with paclitaxel/carboplatin chemotherapy (43\% objective response rate) (Mackay et al. 2010; Dizon, Blessing, et al. 2012; Dizon, Damstrup, et al. 2012) (Table 1.6). 
While response rates with single-agent epigenetic therapies have thus far been low, these agents have been able to at least mitigate platinum resistance and improve response to immunotherapy in pre-clinical studies and some early phase clinical trials, indicating that epigenetic agents may be best used in combination with other therapies (Paller et al. 2014; Smith et al. 2017). One study where low-dose decitabine was used in combination with dose-reduced paclitaxel and platinum chemotherapy in patients with platinum resistant/refractory OC produced a clinical benefit (either partial response or stable disease) in over $70 \%$ of the 17 patients included in the trial (Fu et al. 2011) (Table 1.6). In accordance, epigenetic agents such as DNMT and HDAC inhibitors are being extensively studied and represent an exciting and promising area of OC research. However, the development of new agents for targeting the OC cancer epigenome is also encouraged.

As well as histone de-acetylation, other post-translational histone modifications that might offer new targets for OC treatment are currently being studied (Marsh et al. 2014). Two of these new targets include histone H2B mono-ubiquitination and H3 histone methylation, which are regulated by de-ubiquitinases (DUBs) and histone methyl-transferases (HMTs) respectively (Colland 2010). However, aberrantly expressed histone acetyltransferases, which have been previously linked to tumour growth and cancer progression, have not been investigated in OC settings (Wapenaar \& Dekker 2016).

\subsubsection{Targeting histone acetyltransferases}

The human histone acetyltransferases are classified in two different groups: the type B HATs, which are cytoplasmic enzymes that modify free histones and other proteins in the cytoplasm, and type A HATs, which are (mainly) nuclear and responsible for the acetylation of histones and non-histone proteins in the nucleus (Parthun et al. 1996). Based on their sequence homology, most type A nuclear HATs are organized into three major groups or families: MYST (Utley \& Côté 2003), Gcn5-related N-acetyltransferase (GNAT) family (Poux \& Marmorstein 2003) and p300/CBP (Kalkhoven 2004). 
HAT enzymes acetylate histone and non-histone proteins with various substrate specificities, which are modulated by the incorporation of HATs in large multisubunit complexes (Lee \& Workman 2007). As well as HAT ('writers') and/or HDAC ('erasers') catalytic domains, complexes normally contain different histone 'readers', as well as scaffolding domains that bridge the components of the complex together (Fig. 1.21). The function of the catalytic subunit (HAT or HDAC) in these multi-protein complexes depend largely on the context of the other subunits of the complex (Carrozza et al. 2003).

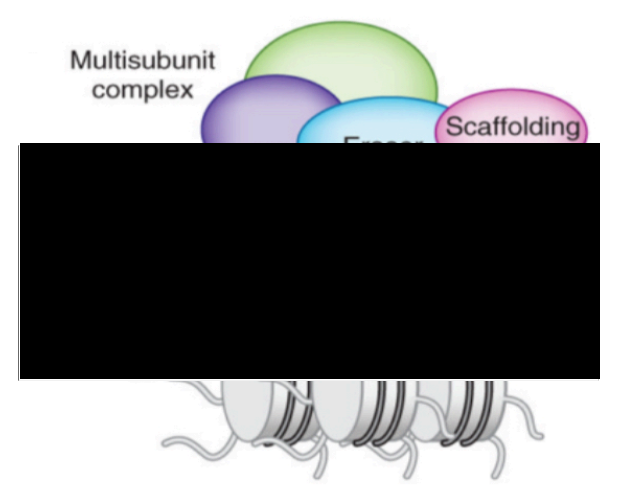
Figure 1.21 Structure of a canonical
protein multi-subunit complex

Multi-subunit protein complexes that exert epigenetic regulatory roles contain a wide variety of molecules, including epigenetic 'writers', 'readers', and 'erasers' as well as scaffolding subunits. Source: 'Perceiving the epigenetic landscape through histone readers' (Musselman et al. 2012).

Histone acetyltransferases have been shown to play a role in malignancies ranging from cancer and inflammatory diseases to neurological disorders (Yang 2004; Sheikh 2014). In cancer, HATs have been shown to suppress as well as to stimulate tumour growth and disease progression, even having opposite effects within the same type of cancer. For example, the 'E1A binding protein p300' gene ( $p 300$, also known as $K A T 3 B$ ) was suggested to function as a tumour suppressor gene in colorectal cancer (Gayther et al. 2000), but high levels of p300 transcripts were correlated with progression of the disease (Ishihama et al. 2007). Same counteracting effects are observed in the acetylation of non-histone targets. The "p300/CBPassociated factor' ( $P C A F$, also known as $K A T 2 B$ ) is known to acetylate c-MYC oncogene increasing its stability, a process that may lead to cancer progression (Patel et al. 2004). At the same time, PCAF is known to acetylate tumour suppressor TP53 protein activating its transcriptional activity, suggesting a protective function for PCAF (Liu et al. 1999). The exact role of HATs in cancer and the regulatory factors 
influencing HAT activity are therefore still under investigation (Wapenaar \& Dekker 2016).

In order to target functional disease-associated HAT activity, research has aimed at developing small molecule inhibitors as potential therapeutic agents (Legube \& Trouche 2003; Yang \& Seto 2007). Potential HAT inhibitors include molecules that interfere with acetyl-CoA and/or substrate binding, which would directly impair HAT activity. Such inhibition would affect the acetylation of histones and associated transcription of oncogenes or downstream oncogene targets, as well as the acetylation of non-histone substrates, such as p53, thereby affecting the stability of this transcription factor (Farria et al. 2015). Alternatively, small molecule inhibitors might be designed to either block interactions between HATs and other proteins, which would affect transcription of downstream targets, or the histone acetyl-lysine binding domain of HAT complexes, impairing the recognition of acetylated residues (Farria et al. 2015; Wapenaar \& Dekker 2016).

Almost all studies on HAT inhibitors are currently still in a pre-clinical phase and only curcumin has been moved forward into prostate cancer clinical trials as a potential anti-cancer therapy (Balasubramanyam et al. 2004). Curcumin is a natural HAT inhibitor that affects cell proliferation of prostate cancer cells through modulation of aberrantly activated Wnt signalling (Shah et al. 2012), suppresses p300 occupancy at androgen receptor $(A R)$ target genes and inhibits xenograft tumour growth of androgen sensitive cells (Diederich et al. 2011). Even though curcumin is indeed a HAT inhibitor, its biological effect cannot be solely appointed to HAT inhibition. For instance, curcumin has been shown to induce apoptosis of OC cell lines in a concentration- and time-dependent manner, via inhibition of the sarco/endoplasmic reticulum calcium-ATPase, which leads to an aberrant influx of cytosolic calcium (Seo et al. 2015). The two major challenges in designing reliable HAT inhibitors with guaranteed physiological effects are the variety of cell substrates (histones, transcription factors, enzymes, nuclear receptors) and the multiprotein complex structure, which determines HAT functions, enzymatic activity and substrate specificity (Wapenaar \& Dekker 2016). 
Surprisingly, most studies on HATs and cancer have been focused on the GNAT and p300/CBP families, leaving aside the highly conserved MYST family, whose members carry out a significant proportion of all nuclear acetylation and play central roles in fundamental cellular mechanisms including gene regulation (Thomas \& Voss 2007). Originally named after its four founding members in yeast and mammals, the MYST family currently comprises five human HATs: 'histone acetyltransferase binding to ORC-1' (HBO1, also known as KAT7 and MYST2), 'Tat interacting protein 60' (Tip60, also known as KAT5), 'lysine acetyltransferase 6B' (KAT6B, also known as MORF), 'lysine acetyltransferase 6A' (KAT6A, also known as MOZ) and the 'human males absent on the first' ( $h M O F$, also known as KAT8) (N Avvakumov \& Côté 2007). MYST HATs are defined by a conserved MYST domain composed of an acetyl-CoA binding motif (LAT domain) and a canonical $\mathrm{C}_{2} \mathrm{HC}$ zinc finger (Cys- $\mathrm{X}_{2}$-Cys- $\mathrm{X}_{12}$-His- $\mathrm{X}_{3}$-Cys) (Utley \& Côté 2003). Zinc fingers are small protein structural motifs, characterized by the coordination of one or more zinc ions that stabilize the fold, whose functions are extraordinarily diverse (Laity et al. 2001). This MYST domain possesses the ability to interact with DNA, RNA, proteins or other molecules through its zinc finger (Klug 2010) and introduce acetyl groups via the LAT motif (Yang 2004). Some family members also have additional chromatin recognition features such as extra plant homeodomain (PHD) zinc fingers and chromo-domains (Yang 2004).

So far, only Tip60 among the MYST family members has been contemplated as a therapeutic target (Ghizzoni et al. 2012). Tip60 is crucial for DNA repair, and it has been shown to be down-regulated in primary melanoma cancer cells and upregulated in drug-resistant prostate cancer cells (Miyamoto et al. 2008; Chen et al. 2012) respectively. Two newly identified Tip60 inhibitors are reported to inhibit cell proliferation and induce apoptosis in prostate cancer cells and breast cancer cells (Coffey et al. 2012; C. Gao et al. 2014). Interestingly, one of the least characterized members of the MYST family, HBO1, has been found overexpressed in OC tissues (Iizuka et al. 2009). 


\subsection{Histone Acetyltransferase Binding to ORC1 - HBO1}

The histone acetyltransferase binding to ORC-1 (HBO1) was originally identified interacting with the largest protein subunit of the human origin recognition complex (ORC) -ORC1 (Iizuka \& Stillman 1999). ORC is a six-subunit protein complex that binds to replicator sequences to initiate DNA replication in eukaryotes (Bell \& Stillman 1992; Dutta \& Bell 1997; Masai et al. 2010). The interacting sequence was identified as a 611 -amino acid protein $(\sim 80 \mathrm{KDa})$ involved in multiple key cellular processes such as replication and transcription regulation. HBO1 is also known as 'lysine acetyltransferase 7' (KAT7) (Allis et al. 2007) and 'MYST histone acetyltransferase 2' (MYST2).

HBO1 has two noteworthy functional domains: a serine-rich region at the aminoterminus domain (NTD) and a 270-amino acid MYST-type domain at the carboxylterminus tail (Iizuka \& Stillman 1999) (Fig. 1.22).

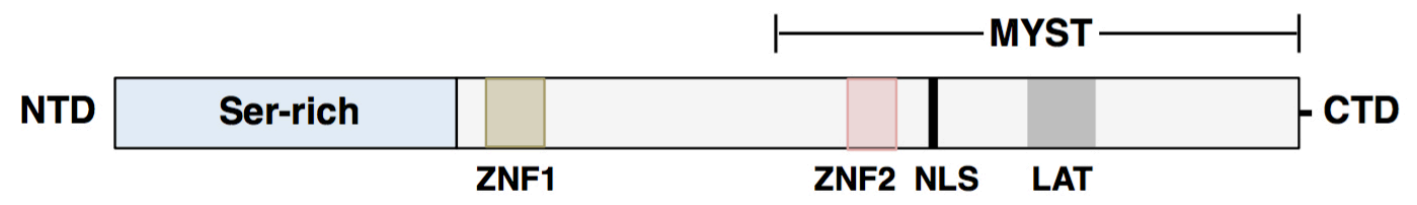

Figure 1. 22 Schematic representation of the structure of human protein HBO1 NTD: Amino-terminus domain; Ser-rich: Serine-rich domain; ZNF1, 2: Zinc finger 1 and 2; NLS: Nuclear localization signal; LAT: Lysine acetyltransferase domain; CTD: Carboxyl-terminus domain; MYST: MYST domain.

\subsubsection{Structure and functions of the N-terminus domain}

The N-terminal domain encompasses a putative zinc finger structure $\left(\mathrm{C}_{2} \mathrm{H}_{2} \mathrm{C}\right.$, amino acids 189-211) (Georgiakaki et al. 2006) and a rather unique serine-rich domain (amino acids 1-169) that had not been reported in any other member of the MYST family (Sharma et al. 2000) (Fig. 1.22). The serine-rich domain is highly enriched with threonine (Thr), tyrosine (Tyr) and serine (Ser) residues and, as such, is prone to protein phosphorylation (Dephoure et al. 2008). In fact, HBO1's Ser-rich domain is hyper-phosphorylated under normal conditions (Miotto \& Struhl 2011) and some well documented phosphorylation sites include: $\operatorname{Ser}^{50}, \mathrm{Ser}^{53}, \mathrm{Ser}^{57}, \mathrm{Thr}^{85}$ and $\mathrm{Thr}^{88}$ 
(Wu \& Liu 2008; Duong et al. 2013; Matsunuma et al. 2016). The phosphorylation of $\mathrm{Thr}^{85} / \mathrm{Thr}^{88}$ is carried out by the CDK1-Cyclin B protein complex, which creates a docking site for further phosphorylation by polo-kinase 1 (Plk1) at $\mathrm{Ser}^{57}$, activating HBO1's enzymatic activity ( $\mathrm{Wu} \&$ Liu 2008). The residue $\mathrm{Thr}^{88}$ can also be phosphorylated by the CDK2-Cyclin E complex, a process that does not activate HBO1's enzymatic activity (Duong et al. 2013). $\mathrm{Ser}^{50}$ and $\mathrm{Ser}^{53}$ are phosphorylated as a consequence of UV radiation (Matsunuma et al. 2016). The 'mitogen-activated protein kinase kinase 1' (Mek1) also phosphorylates HBO1 in vitro, marking HBO1 for deleterious-ubiquitination, although the exact residue has not been specified (Zou et al. 2013). With regard to other PTMs such as ubiquitination (Iizuka et al. 2013; Zou et al. 2013; Matsunuma et al. 2016) and acetylation (Iizuka et al. 2006; Iizuka et al. 2013), which target protein residues alongside the entire HBO1 protein, it is interesting to highlight that many other acetyltransferases have ubiquitin-associated capabilities (Sadoul et al. 2008) and that HBO1 auto-acetylates itself in vitro (Iizuka et al. 2006; Iizuka et al. 2013).

The NTD of HBO1 has been often conjectured as an intrinsic transcriptional repression domain (Sharma et al. 2000; Burke et al. 2001; Georgiakaki et al. 2006; Contzler et al. 2006). This theory is sustained by differing behaviours displayed by different HBO1 constructs in $\beta$-galactosidase assays (Smale 2010). Specifically, Sharma et al. (2000) conducted $\beta$-galactosidase experiments to compare the luciferase activity generated by full-length HBO1 protein, NTD-only or CTD (carboxyl-terminus domain)-only protein constructs. The highest levels of luciferase activity, and therefore the most transcriptionally active HBO1 structure, were detected with the CTD-only construct, followed by full-length HBO1 and finally NTD-only, suggesting the existence of a transcriptional repression function within the NTD domain of HBO1 (Sharma et al. 2000). Georgiakaki et al. (2006) obtained similar results when comparing full-length $\mathrm{HBO} 1$ and CTD-only constructs. Furthermore, when bound to $\mathrm{AR}, \mathrm{HBO} 1$ represses transcription from $\mathrm{AR}$ element containing promoters, an effect totally abrogated by the deletion of the first 230 amino acids of HBO1 (the NTD domain) (Sharma et al. 2000). 


\subsubsection{Structure and functions of the C-terminus domain}

The MYST-type region located at the C-terminus domain is highly conserved and has many structural similarities with several other MYST acetyltransferases (Utley \& Côté 2003). It comprises a canonical zinc finger structure $\left(\mathrm{C}_{2} \mathrm{HC}\right.$, amino acids 365-390), a LAT domain (Iizuka \& Stillman 1999; Burke et al. 2001) and a bipartite nuclear localization signal (NLS, amino acids 400-417) (Georgiakaki et al. 2006).

The MYST-domain, through the action of the LAT motif, acetylates histone H3 (at lysine residue K14 and possibly K9) (Kueh et al. 2011; Mishima et al. 2011; M. S. Kim et al. 2015), histone H4 (at lysine residues K5, K8, K12, K16) (Doyon et al. 2006; Iizuka et al. 2009; M. S. Kim et al. 2015) and other non-histone substrates (Choudhary et al. 2009). Among these, HBO1 acetylates ORC2, the 'minichromosome maintenance complex component 2' (MCM2), 'cell division control protein 6' (Cdc6), geminin, ER $\alpha$ and HBO1 itself (auto-acetylation) (Iizuka et al. 2006; Iizuka et al. 2013). In addition, the MYST domain is involved in the interaction with a myriad of proteins including:

- Nuclear receptors: AR (Sharma et al. 2000), ERa (Iizuka et al. 2013), progesterone receptor (PR) and the 'steroid-receptor coactivator-1' (SRC-1) (Georgiakaki et al. 2006).

- DNA replication complex-related proteins: ORC1 (Iizuka \& Stillman 1999), MCM2 (Burke et al. 2001), the 'chromatin licensing and DNA replication factor 1' (Cdt1) (Miotto \& Struhl 2008) and geminin (Miotto \& Struhl 2010).

- Transcription complex-related proteins: the 'JADE family PHD finger 1-3' (JADE1/2/3) (Avvakumov et al. 2012) and the 'bromodomain and PHD finger containing protein 1-3' (BRPF1/2/3) (Mishima et al. 2011; Lalonde et al. 2013; Feng et al. 2015).

- Other proteins such as CDK11p58 (Zong et al. 2005), Plk1 (Wu \& Liu 2008) and p53 (Iizuka et al. 2008). 
Despite being able to perform all these interactions, the preponderant competence of the MYST domain is the acetylation of histones. Because of this and to facilitate further descriptions, the MYST domain will be named the HAT domain and the capacity of this HAT domain to acetylate, HAT activity.

\subsubsection{HBO1 cell localization}

Northern blots showed HBO1 mRNA expressed ubiquitously in all tested healthy human tissues, with the lowest expression in liver, kidney, spleen and lung (Iizuka \& Stillman 1999; Sharma et al. 2000; Kueh et al. 2011); and the highest in ovaries (Iizuka \& Stillman 1999) or testis (Sharma et al. 2000; Kueh et al. 2011). For the most part, HBO1 is located evenly throughout the nucleoplasm in the nucleus (Sharma et al. 2000; Burke et al. 2001; Zong et al. 2005; Havasi et al. 2013), with the exception of the nucleoli where it does not accumulate (Doyon et al. 2006; Yan et al. 2016), and almost entirely associated with the chromatin fraction (Burke et al. 2001; Miotto \& Struhl 2008). Nonetheless, its degradation by the proteasome happens in the cytoplasm, where the 'Skp-Cullin 1-F box Fbxw15' ( $\mathrm{SCF}^{\mathrm{Fbxw15}}$ ) protein binds and marks HBO1 with poly-ubiquitin chains at the Lys ${ }^{338}$ residue, a process accelerated by HBO1's phosphorylation by the 'mitogen-activated protein kinase kinase 1' Mek1 (Zou et al. 2013). HBO1 protein has a short life span predicted half-life of $\sim 3 \mathrm{~h}-$ (Zou et al. 2013), resembling other acetyltransferases (Jain et al. 2012).

\subsubsection{Roles of HBO1 in DNA replication}

Replication is the mechanism through which our cells duplicate the genome in $\mathrm{S}$ phase (synthesis phase) of the cell cycle, once before each cellular division (Cooper 2000). An efficient replication requires a tight regulation of gene expression and chromatin structure, amongst other aspects, in order to co-ordinately initiate thousands of DNA replication origins (Méchali 2010; Parker et al. 2017).

The initiation of DNA replication can be divided into three tightly regulated steps. First, ORC is recruited onto replication origins as cells exit mitosis (Bell \& Dutta 2002). Second, pre-replication complexes (pre-RC) are assembled via the sequential 
loading of licensing factors Cdt1, Cdc6, and Mcm2-7 in a process termed licensing (Méchali 2010) (Fig. 1.23 A-C). The resulting pre-RC complex is licensed for activation that will occur in the subsequent S phase (Thömmes \& Blow 1997; Bell \& Dutta 2002). Finally, the activation/firing of the pre-RC results in bidirectional DNA replication from the origin, whereupon the pre-RC disassembles, restricting DNA synthesis to once per cycle (DePamphilis 2003; Remus \& Diffley 2009).

Regulation of Cdt1 is the key event in replication licensing that permits the ordered assembly and disassembly of the pre-RC (Arias \& Walter 2006). The Cdt1 protein lacks enzymatic activity and shares little resemblance to any other protein of known molecular function, yet it is essential for origin licensing in eukaryotes. In mammalian cells, small changes in Cdt1 control can lead to catastrophic consequences for genome stability, underscoring the paramount importance of this protein (Pozo \& Cook 2016).

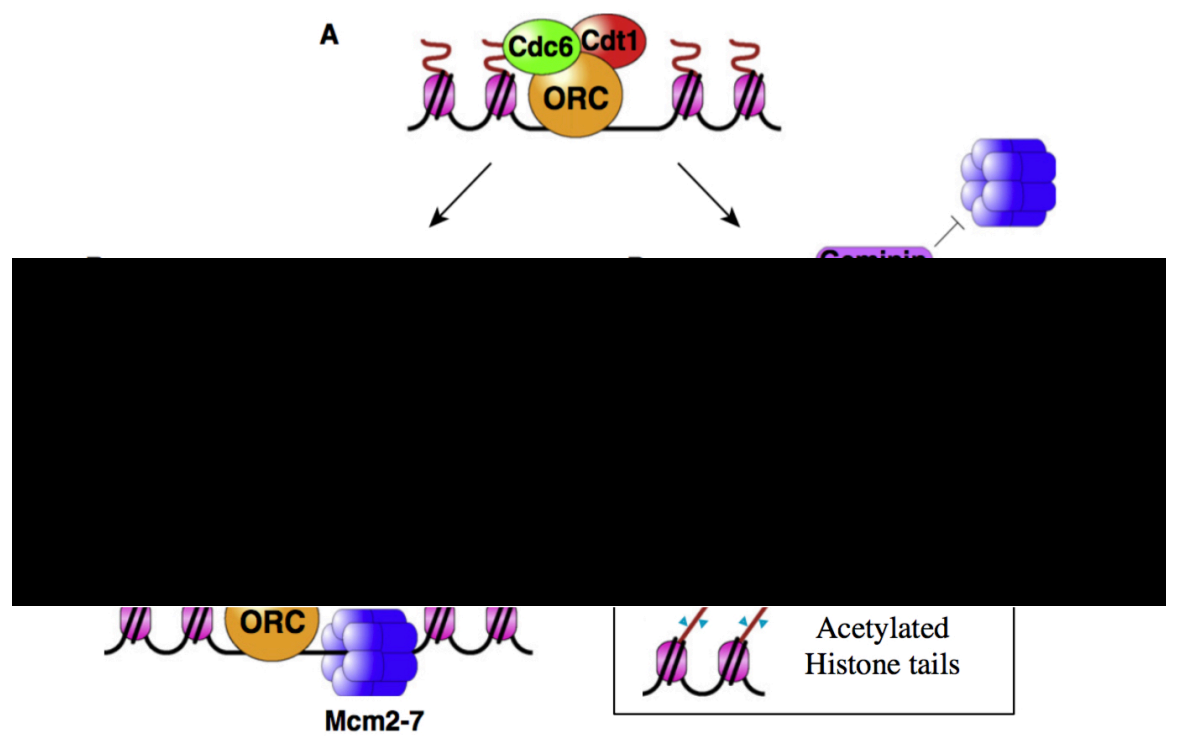

Figure 1. 23 Regulation of DNA replication licensing by $\mathrm{HBO1}$

(A) From late mitosis (M) to Gap1 (G1) phase, ORC, Cdc6 and Cdt1 sequentially load onto chromatin to mark origins of replication. (B) Cdt1-assisted binding of $\mathrm{HBO} 1$ to origins results in acetylation of histone $\mathrm{H} 4$ at origins. (C) The presence of ORC-Cdc6-Cdt1 and acetylated histones facilitates the loading of Mcm2-7 complex. (D) Geminin binds to Cdt1 during S and Gap2 (G2) phases and blocks its interaction with Mcm2-7 as well as inhibiting HBO1-mediated H4-acetylation, thus preventing reloading of Mcm2-7 once replication is initiated. Source: 'Histone acetylation by HBO1 tightens replication licensing' (Chadha \& Blow 2010). 
Histone acetylation is linked to pre-RC assembly and licensing (J. Zhou et al. 2005; Kemp et al. 2005; Lucas et al. 2007; Goren et al. 2008). For instance, early-firing origins are typically localized in hyper-acetylated genomic regions, whereas latefiring origins lie in silenced non-acetylated heterochromatic domains (Karnani et al. 2007).

HBO1 plays an active role in DNA replication and is thought to be required for normal cell cycle progression (Doyon et al. 2006). It is recruited to origins of replication in Gap1 (G1) phase via its interaction with Cdt1, where it acetylates H4 K5/K12 inducing large-scale chromatin de-condensation that stimulates Mcm2-7 complex recruitment thus enabling the pre-RC licensing (Iizuka et al. 2006; Johmura, Osada, et al. 2008; Miotto \& Struhl 2008; Miotto \& Struhl 2010) (Fig. 1.23 B, C). Once in late $\mathrm{S}$ phase, geminin and the 'histone deacetylase 11' (HDAC11) associate with Cdt1-HBO1, inhibiting HBO1's HAT activity, suppressing Mcm2-7 complex loading (likely by lose of $\mathrm{H} 4$ acetylation) and ultimately disassembling the pre-RC (Miotto \& Struhl 2010; Wong et al. 2010). HBO1 may also be implicated in the acetylation of other targets apart from $\mathrm{H} 4$ in DNA replication. For instance, it is partly responsible for H3 hyper-acetylation at terminal repeat regions, latent replication origins of Kaposi's sarcoma-associated herpesvirus (Stedman et al. 2004). The importance of HBO1 in replication licensing is highlighted by difficulties in progression through the replicative (S) phase, accumulation in the Gap2 (G2)/mitosis (M) DNA damage checkpoint and ultimately the reduced cell proliferation rates of HBO1 depleted cells (Doyon et al. 2006; Zou et al. 2013).

Synchronous localization of HBO1 at origins and changes in HAT activity, most active during G1 phase (pre-RC assembly and licensing), are cell cycle regulated (Iizuka et al. 2006). Conversely, mRNA expression and protein level/stability remain fairly constant throughout the whole cell cycle (Zong et al. 2005; Iizuka et al. 2006; Miotto \& Struhl 2008). Nonetheless, the association of HBO1 with non-origin target sites is not affected by cell cycle regulations, limiting these to origin-bound HBO1 (Miotto \& Struhl 2008). In line with this, HBO1 H4-dependent acetylation controls replication licensing independently of global effect at the transcriptional level (Miotto \& Struhl 2010). 
According to Iizuka et al. (2009), in an asynchronous population of normal human fibroblasts immortalized with telomerase there are $0.9 \sim 1.8$ molecules of HBO1 per origin of replication. These results suggest that in normal cells HBO1 is in a critical concentration range and its activity must be tightly regulated to participate in both replication licensing and transcription regulation (Iizuka et al. 2009).

\subsubsection{Roles of HBO1 in transcription}

The first evidence of the role of $\mathrm{HBO} 1$ in transcription regulation was the identification of HBO1-AR mediated transcriptional repression, an attribute that has also been associated to other MYST HATs such as Tip60 (Sharma et al. 2000). In the same publication, Sharma et al. (2000) claimed that, unlike with AR, HBO1 had no inhibitory effect on either ER $\alpha$ or thyroid hormone receptor beta (TR $\beta$ ) mediated transcription. Conversely, HBO1 enhances transcription mediated by PR, the mineralocorticoid receptor (MCR) or the glucocorticoid receptor (GCR) and strongly induces PR and ER $\alpha$ co-activation via interaction with the steroid-receptor coactivator-1 (SRC-1) (Georgiakaki et al. 2006). Furthermore, additional studies suggest that HBO1 enhances the activity of basic leucine zipper domain (bZIP) transcription factors (Miotto \& Struhl 2006), JADE1 (Foy et al. 2008) and p53 (Wright et al. 2016). This dichotomous behaviour of HBO1 displaying both activating and repressing properties was also observed with Chameau $(\mathrm{Chm})$, the drosophila HBO1 homolog (Grienenberger et al. 2002; Miotto et al. 2006). HBO1 expression in adult brain, a tissue consisting almost exclusively of post-mitotic cells, encouraged previous in vitro observations suggesting that $\mathrm{HBO} 1$ is likely to possess cellular functions aside from DNA replication in vivo (Kueh et al. 2011).

Using chromatin immune-precipitation (ChIP) experiments, HBO1 was detected mainly near the transcription starting site (TSS) of genes, whose expression level was strongly correlated with HBO1 signal intensity, suggesting a role of HBO1 in transcription initiation (Mishima et al. 2011; Avvakumov et al. 2012; Feng et al. 2015). Most importantly, HBO1 co-localizes with H3K4me3, a hallmark of actively transcribed protein-coding promoters in eukaryotes, permissive for acetylation and specifically connected to H3K9/14 acetylation (Wang et al. 2008; Hon et al. 2009). 
Interestingly, a great proportion of replication origins also localize at TSSs (Picard et al. 2014), highlighting the importance of HBO1 in both cellular processes.

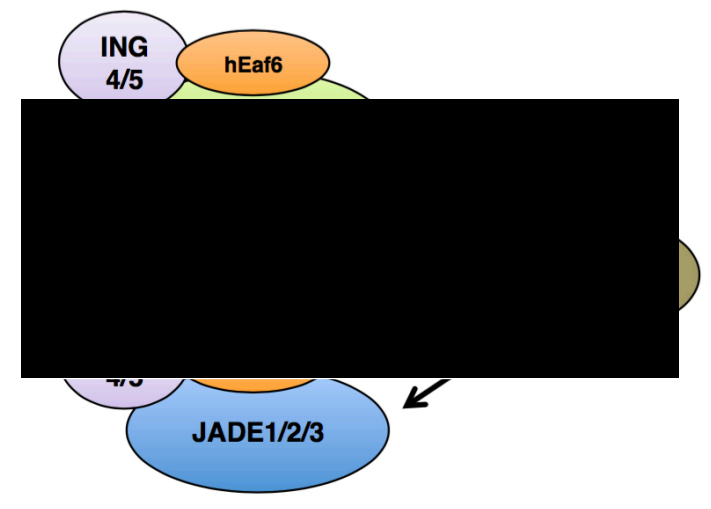

Figure 1. 24 Model for HBO1 assembly in alternate multi-protein complexes The HBO1 catalytic subunit can be associated with different scaffolding proteins, leading to a switch in histone tail specificity for acetylation of chromatin substrates. Source: 'Exchange of associated factors...' (Lalonde et al. 2013).

Other HBO1 complex-interacting proteins that co-localize to either the surroundings of the TSS or the coding region of genes are the 'inhibitor of growth family member 4' (ING4) (Hung et al. 2009), ING5 (Lalonde et al. 2013), JADE1 (Saksouk et al. 2009) and BRPF1/2 (Lalonde et al. 2013). Combinations between these and other protein subunits (JADE2/3, BRPF3 and the 'MYST/Esal associated factor 6' or hEaf6) might form up to 12 distinct HBO1-transcription complexes (Yan et al. 2016) (Fig. 1.24). Despite the complications of having such variety of subunits, all complexes share certain characteristics: (1) JADE1/2/3 or BRPF1/2/3 form the scaffold subunit and determine acetylation specificity (Lalonde et al. 2013), (2) ING proteins direct complex binding towards target epigenetic marks (Hung et al. 2009; Saksouk et al. 2009), (3) HBO1 alone exerts the HAT activity (lizuka et al. 2009) and (4) all complexes contain hEaf6, an uncharacterized protein also present in human Tip60 HAT complexes whose role is unknown (Avvakumov et al. 2012) (Fig. 1.24). 


\subsubsection{The HBO1 complex: JADE/BRPF families and ING4/5}

The 'gene for apoptosis and differentiation in epithelia' small family is comprised of three individual paralogs: JADE1 (PHF17), JADE2 (PHF15) and JADE3 (PHF16) (Ishikawa et al. 1998; Zhou et al. 2002). All three proteins contain two PHD fingers (Tzouanacou et al. 2003), well-characterized histone recognition motifs (Sanchez \& Zhou 2011; Musselman et al. 2012) (Fig. 1.25). Furthermore, the three paralogs interact with HBO1, ING4/5 and hEaf6 subunits (Doyon et al. 2006).

$J A D E 1$ is the most studied gene member of the JADE family (Panchenko 2016) and gives rise to two isoforms with different functions: JADE1L (long) and truncated JADE1S (short) (Tzouanacou et al. 2003) (Fig. 1.25). Apart from its part in histone acetylation (Panchenko et al. 2004), JADE1 is involved in cell cycle regulation (Siriwardana et al. 2015) and interacts with the 'von Hippel-Lindau' TSG (pVHL), suggesting a role in apoptosis and tumour suppression (Zhou et al. 2004; M. I. Zhou et al. 2005; Chitalia et al. 2008). Additionally, overexpression of JADE1L in Hela cells led to decreased colony formation in soft agar, a characteristic of TSGs (Avvakumov et al. 2012).

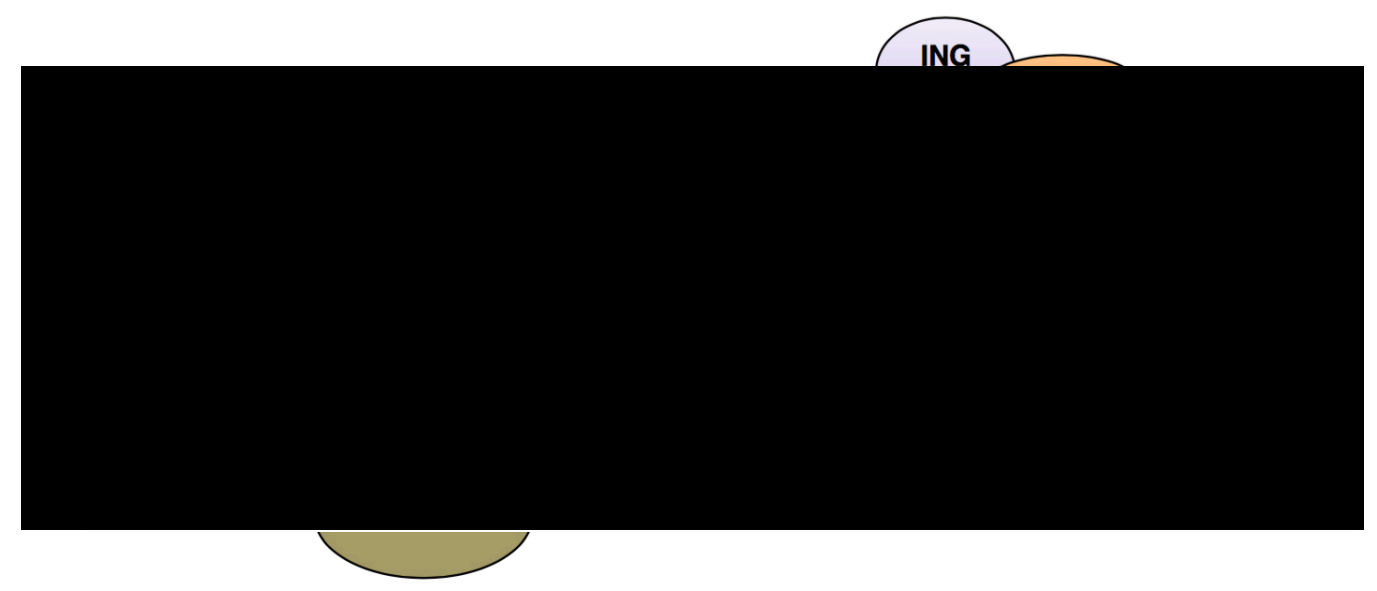

Figure 1. 25 Schematic representation of the structure and interactions of human proteins JADE1L and JADE1S

The picture depicts different models of interactions within JADE1L/S-HBO1 complexes. I, II: JADE interacting domains; PHD-Zn-PHD: nucleosome binding domain. Source: 'Conserved molecular interactions within the HBO1...' (Avvakumov et al. 2012). 
JADE1L functions as scaffold subunit of the HBO1 complex interacting directly and independently with hEaf6/ING4/5 and HBO1 (Avvakumov et al. 2012) (Fig. 1.25). When acting on chromatin, JADE1L's PHD fingers recognize different states of H3K4 methylation and direct the HAT activity to histone 4 lysine residues (Saksouk et al. 2009; Avvakumov et al. 2012). On the contrary, the truncated version JADE1S is unable to interact with ING4/5 or hEaf6 (Saksouk et al. 2009) (Fig. 1.25). This change in subunit conformation promotes a shift on complex specificity toward methylated H3K36me3 (Saksouk et al. 2009), a mark found on coding regions of transcribed genes, just downstream of H3K4 methylation (Bannister et al. 2005).

The 'bromodomain and PHD finger containing protein' family is comprised of three individual paralogs: BRPF1 (Thompson et al. 1994), BRPF2 (commonly known as BRD1) (McCullagh et al. 1999) and BRPF3 (Feng et al. 2015). All three proteins contain two PHD fingers, a bromo-domain and a chromo/Tudor-related Pro-Trp-TrpPro (PWWP) domain (Ullah et al. 2008). BRPF proteins function as scaffolding subunits of $\mathrm{HBO} 1$ complexes interacting, equivalently to JADE1/2/3, with hEaf6/ING4/5 and HBO1 (Doyon et al. 2006; Mishima et al. 2011). When acting on chromatin, BRPF's PHD fingers recognize different states of $\mathrm{H} 3 \mathrm{~K} 4$ methylation and direct the HAT activity to histone 3 lysine residues (Lalonde et al. 2013). The fact that BRPF proteins contain a PWWP domain that recognizes methylated H3K36 (Vezzoli et al. 2010) agrees with HBO1 complexes located not only near the TSS but also across the coding region of genes (Saksouk et al. 2009). In this line, a role for HBO1 complexes in transcription elongation has been proposed in several publications (Saksouk et al. 2009; Kueh et al. 2011; Mishima et al. 2011).

BPRF paralogs have separate functions despite their high similarity and large overlap in chromatin binding (Feng et al. 2015):

- The BRPF1 gene is required for embryonic survival and development (You et al. 2014; You et al. 2015), regulates growth and cell cycle progression (You et al. 2015). BRPF1-HBO1 complexes preferentially target H3K9/14/23 acetylation (Lalonde et al. 2013; You et al. 2015; Feng et al. 2015). Besides HBO1, BRPF1 interacts with MOZ/MORF acetyltransferases and enhances their HAT activity toward histone H3 (Ullah et al. 2008). 
- The BRPF2 gene is required for embryonic survival and foetal liver erythropoiesis (Mishima et al. 2011). BRPF2-HBO1 complexes preferentially target H3K14 acetylation (Mishima et al. 2011; Mishima et al. 2014).

- Unlike BRPF1 and BRPF2, the BRPF3 gene is non-essential for mouse development and survival (Yan et al. 2016). BRPF3-HBO1 complexes preferentially target H3K14 acetylation (Feng et al. 2015). Interestingly, BRPF3 was identified as a candidate gene involved in DNA replication (Feng et al. 2015) and co-localizes with ORC1 (Dellino et al. 2013), HBO1 (Avvakumov et al. 2012) and acetylated H3K14 (accession number: GSM521881). In line with this, Feng et al. (2015) proposed that BRPF3-HBO1 complexes promote H3K14 acetylation near TSSs/origins thus regulating the ability of licensed origins to be activated in $\mathrm{S}$ phase.

The 'inhibitor of growth' family has five members including ING4/5 which are involved in critical biological processes (He et al. 2005). ING4/5 proteins are part of HBO1 complexes and interact with the scaffolding subunits JADE1/2/3 (not JADE1S) and BRPF1/2/3 (Doyon et al. 2006; Mishima et al. 2011). Both ING4 and ING5 have a highly conserved PHD finger that interacts with H3K4me3 (Champagne et al. 2008; Hung et al. 2009), promoting HBO1 HAT activity towards H3K4me3-containing TSSs of active genes (Avvakumov et al. 2012).

ING4/5 are considered TSGs and display different activities (Piche \& Li 2010):

- ING4 harbours several anti-cancer activities such as inhibiting angiogenesis (Garkavtsev et al. 2004; Ozer \& Bruick 2005) and cell migration (Shen et al. 2007) or promoting cell death (Shiseki et al. 2003) and contact inhibition (Kim et al. 2004). Additionally, overexpression of ING4 in HeLa cells led to decreased colony formation in soft agar, a characteristic of TSGs (Avvakumov et al. 2012). ING4 expression is decreased in many cancer types (Liu et al. 2012), and this is often correlated with poor prognosis (Cui et al. 2015).

- ING5 TSG properties include inhibition of cell growth (F. Zhang et al. 2011), abrogation of EMT (F. Zhang et al. 2015) and promotion of cell death (Shiseki et al. 2003). While low levels of ING5 expression correlate with advanced stages and metastases, high levels are associated with better prognoses (F. Zhang et al. 
2015). Interestingly, ING5 depletion in $293 \mathrm{~T}$ cells blocked cell cycle progression through S-phase while no effect was observed when depleting ING4 (Doyon et al. 2006).

\subsubsection{HBO1 at the crossroads}

HBO1 gene knockout in mouse embryos led to loss of bulk H3K14 acetylation at gene coding regions and significant reduction in expression of key regulator genes involved in patterning the early embryo (e.g. MOZ, MORF, the 'lysine acetyltransferase' GCN5 and 'sex determining region Y box 1/2' or SOX1/2) (Kueh et al. 2011). This profile ultimately prompted embryonic lethality but, contrary to expectations, the embryo did not display any dysfunctionality in proliferation or DNA replication (Kueh et al. 2011). Surprisingly, Kueh et al. (2011) neither detected significant increases in expression of predicted HBO1-repressed genes or changes in H4 acetylation levels (Table 1.7).

The preponderance of $\mathrm{H} 3 \mathrm{~K} 14$ as the main target of $\mathrm{HBO} 1$ acetylation was observed in other knockout and stable knockdown studies (Table 1.7). For instance, Mishima et al. (2011) detected a great descence in H3K14 acetylation when transfecting erythroblasts with short hairpin RNAs (shRNA) targeting HBO1. Kim et al. (2015) depleted HBO1 using shRNAs in murine embryonic stem cells, observing acetylation deffects in H3K14 and H4K12 and, in this case, HBO1 knockdown also led to significant up and down regulation of gene targets (M. S. Kim et al. 2015) (Table 1.7). As with the latter, a considerable group of studies have reported H4 acetylation as the main target of HBO1 acetylation, highlighting its deeply specialised role in a tissue specific context (Sauer et al. 2015). Similarly, HBO1 is reported to have multiple and diverse roles, yet is non essential for cancer cell growth, as shown by the majority of knockdown experiments conducted thus far (Table 1.7).

HBO1 complexes play important roles both in DNA replication and transcription. As a consequence, it is reasonable to speculate that defects in its function might induce critical consequences to the cell and can easily lead to carcinogenesis (Nikita Avvakumov \& Côté 2007). For instance, origin hyper-activation in response to 
oncogenic signalling can drive genome instability (Halazonetis et al. 2008; Alver et al. 2014). In line with this, Iizuka et al. (2009) confirmed that HBO1 shows overexpression in testicular germ cell tumours, breast adenocarcinomas and ovarian serous carcinomas.

Table 1. 7 List of previous HBO1 knockdown experiments

\begin{tabular}{|c|c|c|}
\hline Experiment & $\begin{array}{c}\text { Altered } \\
\text { Acetylation (ac) }\end{array}$ & Related phenotype \\
\hline $\begin{array}{c}\text { Small interfering RNA } \\
\text { 293T embryonic kidney cells } \\
\text { (Doyon et al. 2006) }\end{array}$ & $\mathrm{H} 4 \mathrm{ac}$ & $\begin{array}{l}\text {-Reduced proliferation } \\
\text {-Affected DNA synthesis }\end{array}$ \\
\hline $\begin{array}{l}\text { Small interfering RNA } \\
\text { HeLa cervical cancer cells } \\
\text { (Avvakumov et al. } 2012 \text { ) }\end{array}$ & - & $\begin{array}{l}\text {-Down-regulation of p53- } \\
\text { regulated genes }\end{array}$ \\
\hline $\begin{array}{l}\text { Small interfering RNA } \\
\text { MCF7 breast cancer cells } \\
\text { (lizuka et al. 2013) }\end{array}$ & - & $\begin{array}{c}\text {-Enhanced anti-proliferative } \\
\text { effects of tamoxifen }\end{array}$ \\
\hline $\begin{array}{l}\text { Small interfering RNA } \\
\text { MLE-12 murine lung cells } \\
\text { (Zou et al. 2013) }\end{array}$ & H3K14ac & -Reduced cell proliferation \\
\hline $\begin{array}{l}\text { Short hairpin RNA } \\
\text { K562 leukemic cells } \\
\text { (Mishima et al. 2011) } \\
\end{array}$ & $\mathrm{H} 3 \mathrm{~K} 14 \mathrm{ac}$ & - \\
\hline $\begin{array}{l}\text { Short hairpin RNA } \\
\text { 76NE6-LMW-E cells } \\
\text { (Duong et al. 2013) }\end{array}$ & - & $\begin{array}{l}\text {-Reduced mammosphere } \\
\text { formation } \\
\text {-Reduced expression EMT } \\
\text { markers }\end{array}$ \\
\hline $\begin{array}{l}\text { Short hairpin RNA } \\
\text { THP-1 leukemic cells } \\
\text { (Sauer et al. 2015) }\end{array}$ & H4K5ac & -Increased colony formation \\
\hline $\begin{array}{c}\text { Short hairpin RNA } \\
\text { Murine embryonic stem cells } \\
\text { (M. S. Kim et al. 2015) }\end{array}$ & $\begin{array}{l}\mathrm{H} 3 \mathrm{~K} 14 \mathrm{ac} \\
\mathrm{H} 4 \mathrm{~K} 12 \mathrm{ac}\end{array}$ & $\begin{array}{l}\text {-Regulation of the Nanog } \\
\text { homeobox (Nanog) expression }\end{array}$ \\
\hline $\begin{array}{c}\text { KnockOUT } \\
\text {-/- Mice } \\
\text { (Kueh et al. 2011) } \\
\end{array}$ & $\mathrm{H} 3 \mathrm{~K} 14 \mathrm{ac}$ & $\begin{array}{l}\text {-Embryonic lethal E10.5 } \\
\text {-Reduction of key gene } \\
\text { regulators } \\
\end{array}$ \\
\hline \multicolumn{3}{|c|}{$\begin{array}{l}176 \text { NE6-LMW-E cells: human mammary epithelial cells derived from mammoplasty } \\
\text { were transfected with vectors expressing low molecular weight cyclin E isoform } \\
\text { subjected to mice xenograft transplantation and in vivo passaging. Tumours wer } \\
\text { removed and injected back for } 4 \text { successive generations to generate the resultant cel } \\
\text { line (Duong et al. 2013). }\end{array}$} \\
\hline
\end{tabular}




\subsubsection{Pro-oncogenic features of $\mathrm{HBO1}$}

Genome localization is amongst the reasons why $H B O 1$ is often considered an oncogene ( $\mathrm{Hu}$ et al. 2009), since $H B O 1$ maps to $17 \mathrm{q} 21.3$, a region that shows frequent allelic gains in a variety of tumours, including breast cancer (Clark et al. 2002), where this amplification is associated with poor prognosis (Hyman et al. 2002; Pollack et al. 2002). Furthermore, the HBOl locus site has been identified as a common retroviral integration site, leading to myeloid leukaemia and B/T-cell lymphoma in mice (Suzuki et al. 2002). Notably, different lines of study point to a prominent role of $\mathrm{HBO} 1$ in breast cancer tumour progression:

- In line with amplification events happening at the $17 \mathrm{q} 21.3$ locus, $H B O 1$ was found overexpressed in breast cancer tissues (Iizuka et al. 2009; Wang et al. 2010; Iizuka et al. 2013) and several breast cancer cell lines (Clark et al. 2002; Iizuka et al. 2009; Duong et al. 2013).

- Overexpression of HBO1 dramatically enhances the ability of MCF-7 and SKBR3 breast cancer cells to grow without anchorage (anoikis), a crucial step in the acquisition of malignancy (Hu et al. 2009).

- Depletion of HBO1 enhances the anti-proliferative effect of tamoxifen on MCF7 cells (Iizuka et al. 2013) and decreases cancer stem cell-associated properties of several breast cancer cell lines (e.g. mammosphere formation) (Duong et al. 2013).

Approximately two thirds of human breast cancers are dependent on estrogen for proliferation and express ER $\alpha$ (Jordan 1997). As mentioned earlier, HBO1 interacts with ER $\alpha$ and enhances its transcriptional activity, suggesting a role of HBO1 in regulating ER $\alpha$-positive breast cancer progression. In fact, HBO1 has been shown to modulate ER $\alpha$ stability in MCF7 cells (Iizuka et al. 2013), and its expression is regulated by estradiol (E2) (Wang et al. 2010). However, while Wang et al. (2010) found that $\mathrm{HBO} 1$ expression is significantly correlated with $\mathrm{ER} \alpha$ expression in breast cancer tissues, Iizuka et al. (2013) did not.

The tumour suppressor gene TP53 is often mutated in cancer and regulates multiple key cell cycle and apoptotic processes via transcription dependent and independent 
mechanisms (Vogelstein et al. 2000; Harris \& Levine 2005; Moll et al. 2005). In order to efficiently coordinate these actions, the stability and activity of p53 are in turn regulated by several HATs including p300, Tip60, PCAF and HBO1 (Barlev et al. 2001; Sykes et al. 2006; Tang et al. 2006; Sykes et al. 2009; Wright et al. 2016). HBO1 interacts with p53 (Iizuka et al. 2008) and participates of p53-mediated transcription via its HAT activity (Wright et al. 2016), regulating important genes such as the 'cyclin dependent kinase inhibitor 1A' (CDKN1A also known as $p 21)$ (Avvakumov et al. 2012; Wright et al. 2016). Interestingly, the interaction of p53 protein with HBO1 has the ability to inhibit HBO1's HAT activity (Iizuka et al. 2008), linking stress with replication halt.

HBO1 has also been associated with chemotherapy resistance, specifically with gemcitabine resistance in pancreatic cancer. In this setting, excess Plk1 phosphorylates $\mathrm{HBO} 1$ promoting the overexpression of transcription factors like cFos, a feature that eventually contributes to gemcitabine resistance (Song et al. 2013).

\subsubsection{Anti-oncogenic features of $\mathrm{HBO1}$}

The presence of HBO1 in complexes with TSGs ING4/5 and JADE paralogs imply that, as the catalytic subunit of each respective complex, HBO1 itself may contribute to TSG activities and cancer protection (N Avvakumov \& Côté 2007). Nonetheless, this is not the only setting where $\mathrm{HBO} 1$ seems to play a protective role in cancer. For instance, HBO1 expression is suppressed in patients with acute myeloid leukaemia (Sauer et al. 2015). Accordingly, the acetylation of HBO1's main target in this cell background -H4K5- was also suppressed, a circumstance that is associated with poor prognosis (Sauer et al. 2015). Furthermore, depletion of HBO1 in THP-1 and SEM human hematopoietic cell lines enhanced anchorage-independent growth (Sauer et al. 2015), while overexpression of HBO1 in NIH-3T3 mouse cells inhibited the growth of independent colonies (Johmura et al. 2008).

Other features of HBO1 that could indicate TSG behaviour are the cooperation with $\mathrm{CDK} 11 \mathrm{p}^{58}$ and the 'nuclear factor kappa B' $(\mathrm{NF}-\kappa \beta)$. CDK $11 \mathrm{p}^{58}$ is a protein kinase involved in the negative regulation of AR (Zong et al. 2007) whose expression is 
abnormally low in prostate cancer (Chi et al. 2014). Interestingly, CDK $11 p^{58}$ interacts with HBO1, another negative regulator of AR (Sharma et al. 2000), enhancing its HAT activity (Zong et al. 2005), which suggests a cooperative role in growth inhibition.

On the other hand, the NF- $\kappa \beta$ protein complex is a crucial player in tumorigenesis, immunity, inflammation, apoptosis and cell proliferation, and as such, it is constitutively active in many cancers (Hoesel \& Schmid 2013). HBO1 was shown to inhibit NF- $\kappa \beta$ activity, stimulated either by 'tumour necrosis factor alpha' (TNF $\alpha$ ) or by the overexpression of other co-activators of NF- $\kappa \beta$ signalling, in a dosedependent manner. However, HBO1 does not interact with NF- $\kappa \beta$ neither reduces its DNA binding capabilities, and so it has been proposed that HBO1 inhibits NF- $\kappa \beta$ activity by co-activator sequestration (Contzler et al. 2006).

\subsubsection{HBO1 and ovarian cancer}

As previously indicated, HBO1 was found overexpressed in a number of primary OC tissues (Iizuka et al. 2009). Specifically, Iizuka et al (2009) examined two independent panels of tumour samples containing 20 and 7 OC cases each. The first panel consisted of ovarian serous carcinomas (without any further histo-type differentiation), whereas no information about the histology of the second panel was provided. Five OC samples of the first panel (25\%) revealed strong positive signal for $\mathrm{HBO} 1$ and $30 \%$ of cases in the second panel showed more than $50 \%$ positive stained cells (Iizuka et al. 2009).

In accordance with $\mathrm{HBO} 1$ overexpression in $\mathrm{OC}$ tissues, MCM proteins are highly expressed in CICs (Freeman et al. 1999), possibly linking replication abnormalities with cancer progression. The role of HBO1 in replication is also linked with the promotion of adipogenesis (Johmura, Osada, et al. 2008), a critical phenomenon that boosts OC rapid metabolism, growth, and metastasis (Nieman et al. 2011). Additionally, ING4, an important partner of HBO1 and candidate TSG, is frequently mutated in OC (Liu et al. 2012; Cui et al. 2015). 


\subsection{Research Aims}

The main goal of the work reported herein was to explore the impact of HBO1 in OC, looking at its regulatory role of the transcriptome and epigenome in advanced forms of disease. This information will help to determine whether HBO1 would be a suitable target to develop a future therapeutic strategy.

In order to study the functions of HBO1 in $\mathrm{OC}$, an important pre-requisite was the selection of a panel of OC cell lines representative of different disease sub-types, as well as a normal ovarian epithelial non-cancerous control. This panel enabled feature comparisons between cancerous and non-cancerous cells, and, depending on the selected cell lines, comparisons amongst different sub-types or cancer staging. Once the panel of cell lines was established, the first goal was to define the expression pattern of HBO1. Assuming that $\mathrm{HBO} 1$ regulates important $\mathrm{OC}$ tumorigenic pathways, and as previously observed in cancer cell lines and OC tissues, its expression was expected to be higher in OC cell lines compared to the control.

Due to HBO1 known effects on steroid hormone-dependent transcription (e.g. via AR or ER nuclear receptors), its potential overexpression might functionally link DNA replication and transcription affecting growth and cell proliferation in OC. In order to find a possible correlation between the expression of nuclear receptors such as AR/ER and HBO1, their pattern of expression in the OC cell lines was assessed. Additionally, the existence of a potential causative relationship between hormones and HBO1 expression was studied.

Due to its HAT activity, overexpressed HBO1 protein is expected to localize nearby promoters/enhancers of OC-related oncogenes. To test that hypothesis, a chromatin immune-precipitation combined with sequencing (ChIP-Seq) experiment was planned. Prior to ChIP-Seq, a step-wise procedure for the optimization of ChIP experiments following the ENCODE (encyclopaedia of DNA elements) guidelines was observed. The validation of HBO1 protein enrichment in known genome sites was the most crucial step amongst the different stages of optimization. In order to help with this procedure, publicly available databases were perused to generate a battery of candidate $\mathrm{HBO} 1$ targets, which are potentially regulated by $\mathrm{HBO} 1$ in an 
OC setting. The downloaded material was handled using an SQL database system, which allowed for the reliable linkage and comparison of numerous datasets.

One important aim of the work reported herein was to unravel the functions of HBO1 in OC. A number of strategies aimed at knocking down the expression of HBO1 in OC cell lines, either transiently or permanently, were assessed in order to examine the specific roles of HBO1. These strategies included the complete knockout $(\mathrm{KO})$ of $\mathrm{HBOl}$ gene expression, the stable lentiviral transduction of shRNAs and the transient transfection of small interfering RNAs (siRNAs). If the hypothesis is correct, and HBO1 is involved in OC-oncogene up-regulation, the decrease of HBO1 transcript and protein expression levels would lead to the subsequent down-regulation of these potential targets, and more importantly, to the inhibition of HBO1-related OC tumorigenic features. However, a role of HBO1 as a tumour suppressor gene cannot be discounted, as it has been previously observed in the literature.

Successful modulation of HBO1 levels followed by gene profiling experiments, including RNA sequencing (RNA-Seq) and microarrays, was used to identify potential HBO1 targets. These gene transcripts were analysed using a set of bioinformatics tools to decipher signalling pathway involvement, as well as potential biological effects caused by the down-regulation of HBO1. The results obtained from the pathway analysis were used to select and design specific assays to confirm predicted phenotypical variations in vitro (e.g. proliferation assay). As well as the gene names and pathways involved in the OC HBO1 regulatory network, successful knockdown allowed the identification of histone acetylation targets within this setting.

Altogether, the successful completion of these goals would provide significant insights into the roles of $\mathrm{HBO} 1$ in OC progression, determining its suitability as biomarker and/or therapeutic target. 
2. Materials and Methods 


\subsection{Cell Lines and Culturing}

All cell lines purchased and used for experimentation throughout this thesis dissertation are depicted in Table 2.1. As well as multiple OC and cervical cancer derived cell lines; the Human Ovarian Surface Epithelial Cells (HOSEpiC) were used as non-cancerous control.

Table 2. 1 List of cell lines used as in vitro model

\begin{tabular}{|c|c|c|c|}
\hline Origin & Cell line & Product & Provider \\
\hline Non-cancerous & HOSEpiC (Murdoch 1995) & $\# 7310$ & ScienceCell $^{\mathrm{TM}}$ \\
\hline \multirow{7}{*}{ Ovarian Cancer } & OVCAR-3 (Hamilton et al. 1983) & HTB-161 & \multirow{6}{*}{$\operatorname{ATCC}^{\circledR}$} \\
\hline & SKOV-3 (Fogh et al. 1977) & НTB-77тм & \\
\hline & UWB1.289 (DelloRusso et al. 2007) & CRL-2945 TM & \\
\hline & UACC-1598 (Heiskanen et al. 2000) & CRL-3128 & \\
\hline & TOV-112D (Provencher et al. 2000) & CRL-11731 TM & \\
\hline & TOV-21G (Provencher et al. 2000) & CRL-11730 & \\
\hline & COV644 (Berg-Bakker et al. 1993) & 07071908 & Sigma $^{\circledR}$ \\
\hline Cervical Cancer & HeLa (Scherer et al. 1953) & CCL-2TM & $\mathrm{ATCC}^{(\mathbb{R}}$ \\
\hline
\end{tabular}

The different cell lines were maintained in their particular culture media, depending on special nutrient requirements, detailed in Table 2.2. Each media type was supplemented with a variety of compounds including foetal calf/bovine serum (FBS) (SH30071, HyClone ${ }^{\mathrm{TM}}$ ) in different concentrations and 1\% penicillin/streptomycin solution (30-002-CI, Corning), which includes $100 \mathrm{U} / \mathrm{mL}$ of penicillin and 100 $\mu \mathrm{g} / \mathrm{mL}$ of streptomycin (Table 2.2). HOSEpiC cells were grown in extracellular matrix coated-flasks $\left(\mathrm{G} 299, \mathrm{abm}^{\circledR}\right)$ and all cell lines were maintained at $37^{\circ} \mathrm{C}$ in a humidified $5 \% \mathrm{CO}_{2}$ incubator. 
Table 2. 2 List of cell lines culture media

\begin{tabular}{|c|c|c|c|}
\hline Cell line & Culture media & Provider & Supplements \\
\hline HOSEpiC & $\begin{array}{l}\text { Prigrow I } \\
(\mathrm{TM} 001)\end{array}$ & $\mathrm{abm}^{\circledR}$ & $\begin{array}{c}10 \% \text { FBS }+0.01 \% \\
\text { Ovarian Epithelial Cell } \\
\text { Growth Supplement } \\
\left(\# 7352, \text { ScienceCell }{ }^{\mathrm{TM}}\right)\end{array}$ \\
\hline OVCAR-3 & \multirow{2}{*}{$\begin{array}{l}\text { RPMI }^{1}-1640 \\
(30-2001)\end{array}$} & \multirow{2}{*}{$\mathrm{ATCC}^{\circledR}$} & \multirow{2}{*}{$\begin{array}{c}20 \% \mathrm{FBS}+10 \mu \mathrm{g} / \mathrm{mL} \\
\text { insulin solution from } \\
\text { bovine pancreas } \\
\left(\mathrm{I} 0516, \text { Sigma }^{\circledR}\right)\end{array}$} \\
\hline SKOV-3 & & & \\
\hline UWB1.289 & \multirow{2}{*}{$\begin{array}{c}\mathrm{DMEM}^{2} / \mathrm{F}-12 \\
+\mathrm{GlutaMAX}^{\mathrm{TM}} \\
(10565)\end{array}$} & \multirow{2}{*}{$\begin{array}{c}\text { Thermo } \\
\text { Scientific }^{\mathrm{TM}}\end{array}$} & \multirow{2}{*}{$10 \% \mathrm{FBS}$} \\
\hline UACC-1598 & & & \\
\hline TOV-112D & \multirow{2}{*}{$\begin{array}{c}\text { MCDB } 105(\mathrm{M} 6395) \\
+ \text { M199 (M4530) } \\
(1 / 2)\end{array}$} & \multirow{2}{*}{ Sigma $^{\circledR}$} & \multirow{2}{*}{$\begin{array}{c}15 \% \mathrm{FBS}+\mathrm{NaHCO}_{3} \\
(3.7 \mathrm{gr} / \mathrm{L})\end{array}$} \\
\hline TOV-21G & & & \\
\hline COV644 & $\begin{array}{c}\text { DMEM } \\
+ \text { GlutaMAX }^{\mathrm{TM}} \\
(10566) \\
\end{array}$ & $\begin{array}{c}\text { Thermo } \\
\text { Scientific }^{\mathrm{TM}}\end{array}$ & $10 \% \mathrm{FBS}$ \\
\hline HeLa & $\operatorname{EMEM}^{3}(30-2003)$ & $\mathrm{ATCC}^{\circledR}$ & $10 \% \mathrm{FBS}$ \\
\hline
\end{tabular}

${ }^{1}$ RPMI: Roswell park memorial institute medium

${ }^{2}$ DMEM: Dulbecco's modified Eagle's medium

${ }^{3}$ EMEM: Eagle's minimum essential medium

Depending on doubling time and confluence, cells were ordinarily passaged every 23 days using $0.25 \%$ trypsin (25-053-CI, Corning) for cell detachment. A Bright-Line and Hy-Lite counting chamber (267110, Thermo Scientific ${ }^{\mathrm{TM}}$ ), also called hemacytometer, and rectangular cover glasses $\left(2026 \mathrm{H} 001\right.$, Thermo Scientific ${ }^{\mathrm{TM}}$ ) were used for cell counting and seeding. In order to avoid extensive cell sub-culturing, cell pellets were frozen at low passages using 5\% dimethyl-sulfoxide (DMSO) (C6295, Sigma $^{\circledR}$ ) diluted in growth medium (medium supplemented with pertinent amounts of FBS and antibiotics). The stock of frozen cell pellets was preserved either in $80^{\circ} \mathrm{C}$ incubators (maximum 2-3 weeks) or Liquid Nitrogen Dewars (SPI supplies ${ }^{\circledR}$ ). After being thawed, all cells were passaged at least twice prior to their use in experimentation, allowing them to properly recover from the freezing procedure. 


\subsection{Antibodies}

The commercial antibodies described in Table 2.3 were used throughout this thesis in experiments such as western blot (WB), immune-precipitation (IP), immunefluorescence (IF), dot-blot (DB), chromatin immune-precipitation (ChIP) and ChIP combined with sequencing (ChIP-Seq).

Table 2. 3 List of commercial antibodies

\begin{tabular}{|c|c|c|c|c|}
\hline Primary antibodies & Application & Quantity & Product & Provider \\
\hline \multirow{5}{*}{ HBO1 (AB70) } & \multirow{5}{*}{$\begin{array}{c}\text { WB } \\
\text { IP } \\
\text { ChIP } \\
\text { ChIP-Seq } \\
\text { IF } \\
\text { DB } \\
\end{array}$} & $1: 1000$ & \multirow{5}{*}{ ab70183 } & \multirow{5}{*}{ Abcam $^{\circledR}$} \\
\hline & & $1 \mu \mathrm{g}$ & & \\
\hline & & $3-10 \mu \mathrm{g}$ & & \\
\hline & & $1: 100$ & & \\
\hline & & $1: 1000$ & & \\
\hline HBO1 (G-2) & $\begin{array}{c}\text { WB } \\
\text { IP } \\
\text { ChIP }\end{array}$ & $\begin{array}{c}1: 500 \\
1 \mu \mathrm{g} \\
5 \mu \mathrm{g}\end{array}$ & sc-398346 & Santa Cruz ${ }^{\circledR}$ \\
\hline \multirow{2}{*}{$\begin{array}{c}\text { H4ac (pan-acetyl) } \\
\text { H3K14ac }\end{array}$} & \multirow{9}{*}{ WB } & $1: 1000$ & 39244 & \multirow{3}{*}{ Active motif $^{\circledR}$} \\
\hline & & $1: 1000$ & 39599 & \\
\hline Histone H4 & & $1: 1000$ & 39269 & \\
\hline GAPDH & & $1: 1000$ & sc-25778 & \multirow{5}{*}{ Santa Cruz ${ }^{\circledR}$} \\
\hline CyPA/PPIA & & $1: 250$ & sc-133494 & \\
\hline XRCC1 & & $1: 100$ & sc-56254 & \\
\hline EGFR & & $1: 250$ & sc-03-G & \\
\hline \multirow{2}{*}{$\begin{array}{c}\text { Ubiquitin (P4D1) } \\
\text { ING4 }\end{array}$} & & $1: 100$ & sc- 8017 & \\
\hline & & $1: 500$ & ab108621 & Abcam $\left.^{(}\right)$ \\
\hline Anti-V5 tag & \multirow{2}{*}{ DB } & \multirow{2}{*}{$1: 1000$} & ab27671 & \multirow{2}{*}{ Abcam $^{\circledR}$} \\
\hline$\beta$-Actin (AC-15) & & & ab6276 & \\
\hline H3K4me3 & \multirow{6}{*}{ ChIP } & $3 \mu \mathrm{g}$ & 39159 & Active motif $^{(R)}$ \\
\hline HBO1 (T-20) & & $5 \mu \mathrm{g}$ & sc-13284 & \multirow{5}{*}{ Santa Cruz ${ }^{\circledR}$} \\
\hline HBO1 (N-18) & & $5 \mu \mathrm{g}$ & sc-13283 & \\
\hline HBO1 (H-180) & & $5 \mu \mathrm{g}$ & sc-25379 & \\
\hline Normal rabbit IgG & & $5 \mu \mathrm{g}$ & sc-2027 & \\
\hline Normal mouse IgG & & $5 \mu \mathrm{g}$ & sc-2025 & \\
\hline
\end{tabular}

\begin{tabular}{|c|c|c|c|c|}
\hline Secondary antibodies & Application & Quantity & Product & Provider \\
\hline $\begin{array}{l}\text { Alexa Fluor }^{(B)} 488 \\
\quad \text { (anti-rabbit) }\end{array}$ & IF & $1 / 500$ & A-11034 & Invitrogen $^{\mathrm{TM}}$ \\
\hline \multirow{2}{*}{ IgG anti-rabbit } & WB & $1: 2000$ & \multirow{2}{*}{ sc-2004 } & \multirow{2}{*}{ Santa Cruz $^{\circledR}$} \\
\hline & IP & $1 \mu \mathrm{g}$ & & \\
\hline \multirow{2}{*}{ IgG anti-mouse } & WB & $1: 2000$ & \multirow{2}{*}{ sc-2005 } & \multirow{2}{*}{ Santa Cruz $^{\circledR}$} \\
\hline & IP & $1 \mu \mathrm{g}$ & & \\
\hline
\end{tabular}




\subsection{Two Step quantitative Real Time-Polymerase Chain Reaction}

\subsubsection{Extraction of RNA and synthesis of complementary DNA}

First, confluent monolayers of cells were washed with phosphate buffered saline solution (PBS) (SH30256, HyClone ${ }^{\mathrm{TM}}$ ) pre-warmed at room temperature (RT) and then lysed using the RNA extraction RNeasy Kit (74104, Qiagen $\left.{ }^{\circledR}\right)$ RLT buffer. Subsequently, total cell RNA was isolated following manufacturer's instructions and quantified using a Nanodrop ${ }^{\circledR}$ Spectrophotometer ND-1000 (GE Healthcare, Version 3.8.1). Two hundred nanograms of total RNA were reverse transcribed to complementary DNA (cDNA) using SuperScript ${ }^{\circledR}$ II reverse transcriptase, dithiothreitol (DTT, 0.1M) (18064-014, Invitrogen ${ }^{\mathrm{TM}}$ ), random primers (48190-011, Thermo Scientific ${ }^{\mathrm{TM}}$ ), RNAseOUT recombinant ribonuclease inhibitor (10777-019, Invitrogen ${ }^{\mathrm{TM}}$ ) and a mix of deoxy-nucleotide triphosphates (dNTPs, 10mM) (18427013, Thermo Scientific ${ }^{\mathrm{TM}}$ ) following the first-strand synthesis protocol (Invitrogen ${ }^{\mathrm{TM}}$ ). The cDNA synthesis was conducted in a MJ Mini ${ }^{\mathrm{TM}}$ Personal Thermal Cycler (PTC1148EDU, Bio-rad).

\subsubsection{Design and characterization of primers}

At first, the messenger RNA (mRNA) sequence of the transcript of interest was extracted from the Reference Sequences Database (RefSeq) via the National Centre for Biotechnology Information (NCBI) website (O'Leary et al. 2016; NCBI 2017). The consensus coding DNA sequence or CCDS was uploaded into Primer3, a tool that generates batteries of candidate synthetic oligonucleotides, commonly named primers (Untergasser et al. 2012). The constitution and main characteristics of these primers was carefully analysed in order to select the most suitable choices. All quantitative Real Time-Polymerase Chain Reaction (qRT-PCR) primers selected for and used in this thesis share common characteristics:

- Product sizes range from 70 to 160 base pairs.

- Melting temperature close to $60^{\circ} \mathrm{C}$, unless specified otherwise.

- Primer PCR suitability assessed using the online tool 'PCR Primer Stats' from the 'Sequence Manipulation Suite' site, which analyses individual primers and 
returns a report describing their main structural and stability properties (Stothard 2000).

- Guanine-cytosine content lower than $60 \%$.

- When possible, primers span exon-exon boundaries to preclude amplification of genomic DNA (gDNA). The University of California Santa Cruz (UCSC) 'InSilico PCR' tool was used to verify mRNA product amplification (Kent et al. 2002).

- If possible and unless specifically stated, all primers amplify a region shared among all the isoforms of the pertinent gene target. NCBI's 'Primer-BLAST' tool was used to confirm product amplification (Ye et al. 2012).

All primers were purchased from Sigma ${ }^{\circledR}$ and received lyophilized. After a quick spin, each primer was reconstituted to a concentration of $100 \mu \mathrm{M}$ (e.g. $50 \mathrm{nmol} / 500 \mu \mathrm{l}$ ) in $1 \mathrm{X}$ TE buffer solution containing $1 \mathrm{M}$ Tris- $\mathrm{HCl}$ (15568-025, Thermo Scientific $\left.{ }^{\mathrm{TM}}\right)$ and 100mM ethylenediaminetetraacetic acid (EDTA) (AM9260G, Thermo Scientific $\left.{ }^{\mathrm{TM}}\right)$, diluted in $1 \mu \mathrm{M}$ stocks and stored at $-20^{\circ} \mathrm{C}$.

Prior to their experimental use, primers were tested in order to confirm the generation of single melting curves. Two primer pairs were always designed and purchased for each target gene so that at least one would amplify an individual product. All validated primers used for qRT-PCR in this thesis are listed in Table 2.4.

Table 2. 4 List of qRT-PCR primers

\begin{tabular}{|c|l|c|}
\hline \multicolumn{3}{|c|}{ Primers used throughout the thesis } \\
\hline Target / Primer & \multicolumn{1}{|c|}{ Sequence } & Product size \\
\hline $\begin{array}{c}\text { GAPDH } \\
\text { (Reference gene) }\end{array}$ & $\begin{array}{l}\text { F: 5'-TCTTTTGCGTCGCCAGCCGA-3' } \\
\text { R: 3'-CCAGGCGCCCAATACGACCA-5' }\end{array}$ & 91 \\
\hline $\begin{array}{c}\text { RPL5 } \\
\text { (Reference gene) }\end{array}$ & $\begin{array}{l}\text { F: 5'-GCAGGATGGGGTTTGTTAAA-3' } \\
\text { R: 3'-CAAGCGTTTCCGAGCATAAT-5' }\end{array}$ & 113 \\
\hline $\begin{array}{c}\text { HBO1 } \\
\text { (All isoforms) }\end{array}$ & $\begin{array}{l}\text { F: 5'-CTATGGGAACACACGGGAAC-3' } \\
\text { R: 3'-TTGCTTCCCTCTGTGATTTG-5' }\end{array}$ & 138 \\
\hline CDKN1A/p21 & $\begin{array}{l}\text { F: 5'-GCACTCAGAGGAGGCGCCATGT-3' } \\
\text { R: 3'-GCAGCCCGCCATTAGCGCAT -5' }\end{array}$ & 141 \\
\hline
\end{tabular}




\begin{tabular}{|c|c|c|}
\hline \multicolumn{3}{|c|}{ 3. HBO1 Expression and Regulation in Ovarian Cancer } \\
\hline Target / Primer & Sequence & Product size \\
\hline $\begin{array}{c}\text { HBO1 } \\
\text { Isoform } 1\end{array}$ & $\begin{array}{l}\text { F: 5'-TGTCCTACACCAGGCTGTAACT-3' } \\
\text { R: 3'-TGTGCTCTCACCTTGCATTC-5' }\end{array}$ & 122 \\
\hline $\begin{array}{c}\text { HBO1 } \\
\text { Isoform } 2\end{array}$ & $\begin{array}{l}\text { F: 5'-GGCTGTAACTCTCTAGGACACC-3' } \\
\text { R: 3'-CGTTGGTGCCTTGCATT-5' }\end{array}$ & 108 \\
\hline $\begin{array}{c}\text { HBO1 } \\
\text { Isoform } 3\end{array}$ & $\begin{array}{l}\text { F: 5'-GCCAGAGTTCTCAAGGACACC-3' } \\
\text { R: 3'-TGTGCTCTCACCTTGCATTC-5' }\end{array}$ & 109 \\
\hline $\begin{array}{c}\text { HBO1 } \\
\text { Isoform } 4\end{array}$ & $\begin{array}{l}\mathbf{F}: \text { 5'-GCAAGTGGTTGATTTTTCAG-3' } \\
\text { R: 3'-ATACAGTGGGCATCCTGAG-5' }\end{array}$ & 82 \\
\hline $\begin{array}{c}\mathrm{HBO} 1 \\
\text { Isoform } 5\end{array}$ & $\begin{array}{l}\text { F: 5'-GCCAGAGTTCTCAAGGACACC-3' } \\
\text { R: 3'-TTGGTGCCTTGCATTCGT-5' }\end{array}$ & 105 \\
\hline AR & $\begin{array}{l}\text { F: 5'-CGCTGAAGGGAAACAGAAGT-3', } \\
\text { R: 3'-CGAAGACGACAAGATGGACA-5' }\end{array}$ & 93 \\
\hline $\mathrm{ER} 1 / \alpha$ & $\begin{array}{l}\text { F: 5'-TGCTGCTGGCTACATCATCT-3' } \\
\text { R: 3'-TCCAGAGACTTCAGGGTGCT-5' }\end{array}$ & 130 \\
\hline $\mathrm{ER} 2 / \beta$ & $\begin{array}{l}\mathbf{F}: \text { 5'-GTGGTCCATCGCCAGTTATC-3', } \\
\text { R: 3'-ACGGTTCCCACTAACCTTCC-5' }\end{array}$ & 129 \\
\hline CASP4 & $\begin{array}{l}\mathbf{F}: \text { 5'-TCTCCAGCATCCTTGGAAGT-3' } \\
\text { R: 3'-TCTCCAGGACACGTTGTGTG-5' }\end{array}$ & 132 \\
\hline KRT7 & $\begin{array}{l}\mathbf{F}: \text { 5'-CAGGATGTGGTGGAGGACTT-3' } \\
\text { R: 3'-TTGCTCATGTAGGCAGCATC-5' }\end{array}$ & 116 \\
\hline LCN2 & $\begin{array}{l}\text { F: 5'-CTCTACGGGAGAACCAAGGA-3' } \\
\text { R: 3'-CAGGGAAGACGATGTGGTTT-5', }\end{array}$ & 100 \\
\hline TRAP1 & $\begin{array}{l}\mathbf{F}: \text { 5'-CTTGGAAAAACTGCGTCACA-3' } \\
\text { R: 3'-TCCTGTGTCATCCCGATACC-5' }\end{array}$ & 129 \\
\hline JADE1L & $\begin{array}{l}\text { F: 5'-CTGTGTGCAAAGTCCAGGAA-3' } \\
\text { R: 3'-TTTTCCAGTGAGGAGGAGGA-5', }\end{array}$ & 106 \\
\hline JADE1S & $\begin{array}{l}\text { F: 5'-GAAGAGGACAATCTAGCCAAGC-3' } \\
\text { R: 3'-TCAATCATTACCCTCTCCAGGT-5' }\end{array}$ & 98 \\
\hline JADE2 & $\begin{array}{l}\mathbf{F}: \text { 5'-TCGGAGCTTAAGGAGATGGA-3' } \\
\text { R: 3'-CAACATCCTCGTCGTACTCG-5' }\end{array}$ & 148 \\
\hline JADE3 & $\begin{array}{l}\mathbf{F}: \text { 5'-GGAGTTGGCAGCATCTGTTT-3' } \\
\text { R: 3'-TGGCGTTCCAGGACTTCTAC-5' }\end{array}$ & 147 \\
\hline BRPF1 & $\begin{array}{l}\mathbf{F}: \text { 5'-AAGATGCCCCGAGAAGGTAT-3' } \\
\mathbf{R}: \text { 3'-CACTGCCAGGTTCGTTTGTT-5' }\end{array}$ & 149 \\
\hline BRPF2 & $\begin{array}{l}\mathbf{F}: \text { 5'-AGCGGAAGAAGCAGTTTGTG-3' } \\
\text { R: 3'-GCAGCCTTCATCTCCTCATC-5' }\end{array}$ & 154 \\
\hline BRPF3 & $\begin{array}{l}\mathbf{F}: \text { 5'-TCAAACGAGAGCAGGTCAAA-3' } \\
\mathbf{R}: \text { 3'-TGACTGGTTCTGCGAAGATG-5' }\end{array}$ & 132 \\
\hline ING4 & $\begin{array}{l}\text { F: 5'-GGAGAAGAAAGCTGCTCGTG-3' } \\
\text { R: 3'-CTTCTTCTGGGCAGTCTTGG-5' }\end{array}$ & 76 \\
\hline ING5 & $\begin{array}{l}\text { F: 5'-CATCCTGGCTGCAGAGTACA-3' } \\
\text { R: 3'-CACTTGCTGTAGGCGTTCTG-5' }\end{array}$ & 120 \\
\hline
\end{tabular}




\begin{tabular}{|c|l|c|}
\hline \multicolumn{2}{|l|}{ 5. Functional HBO1 Evaluation using a Stable RNAi-based Knockdown System } \\
\hline Target / Primer & \multicolumn{1}{|c|}{ Sequence } & Product size \\
\hline HDAC6 & $\begin{array}{l}\text { F: 5'-CTCAACAGAAACACCGCATC-3' } \\
\text { R: 3'-GCGGTGGATGGAGAAATAGA-5' }\end{array}$ & 119 \\
\hline SNAP47 & $\begin{array}{l}\text { F: 5'-TCAGAAGCGCAAGAACCTCT-3' } \\
\text { R: 3'-GTTCCTGGGTATCTGCCTCA-5' }\end{array}$ & 174 \\
\hline STAT6 & $\begin{array}{l}\text { F: 5'-GGAAGGGCACTGAGTCTGTC-3' } \\
\text { R: 3'-GGCTTTGGCATTGTTGTCTT-5' }\end{array}$ & 155 \\
\hline PCM1 & $\begin{array}{l}\text { F: 5'-CCCAGAGCAGGCAGAATTAG-3' } \\
\text { R: 3'-AAGGGCTTTCGGTTTTCACT-5' }\end{array}$ & 141 \\
\hline PSM2 & $\begin{array}{l}\text { F: 5'-CACTACCTGTGCGCCATAAG-3' } \\
\text { R: 3'-CAGGCTGTCGTTTTCCTTGT-5' }\end{array}$ & 147 \\
\hline TFRC & $\begin{array}{l}\text { F: 5'-TGGACAGCACAGACTTCACC-3' } \\
\text { R: 3'-ACGCCAGACTTTGCTGAGTT-5' }\end{array}$ & 146 \\
\hline
\end{tabular}

\begin{tabular}{|c|c|c|}
\hline \multicolumn{3}{|c|}{$\begin{array}{l}\text { 6. Functional HBO1 Evaluation using a Transient RNAi-based Knockdown } \\
\text { System }\end{array}$} \\
\hline Target / Primer & Sequence & Product size \\
\hline BST2 & $\begin{array}{l}\mathbf{F}: \text { 5'-TCTCCTGCAACAAGAGCTGA-3' } \\
\text { R: 3'-CCСТCAAGCTCCTCCACTTT-5' }\end{array}$ & 150 \\
\hline ISG15 & $\begin{array}{l}\text { F: 5'-GACAAATGCGACGAACCTCT-3' } \\
\text { R: 3'-GCTCACTTGCTGCTTCAGG-5' }\end{array}$ & 111 \\
\hline STAT1 & $\begin{array}{l}\text { F: 5'-TGGGCTCAGCTTTCAGAAGT-3' } \\
\text { R: 3'-CTGGCGTTAGGACCAAGAAG-5' }\end{array}$ & 113 \\
\hline CD46 & $\begin{array}{l}\mathbf{F}: \text { 5'-GTCCAGTGCCTCAGGTCCTA-3' } \\
\text { R: 3'-CACAGCAATGACCCAAACAT-5' }\end{array}$ & 115 \\
\hline FGFR4 & $\begin{array}{l}\text { F: 5'-TGGAGTCCCGGAAGTGTATC-3' } \\
\text { R: 3'-GCCCAAAGTCAGCAATCTTC-5', }\end{array}$ & 90 \\
\hline YAP1 & $\begin{array}{l}\mathbf{F}: \text { 5'-CTCGAACCCCAGATGACTTC-3' } \\
\text { R: 3'-TCCAAGGTCCACATTTGTCC-5' }\end{array}$ & 140 \\
\hline AAK1 & $\begin{array}{l}\text { F: 5'-GGCACAGGCGTATTCTCAGT-3' } \\
\text { R: 3'-GAGGAGAGCCTGATGGAGTG-5' }\end{array}$ & 147 \\
\hline NUCKS1 & $\begin{array}{l}\text { F: 5'-CGGCCTGTCAGAAATAGGAA-3' } \\
\text { R: 3'-GCCCGAATCTCTTCCATAATC-5' }\end{array}$ & 87 \\
\hline STK11IP & $\begin{array}{l}\text { F: 5'-GCTCTTCCTGGATGTTGAGG-3' } \\
\text { R: 3'-CGGTCAGACACAACCACAAG-5' }\end{array}$ & 123 \\
\hline MIB1 & $\begin{array}{l}\text { F: 5'-TGCCAATGGAGATGTTGCTA-3' } \\
\text { R: 3'-GACATCCACGTTTTGCTTCA-5' }\end{array}$ & 154 \\
\hline SPAST & $\begin{array}{l}\text { F: 5'-GGTCCTGCTCCTACCACTCA-3' } \\
\text { R: 3'-AAGGTTGCTGTCCACATTCC-5' }\end{array}$ & 126 \\
\hline SUMO1 & $\begin{array}{l}\text { F: 5'-CAAAGACAGGGTGTTCCAATG-3' } \\
\text { R: 3'-TCCATTCCCAGTTCTTTTGG-5' }\end{array}$ & 103 \\
\hline $\mathrm{CCNG} 2$ & $\begin{array}{l}\mathbf{F}: \text { 5'-GCTACCCCGGAGAATGATAA-3' } \\
\text { R: 3'-AAGAGCCAAGAACCTGTCCA-5', }\end{array}$ & 144 \\
\hline
\end{tabular}




\begin{tabular}{|c|c|c|}
\hline DACT1 & $\begin{array}{l}\mathbf{F}: \text { 5'-ACAGACAGTCGGCCTAGCTC-3' } \\
\text { R: 3'-GTGCTGGAATGACAACTGGA-5' }\end{array}$ & 116 \\
\hline SLC2A12 & $\begin{array}{l}\text { F: 5'-AGGGATGCTCTTTGGAACAA-3' } \\
\text { R: 3'-TGAGGCTGTTTTGGCACTAA-5' }\end{array}$ & 112 \\
\hline NIPAL1 & $\begin{array}{l}\mathbf{F}: \text { 5'-CCTCTGGGTGCTTTGAGTGT-3' } \\
\mathbf{R}: \text { 3'-CCATCACAGTTGACCCCAAT-5' }\end{array}$ & 121 \\
\hline PLAGL2 & $\begin{array}{l}\mathbf{F}: \text { 5'-GCAACCAGAGCAGAGACCAT-3' } \\
\mathbf{R}: \text { 3'-GTCCTTGCGGTGAAACATCT-5' }\end{array}$ & 151 \\
\hline SAR1A & $\begin{array}{l}\text { F: 5'-CAATATCCAATGTGCCAATCC-3' } \\
\text { R: 3'-ATTCCCCTTTCCTGTGGTCT-5' }\end{array}$ & 119 \\
\hline H2AFY & $\begin{array}{l}\text { F: 5'-GCCCTGGCTGATGATAAGAA-3', } \\
\text { R: 3'-TGGAGATGGCCTTCAGAATC-5', }\end{array}$ & 112 \\
\hline TSPAN2 & $\begin{array}{l}\mathbf{F}: \text { 5'-TCCGAACAGGTCCAACCTAC-3' } \\
\mathbf{R}: \text { 3'-CCAATACCGACAATTCCAATG-5' }\end{array}$ & 116 \\
\hline КАТ2B & $\begin{array}{l}\mathbf{F}: \text { 5'-AGATCCTGATGTGGCTGGTT-3' } \\
\text { R: 3'-AGCAAGGGTTTTGTGTTTCG-5' }\end{array}$ & 113 \\
\hline MYC & $\begin{array}{l}\text { F: 5'-CAGCGACTCTGAGGAGGAAC-3' } \\
\text { R: 3'-CTGTGAGGAGGTTTGCTGTG-5' }\end{array}$ & 132 \\
\hline MUC16 & $\begin{array}{l}\mathbf{F}: \text { 5'-CCCTACACCCTGGACAGAAA-3' } \\
\mathbf{R}: \text { 3'-GAGAGGGCCAGCAGATGTAG-5' }\end{array}$ & 153 \\
\hline DDX60 & $\begin{array}{l}\mathbf{F}: \text { 5'-AGGGAGATGACCCAGAGGAT-3' } \\
\mathbf{R}: \text { 3'-TAGCCCTCTTTCACCAGGAA-5' }\end{array}$ & 142 \\
\hline IFI44 & $\begin{array}{l}\text { F: 5'-AGCCGTAGTGGGGTCTGATA-3' } \\
\text { R: 3'-TGTCATCCATGCACAGTCCT-5' }\end{array}$ & 152 \\
\hline RSAD2 & $\begin{array}{l}\mathbf{F}: \text { 5'-CTCGCCAGTGCAACTACAAA-3' } \\
\text { R: 3'-AGAAATGGCTCTCCACCTGA-5' }\end{array}$ & 148 \\
\hline IFNA/ $\alpha$ & $\begin{array}{l}\mathbf{F}: \text { 5'-CTGAAACCATCCCTGTCCTC-3' } \\
\text { R: 3'-ATCACACAGGCTTCCAGGTC-5', }\end{array}$ & 151 \\
\hline $\mathrm{IFNB} / \beta$ & $\begin{array}{l}\mathbf{F}: \text { 5'-CAGCAGTTCCAGAAGGAGGA-3' } \\
\text { R: 3'-AGCCAGGAGGTTCTCAACAA-5' }\end{array}$ & 126 \\
\hline IFNG/ $\gamma$ & $\begin{array}{l}\mathbf{F}: \text { 5'-TGACCAGAGCATCCAAAAGA-3' } \\
\text { R: 3'-TGTATTGCTTTGCGTTGGAC-5' }\end{array}$ & 147 \\
\hline
\end{tabular}

\subsection{3 qRT-PCR}

qRT-PCR experiments were conducted in a LightCycler ${ }^{\circledR} 480$ II instrument (05015243001, Roche) with the LightCycler ${ }^{\circledR} 480$ Software 1.5.0 SP3 (Version 1.5.0.39), using 384-well LightCycler ${ }^{\circledR} 480$ Multi-well Plates (4729749001, Roche) and Thermal Seal RT ${ }^{\mathrm{TM}}$ sealing films (TS2-100, EXCEL Scientific, Inc.). Each qRTPCR reaction was used to detect the presence of a specific cDNA sequence in the target sample by means of primer pair amplification, allowing for the determination of gene transcript enrichment relative to a standard or reference gene (Bustin et al. 2009). 
The different components of each qRT-PCR reaction were assembled in a sample mixture that contained: $5 \mu 1$ of $2 \mathrm{X}$ Roche 480 SYBR Green I MasterMix (04707516001, Roche), $1 \mu 1$ of nuclease-free water (AM9937, Thermo Scientific ${ }^{\mathrm{TM}}$ ), $2 \mu l$ of diluted cDNA sample (1/10 dilution in nuclease-free water unless specified otherwise) and $2 \mu \mathrm{l}$ of primer mix (1 $\mu \mathrm{l}$ of each forward and reverse primer stock $[1 \mu \mathrm{M}])$. An example of the process of preparation of qRT-PCR reaction mixtures is depicted in Table 2.5.

Table 2. 5 Example of master mix sample preparation for qRT-PCR

\begin{tabular}{|c|c|c|c|c|c|}
\hline $\begin{array}{c}\text { Master mix } \\
\text { (MM) }\end{array}$ & Composition & $\begin{array}{l}\text { Vol. } \\
\text { MM }\end{array}$ & $\begin{array}{l}\text { Separation } \\
3 \text { reactions }\end{array}$ & $\begin{array}{l}\text { Addition of } \\
\text { Primer mix }\end{array}$ & $\begin{array}{l}\text { Total Vol. } \\
\text { (Reaction) }\end{array}$ \\
\hline \multirow{3}{*}{$\begin{array}{c}\text { Treatment } \\
\text { (3 reactions) }\end{array}$} & $15 \mu \mathrm{l} \mathrm{SYBR}$ & \multirow{3}{*}{$24 \mu 1$} & $8 \mu \mathrm{l}$ & $2 \mu l$ Reference & \multirow{6}{*}{$10 \mu 1$} \\
\hline & $3 \mu 1$ water & & $8 \mu 1$ & $2 \mu \mathrm{l}$ Query 1 & \\
\hline & $6 \mu \mathrm{l}$ cDNA & & $8 \mu 1$ & $2 \mu 1$ Query 2 & \\
\hline \multirow{3}{*}{$\begin{array}{c}\text { Control } \\
\text { (3 reactions) }\end{array}$} & $15 \mu \mathrm{l}$ SYBR & \multirow{3}{*}{$24 \mu 1$} & $8 \mu \mathrm{l}$ & $2 \mu \mathrm{l}$ Reference & \\
\hline & $3 \mu \mathrm{l}$ water & & $8 \mu 1$ & $2 \mu \mathrm{l}$ Query 1 & \\
\hline & $6 \mu 1 \mathrm{cDNA}$ & & $8 \mu \mathrm{l}$ & $2 \mu l$ Query 2 & \\
\hline
\end{tabular}

In the first place and depending on the number of interrogated transcripts/qRT-PCR reactions, different quantities of SYBR Green, water and cDNA were pooled together in a master mix (Table 2.5). Next, each master mix was separated into a number of $8 \mu 1$ aliquots using 8-Strip Tubes (T-3218, BioExpress) and loaded into the 384-well plate. After this, the correspondent primer mix $(2 \mu \mathrm{l})$ was added to the wells in order to achieve a final volume per reaction of $10 \mu 1$ (Table 2.5). This methodology ensured that every qRT-PCR reaction, both interrogating queried transcripts and reference genes, used the same cDNA.

After the sample preparation, loaded 384-well plates were centrifuged for $3 \mathrm{~min}$ $\left(4^{\circ} \mathrm{C}\right)$ to help mixing of the qRT-PCR reagents. Then, the 384-plate was loaded into the LightCycler ${ }^{\circledR} 480$ II instrument where it was subjected to 40 cycles of amplification. The specific conditions (e.g. temperature, length) of the amplification steps are described in Table 2.6. All samples and controls were assayed in duplicates. 
Table 2. 6 Amplification steps used in qRT-PCR experiments

\begin{tabular}{|c|c|c|c|c|c|}
\hline Steps & Temp $\left({ }^{\circ} \mathrm{C}\right)$ & Length & Ramp $\left({ }^{\circ} \mathrm{C} / \mathrm{s}\right)$ & Acquisition & Cycles \\
\hline Pre-incubation & 95 & $10: 00$ & 4.8 & None & 1 \\
\hline \multirow{3}{*}{ Amplification } & 95 & $00: 10$ & 4.8 & None & \multirow{2}{*}{40} \\
\cline { 2 - 5 } & Variable $^{1}$ & $00: 20$ & 2.5 & None & \\
\cline { 2 - 5 } & 72 & $00: 20$ & 4.8 & Single & \\
\hline \multirow{4}{*}{ Melting curve } & 95 & $00: 05$ & 4.8 & None & \multirow{2}{*}{1} \\
\cline { 2 - 5 } & 65 & $01: 00$ & 2.5 & None & \\
\cline { 2 - 5 } & 97 & - & 0.11 & $\begin{array}{c}\text { Continuous } \\
\left(5 \text { per }{ }^{\circ} \mathrm{C}\right)\end{array}$ & \\
\hline Hold & 40 & $00: 30$ & 2.5 & None & 1 \\
\hline
\end{tabular}

${ }^{1}$ Variable: primer pair-dependent, typically $60^{\circ} \mathrm{C}$.

\subsubsection{Analysis of qRT-PCR data}

Relative gene expression was determined following the comparative double delta cycle threshold method $\left(\Delta \Delta C_{T}\right)$ (Yuan et al. 2006), using 'glyceraldehyde-3phosphate dehydrogenase' $(G A P D H)$ or 'ribosomal protein L5' (RPL5) as housekeeping genes to calculate normalised gene expression. The outcome of this analysis is a fold-change value (FC), an estimation that measures changes in gene expression levels (increase or decrease). An example of the calculation process of fold-change values is depicted in Table 2.7. Fold-change values representing a decrease in gene expression levels ( 0 to 1$)$ were converted using the formula [1/Fold-change] to facilitate comparisons (Table 2.7: Fold-change) (Schmittgen \& Livak 2008). 
Table 2. 7 Example of fold-change calculation following the $\Delta \Delta C_{\mathrm{T}}$ method

\begin{tabular}{|c|c|c|c|c|c|}
\hline Sample & Target & $\begin{array}{c}\text { Average } \boldsymbol{C}_{\boldsymbol{T}} \\
\left(\bar{x} C_{T}\right)\end{array}$ & $\begin{array}{c}\Delta \boldsymbol{C}_{\boldsymbol{T}} \\
\left(C_{T} \text { Query - }\right. \\
\left.C_{T} \text { Reference }\right)\end{array}$ & $\begin{array}{c}\Delta \Delta \boldsymbol{\Delta} \boldsymbol{C}_{\boldsymbol{T}} \\
\left(\Delta C_{T} \text { Treatment }-\right. \\
\left.\Delta C_{T} \text { Control }\right) \\
\end{array}$ & $\begin{array}{l}\text { Fold-change } \\
\left(2^{\wedge}\left(-\Delta \Delta C_{T}\right)\right)\end{array}$ \\
\hline \multirow{3}{*}{ Treatment } & Reference & 19.43 & - & 0 & 1 \\
\hline & Query 1 & 27.495 & 8.06 & -1.3 & 2.46 \\
\hline & Query 2 & 33.78 & 14.35 & 1.61 & $0.32(-3.05)$ \\
\hline \multirow{3}{*}{ Control } & Reference & 19.75 & - & & \\
\hline & Query 1 & 29.11 & 9.36 & & \\
\hline & Query 2 & 32.49 & 12.74 & & \\
\hline
\end{tabular}

The unpaired one-way analysis of variance (ANOVA) statistical analysis was performed on treatment and control $\Delta C_{\mathrm{T}}$ values using Graphpad Prism (Version 6). The Tukey's Honest Significant Difference (HSD) test was used to correct for multiple comparisons testing and a confidence level of 95\% was selected, defining the statistical significance in p-value $<0.05\left(^{*}\right.$ ) (Yuan et al. 2006; Rieu \& Powers 2009). Even though certain assumptions (Gaussian/normal distribution) required for the use of ANOVA are not fulfilled to perfection on small sample sizes such as those used for qRT-PCR, this analysis is commonly used in detriment of more stringent non-parametrical test.

\subsection{End-point Polymerase Chain Reaction}

The Phusion ${ }^{\circledR}$ High-Fidelity DNA Polymerase (M0530S, New England BioLabs ${ }^{\circledR}$ ) was used to perform end-point polymerase-chain reaction (PCR) experiments using a MJ MiniTMPersonal Thermal Cycler (PTC1148EDU, Bio-Rad). Apart from the Phusion ${ }^{\circledR}$ proprietary polymerase and buffer, other components of end-point PCRs were dNTPs $(10 \mathrm{mM})$, specific primers $(10 \mu \mathrm{M})$ and target DNA or cDNA templates ( $\sim 50 \mathrm{ng})$. Negative controls consisting of regular PCR components without the target DNA were conducted alongside every PCR reaction. The concentration of reagents and steps conditions of PCR reactions used throughout this thesis are summarized in Table 2.8. 
Table 2. 8 Basic components and amplification steps used in end-point PCR

\begin{tabular}{|c|c|}
\hline \multicolumn{2}{|c|}{ Basic components } \\
\hline Components & Quantity \\
\hline $\begin{array}{l}\text { Phusion }^{(B)} \\
\text { Polymerase }\end{array}$ & $0.5 \mu l$ \\
\hline $\begin{array}{c}5 \text { X Phusion } \\
\text { Buffer } \\
\end{array}$ & $4 \mu 1$ \\
\hline 10mM dNTPs & $0.4 \mu \mathrm{l}$ \\
\hline $10 \mu \mathrm{M}$ Primers & $1 \mu \mathrm{l}$ \\
\hline $\begin{array}{c}\text { Template DNA } \\
(\sim 50 \mathrm{ng})\end{array}$ & Variable \\
\hline Water & Variable \\
\hline
\end{tabular}

\begin{tabular}{|c|c|c|}
\hline \multicolumn{3}{|c|}{ Amplification } \\
\hline Steps & Temp $\left({ }^{\circ} \mathrm{C}\right)$ & Length \\
\hline Pre-incubation & 98 & $00: 30$ \\
\hline \multirow{2}{*}{$\begin{array}{c}\text { Amplification } \\
(25-35 \text { cycles })\end{array}$} & Variable & $00: 10$ \\
\cline { 2 - 3 } & 72 & Flexible \\
\hline Final extension & 72 & $10: 00$ \\
\hline Hold & 10 & $\infty$ \\
\hline
\end{tabular}

${ }^{1}$ Flexible: Approximately 15-30 seconds per $\mathrm{Kb}$.

The size of PCR reaction products was corroborated running the end-samples in 1$2 \%$ agarose gels $\left(\mathrm{A} 2500\right.$, Apex $^{\mathrm{TM}}$ ) using a $1 \mathrm{X}$ Tris-acetate-EDTA electrophoresis buffer (B49, Thermo scientific ${ }^{\mathrm{TM}}$ ). Ethidium bromide (15585011, Thermo scientific $^{\mathrm{TM}}$ ) was used for DNA staining and the resulting fluorescence was observed using an Alpha Innotech Fluorchem ${ }^{\circledR}$ FC2 gel imager (10732, Cambridge Scientific). Unknown band sizes were determined by comparison with a GeneRuler 100bp DNA ladder (SM0241, Thermo scientific ${ }^{\mathrm{TM}}$ ).

Table 2.9 List of primers used for DNA sequencing

\begin{tabular}{|c|c|c|}
\hline Target & Sequence & Experiment \\
\hline $\begin{array}{l}\text { HBO1-Tag2B } \\
\text { (Fig. 2.10) }\end{array}$ & $\begin{array}{l}\text { F: 5'-CTATGGGAACACACGGGAAC-3' } \\
\text { R: 3'-TTGCTTCCCTCTGTGATTTG-5' }\end{array}$ & $\begin{array}{l}\text { HBO1-expressing } \\
\text { vector }\end{array}$ \\
\hline $\begin{array}{l}\text { Genomic DNA } \\
\text { (Fig. 2.5) }\end{array}$ & $\begin{array}{l}\text { F: 5'-TTGAACTTGGGGTTTTCAGTG-3' } \\
\text { R: 3'-CAGTCAGCCAGATGTGAGGA-5' }\end{array}$ & Gene knock-out \\
\hline
\end{tabular}

When necessary, PCR products were sent to 'Lone Star Labs' for DNA sequencing. Each PCR product ( $5 \mathrm{ng} / \mu \mathrm{l})$ was sequenced twice using specific forward and reverse 
primers $[5 \mu \mathrm{M}]$, achieving a read definition of approximately $600-700$ nucleotides per primer. The same procedure was applied when sequencing an expression vector; only in this case the required product quantity was higher $(200 \mathrm{ng} / \mu \mathrm{l})$ (Table 2.9). The 4Peaks (Version 1.7, Nucleobytes) software was used to visualize the resulting chromatograms and Clustal Omega was used to perform sequence multiple alignment (Sievers et al. 2011).

\subsection{Western immune-blotting}

\subsubsection{Protein extraction and quantification}

Confluent monolayers of cells were washed with ice-cold PBS and then lysed with Radio-Immune-Precipitation Assay (RIPA) buffer. RIPA buffer contains $150 \mathrm{mM}$ $\mathrm{NaCl}$ (AM9760G, Thermo Scientific ${ }^{\mathrm{TM}}$ ), 50mM Tris-HCl pH = 7.5 (15567027, Thermo Scientific $\left.{ }^{\mathrm{TM}}\right), 0.5 \%$ sodium deoxycholate $\left(\mathrm{D} 6750, \mathrm{Sigma}^{\circledR}\right), 0.1 \%$ Sodium Dodecyl Sulfate (SDS) (28312, Thermo Scientific ${ }^{\mathrm{TM}}$ ) and 1\% Triton X-100 (T8787, Sigma $^{\circledR}$ ). Protease inhibitor cocktail (PIC) cOmplete ${ }^{\mathrm{TM}}$ (50X) (11697498001, Roche) was added to the RIPA buffer (50:1) before each protein extraction. The PIC was originally received in individual tablets, which were diluted in $1 \mathrm{~mL}$ of doubledistilled water, separated into aliquots and stored at $-20^{\circ} \mathrm{C}$ as $50 \mathrm{X}$ PIC stock solution. Lysed cells were thoroughly agitated every five minutes for half an hour using a vortex to ensure cell lysis and release of the protein extract. After this, samples were centrifuged at $14,000 \mathrm{rpm}(5415 \mathrm{R}$, Eppendorf $)\left(4^{\circ} \mathrm{C}\right)$ to isolate the protein-containing supernatants.

The concentration of each sample was determined using a standardised bovine serum albumin (BSA) concentration curve (A3803, Sigma ${ }^{\circledR}$ ) following the Bio-Rad DCTM protein assay method (500-0116, Bio-Rad). Both unknown samples and the standard curve were loaded into Corning ${ }^{\mathrm{TM}}$ Clear Polystyrene 96-well micro-plates (Corning ${ }^{\mathrm{TM}}$ ) and tested in triplicates. Finally, a Safire ${ }^{2}$ micro-plate reader (Tecan) and the XFluor4SafireII software (Version 4.62n) were used to calculate samples' absorbance at $750 \mathrm{~nm}$. At this point, protein extracts were either used for western blot or stored at $-80^{\circ} \mathrm{C}$ for future experimentation. 


\subsubsection{Gel electrophoresis}

All western blots were performed with $10 \mu \mathrm{g}$ of total protein and at RT conditions, unless otherwise specified. Protein extracts were mixed 1:1 with $2 \mathrm{X}$ Laemmli Sample Buffer (161-0737, Bio-Rad) containing 2-Mercaptoethanol (M6250, Sigma ${ }^{\circledR}$ ), de-naturalised at $98^{\circ} \mathrm{C}$ for $10 \mathrm{~min}$ and then separated by SDSpolyacrylamide gel electrophoresis (SDS-PAGE) using NuPAGETM Bis-Tris gels (Invitrogen $^{\mathrm{TM}}$ ) and MOPS SDS Running Buffer (NP0001, Thermo scientific ${ }^{\mathrm{TM}}$ ). Four-to-twelve per cent gels (NP0321, Thermo scientific ${ }^{\mathrm{TM}}$ ) were commonly used to detect medium to large sized proteins, while 10\% gels (NP0301, Thermo scientific $^{\mathrm{TM}}$ ) allowed for an improved separation of smaller peptides such as histones. Samples were then transferred to an Immune-blot ${ }^{\circledR}$ Polyvinylidene difluoride (PVDF) membrane (1620177, Bio-Rad), previously activated with High Performance Liquid Chromatography (HPLC)-grade methanol (A452, Fisher Scientific) for 5 minutes and washed with double-distilled water.

After ninety minutes of transference in NuPAGE ${ }^{\circledR}$ Transfer Buffer (NP0006, BioRad) containing 20\% HPLC-grade methanol, membranes were blocked for $2 \mathrm{~h}$ with $5 \%$ Non-Fat powdered milk (P-1400, Boston BioProducts) diluted in TBS-T, a Trisbuffered saline solution (28358, Thermo scientific ${ }^{\mathrm{TM}}$ ) supplemented with $0.1 \%$ of Tween $^{\circledR} 20$ detergent (10113103, Fisher BioReagents $\left.{ }^{\mathrm{TM}}\right)$. All membranes were incubated over-night $(\mathrm{O} / \mathrm{N})$ on a slow rocking platform at $4{ }^{\circ} \mathrm{C}$ with the correspondent primary antibodies diluted in fresh blocking solution $(5 \%$ milk in TBS-T). Prior to the incubation with the pertinent horseradish peroxidase (HRP)conjugated secondary antibody, membranes were heavily washed with TBS-T in a rocking platform for thirty minutes. Membranes were then incubated with the secondary antibody at RT for $2 \mathrm{~h}$ in a slow rocking platform shaker (444-0143, $\mathrm{VWR}^{\circledR}$ ) and thoroughly washed again before the band detection.

\subsubsection{Band detection and analysis}

Target membranes were treated with Luminata ${ }^{\mathrm{TM}}$ Classico (WBLUC0500, Millipore $^{\mathrm{TM}}$ ) for 1 minute and then exposed to Blue basic autorad films (F-9023, Bioexpress) in the dark. The exposure was conducted using Monotec cassettes 
(5706, Spectroline ${ }^{\circledR}$ ) and the duration of the process varied depending on the abundance of the protein of interest and the concentration and specificity of the selected antibody. Exposed films were developed in an X-ray film processor (SRX101A, Konica Minolta) and digital images were captured and saved as Tag Image File Format files (TIFF) using an Alpha Innotech Fluorchem ${ }^{\circledR}$ FC2 gel imager. If needed, membranes were stripped for 10 minutes on a fast-paced rocking platform using PLUS Stripping Buffer (46430, Thermo scientific ${ }^{\mathrm{TM}}$ ) followed by another 10 minutes of PBS wash and a new $\mathrm{O} / \mathrm{N}$ primary antibody incubation.

ImageJ, image processing and analysis in Java software, was used to compare the intensity of bands in WB experiments (Schneider et al. 2012). The files were loaded onto ImageJ and converted into simple 8-bit grey scale images, formed of shades of grey where each pixel carries only intensity information. An example of the process of calculation of relative density values and the ratio of intensity is depicted in Table 2.10.

Table 2. 10 Example of relative density calculations in WB analysis

\begin{tabular}{|c|c|c|c|c|c|}
\hline Antibody & Peaks & Area & $\begin{array}{c}\text { Per cent } \\
\text { (P) }\end{array}$ & $\begin{array}{c}\text { Relative Density (RD) } \\
\text { (P target / P standard) }\end{array}$ & $\begin{array}{c}\text { Ratio } \\
\text { (R, \%) }\end{array}$ \\
\hline \multirow{2}{*}{ Target } & Treatment & 3,451 & 7.91 & 0.283 & 28.79 \\
\cline { 2 - 6 } & Control & 13,883 & 31.82 & 0.983 & 100 \\
\hline \multirow{2}{*}{ Standard } & Treatment & 12,184 & 27.92 & & \\
\cline { 2 - 4 } & Control & 14,111 & 32.34 & & \\
\hline
\end{tabular}

Following the Luke Miller's outline analysis methodology (Miller 2010), each dark band detected on the film was recorded as a peak whose area depended on signal intensity. These areas were assigned a percentage value $(\mathrm{P})$ that was subsequently used to calculate the relative density (RD) of target antibody samples, treatment and control, compared to the correspondent loading standard (Table 2.10). GAPDH protein expression was used as standard control throughout the thesis in all WB experiments. In cases where the RD of the control peak was very low and to avoid 
extremely high ratio values of treatment samples, $\mathrm{R}$ was expressed in natural logarithms.

Even though theoretical investigation points toward a log-normal distribution of WB data instead of a Gaussian type, its actual statistical distribution is unknown (Kreutz et al. 2007), however, it is quite common to assume that WB data falls within parameters close to normality allowing for the use of Student's $t$-test and ANOVA (Fay \& Gerow 2013). Except for those experiments aimed to optimize antibody conditions, all immune-blots were performed three times. Two-tailed unpaired Student's $t$-tests and unpaired one-way ANOVA statistical analyses were performed on protein relative density values using Graphpad Prism (Table 2.10). The Tukey's Honest Significant Difference test was used to correct for multiple comparisons testing and a confidence level of $95 \%$ was selected, defining the statistical significance in $\mathrm{p}$-value $<0.05(*)$.

\subsection{Protein Immune-Precipitation}

Every step of the protein immune-precipitation (IP) protocol was performed at $4^{\circ} \mathrm{C}$ unless specifically stated. Approximately 300,000-350,000 cells were cultured $\left(37^{\circ} \mathrm{C}\right)$ in $\mathrm{TC}$ dishes $\left(60 \times 15 \mathrm{~mm}\right.$, Falcon $\left.^{\circledR}\right)$ aiming for $80-90 \%$ confluence at the moment of extraction. After $24 \mathrm{~h}$, total protein was isolated as explained earlier in the western immune-blot section using IP-RIPA buffer $(150 \mathrm{mM} \mathrm{NaCl}, 50 \mathrm{mM}$ Tris $\mathrm{pH}$ $7.5,1 \%$ Triton X-100). In the meantime, Protein A/G beads (sc-2003, Santa Cruz ${ }^{\circledR}$ ) were centrifuged at 14,000 rpm for one minute (RT), washed twice with PBS and one last time with IP-RIPA buffer before they were finally re-suspended in the aforementioned buffer (prepared beads). Apart from protein $A / G$, protein $G$ beads (sc-2002, Santa $\mathrm{Cruz}^{\circledR}$ ) were also tested for IP but rendered disappointing results.

Next, the whole protein extract was pre-cleared by rotation with 'prepared beads' for $1 \mathrm{~h}$ to reduce non-specific background. Once the clearance step was finished, the sample was centrifuged at $14,000 \mathrm{rpm}$ for one minute and the remaining protein present in the supernatant was quantified as explained in the western immune-blot section. 
One hundred micrograms of whole protein extract were transferred to a new tube labelled 'Input' and kept at $-20^{\circ} \mathrm{C}$ to use as antibody-specificity control. The rest of the sample was split in two halves (50-50) and subjected to $\mathrm{O} / \mathrm{N}$ antibody immuneprecipitation on a rotation platform. The first half was incubated with an antibody that recognizes the target protein of interest, labelled 'IP'. Instead, the second half or negative control was incubated with a non-specific immunoglobulin $G$ ( $\operatorname{IgG}$ ) antibody, labelled 'IgG'. If possible, both selected specific and non-specific antibodies were grown in the same host (e.g. rabbit).

After the O/N IP step, samples were incubated for $2 \mathrm{~h}$ with newly prepared beads on a rotation platform. Immune complexes (bead-antibody-protein) were then centrifuged at 14,000 rpm for one minute and washed three times with IP-RIPA buffer. At this point, the 'Input' was thawed and further processed alongside the 'IP' and ' $I g G$ ' samples. All samples were re-suspended in NuPAGE ${ }^{\circledR}$ LDS Sample Buffer (NP0007, Thermo Scientific ${ }^{\mathrm{TM}}$ ) and boiled at $98^{\circ} \mathrm{C}$ for ten minutes prior addition of $\mathrm{NuPAGE}^{\circledR}$ Sample Reducing agent (NP0004, Invitrogen ${ }^{\mathrm{TM}}$ ). After a quick spin to deposit the beads, isolated proteins in the supernatant were separated by SDS-PAGE, transferred to a PVDF membrane and incubated for antibody detection as indicated previously in the western immune-blot section.

The Clean-Blot IP Detection Kit (21232, Thermo Scientific ${ }^{\mathrm{TM}}$ ) was employed for western immune-blots where the resolution of proteins of interest was interfered by fragments of de-natured IgG (e.g. ING4). This kit contains special blocking buffer, detection reagent and chemo-luminescent substrate, which substitute the western immune-blot materials used in the steps following the transference of proteins to a PVDF membrane. The protocol was performed according to manufacturer's instructions.

\subsection{Hormonal Treatment}

Cells were cultured $\mathrm{O} / \mathrm{N}\left(37^{\circ} \mathrm{C}\right)$ in Costar ${ }^{\mathrm{TM}}$ Corning ${ }^{\mathrm{TM}} 12-\mathrm{Well}$ Clear TC-treated plates until they reached $70-80 \%$ confluence. Prior to the treatment, growth medium was replaced with DMEM/F-12 medium (without phenol red) (11039021, Thermo 
Scientific $^{\mathrm{TM}}$ ) supplemented with 10\% of Charcoal Stripped FBS (12676029, Thermo Scientific $\left.^{\mathrm{TM}}\right)$. After $24 \mathrm{~h}$, cells were treated with estradiol (1-10-100nM) (E2758, Sigma $^{\circledR}$ ) diluted in absolute ethanol (BP2818, Fisher Bioreagents ${ }^{\mathrm{TM}}$ ) for 4, 8, 24 and $48 \mathrm{~h}$, whereupon RNA samples were collected. Cells treated with absolute ethanol alone (vehicle) were used as control.

\subsection{Immune-fluorescence Staining}

Cells were cultured $\mathrm{O} / \mathrm{N}\left(37^{\circ} \mathrm{C}\right)$ in Nunc ${ }^{\mathrm{TM}} \mathrm{Lab}^{-T e k^{\mathrm{TM}}}$ II 4-Well Chamber Slides (154526, Thermo Scientific ${ }^{\mathrm{TM}}$ ) to achieve $50-60 \%$ confluence. After $24 \mathrm{~h}$, cells were washed with PBS twice and fixed with 3.7\% Formaldehyde (F8775, Sigma ${ }^{\circledR}$ ) diluted in PBS for 10 minutes (RT). Then, cells were washed three times for 10 minutes, permeabilized with $0.25 \%$ Triton $\mathrm{X}-100$ and washed three times again. Subsequently, the slides were blocked for $1 \mathrm{~h}$ with $3 \%$ BSA diluted in PBS. Following this step, fixed-cells were incubated with the selected primary antibody diluted in blocking solution for $4 \mathrm{~h}$ in a dark humidified chamber $\left(4^{\circ} \mathrm{C}\right)$. Samples were then washed three times, incubated with secondary antibody for $1 \mathrm{~h}$ (same conditions as with the primary antibody) and washed three times again. One chamber was always incubated with secondary antibody alone as fluorescence control.

Finally, the samples were mounted with Fisherbrand ${ }^{\mathrm{TM}}$ Rectangle Cover Glasses (12-545K, Fisher Scientific), prior addition of a drop of Vectashield ${ }^{\circledR}$ mounting solution containing 4', 6-Diamidine-2'-phenylindole dihydrochloride (DAPI) (H1200, Vector Laboratories). To prevent any coverslip motion and for long-term storage, the edges of the cover glass were secured using Electron Microscopy Sciences nail polish (72180, Fisher Scientific).

Bright-field cell images were taken with a Nikon Eclipse Ti $\left(\right.$ Nikon $\left.^{\circledR}\right)$ microscope supplied with a Photometrics ${ }^{\circledR}$ CoolSNAP HQ ${ }^{2}$ camera (Roper Scientific ${ }^{\mathrm{TM}}$ ) and the NIS-Elements Advanced Research imaging software (Version 4.00.07, Build 787). Additionally, DAPI and fluorescein signal were detected using this instrument. DAPI staining emits blue fluorescence upon binding to adenosine-thymidine regions of DNA, whereas the Alexa Fluor ${ }^{\circledR} 488$ secondary antibody emits green fluorescence. 


\subsection{Subcellular Protein Fractionation}

The Subcellular Protein Fractionation Kit for Cultured Cells (78840, Thermo Scientific ${ }^{\mathrm{TM}}$ ) was used to separate cytoplasmic, membrane, nuclear soluble and chromatin-bound protein extracts in mammalian cells. The protocol was conducted following manufacturer's instructions, using approximately 200,000 cells grown in a Corning ${ }^{\circledR} 100 \mathrm{~mm}$ TC-treated Culture Dish $\left(430167\right.$, Corning $\left.{ }^{\circledR}\right)$ for $24 \mathrm{~h}\left(37^{\circ} \mathrm{C}\right)$. After the extraction, protein extracts were quantified, run through SDS-PAGE electrophoresis and analysed following the protocol described previously in the western blot section. The subcellular protein fractionation was performed three times with three biological replicates of UWB1.289 cells. Percentages of localization were calculated following the indications illustrated in Table 2.11 below these lines.

Table 2. 11 Example of 'percentage of cell localization' calculations

\begin{tabular}{|c|c|c|c|}
\hline Antibody & Peaks & Area & $\overline{\boldsymbol{x}}$ Percentage \\
\hline \multirow{4}{*}{ Target } & Cytoplasmic fraction & 6,053 & $14.76 \pm \mathrm{SD}$ \\
\cline { 2 - 4 } & Membrane fraction & 956 & $2.33 \pm \mathrm{SD}$ \\
\cline { 2 - 4 } & Nuclear soluble fraction & 16,262 & $39.66 \pm \mathrm{SD}$ \\
\cline { 2 - 4 } & Chromatin-bound fraction & 17,730 & $43.24 \pm \mathrm{SD}$ \\
\hline
\end{tabular}

\subsection{Chromatin Immune-precipitation coupled to qRT-PCR}

Different ChIP procedures were used throughout the elaboration of this thesis: EZMagna ChIPтм A/G (17-10086, Millipore $\left.{ }^{\mathrm{TM}}\right)$, Chromatrap ${ }^{\circledR}$ Pro-A ChIP Kit (500189, Chromatrap), Chromatrap ${ }^{\circledR}$ Native ChIP Kit (500237, Chromatrap) and a non-commercial ChIP protocol outlined in the laboratory of Paul Webb in the Houston Methodist Research Institute, Houston, United States. In addition, the Cell Fixation protocol of the Active Motif ${ }^{\circledR}$ Epigenetics Services was followed in order to fix and prepare UWB1.289 cells for shipment to the Active Motif ${ }^{\circledR}$ laboratories. The main protocol stages of the general ChIP procedure are depicted in Figure 2.1 A. 
A

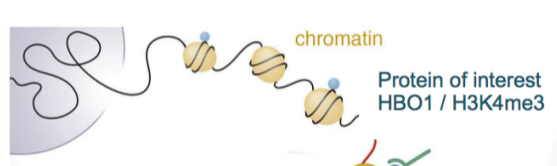

B

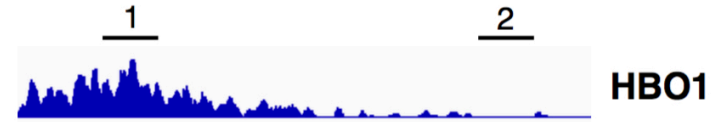

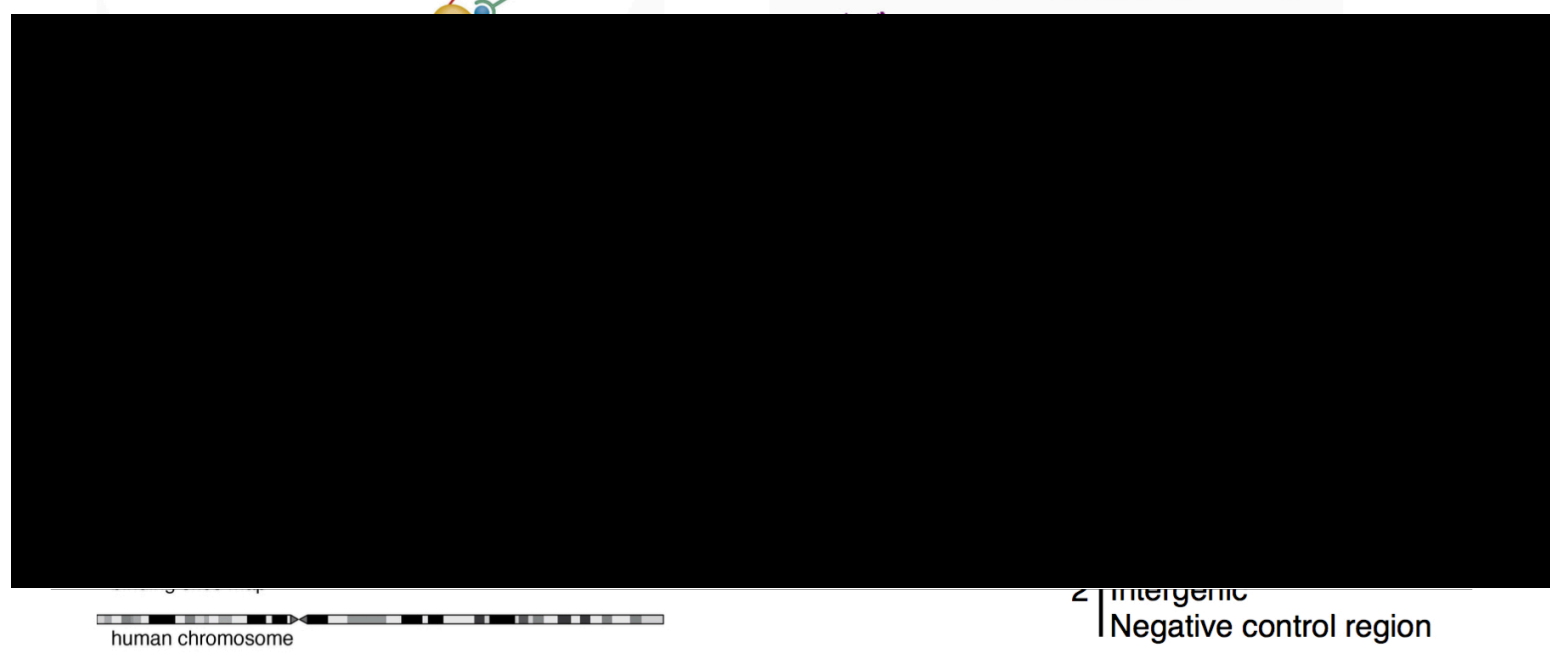

Figure 2. 1 Main characteristics of ChIP and ChIP-qRT-PCR primer design (A) Main protocol stages of general ChIP procedures; image taken from 'First Analysis of Tumor-Suppressor Interactions with Whole Genome...' (McNulty \& Gender 2011). (B) Illustrative depiction of antibody binding for anti-HBO1, anti$\mathrm{H} 3 \mathrm{~K} 4 \mathrm{me} 3$, IgG and 'Input' ChIP samples on a putative HBO1-target gene. Images were obtained using the Integrative Genomics Viewer. (C) Generation of specific ChIP-qRT-PCR primers targeting both enriched and negative control regions.

Common to all ChIP protocols, cells were cultured in 100x20mm TC-treated dishes (25-203, Genesee Scientific) $\left(37^{\circ} \mathrm{C}\right)$ to reach $90-95 \%$ confluence $(\sim 10-12$ million cells). Next, a 1\% formaldehyde solution (F8775, Sigma ${ }^{\circledR}$ ) was used to cross-link chromatin with adjacent interacting proteins and protein complexes, followed by a series of chromatin isolation steps that are detailed in the manual of instructions of each manufacturer. Cross-linked chromatin was then sheared using a Bioruptor ${ }^{\mathrm{TM}}$ (UCD-200, Diagenode) $\left(4^{\circ} \mathrm{C}\right)$ under different sonication settings, which were optimized for each cell line and include varying number, length and power (low, medium or high) of sonication cycles. The sonication efficiency was validated resolving sheared-chromatin fragments by electrophoresis in 1-2\% agarose gels.

All ChIP protocols targeted cross-linked chromatin for immune-precipitation with at least 3 different antibodies: (1) the target antibody (e.g. anti-HBO1) (2) a positive control antibody (e.g. anti-H3K4me3) and (3) a negative control IgG antibody from the same species as the selected target antibody (e.g. rabbit) (Fig. 2.1 B). Likewise, 
all ChIP protocols set aside a fraction of cross-linked sheared chromatin that is not selectively enriched with any antibody, the 'Input'. This chromatin fraction is used as base-line control of antibody enrichment for ChIP-qRT-PCR and ChIP-Seq.

\subsubsection{Chromatrap ${ }^{\circledR}$ and EZ-Magna ChIP protocols}

Unlike the other ChIP protocols, the Chromatrap ${ }^{\circledR}$ Native ChIP protocol did not require chromatin cross-linking and sonication, using an enzymatic approach to segment chromatin naturally bound to protein complexes. The Native ChIP protocol was performed following manufacturer's instructions, using $10 \mathrm{~K}$ MWCO $3 \mathrm{~mL}$ Slide-A-Lyzer ${ }^{\circledR}$ Dialysis Cassettes (66380, Thermo Scientific ${ }^{\text {TM}}$ ), Slide-A-Lyzer ${ }^{\circledR}$ Cassette Syringes (66490, Thermo Scientific ${ }^{\mathrm{TM}}$ ) and Float Buoys (66430, Thermo Scientific $^{\mathrm{TM}}$ ) for the $\mathrm{O} / \mathrm{N}$ dialysis procedure. Additionally, the DNA purification step required the use of Sodium Acetate Solution (3M, pH = 5.2) (R1181, Thermo Scientific $\left.{ }^{\mathrm{TM}}\right)$.

The Chromatrap ${ }^{\circledR}$ Pro-A ChIP Kit (Chromatrap) uses spin columns with an inert, porous polymer covalently bound to Protein A that captures the target chromatin/antibody complex. Protein A was selected in detriment of Protein G for anti-HBO1 antibody purification due to its higher affinity and specificity for the tail region (fragment crystallisable region, Fc) of rabbit (Table 2.3: AB70) and mouse $\operatorname{IgG}_{2 b}$ (Table 2.3: G-2) antibodies, according to New England BioLabs ${ }^{\circledR}$. The protocol was conducted following manufacturer's instructions. In addition to the components provided by Chromatrap ${ }^{\circledR}$, a GeneJET PCR Purification Kit (K0701, Thermo Scientific ${ }^{\mathrm{TM}}$ ) was used for DNA purification.

The EZ-Magna ChIPTM A/G Kit protocol was performed following manufacturer's instructions. As the main feature, it uses a blend of protein $A$ and protein $G$ paramagnetic beads to isolate sheared cross-linked chromatin, which demanded the use of a magnetic article separator DynaMag ${ }^{\mathrm{TM}}-2$ (12321D, Invitrogen ${ }^{\mathrm{TM}}$ ). 


\subsubsection{Non-commercial ChIP protocol}

The buffers and solutions used in this protocol are detailed in Table 2.12. As opposed to the commercial ChIP protocols, this one is designed to immuneprecipitate DNA from $30 \mathrm{M}$ cells $(\sim 3 \times 100 \times 20 \mathrm{~mm}$ TC-treated dishes), and can be scaled to up to $100-200 \mathrm{M}$.

In day one of the protocol, 1mg of Dynabeads ${ }^{\circledR}$ Protein A magnetic beads (1001D, Thermo Scientific $\left.{ }^{\mathrm{TM}}\right)$ were washed with $1 \mathrm{~mL}$ of blocking buffer $(0.5 \%$ BSA diluted in PBS) three times for 5 minutes and incubated $\mathrm{O} / \mathrm{N}$ on a rotation platform $\left(4^{\circ} \mathrm{C}\right)$ with $0.5 \mathrm{~mL}$ of blocking buffer and the appropriate volume of target antibody $(10 \mu \mathrm{g})$. Bead washes were conducted using the magnetic article separator DynaMag ${ }^{\mathrm{TM}_{-}} 2$. The day after, adherent cells were fixed with a $1 \%$ formaldehyde solution $(1 \mathrm{~mL} 11 \%$ formaldehyde solution $+10 \mathrm{~mL}$ cold PBS) for 10 minutes in a slow rocking platform at RT (Table 2.12). Importantly, all buffers and solutions used during the fixation and sonication steps were supplemented with fresh $1 \mathrm{X}$ Halt ${ }^{\mathrm{TM}}$ PIC (78430, Thermo Scientific $\left.{ }^{\mathrm{TM}}\right)$.

The cross-linking process was quenched by the addition of 2.5M Glycine (G7403, Sigma $^{\circledR}$ ) for three minutes whereupon cells were washed with cold PBS twice, scraped and transferred to a Nunc ${ }^{\mathrm{TM}}$ Conical Sterile tube $15 \mathrm{~mL}$ (339651, Thermo Scientific $\left.{ }^{\mathrm{TM}}\right)$. Immediately after, cells were centrifuged at $1350 \mathrm{G}$ for 5 minutes $\left(4^{\circ} \mathrm{C}\right)$, re-suspended in $5 \mathrm{~mL}$ of lysis buffer 1 and incubated 10 minutes on a rotation platform $\left(4^{\circ} \mathrm{C}\right)$. The same procedure was repeated with the lysis buffer 2 (Table 2.12). 
Table 2. 12 Recipes for the non-commercial ChIP protocol

\begin{tabular}{|c|c|c|}
\hline Solution & Composition & Target concentration \\
\hline \multirow{5}{*}{$\begin{array}{l}11 \% \text { Formaldehyde } \\
\text { solution }\end{array}$} & $1 \mathrm{M}$ Hepes-KOH, $\mathrm{pH}=7.5^{1}$ & $50 \mathrm{mM}$ \\
\hline & $5 \mathrm{M} \mathrm{NaCl}$ & $100 \mathrm{mM}$ \\
\hline & $0.5 \mathrm{M}$ EDTA, $\mathrm{pH}=8.0$ & $1 \mathrm{mM}$ \\
\hline & $0.5 \mathrm{M}$ EGTA, $\mathrm{pH}=8.0^{2}$ & $0.5 \mathrm{mM}$ \\
\hline & $37 \%$ Formaldehyde & $11 \%$ \\
\hline \multirow{6}{*}{ Lysis buffer 1} & 1M Hepes-KOH, $\mathrm{pH}=7.5$ & $50 \mathrm{mM}$ \\
\hline & $5 \mathrm{M} \mathrm{NaCl}$ & $140 \mathrm{mM}$ \\
\hline & $0.5 \mathrm{M}$ EDTA, $\mathrm{pH}=8.0$ & $1 \mathrm{mM}$ \\
\hline & Glycerol & $10 \%$ \\
\hline & NP-40 / Igepal CA-630 3 & $0.5 \%$ \\
\hline & Triton X-100 & $0.25 \%$ \\
\hline \multirow{4}{*}{ Lysis buffer 2} & Tris-HCl, $\mathrm{pH}=7.5$ & $10 \mathrm{mM}$ \\
\hline & $5 \mathrm{M} \mathrm{NaCl}$ & $200 \mathrm{mM}$ \\
\hline & $0.5 \mathrm{M}$ EDTA, $\mathrm{pH}=8.0$ & $1 \mathrm{mM}$ \\
\hline & $0.5 \mathrm{M}$ EGTA, $\mathrm{pH}=8.0$ & $0.5 \mathrm{mM}$ \\
\hline \multirow{6}{*}{ Sonication buffer } & 1M Hepes-KOH, $\mathrm{pH}=7.5$ & $50 \mathrm{mM}$ \\
\hline & $5 \mathrm{M} \mathrm{NaCl}$ & $140 \mathrm{mM}$ \\
\hline & $0.5 \mathrm{M}$ EDTA, $\mathrm{pH}=8.0$ & $1 \mathrm{mM}$ \\
\hline & $0.5 \mathrm{M}$ EGTA, $\mathrm{pH}=8.0$ & $1 \mathrm{mM}$ \\
\hline & Triton X-100 & $1 \%$ \\
\hline & Sodium deoxycholate & $0.1 \%$ \\
\hline \multirow{7}{*}{$\begin{array}{l}\text { Sonication buffer } \\
\text { high salt }\end{array}$} & $1 \mathrm{M}$ Hepes-KOH, $\mathrm{pH}=7.5$ & $50 \mathrm{mM}$ \\
\hline & $5 \mathrm{M} \mathrm{NaCl}$ & $500 \mathrm{mM}$ \\
\hline & $0.5 \mathrm{M}$ EDTA, $\mathrm{pH}=8.0$ & $1 \mathrm{mM}$ \\
\hline & $0.5 \mathrm{M}$ EGTA, $\mathrm{pH}=8.0$ & $1 \mathrm{mM}$ \\
\hline & Triton X-100 & $1 \%$ \\
\hline & Sodium deoxycholate & $0.1 \%$ \\
\hline & SDS & $0.1 \%$ \\
\hline \multirow{5}{*}{$\begin{array}{l}\text { Lithium chloride } \\
\text { wash buffer }\end{array}$} & Tris- $\mathrm{HCl}, \mathrm{pH}=7.5$ & $20 \mathrm{mM}$ \\
\hline & $0.5 \mathrm{M}$ EDTA, $\mathrm{pH}=8.0$ & $1 \mathrm{mM}$ \\
\hline & Lithium chloride $^{4}$ & $250 \mathrm{mM}$ \\
\hline & NP-40 / Igepal CA-630 & $0.5 \%$ \\
\hline & Sodium deoxycholate & $0.5 \%$ \\
\hline \multirow{3}{*}{ Elution buffer } & Tris- $\mathrm{HCl}, \mathrm{pH}=7.5$ & $50 \mathrm{mM}$ \\
\hline & $0.5 \mathrm{M}$ EDTA, $\mathrm{pH}=8.0$ & $10 \mathrm{mM}$ \\
\hline & SDS & $1 \%$ \\
\hline
\end{tabular}

\footnotetext{
${ }^{1}$ Hepes-KOH (1M) pH = 7.5 (EK-654013, E\&K Scientific Inc.)

${ }^{2}$ EGTA (0.5M) pH = 8.0 (50-255-956, Bioworld)

${ }^{3}$ NP-40 / Igepal CA-630 (I8896, Sigma ${ }^{\circledR}$ )

${ }^{4}$ Lithium chloride (L121, Fisher Bioreagents ${ }^{\mathrm{TM}}$ )
} 
Following the lysis step, cell pellets were re-suspended in sonication buffer containing SDS $(\sim 0.25 \mathrm{~mL} / 5 \mathrm{M}$ cells $)$ and placed in Bioruptor ${ }^{\circledR}$ Plus TPX tubes (C30010010, Diagenode) for sonication. Subsequently, samples were centrifuged at top speed for 10 minutes, after which a small fraction of the supernatant was stored at $-20^{\circ} \mathrm{C}$ as 'Input' and the rest diluted in sonication buffer without SDS $(\sim 1 \mathrm{~mL} / 5 \mathrm{M}$ cells). At this point, a fresh blocking solution was used to wash bead-antibody complexes (from day one) three times for 5 minutes on a rotation platform $\left(4^{\circ} \mathrm{C}\right)$. Re-suspended bead-antibody complexes were added to the diluted chromatin and incubated on a rotation platform $\mathrm{O} / \mathrm{N}\left(4^{\circ} \mathrm{C}\right)$.

In order to test the sonication efficiency, $10 \mu 1$ of cross-linked chromatin were mixed with $90 \mu \mathrm{l}$ of nuclease-free water and $10 \mu \mathrm{l}$ of $\mathrm{NaCl}(5 \mathrm{M})$, and then boiled for 15 minutes $\left(98^{\circ} \mathrm{C}\right)$. After this, the chromatin sample was incubated with $1 \mu$ l of RNAse A $(10 \mathrm{mg} / \mathrm{mL})$ (EN0531, Thermo Scientific $\left.{ }^{\mathrm{TM}}\right)$ for 15 minutes $\left(37^{\circ} \mathrm{C}\right)$ and immediately after with $1 \mu \mathrm{l}$ of Proteinase K solution $(20 \mathrm{mg} / \mathrm{mL}$ ) (AM2548, Thermo Scientific $\left.{ }^{\mathrm{TM}}\right)$ for another 15 minutes $\left(67^{\circ} \mathrm{C}\right)$. The final chromatin sample was purified using the MinElute Reaction Cleanup Kit (28204, Qiagen $\left.{ }^{\circledR}\right)$ and run through a $1-2 \%$ agarose gel.

On day three of the protocol, the complexes (bead-antibody-protein-chromatin) were collected and washed on a rotation platform for 5 minutes $\left(4^{\circ} \mathrm{C}\right)$ with different buffers in the following order: twice with sonication buffer, once with sonication buffer high salt, lithium chloride wash buffer and NaCl-TE Buffer $(50 \mu 15 \mathrm{M} \mathrm{NaCl}+$ $5 \mathrm{~mL}(1 \mathrm{X}) \mathrm{TE} \mathrm{pH}=8.0$ ) (Table 2.12). After a quick spin (960G for 3 minutes), bead complexes were re-suspended with $200 \mu$ l of elution buffer and eluted on a heat block at $65^{\circ} \mathrm{C}$ for 15 minutes. After a new spin $(20,000 \mathrm{G}$ for 30 seconds at RT), the supernatant, which contained eluted antibody-protein-chromatin complexes, was placed in a heat block at $65^{\circ} \mathrm{C} \mathrm{O} / \mathrm{N}$ with $\mathrm{NaCl}(5 \mathrm{M})$ to reverse cross-linking. At this point, the 'Input' fraction was thawed, supplemented with $150 \mu \mathrm{l}$ of $\mathrm{EB}$ and placed together with the rest of the samples in the heat block $\mathrm{O} / \mathrm{N}$.

On day four of the protocol, $8 \mu \mathrm{l}$ of RNase A $(10 \mathrm{mg} / \mathrm{mL})$ were added to each sample, which were then incubated at $37^{\circ} \mathrm{C}$ for $2 \mathrm{~h}$. Subsequently, $7 \mu \mathrm{l}$ of $\mathrm{CaCl}_{2}$ and $4 \mu \mathrm{l}$ of Proteinase $\mathrm{K}(20 \mathrm{mg} / \mathrm{mL})$ were added to the samples for a 30 minute 
incubation at $55^{\circ} \mathrm{C}$. Next, $400 \mu \mathrm{l}$ of Ultrapure ${ }^{\mathrm{TM}}$ Phenol:Chloroform:Isoamyl Alcohol $(25: 24: 1)$ (15593031, Thermo Scientific $\left.{ }^{\mathrm{TM}}\right)$ were added to the mixture. Samples were then transferred to a MaXtract High Density tube (129046, Qiagen ${ }^{\circledR}$ ) and centrifuged at $14,000 \mathrm{G}$ for 5 minutes (RT). The isolated DNA fraction was transferred to a new tube and mixed with $16 \mu \mathrm{l}$ of $5 \mathrm{M} \mathrm{NaCl}, 1.5 \mu \mathrm{l}$ of Ultrapure ${ }^{\mathrm{TM}}$ Glycogen $(20 \mu \mathrm{g} / \mu \mathrm{L})\left(10814010\right.$, Thermo Scientific $\left.{ }^{\mathrm{TM}}\right)$ and $880 \mu \mathrm{l}$ of $100 \%$ Ethanol (BP2818, Fisher Bioreagents ${ }^{\mathrm{TM}}$ ). At this point, samples were cooled at $-80^{\circ} \mathrm{C}$ for 1 $2 \mathrm{~h}$, centrifuged for 20 minutes at $20,000 \mathrm{G}\left(4^{\circ} \mathrm{C}\right)$, washed with $0.5 \mathrm{~mL}$ of $70 \%$ ethanol and centrifuged again in the same conditions. Finally, DNA pellets were left to air dry for approximately 30 minutes and re-suspended in nuclease-free water.

\subsubsection{Design and characterization of ChIP-qRT-PCR primers}

First of all, the Integrative Genomics Viewer (IGV, Broad Institute, V 2.3.88) was used to localize the target chromosome region to interrogate by ChIP-qRT-PCR (Robinson et al. 2011; Thorvaldsdóttir et al. 2013) (Fig. 2.1 B). The selection of specific chromosome regions was based on preliminary ChIP-Seq data, including positive (expected antibody enrichment) and negative (no expected enrichment) regions (Fig. 2.1 B, C). Coordinates for this exact location in the genome were copied into the UCSC Genome Browser which enables, through the function 'Get DNA', the extraction of raw DNA sequences lying between those coordinates (human genome assembly GRCh37/hg19) (Kent et al. 2002). Once isolated the target DNA region, it was uploaded into Primer3 to, from then on, follow the rest of steps used in the design of qRT-PCR primers, except those applied exclusively to mRNA features.

To test the efficacy of ChIP-qRT-PCR primers, gDNA was extracted from different cell lines using the GeneJET Genomic DNA Purification Kit and sonicated to accurately mimic the DNA status of a normal ChIP-qRT-PCR reaction. All validated primers used for ChIP-qRT-PCR in this thesis can be found in Table 2.13. 
Table 2. 13 List of ChIP-qRT-PCR primers

\begin{tabular}{|c|c|c|}
\hline Target & Sequence & $\begin{array}{c}\text { Coordinates } \\
\text { GRCh37/hg19 }\end{array}$ \\
\hline LMNB2 & $\begin{array}{l}\mathbf{F}: 5^{\prime} \text {-CTCCACCCCCAAGGAAAAAG-3' } \\
\text { R: 3'-GGCAGGGTCCCATGCA-5' }\end{array}$ & $\begin{array}{l}\text { chr19: } 2,428,005- \\
\quad 2,428,081\end{array}$ \\
\hline MCM4 & $\begin{array}{l}\text { F: 5'-TCTGCACTCCGTTCAGCTCCTCTG-3' } \\
\text { R: 3'-GAGTGAGGATGCCAGGTCATCTCC-5' }\end{array}$ & $\begin{array}{l}\text { chr8: } 48,874,080- \\
\quad 48,874,400\end{array}$ \\
\hline SNHG12 & $\begin{array}{l}\text { F: 5'-GCATAGCTGCTGTGGTCAAA-3' } \\
\text { R: 3'-CGTTCTGCTGTTCTGTGGAA-5' }\end{array}$ & $\begin{array}{l}\text { chr1: } 28,907,355- \\
\quad 28,907,535\end{array}$ \\
\hline LUC7L & $\begin{array}{l}\mathbf{F}: 5^{\prime} \text {-CAGCGCTTGACAGTCGTTAG-3', } \\
\text { R: 3'-ATCTGCACAGCCCTGAGAAT-5' }\end{array}$ & $\begin{array}{c}\text { chr16: } 278,536- \\
278,719\end{array}$ \\
\hline IGR 1 & $\begin{array}{l}\text { F: 5'-AAATCCGGTGCTGTATCCTG-3', } \\
\text { R: 3'-TTGATTGTGGGGAAGGTTGT-5' }\end{array}$ & $\begin{array}{c}\text { chr7: } 118,998,664- \\
118,998,812\end{array}$ \\
\hline IGR 2 & $\begin{array}{l}\text { F: 5'-TTGGAGGCTTTGTTCATTCC-3' } \\
\text { R: 3'-TGAGGATTTCGTGACACCTG-5' }\end{array}$ & $\begin{array}{l}\text { chr12: } 63731970- \\
63732119\end{array}$ \\
\hline IGR 3 & $\begin{array}{l}\text { F: 5'-GTGCATGCAAGCCAGAGTTA-3', } \\
\text { R: 3'-GGGCTAATCACCTGAAACCA-5' }\end{array}$ & $\begin{array}{l}\text { chr1: } 80,854,465- \\
\quad 80,854,562\end{array}$ \\
\hline CYR61 & $\begin{array}{l}\text { F: 5'-CCGAGTCTCACGCGTATCTT-3' } \\
\text { R: 3'-AGTCCTCGTTGAGCTGCTTG-5', }\end{array}$ & $\begin{array}{l}\text { chr1: } 86,047,015- \\
\quad 86,047,174\end{array}$ \\
\hline ERBB2 & $\begin{array}{l}\text { F: 5'-CTCCGGGAACTTGTCAAAGA-3' } \\
\text { R: 3'-GAGGCTGCCAACTGGAAAG-5' }\end{array}$ & $\begin{array}{c}\text { chr17: } 37856807- \\
37856949 \\
\end{array}$ \\
\hline $\mathrm{ABCC} 4$ & $\begin{array}{l}\text { F: 5'-GGGGTGTCTCTCTGCTTCTG-3' } \\
\text { R: 3'-TGGGGAGACAATGAAAGTCC-5' }\end{array}$ & $\begin{array}{c}\text { chr16: } 16,044,012- \\
16,044,147\end{array}$ \\
\hline ALCAM & $\begin{array}{l}\text { F: 5'-GAATTTCCAGGACCTGCTCA-3' } \\
\text { R: 3'-GAGGGTGTGGAAAGGGATTT-5' }\end{array}$ & $\begin{array}{l}\text { chr3: } 105,087,011- \\
\quad 105,087,165\end{array}$ \\
\hline TLN1 & $\begin{array}{l}\text { F: 5'-CCCTGACTCTGCATTTCACA-3' } \\
\text { R: 3'-ATGTGCGGATTAAAGGTTGG-5' }\end{array}$ & $\begin{array}{c}\text { chr9: } 35,730,626- \\
35,730,762 \\
\end{array}$ \\
\hline ANKRD1 & $\begin{array}{l}\text { F: 5'- TTTTGCATTGGAGGTTTTCC-3' } \\
\text { R: 3'-ATGCTTGGAAGGACATTTGG-5' }\end{array}$ & $\begin{array}{l}\text { chr10: } 92677412- \\
92677561\end{array}$ \\
\hline CASP7 & $\begin{array}{l}\text { F: 5'-TGGTTGAGTGGGACTGAGTG-3' } \\
\text { R: 3'-ACGAGCCCTCCTCTCCTAAA-5' }\end{array}$ & $\begin{array}{c}\text { chr10: } 115,439,860- \\
115,440,027\end{array}$ \\
\hline PKP4 & $\begin{array}{l}\mathbf{F}: 5^{\prime} \text {-CGGCTGTCTGTCCTTCTAGC-3' } \\
\text { R: 3'-AGTACAGGACTTCGGGGACA-5' }\end{array}$ & $\begin{array}{l}\text { chr2: } 159314186- \\
159314292\end{array}$ \\
\hline MRS2 & $\begin{array}{l}\text { F: 5'-GTCCCACTCGCTCAGAATG-3' } \\
\text { R: 3'-GAGGAGAAGGCAGAGGGTTG-5' }\end{array}$ & $\begin{array}{c}\text { chr6: } 24,403,627- \\
24,403,708\end{array}$ \\
\hline NR2F2 & $\begin{array}{l}\text { F: 5'-GTCGCCTTTATGGACCACAT-3' } \\
\text { R: 3'-CTCAGGTAGGAAGGAGCCCT-5', }\end{array}$ & $\begin{array}{c}\text { chr15: } 96,876,802- \\
96,877,994\end{array}$ \\
\hline
\end{tabular}




\subsubsection{Analysis of ChIP-qRT-PCR data}

ChIP-qRT-PCR experiments were conducted using the same machinery, reagents and protocols as in the qRT-PCR section. However, due to the low abundance of immune-precipitated DNA, samples were not diluted in nuclease-free water during the master mix preparation step (Table 2.5). Antibody enrichment was determined using the percentage of input calculation methodology (Nagaki et al. 2003; Cawley et al. 2004), illustrated below these lines in Table 2.14. Input $C_{T}$ values obtained with the EZ-Magna ChIPTM Kit protocol were adjusted from $1 \%$ to $100 \%$ before the analysis. All samples and controls were assayed in duplicates.

Table 2. 14 Example of 'percentage of input' enrichment calculations

\begin{tabular}{|c|c|c|c|c|}
\hline \multirow{2}{*}{ Sample } & \multicolumn{2}{|c|}{ Antibody } & $\begin{array}{c}\text { Average } \boldsymbol{C}_{\boldsymbol{T}} \\
\left(\overline{\boldsymbol{x}} \boldsymbol{C}_{\boldsymbol{T}}\right)\end{array}$ & $\begin{array}{c}\text { Percentage of Input (\%) } \\
\left(100^{*} 2^{\wedge}\left(\text { Input } C_{T}-\text { IP } C_{T}\right)\right)\end{array}$ \\
\hline \multirow{3}{*}{$\begin{array}{c}\text { Target region } \\
\text { (Table 2.13) }\end{array}$} & \multirow{2}{*}{ IP } & Target protein & 30.88 & 0.10 \\
\cline { 3 - 5 } & & Positive control & 25.89 & 3.34 \\
\cline { 2 - 5 } & No IP & Negative control & 30.98 & 0.098 \\
\hline
\end{tabular}

\subsection{Structured Query Language Relational Database}

Structured query language (SQL) relational databases allow for the efficient and convenient storage of, and access to, massive amounts of information (Rice et al. 2004). Its design is based on the relational model of data, which organizes data into tables of columns and rows with unique keys identifying each column (Codd \& F. 1970). Specifically, an SQL relational database containing information about OC and HBO1 was built, allowing the comparison and association of personal data (e.g. gene profiles) and accessible online material (NCBI databases). The SQL relational database was built using the SQLite library, accessible through the Mozilla Firefox ${ }^{\circledR}$ add-on SQLite Manager. An example of one of the common uses of this SQL database is depicted in Figure 2.2 below these lines. 


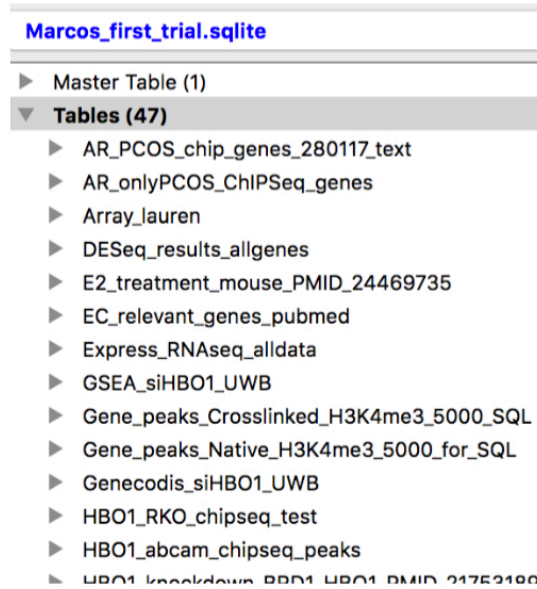

Structure Browse \& Search

Enter SQL

SELECT DISTINCT ID.Gene_symbol, ID.Array_ID

FROM Microarray_OVCAR3

INNER JOIN Microarray ID ID

ON Microarray_OVCAR3.Array_ID = ID.Array_ID

Run SQL Actions $\vee$ Last Error: not an error

Gene_symbol Array ID

ADAM32 $\quad$ ILMN_1806841

AKR1C3

BST2 ILMN_1723480

C19orf66

C20orf103 |ILMN_1713561

C21orf128 ILMN_1663893

CD69 |ILMN_2188333

Figure 2. 2 Example of SQL relational database correlations

The 'Tables' or datasets of an SQL relational database store vast amounts of data, which can be interconnected through the use of SQL language, thus rendering meaningful combinatorial outcomes. In this instance, the database was used to correlate the results of an Illumina ${ }^{\circledR}$ microarray, which provides solely Illumina ${ }^{\circledR}$ probe identifiers for each target gene, with a dataset containing the official gene symbols associated to each Illumina ${ }^{\circledR}$ probe identifier. As a result of this comparison, microarray expression results could be directly associated with official gene symbols, facilitating to a large extent further analyses.

\subsection{ChIP combined with Sequencing}

\subsubsection{Preparation of samples and acquisition of data}

During the development of this thesis, ChIP-Seq data was gathered from multiple sites including: (1) the Gene Expression Omnibus data repository of the NCBI (GEO Datasets), (2) the Epigenetics Services of Active Motif ${ }^{\circledR}$ (AM) and (3) the Epigenomics Core Facility of Weill Cornell Medical College.

The Active Motif ${ }^{\circledR}$ Epigenetics Services ChIP-Seq raw data was obtained performing a ChIP Antibody Validation procedure on a fixed pellet of UWB1.289 cells. According to the AM results report, $30 \mu \mathrm{g}$ of UWB1.289 cell chromatin and $5 \mu \mathrm{g}$ of AB70 HBO1 antibody were used to conduct a ChIP-Seq. The process included the use of a True-Seq ChIP Library Preparation Kit (IP-202-1012, Illumina ${ }^{\circledR}$ ) and a HiSeq 2500 System (Illumina ${ }^{\circledR}$ ) that generated approximately $2 \mathrm{M}$ reads. ChIP-Seq raw data was then deposited to a downloadable File Transfer Protocol (FTP) site. 
Six ChIP samples, two 'Input', two 'HBO1' and two 'H3K4me3' IPs obtained from UWB1.289 cells using the Chromatrap ${ }^{\circledR}$ Pro-A ChIP kit, were sent to the Epigenomics Core Facility at Weill Cornell Medical College to conduct ChIP-Seq. The Quant-iT ${ }^{\text {TM }}$ dsDNA High-Sensitivity Assay Kit (Q-33120, Thermo Scientific ${ }^{\text {TM}}$ ) was used to accurately quantify DNA concentrations. The DNA quantification was performed following manufacturer's instructions, using a Corning ${ }^{\mathrm{TM}}$ Clear Polystyrene 96-well micro-plate (Corning ${ }^{\mathrm{TM}}$ ) to measure samples, blank and standards in triplicates. The standard curve was designed to cover a low DNA quantity range (0-20ng) due to the small amounts of immune-precipitated samples. Resulting fluorescence was measured with a Safire $^{2}$ micro-plate reader and the XFluor4SafireII software at standard fluorescein wavelengths $\left(480_{\mathrm{Ex}} / 530_{\mathrm{Em}} \mathrm{nm}\right)$. The final sample quantities sent to the Epigenomics Core Facility were 60ng of 'Input' (5 $\mathrm{ng} / \mu \mathrm{l})$ and $22-25 \mathrm{ng}$ of 'H3K4me3' and 'HBO1' (1 ng/ $\mu \mathrm{l})$.

Again, the Epigenomics Core used the True-Seq ChIP Library Preparation Kit and a HiSeq 2500 System to conduct Single-Read Clustering DNA Sequencing (1x50 sequencing cycles, maximum 50bps per DNA). All samples were multiplexed into one lane, allowing the sequencing of approximately $30 \mathrm{M}$ reads per sample and the ChIP-Seq raw data was finally deposited to PubShare for downloading.

\subsubsection{Analysis of ChIP-Seq raw data}

ChIP-Seq raw data, including at least a sample and the corresponding 'Input' in FASTQ format, were downloaded and deposited into a Linux high performance computer (HPC) cluster server. Alongside the ChIP-Seq data analysis, the terminal tool for Mac Os X provided a line interface to control the underpinnings of the Linux operating system, while FileZilla 2 was used to transfer HPC data results to a personal computer.

Occasionally, ChIP-Seq GEO DataSets were compiled into Sequence Read Archives files or SRAs, in which case the SRA ToolKit was used to convert them into FASTQ files (Staff 2011). Prior to the ChIP-Seq analysis, the FastQC programme (Leggett et al. 2013) was used to assess the quality of next-generation sequencing data (Fig. 2.3). 


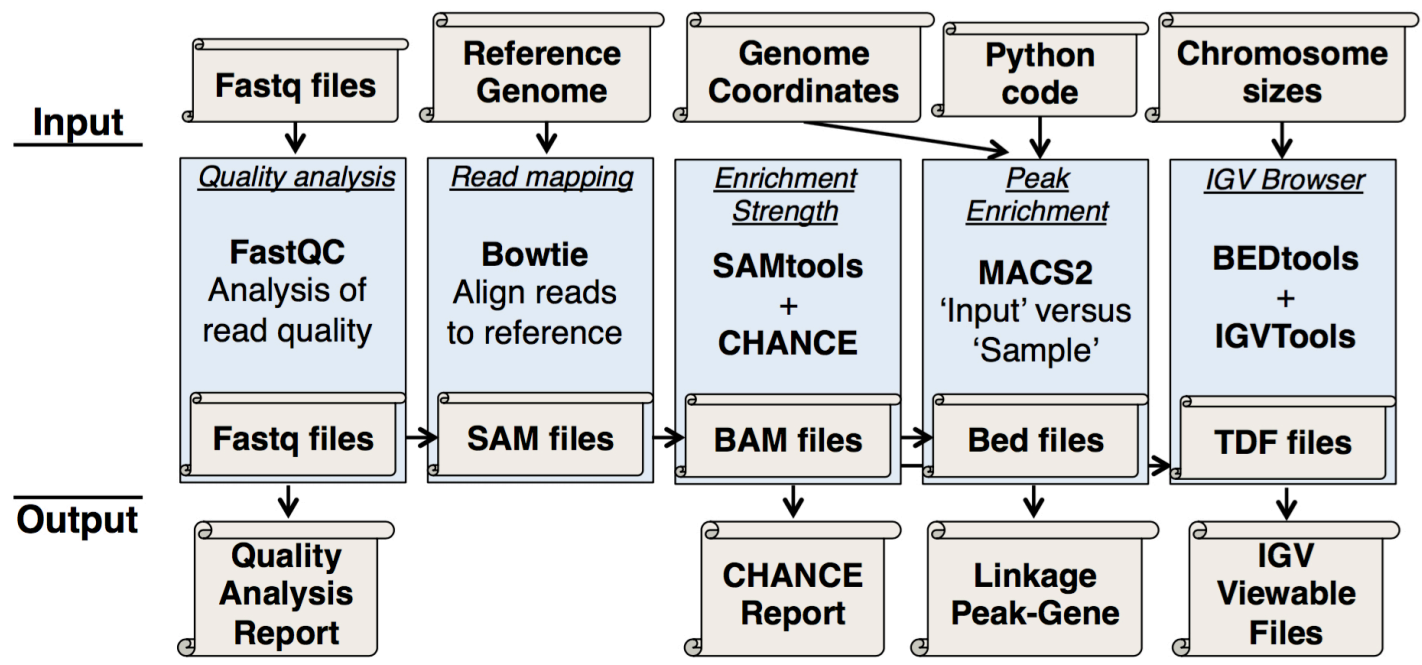

Figure 2. 3 Workflow diagram of the ChIP-Sequencing analysis pipeline

ChIP-Seq reads were aligned to the GRCh37/hg19 genome alignment using Bowtie (Langmead et al. 2009), which generated SAM files that were subsequently converted into BAM format using SAMTools (Li et al. 2009) (Fig 2.3). At this point, the software for ChIP-Seq Analytics and Confidence Estimation (CHANCE) (Diaz et al. 2012) was used to derive genome-wide indications of ChIP enrichment ('Sample' versus 'Input'), estimating the strength and quality of immuneprecipitation to identify potentially failed experiments (Fig. 2.3 \& Fig. 2.4). As well as with the FastQC programme, this report is merely an indication of ChIP-Seq quality, and its result does not preclude from pursuing the whole analysis.

Next, BAM files were analysed using the Model-based Analysis for ChIP-Seq 2 (MACS2) (Y. Zhang et al. 2008; Feng et al. 2012) in order to derive significantly enriched peaks in the pertinent sample compared to the 'Input'. MACS2 package analysis generated Browser Extensible Data (BED) files, which included the genomic localization and intensity of each derived peak. Using a SQL relational database and a Python script (Bassi 2007), genomic peak localizations compiled into BED files were linked to potential gene regulatory mechanisms based on sequence proximity. 
Additionally, BAM files were used to derive individual Trusted Data Format or TDF files, viewable in the IGV browser (Fig. 2.1 B). In doing so, BAM files were processed using BEDTools (Quinlan 2014) and IGVTools (Broad Institute).

A

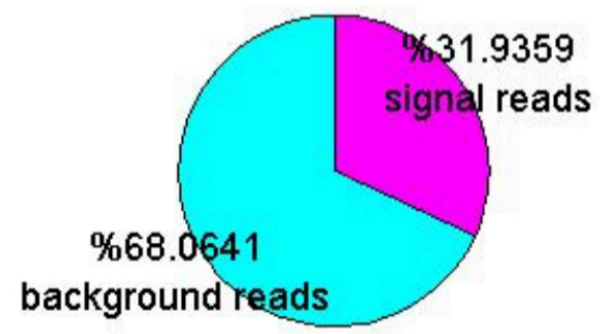

B

The IP appears weak.

The percentage enrichment of IP over Input is not statistically significant

Figure 2. 4 Example of CHANCE analysis on ChIP-Sequencing data

To estimate IP strength, CHANCE decomposes the population of IP reads into two distinct components: those pulled down by the antibody or signal reads, and background reads. The level of IP enrichment strength is used to classify whether an experiment was (A) successful or (B) failed.

\subsection{Plasmid DNA Transfections}

Several plasmid transfection methods were used throughout the elaboration of this thesis. In order to test the efficiency and toxicity of each reagent, a pmaxGFPTM Vector (Lonza) was transfected into target cells. Green fluorescent protein (GFP) signal was then visualized using the imaging equipment described in the immunefluorescence section.

Generally, cells were cultured $\mathrm{O} / \mathrm{N}\left(37^{\circ} \mathrm{C}\right)$ in Costar ${ }^{\mathrm{TM}}$ Corning ${ }^{\mathrm{TM}} 12-$ Well Clear TCtreated plates to reach $60-80 \%$ confluence, depending on the cell line (e.g. UWB1.289 70,000 cells/well), and analysed 24h post-transfection. The amounts of pmaxGFPTM Vector and transfection reagent were selected following manufacturer's indications. The different transfection reagents used in this thesis are listed below these lines (Table 2.15). 
Table 2. 15 List of reagents used for plasmid DNA transfection

\begin{tabular}{|c|c|c|}
\hline Transfection Reagent & Product & Provider \\
\hline FuGENE $^{\circledR}$ HD & E2311 & Promega $\left.{ }^{(}\right)$ \\
\hline Lipofectamine $^{\circledR} 3000$ & L3000 & Invitrogen $^{(B)}$ \\
\hline Lipofectamine $^{\circledR}$ LTX \& PLUS ${ }^{\mathrm{TM}}$ & 15338 & Invitrogen $^{(B)}$ \\
\hline TransFectin $^{\mathrm{TM}}$ Lipid & 170335 & Bio-rad \\
\hline TurboFect ${ }^{\mathrm{TM}}$ & R0531 & Thermo Scientific ${ }^{\mathrm{TM}}$ \\
\hline UltraCruz $\left.^{(}\right)$ & 395739 & Santa Cruz ${ }^{\circledR}$ \\
\hline X-tremeGENETM 9 & 6365779 & Roche \\
\hline X-tremeGENETM HP & 6366244 & Roche \\
\hline
\end{tabular}

Alternatively, certain DNA plasmids were transfected via electroporation using the Nucleofector $^{\mathrm{TM}}$ 2b Device (AAB-1001, Lonza) following manufacturer's instructions. The Amaxa ${ }^{\circledR}$ Cell Line Optimization Nucleofector ${ }^{\circledR}$ Kit (VCO-1001N, Lonza) and the pmaxGFPTM Vector were used to optimize the electroporation conditions of UWB1.289 cells. Efficient transfection of UWB1.289 cells was achieved with the Nucleofector ${ }^{\mathrm{TM}}$ Kit V (Programme A-020).

\subsection{Dot-blot}

The aim of the dot-blot (DB) experiments performed in this thesis was to simply detect either the presence or absence of a target protein in a cell protein lysate; hence there was no need to conduct protein quantification. Total protein extraction was conducted as described previously in the first part of the western immune-blot section and every step of the dot-blot, apart from the aforementioned protein extraction, was conducted at RT.

Immediately after the protein extraction, a small fraction of each sample (usually $2 \mu \mathrm{l})$ was loaded onto two different Amersham $^{\mathrm{TM}}$ Hybond $^{\mathrm{TM}}-\mathrm{ECL}$ nitrocellulose membranes (RPN303D, GE Healthcare) and left to dry in order to fix the proteins for 2h. During the fixation process, membranes were kept still and inside a container to avoid contamination. Next, non-specific sites were blocked using blocking solution (TBS-T with $2 \%$ milk) for 30 minutes in a slow rocking platform. At this point, 
while one membrane was incubated with the target antibody, the other was incubated with a control antibody, both diluted in blocking solution. A $\beta$-Actin antibody was used as control in all DB experiments since the GAPDH antibody did not work very well in this particular setting. Membranes were incubated with primary antibodies for one hour in a slow rocking platform, followed by repeated washes with TBS-T and the incubation with the pertinent secondary antibody for another hour. After several final washes, the absence or presence of protein dots in the membranes was analysed as indicated in the band detection part of the western blot section.

\subsection{HBO1 Knock-OUT}

\subsubsection{Knock-OUT preliminary steps}

The Alt-R ${ }^{\mathrm{TM}}$ CRISPR-Cas9 System (Clustered Regularly Interspaced Short Palindromic Repeats) from IDT ${ }^{\circledR}$ was selected to manipulate the genome of OC cells seeking to constitutively eradicate HBO1 expression (knock-out, KO). The specific aim of CRISPR-Cas9 was to disrupt the $\mathrm{HBOl}$ locus genome sequence, altering the function of the resulting protein and/or completely abolishing its derivate expression. The principal constituents of the CRISPR/Cas-9 mediated gene disruption system were: a CRISPR RNA sequence guide (crRNA guide) specific to the target DNA (Fig. 2.5), a recombinant form of Cas9 protein with endonuclease activity and a trans-activating crRNA sequence (tracrRNA), which interacts with both the Cas9 protein and the crRNA (Fig. 2.5 C). The resulting complex causes target-specific double-stranded DNA cleavage, which is commonly repaired by the NHEJ DNA repair pathway, an error-prone process that could result in insertions and/or deletions (INDELs) that disrupt gene function.

Specific crRNA guides targeting the $\mathrm{HBOl}$ locus were designed using the CRISPR Design tool following manufacturer's recommendations (Haeussler et al. 2016), which pinpointed the importance of early INDEL target sites that could render more altered proteins in case of the introduction of frame-shift mutations. Accordingly, the selected target region for $\mathrm{HBO} 1 \mathrm{KO}$ was the exon 2, common to the five alternative splice variants of HBO1 and localised in chromosome 17 with coordinates 
47,869,247-47,869,395 (GRCh37/hg19) (Fig. 2.5 A). As a result of the 'CRISPR Design' search, eight potential crRNA guides were found in this region, each one with a score depending on individual qualities (Fig. 2.5 A, B). A determining aspect for the selection of crRNA guides is the presence of a 5'-NGG-3' (N: any nucleotide base) proto-spacer adjacent motif (PAM) immediately following the targeted DNA sequence (Fig. 2.5 B, C). This motif is recognized by the Cas9 protein and is indispensable for gene editing.

A
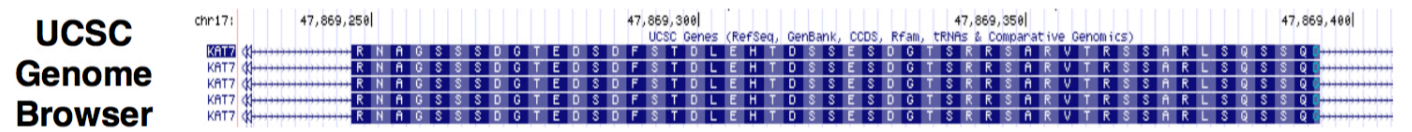

Browser

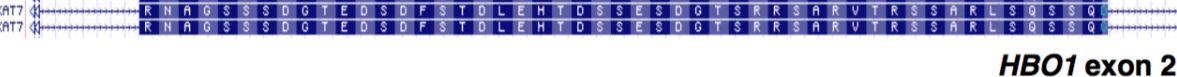

'CRISPR

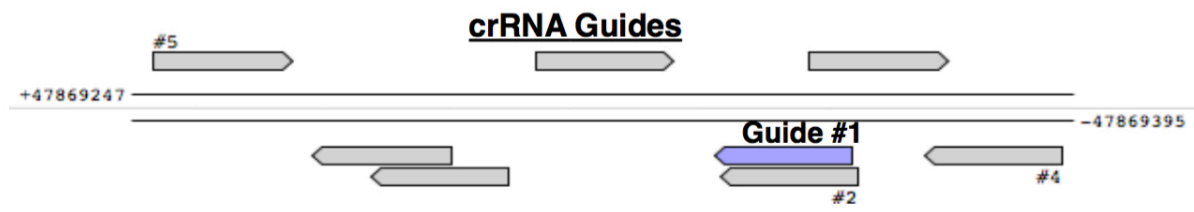

B

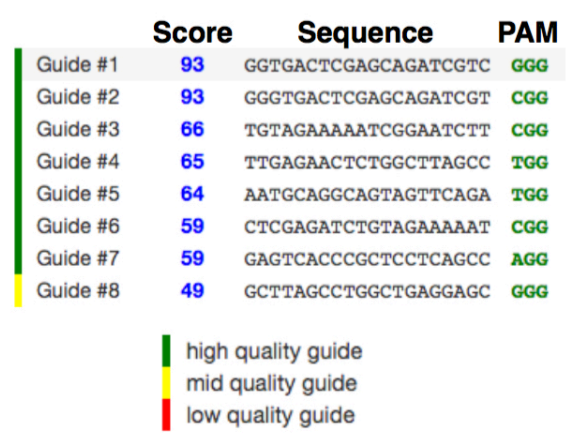

C

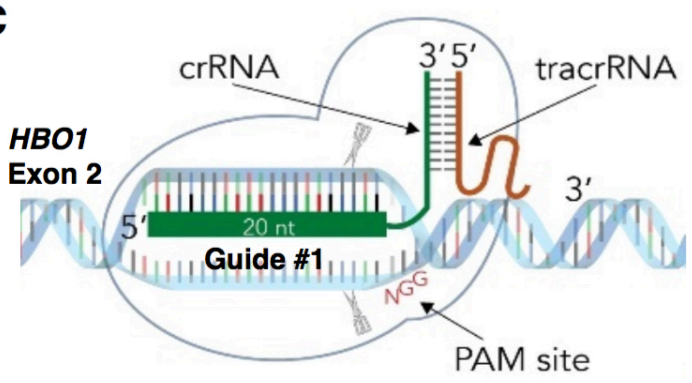

Alt-R ${ }^{\mathrm{TM}}$ crRNA:tracrRNA complex

Figure 2. 5 Selection of CRISPR RNAs targeting the HBO1 locus

(A) Graphical depiction of the localization of target CRISPR RNA guides in regard to the $H B O 1$ exon 2 locus, including (B) respective quality scores provided by the 'CRISPR Design' tool and (C) the main structure of potential crRNA:tracrRNA complexes.

CRISPR RNA guides 1 and 3 (2nmol) (Table 2.16), Alt-R ${ }^{\mathrm{TM}}$ S.p. Cas9 Expression Plasmid (1072566) (Fig. 2.6 A) and the CRISPR-Cas9 Control Kit (1072554) were purchased from IDT $^{\circledR}$. The latter included crRNA targeting positive control 'hypoxanthine phosphoribosyltransferase 1' (HPRT) (2nmol), crRNA Negative Control (2nmol), tracrRNA (5nmol), HPRT PCR Primer Mix (2nmol each primer) 
and Nuclease-Free Duplex Buffer. The Alt-R ${ }^{\mathrm{TM}}$ CRISPR Negative Control crRNA contains a nucleotide sequence that is computationally designed to be non-targeting in the human genome.

Table 2. 16 List of CRISPR RNA sequences and HBO1-knockOUT primers

\begin{tabular}{|c|c|c|}
\hline Target & \multicolumn{1}{|c|}{ Sequence } & Description \\
\hline \multirow{3}{*}{$\begin{array}{c}\text { HBO1 exon 2 } \\
\text { (Fig. 2.5 A) }\end{array}$} & 5'-GGUGACUCGAGCAGAUCGUC-3' & crRNA 1; KO \\
\cline { 2 - 3 } & F: 5'-GAGAGAGAAAAAUCGGAAUCUU-3' & crRNA 3; KO \\
\hline R: 3'- TATCACTGGCCCAGGAAAAG-5' & Primers; T7EI test \\
\hline
\end{tabular}

The Alt-R ${ }^{\mathrm{TM}}$ S.p. Cas9 expression plasmid was transformed into $\mathrm{NEB}^{\circledR}$-alpha competent Escherichia coli (E. coli) cells following manufacturer's instructions. Transformed E. coli cells were spread onto lysogeny broth agar selection plates (BP9724, Fisher BioReagents ${ }^{\mathrm{TM}}$ ) containing $50 \mu \mathrm{g} / \mathrm{mL}$ of ampicillin (A0166, Sigma $^{\circledR}$ ) and incubated $\mathrm{O} / \mathrm{N}$ at $37^{\circ} \mathrm{C}$. Next, positive colonies were grown in $5 \mathrm{~mL}$ of steriliSed lysogeny broth (BP9723, Fisher BioReagents ${ }^{\mathrm{TM}}$ ) containing $50 \mu \mathrm{g} / \mathrm{mL}$ of ampicillin $\left(\mathrm{O} / \mathrm{N}, 37^{\circ} \mathrm{C}\right)$. A considerable fraction of the broth containing $\mathrm{O} / \mathrm{N}$ grown cells (4/5) was mixed 2:1 with 50\% Glycerol (BP229, Fisher BioReagents ${ }^{\mathrm{TM}}$ ) and kept in $-80^{\circ} \mathrm{C}$ as a stock. The remaining cells were subjected to plasmid extraction using a QIAprep Spin Miniprep Kit (27104, Qiagen $\left.{ }^{\circledR}\right)$.

Aside from the Streptococcus pyogenes (S.p.) Cas9 gene (Marraffini 2016), an ampicillin resistance cassette and a cytomegalovirus (CMV) promoter, the Alt-R ${ }^{\mathrm{TM}}$ S.p. Cas9 Expression Plasmid encompasses a V5 epitope tag (Southern et al. 1991), which facilitates the recognition of Cas 9 -expressing cells via dot-blot (Fig. 2.6 A). Prior to dot-blotting V5, the Anti-V5 Tag antibody was tested via western blot to ensure the recognition of a single band. 
A

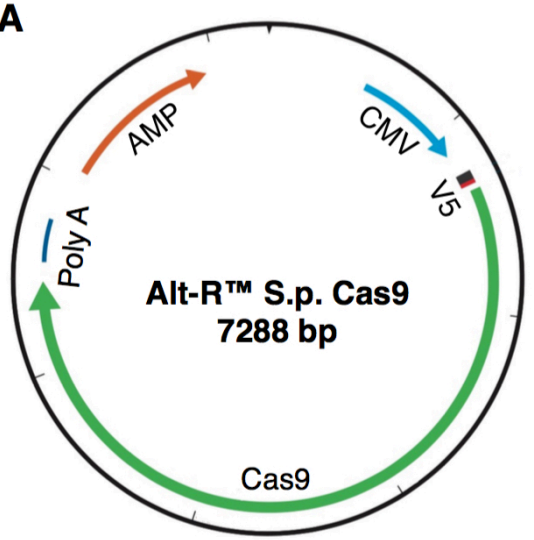

B

Calculation of Ad-GFP-Cas9 volume (Diluted adenoviral stock: $1 \times 10^{8} \mathrm{PFU} / \mathrm{mL}$ )

\begin{tabular}{|c|c|c|c|}
\hline No of cells & MOI & $\begin{array}{c}\text { Total PFU } \\
\text { (no of } \\
\text { cells*MOI) }\end{array}$ & $\begin{array}{c}\text { Volume } \\
\text { (total PFU/ } \\
\text { Stock) }\end{array}$ \\
\hline $\mathbf{1 4 0 , 0 0 0}$ & 10 & $1.4 \times 10^{6}$ & $14 \mu \mathrm{l}$ \\
\hline $\mathbf{1 4 0 , 0 0 0}$ & 100 & $1.4 \times 10^{7}$ & $140 \mu \mathrm{l}$ \\
\hline
\end{tabular}

Figure 2. 6 Methodology to transiently express Cas9 in OC cells

(A) Structure of the Alt-R ${ }^{\mathrm{TM}}$ S.p. Cas 9 expression plasmid. (B) Table summarizing an exemplary calculation of the required volume of Ad-GFP-Cas 9 adenoviruses to successfully infect mammalian cell lines. Poly A: Polyadenylation tail; AMP: Ampicillin resistance cassette; CMV: Cytomegalovirus promoter; V5: Epitope tag; MOI: Multiplicity of infection; PFU: plaque-forming units.

Another approach that aimed to introduce transient Cas 9 expression in UWB1.289 was the use of Cas9-expressing adenoviruses (Ad-GFP-Cas9) (1901, Vector Biolabs) (Gong et al. 2017). To test the suitability of this approach, cells were cultured $\mathrm{O} / \mathrm{N}\left(37^{\circ} \mathrm{C}\right)$ in Costar ${ }^{\mathrm{TM}}$ Corning ${ }^{\mathrm{TM}} 12-$ Well Clear TC-treated plates $(70,000$ cells/mL), followed by adenovirus infection and ascertainment of GFP-expression and concomitant cell cytotoxicity (Nikon Eclipse Ti microscope). The initial titre of pre-packaged Ad-GFP-Cas 9 adenoviruses was $1 \times 10^{10}$ plaque-forming units per $\mathrm{mL}$ $(\mathrm{PFU} / \mathrm{mL})$, a typical measuring unit of the number of infectious virus particles within a sample stock. Different concentrations of polybrene (sc-134220, Santa Cruz ${ }^{\circledR}$ ), a positively charged polycation commonly used to boost virus transduction events, were included in an effort to enhance adenoviral infection efficiency.

In order to select the appropriate number of adenoviruses to successfully infect UWB1.289 cells (total plaque-forming units), different virus/cell ratio were tested (Fig. 2.6 B). The average number of virus particles infecting each cell is commonly known as multiplicity of infection (MOI) and ranges from 10 to 100 or more, depending on the type and number of cells (Gong et al. 2017). In this case and to calculate MOI, the number of cells was established on 140,000 after $24 \mathrm{~h} \mathrm{O} / \mathrm{N}$ culturing, assuming an average of 1 cycle of doubling time (Fig. 2.6 B). 


\subsubsection{Knock-OUT and T7EI surveyor nuclease assay}

In an attempt to knock-OUT HBO1 using Ad-GFP-Cas 9 adenoviruses, cells were cultured $\mathrm{O} / \mathrm{N}\left(37^{\circ} \mathrm{C}\right)$ in Costar $^{\mathrm{TM}}$ Corning ${ }^{\mathrm{TM}}$ 6-Well Clear TC-treated plates and infected with an appropriate MOI of Ad-GFP-Cas 9 for $24 \mathrm{~h}$. After this, cells were transfected with pertinent crRNAs following manufacturer's instructions. Otherwise, approximately 1-2M UWB1.289 cells grown in Corning ${ }^{\circledR} 100 \mathrm{~mm}$ TC-treated Culture Dishes were electroporated with Alt-R ${ }^{\mathrm{TM}}$ S.p. Cas 9 expression plasmid and subsequently split into Costar ${ }^{\mathrm{TM}}$ Corning ${ }^{\mathrm{TM}}$ 6-Well Clear TC-treated plates; each of which was transfected with different concentrations of target crRNAs and controls.

Complexes of crRNA:tracrRNA [30-60nM] were transfected to UWB1.289 cells using $1 \mu 1$ of DharmaFECT Transfection Reagent 4 (T-2004, Dharmacon ${ }^{\mathrm{TM}}$ ) per $\mathrm{mL}$ of medium, following manufacturer's instructions and recommendations. The culture medium was replenished in $24 \mathrm{~h}$, after which samples were grown for another $24-72 \mathrm{~h}$ depending on cell viability and proliferative activity. Finally, gDNA samples were isolated in order to confirm possible mutation events.

The detection of INDELs in CRISPR DNA target regions using the T7 Endonuclease I (T7EI) (M03025, New England BioLabs ${ }^{\circledR}$ ) surveyor nuclease assay was performed following manufacturer's instructions (Qiu et al. 2004). First, Phusion ${ }^{\circledR}$ HighFidelity DNA Polymerase, primers targeting the $\mathrm{HBO} 1$ exon 2 locus region and a MJ MiniтMPersonal Thermal Cycler were used to generate the PCR product encompassing the potential mutation site (Table 2.16 \& Fig. 2.7 A). Next, the resulting material of the previous PCR reaction was subjected to a process denominated heteroduplex formation, using 384-well LightCycler ${ }^{\circledR} 480$ Multi-well Plates and a LightCycler ${ }^{\circledR} 480$ II machine (Fig. 2.7 B). Finally, half of the heteroduplex reaction volume was treated with T7EI enzyme $(1 \mathrm{U} / \mu \mathrm{l})$ and the other half was mixed with NEB Buffer 2 (B7002S, New England BioLabs ${ }^{\circledR}$ ) alone as negative control, following manufacturer's instructions. Agarose gels (1-2\%) were used to visualize T7EI mismatch endonuclease results (Fig. 2.7 C, D) and ImageJ was used to measure the percentage of cleavage comparing the non-cleaved band with the cleaved bands (mutagenesis efficiency), assuming that an efficient digestion would generate the same number of band fractions (Fig. 2.7 C). 
A

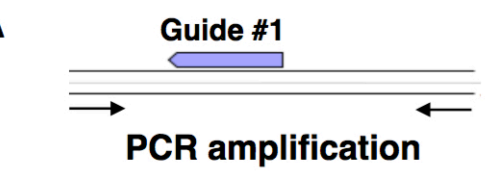

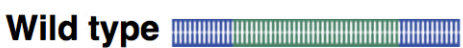

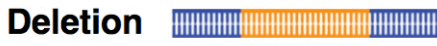

Insertion ${ }^{2}$

C

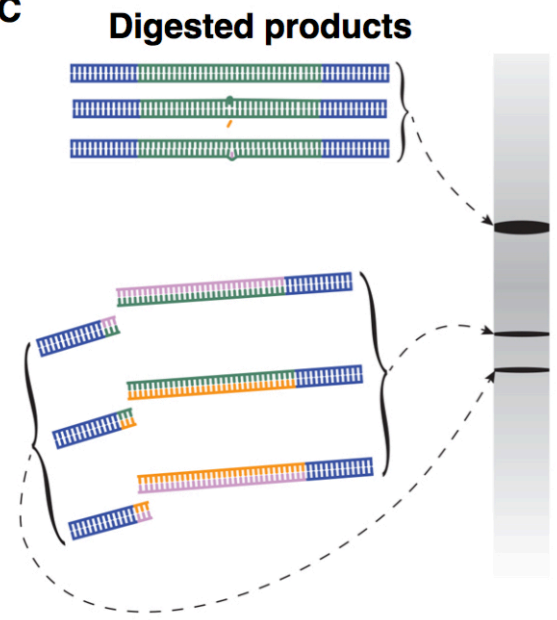

B

\section{Heteroduplex formation and T7EI digestion}

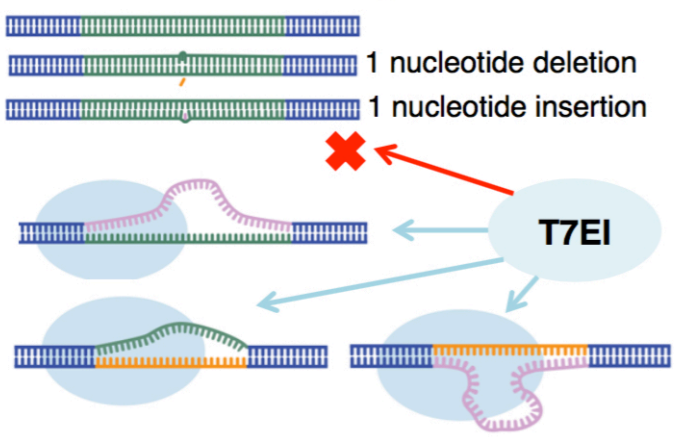

D

\section{Example T7El assay: HBO1 crRNA 1}

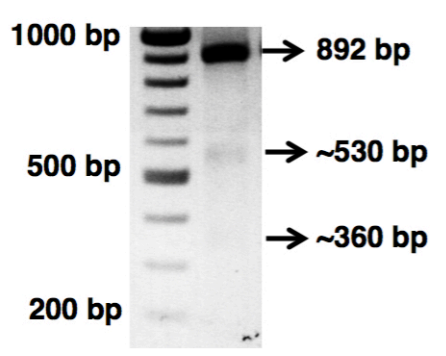

Figure 2. 7 Analysis of CRISPR mutagenesis efficiency using the T7EI assay

(A) Specific PCR amplification generated one or more DNA products, depending on INDEL events. (B) These DNA products were separated into individual strands and pulled back together, giving rise to the formation of potential heteroduplexes, prone to T7EI cleavage. (C) The digestion of T7EI generated different sized DNA products; (D) which ran through electrophoresis gels allow for the observation of distinct bands and thus the calculation of mutagenesis efficiency.

After the T7 Endonuclease testing confirmation, individual cells were cultured in Costar $^{\mathrm{TM}}$ Corning ${ }^{\mathrm{TM}}$ 96-Well Clear TC-treated plates $\left(37^{\circ} \mathrm{C}\right)$ until they reached enough confluence to allow protein/gDNA extractions alongside with further cell proliferation. In addition, and in order to improve the isolation of individual colonies, a single pipette device was used to separate potential UWB1.289 KO cells. In both cases, cells were grown for approximately 3-4 weeks, during which cell culture medium was replenished every 3-4 days. Finally, protein samples were interrogated using dot-blot and/or western blot, whereas gDNA samples were sent for DNA sequencing. These different assays confirmed or denied the presence of DNA mutations in the target $\mathrm{HBOl}$ locus. 


\subsection{Small interfering RNA Knockdown}

The ON-TARGETplus Human KAT7 siRNA-SMARTpool (11143, Dharmacon ${ }^{\mathrm{TM}}$ ) was purchased to transiently knockdown $H B O 1$ and the ON-TARGETplus nontargeting pool (Dharmacon ${ }^{\mathrm{TM}}$ ) was used as negative control (Table 2.17 \& Fig. 2.8). To confirm the specific effect of each one of the four small interfering RNAs (siRNAs) that are part of the pool, the corresponding set of four de-convoluted siRNAs was also acquired. Each non-overlapping siRNA is formed by two RNA strands forming a duplex of 19-25 base pairs where the antisense strand (guide) is a perfect reverse complement of the target $H B O 1$ mRNA (Table 2.17 \& Figure 2.8).

Table 2. 17 List of small interfering RNAs

\begin{tabular}{|c|c|c|c|}
\hline Target & Guide sequence & Code & Product \\
\hline \multirow{4}{*}{ HBO1 } & 5'-GAACCGAAGAUUCCGAUUU-3' & J-017668-05 & \\
\cline { 2 - 3 } & 5'-GAGGGAAGCAACAUGAUUA-3' & J-017668-06 & \multirow{2}{*}{ ON-TARGET } \\
& 5'-UAGGACACCUUACAGGAAA-3' & J-017668-07 & SMARTpool \\
\cline { 2 - 3 } & 5'-GGCAAGAUGCUUAUUGAUU-3' & J-017668-08 & \\
\hline \multirow{3}{*}{$\begin{array}{c}\text { Non- } \\
\text { specific }\end{array}$} & 5'-UGGUUUACAUGUCGACUAA-3' & & \multirow{2}{*}{ ON-TARGET } \\
& 5'-UGGUUUUACAUGUUGUGUGA-3' & D-001810- & Non-targeting \\
& 5'-UGGUUUACAUGUUUUUUCCUA-3' & & Pool \\
\hline
\end{tabular}

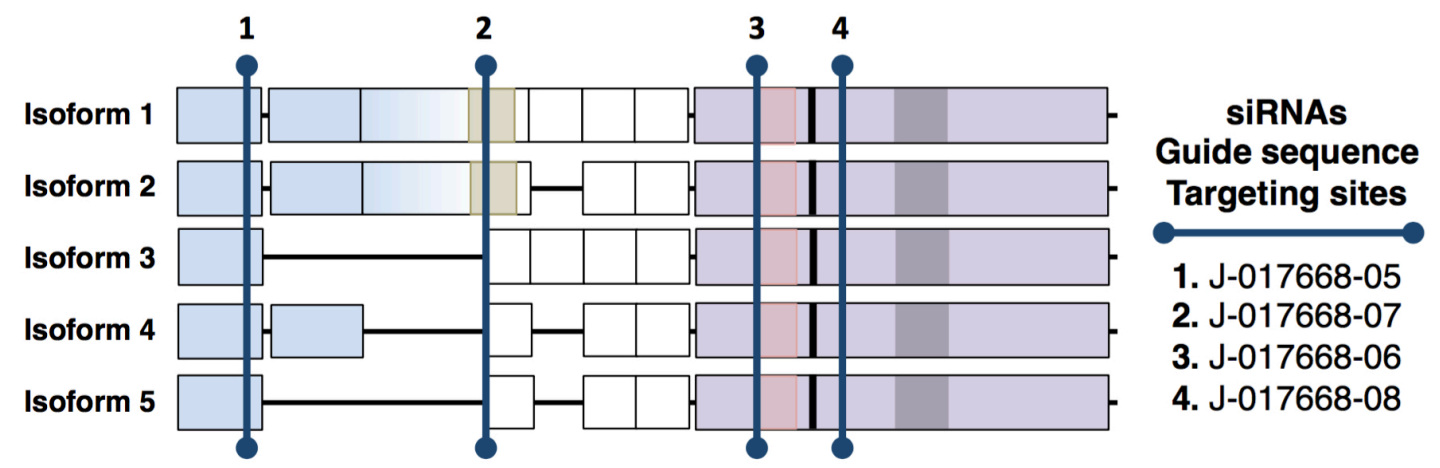

Figure 2. 8 Targeting sites of small interfering RNAs

Each horizontal row represents an HBO1 isoform (depicted as translated mRNA) and the numbered markers indicate sites of siRNA targeting. These isoforms are described in a more detailed manner in chapter 3: Expression and Regulation of HBO1 in Ovarian Cancer. 
Small interfering RNAs delivered in dry state were re-suspended in $1 \mathrm{X}$ siRNA Buffer (B-002000-UB, Dharmacon ${ }^{\mathrm{TM}}$ ) following manufacturer's instructions. The concentration of re-suspended siRNA molecules was confirmed using a Nanodrop ${ }^{\circledR}$ Spectrophotometer ND-1000; siRNA stocks were separated into small aliquots and stored at $-20^{\circ} \mathrm{C}$, up to four weeks, or $-80^{\circ} \mathrm{C}$ for long-term storage.

Cells were cultured $\mathrm{O} / \mathrm{N}\left(37^{\circ} \mathrm{C}\right)$ in Costar ${ }^{\mathrm{TM}}$ Corning ${ }^{\mathrm{TM}}$ Clear $\mathrm{TC}$-treated plates (Fisher Scientific) with pertinent medium without antibiotics and differing plating densities (Table 2.18). Costar $^{\circledR}$ Clear TC-treated plates used in this thesis for siRNA include 12-well (3527, Corning $\left.{ }^{\mathrm{TM}}\right)$, 24-well (3526, Corning $\left.{ }^{\mathrm{TM}}\right)$, 48-well (3548, Corning $\left.{ }^{\mathrm{TM}}\right)$ and 96-well (3585, Corning $\left.{ }^{\mathrm{TM}}\right)$.

Table 2. 18 List of siRNA transfection conditions for OC cell lines

\begin{tabular}{|c|c|c|c|}
\hline Cell line & $\begin{array}{c}\text { Plating density } \\
\text { (cells/mL) }\end{array}$ & $\begin{array}{c}\text { Transfection } \\
\text { Reagent (TR) }\end{array}$ & $\begin{array}{c}\text { TR } \\
\text { Reference }\end{array}$ \\
\hline OVCAR-3 & 67,000 & DharmaFECT 1 & Dharmacon $^{\mathrm{TM}}$ \\
\hline UWB1.289 & 50,000 & DharmaFECT 4 & Chang et al. $2014^{\text {TM }}$ \\
\hline SKOV-3 & 50,000 & DharmaFECT 1 & Dharmacon $^{\mathrm{TM}}$ \\
\hline TOV-112D & 50,000 & DharmaFECT 1 & Test 1 and 4 \\
\hline UACC-1598 & 150,000 & DharmaFECT 1 & Test 1 and 4 \\
\hline
\end{tabular}

After $24 \mathrm{~h}$, cells were transfected with the siRNA pool [25nM] or individual siRNAs $[6.25 \mathrm{nM}]$ using the correspondent DharmaFECT Transfection Reagent following manufacturer's instructions (Table 2.18). One microliter of DharmaFECT 4 or DharmaFECT 1 (T-2001, Dharmacon ${ }^{\mathrm{TM}}$ ) was included per millilitre of transfection medium. In addition to the transfection of siRNAs targeting $\mathrm{HBO1}$, cells were also transfected with non-targeting siRNAs ('siCtrl') and transfection reagent alone ('Control') in order to minimize off-target effects (Jackson \& Linsley 2010). Whole cell lysates were then harvested 24,48 or $72 \mathrm{~h}$ post- transfection. 


\subsection{Lentiviral transduction of short hairpin RNAs}

\subsubsection{Characteristics and structure of selected molecules}

A set of three SMARTvector ${ }^{\text {TM }}$ Lentiviral Human KAT7 hCMV-TurboGFP short hairpin RNA (shRNA) particles (V3SH7596-00EG11143, Dharmacon ${ }^{\mathrm{TM}}$ ) was purchased in order to constitutively knockdown $H B O 1$ (Table $2.19 \&$ Fig. 2.9). The SMARTvector Non-targeting hCMV-TurboGFP Control particles (Dharmacon ${ }^{\mathrm{TM}}$ ) were used as negative control (Table 2.19). All vials of lentiviral particles contained $10^{8}$ transducing units per millilitre $(\mathrm{TU} / \mathrm{mL})$, a tittering calculation based on the identification, through fluorescent-activated cell sorting, of functional particles that delivered the packaged RNA of interest in human embryonic kidney (HEK) $293 \mathrm{~T}$ cells.

The main structure of the SMARTvector ${ }^{\mathrm{TM}}$ lentiviral shRNA vector is illustrated in Figure 2.9 A, below these lines. Briefly, each vector contains the machinery for lentiviral integration in the host cell genome, a human CMV promoter, a green fluorescent protein reporter (TurboGFP) for visual tracking, a puromycin resistance $\left(\right.$ Puro $^{\mathrm{R}}$ ) element and a microRNA-adapted shRNA. The microRNA-adapted shRNA consists of an shRNA stem structure surrounded by a loop and flanking sequences of an endogenous microRNA (Fig. 2.9 A). The shRNA structure contains the guide sequence that is a perfect reverse complement of the target $H B O 1$ mRNA and the endogenous microRNA scaffold enhances the efficiency and reduces the cytotoxicity of the process (Table 2.19 \& Fig. 2.9).

Table 2. 19 List of short hairpin RNAs

\begin{tabular}{|c|c|c|c|}
\hline Target & Guide Sequence & Code & Product \\
\hline \multirow{3}{*}{ HBO1 } & 5'-UUCUAAAAGAGGUUCCCGU-3' & 227169180 & \begin{tabular}{c} 
SMARTvector \\
Lentiviral Human \\
\cline { 2 - 3 }
\end{tabular} \\
\cline { 2 - 4 } & 5'-UUUGCGAUAUAUCUCAUCA-3' & 227619297 & V3SH7590 \\
\hline $\begin{array}{c}\text { Non- } \\
\text { specific }\end{array}$ & 5'-UGGUUGUACUUGCCAAUCAC-3' & 230346186 & $\begin{array}{c}\text { SMARTvector } \\
\text { Non-targeting }\end{array}$ \\
\hline
\end{tabular}


A

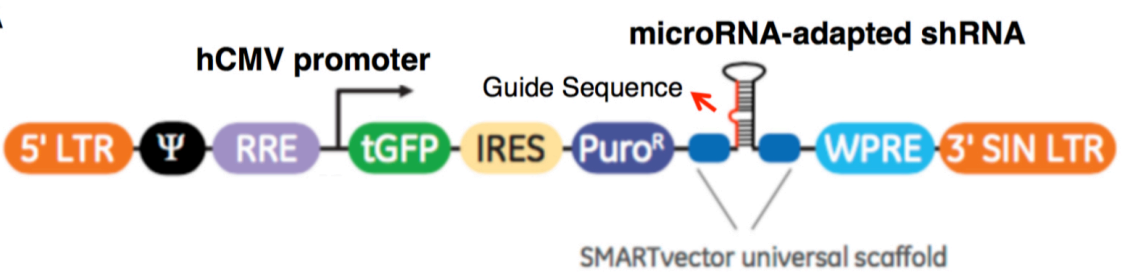

B

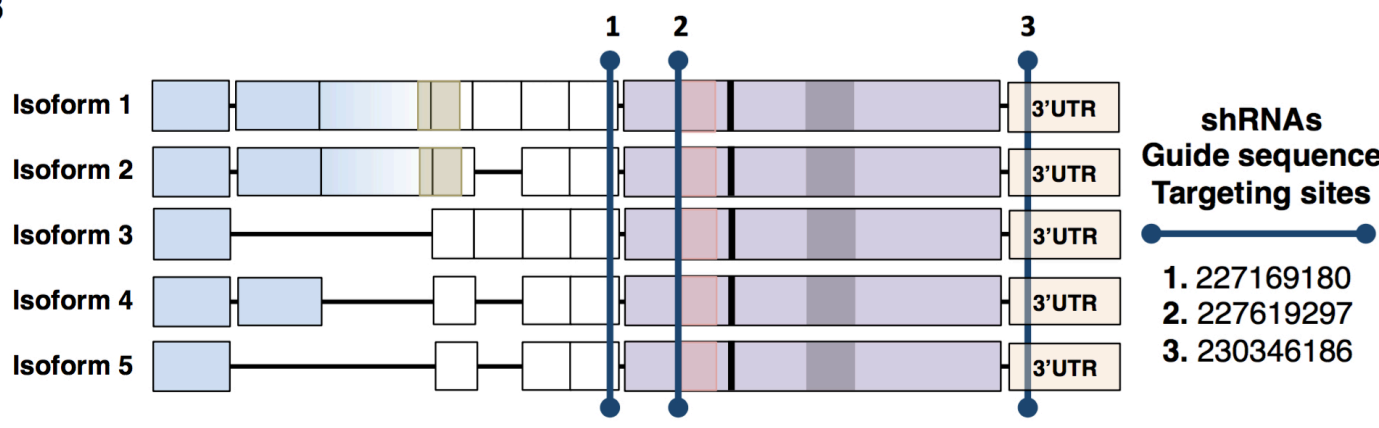

Figure 2. 9 Short hairpin RNAs targeting HBO1

(A) Schematic representation of the structure of a SMARTvector ${ }^{\mathrm{TM}}$ lentiviral shRNA vector. LTR: Long terminal repeat; $\Psi$ : Psi packaging sequence; RRE: Rev response element; tGFP: TurboGFP; hCMV: human cytomegalovirus; IRES: Internal ribosomal entry site; Puro ${ }^{\mathrm{R}}$ : Puromycin resistance, WPRE: Woodchuck hepatitis post-transcriptional regulatory element; SIN LTR: self-inactivating LTR; (B) Each horizontal row represents an HBO1 isoform (depicted as translated mRNA plus the 3'UTR region) and the numbered markers indicate sites of shRNA targeting. These isoforms are described in a more detailed manner in chapter 3: Expression and Regulation of HBO1 in Ovarian Cancer. UTR: Un-translated region.

\subsubsection{Optimization of the lentiviral transduction}

Prior to the utilization of lentiviral particles, target cells were tested for optimal transduction conditions and resistance to puromycin. First, non-infected cells were cultured $\mathrm{O} / \mathrm{N}\left(37^{\circ} \mathrm{C}\right)$ in $\operatorname{Costar}^{\mathrm{TM}}$ Corning ${ }^{\mathrm{TM}}$ 96-Well Clear TC-treated plates at different platting densities $(5,000-7,500-10,000$ cells/well) with normal growth medium. After 24h, growth medium was replaced with FBS and FBS-free 'Transduction Medium' containing different concentrations of polybrene (0-14 $\mu \mathrm{g} / \mathrm{mL})$. Next, after 2-3 days of culture, cell confluence and morphologic/phenotypic alterations were assessed using a Nikon Eclipse Ti microscope. This test allowed for the selection of the optimal conditions for UWB1.289 transduction: 5,000 initial cell density and 'Transduction Medium' containing FBS and $10 \mu \mathrm{g} / \mathrm{mL}$ of polybrene. 
Next, non-infected cells were cultured $\mathrm{O} / \mathrm{N}\left(37^{\circ} \mathrm{C}\right)$ in Costar ${ }^{\mathrm{TM}}$ Corning ${ }^{\mathrm{TM}}$ 96-Well Clear TC-treated plates with normal growth medium containing different concentrations of puromycin $(0.1-10 \mu \mathrm{g} / \mu \mathrm{l})$ (sc-108071, Santa $\left.\mathrm{Cruz}^{\circledR}\right)$. Each well was seeded with $\sim 20,000$ cells, assuming that a potential initial seeding of 5,000 cells would achieve that number after 3-4 days of culture ( $24 \mathrm{~h}$ doubling time). Cells were grown for $48 \mathrm{~h}$ and confluence was measured 6, 24 and $48 \mathrm{~h}$ after the addition of puromycin. The UWB1.289 optimal puromycin concentration, $1 \mu \mathrm{g} / \mu \mathrm{l}$, achieved a $95 \%$ cell death after $48 \mathrm{~h}$ of antibiotic treatment.

\subsubsection{Transduction of shRNAs targeting HBO1}

Cells were cultured $\mathrm{O} / \mathrm{N}\left(37^{\circ} \mathrm{C}\right)$ in Costar ${ }^{\mathrm{TM}}$ Corning ${ }^{\mathrm{TM}}$ 96-Well Clear TC-treated plates at the specific density $(5,000$ cells/well) determined on previous lentiviral transduction sections. The next day, non-targeting control particles were diluted in 'Transduction Medium' (FBS $+10 \mu \mathrm{g} / \mathrm{mL}$ polybrene) and added to the cells, prior removal of normal growth medium, for $6-8 \mathrm{~h}$ incubation. Decreasing quantities of lentiviral particles were added to different wells in order to find the optimal conditions for transduction, including lower cytotoxicity and higher transduction efficiency. After the 6-8h incubation, cells were supplemented with normal growth medium and cultured for another $48-72 \mathrm{~h}$.

Finally, the efficacy of each lentiviral transduction was assessed via GFP observation in a Nikon Eclipse Ti microscope. Low quantities of lentiviral particles resulted in limited GFP expression, whereas high quantities displayed noticeable cytotoxic effects, leaving one remaining sample as the ideal condition for optimal transduction $\left(\sim 2.5 \times 10^{4}\right.$ viral particles). In this particular case, and assuming a total number of $\sim 1 \times 10^{4}$ cells per well at the time of adding the viruses, it could be considered that each cell was targeted by approximately 2-3 lentiviral particles, thus limiting the number of insertions per genome to minimise potential off-target gene disruptions.

Once established the optimal conditions for lentiviral transduction, new cells were cultured and transduced with both HBO1 and non-targeting shRNA lentiviral vectors following the guidelines described earlier in this section. Forty-eight hours after the 
addition of lentiviral particles, the culture medium was removed and substituted with selection medium containing puromycin $(1 \mu \mathrm{g} / \mu \mathrm{l})$. Infected cells were cultured in this medium for $72 \mathrm{~h}$, trypsinized and expanded into a larger culture vessel with new medium containing puromycin $(0.5 \mu \mathrm{g} / \mu \mathrm{l})$. After $48 \mathrm{~h}$, infected cells were tested for HBO1 knockdown, as well as GFP expression and expanded until an adequate cell number was achieved. At this point, cells were either used for further experimentation or frozen and stored as described in the cell culture section.

\subsection{Design and construction of an HBO1-expressing vector}

\subsubsection{Plasmid design}

A pCMV-Tag2B epitope tagged mammalian expression vector (211172, Agilent Technologies) was selected for $\mathrm{HBOl}$ sequence insertion (Fig 2.10). First of all, the pCMV-Tag2B multiple cloning site region was interrogated in search for restriction enzymes that could create sticky ends in the DNA backbone (Fig 2.10 B, C).

A

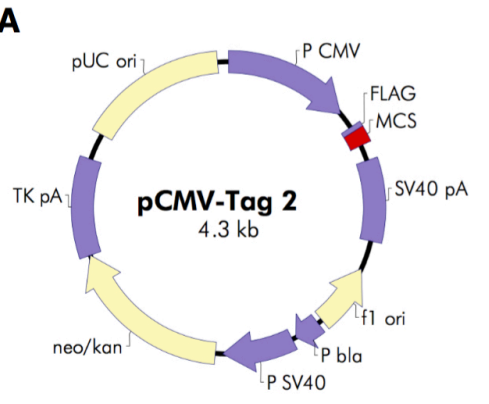

C

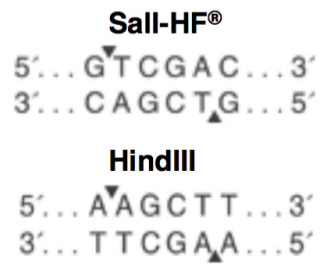

B pCMV-Tag2B Multiple Cloning Site Region

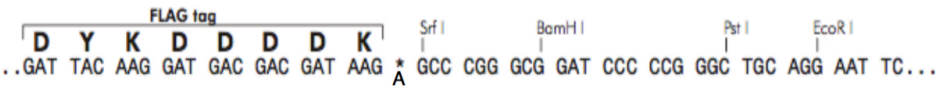

EcoRV Hind III Accl/Soll Xhol Apol

...

T7 promoter

... TAATTAATTAAggtaCCAggtAagtgtacCCAATTCGCCCTATAGTGAGTCGTATTA MULTIPLE STOP CODONS

Figure 2. 10 Design and construction of an HBO1-expressing plasmid (A) Structure of the pCMV-Tag2B vector. (B) Illustrative depiction of the pCMVTag2B multiple cloning site region. (C, D) Selected restriction enzymes cleavage sites $(\mathrm{C})$ and digestion conditions (D). 
Laboratory stock availability and buffer activity complementation led to the election of HindIII (R0104, New England BioLabs ${ }^{\circledR}$ ) and SalI-HF ${ }^{\circledR}$ (R3138, New England BioLabs $^{\circledR}$ ) restriction enzymes, as well as NEBuffer 2.1 (B7202S, New England BioLabs $^{\circledR}$ ), for plasmid digestion (Fig. 2.10 D). The $H B O 1$ sequence was obtained from cDNA of HOSEpiC cells.

\subsubsection{Plasmid construction}

The production of an $H B O 1$ insert from HOSEpiC cDNA was undertaken using a pair of primers designed to bind un-translated regions (UTR) of the $H B O 1$ transcript to create a template for nested PCR (Table 2.20). This template was subjected to a new PCR using specific primers pre-designed to introduce target sites for the selected restriction enzymes (Table 2.20). Both the empty pCMV-Tag2B plasmid $(1 \mu \mathrm{g})$ and the $\mathrm{HBOl}$ template (500ng) were digested at $37^{\circ} \mathrm{C}$ for $90 \mathrm{~min}$ following manufacturer's recommendations. The resultant digested products were then purified using a GeneJET PCR Purification Kit (K0701, Thermo Scientific ${ }^{\mathrm{TM}}$ ).

Table 2. 20 List of primers used to construct an HBO1-expression plasmid

\begin{tabular}{|c|l|c|}
\hline Name & \multicolumn{1}{|c|}{ Sequence } & \multicolumn{1}{|c|}{ Step } \\
\hline HBO1- & F: 5'-GCAGGATTGGGACTGATACAG-3' & Nested \\
UTR & R: 3'-CTCACTGAGGCCGAAACAAC-5' & PCR 1 \\
\hline Tag2B- & F: 5'-GCAAAGCTTATGCCGCGAAGGAAG-3' & Nested \\
HBO1 & R: 3'-GGTTCCCGTGAATTCAGCTGTCA-5' & PCR 2 \\
\hline \multirow{2}{*}{ FLAG- } & F: 5'-CACCATGGATTACAAGGATGAC & Colony \\
HBO1 & $\begin{array}{l}\text { GACGATAAG-3' } \\
\text { R: 3'-TTGCTTCCCTCTGTGATTTG-5' }\end{array}$ & Check \\
\hline \multirow{2}{*}{ G485A- } & F: 5'-CTCAGTACATGAGACAGGCCTATGGC & Mutagenesis \\
HBO1 & RAGATGCTTATTG-3' 3'-CAATAAGCATCTTGCCATAGGCCTGT & wt: GGC (G) \\
& CTCATGTACTGAG-5' & GCC (A) \\
\hline
\end{tabular}

Once digested, both products were ligated $\mathrm{O} / \mathrm{N}\left(16^{\circ} \mathrm{C}\right)$ using $\mathrm{T} 4$ DNA Ligase (M0202, New England BioLabs ${ }^{\circledR}$ ). The NEBioCalculator was used to determine the appropriate amounts of plasmid/DNA for the T4 DNA ligation (1:3, vector/insert). Empty pCMV-Tag2B plasmid was used as negative control (pCMV-Tag2B-Ø). 
After the $\mathrm{O} / \mathrm{N}$ incubation, $\mathrm{T} 4$ ligase was inactivated for $10 \mathrm{~min}\left(65^{\circ} \mathrm{C}\right)$ and the mixture was cooled on ice. Finally, a fraction of the newly synthesized HBOIexpressing plasmid ( $5 \mu \mathrm{l})$ was transformed into $\mathrm{NEB}^{\circledR}$ 5-alpha competent E.coli cells following manufacturer's instructions.

\subsubsection{Plasmid isolation}

Transformed E.coli cells were spread onto lysogeny broth agar selection plates containing $50 \mu \mathrm{g} / \mathrm{mL}$ of kanamycin $\left(\mathrm{K} 0253\right.$, Sigma $\left.{ }^{\circledR}\right)$ and incubated $\mathrm{O} / \mathrm{N}$ at $37^{\circ} \mathrm{C}$. Colonies derived from the $\mathrm{O} / \mathrm{N}$ incubation were re-suspended with super optimal broth with catabolite repression medium or SOC $\left(15544034\right.$, Invitrogen $\left.{ }^{\circledR}\right)$ and a small fraction $(1 \mu \mathrm{l})$ was subjected to PCR in order to confirm presence or absence (Ø) of plasmid-template ligation (Table 2.20). Positive colonies were grown $\mathrm{O} / \mathrm{N}$ $\left(37^{\circ} \mathrm{C}\right)$ in $5 \mathrm{~mL}$ of sterilized lysogeny broth containing $50 \mu \mathrm{g} / \mathrm{mL}$ of kanamycin. A considerable fraction of the broth containing $\mathrm{O} / \mathrm{N}$ grown cells (4/5) was mixed 2:1 with $50 \%$ Glycerol and kept in $-80^{\circ} \mathrm{C}$ as a stock. The remaining cells (1/5) were subjected to plasmid extraction using a QIAprep Spin Miniprep Kit. Plasmid DNA sequencing and western experiments were conducted to re-assure the integrity of the HBO1-expression plasmid.

\subsubsection{Directed-site mutation of the HBO1-expression plasmid}

The QuickChange Primer Design software (Agilent Technologies) was used to generate a pair of primers that introduce a mutation in the $\mathrm{HBOl}$ template inserted in the pCMV-Tag2B plasmid. The specific mutation, glycine to alanine (G485A) (Yan et al. 2000; Iizuka et al. 2009), was introduced using the QuickChange II SiteDirected Mutagenesis Kit (200523, Agilent Technologies). The pCMV-Tag2BhHBOI plasmid was subjected to end-point PCR (Table 2.20) followed by the incubation with DPN I enzyme $(10 \mathrm{U} / \mu \mathrm{l})$ for $1 \mathrm{~h}\left(37^{\circ} \mathrm{C}\right)$. After this, $5 \mu \mathrm{l}$ of the DPN I reaction were transformed into new $\mathrm{NEB}^{\circledR}$ 5-alpha competent E.coli cells which were then incubated $\mathrm{O} / \mathrm{N}\left(37^{\circ} \mathrm{C}\right)$ on lysogeny broth agar selection plates $(50 \mu \mathrm{g} / \mathrm{mL}$ kanamycin). Finally, isolated plasmids were sequenced to confirm mutation events (Fig. 2.11). 
(Gly) GGC $\rightarrow$ (Ala) GCC

wt Colony CTATGCCTCAGTACATGAGACAGGGCTATGGCAAGATGCTTATTGATTTCA

mut Colony 1 CTATGCCTCAGTACATGAGACAGGCCTATGGCAAGATGCTTATTGATTTCA

mut Colony 2 CTATGCCTCAGTACATGAGACAGGCCTATGGCAAGATGCTTATTGATTTCA

mut Colony 3 CTATGCCTCAGTACATGAGACAGGCCTATGGCAAGATGCTTATTGATTTCA

************************** *******************************

Figure 2. 11 Generation of an HBO1-mutant via site-directed mutagenesis

Wild type (wt) and three mutant (mut) colonies of E.coli expressing pCMV-Tag2B$h H B O 1$ and potential pCMV-Tag2B-hHBOl (G485A) plasmids respectively were sequenced to confirm the introduction of the selected mutation.

\subsection{Sequencing of messenger RNA}

\subsubsection{Sample collection and sequencing}

Total RNA samples were obtained following the method described previously in the

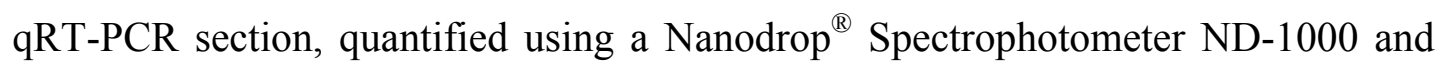
sent to the Epigenomics Core Facility at Weill Cornell Medical College to conduct RNA-seq. Specifically, total RNA samples were extracted from three biological replicates of UWB1.289 cells transduced with the shRNA vector numbered 227169180 targeting $\mathrm{HBOl}$ and the non-targeting vector (Table 2.19). The required sample concentration for each replicate was $50 \mathrm{ng} / \mu \mathrm{l}$ in a volume of $10 \mu \mathrm{l}$.

The Epigenomics Core performed 'TruSeq Stranded RNA-seq (polyA) Library Preparation' for each sample using the Illumina TruSeq Stranded mRNA Library Preparation kit (RS-122-21, Illumina ${ }^{\circledR}$ ). This approach retains the strand orientation of the transcripts and captures both known and novel mRNA features. Samples were multiplexed into one sequencing lane, allowing for approximately 40-45 million reads per sample. The Epigenomics Core conducted Paired-End RNA Sequencing (2x50 sequencing cycles, maximum 100bps per RNA) on a HiSeq 2500 System (Illumina $^{\circledR}$ ). RNA-Seq raw data was finally deposited to PubShare for downloading. 


\subsubsection{Data analysis}

Resulting RNA-Seq raw data (FASTQ files) was downloaded and deposited into a Linux HPC cluster server. Prior to the RNA-Seq analysis, the UCSC goldenPath repository website was used to download the hg19 transcriptome (reference) to which the reads were aligned to. Also, next-generation sequencing data was run through the FastQC programme (Leggett et al. 2013) to pre-assess sequencing quality.

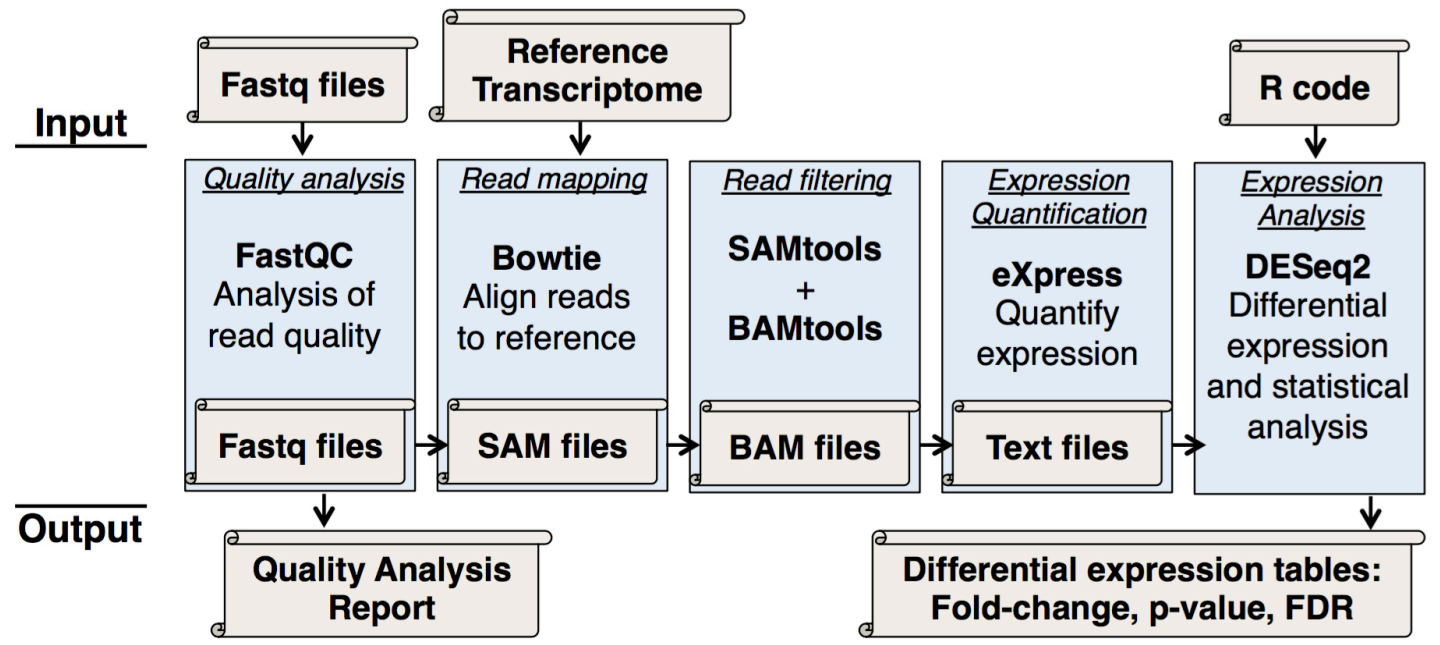

Figure 2. 12 Workflow diagram of the RNAseq analysis pipeline

RNA-seq reads were aligned onto the hg19 indexed reference transcriptome using Bowtie (Langmead et al. 2009), which generated 'Sequence Alignment Map' (SAM) files, a text-based format to store biological sequences aligned to a reference ( $\mathrm{Li}$ et al. 2009) (Fig. 2.12). In order to continue the analysis, SAM files were converted into BAM files (binary SAM files) using SAMTools (Li et al. 2009) and subsequently filtered to exclude reads with unmapped mates (RNA samples only read in one direction) using BAMTools (Barnett et al. 2011). Next, eXpress (Roberts $\&$ Pachter 2013) was used to quantify the expression level, generating a single text file that contains the effective count of every single RNA transcript in each one of the 6 samples (Fig. 2.12). 
Finally, the RNA-Seq differential expression tool package DESeq2 (Love et al. 2014), implemented within R, was used to determine significantly modified transcripts comparing the eXpress results (Fig. 2.12). Specifically, DESeq2 normalized the raw counts, applied a modified unpaired statistical Student's $t$-test (Wald Test) and corrected for multiple hypothesis testing via false discovery rate (FDR) estimation. The outcome of the DESeq2 package analysis included FC values, p-values and q-values (FDR adjusted p-values).

\subsection{Gene Expression Profiling - Microarrays}

\subsubsection{Synthesis of complementary RNA}

Selected total RNA samples $(450 \mu \mathrm{g})$ were used to generate biotinylated, amplified complementary RNA (cRNA) for hybridization with human whole genome expression Illumina ${ }^{\circledR}$ arrays (HumanHT-12 v4). The synthesis and labelling of

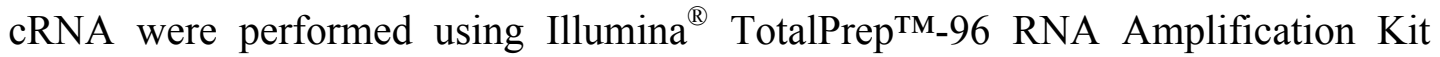
$\left(4393543\right.$, Ambion $\left.^{\circledR}\right)$ following manufacturer's instructions.

All incubation steps were conducted in a MJ Mini ${ }^{\mathrm{TM}}$ Personal Thermal Cycler (Biorad). A 96-well Magnetic-Ring Stand (AM10050, Ambion ${ }^{\circledR}$ ) and a Heidolph Titramax 101 shaker (544-11300-00, Heidolph) were used during the cDNA and cRNA purification steps. The labelling in vitro transcription reaction was performed at $37^{\circ} \mathrm{C}$ for $14 \mathrm{~h}$. Purified cRNA was quantified with a Quant-iT ${ }^{\mathrm{TM}}$ RNA Assay Kit (Q33140, Invitrogen ${ }^{\mathrm{TM}}$ ) using Corning ${ }^{\mathrm{TM}}$ Clear Polystyrene 96-well micro-plates (Corning ${ }^{\mathrm{TM}}$ ), a Safire ${ }^{2}$ micro-plate reader at $644_{\mathrm{Ex}} / 673_{\mathrm{Em}} \mathrm{nm}$ and the XFluor4SafireII software. After this, cRNA samples were either stored at $-20^{\circ} \mathrm{C} \mathrm{O} / \mathrm{N}$ for next-day hybridization or at $-80^{\circ} \mathrm{C}$ for long-term storage. The required cRNA quantity for a 12-Sample BeadChip is 750ng and the total sample volume is $5 \mu 1$. In order to concentrate the cRNA to meet this criteria, samples were occasionally vacuum centrifuged using a Vacufuge plus (022822993, Eppendorf). 


\subsubsection{BeadChip hybridization, scan and analysis}

Biotinylated cRNA samples were hybridized to arrays at $58^{\circ} \mathrm{C}$ for $18 \mathrm{~h}$ in accordance with manufacturer's instructions. The whole hybridization process was conducted using Illumina ${ }^{\circledR}$ equipment (Hyb Oven, Hyb Chamber, Hyb Chamber gasket/inserts, Hybex Waterbath, Hybex Heating Base, Transfer Wash Rack). The BeadArray Reader (iScan System, Illumina ${ }^{\circledR}$ ) was used to scan the arrays and the GenomeStudio software converted the scanned data into meaningful results. The output consisted of text files containing approximately 47,000 rows; each one representing different probes (gene transcripts). For each probe, GenomeStudio provided a non-normalized expression level value (AVG_Signal), the standard error of the bead replicates (BEAD_STDERR), the number of beads (Avg_NBEADS) and a detection p-value (Detection_pval), which estimates the probability of a gene being detected above the background level.

All microarrays were conducted on at least three biological replicates of treatment and control samples. Data obtained from GenomeStudio was background-subtracted and quantile-normalized using the lumi package (Du et al. 2008) within R. The lumi package is a tool for the analysis and comprehension of high-throughput genomic data and it was downloaded from an open-source software site named Bioconductor. The Multiple Experiment Viewer (MEV) (Chu et al. 2008) was utilized to perform statistical analyses and correct for multiple hypotheses testing via FDR estimation. MEV provides several statistical analysis methods for microarray data, being the Linear Models for Microarray Data or LIMMA the most frequently used (Smyth 2004). Nonetheless, the two-class paired rank product method is less stringent and more appropriate for the elevated variability of the biological replicates in this study and hence it was applied to all analysis (Breitling et al. 2004; Koziol 2010). Consequently, MEV provided a list of differentially expressed genes together with their associated FC and q-values (FDR adjusted p-values) (Noble 2009). The SQL database was used to link Illumina ${ }^{\circledR}$ probe identifiers with standardised gene symbols.

The lists of differentially expressed genes were compared graphically using Venny (Hulsen et al. 2008). 


\subsection{Pathway Analysis of Gene Expression Profiles}

\subsubsection{Gene Set Enrichment Analysis}

The role of Gene Set Enrichment Analysis (GSEA, Broad Institute) is to interpret results obtained in gene expression profiling experiments comparing two sample classes (e.g. Treatment and Control), in order to gain insights into driver biological mechanisms (Subramanian et al. 2005). For this purpose, the GSEA algorithm is designed to determine whether members of a Gene-Set (GS) are randomly distributed or primarily found at the top (up-regulated) or bottom (down-regulated) of a ranked list of differentially expressed genes. Gene-Sets are groups of genes that share common characteristics (e.g. biological function, chromosomal location, pathway regulation) and are defined based on prior biological knowledge. They are grouped in collections, which can be downloaded from the Molecular Signatures Database or MSigDB. Specifically, the Hallmarks collection, which contains 50 GSs representing well-defined biological states, was intersected with expression profile data (microarrays) to anticipate alterations of main canonical pathways (e.g. Wnt/ $\beta$ catenin signalling) and processes (e.g. cell apoptosis) caused by gene expression changes.

First, text files containing microarray quantile-normalized data were uploaded to the GSEA proprietary programme, which ranked the $\sim 47,000$ genes detected by the Illumina ${ }^{\circledR}$ HumanHT-12 v4 array based on differential expression levels between the classes (e.g. Treatment and Control) (Fig. 2.13 A). Next, the GSEA algorithm calculated an enrichment score (ES) reflecting the degree to which a defined GS is overrepresented at the top or bottom of the entire ranked gene list (Fig. 2.13 B). The ES was calculated by walking down the entire list of genes incrementing or decreasing the sum-value when confronting or not GS hits. The magnitude of the increment depended on the correlation of the gene with the phenotype (higher if the gene is found at the extremes of the ranked list) and the final ES was the maximum deviation from zero encountered in the walk. The leading-edge subset was interpreted as the core of a GS that accounted for the ES signal (Fig. 2.13 B) (Subramanian et al. 2005). 
A

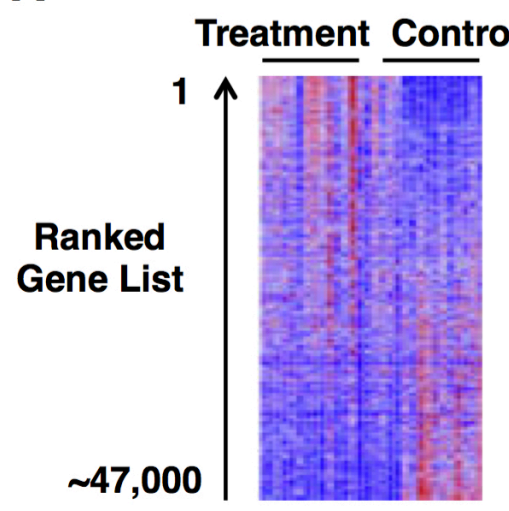

B

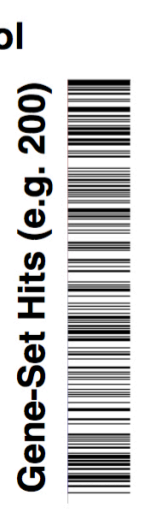

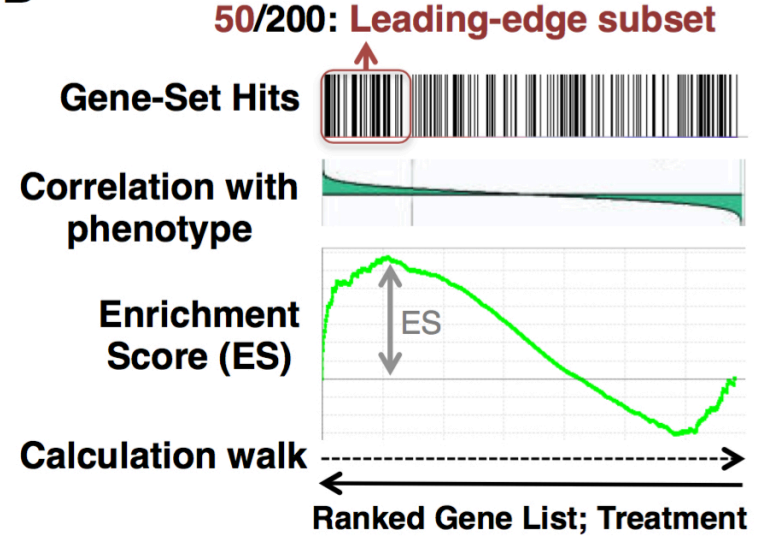

Figure 2. 13 Example of GSEA analysis comparing two samples

(A) Approximately 47,000 genes analysed by the Illumina ${ }^{\circledR}$ arrays were ranked according to their differential expression between the samples 'Treatment' and 'Control'. The 200 genes that belong to the studied Gene-Set displayed a characteristic distribution along the target ranked gene list. (B) Out of the 200 genes of the studied Gene-Set, 50 were located at the top of the ranked gene list (leadingedge subset). This tendency to be grouped at the top or the bottom of a given ranked gene list is converted into enrichment score values by GSEA.

Finally, ES values were subjected to statistical analysis and adjusted for multiple hypothesis testing, which estimated the probability that a normalized Gene-Set ES (NES) represented a false positive finding (Subramanian et al. 2005). The outcome generated by GSEA included the GSs displaying higher NES, either at the top (gene up-regulation) or the bottom (down-regulation) of the ranked gene list, their associated q-value, an enrichment plot (Fig. 2.13 B) and the list of GS genes involved in such biological pathway or function.

\subsubsection{Ingenuity Pathway Analysis}

Ingenuity Pathway Analysis (IPA, Ingenuity Systems ${ }^{\circledR}$, Qiagen ${ }^{\circledR}$ ) is a web-based software application that enables analysis, integration, and understanding of data from gene expression microarrays or RNA-seq. Using prior biological knowledge and a refined suite of algorithms and tools, IPA is able to predict alterations of main canonical pathways (e.g. Wnt/ $\beta$-catenin signalling) and downstream effects on biological functions (e.g. cell proliferation) caused by the observed gene expression changes (Krämer et al. 2014). 
Files containing differentially expressed genes and their associated FC values were uploaded into the IPA proprietary software. Unlike GSEA, where the input consisted of entire normalized gene microarrays ( 47,000 genes), IPA generated significant predictions using selected cohorts of differentially expressed genes only. The outcome generated by the IPA 'Canonical pathways' feature included the most significant affected pathways, their associated p-value, z-score and a list of the modified genes involved in such pathway. Significance values (p-values) were calculated using a Fisher's exact test right-tailed statistical analysis and z-scores predicted either an overall increase or decrease in the activity of the pathway.

A

\begin{tabular}{|c|c|l|c|}
\hline Categories & Function & Prediction & Z-score \\
\hline Cell growth & Proliferation & & -0.121 \\
\hline Cancer & Metastasis & Increased & $\mathbf{2 . 7 4 3}$ \\
\hline Cell death & Apoptosis & Decreased & $\mathbf{- 2 . 1 8 6}$ \\
\hline Cell cycle & Interphase & & 0.512 \\
\hline
\end{tabular}

B

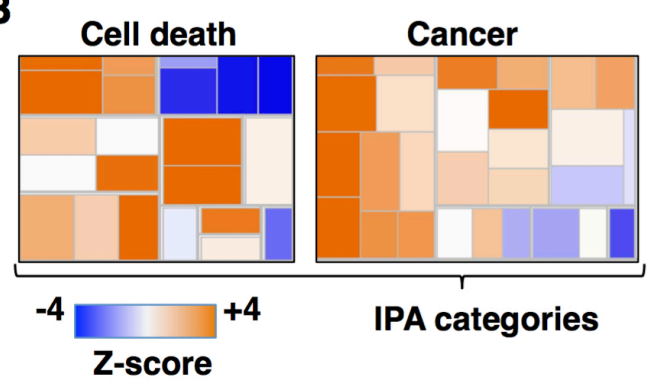

Figure 2. 14 Example of an IPA 'Downstream effects' outcome

(A) Table summarizing an exemplary outcome of the IPA 'Downstream effects' analysis. (B) IPA illustrative depiction of the biological effects caused by observed gene expression changes. Specific biological functions are represented as coloured rectangles enclosed in 'IPA categories', whose size is proportional to the number of modified genes associated to that function. Scale is shown at the bottom.

The IPA 'Downstream effects' feature anticipated the increase or decrease of certain biological functions, foretelling the development of biological processes and diseases. Similar to the results obtained with the 'Canonical pathways', the outcome of the IPA 'Downstream effects' feature included the affected biological function, a significance $\mathrm{p}$-value, $\mathrm{z}$-score, a prediction of the activation state (increased or decreased), and the list of genes involved in the biological process. A summarized exemplary outcome of the IPA 'Downstream effects' feature is depicted in Figure 2.14 A above these lines. As well as the aforementioned report, IPA 'Downstream effects' analysis provided illustrative pictures showing the effect of gene expression changes on broad biological areas or 'IPA categories' (e.g. cell death) (Fig. 2.14 B). 


\subsection{Atomic Force Microscopy}

Atomic Force Microscopy (AFM) was selected to study cell mechanics, which affect important factors of cellular function, including shape, motility, differentiation, division or adhesion, and, most importantly, can act as a reliable indicator of the structure and function of the underlying cytoskeleton (Moeendarbary \& Harris 2014). The AFM technique is based on measuring the attractive and repulsive forces acting between the atoms of a tip and those of the sample's surface. AFM instruments developed for cell biology research setups are known as bio-AFMs and are capable of combining live-cell fluorescence with force measurements at desired cellular locations (Gavara 2017).

Cells were cultured $\mathrm{O} / \mathrm{N}$ in $60 \times 15 \mathrm{~mm}$ TC dishes $\left(\right.$ Falcon $\left.{ }^{\circledR}\right)\left(37^{\circ} \mathrm{C}\right)$ until they reached $\sim 70 \%$ confluence. After $24 \mathrm{~h}$, cells were analysed in growth medium at $37^{\circ} \mathrm{C}$ using a BioScope Catalyst ${ }^{\mathrm{TM}}$ BioAFM (Bruker Instruments). Borosilicate colloidal probes (Novascan) with a sphere radius of $2.5 \mu \mathrm{m}$, a spring constant of $0.17 \mathrm{~N} / \mathrm{m}$ and a deflection sensitivity of $20.11 \mathrm{~nm} / \mathrm{V}$ were used to analyse a total of 40 cells per sample. Three force curves were acquired on the centre of each cell, using a ramp size of $3.5 \mu \mathrm{m}$, a ramp speed of $1 \mathrm{~Hz}$ and an applied force of $400 \mathrm{pN}$. The region of contact in each approach curve was fitted with the Hertz model using the Nanoscope Analysis software (Version 1.5, Bruker Instruments). Each retraction curve was analysed for the presence of adhesion events and only data displaying a goodness-offit higher than 0.9 was taken into consideration. Data distribution and statistical analysis were performed using Wolfram Mathematica 10 and Minitab (Version 17).

All data was analysed for normality using the Kolmogorov-Smirnov test with datasets shown to be statistically different to normal distribution ( $p$-value $<0.05$ ) designated as non-parametric. Following the assumption of variance, pairwise data comparisons were undertaken using the Mann-Whitney $U$ test, with statistical difference stated at 95\% confidence intervals ( $p$-value $<0.05$ ). 


\subsection{Scratch/Wound-healing and Cell Invasion Assays}

The stages of the assay described by Straatman (Straatman 2008) were followed in order to perform a scratch/wound-healing assay. First, cells were cultured in Costar $^{\mathrm{TM}}$ Corning ${ }^{\mathrm{TM}}$ 24-Well Clear TC-treated plates to reach $95-100 \%$ confluence after $24 \mathrm{~h}\left(37^{\circ} \mathrm{C}\right)$. Then, two 'scratches' were made on the surface of the plate (one horizontal, one vertical) using a small pipette tip, followed by immediate replacement of the medium to eliminate debris generated by the scratch. The intersection between the two lines was used as a reference to position the microscope camera. Finally, pictures were taken at different time points (including time $=0$ ) using a Nikon Eclipse Ti microscope.

A

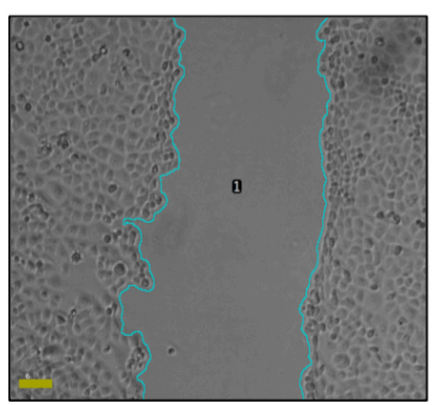

B

\begin{tabular}{|c|c|c|c|}
\cline { 2 - 4 } \multicolumn{1}{c|}{} & Time (t) & Cell-free Area & $\begin{array}{c}\text { Relative Area } \\
\text { (compared to t = 0) }\end{array}$ \\
\hline \multirow{3}{*}{ Control } & $0 \mathrm{~h}$ & 50000 & 0 \\
\cline { 2 - 4 } & $4 \mathrm{~h}$ & 35000 & 15000 \\
\cline { 2 - 4 } & $8 \mathrm{~h}$ & 25000 & 25000 \\
\hline \multirow{3}{*}{ Sample } & $0 \mathrm{~h}$ & 60000 & 0 \\
\cline { 2 - 4 } & $4 \mathrm{~h}$ & 20000 & 40000 \\
\cline { 2 - 4 } & $8 \mathrm{~h}$ & 2000 & 58000 \\
\hline
\end{tabular}

Figure 2. 15 Example of relative area calculations for wound-healing assays (A) Images were taken immediately after the scratch $(\mathrm{t}=0 \mathrm{~h})$ and after $4-8 \mathrm{~h}$ (in this particular example) with a Nikon Eclipse Ti $\left(\mathrm{Nikon}^{\circledR}\right)$ microscope. (B) The ImageJ software was used to calculate cell migration by measuring changes in cell-free occupation area over time. Scale represents $100 \mu \mathrm{m}$.

The ImageJ software (Schneider et al. 2012) including the wound healing tool was used to analyse images and compare changes in cellular properties between treatments (Fig. 2.15). As previously described in the WB section, images representing different time points of the scratch assay were loaded onto ImageJ and converted to 8-bit grey scale images. Subsequently, the 'cell-free' area of target images was calculated applying default ImageJ parameters (Straatman 2008). The difference between the final (e.g. after $4 \mathrm{~h})$ and the starting $(\mathrm{t}=0)$ 'cell-free' value was denominated relative area (RA) and represented the field size that had been taken over by migrating cells (Fig. 2.15). The unpaired one-way ANOVA statistical 
analysis was performed on RA values of three biological replicates using Graphpad Prism. The Tukey's Honest Significant Difference test was used to correct for multiple comparisons testing and a confidence level of 95\% was selected, defining the statistical significance in p-value $<0.05(*)$.

The CytoSelect ${ }^{\mathrm{TM}}$ 24-Well Cell Invasion Assay (Basement Membrane, Colorimetric Format) (CBA-111, Cell Biolabs Inc.) was used to measure the invasiveness of cancer cells. Cells were cultured $\mathrm{O} / \mathrm{N}\left(37^{\circ} \mathrm{C}\right)$ in Costar $^{\mathrm{TM}}$ Corning ${ }^{\mathrm{TM}}$ 6-Well Clear TC-treated plates prior to the addition on the 'Extracellular Matrix Invasion Chamber Plate' $\left(7 \times 10^{5}\right.$ cells $\left./ \mathrm{mL}\right)$, where they were incubated for $24-48 \mathrm{~h}$. The protocol was conducted following manufacturer's instructions and the final absorbance signal was measured with a Safire $^{2}$ micro-plate reader and the XFluor4SafireII software at $560 \mathrm{~nm}$.

\subsection{Enzyme-Linked Immune-Sorbent Assays}

Several commercially available kits were used to perform enzyme-linked immunesorbent assay (ELISA) experiments: VeriKine ${ }^{\mathrm{TM}}$ Human IFN- $\alpha$ Multi-Subtype ELISA Kit (41110, PBL Assay Sciences), VeriKine ${ }^{\mathrm{TM}}$ Human IFN Beta ELISA Kit (41410, PBL Assay Sciences), Human IFN- $\gamma$ ELISA Kit (KHC4021, Invitrogen ${ }^{\mathrm{TM}}$ ) and BrdU Cell Proliferation ELISA Kit (colorimetric) (ab126556, Abcam ${ }^{\circledR}$ ). All protocols were performed following manufacturer's instructions using EDP3-Plus ${ }^{\mathrm{TM}}$ Electronic Pipettes (17013796, Rainin) and common reagent reservoirs.

The interferon alpha (IFNA/ $\alpha$ ), interferon beta (IFNB/ $\beta$ ) and interferon gamma (IFNG/ $\gamma$ ) ELISA protocols were used to measure the amount of these signalling proteins in TC medium. For this purpose, cells were cultured in Costar ${ }^{\mathrm{TM}}$ Corning ${ }^{\mathrm{TM}}$ 12-Well Clear TC-treated plates $\left(37^{\circ} \mathrm{C} ; 80,000\right.$ cells $\left./ \mathrm{mL}\right)$ and the TC medium was extracted after $24 \mathrm{~h}$; unless cells were transfected with siRNA, in which case the cell seeding was lower $(50,000$ cells $/ \mathrm{mL})$ and the TC medium was extracted $48 \mathrm{~h}$ posttransfection. The isolated TC medium was immediately centrifuged at 1,500 rpm (10 minutes, $4^{\circ} \mathrm{C}$ ), separated into aliquots and stored at $-80^{\circ} \mathrm{C}$ until use. 
The Bromodeoxyuridine (BrdU) ELISA Kit was used to study cell proliferation through the incorporation of BrdU to cultured cells. Approximately 20,000 cells were cultured $\mathrm{O} / \mathrm{N}$ in $\mathrm{Costar}^{\circledR}$ 96-Well Clear TC-treated plates $\left(37^{\circ} \mathrm{C}\right)$ and the $\mathrm{BrdU}$ labelling times were 4 and 24h. Again, cells transfected with siRNA were seeded in lower quantities $(\sim 12,000$ cells/well) and the BrdU addition was conducted $48 \mathrm{~h}$ posttransfection.

The absorbance of each sample, blank and standard was measured in duplicate using a Safire ${ }^{2}$ micro-plate reader and the XFluor4SafireII software at $450 \mathrm{~nm}$. In the case of the VeriKineTM Human IFN- $\alpha$ Multi-Subtype ELISA kit, the protocol was performed as per manufacturer's instructions using the High-Sensitivity range of the standard curve $(12.5-500 \mathrm{pg} / \mathrm{mL})$. Sample values lower than the standard were back extrapolated. The unpaired one-way ANOVA statistical analysis was performed on raw absorbance values of three biological replicates using Graphpad Prism. The Tukey's Honest Significant Difference test was used to correct for multiple comparisons testing and a confidence level of $95 \%$ was selected, defining the statistical significance in p-value $<0.05(*)$.

\subsection{ApoTox-Glo Triplex Assay}

The ApoTox-Glo ${ }^{\mathrm{TM}}$ Triplex Assay (G6320, Promega ${ }^{\circledR}$ ) allows assessing of cell viability, apoptosis (caspase activity) and cell death (cytotoxicity) in the same experimental plate. Viability and cytotoxicity are measured by live-cell/dead-cell protease activity with the addition of glycyl-phenylalanyl-amino-fluorocoumerin (GF-AFC) and bis-Ala-Ala-Phe-rhodamine 110 (bis-AAF-R110) substrates respectively. Caspase activation is measured by luciferase activity dependent on caspase cleavage of the substrate present in the Caspase-Glo ${ }^{\circledR}$ 3/7 Reagent.

Cells were cultured in Costar ${ }^{\mathrm{TM}}$ Corning ${ }^{\mathrm{TM}}$ 96-Well Clear TC-treated plates for $24 \mathrm{~h}$ $\left(37^{\circ} \mathrm{C}\right)(\sim 12,000$ cells/well) followed by the sequential addition of substrates and measuring of the resulting signal. After 30 minutes, cells were exposed to different excitation wavelengths (400/485), whose fluorescence emission was recorded as viability/cytotoxicity measurements respectively (505/520) using a Safire ${ }^{2}$ micro- 
plate reader and the XFluor4SafireII software. Luciferase activity was measured after $1 \mathrm{~h}$ of substrate incubation and all samples were tested in triplicates. In case cells were transfected with siRNA prior to the assay, they were seeded in lower concentrations $(6,000$ cells/well $)$ and the substrates were added $48 \mathrm{~h}$ posttransfection. In all circumstances and as additional controls, substrates were always added to un-treated wells and wells without cells.

Table 2. 21 Example of relative cell calculations for ApoTox-Glo ${ }^{\mathrm{TM}}$ assays

\begin{tabular}{|c|c|c|}
\hline Sample & $\begin{array}{c}\overline{\boldsymbol{x}} \text { Raw values }-\overline{\boldsymbol{x}} \text { no } \\
\text { substrate control }\end{array}$ & Relative cell (\%) activity \\
\hline Treatment & 33,903 & 110.12 \\
\hline Control & 30,845 & 100 \\
\hline Treatment & 51,844 & 87.13 \\
\hline Control & 59,496 & 100 \\
\hline
\end{tabular}

The unpaired one-way ANOVA statistical analysis was performed on raw fluorescence/luminescence values of three biological replicates using Graphpad Prism. The Tukey's Honest Significant Difference test was used to correct for multiple comparisons testing and a confidence level of $95 \%$ was selected, defining the statistical significance in p-value $<0.05(*)$. The graphical depiction of relative cell viability, cytotoxicity and caspase activity represents the proportional difference (\%) between a treatment and its control (Table 2.21). 


\section{Expression and Regulation of HBO1 in Ovarian Cancer}




\subsection{Introduction and Aims}

Disturbances in epigenetic regulatory mechanisms are decisive in tumorigenesis and, particularly, in OC development (Balch et al. 2009; Brien et al. 2016). The widereaching opportunities of epigenetic agents, which can reverse these alterations via multiple signalling pathways without relying on single gene mutations, open the door to new potential promising OC therapies (Azad et al. 2013). In this regard, one epigenetic regulator named 'Histone acetyltransferase binding to ORC-1' (HBO1, also known as KAT7 or MYST2) could be a potential target for OC treatment.

The vast majority of studies on $\mathrm{HBO} 1$ in relation to tumour progression are based on breast cancer settings. In this context, HBO1 was found overexpressed in breast cancer tissues and cell lines (Iizuka et al. 2009; Duong et al. 2013), where it displayed pro-oncogenic properties (Iizuka et al. 2013). Importantly, Iizuka et al. (2009) detected strong HBO1 expression in a cohort of OC samples, pointing to a prominent role of the protein in OC development.

The first objective of this project was to establish valid cell model systems for the study of HBO1 in OC. These models included the most prominent OC sub-types (serous, endometrioid, mucinous and clear-cell), which represent approximately $90 \%$ of all OC cases (Meinhold-Heerlein \& Hauptmann 2014), alongside an appropriate non-cancerous control for comparison. Once established, the OC models were analysed for HBO1 expression both at transcript (qRT-PCR) and protein level (WB). HBO1's precursor mRNA is spliced into 5 different isoforms, the expression of which was also analysed in the model systems. Furthermore, the study of HBO1 expression allowed for the optimization of appropriate tools (e.g. antibodies) used in subsequent HBO1 functional studies.

The next objective was to evaluate potential factors that may lead to HBO1 overexpression in the OC models. For this purpose, the proximal promoter region of the HBOl locus was analysed for well-known binding sites of possible nuclear receptor (NR) gene regulators. 
The final aim of this chapter was to study the localization of HBO1 in the 'OC genome' via ChIP-Seq, in order to provide insights into HBO1 regulatory mechanisms, including potential gene targets and the association of HBO1 with repressive or activating genomic domains. In addition, features including subcellular protein localization and multi-protein complex associations were investigated.

\subsection{Ovarian Cancer Cell Line Model Systems}

There are two major approaches to study the mechanics of OC: immortalized cell lines and primary cells isolated directly from malignant ovarian tissues. Even though primary cells are more biologically relevant due to their genetic resemblance to the original patient, OC cell lines offer multiple other advantages including reproducibility, which allows the direct comparison of results between studies (Kaur \& Dufour 2012; Geraghty et al. 2014). Based on this premise and to elucidate the role of HBO1 in OC, a representative panel of cell lines was selected (Table 3.1) (Beaufort et al. 2014). The chosen cell lines represented all the major OC histotypes, but emphasized the predominance of serous histology.

Table 3. 1 Ovarian cancer cell line model system

\begin{tabular}{|c|c|c|c|c|}
\hline Cell line & Tissue of origin & Histology & Grade & Stage \\
\hline HOSEpiC & Ovary & OSE $^{1}$ & & \\
\hline OVCAR-3 & Ascites & High-grade serous & 3 & und $^{2}$ \\
\hline SKOV-3 & Ascites & Serous & $1 / 2$ & und \\
\hline UWB1.289 & Ovary & Serous & und & und \\
\hline UACC-1598 & Ovary & Serous & 3 & IV \\
\hline TOV-112D & Ovary & Endometrioid & 3 & IIIC \\
\hline TOV-21G & Ovary & Clear-cell & 3 & III \\
\hline COV644 & Ovary & Mucinous & und & und \\
\hline
\end{tabular}

Cell line characteristics based on Beaufort et al. (2014) and the ATCC ${ }^{\circledR}$ website ${ }^{1}$ OSE: Ovarian surface epithelium ${ }^{2}$ und: Undetermined grade or stage 
The HOSEpiC line is composed of human ovarian surface epithelial (OSE) cells extracted from healthy ovaries (Murdoch 1995). As such, they are not immortalized and hence expanded for a handful of population doublings. In this study, HOSEpiC cells were used as non-cancerous control and their properties were compared to those of OC cell lines.

Amongst the OC cell lines, serous histology, the most prevalent subtype of OC (Soslow 2008), is represented by OVCAR-3, SKOV-3, UWB1.289 and UACC-1598 (Table 3.1). Considering that this study was predominantly focused on this histotype, the selection of the most suitable serous-like cell lines was paramount. In this regard, it is noteworthy that SKOV-3 and OVCAR-3 together account for more than $50 \%$ of PubMed citations for HGSC cell line models (Domcke et al. 2013). Domcke et al. (2013) and Beaufort et al. (2014) have extensively studied the main genomic characteristics and usefulness of SKOV-3, OVCAR-3 and UWB1.289 as HGSC tumour models. However, there is little publicly available information regarding the UACC-1598 cell line, probably due to its handling difficulties, with very slow doubling rates and preference for hypoxic growth.

All serous-like cell lines carry mutated TP53, a characteristic feature of HGSCs (Beaufort et al. 2014). Another frequent alteration linked to HGSC, the mutation of genes related to HRR pathways (Lord \& Ashworth 2016), is found in OVCAR-3 ('partner and localizer of BRCA2' or PALB2) and SKOV-3 (PALB2 and the 'BRCA1 associated RING domain 1' or BARD1) (Beaufort et al. 2014). Additionally, the UWB1.289 cell line carries a null BRCAl gene mutation (DelloRusso et al. 2007). Other relatively common features of HGSC such as CCNE1 or ERBB2 amplification are also present in OVCAR-3 and SKOV-3 cell lines respectively (Domcke et al. 2013). Interestingly, the high-grade endometrioidlike cell line TOV-112D presents a very clear mutational spectrum (carrying only mutations of TP53 and 'catenin beta 1' or CTNNB1) and probably falls into the type II OC classification, together with the rest of serous-like cell lines. It is important to note that even though the four serous-like cell lines have been catalogued as 'serous' and not 'HGSC', they all display HGSC characteristic features and none resemble the low-grade serous histo-type (Table 3.1). 
The majority of OC cell lines used in this study, except SKOV-3 and OVCAR-3, were obtained from the primary localization of OC, the ovaries (Table 3.1). COV644, TOV-112D and TOV-21G were derived from un-treated patients with primary disease; OVCAR-3 and UWB.1289 were collected from relapsed platinumtreated patients. The SKOV-3 cell line was derived from a patient treated with nonplatinum chemotherapy from which there is no clear information regarding the state of the disease (Beaufort et al. 2014). However, neither OVCAR-3 nor SKOV-3 nor UWB1.1289 developed strong resistance against broad chemotherapy, according to Beaufort et al. (2014). There is no information on the exact origin, treatment or resistance capabilities of UACC-1598.

\subsection{Basal Expression of HBO1 Transcripts}

One of the main hypotheses put forward advocates the involvement of HBO1 in the progression of OC. In order to evaluate the comparative levels of HBO1 in each OC cell line compared to the non-cancerous control, isolated RNA samples were analysed for $\mathrm{HBOl}$ transcript expression. According to NCBI databases (NCBI Resource Coordinators 2017), the HBOl locus codes for five alternative splicing variants that give rise to different protein isoforms. Consequently, and in order to quantify the total amount of transcript (mRNA) regardless of the splicing variant, primer pairs were design to amplify all isoforms of $\mathrm{HBOl}$.

The whole panel of $\mathrm{OC}$ cell lines displayed increased $\mathrm{HBOl}$ mRNA relative expression levels compared to the non-cancerous HOSEpiC control (Fig. 3.1). The two cell lines that showed the lowest levels of $H B O 1$ expression, yet still greater than HOSEpiC, were the clear-cell TOV-21G $\left(\mathrm{FC}=1.76,{ }^{*} \mathrm{p}=0.0170\right)$ and the mucinous COV644 $\left(\mathrm{FC}=2.21,{ }^{*} \mathrm{p}=0.0328\right)$ (Fig. 3.1). Interestingly, all serous-like cell lines including, OVCAR-3 $\left(\mathrm{FC}=2.34,{ }^{* * *} \mathrm{p}=0.0002\right)$, SKOV-3 $(\mathrm{FC}=3.67, * * * * \mathrm{p}=<$ 0.0001), UWB1.289 (FC $=4.50, * * * * \mathrm{p}=<0.0001)$ and UACC-1598 (FC = 4.08, $* * * \mathrm{p}=<0.0001)$, in addition to the endometrioid TOV-112D $\left(\mathrm{FC}=3.64,{ }^{* * * *} \mathrm{p}=<\right.$ 0.0001) displayed significant high levels of $\mathrm{HBOl}$ transcripts compared to the HOSEpiC control (Fig. 3.1). 


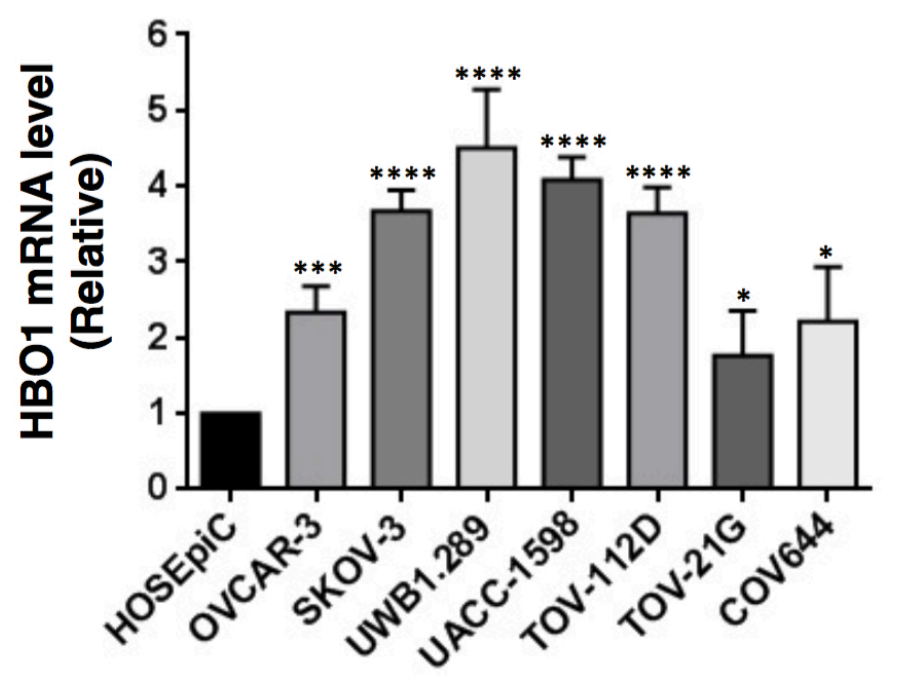

Figure 3. 1 Comparative analysis of $\mathrm{HBO} 1$ transcript expression

RNA lysates from HOSEpiC and 7 OC cell lines were prepared and analysed by qRT-PCR. Relative levels of $H B O 1$ expression were compared to those of the HOSEpiC non-cancerous control. GAPDH expression was used as control. Statistical analysis consisted of one-way ANOVA and the Tukey's multiple comparison test. All values represent the mean \pm standard deviation (SD) of three biological samples $\left(* \mathrm{p}<0.05 ;{ }^{* * *} \mathrm{p}<0.001 ;{ }^{* * * *} \mathrm{p}<0.0001\right)$.

These results show a general augmentation of all $\mathrm{HBO} 1 \mathrm{mRNA}$ isoforms in OC lines compared to the non-cancerous control. Notably, there is growing evidence linking aberrant use of alternative mRNA isoforms with cancer phenotypes (Sveen et al. 2016) and the aberrant use of one gene isoform over another has been directly linked to cancer cell growth (Rajan et al. 2009). In OC, one overexpressed splice variant of the 'baculoviral inhibitor of apoptosis repeat-containing 5' gene (BIRC-5 or 'survivin') was associated with tumour growth in a taxane-resistant setting (VivasMejia et al. 2011). These links raised question as to whether $H B O 1$ overexpression and its potential effects may be associated with the aberrant expression of specific HBO1 splice isoforms. 


\subsection{Basal Expression of HBO1 Isoforms}

\subsubsection{Structure and characteristics of the $\mathrm{HBO1}$ isoforms}

According to the NCBI, HBOl codes a precursor mRNA transcript that is differentially spliced giving rise to five mature mRNA $H B O 1$ isoforms, whose main characteristics are shown in Table 3.2. The specific isoform nomenclature was selected from the consensus coding DNA sequence or CCDS database of the NCBI (Pruitt et al. 2009), as opposed to other designations used by databases such as UniProt (UniProt Consortium 2008). A graphical depiction and comparison of the structure of each one of the HBO1 protein variants is illustrated in Figure 3.2.

Table 3. 2 Structural composition of the five HBO1 isoforms

\begin{tabular}{|c|c|c|c|c|}
\hline Nomenclature & DNA (bp) & Protein (aa) & MW $^{1}$ (kDa) & RefSeq $^{2}$ \\
\hline Isoform 1 & 1836 & 611 & 70 & NP_008998.1 \\
\hline Isoform 2 & 1746 & 581 & 67 & NP_0.001186084.1 \\
\hline Isoform 3 & 1419 & 472 & 55 & NP_001186085.1 \\
\hline Isoform 4 & 1506 & 501 & 58 & NP_001186086.1 \\
\hline Isoform 5 & 1329 & 442 & 51 & NP_001186087.1 \\
\hline
\end{tabular}

${ }^{1} \mathrm{MW}$ : Molecular weight in kilodaltons (unified atomic mass units)

${ }^{2}$ RefSeq: Reference Sequence (O’Leary et al. 2016)

All five HBO1 variants retain a full-length MYST domain but differ in the configuration of the N-terminus (Fig. 3.2). While HBO1 isoform 1 encodes the longest transcript and as a consequence the largest protein, isoform 2 lacks the small alternate in-frame exon 6 (Table $3.2 \&$ Fig. 3.2). Both isoforms 1 and 2 share the same $\mathrm{N}$ and $\mathrm{C}$ terminus, as well as the main functional structures: Ser-rich and MYST domains, zinc fingers, nuclear localization signal (NLS) and the lysine acetyltransferase domain (LAT). HBO1 isoform 3 lacks the alternate in-frame exons 3 and 4, which are part of the Ser-rich domain and are involved in the configuration of a zinc finger structure $\left(\mathrm{C}_{2} \mathrm{H}_{2} \mathrm{C}\right.$, amino acids 189-211) (Fig. 3.2). Similarly, isoform 5 lacks exons 3 and 4 and therefore the zinc finger, in addition to in-frame exon 6. HBO1 isoform 4 lacks in-frame exons 4 and 6 , losing the zinc finger structure but conserving a bigger portion of the Ser-rich domain, as oppose to 
isoforms 3 and 5 (Fig. 3.2). The N-terminus region of HBO1 (amino acids 1-230) is commonly regarded as an intrinsic repression domain (Sharma et al. 2000; Georgiakaki et al. 2006; Contzler et al. 2006). Consequently, splice variants that lack distinct fragments of this domain, such as isoforms 3, 4 and 5 (Fig. 3.2), might lose the corresponding repression function.

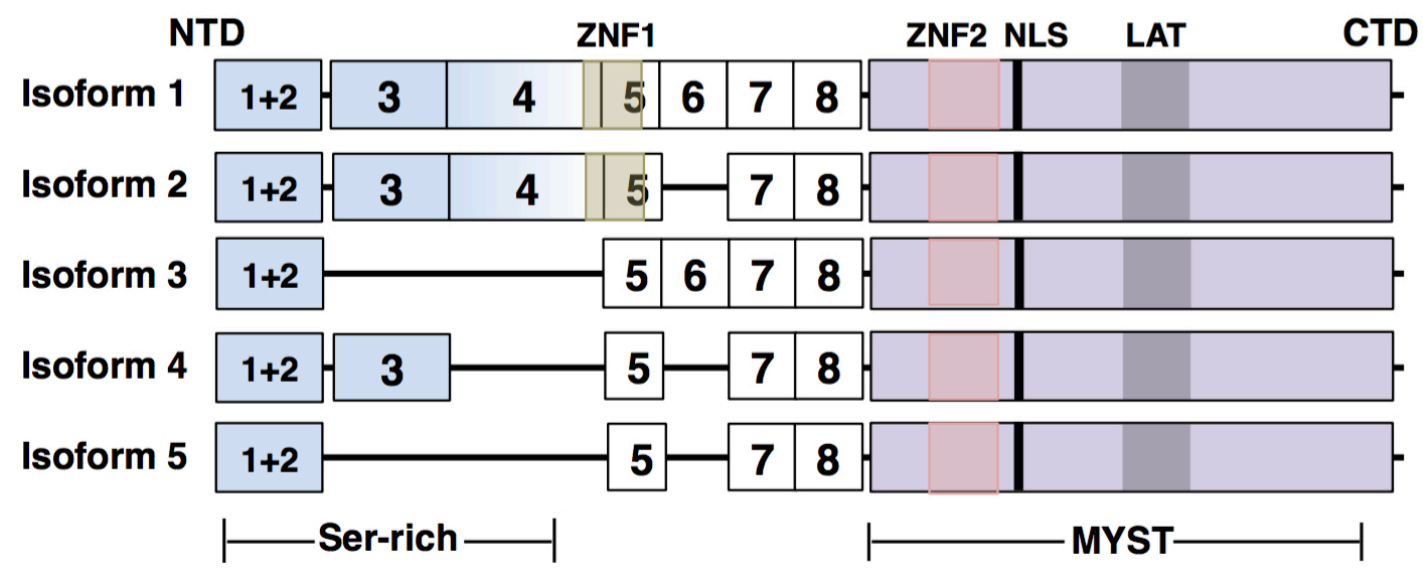

Figure 3. 2 Schematic depiction of the human HBO1 protein variants

The five HBO1 protein isoforms are separated into numbered exon sequences (1-8), except for their C-terminus MYST region (MYST). The serine-rich domain (Serrich) located in the NTD (amino-terminus domain) of the HBO1 protein is represented in clear blue colour and comprises different exons depending on the specific isoform. $\mathrm{ZNF} 1: \mathrm{C}_{2} \mathrm{H}_{2} \mathrm{C}$ zinc finger; $\mathrm{ZNF}$ : $\mathrm{C}_{2} \mathrm{HC}$ zinc finger; NLS: Nuclear localization signal; LAT: Lysine acetyltransferase domain; CTD: Carboxyl-terminus domain.

\subsubsection{Differential expression of HBO1 isoforms in OC cell lines}

Primer pairs spanning isoform-specific exon boundaries were designed to detect and quantify each $\mathrm{HBO}$ isoform transcript. The expression level of each of the five isoforms in the non-cancerous HOSEpiC control was compared to the expression level of those isoforms in each one of the 7 OC cell lines (Fig. 3.3 \& Appendix 1). 


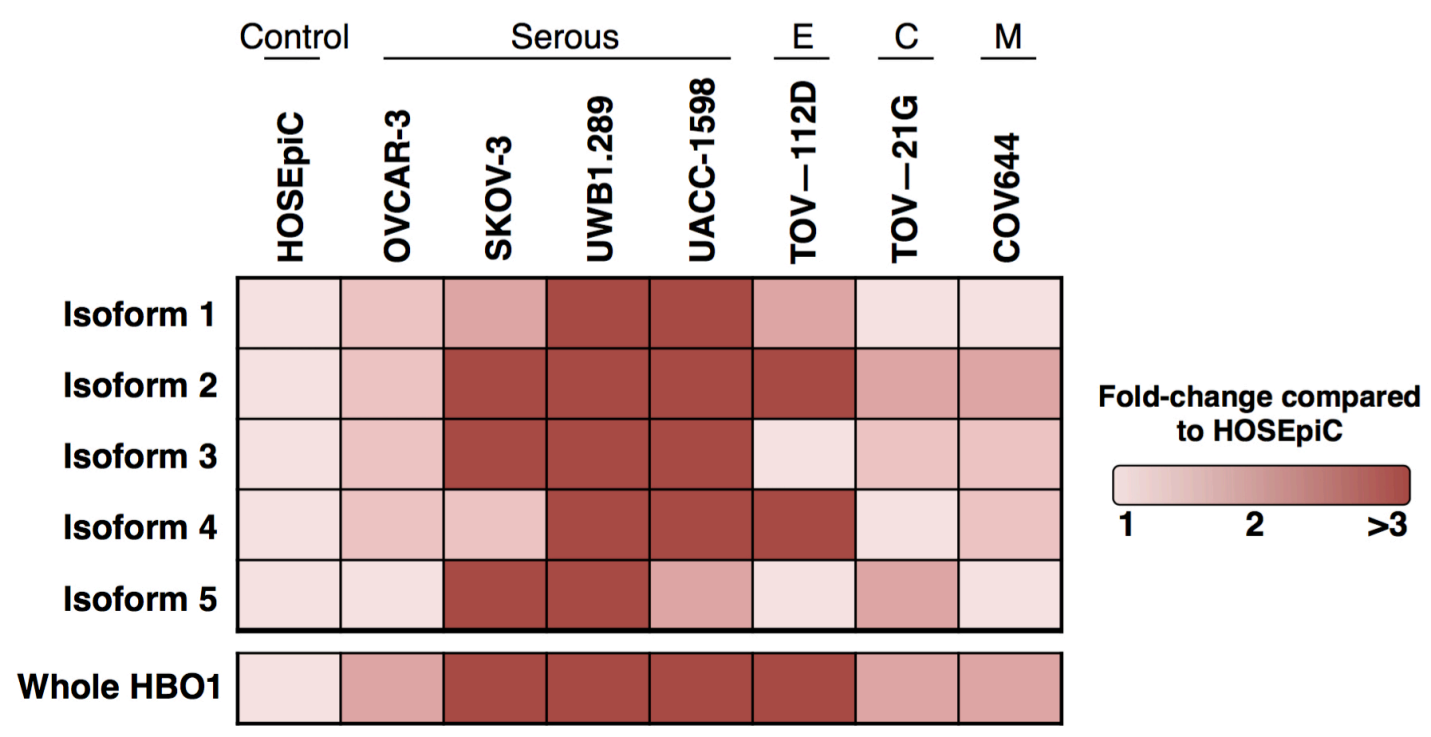

Figure 3. 3 Comparative analysis of HBO1 isoform expression

Graphical depiction of changes in $\mathrm{HBOl}$ isoform transcript abundance comparing HOSEpiC non-cancerous control and the 7 OC cell lines. The red gradation diagram on the right of the panel represents qRT-PCR fold-change values. Individual graphs representing differential isoform expression can be found in Appendix 1 A-E. E: Endometrioid; C: Clear-cell; M: Mucinous.

In general terms and following the trend observed in Figure 3.1, $\mathrm{HBOl}$ isoforms were overexpressed in the panel of OC cell lines compared to the HOSEpiC control (Fig. 3.3). Interestingly, the majority of cell lines with high $\mathrm{HBOl}$ expression levels (SKOV-3, UWB1.289, UACC-1598 and TOV-112D) displayed strong increase of several, if not all HBOl variants, compared to the HOSEpiC control (Fig. 3.3). HOSEpiC control cells did not show higher levels of expression of any $\mathrm{HBOl}$ isoform in comparison with any of the OC cell lines. However, HOSEpiC cells did display a basal level of expression of all isoforms, implying that whatever the source of $\mathrm{HBOl}$ overexpression in $\mathrm{OC}$ is, it does not rely on the expression of a distinctive, cancer-related unique $\mathrm{HBOl}$ variant.

OVCAR-3 cell line displayed moderate to high levels of isoform $1\left(\mathrm{FC}=1.75,{ }^{*} \mathrm{p}=\right.$ $0.0414)$, isoform $2(\mathrm{FC}=1.49, \mathrm{p}=0.1628)$, isoform $3(\mathrm{FC}=1.66, \mathrm{p}=0.0579)$ and isoform $4\left(\mathrm{FC}=1.75,{ }^{*} \mathrm{p}=0.011\right)$, compared to the HOSEpiC control (Fig. 3.3). The rest of the serous-like cell lines, SKOV-3, UWB1.289 and UACC-1598, exhibited much greater up-regulation of most $\mathrm{HBOl}$ isoforms. For instance, SKOV-3 displayed high levels of isoform $1\left(\mathrm{FC}=2.77,{ }^{* *} \mathrm{p}=0.0029\right)$, isoform $2(\mathrm{FC}=3.2$, 
$\left.{ }^{* * *} \mathrm{p}=0.0002\right)$, isoform $3\left(\mathrm{FC}=5.77,{ }^{* * *} \mathrm{p}=0.0007\right)$, isoform $4\left(\mathrm{FC}=2.36,{ }^{* * *} \mathrm{p}=\right.$ $0.0001)$ and isoform $5\left(\mathrm{FC}=7.37,{ }^{* *} \mathrm{p}=0.00721\right)$. Likewise, UACC-1598 exhibited high levels of isoform $1(\mathrm{FC}=4.3, * * * * \mathrm{p}=<0.0001)$, isoform $2(\mathrm{FC}=3.92, * * * * \mathrm{p}=$ $<0.0001)$, isoform $3\left(\mathrm{FC}=3.14,{ }^{*} \mathrm{p}=0.0461\right)$, isoform $4(\mathrm{FC}=3.65, * * * * \mathrm{p}=<$ $0.0001)$ and isoform $5\left(\mathrm{FC}=2.47,{ }^{*} \mathrm{p}=0.042\right)$ (Fig. 3.3).

All isoforms are overexpressed in the UWB1.289 cell line, which showed the highest expression levels of HBO1 (Fig. 3.1), compared to the HOSEpiC control: isoform 1 $\left(\mathrm{FC}=5.27,{ }^{* * * *} \mathrm{p}=<0.0001\right)$, isoform $2\left(\mathrm{FC}=3.32,{ }^{* * *} \mathrm{p}=0.0003\right)$, isoform $3(\mathrm{FC}$ $\left.=3.28,{ }^{* *} \mathrm{p}=0.0078\right)$, isoform $4\left(\mathrm{FC}=3.51,{ }^{* *} \mathrm{p}=0.00741\right)$ and isoform $5(\mathrm{FC}=$ 4.11, $\left.{ }^{*} \mathrm{p}=0.036\right)($ Fig. 3.3). The TOV-112D endometrioid-like cell line showed upregulation of isoform $1\left(\mathrm{FC}=2.48,{ }^{*} \mathrm{p}=0.0107\right)$, isoform $2\left(\mathrm{FC}=4.15,{ }^{* *} \mathrm{p}=\right.$ $0.0076)$ and isoform $4\left(\mathrm{FC}=4.21,{ }^{* *} \mathrm{p}=0.0011\right)$, compared to the HOSEpiC control (Fig. 3.3).

The clear-cell TOV-21G cell line displayed non-significant moderate levels of whole HBO1 (Fig. 3.1), probably caused by the slight increase in isoform $2\left(\mathrm{FC}=2.05,{ }^{*} \mathrm{p}\right.$ $=0.0489)$, isoform $3(\mathrm{FC}=1.76, \mathrm{p}=0.5228)$ and isoform $5\left(\mathrm{FC}=2.82,{ }^{*} \mathrm{p}=\right.$ 0.0375), compared to the HOSEpiC control (Fig. 3.3). Similarly, isoform 2 (FC = $\left.1.96,{ }^{*} \mathrm{p}=0.0485\right)$, isoform $3\left(\mathrm{FC}=1.99,{ }^{*} \mathrm{p}=0.0371\right)$ and isoform $4 \quad(\mathrm{FC}=2.13$, $\left.{ }^{* *} \mathrm{p}=0.0028\right)$ are up regulated in the mucinous COV644 cell line, compared to the HOSEpiC control (Fig. 3.3).

\subsection{Basal Expression of HBO1 Protein in OC}

\subsubsection{Selection of antibodies targeting HBO1}

Western blotting is the most common experiment used to interrogate protein abundance and there are a number of commercially available validated HBO1 antibodies such as those from Abcam $^{\circledR}$ (AB70) or Santa Cruz ${ }^{\circledR}$ (G-2, T-20, H-180, N-18); AB70 and G-2 antibodies were used in this study. 
The monoclonal HBO1 antibody G-2 is specific for an epitope encoded by exon 2, mapping between amino acids 20-47, and therefore should recognize all 5 HBO1 protein isoforms (Fig. 3.2). The AB70 polyclonal HBO1 antibody binds to an internal (and undetermined) region of the N-terminal domain, suggesting it may not recognize all protein variants, however, it was widely used for WB experiments in the literature (Miotto \& Struhl 2008; Avvakumov et al. 2012; Zou et al. 2013; Lalonde et al. 2013).

In order to test the performance of anti-HBO1 G-2 and AB70 antibodies, protein extracts from UWB1.289 and UACC-1598 cell lines were analysed by western blot (Fig. 3.4). Proteins were separated using SDS-PAGE gel electrophoresis on 4-12\% gradient gels to ensure separation of HBO1 isoforms. An anti-GAPDH antibody was used as loading standard.

A

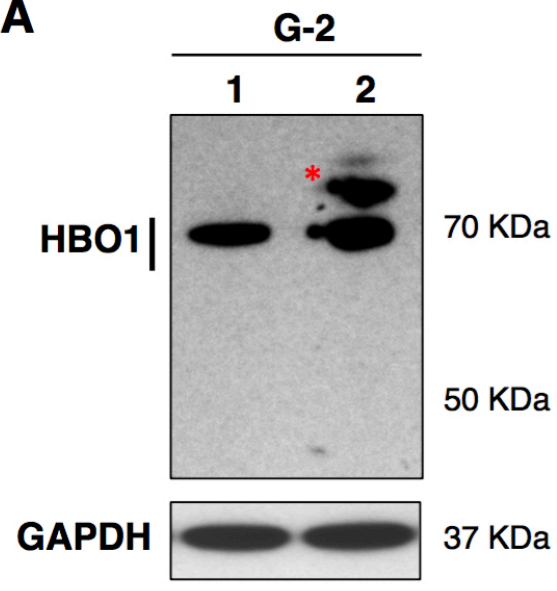

B

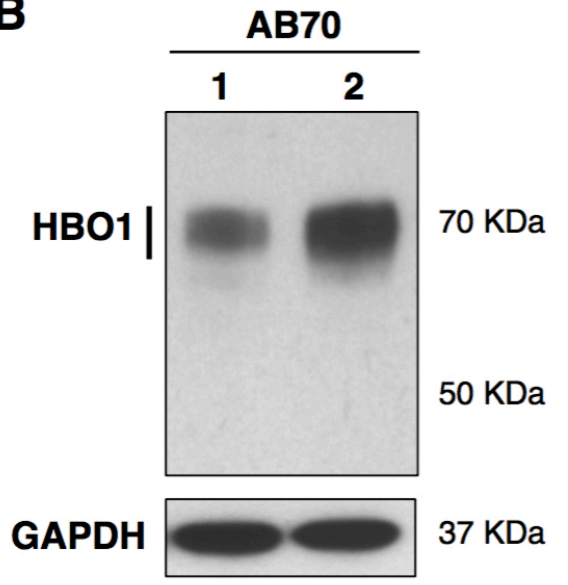

Figure 3. 4 Western blot analyses of $\mathrm{HBO} 1$ antibodies performance

Protein extracts of UWB1.289 and UACC-1598 were analysed by western blot and probed with: (A) G-2 and (B) AB70 anti-HBO1 antibodies. GAPDH was used as control. 1: UWB1.289; 2: UACC-1598; *Unknown bands.

Both antibodies G-2 and AB70 revealed a $\sim 70 \mathrm{KDa}$ band identified as $\mathrm{HBO} 1$ isoforms 1 and 2 (Fig. 3.4). The smeared appearance of this band is probably due to the presence of two similar proteins, isoform $1(70 \mathrm{KDa})$ and isoform $2(67 \mathrm{KDa})$, but also as a result of $\mathrm{HBO} 1$ post-translational modifications such as phosphorylation (Miotto \& Struhl 2011). The absence of bands in the 50-60 KDa 
range suggests that isoforms 3, 4 and 5 are expressed in low levels (Fig. 3.4). Other possibilities can be that the mature mRNA of these isoforms does not translate into protein or that the antibodies do not recognize these specific isoforms.

The unknown bands present in the UACC-1598 lysate (Fig. 3.4 A) might be caused by high weight PTMs such as poly-ubiquitination (Sadowski et al. 2012) or SUMOylation (Flotho \& Melchior 2013), nonspecific antibody binding, noncatalogued HBO1 isoforms or cell line artefacts. HBO1 has been previously linked to ubiquitin-related activity, as it is marked by poly-ubiquitin chains for degradation in the cytoplasm (Zou et al. 2013), so in order to investigate this possibility, the G-2 antibody was used to immune-precipitate (IP) HBO1 prior to ubiquitin detection (Fig. 3.5).

A

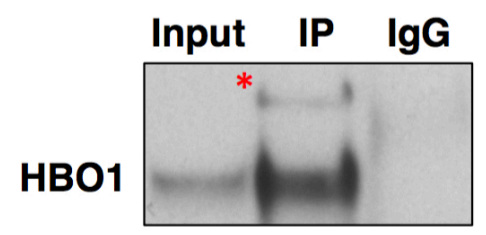

B

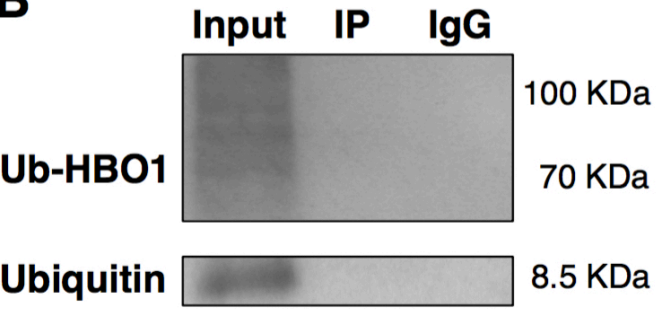

Figure 3. 5 Analysis of HBO1 ubiquitination state via IP using the G-2 antibody UACC-1598 protein lysates were immune-precipitated using anti-HBO1 G-2 antibody, separated using SDS-PAGE and probed with (A) anti-HBO1 and (B) antiubiquitin antibodies. *Unknown bands.

The G-2 antibody was able to specifically IP HBO1 protein $(\sim 70 \mathrm{KDa})$, while no product was isolated with the immunoglobulin $\mathrm{G}(\mathrm{IgG})$ control (Fig. 3.5 A). Again, and as seen in previous results (Fig. 3.4), G-2 detected a $\sim 90-100 \mathrm{KDa}$ undetermined band (Fig. 3.5 A). Surprisingly, the IP sample did not reveal any ubiquitin signal (Fig. 3.5 B), suggesting that the 90-100 KDa band does not represent ubiquitinated protein. The input control revealed multiple ubiquitinated proteins and an $8.8 \mathrm{KDa}$ band representing monomeric ubiquitin protein (Fig. 3.5 B) (Parker et al. 2010). 
Although both antibodies seemed to perform well, some drawbacks of the G-2 Santa Cruz $^{\circledR}$ antibody include the un-recognized bands (Fig. 3.4 A \& Fig. 3.5 A) and the fact that its use has not been previously referred in the literature. In contrast, AB70 specifically recognized isoforms 1 and 2 (inferred by doublets in Figure 3.4 B), did not detect un-expected proteins and has been extensively used in the literature (Miotto \& Struhl 2008; Avvakumov et al. 2012; Zou et al. 2013; Lalonde et al. 2013). AB70 antibody was therefore selected to assess HBO1 protein levels.

\subsubsection{Differential expression of $\mathrm{HBO} 1$ protein in $\mathrm{OC}$ cell lines}

Protein extracts from 7 OC cell lines and the HOSEpiC control were separated using gel electrophoresis (4-12\% gradient gel), transferred to PVDF membranes and probed with anti-HBO1 AB70 antibody. An anti-GAPDH antibody was used as loading standard.

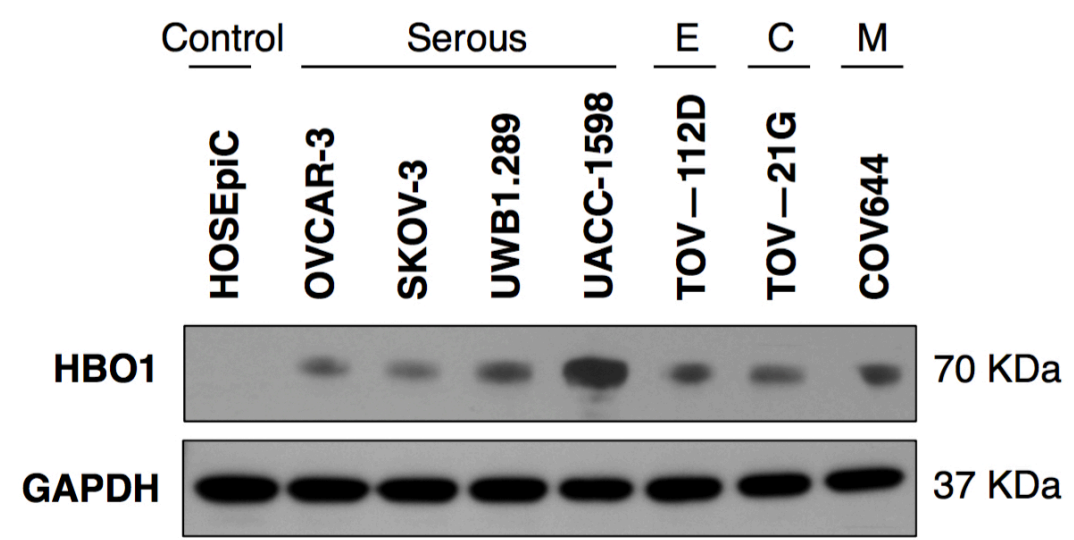

Figure 3. 6 Comparative analysis of HBO1 protein expression

Protein extracts of HOSEpiC and 7 OC cell lines were analysed by western blot and probed with anti-HBO1 (AB70) and anti-GAPDH antibodies. E: Endometrioid; C: Clear-cell; M: Mucinous.

All OC cell lines displayed higher HBO1 expression compared to the non-cancerous HOSEpiC control (Fig. 3.6). However, HBO1 protein intensity did not correlate exactly with previously determined mRNA levels in the OC cell lines. For instance, the SKOV-3 cell line, which expressed $H B O 1$ transcript 3.67 fold-greater than the 
HOSEpiC control (Fig. 3.1), displayed protein intensities analogous to COV644 (2.21 fold-change over HOSEpiC) (Fig. 3.1 \& Fig. 3.6).

HBO1 protein expression in HOSEpiC cells was very low, preventing accurate comparative quantification, therefore, a per cent ratio of relative density (RD) values was expressed in natural logarithms (assuming HOSEpiC per cent ratio $=1$ ). The serous-like cell lines displayed the highest RD compared to the HOSEpiC control: UACC-1598 $\left(\mathrm{RD}=5.87,{ }^{* * * *} \mathrm{p}<0.0001\right), \mathrm{UWB} 1.289\left(\mathrm{RD}=5.31,{ }^{* * * *} \mathrm{p}<0.0001\right)$; with TOV-112D (endometrioid, RD $=5.10, * * *$ p $<0.0001$ ), TOV-21G (clear-cell, $\mathrm{RD}=4.86,{ }^{* * *} \mathrm{p}=0.0009$ ), COV644 (mucinous, $\mathrm{RD}=4.75,{ }^{* *} \mathrm{p}=0.0036$ ), OVCAR-3 (serous, RD $=4.58,{ }^{* *} \mathrm{p}=0.0019$ ) and SKOV-3 (serous, RD $=4.47,{ }^{* *} \mathrm{p}$ $=0.0041)$ all having an $\mathrm{RD}>4$ (Fig. 3.6).

\subsection{Analysis of Potential Sources of HBO1 De-regulation in OC}

The proximal promoter region of $H B O 1$ contains numerous experimentally proven binding sites (Transfac ${ }^{\circledR}$ Professional). Amongst those, the large numbers of nuclear receptor binding sites, such as AR, ER, PR and glucocorticoid receptor, was striking. Other relevant putative binding sites include sequences targeted by p53 and NF- $\kappa \beta$, transcription factors previously linked to $\mathrm{HBO} 1$ function and regulation (Contzler et al. 2006; Iizuka et al. 2008). The presence of nuclear receptor binding sites in the proximal promoter of $\mathrm{HBOl}$ suggests that $\mathrm{HBO} 1$ expression may be modulated by those nuclear receptors, whose up-regulation could be the trigger for HBO1 overexpression. To assess this possibility, the transcript expression of $E R A, E R B$ and $A R$ in the panel of OC cell lines was analysed (Fig. $3.7 \&$ Appendix 2).

The augmentation of HBOI transcript levels compared to the HOSEpiC control did not show any clear correlation with the levels of nuclear receptor ERA mRNA expression (Fig. 3.7). While some OC cell lines with high $\mathrm{HBOl}$ expression levels displayed no ERA transcript: UWB1.289 $\left(\mathrm{FC}=-17.26,{ }^{* *} \mathrm{p}=0.0032\right)$ and TOV112D $\left(\mathrm{FC}=-22.69,{ }^{* *} \mathrm{p}=0.0026\right)$; others, OVCAR-3 $\left(\mathrm{FC}=5.13,{ }^{*} \mathrm{p}=0.0364\right)$, SKOV-3 $\left(\mathrm{FC}=123,{ }^{* *} \mathrm{p}=0.0015\right)$ and COV644 $\left(\mathrm{FC}=13.73,{ }^{* *} \mathrm{p}=0.0043\right)$ showed significant overexpression of the nuclear receptor (Fig. 3.7). 


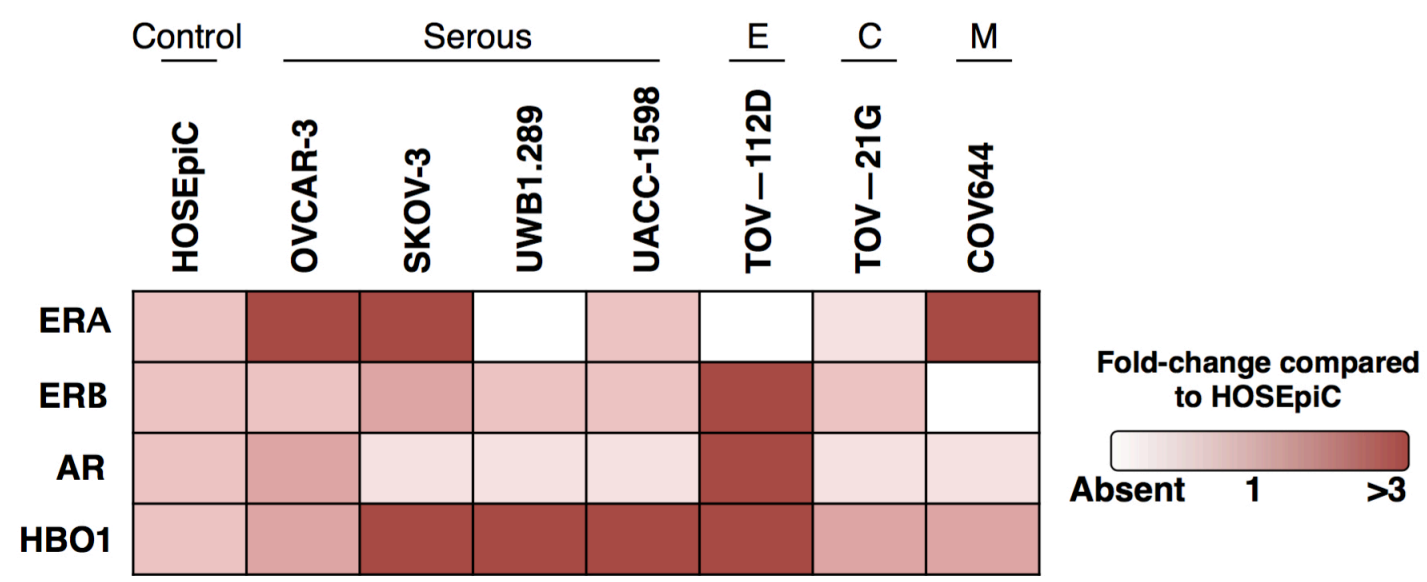

Figure 3. 7 Comparative analysis of nuclear receptor expression levels

Graphical depiction of changes in nuclear receptors transcript abundance comparing HOSEpiC non-cancerous control and the 7 OC cell lines. The red gradation diagram on the right of the panel represents qRT-PCR fold-change values; absent value corresponds to cycle threshold 40. Individual graphs representing differential nuclear receptor expression can be found in Appendix 2 A-C. E: Endometrioid; C: Clearcell; M: Mucinous.

High $H B O 1$ expression did not display a clear correlation with nuclear receptor $E R B$ transcript levels either, with most OC cell lines and the HOSEpiC control expressing similar amounts of the nuclear receptor: OVCAR $(\mathrm{FC}=1.17, \mathrm{p}=0.867)$, UWB1.289 (FC = -3.23, p = 0.4033), UACC-1598 (FC = -1.8, p = 0.9853), TOV$21 \mathrm{G}(\mathrm{FC}=1.57, \mathrm{p}=0.7483)($ Fig. 3.7).

On the contrary, with the exception of the endometrioid cell line TOV-112D (FC = $3.37, \mathrm{p}=0.40)$ and OVCAR-3 $(\mathrm{FC}=2.81, \mathrm{p}=0.324)$, OC cell lines with high levels of $H B O 1$ mRNA expression displayed very low levels of nuclear receptor $A R$ : SKOV-3 $(\mathrm{FC}=-220.59, * * * * \mathrm{p}=<0.0001), \mathrm{UWB1} 1.289(\mathrm{FC}=-60.78, * * * * \mathrm{p}=<$ 0.0001), UACC-1598 (FC $=-113.82, * * * \mathrm{p}=<0.0001)$, TOV21-G $(\mathrm{FC}=-44.72$, $\left.{ }^{* *} \mathrm{p}=0.0062\right)$ and COV644 $\left(\mathrm{FC}=-22.49,{ }^{* *} \mathrm{p}=0.00356\right)($ Fig. 3.7).

The existence of genomic HBOl modulation by ERA, ERB and AR appears unlikely, based on the expression analyses (Fig. 3.7). Nonetheless, additional forms of regulation cannot be discarded, e.g. protein-protein interactions. High levels of $H B O 1$ seem to correlate with low levels of $A R$, suggesting that $\mathrm{HBO} 1$ negatively 
regulates the expression of $A R$, a possibility that agrees with previous studies linking HBO1 with $A R$-mediated transcriptional repression (Sharma et al. 2000).

Estradiol (E2) treatment induced HBO1 transcript expression in T47D and MCF7 breast cancer cell lines through ER $\alpha$ and the 'extracellular signal-regulated kinase' or ERK1/2 signalling pathways (Wang et al. 2010). In order to investigate whether $\mathrm{HBO} 1$ overexpression is linked to E2-related induction of the ER $\alpha$ receptor in serous OC, ER $\alpha$ positive cell lines OVCAR-3 and UACC-1598 were treated with E2. The SKOV-3 cell line was not selected for treatment due to its known nonresponsiveness to E2 (Hua et al. 1995; Lau et al. 1999). A set of gene transcripts that have previously been shown to be modified by E2 treatment in serous OC cell lines were used as positive controls, including caspase 4 (CASP4), keratin 4 (KRT4), lipocalin 2 (LCN2) and the 'TNF receptor associated protein 1' (TRAP1) (O'Donnell et al. 2005).

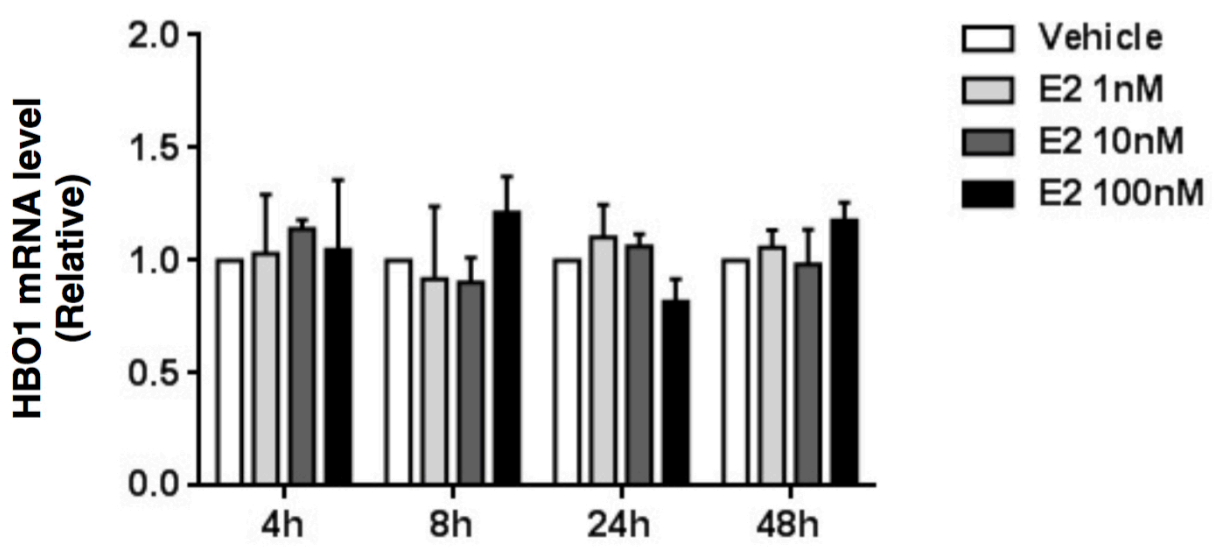

Figure 3. 8 E2 treatment of UACC-1598 cells

UACC-1598 cells were treated with E2 (1-10-100nM) and the control vehicle (absolute ethanol). Cell lysates were extracted after 4, 8, 24, and 48h whereupon RNA samples were prepared and analysed by qRT-PCR. Relative levels of $H B O 1$ expression in E2-treated cells were compared to those of cells treated with the control vehicle. RPL5 expression was used as control. Results represent the mean \pm $\mathrm{SD}$ of two biological replicates.

The expression levels of $\mathrm{HBOl}$ and the set of genes were tested 4, 8, 24 and $48 \mathrm{~h}$ after the treatment with a range of E2 doses, including physiologic concentrations (110nM) (O'Donnell et al. 2005; Celojevic et al. 2011), but no significant changes 
were observed (Fig. 3.8). These results suggest $\mathrm{HBOl}$ overexpression is not driven by E2 in these OC cell lines. The absence of target gene induction after E2 treatment could imply ER $\alpha / \beta$ signalling deficiencies or differing gene modulation patterns amongst OC cell lines.

\subsection{HBO1 Genome Wide Localization}

HBO1 is located in the cellular nucleus (Sharma et al. 2000; Burke et al. 2001) and associated with chromatin (Miotto \& Struhl 2008). Therefore, if HBO1 is an important regulator involved in global OC development, the increased levels of HBO1 protein observed in OC cell lines compared to non-cancerous control (Fig. 3.6) might cause an associated increase in $\mathrm{HBO} 1$ occupancy at promoter/enhancer regions of cancer-associated genes.

The most common tool to study the interaction between proteins such as HBO1 and the DNA is ChIP, which coupled with qRT-PCR can be used to investigate protein enrichment in localized binding sites (Gade \& Kalvakolanu 2012). Combined with massively parallel DNA sequencing (ChIP-Seq), this tool also allows for the genome wide mapping of DNA-binding proteins (Furey 2012). For instance, the genome wide localization of HBO1 has been characterized via ChIP-Seq using the RKO human colon cancer cell line (Avvakumov et al. 2012). Many other studies have also evaluated HBO1 localization at defined loci using ChIP-qRT-PCR (Miotto \& Struhl 2008; Saksouk et al. 2009; Miotto \& Struhl 2010; Mishima et al. 2011; Avvakumov et al. 2012; Lalonde et al. 2013; Song et al. 2013). However, a consensus site associated with $\mathrm{HBO} 1$ binding has not been identified so far.

\subsubsection{ChIP optimization}

The quality of an HBO1 ChIP-Seq experiment is governed by the specificity of the antibody, as well as the degree of enrichment achieved in the affinity precipitation step (Park 2009). Several commercially available HBO1 antibodies have already been used for ChIP-qRT-PCR in the literature (Miotto \& Struhl 2008; Lalonde et al. 2013; Feng et al. 2015), but only one has been used for ChIP-Seq -Abcam ${ }^{\circledR}$ AB70 
(Avvakumov et al. 2012). This antibody has also been used to perform ChIP-qRTPCR in numerous occasions (Miotto \& Struhl 2008; Miotto \& Struhl 2011; Avvakumov et al. 2012; Lalonde et al. 2013), and thus represented the best possible choice to start a ChIP-Seq optimization process.

UWB1.289 was selected as a model to optimize ChIP-Seq based on the fact that this cell line represents the serous histology, exhibits high levels of HBO1 expression at both the mRNA and protein levels (Fig. 3.1 \& Fig. 3.6), and grows rapidly (an important aspect given that ChIP experiments require large cell numbers).

\subsubsection{Antibody validation}

According to the ENCODE consortia, an antibody that has been validated for ChIP in at least three different cell types (e.g. AB70 in HEK 293T, HeLa, CCL-156) (Miotto \& Struhl 2008) does not need further testing (Landt et al. 2012). However, since ChIP-Seq is an expensive and complicated procedure, a series of experiments were performed in order to characterize the suitability of AB70 to accomplish ChIPSeq in an OC cell line.

Following the guidelines recommended by the ENCODE consortia, antibodies directed against transcription factors have to pass several tests to reassure their recognition of the antigen of interest with minimal cross-reactivity toward other DNA-associated proteins. The effective use of an antibody for IP is usually the best indicator of ChIP success, since the antibody recognizes an epitope in its native conformation and within the context of any complexes that the epitope may be found in. In this regard, polyclonal antibodies, which recognize a number of different epitopes of the same protein, have higher probabilities to accomplish strong ChIP experiments.

Consequently, the AB70 antibody was used to perform IP with UWB1.289 cell protein lysates, followed by SDS-PAGE separation and HBO1 antibody detection (Fig. 3.9 A). In addition, the AB70-IPed fraction was probed with an antibody targeting ING4, an important member of the HBO1 complex whose main functions 
were described earlier (Doyon et al. 2006), in order to evaluate the capacity of AB70 to pool specific targets (HBO1) alongside well-known associated proteins (e.g. ING4) (Fig. 3.9 B).

A

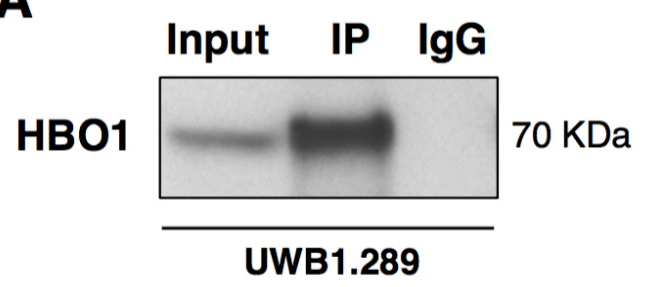

B

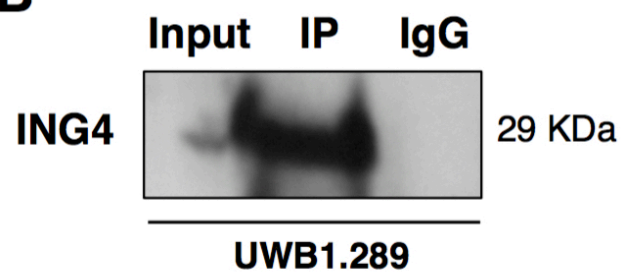

Figure 3.9 HBO1 IP and ING4 co-IP using AB70 antibody

UWB.289 protein lysates were immune-precipitated using anti-HBO1 AB70 antibody, separated using SDS-PAGE and probed with (A) anti-HBO1 and (B) antiING4 antibodies.

AB70 specifically immune-precipitated the HBO1 protein ( 70 KDa) in UWB1.289 cell lysates, while no product was isolated with the $\operatorname{IgG}$ control (Fig 3.9 A). Moreover, HBO1 co-precipitates with ING4 ( 29 KDa) when immune-precipitated with AB70 (Fig 3.9 B), further demonstrating the specificity of the anti-HBO1 antibody. HBO1 nuclear localization was then investigated.

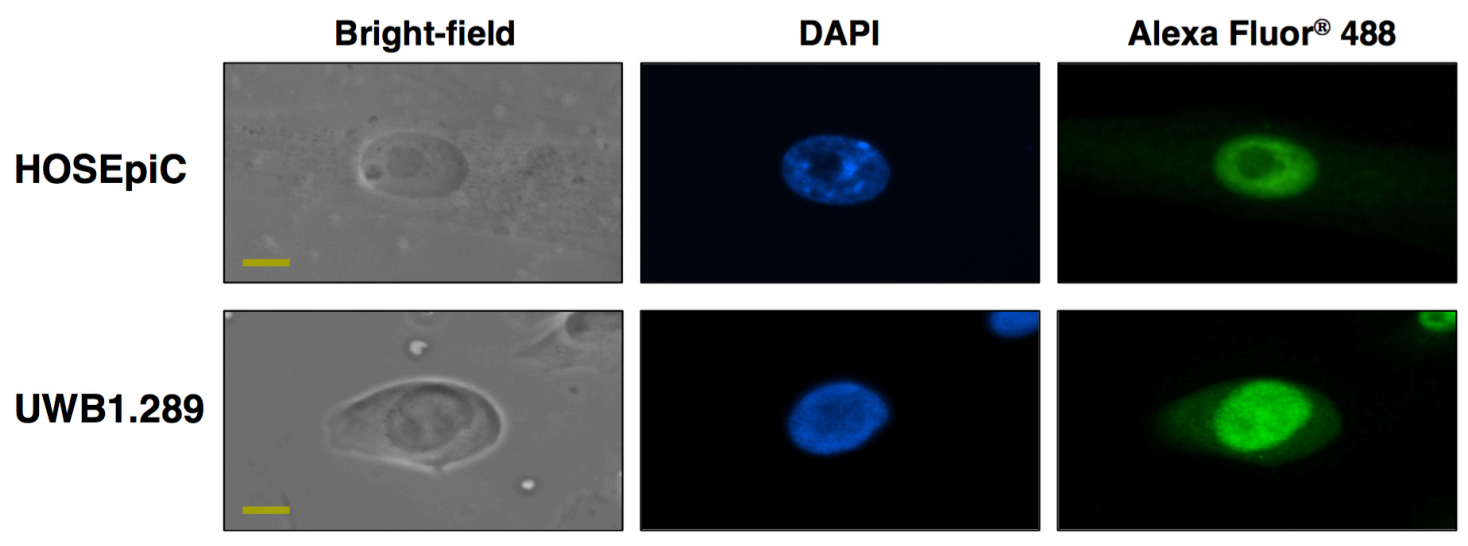

Figure 3. 10 HBO1 immune-fluorescence staining

First column (Bright-field) displays optical microscopic images of UWB1.289 and HOSEpiC fixed-cells; second (DAPI: 4', 6-diamidine-2'-phenylindole dihydrochloride) and third (Alexa Fluor ${ }^{\circledR} 488$ ) columns show fluorescent-labelled DNA and HBO1 images respectively. Scale represents $10 \mu \mathrm{m}$. 
HBO1 was found to localize mainly to the nucleus of HOSEpiC and UWB1.289 cells (Fig. 3.10), with the exception of the area corresponding to the nucleoli, a feature that has been previously described (Doyon et al. 2006; Yan et al. 2016). This phenomenon can be perceived more clearly in the HOSEpiC cell (Fig. 3.10), which has lower HBO1 expression compared to the UWB1.289 cell, in accordance with earlier mRNA and protein analyses (Fig. 3.1 \& Fig. 3.6). HBO1 was not solely localized to the nucleus, but was also observed in the cytoplasm of the cells, where it accumulated at lower levels (Fig. 3.10).

Subcellular protein fractionation was undertaken to demonstrate the association of HBO1 with nuclear chromatin. Total UWB1.289 protein was subcellularly fractionated and probed with different antibodies: anti-HBO1 (AB70), anti-EGFR, anti-XRCC1 and anti-histone H4. Antibodies against the 'epidermal growth factor receptor 1' (EGFR), the 'X-ray repair cross complementing 1' (XRCC1) and histone H4 were used as positive controls, since their targets are commonly restricted to specific cellular fractions: membrane (EGFR), nucleus (XRCC1) and chromatin (histone H4) (Lemmon et al. 2014; London 2015).

A

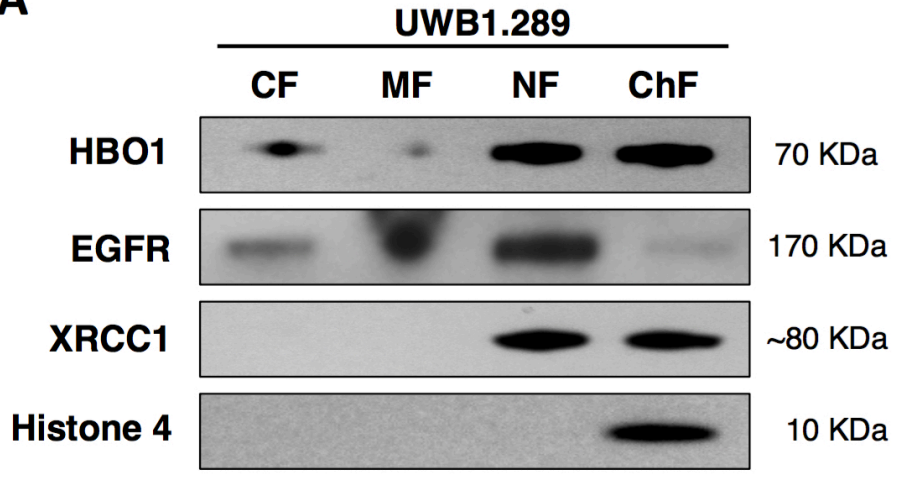

B

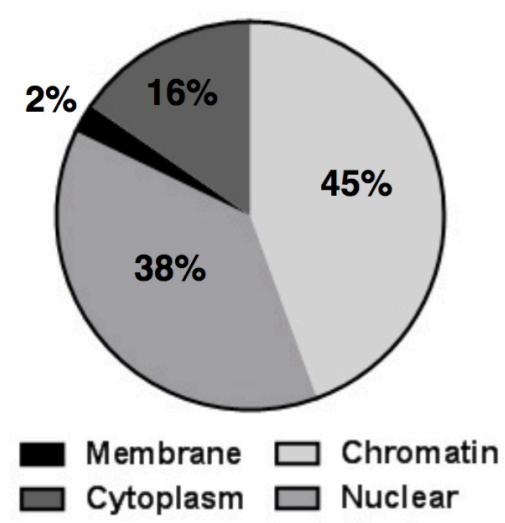

Figure 3. 11 Subcellular protein localization of $\mathrm{HBO1}$ in UWB1.289 cells

(A) UWB1.289 protein lysates were segregated into subcellular fractions, subsequently separated using SDS-PAGE and probed with anti-HBO1 (AB70), antiEGFR, anti-XRCC1 and anti-Histone H4. CF: Chromatin Fraction; MF: Membrane Fraction; NF: Nuclear fraction; ChF: Chromatin-bound fraction. (B) Cellular distribution of $\mathrm{HBO} 1$ protein according to the subcellular protein fractionation. Localization percentages were calculated using ImageJ. 
Approximately half of the HBO1 protein $(44.38 \pm 2.57 \%)$ was found associated with chromatin in UWB1.289 protein lysates, with $37.73 \pm 2.73 \%$ of protein localized to the nucleus but un-bound to chromatin, while only $2.44 \pm 0.67 \%$ and $15.39 \pm 0.76 \%$ was located in the membrane and cytoplasmic fractions respectively (Fig. 3.11).

EGFR, a trans-membrane protein (Lemmon et al. 2014) was localized in the membrane fraction, where the high lipid content in this fragment causes the formation of the large dot seen on the gel (Fig. 3.11 A). All of the DNA repairprotein XRCC1 (London 2015) localized to the nucleus: $52.30 \pm 4.17 \%$ in the socalled nuclear fraction and $47.14 \pm 2.17 \%$ in the chromatin-bound fraction, and 100\% of histone $\mathrm{H} 4$ signal was detected in the chromatin fraction (Fig. 3.11 A), validating the subcellular localization of $\mathrm{HBO} 1$.

\subsubsection{Chromatin shearing}

UWB1.289 cells contain high levels of chromatin-bound HBO1 protein (Fig. 3.6 \& Fig. 3.11), which can be specifically targeted by the anti-HBO1 antibody AB70 (Fig. 3.4 B \& Fig. 3.6). A Chromatrap ${ }^{\circledR}$ kit was therefore used to perform ChIP-qRT-PCR validation experiments and test whether AB70 is able to selectively IP cross-linked HBO1-chromatin fragments. Chromatin shearing was optimized using the random sonication procedure to attain optimal size range of chromatin fragments for ChIPqRT-PCR and ChIP-Seq (100-500 bps, approximately the length of DNA wrapping 2-3 nucleosomes). 10-15 million UWB1.289 cells, the highest number recommended by Chromatrap ${ }^{\circledR}$, were cross-linked and samples sonicated for different time periods, power settings (medium-high) and number of cycles, reverse cross-linked and analysed by agarose gel electrophoresis to evaluate sonication efficiency (Fig. 3.12).

The sonication setting 6 (high-power setting and 10 one-minute sonication cycles with 30 seconds resting time each cycle) resulted in the most appropriate distribution of DNA fragmentation, with most DNA fragments uniformly placed in the one hundred to five hundred base pair (Fig. 3.12). 


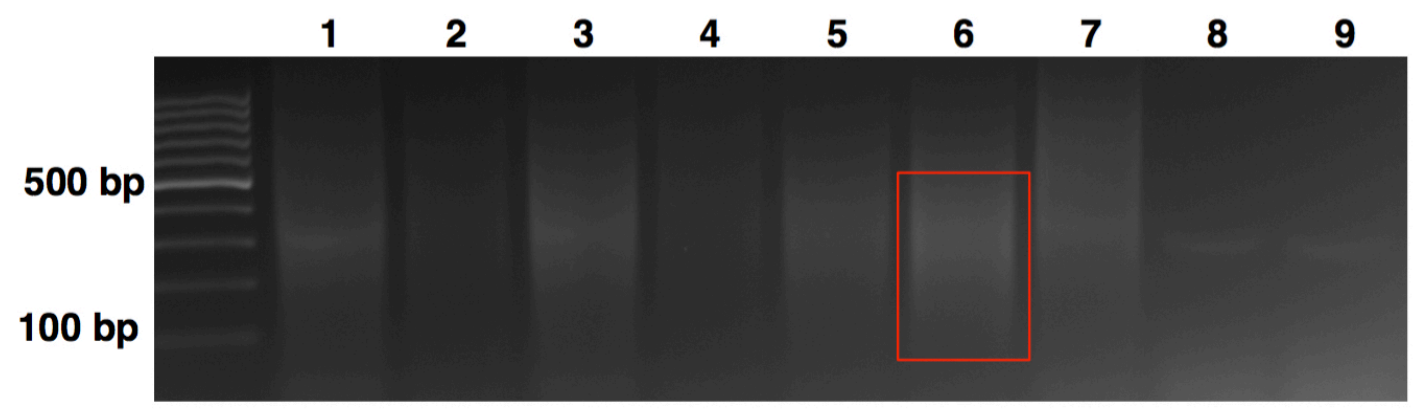

Figure 3. 12 Sonication efficiency of UWB1.289 cross-linked chromatin samples Pellets of 10-15 million UWB1.289 cells were subjected to sonication using different conditions. Sonication settings: (1) MP, 15c, 1-1; (2) MP, 10c, 1-1; (3) MP, 15c, 10.5 ; (4) MP, 10c, 1-0.5 (5) HP, 15c 1-0.5; (6) HP, 10c, 1-0.5; (7) HP, 10c, 1-1.5; (8) HP, 10c, 1-1; (9) HP, 15c, 1-1. HP = High power; MP = Medium power; c = cycles; $1-0.5 / 1 / 1.5=1$ minute sonication-0.5/1/1.5 minutes rest.

\subsubsection{ChIP-qRT-PCR enrichment}

Following the optimization of the shearing procedure, a final validation experiment prior to ChIP-Seq, which consisted of identifying HBO1-interacting genomic regions specifically enriched by the AB70 antibody via ChIP-qRT-PCR, was performed. In this experiment, non-specific $\operatorname{IgG}$ and anti-H3K4me3 antibodies were used as negative and positive enrichment controls respectively. $\mathrm{H} 3 \mathrm{~K} 4 \mathrm{me} 3$ is a histone mark that maps to promoters of actively transcribed genes and, more importantly, colocalizes with HBO1 (Hon et al. 2009; Avvakumov et al. 2012).

There are several well-characterized eukaryotic origins of replication known to be targeted by $\mathrm{HBO} 1$ in different cellular backgrounds, which are named after the nearest gene and include: laminB2 (LMNB2), (Lee \& Romero 2012), MCM4 (Gundelach et al. 2007), 'small nucleolar RNA host gene 12' (SNHG12) and 'putative RNA-binding protein Luc7-like' (LUC7L) (Feng et al. 2015). Feng et al. (2015) designed specific primer pairs that can be used to evaluate target occupancy on such genomic locations. As a consequence, the Chromatrap ${ }^{\circledR}$ Pro-A kit was used to perform ChIP followed by ChIP-qRT-PCR using (1) Feng et al. (2015) primer pairs and (2) three intergenic negative primers. These probes target intergenic regions (IGR) where neither $\mathrm{HBO} 1$ nor $\mathrm{H} 3 \mathrm{~K} 4 \mathrm{me} 3$ are expected to be found and therefore allow evaluating whether detected enrichment is localized or non-specific. 
Each primer pair was used to amplify DNA samples from two biological replicates of different UWB1.289 chromatin extractions with two technical replicates per antibody IP (Fig. 3.13).

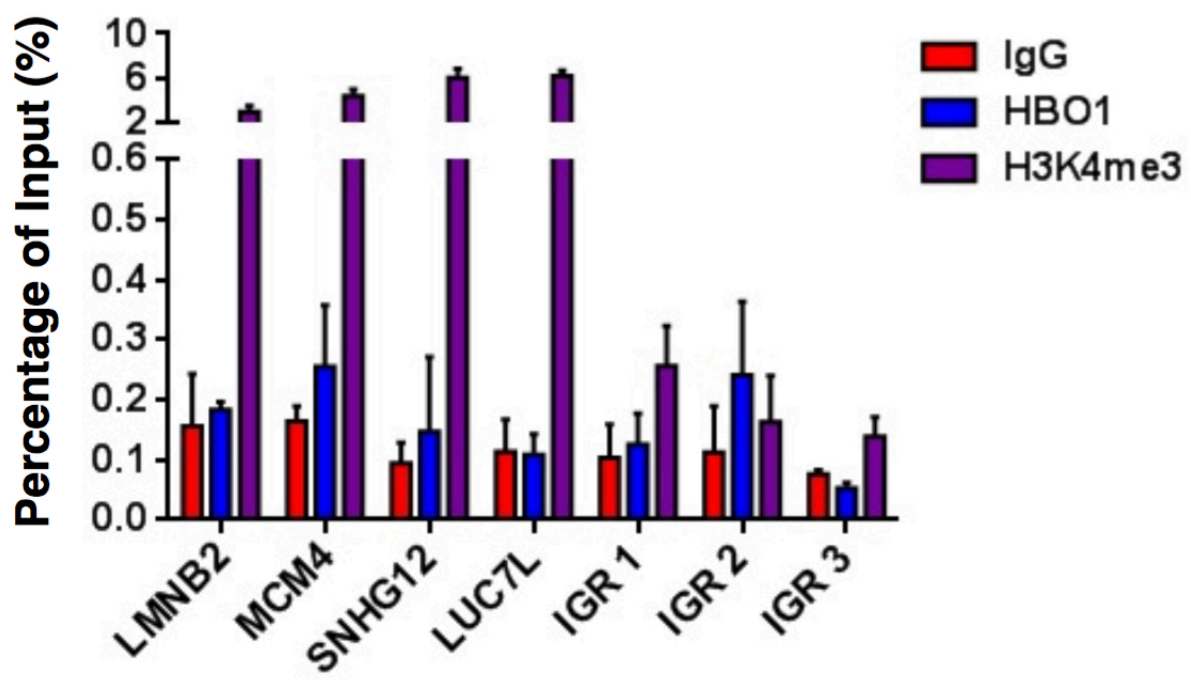

Figure 3. 13 Enrichment of potential HBO1 targets: origins of replication UWB1.289 cells were subjected to ChIP using IgG, anti-HBO1 (AB70) and antiH3K4me3 antibodies, followed by qRT-PCR amplification of LMNB2, MCM4, $S N H G 12$ and $L U C 7 L$ genomic regions. IGRs 1, 2 and 3 were used as controls for antibody-specificity. Final antibody enrichment, expressed as percentage of input, was calculated comparing sample qRT-PCR values with 'Input' control values. Results represent the mean $\pm \mathrm{SD}$ of two biological replicates. IGR: Intergenic region.

The percentage of input (PI) enrichment displayed by AB70 in the IGR 2 region (PI $=0.24 \pm 0.12 \%$ ) was higher than, or at least as high as, the enrichment found at the LMNB2 (PI $=0.18 \pm 0.01 \%)$, MCM4 (PI $=0.25 \pm 0.10 \%)$, SNHG12 (PI $=0.15 \pm$ $0.12 \%)$ and $L U C 7 L(\mathrm{PI}=0.11 \pm 0.03 \%)$ loci (Fig. 3.13); suggesting that $\mathrm{AB} 70$ performed a nonspecific IP. In view of the data obtained, it was concluded that the anti-HBO1 AB70 antibody did not show significant enrichment compared to the negative controls (IgG antibody and IGR primer pairs) in any of the interrogated origin of replication regions.

H3K4me3 antibody showed significant input enrichment in LMNB2 (PI = $3.04 \pm$ $0.52 \%), M C M 4(\mathrm{PI}=4.42 \pm 0.62 \%), S N H G 12(\mathrm{PI}=6.04 \pm 0.83 \%)$ and $L U C 7 L$ $(6.22 \pm 0.51 \%)$ regions (Fig 3.13), suggesting an important presence of the histone 
mark in those genomic regions. On the contrary, H3K4me3 did not show enrichment in any of the three interrogated IGRs: IGR $1(\mathrm{PI}=0.26 \pm 0.07 \%)$, IGR $2(\mathrm{PI}=0.16 \pm$ $0.08 \%)$ and IGR $3(\mathrm{PI}=0.14 \pm 0.03 \%)$ (Fig. 3.13). The results obtained with H3K4me3 antibody demonstrate that the ChIP experiment itself worked, albeit AB70 IP either did not work or needed further optimization. In line with this, small variations of the ChIP protocol such as changes in AB70 or chromatin quantities per IP and IP duration were tested. However, these too failed to give any HBO1 specific enrichment at the tested loci.

In order to evaluate the reasons for the lack of enrichment, origin-of-replication primer binding sites were assessed using the Integrative Genomics Viewer (IGV) (Fig. 3.14). For that, the only HBO1 ChIP-Seq available data and its correspondent 'Input' were downloaded from the Gene Expression Omnibus database (GSE33221) (Avvakumov et al. 2012) and analysed following the procedure detailed in Figure 2.3. Additionally, the raw data of an $\mathrm{H} 3 \mathrm{~K} 4 \mathrm{me} 3 \mathrm{ChIP}-\mathrm{Seq}$ performed in the same cellular background as the HBO1 ChIP-Seq, RKO colon carcinoma cells, was downloaded (GSE47190) and analysed (Lalonde et al. 2013).

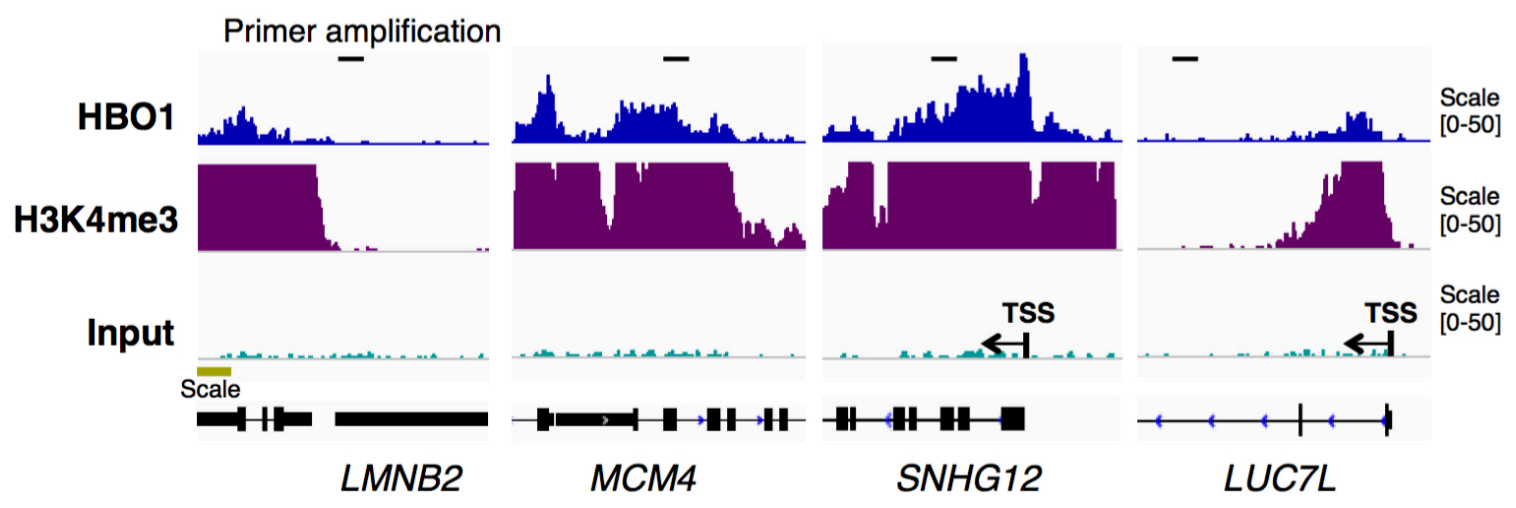

Figure 3. 14 IGV analysis of alleged HBO1-targeted origins of replication

The first and second panels (starting from the top) represent peak enrichment obtained with anti-HBO1 and anti-H3K4me3 antibodies in ChIP-Seq experiments performed with the RKO cell line. The third panel (Input) represents unspecific peaks obtained from RKO chromatin samples that have not been subjected to antibody enrichment. Images were extracted from the Integrative Genomics Viewer (IGV). Selected origins of replication are named after the nearest gene, depicted at the bottom as longitudinal lines where black squares/rectangles symbolise exons. Specific primer amplification sites are illustrated on top of the first panel. TSS = Transcription Starting Site. X-axis scale represents $2 \mathrm{~Kb}$; y-axis scales represent peak enrichment value ranges. 
As depicted in Figure 3.14, LMNB2 and LUC7L primer pairs amplify regions that, although close, do not show enrichment of either HBO1 or H3K4me3 in the RKO ChIP-Seq data, and could help explain the observed lack of enrichment (Fig. 3.13). However, MCM4 and SNHG12 primer pairs amplify regions that lie between the enriched areas of both $\mathrm{HBO} 1$ and $\mathrm{H} 3 \mathrm{~K} 4 \mathrm{me} 3$ (Fig. 3.14). The design of new more accurate primers, closer to the region of $\mathrm{HBO} 1 / \mathrm{H} 3 \mathrm{~K} 4 \mathrm{me} 3$ enrichment at $L M N B 2$ and LUC7L loci was problematic because of the area's high guanine-cytosine content.

\subsubsection{Potential HBO1 targets for ChIP-qRT-PCR enrichment}

As the origins of replication did not reveal any specific recruitment of $\mathrm{HBO}$, promoter regions of genes transcriptionally regulated by HBO1 were investigated. In order to predict genes modulated by HBO1, an SQL database was built.

First, publicly available literature was interrogated in order to obtain as much information as possible related with $\mathrm{HBO} 1$ transcriptional activity, links with cancer in general and/or OC in particular. Using this approach, a number of datasets were identified and downloaded from the NCBI website, the majority consisting of excel or plain text files that contained gene name lists with associated gene expression values (e.g. fold-change or peak enrichment values). These datasets make up the 'Tables' of the SQL database, of which some examples are described in Table 3.3 and depicted in Figure 3.15 A. The information contained in each one of these exemplary 'Tables' is detailed in Table 3.3.

Table 3. 3 Relevant HBO1-related datasets integrated into the SQL database

\begin{tabular}{|c|c|c|}
\hline Dataset & Background / Origin & Dataset information \\
\hline $\begin{array}{c}\text { HBO1 } \\
\text { ChIP-Seq }\end{array}$ & $\begin{array}{c}\text { RKO colon carcinoma } \\
\text { (Avvakumov et al. 2012) }\end{array}$ & HBO1-related peak localization \\
\hline $\begin{array}{c}\text { HBO1 } \\
\text { Knockdown }\end{array}$ & $\begin{array}{c}\text { HeLa cervical cancer } \\
\text { (Avvakumov et al. 2012) }\end{array}$ & $\begin{array}{c}\text { Fold change of genes affected by } \\
\text { HBO1 knockdown }\end{array}$ \\
\hline $\begin{array}{c}\text { HGSC } \\
\text { Sub-types }\end{array}$ & $\begin{array}{c}\text { 489 HGSC tumours } \\
\text { (Network 2011) }\end{array}$ & $\begin{array}{c}\text { Fold change of genes differentially } \\
\text { expressed in 4 HGSC signatures }\end{array}$ \\
\hline $\begin{array}{c}\text { OC relevant } \\
\text { genes }\end{array}$ & $\begin{array}{c}\text { Search results for 'Ovarian } \\
\text { cancer' in NCBI databases }\end{array}$ & List of genes implicated in OC \\
\hline
\end{tabular}


A

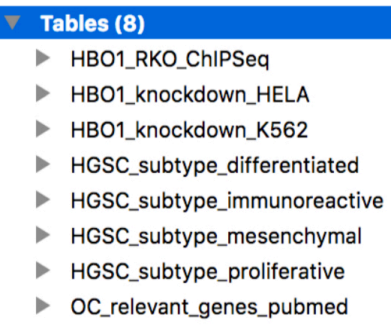

> HBO1_RKO_ChIPSeq

- HBO1_knockdown_HELA

> HBO1_knockdown_K562

> HGSC_subtype_differentiated

- HGSC_subtype_immunoreactive

- HGSC_subtype_mesenchymal

- HGSC_subtype_proliferative

- OC_relevant_genes_pubmed

C

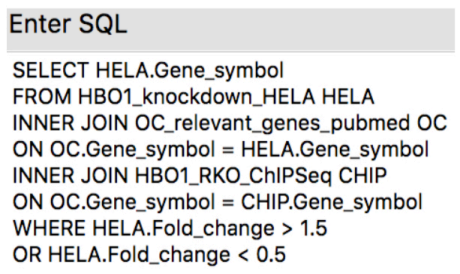

B

\begin{tabular}{|c|c|c|}
\hline Gene_symbol & Fold_change & pvalue \\
\hline PSG4 & 0.271411651 & |7.61069e-8 \\
\hline DUSP2 & 0.302828288 & $3.64751 e-9$ \\
\hline MMP12 & 0.307642461 & $5.46486 \mathrm{e}-10$ \\
\hline NKD2 & | 0.319126361 & |3.64751e-9 \\
\hline CYBRD1 & 0.340736479 & 7.54763e-12 \\
\hline CDKN1A & |0.358503519 & |5.46486e-10 \\
\hline CASP9 & 0.358841156 & $2.76182 \mathrm{e}-10$ \\
\hline GCNT3 & $\mid 0.3610558$ & | $4.51049 \mathrm{e}-10$ \\
\hline & Records: 84 & \\
\hline
\end{tabular}

D

\begin{tabular}{l|l} 
Gene_symbol & \\
ABCC4 & \\
ALCAM & Database \\
ANKRD1 & gene list \\
CYR61 & search result \\
ERBB2 & \\
TLN1 &
\end{tabular}

\section{Figure 3. 15 SQL isolation of potential HBO1 targets}

HBO1-related datasets (A) containing gene names and associated values (B) were stored in an SQL database. Using SQL language $(\mathbf{C})$, these datasets were linked together in order to obtain potential HBO1 targets (D).

The 'Tables' incorporated into the SQL database were queried using SQL language (Fig. 3.15 C) in order to retrieve potential HBO1-modulated genes (Fig. 3.15 D). Using the SQL search engine, it is possible to compare thousands of data and filter them according to multiple variables. For instance, this database was used to narrow down the search of HBO1 targets from hundreds to as low as 6 gene transcripts (Fig. 3.15 D \& Table 3.4).

Table 3. 4 List of potential HBO1 target genes derived from SQL correlations

\begin{tabular}{|c|c|c|}
\hline Gene & Function & Relation with OC \\
\hline $\begin{array}{c}\text { Cysteine rich angiogenic } \\
\text { inducer 61 (CYR61) }\end{array}$ & Cell adhesion & $\begin{array}{c}\text { Overexpression, prognosis } \\
\text { (Shen et al. 2014) }\end{array}$ \\
\hline ERBB2 & Receptor & $\begin{array}{c}\text { Overexpression } \\
\text { (Teplinsky \& Muggia 2014) }\end{array}$ \\
\hline $\begin{array}{c}\text { ATP binding cassette subfamily } \\
\text { C member 4 }(A B C C 4)\end{array}$ & $\begin{array}{c}\text { Protein } \\
\text { transport }\end{array}$ & $\begin{array}{c}\text { Resistance, prognosis } \\
\text { (Ahmed et al. 2016) }\end{array}$ \\
\hline $\begin{array}{c}\text { Activated leukocyte cell } \\
\text { adhesion molecule }(A L C A M)\end{array}$ & Cell migration & $\begin{array}{c}\text { Overexpression } \\
\text { (Carbotti et al. 2013) }\end{array}$ \\
\hline Talin 1 (TLN1) & $\begin{array}{c}\text { Cell-cell } \\
\text { contact }\end{array}$ & $\begin{array}{c}\text { Overexpression, metastasis } \\
\text { (Xu et al. 2013) }\end{array}$ \\
\hline $\begin{array}{c}\text { Ankyrin repeat domain 1 } \\
(A N K R D 1)\end{array}$ & $\begin{array}{c}\text { Transcription } \\
\text { factor }\end{array}$ & $\begin{array}{c}\text { Overexpression, prognosis } \\
\text { (Lei et al. 2015) }\end{array}$ \\
\hline
\end{tabular}


The common characteristics of the selected genes included: (1) modified expression after HBO1 depletion in HeLa cells, (2) presence of enrichment peaks within the transcription starting site area of the pertinent gene in the RKO ChIP-Seq (TSS \pm $1 \mathrm{~Kb}$ ) and (3) the existence of at least 1 citation in NCBI databases linking their expression to OC (Table 3.3 \& Table 3.4).

Following the selection of predicted HBO1 targets, ChIP-qRT-PCR primer pairs were designed based on the genomic coordinates from RKO ChIP-Seq peaks. Two/three primer pairs were designed for each location, in an effort to cover as much genomic space as possible. Most probes target areas surrounding the TSS, an area where H3K4me3 marks were also observed in the RKO ChIP-Seq data and therefore could be used as positive controls. ChIP experiments were performed on two biological replicates and with 2 technical replicates using the newly designed probes together with the three negative control primer pairs (IGR) (Fig. 3.16).

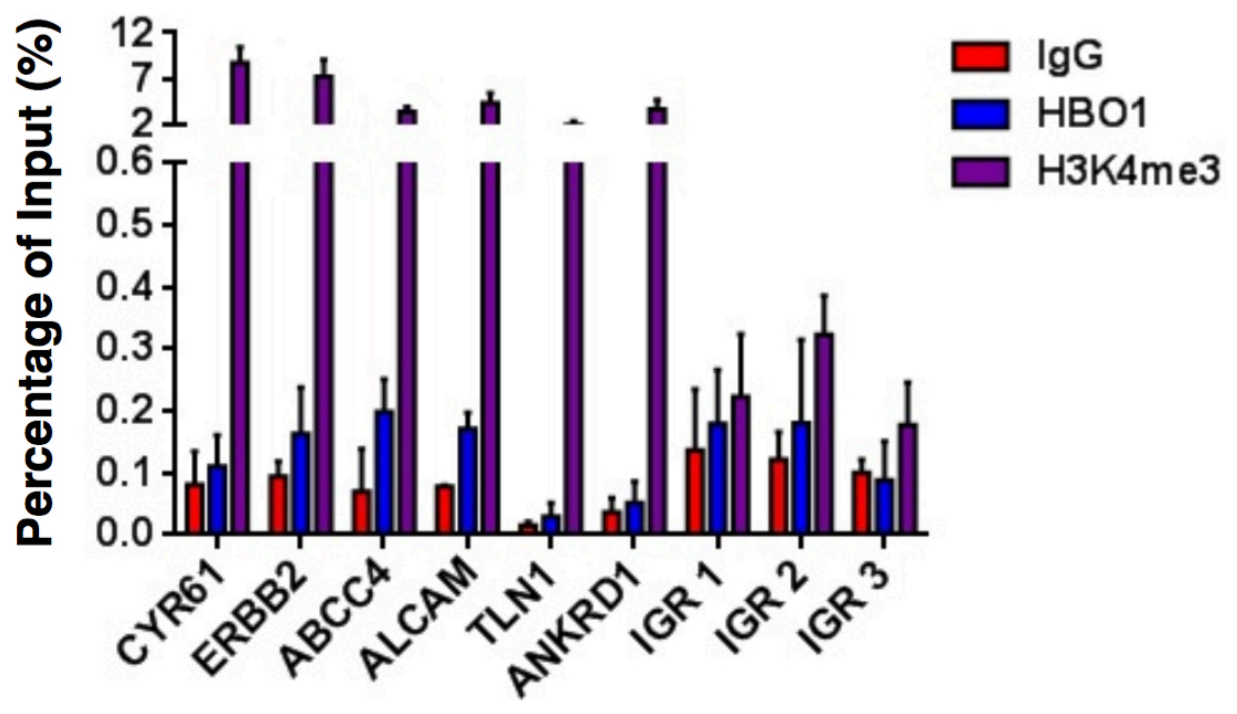

Figure 3. 16 Enrichment of potential HBO1 targets: SQL-derived genes

UWB1.289 cells were subjected to ChIP using IgG, anti-HBO1 (AB70) and antiH3K4me3 antibodies, followed by qRT-PCR amplification of CYR61, ERBB2, $A B C C 4, A L C A M, T L N 1$ and ANKRD1 genomic regions. IGRs 1, 2 and 3 were used as controls for antibody-specificity. Final antibody enrichment, expressed as percentage of input, was calculated comparing sample qRT-PCR values with 'Input' control values. Results represent the mean \pm SD of two biological replicates. IGR: Intergenic region. 
AB70 antibody did not show significant enrichment compared to the negative controls (IgG and IGRs) in any of the interrogated loci (Fig. 3.16). The PI enrichment displayed in IGRs such as IGR 1 (PI $=0.17 \pm 0.08 \%)$ or IGR 2 (PI = $0.18 \pm 0.13 \%$ ) was higher than, or at least as high as the PI enrichment shown at CYR61 $(\mathrm{PI}=0.11 \pm 0.05 \%), E R B B 2(\mathrm{PI}=0.16 \pm 0.07 \%), A B C C 4(\mathrm{PI}=0.19 \pm$ $0.05 \%), A L C A M(\mathrm{PI}=0.17 \pm 0.03 \%), T L N 1(\mathrm{PI}=0.03 \pm 0.02 \%)$ and $A N K R D 1(\mathrm{PI}=$ $0.05 \pm 0.03 \%$ ) loci (Fig. 3.16). Leaving aside the IGR controls, ABCC4 and ALCAM loci showed higher enrichment with HBO1 than their IgG counterparts (ABCC4 IgG PI: $0.07 \pm 0.06$; ALCAM IgG PI: $0.08 \pm 0.002$ ).

Importantly, the $\mathrm{H} 3 \mathrm{~K} 4 \mathrm{me} 3$ antibody displayed significant input enrichment in CYR61 (PI $=8.76 \pm 1.72 \%), E R B B 2(\mathrm{PI}=7.29 \pm 1.79 \%), A B C C 4(\mathrm{PI}=3.50 \pm$ $0.56 \%), \operatorname{ALCAM}(\mathrm{PI}=4.46 \pm 1.07 \%), T L N 1(\mathrm{PI}=2.11 \pm 0.40 \%)$ and $A N K R D 1(\mathrm{PI}=$ $3.77 \pm 1.01 \%$ ) queried regions (Fig. 3.16). These results are in line with the localization of H3K4me3 peaks in the ChIP-Seq dataset. Furthermore, as H3K4me3 generally maps to the promoter of actively transcribed genes, it is reasonable to expect this histone mark to be found along the promoters of OC highly expressed genes (Table 3.4).

\subsubsection{ChIP-Sequencing}

Although the previous ChIP-qRT-PCR trials did not yield HBO1 specific enrichment (Fig. 3.13 \& Fig. 3.16), because the AB70 antibody had passed multiple tests (Fig. 3.9, Fig. 3.10 \& Fig. 3.11) and had been used previously for ChIP-qRT-PCR and ChIP-Seq, a ChIP-Seq experiment was carried out. Six chromatin samples (2 'Input', 2 'HBO1' IPs and 2 'H3K4me3' IPs) were sent for DNA sequencing.

Each of the samples met library preparation and sequencing quality control parameters and generated approximately $30 \mathrm{M}$ reads. The resulting raw data was then analysed following the procedure described in Figure 2.3, but this analysis did not reveal any significant peaks in any of the IP samples ('HBO1' or H3K4me3') when comparing them with the non-specific 'Input'. The negative 'HBO1' result reflected the prior unsuccessful ChIP-qRT-PCR experiments (Fig. 3.13 \& Fig. 3.16), 
however, the absence of enrichment of the 'H3K4me3' sample was surprising given the successful results obtained for gene specific ChIP.

In order to determine whether the lack of positive outcome to the HBO1 ChIP-Seq experiment was due to experimental procedures, a frozen pellet of fixed UWB1.289 cells $(\sim 13-15 \mathrm{M})$ was sent, along with a small aliquot of the AB70 antibody (10 $\mu \mathrm{g})$, to the Active Motif $^{\circledR}$ (AM) laboratories to perform an 'Antibody Validation Service'. This service consisted of a small-scale ChIP-Seq followed by data analysis and a simple yes/no answer to whether the antibody is valuable for ChIP-Seq purposes. AM confirmed that the HBO1 ChIP-Seq data looked more similar to the input control than to the positive/successful ChIP-Seq used for comparison, thus calling this assay unsuccessful (Fig. 3.17). The AM data analysis did, however, return a very limited number of $\mathrm{HBO} 1$ specific peaks that were interrogated further.

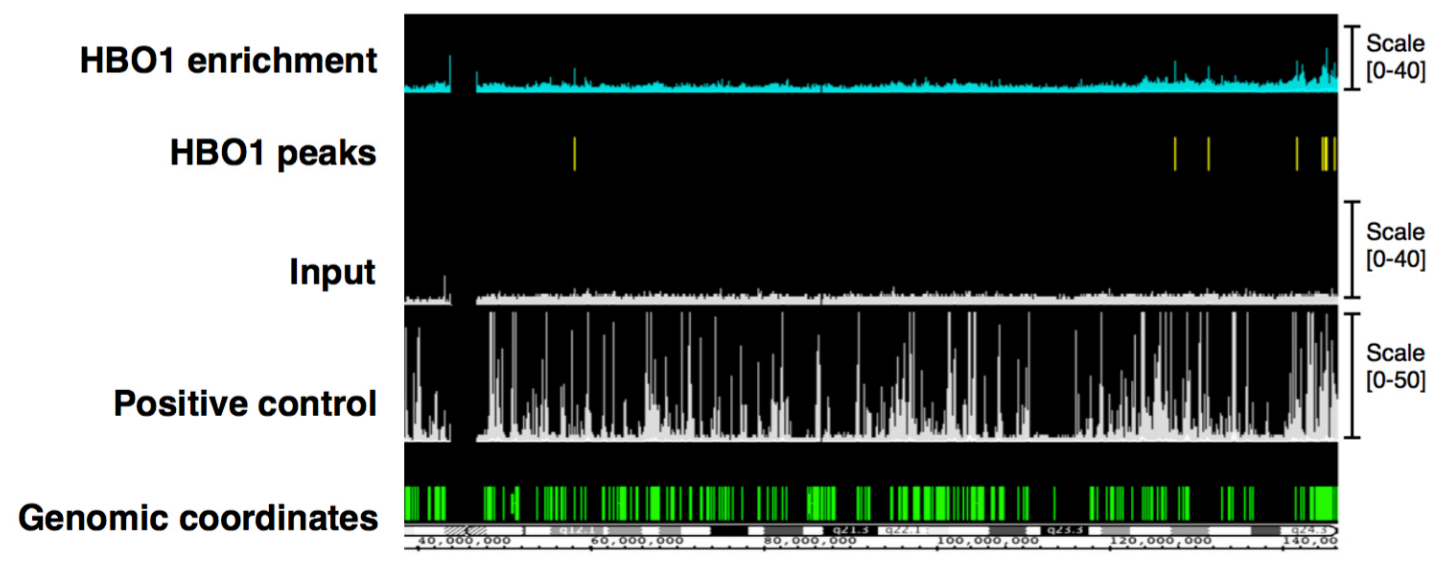

Figure 3. 17 Graphical representation of Active Motif ${ }^{\circledR}$ ChIP-Seq results

Active Motif ${ }^{B}$ Epigenetics Services conducted a small scale ChIP-Seq using the AB70 anti-HBO1 antibody and generated a signal map showing peak enrichment densities along the genome. The top track represents HBO1 enrichment, followed by a track with yellow marks indicating the specific location of HBO1 peaks. The middle/third (Input) and forth (Positive Control) tracks represent negative and positive ChIP-Seq assays for comparison. The bottom track shows the corresponding RefSeq gene annotations. Y-axis scales represent peak enrichment value ranges.

Two hundred and fifty seven peaks were identified using AM's default cut-off $p$ value $\left(1 \times 10^{-07}\right)$. This number of peaks is often found at background level, since 100250 peaks are also typically obtained with input DNA. Indeed, 128 of these peaks 
are enlisted in the ENCODE blacklist of known false ChIP-Seq peaks (Kundaje 2013), leaving a list of only 129 filtered potential HBO1 targets. The peak list contained approximate genomic coordinates accompanied by a number of genes $(0$ to 4 ) in closest proximity to such peak. The establishment of this relationship was evident in cases where the peak was mapped inside a gene locus or in the nearby promoter area. However, some genes were linked to peaks located up to $181 \mathrm{~Kb}$ upstream or downstream the gene locus, suggesting that they are actually enhancer sites. All gene names regardless of their related peak were extracted to a new file, rendering a 173-gene list.

The list of 173 candidate HBO1-regulated genes was added to the SQL database and compared with the existing datasets to select new targets for ChIP-qRT-PCR validation. Ultimately, SQL correlations rendered four genes that showed significant enrichment peaks within the gene body in both HBO1 ChIP-Seq experiments, RKO and UWB1.289, and also displayed significant H3K4me3 peaks in similar locations (RKO ChIP-Seq): Caspase 7 (CASP7), plakophilin 4 (PKP4), the 'MRS2-like magnesium transporter' (MRS2) and the 'nuclear receptor subfamily 2 group $\mathrm{F}$ member 2' (NR2F2) (Table 3.5).

Table 3. 5 List of potential HBO1 target genes derived from ChIP-Seq

\begin{tabular}{|c|c|c|c|c|}
\hline \multirow{2}{*}{ Gene } & \multirow{2}{*}{ Function } & \multicolumn{4}{|c|}{ Average peak enrichment values } \\
\cline { 3 - 5 } & & RKO & UWB1.289 & ${ }^{1}$ H3K4me3 \\
\hline CASP7 & Apoptosis & 19.5 & 4.20 & 76.4 \\
\hline$P K P 4$ & Cell adhesion & 15.23 & 3.87 & 88.23 \\
\hline$M R S 2$ & Protein transport & 15.95 & 5.76 & 106.2 \\
\hline$N R 2 F 2$ & Nuclear receptor & 10.92 & 4.01 & 33.14 \\
\hline
\end{tabular}

${ }^{1} \mathrm{H} 3 \mathrm{~K} 4 \mathrm{me} 3$ data extracted from the RKO ChIP-Seq

ChIP-qRT-PCR primer pairs were designed using the coordinates from the AM ChIP-Seq data for each of the peaks related to the set of 4 genes (Table 3.5). Similar to the process followed previously, two primer pairs were designed for each genomic location (Fig. 3.18). 


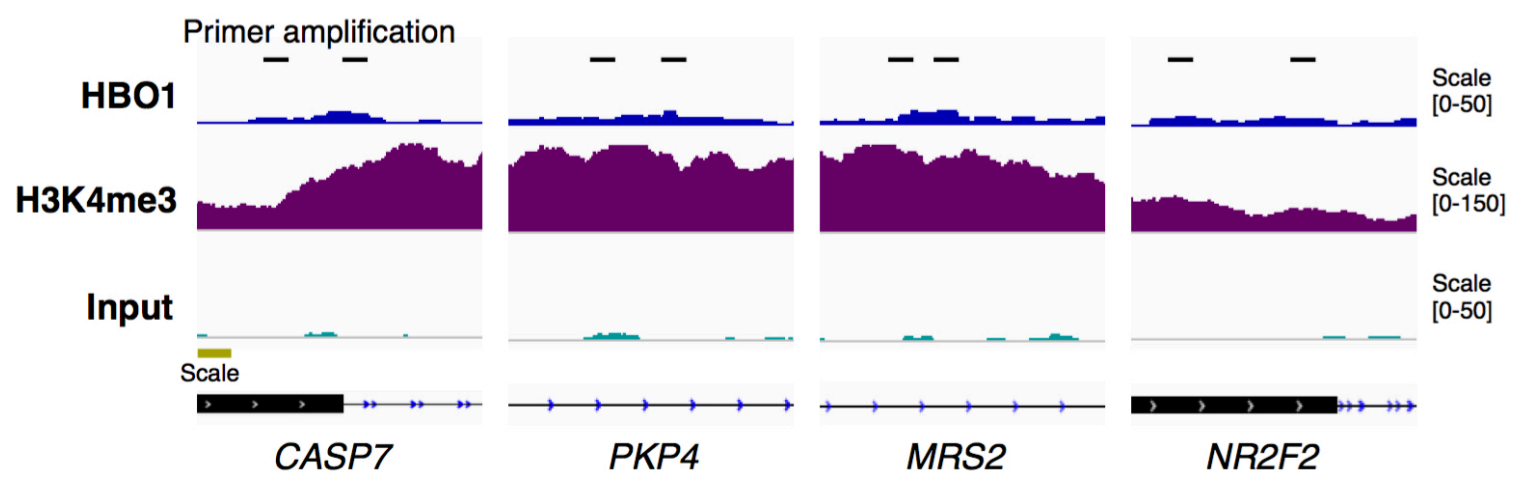

Figure 3. 18 IGV analysis of potential HBO1 targets derived from ChIP-Seq

The first and second panels (starting from the top) represent peak enrichment obtained with anti-HBO1 and anti-H3K4me3 antibodies in ChIP-Seq performed on UWB1.289 and RKO cell lines respectively. The third panel (Input) represents unspecific peaks obtained from UWB1.289 chromatin samples that have not been subjected to antibody enrichment. Images were extracted from the IGV browser. Selected genomic regions are depicted at the bottom as longitudinal lines where black squares/rectangles symbolise exons. Specific primer amplification sites are illustrated on top of the first panel. X-axis scale represents $2 \mathrm{~Kb}$; $\mathrm{y}$-axis scales represent peak enrichment value ranges.

In order to validate the peak enrichment observed in the AM ChIP-Seq (Fig. 3.18), ChIP experiments were performed on UWB1.289 cells followed by ChIP-qRT-PCR using the recently designed probes together with the three negative control primer pairs (IGR) (Fig. 3.19). Again, the anti-HBO1 antibody did not show significant enrichment compared to the negative controls in any of the loci interrogated (Fig. 3.19). The PI enrichment displayed by $A B 70$ in IGRs such as IGR 1 ( $P I=0.13 \pm$ $0.1 \%)$ or IGR $2(\mathrm{PI}=0.11 \pm 0.065 \%)$ was higher than, or at least as high as the PI enrichment shown in PKP4 (PI $=0.11 \pm 0.062 \%), M R S 2$ (PI $=0.03 \pm 0.01 \%)$ and $N R 2 F 2$ (PI $=0.10 \pm 0.04 \%$ ) loci (Fig. 3.19). Interestingly, AB70 displayed strong enrichment of the $C A S P 7$ locus $(\mathrm{PI}=0.26 \pm 0.03)$, although it was matched by $\operatorname{IgG}$ $(\mathrm{PI}=0.28 \pm 0.08)$, and therefore does not represent a specific HBO1 target.

The H3K4me3 antibody displayed significant input enrichment in CASP7 (PI = 7.01 $\pm 2.01 \%)$, PKP4 $(\mathrm{PI}=5.24 \pm 1.56 \%), \operatorname{MRS} 2(\mathrm{PI}=4.52 \pm 1.31 \%)$ and NR2F2 $(\mathrm{PI}=$ $3.01 \pm 1.07)$ regions (Fig. 3.19). These results agree with the localization of H3K4me3 peaks in the ChIP-Seq dataset (Table 3.5 \& Fig. 3.18). 


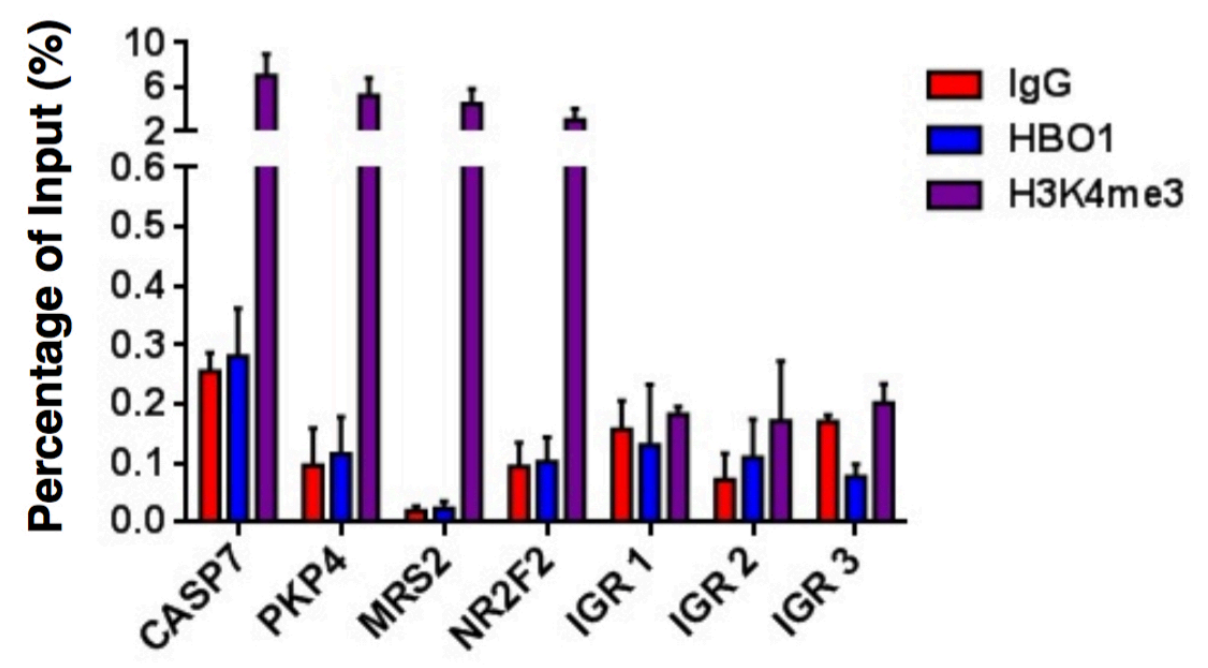

Figure 3. 19 Enrichment of potential HBO1-targets: AM-derived genes

UWB1.289 cells were subjected to ChIP using IgG, anti-HBO1 (AB70) and antiH3K4me3 antibodies, followed by qRT-PCR amplification of CASP7, PKP4, MRS2 and $N R 2 F 2$ genomic regions. IGRs 1, 2 and 3 were used as controls for antibodyspecificity. Final antibody enrichment, expressed as percentage of input, was calculated comparing sample qRT-PCR values with 'Input' control values. Results represent the mean $\pm \mathrm{SD}$ of two biological replicates. IGR: Intergenic region.

\subsubsection{Further ChIP-qRT-PCR optimization}

The anti-HBO1 AB70 antibody, which has been previously used for ChIP and ChIPSeq experiments in a variety of cell line backgrounds (Miotto \& Struhl 2008; Saksouk et al. 2009; Avvakumov et al. 2012), consistently failed to achieve HBO1 positive enrichment over input in UWB1.289 cells (Fig. 3.13, Fig. 3.16 \& Fig. 3.19). As a consequence, new ChIP experiments were performed using Santa Cruz ${ }^{\circledR}$ antibodies including, G-2, T-20, H-180 and N-18, some of which had been previously used by Saksouk et al. (2009), Miotto \& Struhl (2010) or Mishima et al. (2011), as well as a pool of all these antibodies.

Following ChIP (Chromatrap ${ }^{\circledR}$ Pro-A kit), a set of 6 primers targeting potential HBO1-binding sites and the intergenic negative controls were used for ChIP-qRTPCR. This set of primers included a random selection of two probes targeting HBO1-related origins of replication (Fig. 3.14), another two targeting potential HBO1-targets derived from the SQL database (Table 3.4) and two more targeting potential HBO1-targets derived from the AM ChIP-Seq data (Table 3.5). None of 
the individual or pooled HBO1 antibodies succeeded in giving an HBO1 specific enrichment in the selected loci in UWB1.289 cells. Additionally, this approach was attempted in OVCAR-3 and HeLa cells, obtaining similar negative results.

Apart from using different antibody combinations to successfully IP enriched HBO1-chromatin fractions, new approaches included the use of alternative ChIP protocols such as EZ-Magna ChIPTM A/G (Millipore) or the Chromatin ${ }^{\circledR}$ Native ChIP kit, which tackle the IP using different methodologies. New ChIP experiments were followed by ChIP-qRT-PCR using the set of 6 primers targeting HBO1potential binding sites and the pertinent negative controls. However, none of these methods succeeded in achieving HBO1 specific enrichment, even though the H3K4me3 positive antibody performed as outstandingly as how it did with the Chromatin ${ }^{\circledR}$ Pro-A kit (Fig. 3.13, Fig. 3.16 \& Fig. 3.19).

Lastly, a non-commercial protocol was used to perform ChIP on UWB1.289 cells. The procedure was based on the same principles followed by Avvakumov et al. (2012) to achieve the first and only publicly available HBO1 ChIP-Seq, which are explained in the materials and methods section of the publication. In general terms, the protocol hinges on the use of magnetic beads, analogous to the EZ-Magna $\mathrm{ChIP}^{\mathrm{TM}} \mathrm{A} / \mathrm{G}$ from Millipore ${ }^{\mathrm{TM}}$, but allows to scale-up and include millions of cells in each assay in contrast with Chromatrap ${ }^{\circledR}$ and Millipore kits, which can only evaluate target enrichment using 10-15 million cells per sample. Due to the huge number of cells required for each IP reaction, the IgG antibody was not used as negative control in this occasion.

The non-commercial protocol was first tested using 30M of UWB1.289 cells and the H3K4me3 antibody, which displayed significant enrichment over the input sample, compared to the IGR negative controls (data not shown). Subsequently, the HBO1 AB70 antibody was used with 30,60 and 80M UWB1.289 cells, yet it was still unable to give specific enrichment in the tested loci. 
HBO1 exerts its epigenetic regulatory role as part of a protein complex and as so, depends on the spatial and temporal conformation of this structure to bind DNA. Accordingly, changes in the configuration of the histone acetyltransferase complex may affect the way HBO1 binds to potential targets in UWB1.289 cells, and therefore the capability of ChIP procedures to capture and detect these interactions. In order to evaluate alterations in the abundance of components of the HBO1complex in UWB1.289 cells, JADE1-3, BRPF1-3 and ING4-5 gene transcripts were evaluated using qRT-PCR (Fig. 3.20).

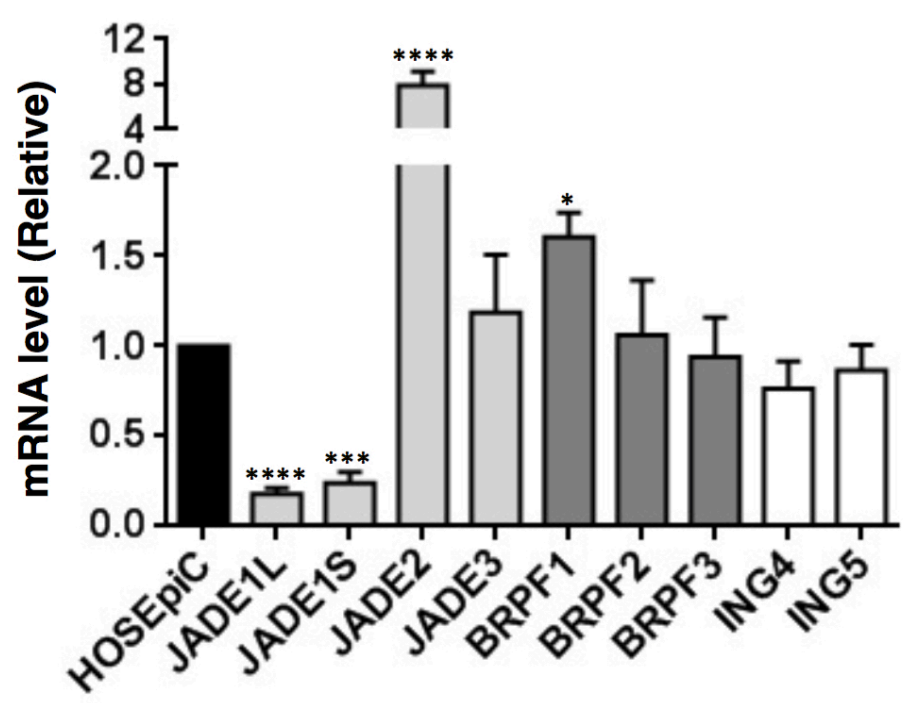

Figure 3. 20 Comparative analysis of HBO1-complex components

RNA lysates from UWB1.289 and HOSEpiC cells were prepared and analysed by qRT-PCR. Relative levels of $J A D E 1 L-S / 2 / 3, B R P F 1 / 2 / 3$ and $I N G 4 / 5$ expression were compared to those of the HOSEpiC non-cancerous control. GAPDH expression was used as control. Statistical analyses consisted of one-way ANOVA and the Tukey's multiple comparison test. All values represent the mean $\pm \mathrm{SD}$ of three biological samples $(* \mathrm{p}<0.05 ; * * * \mathrm{p}<0.001 ; * * * *<0.0001)$.

The expression level of isoforms JADE1L $\left(\mathrm{FC}=-5.64,{ }^{* * * *} \mathrm{p}=<0.0001\right)$ and $J A D E 1 S\left(\mathrm{FC}=-4.34,{ }^{* * *} \mathrm{p}=0.0002\right)$ was significantly lower in UWB1.289 cells compared to the HOSEpiC non-cancerous control (Fig. 3.20). In contrast, the transcript abundance of alternative scaffolding subunits such as $J A D E 2$ (FC $=7.90$, $* * * \mathrm{p}=<0.0001)$ and to a lesser extent BRPF1 $\left(\mathrm{FC}=1.6,{ }^{*} \mathrm{p}=0.0485\right)$ was significantly higher in the cancerous subtype (Fig. 3.20). The expression level of other transcripts, JADE3 $(\mathrm{FC}=1.18, \mathrm{p}=0.995), B R P F 2(\mathrm{FC}=1.06, \mathrm{p}=>0.9999)$, 
BRPF3 $(\mathrm{FC}=-1.06, \mathrm{p}=>0.9999), I N G 4(\mathrm{FC}=-1.3, \mathrm{p}=0.7627)$ and ING5 $(\mathrm{FC}=-$ $1.15, p=0.7235$ ) was not significantly different between the cell lines. Based on the observed patterns, it is possible that the very high levels of $J A D E 2$, for example, may cause the formation of an HBO1 complex where HBO1, whilst recruited to the promoter of target genes, is essentially masked by JADE2 and other components of the complex, rendering HBO1 inaccessible to the AB70 antibody thus interfering with the ChIP experiments.

\subsection{Discussion}

In order to efficiently investigate the role of $\mathrm{HBO} 1$ in $\mathrm{OC}$, a number of model cell lines were evaluated, including a panel of OC cell lines representing the most common OC sub-types (Table 3.1) and a non-cancerous ovarian control. Importantly, four OC cell lines (OVCAR-3, SKOV-3, UWB1.289 and UACC-1598) were representatives of the serous histo-type, the most aggressive and prevalent form of OC. The principal concern regarding the use of cell lines to study OC is related to the genetic and phenotypical drifts, which raise doubts about their suitability since they do not exactly subscribe to the behaviours of original cancer cells. Even so, and assuming these limitations, the chosen cell lines compose a strong informative model that spans major HGSC-associated features, e.g. BRCA1/2 mutation (UWB1.289) or CCNE1 amplification (OVCAR-3). In fact, mutation, copy number analysis and others have confirmed the suitability of some of these cell lines to model OC (Network 2011).

Following the trend previously observed in numerous cancer cell lines (e.g. MCF7 and Saos-2), breast and ovarian cancer tissues, the whole panel of OC cell lines displayed $H B O 1$ transcript overexpression, compared to the non-cancerous control cells (Fig. 3.1). This increased expression was also observed when individual splice isoforms of $\mathrm{HBOl}$ were assessed (Fig. 3.3 \& Appendix 1). Consistent high levels of HBO1 transcript expression amongst OC cell lines point to alterations in DNA replication and/or transcription, key cellular processes allegedly modulated by HBO1. 
The overexpression of $H B O 1$ transcripts in the panel of OC cell lines compared to the non-cancerous control was accompanied by similar HBO1 protein level increase (Fig. 3.6). Therefore, it can be assumed that increased $\mathrm{HBOl}$ gene expression also occurs at the protein level. In order to investigate HBO1 protein abundance, antibodies G-2 and AB70 were optimized for use in western blot experiments (Fig. 3.5), providing an essential tool for further $\mathrm{HBO} 1$ functional analyses. Both antibodies performed well, however, as UACC-1598 protein extracts probed with the G-2 antibody displayed un-identified bands (Fig. 3.4 \& Fig. 3.5); AB70 was used for all further experiments.

A significant number of nuclear receptor binding sites were found located in the $H B O 1$-proximal promoter region (AR, ER, PR, GR), suggesting that $H B O 1$ may undergo hormone-mediated regulation, which could provide a link between OC progression and specifically estrogen exposure. These results differ from previous studies, in which ER $\alpha$ binding sites were not found nearby the proximal promoter region of HBOl using the TRSEARCH software (Wang et al. 2010). However, estradiol (E2) treatment, which induces ER $\alpha$-mediated transcription, did not influence $\mathrm{HBOl}$ transcript expression levels (Fig. 3.8).

Finally, a ChIP-Seq experiment aimed at unravelling HBO1 widespread localization on the OC genome was attempted. In doing so, and throughout the relevant optimization process, HBO1 was found to localize mainly to the nucleus of OC cell lines, exemplified in UWB1.289 and the non-cancerous HOSEpiC control cells (Fig. 3.10). Furthermore, approximately $45 \%$ of the whole HBO1 cellular protein was detected binding to the chromatin fraction (Fig. 3.11), confirming the presupposed relevance of HBO1 in nuclear phenomena such as DNA replication and transcription. However, HBO1 was not solely localized to the nucleus, but was observed in the cytoplasm of the cells (Fig. 3.10 \& Fig. 3.11). Apart from synthesis, like any other protein, and degradation (Zou et al. 2013), the cytoplasmic localization of HBO1 molecules suggests other non-DNA-related functions. 
Prior to ChIP-Seq, ChIP-qRT-PCR experiments using AB70 antibody did not detect any significant enrichment of HBO1 at the LMNB2, MCM4, SNHG12, LUC7L, CYR61, ERBB2, ABCC4, ALCAM, TLN1, ANKRD1 loci (Fig. 3.13 \& Fig. 3.16). Nonetheless, certain genomic loci $(A B B C 4, A L C A M)$ did display differences amongst the enrichment attained by HBO1 and IgG (Fig. 3.16). Although the goal of finding a positive ChIP-qRT-PCR control was not achieved, successful steps in the optimization process offer a solid ground on which to base future experiments. For example, the specific enrichment of the H3K4me3 histone marker in UWB1.289 cells (Fig. 3.13, Fig. 3.16 \& Fig. 3.19).

As expected from the absence of anti-HBO1 enrichment in various ChIP-qRT-PCR experiments, both ChIP-Seq performed by the Epigenomics Core and AM were deemed unsuccessful. Unexpectedly, the HBO1 ChIP-Seq carried out by the Weill Cornell Epigenomics Core was ineffective for H3K4me3 IP, which had previously given significant enrichment in numerous occasions and diverse loci (Fig. 3.13 \& Fig. 3.16), suggesting sequencing related issues. Whilst the AM ChIP-Seq was deemed unsuccessful due to the very low number of peaks returned for HBO1 (hundreds of peaks would normally be expected in a successful ChIP-Seq experiment), a limited number of HBO1 binding sites that represented 173 potential target genes were identified. Although these targets could not be validated via ChIPqRT-PCR (Fig. 3.19), they provide a starting point for future studies, e.g. these loci could be queried in order to assess HBO1-related changes in histone marks such as histone $\mathrm{H} 3$ or $\mathrm{H} 4$ acetylation.

More experiments using new antibodies, different cell lines or methodology approaches could not give a positive enrichment of HBO1 in any of the queried loci, suggesting that the problem goes beyond technical issues. One possibility is that HBO1 is exerting its action at such fast pace that formaldehyde is not able to properly fix it to the chromatin. In this regard, it is worth considering the use of other fixing compounds such as glutaraldehyde for future studies. Also, the functions of HBO1 are highly tissue specific, meaning that its genomic localization may vary between differing backgrounds, a fact that could explain for the failure to detect ChIP-qRT-PCR enrichment. 
Another possibility is that the isolation of fixed HBO1-chromatin complexes is somehow masked, as non-chromatin immune-precipitation of HBO1 worked well with two different antibodies (Fig. 3.5 A \& Fig. 3.9 A). A simple qRT-PCR experiment was performed in order to analyse the transcript levels of different compounds of the HBO1 multi-subunit complex that might interfere with antibody isolation in the UWB1.289 cell line. Interestingly, the transcript levels of JADE2 were very elevated in UWB1.289 compared to non-cancerous HOSEpic cells, while $J A D E$ isoforms $J A D E 1 L$ and $J A D E 1 S$ transcript levels were decreased (Fig. 3.20).

The $J A D E 2$ paralog encodes a slightly smaller protein than $J A D E 1$, constituted by 790 amino acids $(\sim 87.5 \mathrm{kDa})$ as opposed to the 834 amino acids $(\sim 94 \mathrm{kDa})$ of $J A D E 1$, of which little is known. The variable region of JADE2 compared to JADE1L is the C-terminal fragment, similar to JADE1S (Fig. 1.25), yet it still contains a full domain able to interact with ING4/5 and hEaf6 (Panchenko 2016). This initial observation suggests that a change in the conformation of the HBO1 multi-protein complex could interfere the ChIP through preventing access of the AB70 to HBO1, 'buried' in a JADE2 dominated complex.

Finally, it is worth mentioning that all previous HBO1 ChIP-qRT-PCR experiments, whereas not the ChIP-Seq, were performed on HBO1-transfected cell types, hinting previous struggles when conducting ChIP on physiological levels of HBO1. 
4. Optimization of Genetic Tools for the Study of HBO1 Function 


\subsection{Introduction and Aims}

Some of the most direct ways to determine $\mathrm{HBOl}$ functions in an $\mathrm{OC}$ background include the knockdown (KD) of the gene, as well as its up-regulation via expression vectors. Both processes are used to evaluate the effects of gene alteration through reverse genetics (Silva et al. 2004).

There are several approaches to $\mathrm{KD} /$ silence the expression of a target gene that involve RNA interference (RNAi), including the use of small interfering RNAs (siRNAs) and short hairpin RNAs (shRNAs). These methodologies result in the post-transcriptional down-regulation of gene expression without changing the target cell genetic code (Mocellin \& Provenzano 2004). Low amounts of target RNA and protein are always expressed however, the result being that gene-related functions are reduced, but may not be completely eliminated (Mittal 2004). Genome editing is an alternative approach, resulting in genetic code alterations that ultimately cause the complete elimination of gene function or knock-out (KO) (Shalem et al. 2014). Though there are several genome-editing tools available, a system that involves Clustered Regularly Interspaced Palindromic Repeats (CRISPR) and the CRISPRassociated protein 9 (Cas9) is the most widely used. Both KD and KO approaches are widely used to modify in vitro gene expression and each has certain advantages and disadvantages (Table 4.1).

The CRISPR/Cas systems (I-III) are naturally occurring prokaryotic immune defence strategies among which the type II system has been adapted for RNA-directed DNA editing of mammalian genomes (Jinek et al. 2012). The basal CRISPR machinery has been engineered to deliver the nuclease Cas9 to any locus in the genome, whereupon it originates double strand DNA breaks that are repaired by either nonhomologous end joining (NHEJ) or homology directed repair (HDR) pathways (Mao et al. 2008). Most commonly, DNA double breaks are repaired via the NHEJ pathway, an error-prone process that can result in the introduction of stable insertions or deletions (INDELs) in the target region (Lieber 2010), leading to the disruption of the codon-reading frame and ultimately inducing erroneous transcripts and the ablation of gene expression (Unniyampurath et al. 2016). Newer technologies, e.g. CRISPR interference or CRISPRi, are able to repurpose the 
CRISPR machinery for reversible and incomplete modification of gene expression (Qi et al. 2013), references to CRISPR hereafter refer only to the KO variation.

Table 4. 1 Advantages and disadvantages of KD and KO available techniques

\begin{tabular}{|c|c|c|}
\hline Technique & Advantages & Disadvantages \\
\hline RNAi & $\begin{array}{l}\text { - Target any transcript regardless of } \\
\text { ploidy (number of chromosomes). } \\
\text { - Not inhibited by chromatin } \\
\text { context. } \\
\text { - Predictable, no genome mutations } \\
\text { involved. } \\
\text { - Rapid; does not require single } \\
\text { clone isolation. }\end{array}$ & $\begin{array}{l}\text { - High off-target effect based } \\
\text { on mismatch tolerance and } \\
\text { interference with } \\
\text { endogenous RNAi } \\
\text { pathways. } \\
\text { - Finds less true positive } \\
\text { essential genes involved in } \\
\text { fundamental pathways. }\end{array}$ \\
\hline CRISPR & $\begin{array}{l}\text { - Better at finding true positive } \\
\text { essential genes. } \\
\text { - Higher specificity. } \\
\text { - Reduced mismatch tolerance and } \\
\text { consequent off-targets effects. No } \\
\text { interference with endogenous } \\
\text { RNAi pathways. } \\
\text { - More robust phenotypes. }\end{array}$ & $\begin{array}{l}\text { - Comparatively slower; } \\
\text { requires single clone } \\
\text { isolation. } \\
\text { - Relies on presence of } \\
\text { appropriate PAM sites. } \\
\text { - Can be stymied by ploidy or } \\
\text { by specific chromatin } \\
\text { structures. }\end{array}$ \\
\hline
\end{tabular}

(Shalem et al. 2014; Wang et al. 2015; Boettcher \& McManus 2015; Housden \& Perrimon 2016).

RNA interference utilizes a naturally occurring double stranded RNA-mediated homology based mechanism that has evolved to post-transcriptionally regulate eukaryotic gene expression (Downward 2004). The core component of RNAi is the utilization of small interfering RNAs that are processed by the cellular RNA-induced silencing complex or RISC, which in turn uses the siRNAs as template to localize the correspondent complementary target mRNA for degradation, achieving a specific temporary/transient or stable gene KD effect (Wilson \& Doudna 2013). Non-natural RNAi can also be induced by direct exogenous supply of short double stranded RNAs or small interfering RNAs ( $\sim 21$ nucleotides), achieving a transient gene inhibition effect. Alternatively, RNAi can also be induced in cells using plasmid delivery of shRNAs, which are processed in a similar way to siRNAs (Rao et al. 2009). 
The expression levels of shRNAs depend on the associated promoter driving their expression and can result in permanent (constitutive promoter) or induced (inducible on-off promoter) degradation of target mRNAs. RNAi-mediated KD of HBO1 is, thus far, the most widely used methodology to study gene functionality in numerous cell backgrounds (Table 1.7). The successful delivery of siRNAs or shRNAs to OC cell lines would provide a rapid and efficient system to study HBO1 functionality. Meanwhile, knockout studies on mice point to essential functions of $\mathrm{HBOl}$ in embryo development (Kueh et al. 2011), yet most KD studies suggest that $\mathrm{HBOl}$ is a non-essential gene and therefore susceptible to CRISPR-Cas9 editing (Table 1.7). The successful generation of a CRISPR-edited OC cell line would provide a robust and consistent HBO1 loss-of-function 'phenotype' (Shalem et al. 2014). To assess HBO1 functionality, both approaches were undertaken.

A common tool to assess gene functionality, apart from the previously discussed silencing methodologies, is the use of vectors (e.g. plasmids) designed for gene expression. This approach is based on the delivery of expression vectors engineered to contain the target gene sequence as well as regulatory regions that lead to efficient transcription of the latter in transfected cells. In the case of study, the introduction of exogenous $\mathrm{HBOl}$ could help to validate the effects of prior gene silencing methods, as well as regaining gene expression while potentially restoring normal cell phenotype in line with that identified prior to HBO1 KD. Consequently, the last goal of this chapter was to design a mammalian expression vector to drive $\mathrm{HBOl}$ gene expression in OC cells.

\subsection{Generation of an HBO1 Knock-OUT Cell Line}

The UWB1.289 cell line, which represents a model of HGSC with high levels of HBO1, was selected to perform CRISPR-Cas9 KO.

\subsubsection{Cas9 transfection into UWB1.289 cells}

The transient or constitutive expression of ectopic Cas9 protein was the first step in achieving a successful $\mathrm{HBOl} \mathrm{KO}$. Initially, the efficiency of different transfection 
reagents to transfect sub-confluent populations of UWB1.289 cells was tested using a GFP-expression plasmid. Visual inspection of transfected cells resulted in the selection of the X-tremeGENE ${ }^{\mathrm{TM}}$ HP DNA transfection reagent (Roche), which showed high levels of GFP expression and very low cytotoxicity (Fig. 4.1), to pursue the Cas9 transfection in UWB1.289 cells. On the contrary, Lipofectamine ${ }^{\circledR} 3000$ presented higher cell cytotoxicity and X-tremeGENE ${ }^{\mathrm{TM}} 9$ showed lower GFPexpression levels compared to the selected reagent (Fig. 4.1).
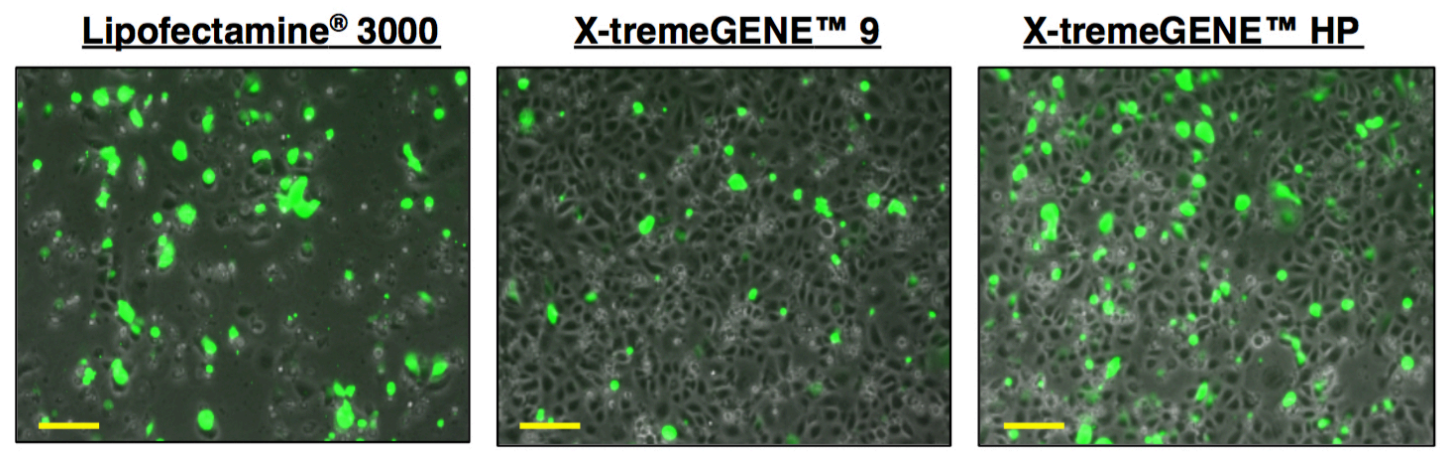

Figure 4. 1 UWB1.289 transfection efficiency test

Sub-confluent populations of UWB1.289 cells were transfected with GFP-expressing plasmid $[1.5 \mu \mathrm{g} / \mathrm{mL}]$ and different transfection reagents. Images were taken $24 \mathrm{~h}$ post-transfection using a fluorescent microscope. Scale represents $100 \mu \mathrm{m}$.

Different concentrations of the Cas9 IDT $^{\circledR}$ expression plasmid $(0.5-1.5-3 \mu \mathrm{g} / \mathrm{mL})$ were transfected into sub-confluent populations of UWB1.289 cells using XtremeGENE ${ }^{\mathrm{TM}} \mathrm{HP}$. HeLa cells were also transfected with the same concentrations of Cas9-expressing plasmid and used as positive control due to their high transfection efficiency. Whole-cell protein lysates were isolated, processed and analysed via dotblot to assess Cas9 transfection levels (Fig. 4.2).

Whilst all transfected HeLa cells rendered considerable V5-Cas9 expression, none of the UWB1.289 transfections displayed any signal (Fig. 4.2). These results suggest plasmid-specific transfection issues in UWB1.289 cells, possibly due to the substantial size of the Cas9-expression plasmid in comparison with GFP (Cas9: $\sim 7.3 \mathrm{~Kb}>$ GFP: $\sim 3.4 \mathrm{~Kb}$ ). Adenoviruses expressing Cas9 protein were therefore used as an alternative method. 


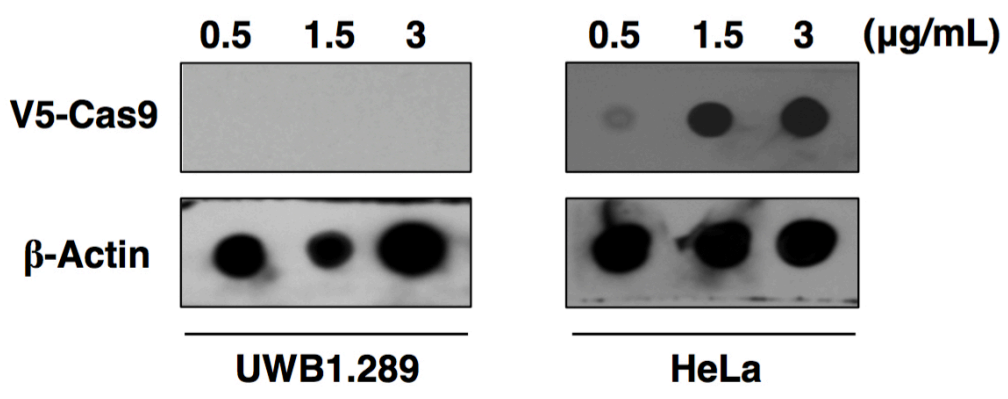

Figure 4. 2 Transfection of a Cas9-expressing plasmid in UWB1.289 and HeLa Protein extracts of UWB1.289 and HeLa cells transfected with different concentrations of Cas9-expressing plasmid [0.5-1.5-3 $\mu \mathrm{g} / \mathrm{mL}]$ were analysed by dotblot and probed with V5-Cas9 and $\beta$-Actin antibodies. $\beta$-Actin was used as loading control.

The Ad-GFP-Cas 9 adenoviruses (Vector BioLabs) express GFP together with Cas 9 , providing a convenient system to corroborate the expression of a protein. First, varying concentrations of adenovirus particles were used to infect sub-confluent populations of UWB1.289 cells (10-500 MOI, multiplicity of infection). A preliminary infection test resulted in very low efficiencies (approximately 5\% cells expressing GFP), so polybrene, a positively charged polycation that reduces the repulsion forces between cells and viruses enhancing transduction efficiency (Clark et al. 1999), was included to intensify the process $(5-20 \mu \mathrm{g} / \mathrm{mL})$.

Optimal transduction conditions (low cytotoxicity and high GFP signal) were identified using $500 \mathrm{MOI}$ and $10 \mu \mathrm{g} / \mathrm{mL}$ of polybrene. However, even though these conditions were innocuous to UWB1.289 cells after as long as 96h (overconfluence), the subsequent transfection of CRISPR RNA (crRNA) complexes resulted in high levels of cell death, and the inability of UWB1.289 cells to proliferate after sub-culture. These results suggested cells were being subjected to excess stress (virus transduction plus immediate transfection), and thus prompted the need to try a different approach.

One efficient approach that is commonly used to transfect plasmids into mammalian cells is electroporation (Potter \& Heller 2003). First, confluent populations of UWB1.289 cells were transfected with a GFP-expressing plasmid [1.5 $\mu \mathrm{g} / \mathrm{mL}]$ using different electroporation programmes (Nucleofector ${ }^{\mathrm{TM}}$, Lonza) in order to optimize 
the conditions for Cas9 transfection. Twenty-four hours after the electroporation, treated samples were observed under the microscope to assess cell cytotoxicity and transfection efficiency (GFP-expression) (Figs. 4.3).
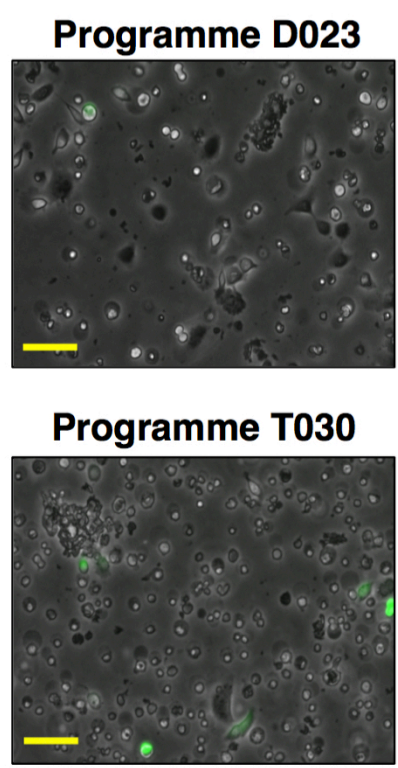

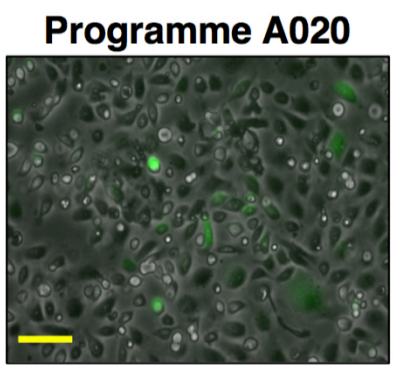

Programme X5
Programme L029

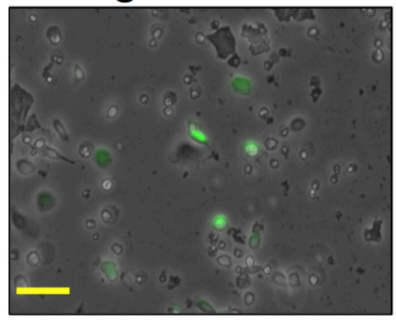

Programme X1
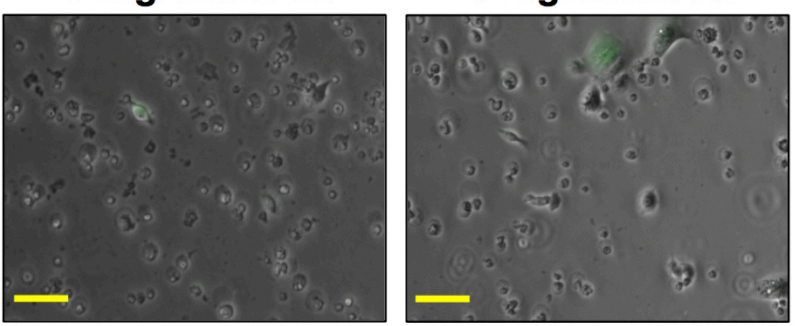

Figure 4. 3 UWB1.289 electroporation efficiency test

Sub-confluent populations of UWB1.289 cells were subjected to electroporation of $1.5 \mu \mathrm{g} / \mathrm{mL}$ of GFP-expression plasmid under different electroporation programmes (D023, A020, L029, T030, X5) (Nucleofector ${ }^{\mathrm{TM}}$, Lonza). Images were taken 24h post-transfection using a fluorescent microscope. Scales represent $100 \mu \mathrm{m}$.

The majority of electroporation programs tested (D023, L029, T030, X5 and X1) rendered high levels of cell cytotoxicity (Fig. 4.3). However, programme A020 resulted in efficient transfection of the GFP vector into viable UWB1.289 cells (Fig. 4.3). This programme was therefore used to transfect different amounts of Cas9expression plasmid (1.5-3 $\mu \mathrm{g} / \mathrm{mL})$ into sub-confluent populations of UWB1.289 cells. Subsequent analysis with dot-blot revealed that $\operatorname{Cas} 9$-electroporated UWB1.289 cells were able to express Cas9 (Fig. 4.4). However, higher concentrations of the Cas9 plasmid (e.g. 5 or $7.5 \mu \mathrm{g} / \mathrm{mL}$ ) did not significantly increase Cas9 transfection efficiency, and also resulted in elevated cell death rates. Consequently, the electroporation methodology was adopted to transfect $3 \mu \mathrm{g} / \mathrm{mL}$ of Cas9-expressing plasmid into UWB1.289 cells. 


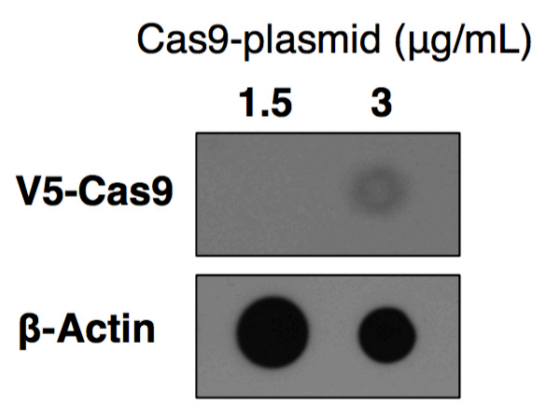

Figure 4. 4 Electroporation of a Cas9expression plasmid in UWB1.289 cells Protein extracts of Cas9-electroporated UWB1.289 cells (Programme A020, Nucleofector ${ }^{\mathrm{TM}}$, Lonza) were analysed using dot-blot and probed with V5-Cas9 and $\beta$ Actin antibodies. $\beta$-Actin was used as loading control.

\subsubsection{CRISPR RNA transfection and confirmation of mutational events}

The introduction of Cas9-expression plasmids in UWB1.289 cells (Fig. 4.4) represented the first step required to achieve successful HBO1 KO. Twenty-four hours after the electroporation with Cas9 plasmid, cells were transfected with crRNA 1-3:tracrRNA complexes targeting $\mathrm{HBOl}$ exon 2 using DharmaFECT 4 $\left(\right.$ Dharmacon $^{\mathrm{TM}}$ ) transfection reagent (Chang et al. 2014). Additionally, Cas9electroporated cells were transfected with crRNA:tracrRNA complexes targeting the positive control gene locus HPRT1 and the non-targeting negative control, computationally designed not to target any fragment of the human genome. All complexes were transfected to a final concentration of $30-60 \mathrm{nM}$ per well in a 6 -well plate, as recommended by the manufacturer. Using this approach, cells retained their ability to proliferate and achieved full confluence 24 hours after the addition of the guide RNA.

To confirm the generation of random INDELs in UWB1.289 cell genomes as a result of Cas9 cleavage, genomic DNA (gDNA) samples were extracted 24h after the transfection with crRNAs and subjected to T7EI digestion as part of a surveyor nuclease assay (Qiu et al. 2004). The region of interest containing potential mutational sites was amplified by end-point polymerase chain reaction. Subsequently, the PCR product was denatured and re-annealed to form DNA heteroduplexes containing a mismatch at the point of the mutation, which can be identified by surveyor nuclease treatment (T7 Endonuclease I or T7EI). Finally, T7-treated PCR samples were analysed by agarose electrophoresis gel (1\%) (Fig. 4.5). 


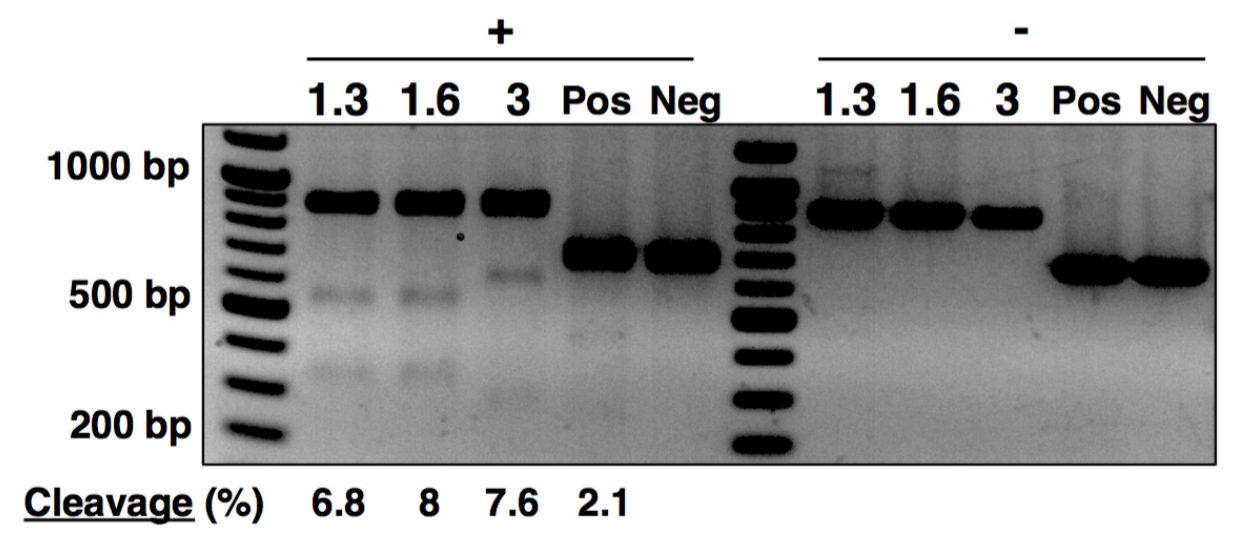

Figure 4. 5 T7EI surveyor assay of Cas9-electroporated UWB1.289 cells Cas9-electroporated UWB1.289 cells were transfected with different CRISPR RNA combinations. 1.3: crRNA $1(3 \mu \mathrm{M})$, 1.6: crRNA $1(6 \mu \mathrm{M})$, 3: crRNA $3(3 \mu \mathrm{M})$, Pos: crRNA HPRT $(3 \mu \mathrm{M})$, Neg: crRNA Negative $(3 \mu \mathrm{M})$. Genomic DNA samples of transfected UWB1.289 cells were subjected to end-point PCR amplification of the region encompassing potential mutational sites (HBO1: $892 \mathrm{bp}, H P R T$ and Negative: $692 \mathrm{bp}$ ). End-point PCR constructs were denatured and re-annealed to form DNA heteroduplexes. Next, an equal fraction of each sample was either treated with T7EI nuclease $(+)$ or left untreated (-). Digested DNA fragments were analysed by gel electrophoresis. The transfection of crRNA molecules followed by specific end-point PCR amplification generated diverse digestion bands: crRNA $1 \quad(\sim 530$ and $\sim 360$ bps), crRNA 3 ( $\sim 600$ and $\sim 290$ bps) and HPRT ( 430 and $\sim 260$ bps). Cleavage percentage was calculated using ImageJ.

The transfection of crRNAs 1-3 led to the T7EI cleavage of the HBOl exon 2 locus, suggesting the successful introduction of INDELs. Potential INDELs generated by crRNA 1 gave rise to two different DNA bands of $\sim 530 \mathrm{bp}$ and $\sim 360 \mathrm{bp}$, as was expected given the size of the PCR product and the specific crRNA 1 binding site (Fig. 4.5). Different concentrations of crRNA $1(3 \mu \mathrm{M}$ and $6 \mu \mathrm{M})$ did not alter the efficiency of the cleavage significantly $(3 \mu \mathrm{M}: 6.8 \pm 1.5 \% ; 6 \mu \mathrm{M}: 8.06 \pm 0.91 \%)$ (Fig. 4.5).

The transfection of crRNA 3 and subsequent T7EI analysis resulted in different digestion products ( $\sim 600 \mathrm{bp}$ and $\sim 290 \mathrm{bp}$ ), as was expected given the crRNA 3 binding site within the $\mathrm{HBOl}$ exon 2 locus, and presented a low percentage of DNA cleavage (7.6 $\pm 1.14 \%)$ (Fig. 4.5). 
The positive control HPRT product was cleaved $(2.1 \pm 0.71 \%)$ into the two expected size digestion products ( $\sim 30$ and $\sim 260 \mathrm{bp}$ ), and the transfection of negative crRNA did not introduce any detectable alteration in the control PCR product (Fig. 4.5). Digestion products were not detected if sample PCRs were not treated with TE7I enzyme (Fig. 4.5: T7EI treatment '-'). Low percentages of DNA cleavage may be due to shortage of INDEL events, as well as other technical issues such as lack of T7 enzyme or inadequate hetero-duplex formation, however, the presence of cleavage confirms the existence of INDELs. Consequently, UWB1.289 cells transfected with crRNA 1, which displayed the highest TE7I cleavage compared to other treatments, were selected to investigate the result of $\mathrm{HBOl} \mathrm{KO}$.

The TE7I assay identified UWB1.289 cells carrying INDELs in the HBO1 locus, possibly due to frame-shift mutations (Fig. 4.5). Two different approaches were used to efficiently isolate individual crRNA 1-treated $\mathrm{HBOl} \mathrm{KO}$ colonies: standard dilution and a single-cell pipetting device. The isolation and separate growth of individual $\mathrm{HBO} \mathrm{KO}$ cells allowed for the propagation of single clones for analysis of HBO1 function. Isolated individual clones were therefore propagated for approximately 3-4 weeks, whereupon protein lysates were extracted to assess HBO1 protein levels (Fig. 4.6).

A

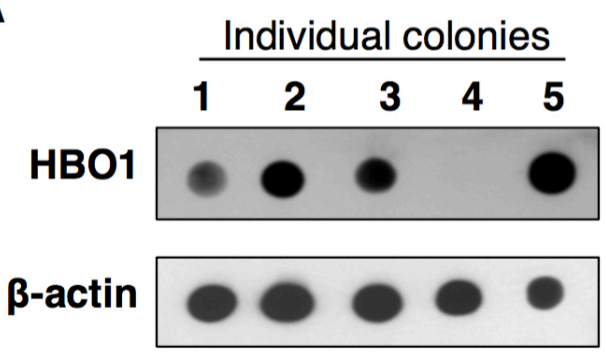

B

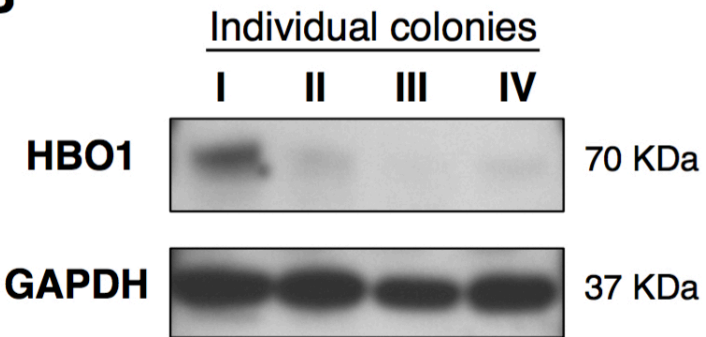

Figure 4. 6 Analysis of potential UWB1.289 knock-OUT individual colonies (A) Protein extracts of UWB1.289 individual colonies propagated for 3-4 weeks were analysed by dot-blot and probed with anti-HBO1 and $\beta$-Actin antibodies. $\beta$ Actin was used as loading control. (B) Protein extracts of UWB1.289 individual colonies that did not show HBO1 expression in dot-blot were analysed by western blot and probed with anti-HBO1 and anti-GAPDH antibodies. GAPDH was used as loading control. 
Protein lysates were isolated from 48 individual UWB1.289 colonies and analysed using protein dot-blotting to determine the presence or absence of HBO1 protein, a process exemplified in Figure 4.6 A. Four out of forty-eight colonies expressed $\beta$ actin but not HBO1 (e.g. Fig. 4.6 A: Colony 4). These samples (I-IV) were analysed further by western blot and relative densities (RD) were calculated considering HBO1 signal of colony I as the control sample $(\mathrm{RD}=100 \%)$ (Fig. 4.6 B). Even though HBO1 signal was null when dot-blotting individual colonies I-IV, western blot displayed different $\mathrm{HBO} 1$ protein levels, ranging from almost absent (III: RD = $1.93 \%)$, to very low (IV: $\mathrm{RD}=7.04 \%)$ and low (II: $\mathrm{RD}=25.26 \%)($ Fig. 4.6 B).

A

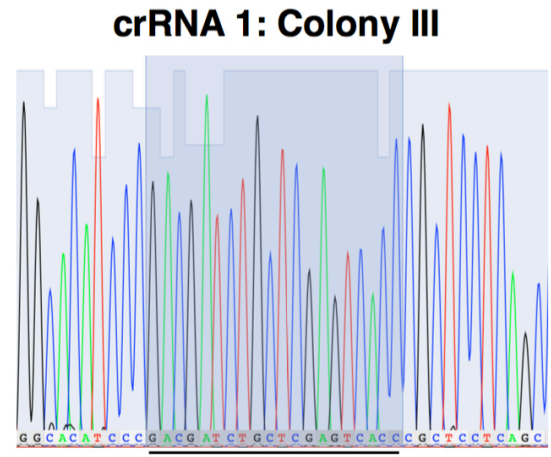

B

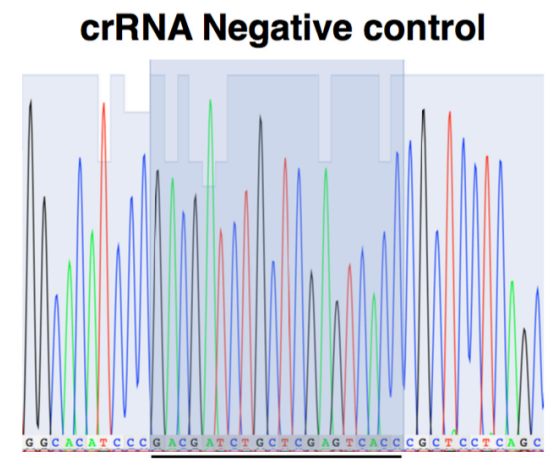

C crRNA 1 Target region

Control AAGTGATGGCACATCCCGACGATCTGCTCGAGTCACCCGCTCCTCAGCCAGGCTAAGC Colony I AAGTGATGGCACATCCCGACGATCTGCTCGAGTCACCCGCTCCTCAGCCAGGCTAAGC Colony II AaGTGATGGCACATCCCGACGATCTGCTCGAGTCACCCGCTCCTCAGCCAGGCTAAGC Colony III AAGTGATGGCACATCCCGACGATCTGCTCGAGTCACCCGCTCCTCAGCCAGGCTAAGC Colony IV AAGTGATGGCACATCCCGACGATCTGCTCGAGTCACCCGCTCCTCAGCCAGGCTAAGC

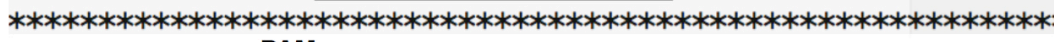
PAM

\section{Figure 4. 7 Sequencing analysis of the targeted HBO1 locus}

Genomic DNA samples of individual UWB1.289 colonies were amplified using endpoint PCR and the product of this reaction was sent for DNA sequencing. The sequencing chromatograms represent the nucleotide composition of the crRNA 1 target region of: (A) the individual colony III and (B) a negative control colony. (C) The sequences of 4 individual colonies and the negative control were aligned using the Clustal Omega software. PAM = Protospacer Adjacent Motif.

Conflicting results between dot-blot and western blot experiments may have occurred for various reasons, such as incomplete separation of individual colonies (mixture of two genetic backgrounds) or haplo-insufficiency (one mutated allele). Genomic DNA samples of individual UWB1.289 colonies I-IV were analysed using 
end-point PCR to amplify the $H B O 1$ locus target region, which was then sent to SeqWright (GE Healthcare) for DNA sequencing together with crRNA negativegenomic DNA as non-mutated $\mathrm{HBOl}$ locus sequence control.

Very surprisingly, sequencing of crRNA 1-target regions within the $\mathrm{HBOl}$ locus, the potential site of INDEL introduction, revealed no differences between the control and the so-called $\mathrm{HBOI} \mathrm{KO}$ colonies (I-IV) (Fig. 4.7). Further analysis of the 4Peaks trace viewer revealed that every peak in the sequence chromatogram was a single nucleotide, rejecting any possibility of heterozygotic mutations (Figs. 4.7 A, B). These results imply that no genomic mutation was introduced into the HBOl locus, yet the presence of TE7I mismatches in crRNA-treated gDNA samples (Fig. 4.5) and the absence of HBO1 protein observed in both dot-blot and western blot experiments (Figs. 4.6) indicated otherwise.

Multiple sequence alignment of the samples revealed no differences between the control and the putative $\mathrm{HBOl} \mathrm{KO}$ colonies (I-IV) in an area of approximately 600 nucleotides surrounding the predicted crRNA 1 mutation site, which showed that any off-target mutations that resulted in the loss of HBO1 expression must be at a distal location. However, as the T7EI digestion of crRNA 1-targeted gDNA samples produced 3 DNA bands matching the expected sizes obtained as a result of the introduction of small INDELs in the target $\mathrm{HBOl}$ locus (Fig. 4.5), it is unlikely that mutations have occurred off-target, since this would have resulted in different sized DNA bands after T7EI digestion. Even so, further testing may include the sequencing of the entire $\mathrm{HBOl}$ gene to certify the absence of off-target mutations.

As the presence of T7EI-cleaved bands (Fig. 4.5) and the absence of HBO1 protein expression in dot-blot (Fig. 4.6 A) and western blot experiments (Fig. 4.6 B), suggest the existence of UWB1.289 KO cells, it is possible that the DNA sequencing was erroneous, or that samples were contaminated with wild type cells. 


\subsection{Transduction of shRNAs to Constitutively Knockdown HBO1}

Given the conflicting results obtained with the CRISPR approach and in order to generate a stable HBO1 KD model, a lentiviral approach was undertaken using particles encompassing shRNAs targeting HBOl. Three different lentiviral shRNAs targeting HBOl (shHBO1 1-3) and a non-targeting control (shCtrl) were used.

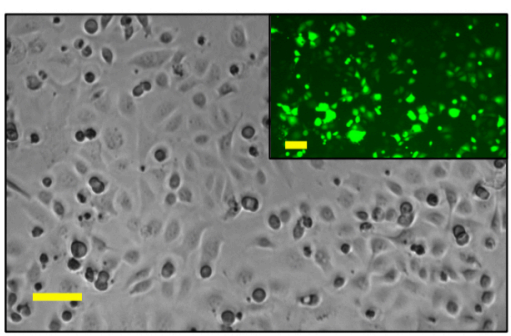

shHB01 1

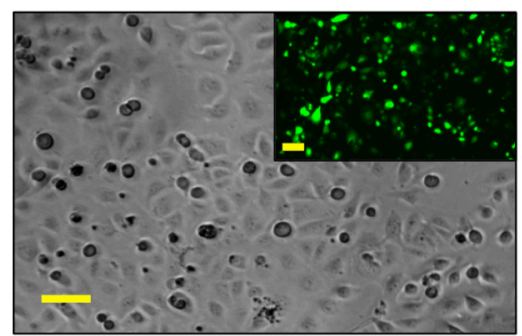

shCtrl

Figure 4. 8 Lentiviral transduction of shRNAs into UWB1.289 cells

UWB1.289 cells were transduced with lentiviral vectors expressing 'shHBO1 1' and non-targeting shRNA ('shCtrl'). Images were taken $72 \mathrm{~h}$ after the transduction using a fluorescent microscope. Scales represent $100 \mu \mathrm{m}$.

The transduction of either 'shHBO1 1-3' or 'shCtrl' in UWB1.289 cells was successful, as can be observed in Figure 4.8, where the majority of transduced cells express GFP protein. Furthermore, none of the populations displayed obvious changes in cell morphology, suggesting that the viruses have no overall effect. To assess the efficiency of lentivirus-delivered shRNA molecules ('shHBO1 1-3', 'shCtrl'), whole cell lysates were analysed by qRT-PCR (Fig. 4.9).

UWB1.289 cells constitutively expressing shRNAs targeting HBOI displayed significant reduction of $\mathrm{HBOl}$ transcript compared to the 'shCtrl'-transduced cells: shHBO1 $1\left(\mathrm{FC}=-7.16,{ }^{* *} \mathrm{p}=0.0014\right), \operatorname{shHBO} 12\left(\mathrm{FC}=-2.71,{ }^{*} \mathrm{p}=0.0261\right)$, $\operatorname{shHBO} 3\left(\mathrm{FC}=-1.87,{ }^{*} \mathrm{p}=0.0483\right)$ (Fig. 4.9). 


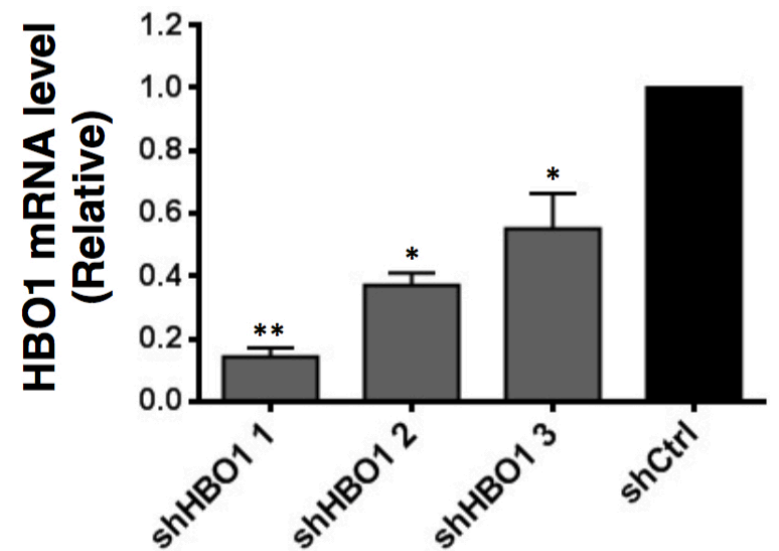

Figure 4. 9 HBO1 mRNA relative levels in transduced UWB1.289 cells

UWB1.289 cells were transduced with 'shHBO1 1-3' targeting HBOl and nontargeting shRNA ('shCtrl'). RNA samples were prepared and analysed by qRT-PCR. Relative levels of $\mathrm{HBOl}$ expression in cells expressing 'shHBO1 1-3' were compared to those of cells expressing 'shCtrl'. GAPDH expression was used as control. Statistical analysis consisted of one-way ANOVA and the Tukey's multiple comparison test. All values represent the mean \pm SD of three biological samples $(* \mathrm{p}$ $\left.<0.05 ;{ }^{* *} \mathrm{p}<0.01\right)$.

As with $\mathrm{HBO1}$ transcript expression, $\mathrm{HBO} 1$ protein levels were significantly lowered following 'shHBO1' lentiviral infection compared to the 'shCtrl' control: shHBO1 $1\left(\mathrm{RD}=6.33 \pm 4.96 \%,{ }^{* *} \mathrm{p}=0.0025\right), \operatorname{shHBO} 12(\mathrm{RD}=25.2 \pm 10.97 \%$, $* p=0.0383), \operatorname{shHBO} 13(\mathrm{RD}=58.93 \pm 20.38 \%, \mathrm{p}=0.0836)($ Fig. 4.10).

A

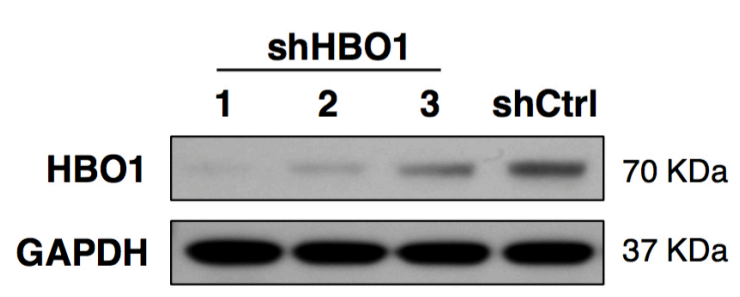

B

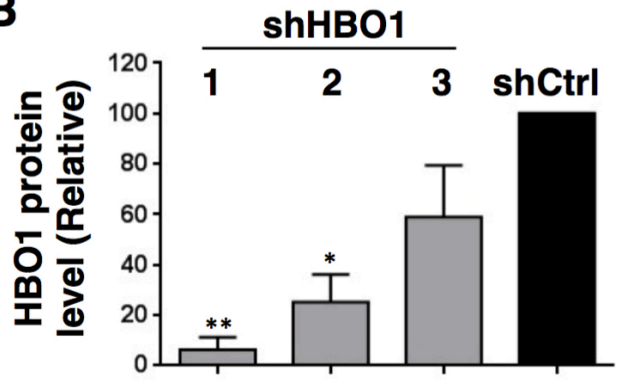

Figure 4. 10 HBO1 protein relative levels in transduced UWB1.289 cells

UWB1.289 cells were transduced with 'shHBO1 1-3' targeting $H B O 1$ and nontargeting shRNA ('shCtrl'). (A) Protein extracts were analysed by western blot and probed with anti-HBO1 and anti-GAPDH antibodies. (B) The relative level of HBO1 protein expression was calculated using ImageJ. GAPDH was used as loading control. Statistical analysis consisted of one-way ANOVA and the Tukey's multiple comparison test. All values represent the mean \pm SD of three biological samples $\left({ }^{*} p\right.$ $<0.05 ; * * \mathrm{p}<0.01)$. 


\subsection{Delivery of siRNAs to Transiently Knockdown HBO1}

As well as achieving a stable KD phenotype via shRNA expression, an RNAi approach to transiently deplete HBO1 cellular levels was also implemented. To achieve this, siRNAs targeting $H B O 1$ were exogenously introduced into five OC cell lines, including OVCAR-3, UWB1.289, SKOV-3, TOV-112D and UACC-1598. A pool of 4 commercial siRNAs targeting HBOl ('siHBO1') was purchased and utilized to accomplish robust and specific HBO1 KD. Using a pool of siRNAs over individual duplexes gives several advantages such as whole-gene KD (as opposed to partial KD, which may lead to the expression of non-targeted isoforms), increased phenotypic penetrance and higher efficiency (Parsons et al. 2009). Importantly, the use of several siRNAs guaranteed that all $H B O 1$ mRNA transcript isoforms were targeted for degradation (Fig. 2.8).

Two different experimental controls were included alongside each $\mathrm{HBO} 1 \mathrm{KD}$ to ensure that any effects observed were due to $H B O 1$ mRNA degradation, rather than off-target effects ('siCtrl') and/or the mere presence of exogenous RNA ('Control') in the cell. A pool of non-specific siRNAs was used as 'siCtrl' negative control, while the 'Control' cells were only treated with the transfection reagent.

The serous-like OVCAR-3 was initially selected for transient KD of HBO1. This was due to it representing a HGSC cell line model (Domcke et al. 2013; Beaufort et al. 2014), its easy handling and the moderate-to-high HBO1 expression levels (Fig. 3.1). In addition, OVCAR-3 KD transfection conditions had already been determined for the Dharmacon ${ }^{\circledR}$ siRNA transfection system used.

\subsubsection{Optimization of the siRNA delivery to OC cells}

To determine the post-transfection time point at which both mRNA and protein levels were lowest, whole-cell lysates from OVCAR-3 cells transfected with 'siHBO1', 'siCtrl' and 'Control' were harvested $24 \mathrm{~h}, 48 \mathrm{~h}$ and $72 \mathrm{~h}$ post-transfection for analysis. Whole-cell lysates were then analysed for $H B O 1$ mRNA levels by qRT-PCR (Fig. 4.11). 
A

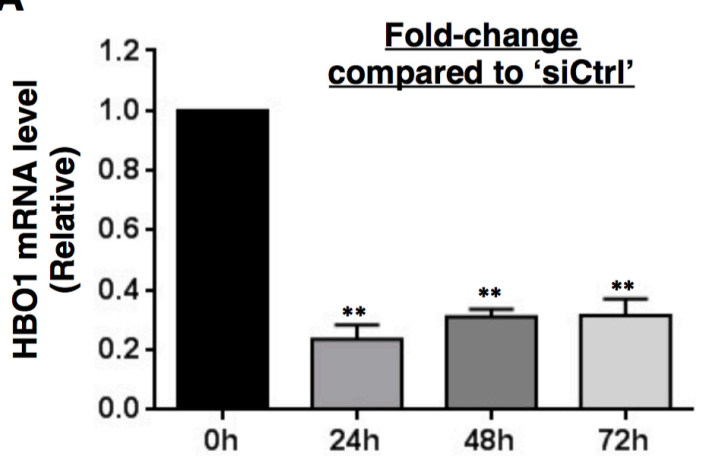

B

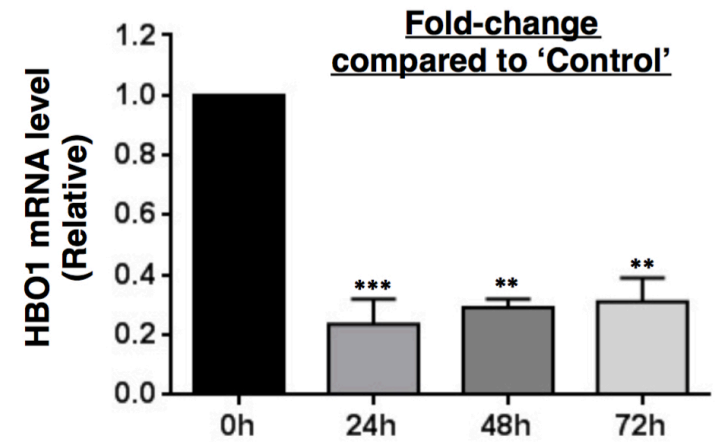

Figure 4. 11 HBO1 mRNA relative levels following siRNA KD in OVCAR-3 OVCAR-3 cells were transfected with a pool of siRNAs targeting $\mathrm{HBOI}$ ('siHBO1'), as well as with non-targeting siRNAs ('siCtrl') and transfection reagent alone ('Control'). Cell lysates were extracted after 24, 48 and 72h whereupon RNA samples were prepared and analysed by qRT-PCR. Relative levels of $H B O 1$ expression in cells treated with 'siHBO1' were compared to those of cells treated with: (A) 'siCtrl' and (B) 'Control'. RPL5 expression was used as control. Statistical analyses consisted of one-way ANOVA and Tukey's multiple comparison tests. All values represent the mean $\pm \mathrm{SD}$ of three biological samples $\left({ }^{*} \mathrm{p}<0.05 ;{ }^{* *} \mathrm{p}<0.01\right)$.

The transcript abundance of $\mathrm{HBOl}$ was lowest $24 \mathrm{~h}$ after the transfection with 'siHBO1', compared to the 'siCtrl' sample control ( $\left.\mathrm{FC}=-4.94,{ }^{* *} \mathrm{p}=0.0078\right)$ (Fig. $4.11 \mathrm{~A}) . \mathrm{HBO1} \mathrm{mRNA}$ levels remained reduced at $48 \mathrm{~h}\left(\mathrm{FC}=-3.21,{ }^{* *} \mathrm{p}=0.0025\right)$ and $72 \mathrm{~h}\left(\mathrm{FC}=-3.23,{ }^{* *} \mathrm{p}=0.0026\right)$ post-transfection $($ Fig. $4.11 \mathrm{~A})$. A similar decrease in $\mathrm{HBO}$ transcript abundance was observed when 'siHBO1' was compared with the un-transfected 'Control' samples (Fig. 4.11 B). Here, HBOI transcript levels remained low at $24 \mathrm{~h}\left(\mathrm{FC}=-4.67,{ }^{* * *} \mathrm{p}=0.0002\right), 48 \mathrm{~h}\left(\mathrm{FC}=-3.45,{ }^{* *} \mathrm{p}=\right.$ $0.0012)$ and $72 \mathrm{~h}\left(\mathrm{FC}=-3.34,{ }^{* *} \mathrm{p}=0.0016\right)$ post-transfection.

Whole-cell lysates of 'siHBO1' and 'siCtrl' samples extracted 24, 48 and 72h posttransfection were then analysed by western blot. Protein extracts were separated using gel electrophoresis gradient gels (4-12\%), following transfer to PVDF membranes, and were probed with anti-HBO1 (AB70) and anti-GAPDH antibodies (Fig. 4.12). The levels of $\mathrm{HBO} 1$ protein decreased after $24 \mathrm{~h}(\mathrm{RD}=40.64 \pm 10.12 \%$, $\mathrm{p}=0.0642), 48 \mathrm{~h}\left(\mathrm{RD}=26.47 \pm 6.87 \%,{ }^{*} \mathrm{p}=0.0382\right)$ and $72 \mathrm{~h}(\mathrm{RD}=37.84 \pm 8.59$ $\left.\%,{ }^{*} \mathrm{p}=0.0368\right)$ of siRNA transfection (Fig. 4.12), demonstrating the effectiveness of the siRNA approach. 


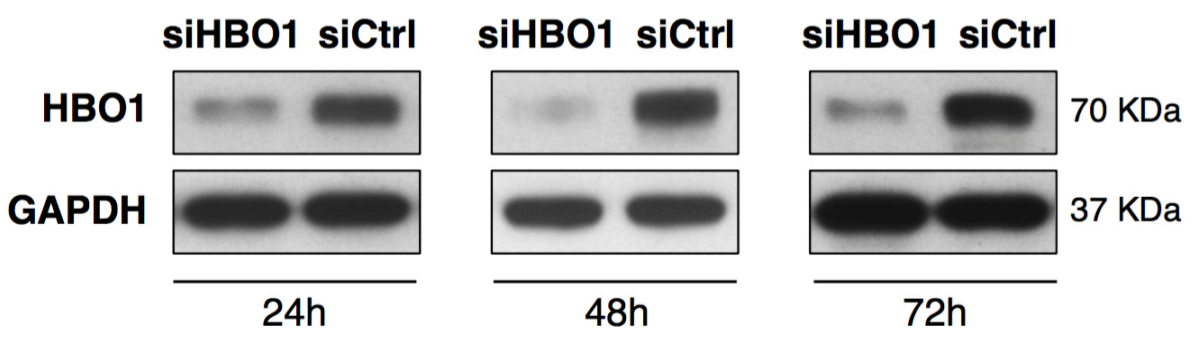

Figure 4. 12 HBO1 protein relative levels following siRNA KD in OVCAR-3

OVCAR-3 cells were transfected with a pool of siRNAs targeting HBOl ('siHBO1') and non-targeting siRNAs ('siCtrl') and cell lysates were extracted 24, 48 and 72 hours post-transfection. Protein extracts were analysed by western blot and probed with anti-HBO1 and anti-GAPDH antibodies. GAPDH was used as loading control.

Interestingly, the maximal reduction of $\mathrm{HBO} 1$ protein was obtained $48 \mathrm{~h}$ after the transfection of 'siHBO1' (Fig. 4.12), suggesting that protein turnover occurs more slowly than mRNA degradation. The reduction of HBO1 both at transcript and protein level was strong and followed a similar pattern when HBO1 fold-changes (FC) were calculated as percentages (Fig. 4.13).

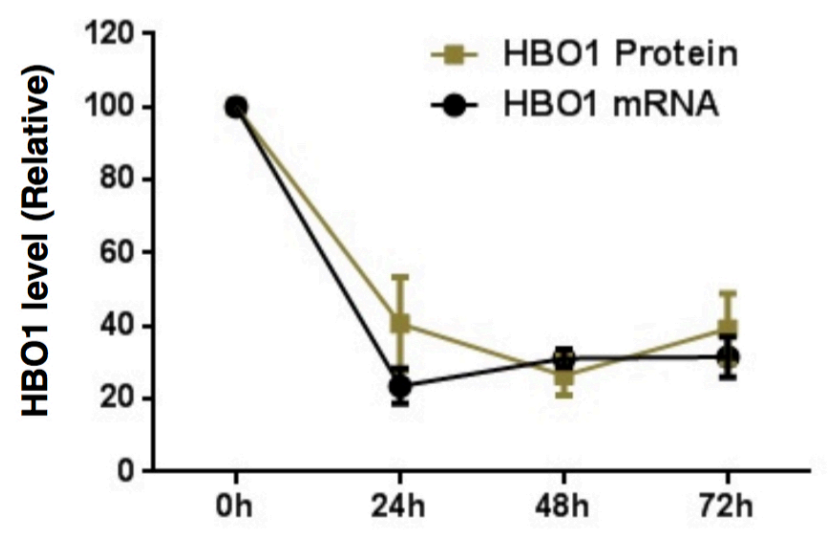

Figure 4. 13 Time-course analysis of HBO1 levels following siRNA KD

OVCAR-3 cells were transfected with a pool of siRNAs targeting HBOl ('siHBO1'), and non-targeting siRNAs ('siCtrl'). Cell lysates were extracted after 24, 48 and 72 hours whereupon RNA and protein samples were prepared and analysed by qRT-PCR and western blot respectively. Relative levels of HBO1 mRNA and protein expression in cells treated with 'siHBO1' were compared to those of cells treated with 'siCtrl'. Fold-changes were transformed into proportional percentages to fit the comparisons. 


\subsubsection{Delivery of siRNAs to OC cell lines}

Small interfering RNA molecules were also delivered to UWB1.289 and SKOV-3 OC cell lines, expressing high and intermediate-high mRNA and protein HBO1 levels respectively (Figs. $3.1 \&$ 3.6). UWB1.289 and SKOV-3 cells were transfected with 'siHBO1' alongside with the two control samples, 'siCtrl' and 'Control'. Whole-cell lysates were then harvested after $48 \mathrm{~h}$ and analysed by qRT-PCR and western blot to confirm HBO1 knockdown both at transcript and protein levels (Fig. 4.14).

A

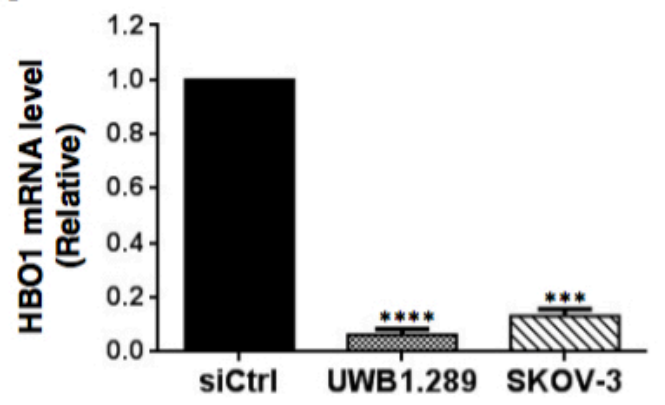

B

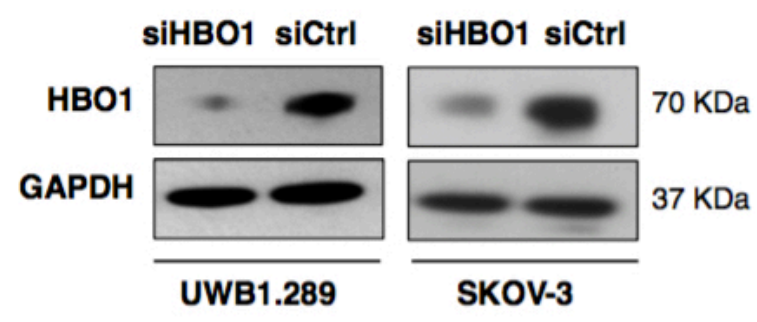

Figure 4. 14 HBO1 relative levels following siRNA KD in OC cell lines

UWB1.289 and SKOV-3 cells were transfected with a pool of siRNAs targeting HBOl ('siHBO1') and non-targeting siRNAs ('siCtrl'). Cell lysates were extracted after $48 \mathrm{~h}$ whereupon RNA and protein samples were prepared and analysed by: (A) qRT-PCR and (B) western blot respectively. Relative levels of $\mathrm{HBOl}$ expression in cells treated with 'siHBO1' were compared to those of cells treated with 'siCtrl'. Statistical analysis consisted of one-way ANOVA and the Tukey's multiple comparison test. All values represent the mean \pm SD of three biological samples $(* * * p<0.001 ; * * * * p<0.0001)$. PVDF membranes were probed with anti-HBO1 and anti-GAPDH antibodies. GAPDH expression was used as control in both experiments.

HBO1 mRNA abundance decreased significantly in both UWB1.289 (FC $=-15.65$, $* * * * \mathrm{p}<0.0001)$ and SKOV-3 $\left(\mathrm{FC}=-8.02,{ }^{* * *} \mathrm{p}=0.0004\right)$ cell lines after the treatment with 'siHBO1' compared to the 'siCtrl' negative control sample (Fig. 4.14 A). Similarly, protein levels of UWB1.289 $\left(\mathrm{RD}=23.10 \pm 7.67 \%,{ }^{*} \mathrm{p}=\right.$ $0.0112)$ and SKOV-3 $\left(\mathrm{RD}=34.64 \pm 4.95 \%,{ }^{*} \mathrm{p}=0.0225\right)$ were significantly reduced, demonstrating the ability of siRNA to knockdown HBO1 in two different OC cell backgrounds (Fig. 4.14 B). 
In addition, siRNAs were also used to transfect the OC cell lines UACC-1598 (FC $\left.=-2.18 \pm 0.59,{ }^{* *} \mathrm{p}=0.0027\right)$ and TOV-112D $(\mathrm{FC}=-4.87 \pm 1.63, * * * \mathrm{p}<0.0001)$ (not shown). The successful delivery of siRNAs targeting HBOl in OVCAR-3, UWB1.289, SKOV-3, UACC-1598 and TOV-112D provided a crucial tool for the study of HBO1 functions in OC.

\subsubsection{Validation of siRNA efficiency}

One of the possible origins of siRNA off-target effects is the use of a siRNA pool instead of individual, more specific siRNAs. This assumption is based on the fact that the greater the number of delivered siRNAs, the higher the mismatch tolerance of the pool, as well as the higher possibilities of interfering with endogenous RNAi pathways. In order to assess this, the four individual RNAs that constitute the siRNA pool were purchased independently and used to transfect UWB1.289 cells separately (Fig. 4.15).
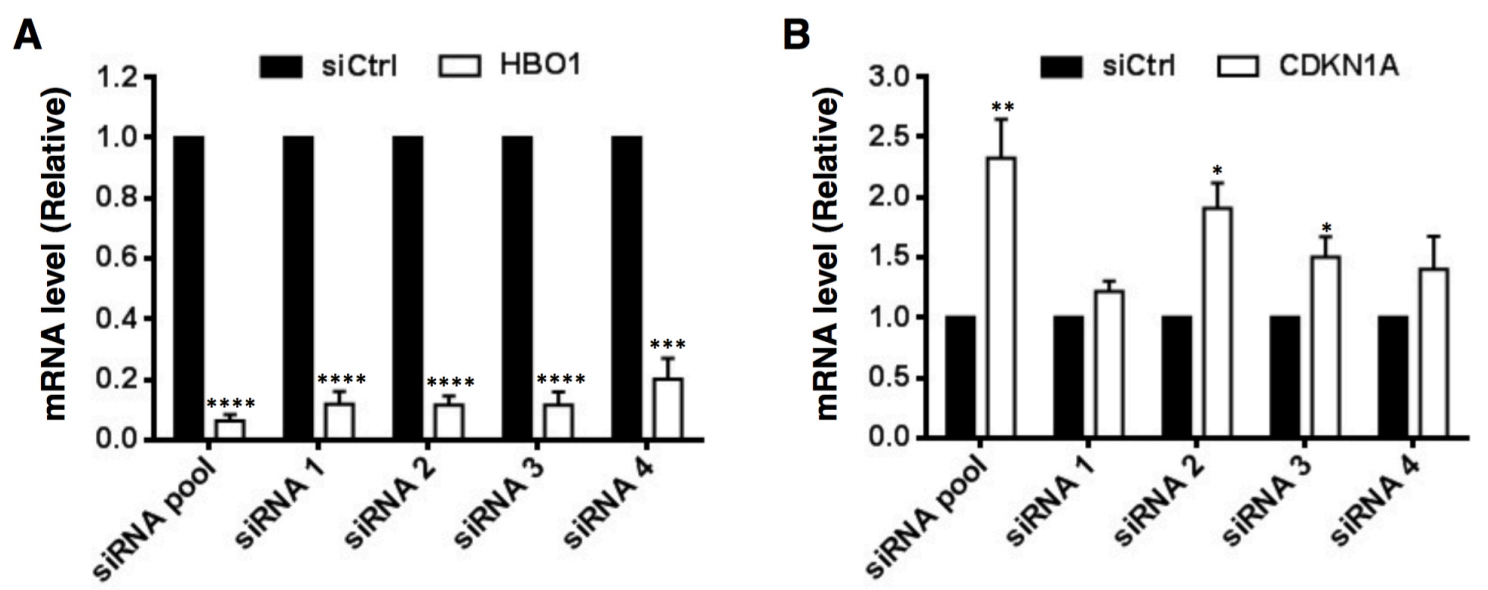

Figure 4. 15 Validation of HBO1 KD using individual siRNAs

UWB1.289 cells were transfected with individual siRNAs targeting $\mathrm{HBO1}$, a pool of siRNAs targeting $\mathrm{HBOl}$ and a non-targeting pool ('siCtrl'). Cell lysates were extracted after $48 \mathrm{~h}$ whereupon RNA samples were prepared and analysed by qRTPCR. Relative levels of $H B O 1$ and $C D K N 1 A$ expression in cells treated with 'siHBO1' were compared to those of cells treated with 'siCtrl'. GAPDH expression was used as control. Statistical analyses consisted of one-way ANOVA and Tukey's multiple comparison tests. All values represent the mean $\pm \mathrm{SD}$ of three biological samples $\left({ }^{*} \mathrm{p}<0.05 ;{ }^{* *} \mathrm{p}<0.01 ;{ }^{* * *} \mathrm{p}<0.001,{ }^{* * * *} \mathrm{p}<0.0001\right)$. 
The transfection of single siRNAs comprised in the siRNA pool led to the significant down-regulation of HBO1 in UWB1.289 cells, compared to the 'siCtrl' control: siRNA $1(\mathrm{FC}=-9.3, * * * * \mathrm{p}=<0.0001)$, siRNA $2(\mathrm{FC}=-9.01, * * * * \mathrm{p}=<0.0001)$, siRNA $3(\mathrm{FC}=-9.63, * * * * \mathrm{p}=<0.0001)$ and siRNA $4(\mathrm{FC}=-5.39, * * * \mathrm{p}=0.0002)$

(Fig. 4.15 A). To validate specific siRNA efficiency, $C D K N 1 A / p 21$, an HBO1-target previously identified in HBO1 KD experiments with HeLa cells (Avvakumov et al. 2012), was analysed by qRT-PCR (Fig. 4.15 B).

CDKN1A was significantly up-regulated when transfecting UWB1.289 cells with individual siRNAs $2\left(\mathrm{FC}=1.90,{ }^{*} \mathrm{p}=0.025\right)$ and $3\left(\mathrm{FC}=1.5,{ }^{*} \mathrm{p}=0.0375\right)$ (Fig. 4.15 B). In the case of transfection with individual siRNAs $1(\mathrm{FC}=1.21, \mathrm{p}=0.0981)$ and $4(\mathrm{FC}=1.4, \mathrm{p}=0.0985)$, even though the results were not statistically significant under the established parameters for qRT-PCR $(\mathrm{p}<0.05)$, the displayed p-values were situated above the $90 \%$ of confidence level $(\mathrm{p}<0.1)$ and the expression change followed an up-regulatory trend, suggesting a strong and specific modulation of HBO1. Altogether, these results confirm the efficiency of individual siRNAs to KD HBO1 and elicit durable gene expression changes.

\subsection{Gene Expressing Vector: a Tool to Up-regulate HBO1 Levels}

Theoretically, an increase in HBO1 cellular levels could induce the opposite effect to HBO1 silencing and reverse prior KD-related events if utilized subsequent to HBO1 KD. To evaluate this, a pCMV-Tag2B-hHBOl expression plasmid was constructed. A mutated version of this plasmid, pCMV-Tag2B- $h H B O 1(G 485 A)$ was also constructed to investigate whether potential effect modulation is linked to HBO1's intrinsic acetyltransferase activity. Lastly, a pCMV-Tag2B-Ø empty plasmid was constructed to use as a control.

OVCAR-3 cells, whose plasmid transfection efficiency had been previously optimized when trying to obtain an HBO1 KO (not shown), were selected to test the transfection of the $\mathrm{HBOl}$-expression plasmid. In this case, the X-tremeGENETM 9 (Roche) provided the best transfection conditions (low cytotoxicity and high GFP expression, data not shown). Consequently, different concentrations of pCMV- 
Tag2B-hHBOl (0.1-1-10-50 ng/mL) were transfected into OVCAR-3 cells using XtremeGENETM 9. Twenty-four hours post-transfection, mRNA and whole-cell protein lysates were isolated and analysed (Fig. 4.16).

A

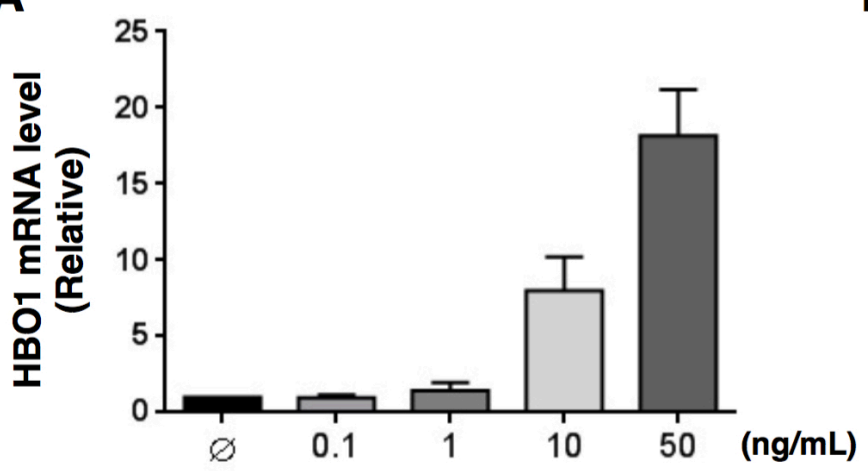

B

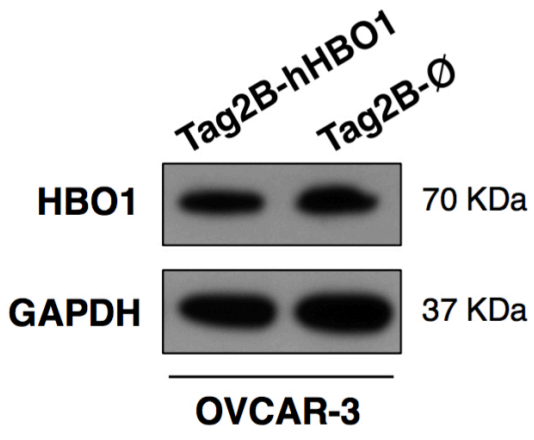

Figure 4. 16 Transfection of an HBO1-expressing vector into OVCAR-3 cells (A) OVCAR-3 cells were transfected with pCMV-Tag2B-hHBOl and the empty pCMV-Tag2B- $\varnothing$ plasmid in different concentrations [0.1-1-10-50 ng/mL]. After 24h, RNA samples were prepared and analysed by qRT-PCR. Relative levels of HBOl expression in Tag2B-hHBOl-transfected cells were compared to those of cells transfected with the empty plasmid. All values represent the mean \pm SD of 2 biological replicates. (B) Protein extracts of OVCAR-3 transfected with Tag2B$h H B O 1$ and Tag2B- $\varnothing[10 \mathrm{ng} / \mathrm{mL}]$ were analysed by western blot and probed with anti-HBO1 and anti-GAPDH antibodies. GAPDH expression was used as control in both experiments.

Low plasmid concentrations, $0.1 \mathrm{ng} / \mathrm{mL}(\mathrm{FC}=0.96 \pm 0.19)$ and $1 \mathrm{ng} / \mathrm{mL}(\mathrm{FC}=1.45$ \pm 0.53 ), did not increase $H B O 1$ expression (Fig. 4.16 A). Transfection of $10 \mathrm{ng} / \mathrm{mL}$ of $H B O 1$-expressing plasmid in OVCAR-3 cells led to a convenient $7.1 \pm 2.1$ foldchange increase in $H B O 1$ mRNA (Fig. 4.16 A). Very high plasmid concentrations [50 ng/mL] resulted in large increases in $H B O 1 \mathrm{mRNA}$ levels $(\mathrm{FC}=18.17 \pm 3.01)$ (Fig. 4.16 A), which were considered too high to study the effect of $H B O 1 \mathrm{mRNA}$ at physiological levels and were not used further.

In order to investigate whether increased $\mathrm{HBO} 1$ transcript levels led to an increase in HBO1 protein levels, whole-cell lysates of 24h-transfected OVCAR-3 cells [10 $\mathrm{ng} / \mathrm{mL}]$ were analysed by western blot (Fig. 4.16 B). The incubation with anti-HBO1 AB70 antibody showed that although the quantity of mRNA increased in the samples transfected with $H B O 1$-expression plasmid compared to the empty control, 
protein levels remained invariable $(\mathrm{RD}=95.1 \pm 6.52)($ Fig. 4.16 B $)$. A similar result was also obtained when OVCAR-3 cells were transfected for 48h (data not shown). These results suggest that the plasmid mRNA is not translated to protein, perhaps due to mistakes during the cloning process. Alternatively, HBO1 levels could be tightly regulated to prevent over-accumulation of the protein to either impede abnormal translation of $\mathrm{HBOl}$ or promote rapid protein turnover.

A

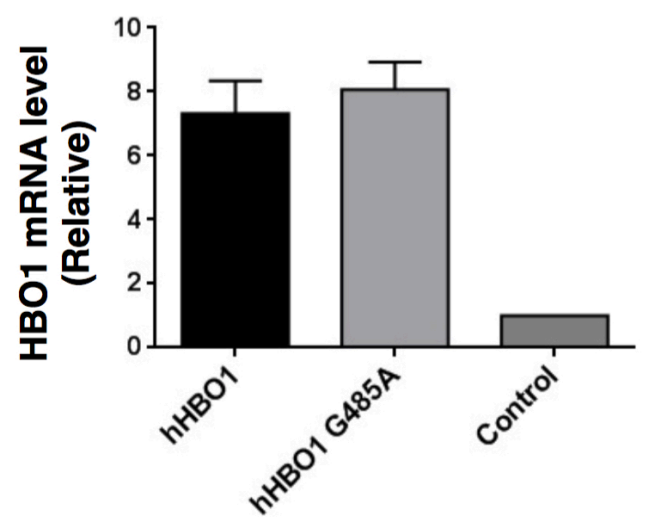

B

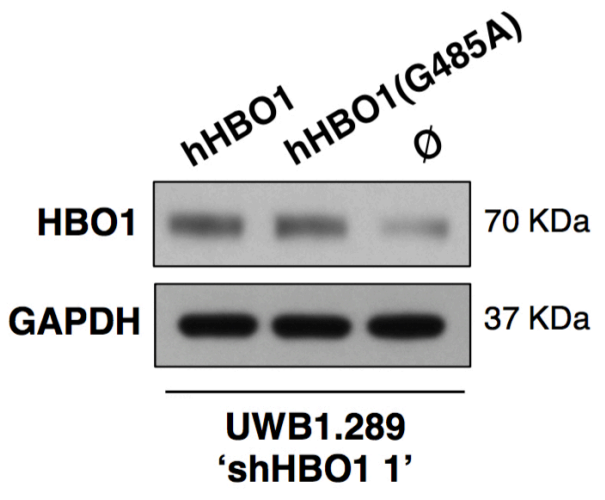

Figure 4. 17 Ectopic expression of HBO1 in shRNA-transduced UWB1.289 cells UWB1.289 cells expressing 'shHBO1 1' were transfected with $10 \mathrm{ng} / \mathrm{mL}$ of $\mathrm{pCMV}$ Tag2B-hHBO1, pCMV-Tag2B-hHBOl(G485A) and the empty pCMV-Tag2B-Ø plasmid. After $24 \mathrm{~h}, \mathrm{RNA}$ and protein extracts were analysed by: (A) qRT-PCR and (B) western blot respectively. Relative levels of $\mathrm{HBO} 1$ expression in Tag2B-hHBO1 and Tag2B-hHBOl(G485A)-transfected cells were compared to those of cells transfected with the empty plasmid. All values represent the mean \pm SD of 2 biological replicates. Protein extracts were probed with anti-HBO1 and anti-GAPDH antibodies. GAPDH expression was used as control.

To further assess the feasibility of the $\mathrm{HBOl}$-expressing vector, the pCMV-Tag2BhHBO1 plasmid, alongside with its mutated variant and the empty control were transfected into UWB1.289 cells that constitutively express 'shHBO1 1' (Fig. 4.17). The introduction of $10 \mathrm{ng} / \mathrm{mL}$ of pCMV-Tag2B-hHBOl and pCMV-Tag2B$h H B O 1(G 485 A)$ plasmids increased $\mathrm{HBO1}$ transcript levels $(\underline{\mathrm{hHOO}}$ : $\mathrm{FC}=7.32 \pm$ $1.03 ; \underline{h H O 1(G 845 A):} \mathrm{FC}=8.07 \pm 0.86)$. HBO1 protein levels were similarly increased following transfection with either plasmid $(\underline{h H B O 1}$ : $\mathrm{RD}=156 \pm 28.4 \%$;

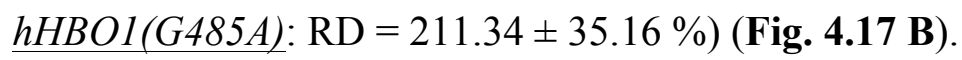




\subsection{Discussion}

In this chapter, three tools were developed in order to (a) constitutively KD HBO1 in the UWB1.289 cell line (b) transiently KD HBO1 in a variety of OC cell lines and (c) up-regulate the expression of $\mathrm{HBO} 1$ via the introduction of an $\mathrm{HBO}$-expressing vector. These strategies are then used, in later chapters, to analyse the functional roles of HBO1 in these cells.

Genome editing of the $\mathrm{HBOI}$ locus using the CRISPR-Cas9 system was attempted in order to generate an UWB1.289 cell line that did not express HBO1. After a number of optimization steps, including efforts to infect OC cells with adenoviruses expressing Cas9 and transfection of a Cas9-expression plasmid using either common transfection reagents (Fig. 4.2) or electroporation procedures (Fig. 4.3), electroporation using the Nucleofector ${ }^{\mathrm{TM}}$ system (Programme A020) proved successful (Fig. 4.4). This achievement was followed by the observation of successful T7EI band digestion (Fig. 4.5), confirming INDEL events, after the addition of crRNA:tracrRNA complexes targeting the $\mathrm{HBOl}$ locus. Moreover, further colony isolation rendered several UWB1.289 colonies allegedly devoid of HBO1 protein expression (Fig. 4.6). Notwithstanding, DNA sequencing of target locus gDNA belonging to these UWB1.289 colonies did not display appreciable INDELs (Fig. 4.7).

The inability to isolate individual clones carrying detectable INDELs, even though a number of experiments seem to indicate otherwise, could be due to a number of possibilities summarized in Table 4.2. Among all these options, and based on the knowledge and experience acquired in the development of this methodology, we favour the fact that the experiment did not achieve INDEL introduction in the $\mathrm{HBOI}$ locus. According to this hypothesis, the absence of $\mathrm{HBO} 1$ protein expression, both in DB and WB experiments (Fig. 4.6), could be explained as an artefact, consequence of the single cell cloning procedure. Since UWB1.289 cells were submitted to an abnormal type of growth, that is, without neighbouring cells, this may affect their development, thus enabling genomic and non-genomic alterations in order to achieve adaptation. As a consequence of these changes, it may happen that these cells reduce HBO1 expression, e.g. via promoter methylation, as suggested in Table 4.2. 
Low levels of Cas9 protein expression in electroporated-UWB1.289 cells (Fig. 4.4) and the poor cleavage efficiency obtained in enzymatic surveyor assays (Fig. 4.5) further support this hypothesis. In addition, the ability of CRISPR-Cas9 to edit mammalian genes is known to vary widely, with some studies reporting varying efficiency in the range of $1 \%$ to $79 \%$ (Duda et al. 2014; Swiech et al. 2014; Liang et al. 2015), highlighting the complications currently associated with this approach, which appear to be cell line specific.

Table 4. 2 List of possible outcomes of the CRISPR-Cas9 genome editing

\begin{tabular}{|c|c|}
\hline Hypotheses & Controversies \\
\hline 1. There was no INDEL introduction. & $\begin{array}{l}\text { Positive T7EI digestion and negative } \\
\text { HBO1 signal (dot-blot, western blot). }\end{array}$ \\
\hline $\begin{array}{l}\text { 2. There was no INDEL introduction. } \\
\text { However, the clonal isolation selected a } \\
\text { highly } H B O 1 \text {-methylated-promoter } \\
\text { clone. }\end{array}$ & Positive T7EI digestion. \\
\hline $\begin{array}{l}\text { 3. INDELs were introduced in the } \\
\text { expected } H B O 1 \text { locus. However, } \\
\text { isolated } \mathrm{KO} \text { colonies died because } \\
\mathrm{HBOl} \text { is an essential gene in OC cells. }\end{array}$ & $\begin{array}{l}\text { Negative HBO1 signal of isolated } \\
\text { colonies (dot-blot, western blot). }\end{array}$ \\
\hline $\begin{array}{l}\text { 4. INDELs were introduced in the } \\
\text { expected location within the } H B O 1 \\
\text { locus. However, the isolation of } \\
\text { individuals was inaccurate and more } \\
\text { than one clone co-existed or cell- } \\
\text { specific ploidy confers the cell several } \\
\text { copies of the } H B O 1 \text { locus. }\end{array}$ & \multirow[t]{2}{*}{$\begin{array}{l}\text { Sequencing chromatograms are clear and } \\
\text { do not suggest mixed populations or } \\
\text { heterozygosis. }\end{array}$} \\
\hline $\begin{array}{l}\text { 5. INDELs were introduced in the } \\
\text { expected location within the } H B O 1 \\
\text { locus in only one allele. }\end{array}$ & \\
\hline $\begin{array}{l}\text { 6. INDELs were introduced in off-target } \\
\text { locations within the } H B O 1 \text { locus. }\end{array}$ & $\begin{array}{l}\text { TE7I digestion displayed predicted sized } \\
\text { bands and sequencing chromatograms } \\
\text { covered as much as } 600 \text { base pairs of the } \\
H B O 1 \text { locus }\left(\sim 30 \% \text { of } H B O 1 \text { 's ORF }^{1}\right) \text {. }\end{array}$ \\
\hline $\begin{array}{l}\text { 7. INDELs were introduced in off-target } \\
\text { locations outside the } H B O l \text { locus. }\end{array}$ & $\begin{array}{l}\text { Negative HBO1 signal of isolated } \\
\text { colonies (dot-blot, western blot). }\end{array}$ \\
\hline $\begin{array}{l}\text { 8. INDELs were introduced in the } \\
\text { expected location within the } H B O 1 \\
\text { locus. However, the DNA sequencing } \\
\text { procedure was erroneous. }\end{array}$ & \\
\hline
\end{tabular}

${ }^{1} \mathrm{ORF}=$ Open Reading Frame. 
A different approach, based on the expression of shRNAs, was developed to constitutively reduce cellular levels of HBO1. The successful integration of lentiviral particles carrying shRNA structures ('shHBO1 1-3' and 'shCtrl') in UWB1.289 cells was confirmed via fluorescent microscopy (Fig. 4.8). Furthermore, the expression of shRNAs and the subsequent reduction in HBO1 mRNA and protein levels when compared with cells infected with the 'shCtrl' control was confirmed for three different 'shHBO1'-transduced cells (1-3) (Fig. 4.9 \& Fig. 4.10).

Another tool based on the use of RNAi, in this case the delivery of siRNAs to OC cells to reduce HBO1 expression, was also successful. This approach resulted in the transient KD of HBO1 in OVCAR-3, UWB1.289, SKOV-3, UACC-1598 and TOV112D OC cell lines (Fig. 4.11 \& Fig. 4.14), providing valuable tools for the study of HBO1 function in OC. The decrease of HBO1 expression in OVCAR-3 cells, both at mRNA and protein level, was monitored for 24, 48 and 72 hours (Fig. 4.12) and was most effective at 48 hours post-delivery of siRNAs. Whilst previous shRNA-based experiments evaluated cellular changes promoted in the long-term absence of HBO1, this methodology allows the assessment of transient deficiencies. Together, these approaches will enable the functions of $\mathrm{HBO} 1$ in $\mathrm{OC}$ to be further characterised.

In order to increase HBO1 levels, a pCMV-Tag2B- $h H B O 1$ expression plasmid was utilised. Although the transfection of the vector resulted in a significant fold increment of HBO1 mRNA levels in OVCAR-3 cells, protein quantities were not altered (Fig. 4.16). This may be due to a number of reasons, including an inefficient translation of $H B O 1$ mRNAs, a short mRNA lifetime, or a quick degradation of newly synthesised HBO1 protein, preventing its detection by immune blot. These effects could be related to defects in plasmid construction, but may also stem from specific cellular responses. Accordingly, it is possible that UWB1.289 cells may exert some form of regulation at the translational level or with PTMs, with the objective of maintaining the protein expression levels of this important HAT under control. In a similar study, however, where the pCMV-Tag2B-hHBO1 plasmid was transfected into stable HBO1 KD UWB1.289 cells, the levels of HBO1 protein were significantly increased (Fig. 4.17). These findings suggest that the plasmid is actually capable of synthesizing $\mathrm{HBO}$ transcripts that translate into protein under certain conditions. The question remains as to whether this protein is active, capable 
Results

of folding correctly, receive the appropriate PTMs and work in a coordinated way with the rest of complex components to perform its functions. 


\section{Functional HBO1 Evaluation}

using a Stable RNAi-based Knockdown System 


\subsection{Introduction and Aims}

$\mathrm{HBO} 1$ is reported to have multiple and diverse tissue specific roles that are attributed to its involvement in major cellular processes such as DNA replication and transcription (Doyon et al. 2006; Kueh et al. 2011). For example based on previous KD studies (Table 1.7), one of the most obvious features of HBO1 is its ability to modulate cell proliferation in certain cellular backgrounds e.g. 293T embryonic kidney cells or MLE-12 murine lung cells (Doyon et al. 2006; Zou et al. 2013). In order to investigate the particular roles of $\mathrm{HBO} 1$ in OC, the effect of constitutive $\mathrm{KD}$ using lentivirus-infected UWB1.289 cells was investigated.

Loss or gain of HAT activity is obviously reflected in the pattern of histone modifications, and several histone mark signatures have been associated with cancerous phenotypes e.g. reduction in histone $\mathrm{H} 4 \mathrm{~K} 16$ acetylation is thought to be a general hallmark of cancer cells (Fraga et al. 2005). Accurate screening of the changes of these marks could reveal tissue status and help predict the clinical outcome of a tumour (Di Cerbo \& Schneider 2013). Accordingly, many studies have proposed general prognostic rules for a diverse panel of histone marks in different cancers types, including prostate (Seligson et al. 2005) and renal (Mosashvilli et al. 2010) cancers. In ovarian tumours, the decrease of H3K9 acetylation has been linked with tumour progression (Zhen et al. 2010), a result that follows the established tendency to relate global loss of histone marks with a poorer prognosis (Kurdistani 2011). HBO1 has been previously linked to histone $\mathrm{H} 4$ (K5, K8, K12 and K16) and histone $\mathrm{H} 3 \mathrm{~K} 14$ acetylation, whereas no significant association has been established with other H3 residues, histone H2A or H2B (Iizuka et al. 2009; Kueh et al. 2011). Based on these previous observations, the effect of reducing HBO1 levels in OC cell lines was analysed for changes in bulk histone $\mathrm{H} 4$ and H3K14 acetylation status.

In order to evaluate changes in gene mRNA expression, RNA samples from UWB1.289 cells expressing either non-targeting shRNAs or shRNAs targeting HBOI were sequenced (RNA-seq). Statistical analysis of the outcome resulted in a list of significantly up- and down-regulated transcripts, which, after compelled qRTPCR validation, were considered as candidate HBO1-target genes. The resultant gene list was further analysed to identify any cellular pathways governed or 
influenced by HBO1 using Ingenuity Pathway Analysis ${ }^{\circledR}$ (IPA) (Krämer et al. 2014). Finally, the results of the in silico pathway analysis were tested in vitro to confirm any role of $\mathrm{HBO} 1$ in predicted pathways. The combination of pathway analysis and in vitro confirmation of predicted roles contributed to understanding the functions of $\mathrm{HBO} 1$ in $\mathrm{OC}$.

\subsection{HBO1 Histone Acetylation Function}

The main nucleosome-substrates of HBO1 acetylation are histone $\mathrm{H} 4$ and histone H3K14 (Doyon et al. 2006; Iizuka et al. 2009; Kueh et al. 2011; Mishima et al. 2011). Thus, as a consequence of stable HBO1 KD and therefore the reduction of acetyltransferase activity in OC cells, broad acetylation of histone $\mathrm{H} 4$ and specific acetylation of histone H3K14 would be anticipated to decline. In order to test these hypotheses, protein extracts from UWB1.289 cells expressing 'shHBO1' 1-3 and 'shCtrl' were analysed using acetylation-specific antibodies (Fig. 5.1).

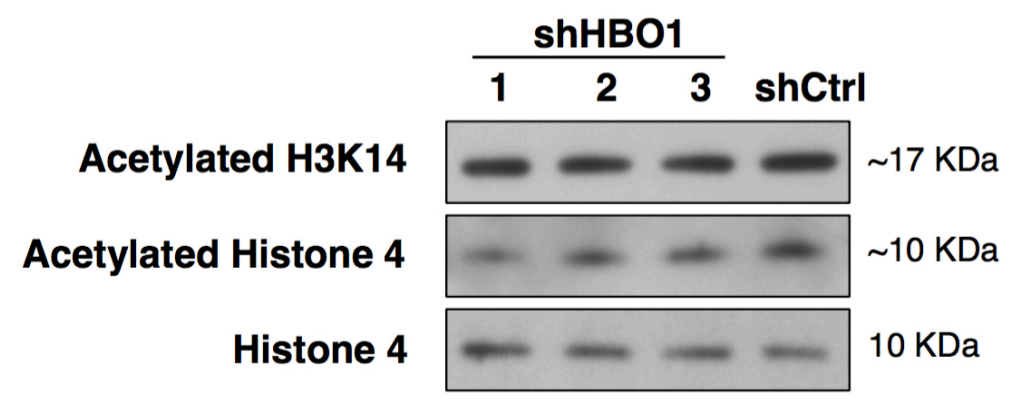

Figure 5. 1 Histone acetylation patterns after stable HBO1 KD

UWB1.289 cells were transduced with 'shHBO1 1-3' targeting HBOl and nontargeting shRNA ('shCtrl'). Protein lysates were analysed by western blot and probed with anti-acetylated H3K14, anti-acetylated histone $4(\mathrm{H} 4)$ and anti-H4 antibodies. H4 was used as loading control.

The constitutive delivery of shHBO1 $1\left(\mathrm{RD}=59.53 \pm 7.59 \%,{ }^{*} \mathrm{p}=0.0437\right)$ and 2 $\left(\mathrm{FC}=72.91 \pm 8.82 \%,{ }^{*} \mathrm{p}=0.0489\right)$ resulted in significant reductions in bulk histone H4 acetylation compared to the parental OC cell line, demonstrating that H4 is a major HBO1 target in these tumour derived cells (Fig. 5.1 \& Fig. 5.2). ShHBO1 3 $(\mathrm{RD}=93.77 \pm 6.87 \%, \mathrm{p}=0.3980)$ did not cause any significant changes in 
acetylation levels of $\mathrm{H} 4$, probably due to $\mathrm{HBO} 1$ expression levels being reduced by less than 40\%, meaning functional HBO1 was still present in cells (Fig. 5.1 \& Fig. 5.2). In contrast, histone $\mathrm{H} 3 \mathrm{~K} 14$ acetylation levels did not display significant changes with any of the shHBO1 constructs that were tested: shHBO1 1 (RD = $94.37 \pm 7.15 \%, \mathrm{p}=0.8752)$, shHBO1 $2(\mathrm{RD}=92.67 \pm 8.36 \%, \mathrm{p}=0.8608)$, shHBO1 $3(\mathrm{RD}=87.77 \pm 6.16 \%, \mathrm{p}=0.9255)$ (Fig. 5.1 \& Fig. 5.2). These data suggest $\mathrm{HBO} 1$ specific regulation of $\mathrm{H} 4$ acetylation but not $\mathrm{H} 3 \mathrm{~K} 14$ acetylation in UWB1.289 cells.

A

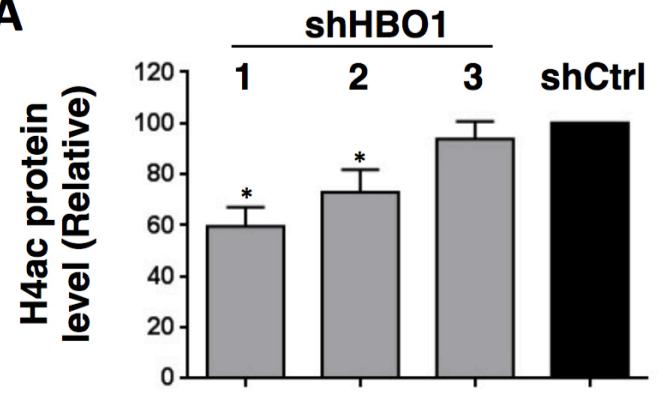

B

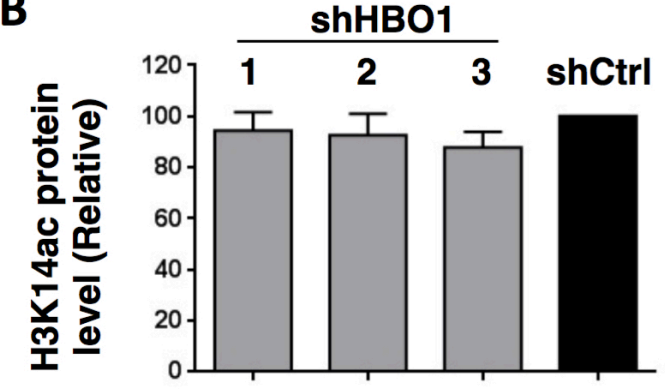

Figure 5. 2 Histone acetylation relative levels after stable HBO1 KD

UWB1.289 cells were transduced with 'shHBO1 1-3' targeting $\mathrm{HBOl}$ and nontargeting shRNA ('shCtrl'). The relative levels of acetylated $\mathrm{H} 4$ (H4ac) and acetylated H3K14 (H3K14ac) protein expression were calculated using ImageJ; H4 was used as loading control. Statistical analyses consisted of one-way ANOVA and Tukey's multiple comparison tests. All values represent the mean $\pm \mathrm{SD}$ of three biological samples $(* \mathrm{p}<0.05)$.

In line with these findings, the ability of HBO1 to acetylate histone 4 substrates was used to evaluate the activity of HBO1 protein synthesized by the pCMV-Tag2BhHBOl expression plasmid (Fig. 5.3). The increase in HBO1 protein levels that were measured following transfection of pCMV-Tag2B-hHBOl and pCMV-Tag2BhHBOl (G485A) plasmids into transduced-UWB1.289 cells was not accompanied with significant changes in $\mathrm{H} 4$ acetylation (hHBO1: $\mathrm{FC}=71.47 \pm 20.48$; hHBO1 (G485A): $\mathrm{FC}=68.15 \pm 20.61)$ (Fig. 5.3). These results suggest that HBO1 protein synthesized by the $H B O 1$-expressing vector is not active at cellular level, and therefore cannot perform regular $\mathrm{HBO} 1$ functions. 
A

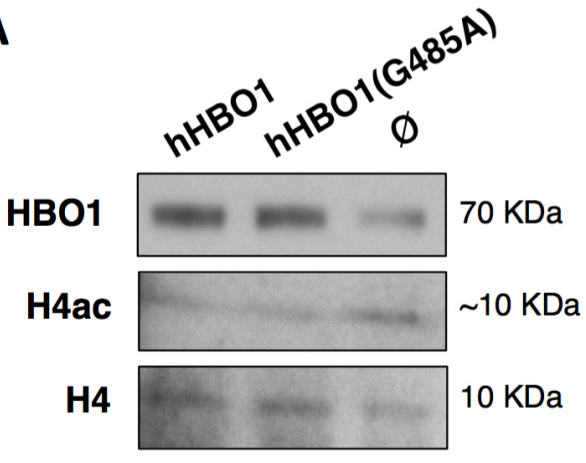

B

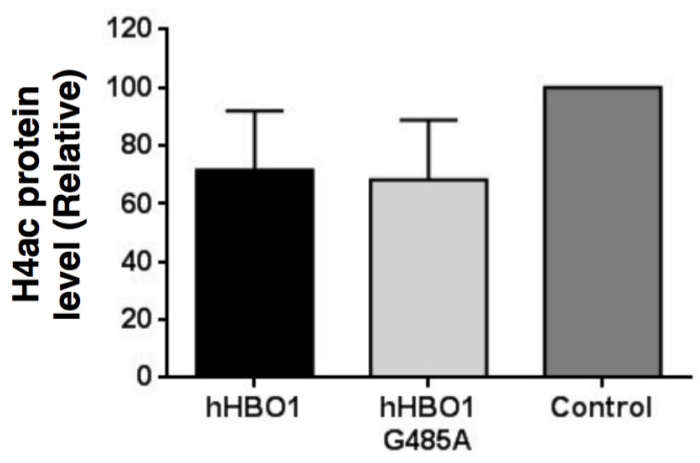

Figure 5. 3 Histone acetylation patterns linked to HBO1 ectopic expression UWB1.289 cells expressing 'shHBO1 1' were transfected with $10 \mathrm{ng} / \mathrm{mL}$ of $\mathrm{pCMV}$ Tag2B-hHBO1, pCMV-Tag2B-hHBO1 (G485A) and the empty pCMV-Tag2B-Ø plasmid. (A) After 24h, protein extracts were analysed by western blot and probed with anti-HBO1, anti-acetylated $\mathrm{H} 4$ and anti-H4 antibodies. (B) The relative level of $\mathrm{H} 4 \mathrm{ac}$ protein expression was calculated using ImageJ; H4 expression was used as control.

\subsection{Gene Expression Profiling of Transduced UWB1.289 Cells}

The transcriptional targets of HBO1 in UWB1.289 cells at a global gene expression level were determined using RNA-seq analysis by comparing transcript profiles from native and HBO1 KD cells. RNA-seq enables rapid transcriptome profiling and can detect novel transcripts and splicing isoforms, features that are not measurable in gene profiles performed by microarray. Sequencing yielded a total of 58,215 different transcripts in each and every one of the samples examined ( $3 \mathrm{KD}$ and 3 control), whose expression was quantified and compared to determine significantly modified transcripts. Data analysis identified a list of 68 differentially expressed genes $(\mathrm{FC} \pm 1.5 ;$ q-value $<0.05)$, including 30 up-regulated and 38 down-regulated gene transcripts (Table 5.1). The low numbers of HBO1-regulated targets point to a highly specific role of HBO1 in UWB1.289 cells.

HBOl isoforms $1\left(\mathrm{FC}=-3.38, \mathrm{q}\right.$-value $\left.=1.14 \times 10^{-45}\right)$ and $2(\mathrm{FC}=-2.73, \mathrm{q}$-value $=$ $1.59 \times 10^{-06}$ ) were detected amongst the 38 down-regulated gene transcripts, confirming the success of the experimental approach. Even though isoforms 3, 4 and 5 were detected amongst the 58,215 identified transcripts, their effective count, which could be interpreted as expression level, was very low compared to isoforms 1 
and 2, confirming previous observations that suggested the predominance of isoforms 1 and 2 in the pool of HBOl transcripts (Fig. 3.4). Furthermore, isoforms 3, 4 and 5 did not display significant differences between samples.

Table 5. 1 List of altered transcripts after constitutive KD of HBO1

Up-regulated transcripts (30) are listed in descending order, with the 'histone deacetylase 6' (HDAC6) displaying the highest FC (2.48). Down-regulated transcripts (38) are listed in ascending order, with MYST2 (1) displaying the lowest FC (-3.38). Corresponding isoform numbers are represented in brackets.

\begin{tabular}{|c|c|c|c|}
\hline \multicolumn{2}{|c|}{ Up-regulated transcripts (30) } & \multicolumn{2}{|c|}{ Down-regulated transcripts (38) } \\
\hline HDAC6 & DCTN1 & MYST2 (1) & IAH1 \\
CEP131 & SLIT2 & MYST2 (2) & FBXL12 \\
MAMLD1 & TPRKB & PRDM2 & EPB4 \\
SNAP47 & HDHD3 & TFRC & GAA \\
SEC14L1 & PPPIR12A & AP351 & UBALD1 \\
DAZAP2 & STAT6 & COL26A1 & PPHLN1 \\
PPP6R3 & FAM43A & MED15 & PCID2 (2) \\
SMG5 & PKIB & GUCD1 & ZNF562 \\
ZNF133 & FOXQ1 & GBGT1 & NPERL1 \\
SNX10 & REST & PGAP2 & FAM111A \\
PDE2A & VIM & PMS2 & ALG9 \\
PDP2 & & FAM114A1 & SUGP2 \\
DDAH1 & & ADAL & PCID2 (1) \\
FGDS-AS1 & & NAT9 & SF1 \\
ARHGAP44 & & ZNF426 & PXRD3 \\
NCAPG & & CRCP & FBLN1 \\
CDK8 & & PHTF2 & P3H2 \\
POLRIB & & ZNF12 & PCM1 \\
PGAP1 & & C19orf66 & DSN1 \\
\hline
\end{tabular}

In order to validate these results, three of the top up and down-regulated targets were analysed using qRT-PCR, including the 'histone deacetylase 6' (HDAC6), the 'synaptosome associated protein 47' (SNAP47), the 'signal transducer and activator of transcription 6' (STAT6), 'periocentriolar material 1' (PCM1), PMS2 and the 'transferrin receptor' (TFRC) (Fig. 5.4).

Up-regulated gene transcripts identified in the RNA-seq: $H D A C 6\left(\mathrm{FC}=1.62,{ }^{*} \mathrm{p}=\right.$ 0.0365), SNAP47 $(\mathrm{FC}=1.59, \mathrm{p}=0.0642)$ and STAT6 $\left(\mathrm{FC}=1.62,{ }^{*} \mathrm{p}=0.0455\right)$ were similarly up-regulated when analysed by qRT-PCR (Fig. 5.4 A). Conversely, downregulated gene transcripts identified in the RNA-seq: PCMI $(\mathrm{FC}=-1.26, \mathrm{p}=$ 
0.0576), PMS2 (FC $\left.=-1.27,{ }^{*} \mathrm{p}=0.0388\right), \operatorname{TFRC}\left(\mathrm{FC}=-1.33,{ }^{*} \mathrm{p}=0.0644\right)$ were similarly down-regulated when analysed by qRT-PCR (Fig. 5.4 B). All genes selected to validate the expression profile of stable HBO1 KD in UWB1.289 cells displayed similar values in both the qRT-PCR experiments and the RNA-seq analysis, suggesting that the 68 altered genes are direct or indirect targets of the effects of constitutive HBO1 KD.

A

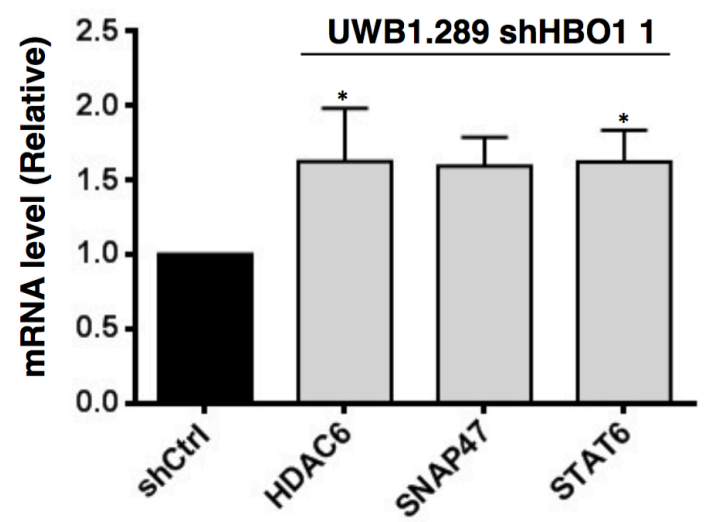

B

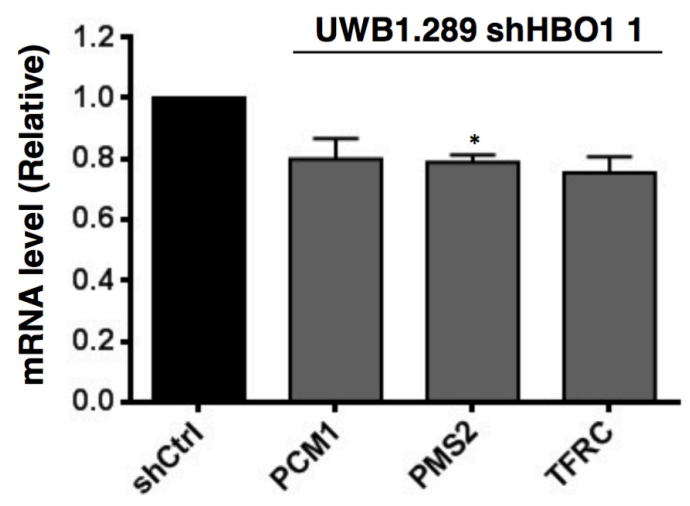

Figure 5. 4 Validation of gene expression changes caused by stable HBO1 KD UWB1.289 cells were transduced with 'shHBO1 1' and non-targeting shRNA ('shCtrl'). RNA samples were prepared and analysed by qRT-PCR to confirm the: (A) up-regulation of HDAC6, SNAP47, STAT6 and (B) the down-regulation of PCM1, PMS2 and TFRC. Relative levels of expression in cells expressing 'shHBO1 1 ' were compared to those of cells expressing 'shCtrl'. RPL5 expression was used as control. Statistical analyses consisted of one-way ANOVA and Tukey's multiple comparison tests. All values represent the mean \pm SD of three biological samples $\left({ }^{*} \mathrm{p}\right.$ $<0.05)$.

\subsection{Pathway Analysis of the Stable HBO1 Knockdown Profile}

In order to identify biological pathways affected by constitutive $\mathrm{KD}$ of $\mathrm{HBO} 1$ in UWB1.289 cells, the list of differentially expressed genes was analysed using the pathway-prediction programme IPA. HBO1 KD in UWB1.289 cells was found to cause no significant alterations of pathway activity using the 'Canonical pathways' feature of IPA, probably due to an insufficient number of differentially expressed genes (68). However, the IPA feature 'Downstream effects' identified several biological functions activated upon HBO1 depletion (Table 5.2). 
Table 5. 2 List of predicted IPA alterations following stable HBO1 KD

\begin{tabular}{|c|c|c|c|}
\hline Categories & Biological function & Prediction & Z-score \\
\hline Cellular organization & Formation of protrusions & Increased & 1.81 \\
Cellular organization & Organization of cytoplasm & Increased & 2.23 \\
Cellular organization & Microtubule dynamics & Increased & 2.24 \\
Cancer & Neoplasia of epithelial tissue & Increased & 2.41 \\
Cancer & Tumorigenesis of tissue & Increased & 2.41 \\
\hline
\end{tabular}

The highest ranked IPA predictions were related to the activation of cancerous functions, including an increment in the 'tumorigenesis of tissue' $\left(p=8.92 \times 10^{-03}\right)$ and 'neoplasia of epithelial tissue' $\left(\mathrm{p}=1.25 \times 10^{-02}\right)$ (Table 5.2). The predicted activation of oncogenic traits upon $\mathrm{HBO} 1$ depletion suggests a role for $\mathrm{HBO} 1$ in the inhibition of cancer-promoting pathways.

Additionally, IPA predicted an association with cellular organization: microtubule dynamics $\left(p=8.97 \times 10^{-04}\right)$, organization of cytoplasm $\left(p=1.46 \times 10^{-03}\right)$ and the formation of cellular protrusions $\left(p=2.68 \times 10^{-03}\right)$ (Table 5.2). These results suggest the involvement of HBO1 in the regulation of cellular assembly and organization processes, a feature that has not previously been reported. Furthermore, the formation of protrusions on the membrane surface, as well as the activation of microtubule dynamics, are commonly linked with cell locomotion (Xue et al. 2010; Kaverina \& Straube 2011), thus suggesting a role for HBO1 in cell movement and migration.

\subsection{In vitro Evaluation of HBO1-predicted Biological Functions}

The reported role of HBO1 in regulating cell proliferation (Doyon et al. 2006; Zou et al. 2013) motivated the first approach to better understand HBO1 functionality in OC, which was to evaluate the proliferative capacities exhibited by transduced UWB1.289 cells. Subsequently, different in vitro assays were undertaken in order to assess changes in cell motility and organization, both biological processes predicted to be activated following HBO1 KD in UWB1.289 cells according to IPA in silico analysis (Table 5.2). 


\subsubsection{Assessment of HBO1 suggested role in cell proliferation}

The essential role of HBO1 in DNA replication (Miotto \& Struhl 2010) suggested the prospect of proliferative changes as a result of constitutive HBO1 KD. To investigate this possibility, shRNA-transduced cells were subjected to proliferation assays, which measured the incorporation of a labelled nucleoside (5'-Bromo-2'deoxyuridine or BrdU) into newly synthesized DNA of replicating cells. Since DNA replication must happen before cell division, measurement of DNA synthesis is considered synonymous with measurement of cell proliferation (Madhavan 2007). BrdU incorporation was followed by monoclonal antibody binding (anti-BrdU) and immune-peroxidase identification of BrdU-labelled replicative cells (Fig. 5.5).

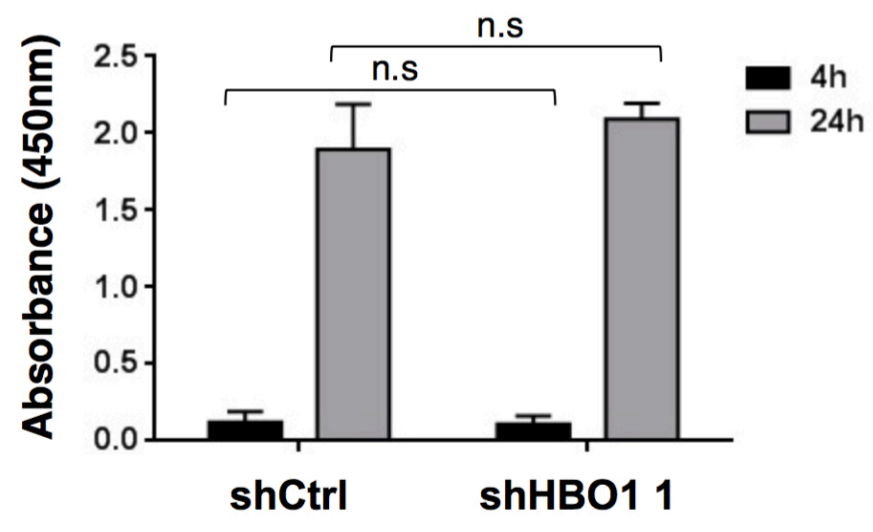

Figure 5. 5 Study of cell proliferation after constitutive HBO1 KD

UWB1.289 cells were transduced with 'shHBO1 1' and non-targeting shRNA ('shCtrl'). BrdU was added to the cells for $4 \mathrm{~h}$ and $24 \mathrm{~h}$ to assess cell proliferation. Statistical analysis consisted of one-way ANOVA and the Tukey's multiple comparison test. All values represent the mean \pm SD of three biological samples (n.s. not significant).

UWB1.289 cells were treated with BrdU for $4 \mathrm{~h}$ and 24h, in order to observe changes in DNA synthesis rates following reduction in HBO1 in OC cells. Analysis of final absorbance values revealed that HBO1 depletion did not appear to affect the replication process (Fig. 5.5), suggesting that HBO1 is not essential for DNA replication in OC cells and that its overexpression most likely do not function as a cancerous mechanism to enhance oncogenic cell proliferation. These results confirm IPA predictions, which did not identify cell proliferation changes as a consequence of $\mathrm{HBO} 1$ constitutive decline. 


\subsubsection{Assessment of HBO1 suggested involvement in cell motility}

In order to evaluate whether the predicted changes in cell organization (e.g. formation of protrusions) result in cell motility alterations, phenotypical changes were measured using a scratch/wound-healing assay, which measures cell migration in vitro (Fig. 5.6). This method is based on the observation that, upon creation of a new artificial gap, a so called 'scratch', on a confluent cell monolayer, the cells on the edge of the newly created gap will move toward the opening to close the 'scratch' until new cell-cell contacts are established again. If HBO1 was involved in the regulation of cell movement, UWB1.289-transduced cells would migrate differently based on the different levels of HBO1 expression.

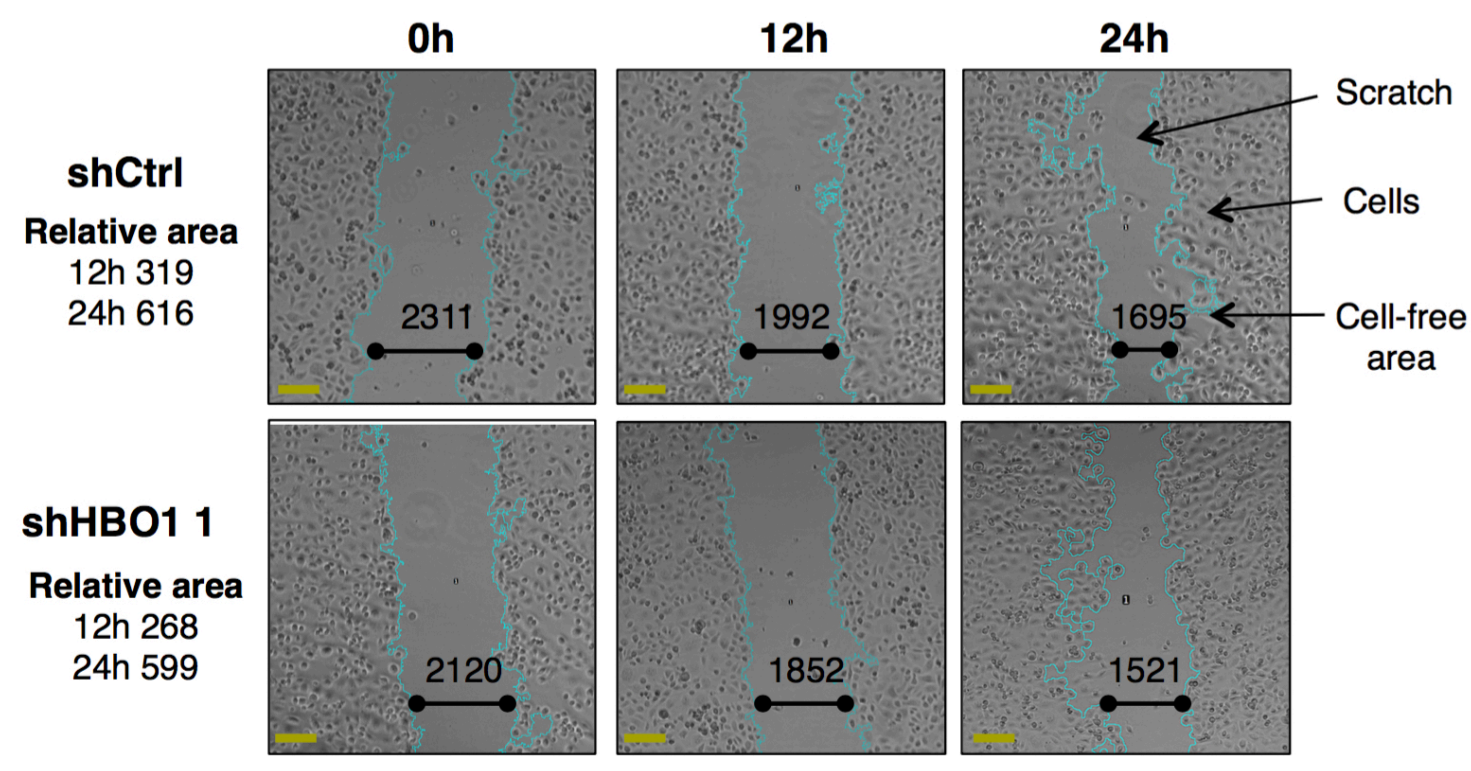

Figure 5. 6 Example of scratch assay with stable HBO1 KD UWB1.289 cells UWB1.289 cells were transduced with 'shHBO1 1' and non-targeting shRNA ('shCtrl'). After $48 \mathrm{~h}$, the confluent cell monolayer was partially removed by a pipette tip (scratch). Images were taken immediately after the scratch $(\mathrm{t}=0 \mathrm{~h})$ and after 12-24h; ImageJ software was used to calculate cell-free area values. The relative area was calculated subtracting cell-free area values at $12-24 \mathrm{~h}$ from cell-free area values at $\mathrm{t}=0 \mathrm{~h}$. Scales represent $100 \mu \mathrm{m}$.

Analysis of wound-healing data did not reveal any differences in scratch closure as determined by the relative areas (RA) of 'shHBO1 1' and 'shCtrl' transduced cells 12 or 24 hours after the scratch (Fig. 5.6). These results suggest that even though HBO1 depletion may enable cells to alter their internal sub-cellular organization as 
predicted in silico, in vitro changes in cell motility were not observed. Therefore, scratch assay results agree with predicted IPA biological alterations of stable KD, which did not anticipate changes in cell movement per se.

One common issue when performing wound-healing assays is to determine whether the observed changes are due to cell migration or cell proliferation. Typically, and in order to control interferences associated with cell propagation, target cells may undergo a period of serum-starvation prior to the assay, enabling cell cycle synchronisation. In this case however, proliferation rates do not change between samples (Fig. 5.5), so serum-starvation was considered unnecessary.

\subsubsection{Assessment of HBO1 suggested involvement in cell organization}

In order to evaluate the IPA predicted role for HBO1 in cellular organization, phenotypical changes were measured using Atomic Force Microscopy (AFM). AFM offers the possibility to perform force measurements at desired cellular locations, measuring mechanical properties of adherent cells and thus acting as a reliable indicator of the structure and function of the underlying cytoskeleton (Gavara 2017). Notably, AFM is emerging as a valuable tool for studying cellular mechanics because of its ability to maintain cell viability, so that living cells may be analysed in their culture medium without inducing irreversible damage.

The cell cytoskeleton is a dynamic network of three main fibrous structures, namely actin filaments, microtubules and intermediate filaments, each type specifically distributed inside the cell but intricately linked (Cooper 2000). It has a central role in cell structure and intracellular organization, and thus affects cell mechanical properties that ultimately dictate cell functionality (Fletcher \& Mullins 2010). Interestingly, oncogenic transformation not only leads to changes in cellular growth and differentiation, but also results in the modification of internal cytoskeleton structures (Jinka et al. 2012). Cell stiffness, the resistance of a single cell to an externally induced deformation, depends on the level of structure and organization of the cytoskeleton and can be measured using AFM, which delivers quantitative measurements of cellular elastic responses expressed as specific Young's modulus E (Lekka 2016). Additionally, the application of external forces through AFM enables 
the acquisition of quantitative information on the adhesion strength of living cells (Simon \& Durrieu 2006).

Sub-confluent populations of UWB1.289-transduced cells were analysed using AFM to measure mechanical cellular properties (on about 40 cells per cell type), including cell stiffness and cell adhesiveness (Fig. 5.7).

A

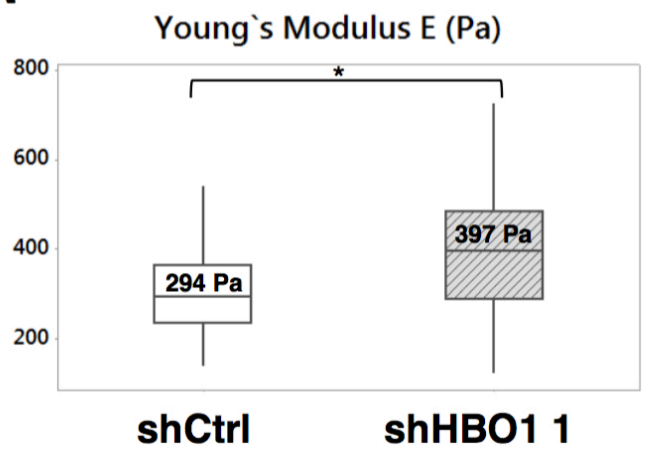

B

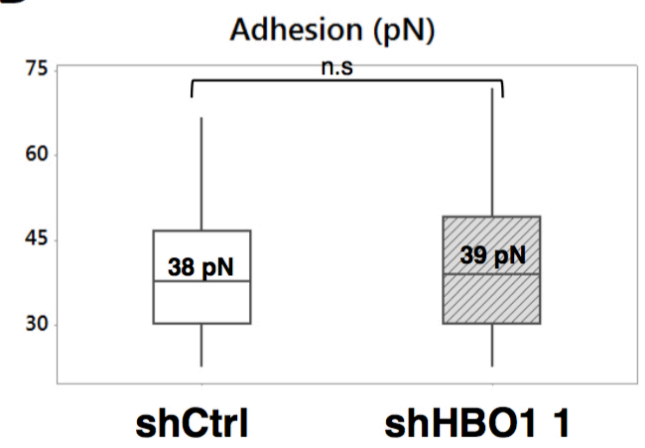

Figure 5. 7 Study of cell organization after stable HBO1 KD in UWB1.289 cells UWB1.289 cells were transduced with 'shHBO1 1' and non-targeting shRNA ('shCtrl'). After 24h, Atomic Force Microscopy (AFM) was used to measure: (A) cell stiffness and (B) cell adhesiveness of 40 transduced cells per type. All AFM values are expressed as median \pm interquartile range $\left({ }^{*} \mathrm{p}<0.05\right.$; n.s. not significant $)$. Pa: Pascal, pN: Piconewton.

As shown in Figure 5.7 A, UWB1.289 cells were significantly more elastic (294 Pa) than cells subjected to $\mathrm{HBO} 1 \mathrm{KD}$ (397 Pa), which were stiffer, indicating that UWB1.289 cells require HBO1 to retain their oncogenic sub-cellular phenotype. When cell surface adhesiveness was analysed, no significant difference was observed between the samples suggesting that HBO1 does not play a part in cell-cell adhesion (Fig. 5.7 B). These findings point to a role for HBO1 in cytoskeletal and/or cytoplasmic re-organization, which were altered following its depletion (Table 5.2). Accordingly, these results suggest a role for $\mathrm{HBO} 1$ promoting the disorganization of microtubules, thus stimulating less organised/differentiated and more elastic phenotypes in OC cells, a common characteristic of invasive tumour cells (Lekka 2016; Muroyama \& Lechler 2017). 


\subsection{Discussion}

In order to extend our knowledge about the roles of HBO1 in OC, UWB1.289 cells were transduced with shRNAs constitutively targeting $H B O 1$ to significantly reduce its expression, and subsequently monitored for gene and phenotypical alterations.

First, previously described nucleosome targets of HBO1 H4 and H3K14, identified in other cell systems including 293T embryonic kidney cells and K562 leukemic cells (Doyon et al. 2006; Mishima et al. 2011), were evaluated using acetylationspecific antibodies. Constitutive HBO1 KD resulted in the decrease of $\mathrm{H} 4$ acetylated residues (Fig. 5.1 \& Fig. 5.2), supporting a major role for HBO1 in maintaining histone $\mathrm{H} 4$ acetylation throughout the genome of UWB1.289 cells. These results agree with a number of previous studies performed with siRNA (Doyon et al. 2006) and shRNA approaches (Sauer et al. 2015; M. S. Kim et al. 2015) (Table 1.7). Notably, one nucleosome target commonly associated with cancerous phenotypes, H4K16 (Fraga et al. 2005), appears to be specifically modulated by HBO1 HAT activity. Still, further studies are required in order to pinpoint HBO1 specificity within the group of $\mathrm{H} 4$ acetylated residues targeted by the antibody that was used.

Constitutive HBO1 KD did not, however, affect H3K14 acetylation (Fig. 5.1 \& Fig. 5.2), in contraposition with several studies performed with siRNA (Zou et al. 2013), shRNA (Mishima et al. 2011; Kim et al. 2015) and KO mice (Kueh et al. 2011) (Table 1.7). These findings suggest that $\mathrm{H} 3 \mathrm{~K} 14$ is not a target of $\mathrm{HBO} 1$ acetylation in UWB1.289 cells. However, it is interesting to consider whether the preservation of H3K14 acetylation levels following HBO1 KD could be due to the action of other proteins that may compensate for an HBO1-related loss of H3K14 acetylation. This phenomenon could be derived from the activation/overexpression of different HATs, e.g. known regulators of H3K14 acetylation like Gcn5 (Kuo \& Andrews 2013) or p300/CBP (Henry et al. 2013), HAT complex members or other regulatory proteins. The RNA-seq transcriptome analysis revealed no alterations in the expression level of HATs or known members of HAT complexes ( $\mathrm{FC} \pm 1.3$, q-value $<0.25)$, and thus if this compensation were to occur, it would be most likely driven through nongenomic pathways (e.g. increased HAT activity). 
RNAseq analysis was used to determine gene expression changes elicited by HBO1 depletion and identified 68 altered genes (Table 5.1). The number of targets is surprisingly low, considering previous HBO1 KD studies (Avvakumov et al. 2012), which could be a consequence of a partial effect on altering gene regulation due to only a 40\% reduction in histone $\mathrm{H} 4$ acetylation (Fig. 5.1 \& Fig. 5.2). In contrast, Avvakumov et al. (2012) reported the identification of 782 HBO1 targets (397 upregulated and 385 down-regulated) following transient $\mathrm{KD}$ in HeLa cells. The differences in the number of targets identified in the experiment performed by Avvakumov et al. (2012) and the RNA-seq (782 as opposed to 68 altered genes) can be due to many factors, including cell-type specificity, the silencing approach (transient siRNA silencing versus constitutive shRNA expression) or the presence of compensatory effects. In this regard, previous studies have suggested that the upregulation of HAT complex components after HBO1 KD could compensate the effects of protein depletion, e.g. up-regulation of BRPF1 in HeLa cells after HBO1 KD could be due to loss of HBO1 activity being compensated for by the MOZ/MORF HAT complex (Avvakumov et al. 2012).

The list of differentially expressed genes was examined using SQL queries, which identified 3 potential OC-related target genes: 'slit guidance ligand 2' (SLIT2) $(\mathrm{FC}=$ 1.72 , q-value $\left.=7.84 \times 10^{-04}\right)$, the 'globoside alpha-1,3-N-acetyl-galactosaminyltransferase 1' $(G B G T 1)\left(\mathrm{FC}=-1.94, \mathrm{q}\right.$-value $\left.=1.46 \times 10^{-05}\right)$ and $P M S 2(\mathrm{FC}=-1.88$, qvalue $\left.=3.94 \times 10^{-05}\right)$. SLIT2 proteins have highly conserved roles in axon guidance and cell migration (Brose \& Tessier-Lavigne 2000) and the SLIT2 gene is regarded as a TSG whose promoter has been reported to be hyper-methylated in OC (Qiu et al. 2011). The up-regulation of SLIT2 as a consequence of HBO1 KD suggests that HBO1 may have a role in regulating its expression in UWB1.289 cells. On the contrary, GBGT1, another candidate TSG epigenetically silenced through promoter hyper-methylation in OC (Jacob et al. 2014), was down-regulated upon HBO1 depletion, thus suggesting that HBO1 has a differential role in modulating different TSGs. GBGT1 is involved in the synthesis of a specific cell membrane antigen, whose function is not well understood yet. However, other members of this family have been associated with various biological processes, including multidrug resistance and cell proliferation (Behnam-Motlagh et al. 2010; Park et al. 2012). The DNA repair gene PMS2 (Jenkins 2009) is recurrently mutated in family cases with 
Lynch syndrome (hereditary non-polyposis colorectal cancer) (Weissman et al. 2011), which increases the risk of Lynch-associated cancers like OC (Daniels \& Lu 2015). Again, the down-regulation of putative TSG PMS2 suggests a role for HBO1 in controlling the expression level of TSGs.

Prior to the analysis of predicted activities and based on previous reports on $\mathrm{HBO} 1$ function (Doyon et al. 2006; Mishima et al. 2011), UWB1.289 cells expressing 'shHBO1 1' and 'shCtrl' were subjected to cell proliferation assays, which measured the incorporation of BrdU into newly synthesized DNA of replicating cells. However, cell proliferation assays revealed no changes in proliferative capabilities between KD and control samples (Fig. 5.5). These results differ from previous studies where HBO1 KD: (1) significantly affected DNA synthesis and cell proliferation of HEK $293 \mathrm{~T}$ cells (Doyon et al. 2006) and (2) reduced cell proliferation rates of MLE-12 cells (Zou et al. 2013). Conversely, these findings agree with other studies that point to a non-essentiality of $\mathrm{HBO} 1$ for cell proliferation or DNA replication (Kueh et al. 2011; M. S. Kim et al. 2015).

The increased formation of cellular protrusions, as well as increments in microtubule dynamics, are phenomena commonly linked to the generation of traction force that ultimately results in cell motility (Parsons et al. 2010; Sens \& Plastino 2015). Consequently, transduced UWB1.289 cells were subjected to scratch/wound-healing assays in order to evaluate potential changes in cell migration prompted by HBO1 KD. In silico predicted increase in cellular organization did not translate into variations in cell migration between KD and control samples (Fig. 5.6).

Increased cytoplasm organization and microtubule dynamics are consistent with cytoskeletal rearrangements towards higher levels of cellular organization, a feature that was confirmed using AFM (Fig. 5.7). Interestingly, alterations in the cytoskeleton structure, which drive changes in the mechanical properties of individual cells, are associated with disease pathogenesis and progression (Lekka 2016). As cells transform from non-malignant to cancerous states, their cytoskeletal structure changes from an organized to an irregular network, reducing the stiffness of single cells, a feature that has been reported for a variety of cancer cells including OC cells (Guck et al. 2005; Xu et al. 2012). For instance, singular cells belonging to 
OC cell lines HEY and HEY A8 displayed very low Young's modulus (884 Pa and $494 \mathrm{~Pa}$ respectively) compared to non-malignant immortalised ovarian surface epithelial cells (2472 Pa) (Xu et al. 2012). Accordingly, the augmentation of single cell stiffness of $\mathrm{HBO} 1 \mathrm{KD}$ cells point to a role for overexpressed $\mathrm{HBO} 1$ in the maintenance of an elastic cancerous cell structure, perhaps promoting the acquisition of mesenchymal properties that would drive further tumorigenic growth. 


\section{Functional HBO1 Evaluation using a Transient RNAi- based Knockdown System}




\subsection{Introduction and Aims}

Despite consistent HBO1 level reductions, the implementation of a stable KD system in OC cells yielded somewhat inconclusive results using the functional assays reported in chapter 5 . The effects of transient $\mathrm{HBO} 1 \mathrm{KD}$ were therefore evaluated in order to further investigate the roles of $\mathrm{HBO} 1 \mathrm{in}$ OC. The most significant advantage of this approach is that it allows overcoming problems associated with the application of a constant selective pressure onto the cells, which may lead to offtarget effects. Consequently, gene and phenotypical alterations were monitored following transient $\mathrm{HBO} 1 \mathrm{KD}$ using a similar study design as the one applied in the preceding chapter.

After the examination of nucleosome targets of $\mathrm{HBO} 1$ acetylation (H4 and $\mathrm{H} 3 \mathrm{~K} 14$ ) in UWB1.289, OVCAR-3 and SKOV-3 OC cell lines, and in order to evaluate changes in gene mRNA expression, RNA samples from siRNA-transfected cells were hybridized to human whole-genome expression Illumina ${ }^{\circledR}$ microarrays. This type of microarray provided genome-wide transcriptional coverage of as many as 47,323 well-characterized human genes, gene candidates and splice variants, generating specific gene expression profiles that help shape the understanding of HBO1 cellular functions in OC. Statistical analysis of the outcome resulted in lists of significantly up and down-regulated transcripts, which, after qRT-PCR validation, were compared in search for common regulated transcripts.

Following the study of transient HBO1 KD effects at transcript level, derived gene lists were analysed to enable the identification of cellular pathways governed or influenced by HBO1 using IPA as well as Gene Set Enrichment Analysis (GSEA) predictor programmes (Subramanian et al. 2005). The results of the in silico pathway analysis were then tested in vitro to confirm any role of HBO1 in predicted pathways. The combination of pathway analyses and in vitro confirmation of predicted roles enabled a further understanding of $\mathrm{HBO} 1$ functions in $\mathrm{OC}$. 


\subsection{HBO1 Histone Acetylation Function in OC Cells}

Histone $4(\mathrm{~K} 5, \mathrm{~K} 8, \mathrm{~K} 12, \mathrm{~K} 16)$ and histone 3 (K14) nucleosome substrates are considered the main targets of HBO1 acetylation (Doyon et al. 2006; lizuka et al. 2009; Kueh et al. 2011; Mishima et al. 2011), and H4 acetylation levels were shown to be significantly reduced in UWB1.289 cells constitutively expressing shRNAs in the previous chapter. In order to further substantiate this function, protein extracts of siRNA-treated UWB1.289, OVCAR-3 and SKOV-3 cell lines were analysed using acetylation-specific antibodies (Fig. 6.1).

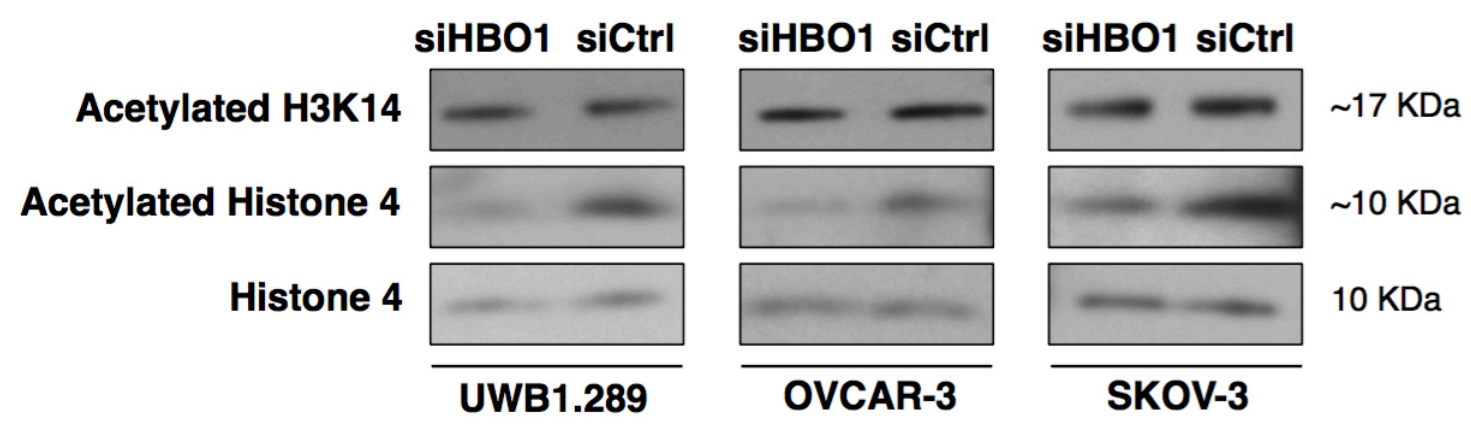

Figure 6. 1 Histone acetylation patterns after transient HBO1 KD

UWB1.289, OVCAR-3 and SKOV-3 cells were transfected with a pool of siRNAs targeting $\mathrm{HBOl}$ ('siHBOl') and non-targeting siRNAs ('siCtrl'). Cell lysates were extracted after $48 \mathrm{~h}$ whereupon protein samples were analysed by western blot and probed with anti-acetylated $\mathrm{H} 3 \mathrm{~K} 14$, anti-acetylated histone $4(\mathrm{H} 4)$ and anti-H4 antibodies. H4 was used as loading control.

HBO1 KD led to a significant depletion of acetylated histone $\mathrm{H} 4$ in all studied OC cell lines: UWB1.289 $\left(\mathrm{RD}=28.47 \pm 3.37 \%,{ }^{* *} \mathrm{p}=0.0088\right)$, OVCAR-3 $(\mathrm{RD}=23.11$ $\left.\pm 4.11 \%,{ }^{*} \mathrm{p}=0.0375\right)$, SKOV-3 $\left(\mathrm{RD}=31.28 \pm 2.85 \%,{ }^{*} \mathrm{p}=0.0429\right)($ Fig. $6.1 \&$ Fig. 6.2). In contrast, there were no significant changes in $\mathrm{H} 3 \mathrm{~K} 14$ acetylation status as a result of the KD in UWB1.289 $(\mathrm{RD}=85.48 \pm 2.51 \%, \mathrm{p}=0.550)$, OVCAR-3 $(\mathrm{RD}=88.91 \pm 3.66 \%, \mathrm{p}=0.7615)$ or SKOV-3 $(\mathrm{RD}=94.67 \pm 2.98 \%, \mathrm{p}=0.9261)$ (Fig. 6.1 \& Fig. 6.2). These data suggest specific regulation of $\mathrm{H} 4$ acetylation but not $\mathrm{H} 3 \mathrm{~K} 14$ acetylation in $\mathrm{OC}$ cell lines and agree with previous results on HBO1 stable KD UWB1.289 cells (Fig. 5.1 \& Fig. 5.2). 


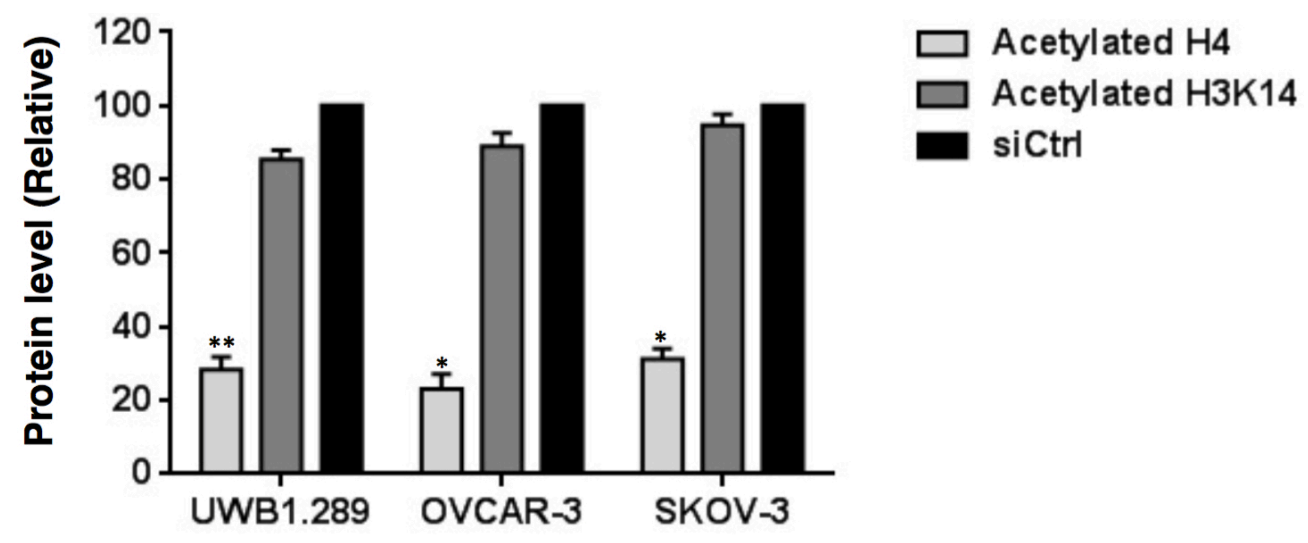

Figure 6. 2 Histone acetylation relative levels after transient HBO1 KD

UWB1.289, OVCAR-3 and SKOV-3 cells were transfected with a pool of siRNAs targeting $\mathrm{HBO}$ l and non-targeting siRNAs ('siCtrl'). Protein lysates were extracted after $48 \mathrm{~h}$ whereupon they were analysed by western blot. The relative levels of acetylated $\mathrm{H} 4$ and acetylated $\mathrm{H} 3 \mathrm{~K} 14$ protein expression were calculated using ImageJ; H4 was used as loading control. Statistical analysis consisted of one-way ANOVA and the Tukey's multiple comparison test. All values represent the mean \pm $\mathrm{SD}$ of three biological samples $\left({ }^{*} \mathrm{p}<0.05 ;{ }^{* *} \mathrm{p}<0.01\right)$.

\subsection{Microarray Analysis of Transient HBO1 Knockdown Profiles}

The effect of transient HBO1 KD on global gene expression was determined using whole-genome Illumina ${ }^{\circledR}$ microarrays. As the Illumina ${ }^{\circledR}$ microarrays contain only one probe for HBO1 that specifically recognizes the isoform 1 of the HAT, and lack probes for isoforms 2, 3, 4 and 5, the scope of HBO1 analysis is somewhat limiting. For each studied OC cell line, OVCAR-3, SKOV-3 and UWB1.289, three biological replicates transfected with 'siHBO1' and 'siCtrl' were used to generate specific gene expression profiles, which contained lists of differentially expressed genes, their associated fold-change values and statistical probabilities (q-value).

\subsubsection{Gene expression profiling of OVCAR-3 cells}

The depletion of HBO1 in OVCAR-3 cells caused the up-regulation and downregulation of 110 and 45 gene transcripts respectively, rendering a total of 155 genes altered by $\mathrm{HBO} 1 \mathrm{KD}(\mathrm{FC} \pm 1.5$; q-value $<0.1)$ (Table 6.1). As a positive control, the HBO1 transcript (MYST2) was one of the 45 down-regulated targets $(\mathrm{FC}=-1.55$, $\mathrm{q}$-value $\left.=1.65 \times 10^{-02}\right)$. 
Table 6. 1 List of altered genes after transient HBO1 KD in OVCAR-3

Up-regulated genes (110) are listed in descending order of expression, with the ' 2 '5'-oligoadenylate synthetase 2' $(O A S 2)$ displaying the greatest up-regulation ( $\mathrm{FC}=$ 4.16). Down-regulated genes (45) are listed in ascending order of expression, with the 'lysine acetyltransferase 7 pseudogene' (LOC648927) displaying the greatest down-regulation $(\mathrm{FC}=-2.1)$.

\begin{tabular}{|c|c|c|c|c|c|}
\hline \multicolumn{7}{|c|}{ Up-regulated genes (110) } & \multicolumn{2}{c|}{ Down-regulated genes (45) } \\
\hline OAS2 & IRF7 & ZC3HAV1 & LOX & LOC648927 & AXL \\
IFI6 & OASL & VCAN & LOC389386 & ADRB2 & NNAT \\
TRIM22 & HERC5 & LEF1 & UBE2E2 & LIFR & MBNL3 \\
IFI44L & DHX58 & GBP4 & ElF2AK2 & SMPDL3B & AQP6 \\
IFITM1 & STAT1 & HLA-B & TSPAN7 & C3orf64 & KRT4 \\
IFIT1 & CMPK2 & LOC643384 & NBPF20 & MIR369 & SMC4 \\
IFI27 & IFIT5 & TMEM140 & C19orf66 & KRT81 & RNF141 \\
CXCL10 & LAMP3 & LOC6465562 & HSPE1 & NPFFR2 & CDR2 \\
SLC15A3 & PSMB9 & GBP5 & KCNE1 & EDN2 & TRIM47 \\
IFIT3 & SP110 & LAMA1 & IRX3 & CA12 & WDR89 \\
LOC100 & PARP14 & TSPAN2 & LOC646146 & RABL2B & MYADML \\
BST2 & LBA1 & TDRD7 & FLJ21986 & CD46 & LOH3CR2A \\
RSAD2 & PLSCR1 & IFNB1 & SLNF5 & SCARNA27 & CCL28 \\
ISG15 & EPST11 & CPA6 & HRH1 & GRIP2 & PRPF40A \\
DDX60 & PRIC285 & GBP1 & PRSS35 & CHORDC1 & C11orf47 \\
IFIT2 & IFI16 & UBE2L6 & PRM1K & TRIM23 & SPIRE2 \\
HERC6 & CXCL11 & PARP12 & C21orf128 & S100A2 & RUNX1T1 \\
IFI44 & IFI35 & CMYAS & SP100 & TNFRSF10D & \\
SAMD9L & C20orf103 & LTBP1 & TLE4 & H2AFY & \\
USP18 & PARP9 & USP41 & HLA-F & RABL2A & \\
OAS1 & MX1 & LPIN2 & MAP2 & MYST2 & \\
OAS3 & 2LOC100 & GABRA2 & CD69 & FOXS1 & \\
DDX60L & TAP1 & ST8SIA1 & SAMD11 & YAP1 & \\
RASGRP3 & SPP1 & IFITM3 & NMNAT2 & AGFG1 & \\
IDO1 & IRF9 & TBX2 & LOC401093 & GREM1 & \\
DDX58 & ADAM32 & AKR1C3 & THAP7 & TMEM55A & \\
IFIH1 & LGALS9 & MAP2K3 & & TNRC18 & \\
SAMD9 & INDO & RASSF2 & & KRT16 & \\
\hline
\end{tabular}

${ }^{1}$ LOC100129681, ${ }^{2}$ LOC 100128274

In order to identify potential meaningful OC-related genes that could be used to validate the microarray results, the list of differentially expressed transcripts was incorporated into the SQL relational database. Using SQL queries, the search for validation targets was narrowed down to 6 genes, each with functions involved in OC-related processes and which had been identified as HBO1 targets in a previously published RKO ChIP-Seq experiment (Avvakumov et al. 2012) (Table 6.2). The transcript abundance of these targets was confirmed using qRT-PCR (Fig. 6.3). 
Table 6. 2 List of gene validation targets for the OVCAR-3 expression profile

\begin{tabular}{|c|c|c|c|}
\hline Gene target & FC & q-value & Ovarian Cancer Link \\
\hline $\begin{array}{c}\text { Bone marrow stromal cell } \\
\text { antigen 2 (BST2) }\end{array}$ & 2.79 & $1 \times 10^{-8}$ & $\begin{array}{c}\text { Up-regulated in metastasis } \\
\text { (Walter-Yohrling et al. 2003) }\end{array}$ \\
\hline $\begin{array}{c}\text { Interferon stimulated protein } \\
15(I S G 15)\end{array}$ & 2.66 & $1 \times 10^{-7}$ & $\begin{array}{c}\text { Linked to NF- } \beta \beta \text { and survival } \\
\text { (Darb-Esfahani et al. 2014) }\end{array}$ \\
\hline $\begin{array}{c}\text { Signal transducer and activator } \\
\text { of transcription 1 (STAT1) }\end{array}$ & 1.97 & $1 \times 10^{-8}$ & $\begin{array}{c}\text { Linked to platinum resistance } \\
\text { (Stronach et al. 2011) }\end{array}$ \\
\hline $\begin{array}{c}\text { CD46 molecule/antigen } \\
(C D 46)\end{array}$ & -1.62 & $2.6 \times 10^{-2}$ & $\begin{array}{c}\text { Up-regulated in OC tissues } \\
\text { (Fishelson et al. 2003) }\end{array}$ \\
\hline $\begin{array}{c}\text { Fibroblast growth factor } \\
\text { receptor 4 (FGFR4) }\end{array}$ & -1.56 & $1.6 \times 10^{-3}$ & $\begin{array}{c}\text { Linked to HGSC prognosis } \\
\text { (Zaid et al. 2013) }\end{array}$ \\
\hline $\begin{array}{c}\text { Yes-associated protein 1 } \\
(Y A P 1)\end{array}$ & -1.51 & $3.5 \times 10^{-3}$ & $\begin{array}{c}\text { Up-regulated in OC tissues } \\
\text { (Steinhardt et al. 2008) }\end{array}$ \\
\hline
\end{tabular}

Up-regulated gene transcripts identified in the OVCAR-3 microarray: BST2 (FC = $\left.4.19,{ }^{* *} \mathrm{p}=0.0025\right), \operatorname{ISG} 15\left(\mathrm{FC}=4.22,{ }^{* *} \mathrm{p}=0.0021\right)$ and STAT1 $\left(\mathrm{FC}=2.37,{ }^{*} \mathrm{p}=\right.$ 0.0268), were similarly up-regulated when analysed by qRT-PCR (Fig. 6.3 A). Conversely, $C D 46\left(\mathrm{FC}=-1.74,{ }^{*} \mathrm{p}=0.0278\right), F G F R 4\left(\mathrm{FC}=-1.68,{ }^{*} \mathrm{p}=0.042\right)$ and YAPl $\left(\mathrm{FC}=-1.8,{ }^{*} \mathrm{p}=0.0355\right)$, were significantly reduced (Fig. 6.3 B).

A

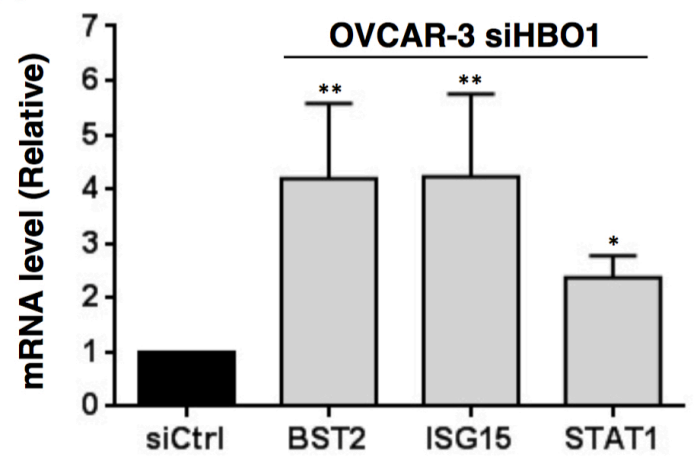

B

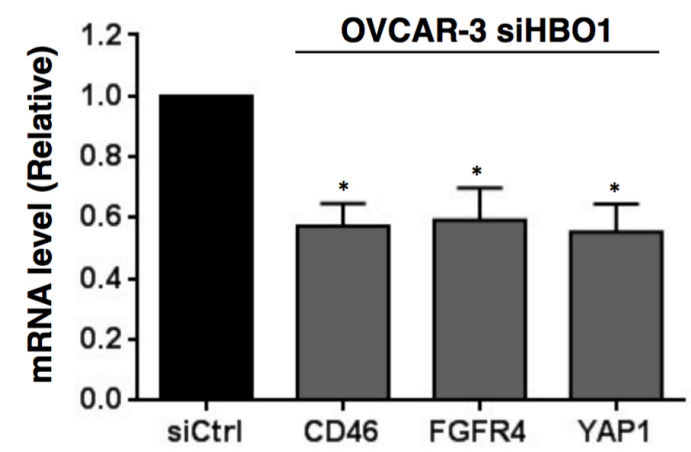

Figure 6. 3 Validation of the HBO1 KD OVCAR-3 gene expression profile OVCAR-3 cells were transfected with a pool of siRNAs targeting HBOl ('siHBO1') and non-targeting siRNAs ('siCtrl'). Cell lysates were extracted after $48 \mathrm{~h}$ whereupon RNA samples were prepared and analysed by qRT-PCR to confirm: (A) the upregulation of BST2, ISG15, STAT1 and (B) the down-regulation of CD46, FGFR4 and YAP1. Relative levels of expression in cells treated with 'siHBO1' were compared to those of cells treated with 'siCtrl'. RPL5 expression was used as control. Statistical analyses consisted of one-way ANOVA and Tukey's multiple comparison tests. All values represent the mean \pm SD of three biological samples $\left({ }^{*} p\right.$ $<0.05 ;{ }^{* *} \mathrm{p}<0.01$ ). 
The gene expression of a number of the target genes remained altered at additional time points ( 24 and $72 \mathrm{~h}$ post-transfection). Up-regulation of $B S T 2\left(\mathrm{FC}=2.46{ }^{*} \mathrm{p}=\right.$ $0.0344), \operatorname{ISG} 15\left(\mathrm{FC}=2.82,{ }^{*} \mathrm{p}=0.0305\right)$ and STAT1 $\left(\mathrm{FC}=1.59,{ }^{*} \mathrm{p}=0.0489\right)$ were maintained after $72 \mathrm{~h}$, but to a lesser extent than at $48 \mathrm{~h}$ (Fig. 6.4 A), demonstrating the transient nature of the siRNA treatment.

The down-regulation of $C D 46\left(\mathrm{FC}=-1.83,{ }^{* *} \mathrm{p}=0.0021\right)$ and $Y A P 1\left(\mathrm{FC}=-1.41,{ }^{*} \mathrm{p}\right.$ $=0.0236$ ) was significantly reduced after $24 \mathrm{~h}$, whereas reduction in FGFR4 expression ( $\mathrm{FC}=-1.75, \mathrm{p}=0.1336)$ was not statistically significant despite the downward trend (Fig. 6.4 B). The effect of siRNA treatment was sustained for the $72 \mathrm{~h}$ of the experiment: $C D 46\left(\mathrm{FC}=-1.69,{ }^{*} \mathrm{p}=0.0429\right), F G F R 4\left(\mathrm{FC}=-1.45,{ }^{*} \mathrm{p}=\right.$ $0.0124)$ and YAPl $\left(\mathrm{FC}=-1.39,{ }^{*} \mathrm{p}=0.0477\right)$ (Fig. 6.4 B). These results suggest that the expression of this gene set (Table 6.2) is extensively regulated by HBO1 in OVCAR-3 cells. Interestingly, none of these genes were identified in the previous shRNA approach using UWB1.289 cells (Table 5.1).

A

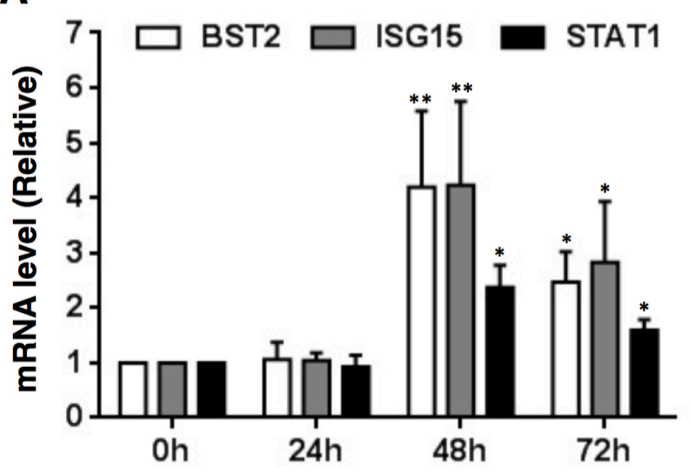

B

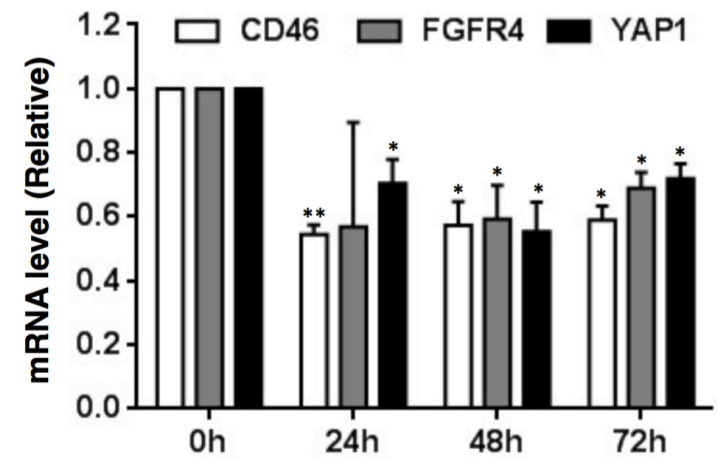

Figure 6. 4 Time-course analysis of the transient HBO1 KD effect in OVCAR-3 OVCAR-3 cells were transfected with a pool of siRNAs targeting HBOl ('siHBO1') and non-targeting siRNAs ('siCtrl'). Cell lysates were extracted after 24, 48 and $72 \mathrm{~h}$ whereupon RNA samples were prepared and analysed by qRT-PCR to confirm: (A) the up-regulation of BST2, ISG15, STAT1 and (B) the down-regulation of CD46, FGFR4 and YAP1. Relative levels of expression in cells treated with 'siHBO1' were compared to those of cells treated with 'siCtrl'. RPL5 expression was used as control. Statistical analyses consisted of one-way ANOVA and Tukey's multiple comparison tests. All values represent the mean \pm SD of three biological samples $\left({ }^{*} \mathrm{p}\right.$ $<0.05 ;{ }^{* *} \mathrm{p}<0.01$ ). 


\subsubsection{Gene expression profiling of SKOV-3 cells}

Prior to the gene expression profiling study in SKOV-3 cells, gene targets identified in OVCAR-3 cells (Table 6.2) were assessed for expression in the SKOV-3 HBO1 KD model. CD46 and YAP1 genes displayed significant down-regulation compared to the control, whereas BST2, ISG15, STAT1 and FGFR4 remained unchanged. These results indicate that successful HBO1 KD in SKOV-3 cells again perturbed HBO1 regulatory mechanisms, with some of the changes in target modulation conserved amongst cell lines and others displaying higher specificity.

The depletion of HBO1 in SKOV-3 cells resulted in the up-regulation and downregulation of 20 and 60 gene transcripts respectively, a total of 80 genes products altered by $\mathrm{HBO} 1 \mathrm{KD}(\mathrm{FC} \pm 1.5$; q-value $<0.1)$ (Table 6.3).

\section{Table 6. 3 List of altered genes after transient HBO1 KD in SKOV-3}

Up-regulated genes (20) are listed in descending order of expression, with the 'p21 protein activated-kinase 2' ( $P A K 2)$ displaying the greatest up-regulation $(\mathrm{FC}=2.31)$. Down-regulated genes (60) are listed in ascending order of expression, with the 'cysteine and histidine rich domain containing 1' (CHORDC1) displaying the greatest down-regulation $(\mathrm{FC}=-2.5)$.

\begin{tabular}{|c|c|c|c|}
\hline Up-regulated genes (20) & \multicolumn{3}{|c|}{ Down-regulated genes (60) } \\
\hline PAK2 & CHORDC1 & LOC442153 & LOC100129379 \\
LOC729513 & RNF141 & YAP1 & LOC100133803 \\
CAST & RABL2A & LOC100134504 & LOC645630 \\
SLFN5 & LOC648927 & SUMO1 & LOC728060 \\
PLAT & LIFR & MYST2 & PRMT6 \\
FBXW27 & SAR1A & SPAST & LOC100132499 \\
ABTB1 & GCNT2 & TOP1 & LOC100133211 \\
GOLGA8A & RABL2B & NAPEPLD & VPS36 \\
AAK1 & CD46 & LOC728602 & SSR2 \\
CDCP1 & C3orf64 & ARL6IP1 & ZNF268 \\
TSPAN2 & LOC728843 & PTMA & LOC727821 \\
CNTNAP1 & TOP1P2 & LOC728026 & LOC345041 \\
CHRNA9 & C6orf192 & DCTN4 & PSG6 \\
PGM2L1 & H2AFY & LOC729362 & PRPF38A \\
LOC100133800 & CDR2 & LOC643668 & LOC646966 \\
TOB1 & NEK1 & MIB1 & LOC649839 \\
NUCKS1 & LOC100131713 & KPNA2 & F3 \\
LOC643977 & LOC728484 & LOC100128060 & LOC643308 \\
STK11IP & AGFG1 & LOC644889 & ATMIN \\
OLFML3 & ASPH & LOC100132547 & UFM1 \\
\hline
\end{tabular}


The HBO1 transcript (MYST2) was detected amongst the 60 down-regulated genes $(\mathrm{FC}=-1.63, \mathrm{q}$-value $=0.047)$, confirming the success of the experimental approach. To further validate the results, three of the top up- and down-regulated targets were re-analysed using qRT-PCR, including the 'AP2 associated kinase 1' (AAK1), the 'nuclear casein kinase and cyclin dependent kinase substrate 1' (NUCKS), the 'serine/threonine kinase 11 interacting protein' (STK11IP), the 'ubiquitin ligase mindbomb' $(M I B 1)$, spastin $(S P A S T)$ and the 'small ubiquitin-like modifier 1' (SUMO1) (Fig. 6.5).

A

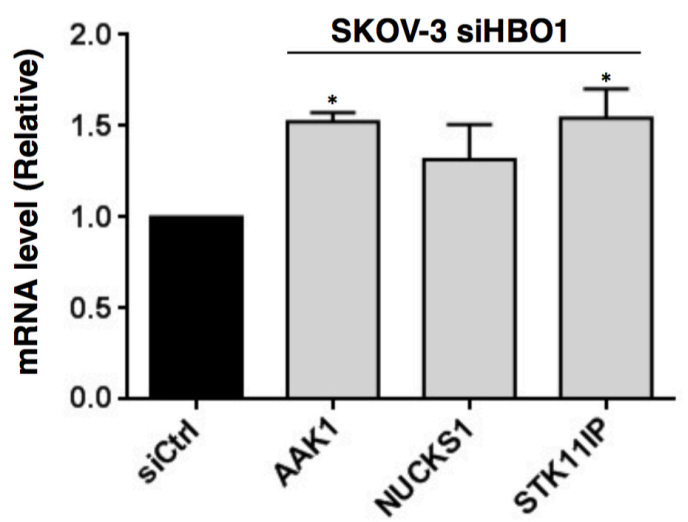

B

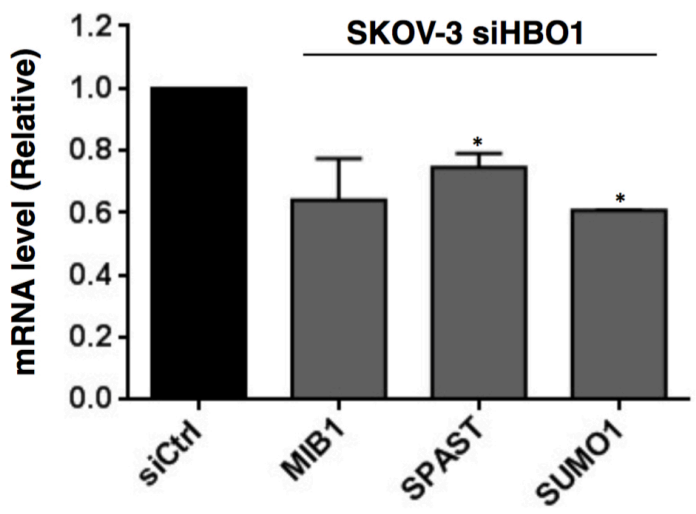

Figure 6. 5 Validation of the HBO1 KD SKOV-3 gene expression profile

SKOV-3 cells were transfected with a pool of siRNAs targeting HBOl ('siHBO1') and non-targeting siRNAs ('siCtrl'). Cell lysates were extracted after $48 \mathrm{~h}$ whereupon RNA samples were prepared and analysed by qRT-PCR to confirm: (A) the upregulation of $A A K 1, N U C K S 1, S T K 11 I P$ and (B) the down-regulation of MIB1, $S P A S T$ and SUMO1. Relative levels of expression in cells treated with 'siHBO1' were compared to those of cells treated with 'siCtrl'. GAPDH expression was used as control. Statistical analyses consisted of one-way ANOVA and Tukey's multiple comparison tests. All values represent the mean \pm SD of three biological samples $(* p$ $<0.05)$.

Up-regulated gene transcripts identified in the SKOV-3 microarray: $A A K 1$ (FC = $\left.1.6,{ }^{*} \mathrm{p}=0.0259\right), \operatorname{NUCKS1}(\mathrm{FC}=1.42, \mathrm{p}=0.0788)$ and $\operatorname{STK1} 11 P\left(\mathrm{FC}=1.51,{ }^{*} \mathrm{p}=\right.$ 0.0452), were similarly up-regulated when analysed by qRT-PCR (Fig. 6.5 A), and MIB1 $(\mathrm{FC}=-1.56, \mathrm{p}=0.053), \operatorname{SPAST}\left(\mathrm{FC}=-1.34,{ }^{*} \mathrm{p}=0.0433\right)$ and SUMO1 $(\mathrm{FC}=$ $-1.64,{ }^{*} p=0.0297$ ) were also significantly reduced (Fig. 6.5 B). 


\subsubsection{Gene expression profiling of UWB1.289 cells}

Prior to the gene expression profiling study, previously defined OVCAR-3 targets (Table 6.2) were assessed for expression in the UWB1.289 HBO1 KD model. As in OVCAR-3 and SKOV-3, CD46 and YAP1 displayed significant down-regulation compared to the control, whereas BST2, ISG15, STAT1 or FGFR4 expression was unchanged, suggesting a tight $\mathrm{HBO} 1$ regulation of selected genes across the OC cell model. The depletion of HBO1 in UWB1.289 cells resulted in the up-regulation and down-regulation of 507 and 382 gene transcripts respectively, a total of 889 altered gene products $(\mathrm{FC} \pm 1.5 ;$ q-value $<0.1)$ (Table 6.4).

Table 6. 4 Top altered genes after transient HBO1 KD in UWB1.289

Top up-regulated genes are listed in descending order of expression, with 'chromosome 8 ORF 4' (C8orf4) displaying the greatest up-regulation $(\mathrm{FC}=4.34)$. Top down-regulated genes are listed in ascending order of expression, with the 'lysine acetyltransferase 7 pseudogene' (LOC648927) displaying the greatest downregulation $(\mathrm{FC}=-4.01)$.

\begin{tabular}{|c|c|c|c|c|c|}
\hline \multicolumn{2}{|c|}{ Top up-regulated genes (78) } & \multicolumn{2}{c|}{ Top down-regulated genes (78) } \\
\hline C8orf4 & TSHZ2 & TSC22D3 & LOC648927 & FLI12684 & SLC6A15 \\
MAFB & COL22A1 & SLC13A5 & MYST2 & GCNT2 & KAT2B \\
SLC2A12 & CDKN1A & LOC644612 & SAR1A & NEIL2 & SNORD104 \\
PROC & PROM1 & RARRES2 & CHORDC1 & PLCB4 & SLC7A2 \\
RASGRP1 & SRGAP3 & ATOH8 & C16orf73 & SERPINE1 & RRP9 \\
DACT1 & TNFRSF19 & ITGB6 & S1PR1 & ADRB2 & AADAC \\
KCNK12 & BEND5 & ZBTB46 & SNAPC1 & HBEGF & LHX6 \\
FAM107A & TNFRSF10 & SOX4 & FOSL1 & YAP1 & LOC732371 \\
TSPAN8 & HAPLN3 & LOC606724 & ESM1 & C3orf64 & MARS2 \\
KLHL24 & TUBB3 & CTDSP2 & H2AFY & IL1RL1 & ZNF699 \\
PLA2G10 & ASAP3 & FLJ11235 & AFAP1L1 & CD46 & JAG2 \\
CDH6 & SGPP2 & RASSF2 & LOC729231 & CREB5 & CR2 \\
MUC16 & FXYD6 & LBH & RABL2A & SLC25A43 & TLR4 \\
MAP2 & D4S234E & HIST2 & PDE1C & BNC1 & AOX1 \\
TP53INP1 & VSNL1 & YPEL2 & C6orf192 & GOLM1 & SDCCAG3 \\
RASD1 & RASD2 & MFAP2 & EDG1 & MCOLN2 & PRKAA1 \\
C5orf13 & SYT11 & NCCRP1 & RABL2B & PRNP & CCND2 \\
ALPL & ZMYND15 & PCDHB5 & NIPAL1 & LOC647691 & MLKL \\
ID2 & ANKRD34B & PBX1 & PLAGL2 & S100A2 & LOC732419 \\
SCNN1A & SAMD14 & PROX1 & ADAMT56 & NIN & LOC201651 \\
JAM2 & GRHL3 & EDAR & TNFRSF8 & TOP1 & STYK1 \\
RCAN2 & CXXC5 & MXD4 & 2LOC100 & RASL11B & HPDL \\
CCNG2 & TRIM2 & PGM2L1 & LETM2 & TAF1A & SNAI2 \\
ARID5B & GPR64 & DLX5 & TMEM184A & SFXN1 & FCF1 \\
CLIC3 & EPN3 & WNT10A & PRPF38A & VPS36 & DCLK1 \\
BAPX1 & CSDC2 & CX3CL1 & CDR2 & ADPGK & ACTR3B \\
\hline
\end{tabular}

${ }^{1}$ HIST2H2AA3, ${ }^{3}$ LOC 100132240 
HBO1 transcript (MYST2) was detected amongst the 382 down-regulated genes (FC $=-2.7$, q-value $\left.=1.1 \times 10^{-09}\right)$, validating the experimental approach. To further validate these results, three of the top up and down-regulated targets were analysed using qRT-PCR, including cyclin G2 (CCNG2), the 'Dapper antagonist of catenin 1' (DACT1), the 'solute carrier family 2 member 12' (SLC2A12), the 'NIPA like domain containing 1' (NIPAL1), the 'pleiomorphic adenoma gene-like 2' (PLAGL2) and the 'secretion associated Ras related GTPase 1A' (SAR1A) (Fig. 6.6).

A

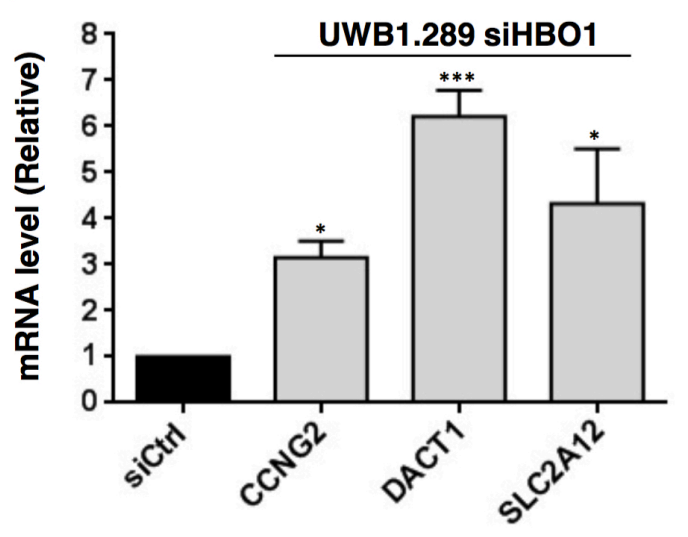

B

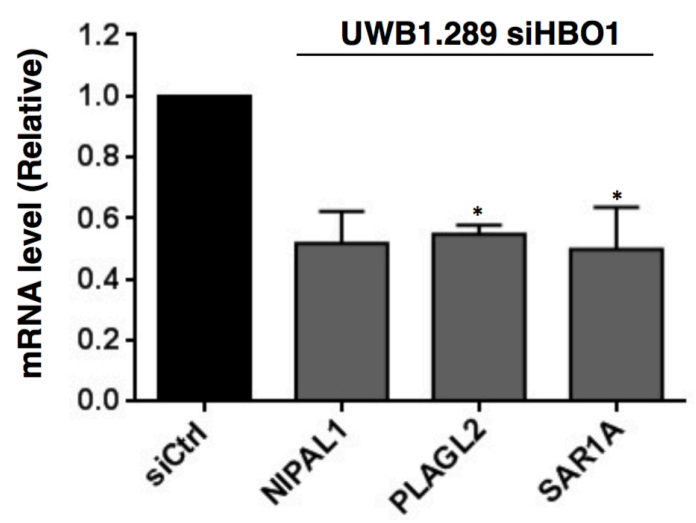

Figure 6. 6 Validation of the HBO1 KD UWB1.289 gene expression profile UWB1.289 cells were transfected with a pool of siRNAs targeting HBOl ('siHBO1') and non-targeting siRNAs ('siCtrl'). Cell lysates were extracted after 48h whereupon RNA samples were prepared and analysed by qRT-PCR to confirm: (A) the up-regulation of CCNG2,DACT1, SLC2A12 and (B) the down-regulation of NIPAL1, PLAGL2 and SAR1A. Relative levels of expression in cells treated with 'siHBO1' were compared to those of cells treated with 'siCtrl'. GAPDH expression was used as control. Statistical analyses consisted of one-way ANOVA and Tukey's multiple comparison tests. All values represent the mean \pm SD of three biological samples $(* \mathrm{p}<0.05 ; * * * \mathrm{p}<0.001)$.

Up-regulated gene transcripts identified in the UWB1.289 microarray: CCNG2 (FC $\left.=3.22,{ }^{*} \mathrm{p}=0.0232\right), D A C T 1\left(\mathrm{FC}=6.21,{ }^{* * *} \mathrm{p}=0.0008\right)$ and $\operatorname{SLC} 2 A 12(\mathrm{FC}=4.31$, $* \mathrm{p}=0.0143$ ), were similarly up-regulated when analysed by qRT-PCR (Fig. 6.6 A), and NIPAL1 $(\mathrm{FC}=-1.92, \mathrm{p}=0.0672)$, PLAGL2 $\left(\mathrm{FC}=-1.82,{ }^{*} \mathrm{p}=0.0423\right)$ and $\operatorname{SAR} 1 A\left(\mathrm{FC}=-2.08,{ }^{*} \mathrm{p}=0.0478\right)$ were also significantly reduced (Fig. 6.6 B). 


\subsubsection{Study of $\mathrm{HBO1}$ responsive genes in multiple $\mathrm{OC}$ cell lines}

The transient KD of HBO1 in OVCAR-3, SKOV-3 and UWB1.289 OC cell lines revealed some similarities, but also many differences in the numbers and identities of altered targets following HBO1 decline. Genome-wide comparisons of the effects of $\mathrm{KD}$ in the three cell lines are represented as scatter-plots in Appendix 3, illustrating the consistency of HBO1 KD amongst the studied cell lines.

The number of genes that had significantly altered expression as a consequence of HBO1 KD could be linked to different levels of HBO1 expression in each cell line, as well as different stages of OC from which they were isolated. UWB1.289, which expressed the highest level of HBO1 protein compared to OVCAR-3 and SKOV-3 (Fig. 3.6), had the highest number of altered target genes after transient HBO1 KD (Table 6.5).

Table 6. 5 Summary of gene expression changes caused by transient HBO1 KD

\begin{tabular}{|c|c|c|c|}
\hline Cell line & Total genes altered & Up-regulated & Down-regulated \\
\hline UWB1.289 & 889 & 507 & 382 \\
\hline OVCAR-3 & 155 & 110 & 45 \\
\hline SKOV-3 & 80 & 20 & 60 \\
\hline
\end{tabular}

The majority of genes identified were up-regulated following loss of HBO1, UWB1.289 (57\%) and OVCAR-3 (71\%), suggesting either a direct role for HBO1 in transcription repression or an indirect effect based on the activation of potential repressors (Table 6.5). To compare the overlap of HBO1 responsive gene transcripts in the three studied cell lines, UWB1.289, OVCAR-3 and SKOV-3, a Venn diagram was constructed from the lists of differentially expressed genes (Fig. 6.7 A). A set of 13 genes including $\mathrm{HBOl}$ and the 'lysine acetyltransferase 7 pseudogene' or LOC648927 (1.2\% of the total number of HBO1-modulated genes) were common between the three OC cell lines (Fig. 6.7 B), a number of which were validated using qRT-PCR, including CD46, the 'H2A histone family member $\mathrm{Y}$ ' (H2AFY), tetraspanin $2(T S P A N 2)$ and $Y A P 1$ (Fig. 6.8). 
A

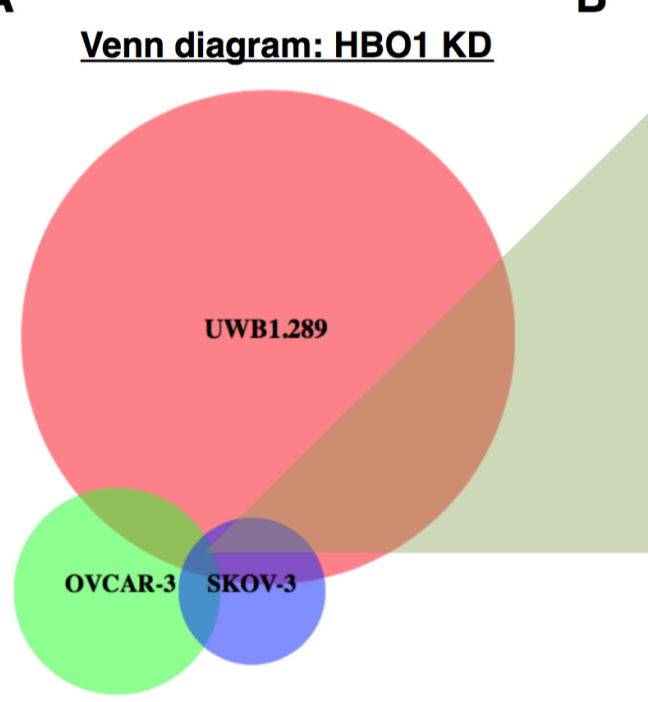

B

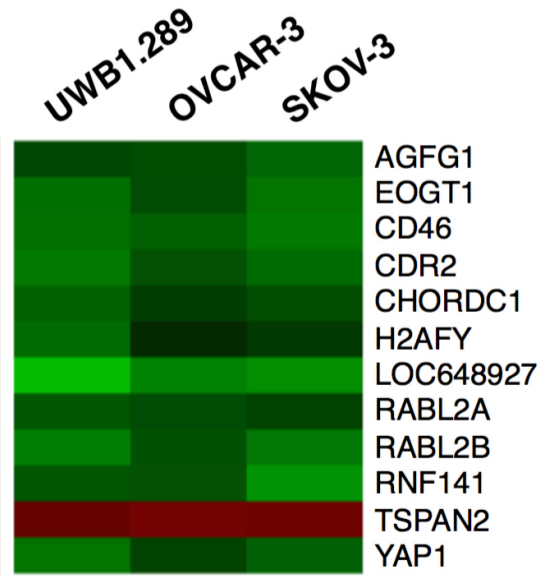

Fold-change

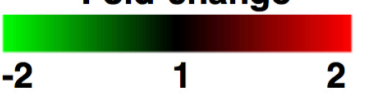

Figure 6. 7 Common response to $\mathrm{HBO1} \mathrm{KD}$ in $\mathrm{OC}$ cell lines

(A) Microarray-derived lists of differentially expressed genes following $\mathrm{HBO} 1 \mathrm{KD}$ in UWB1.289, OVCAR-3 and SKOV-3 cell lines were collated using a Venn diagram. (B) The relative expression of genes that showed a common response to HBO1 KD in the three cell lines are represented in a heat map elaborated with MEV. Scale is shown at the bottom, where red represents gene up-regulation and green represents gene down-regulation compared to the control. $A G F G 1$ : arfGAP with FG repeats 1; C3orf64/EOGT: EGF domain specific O-linked Nacetylglucosamine transferase; $C D R 2$ : cerebellar degeneration related protein 2; CHORDC1: cysteine and histidine rich domain containing 1; RABL2A/B: RAB, member of RAS oncogene family like 2A/B; RNF141: ring finger protein 141.

The gene transcript for $C D 46$, a membrane protein with roles in immunity and cell adhesion (Tang et al. 2016), was down-regulated in OVCAR-3 HBO1 KD cells (FC $\left.=-1.74,{ }^{*} \mathrm{p}=0.0278\right), \mathrm{UWB} 1.289\left(\mathrm{FC}=-2.09,{ }^{*} \mathrm{p}=0.0337\right)$ and SKOV-3 $(\mathrm{FC}=$ $2.12,{ }^{*} \mathrm{p}=0.0462$ ) (Fig. 6.8). Similarly, YAP1, a main downstream nuclear effector of the Hippo signalling pathway involved in tissue homeostasis and cell proliferation (Moroishi et al. 2015), was down-regulated in OVCAR-3 (FC $\left.=-1.8,{ }^{*} \mathrm{p}=0.0355\right)$, UWB1.289 $\left(\mathrm{FC}=-1.57,{ }^{*} \mathrm{p}=0.0371\right)$ and SKOV-3 $\left(\mathrm{FC}=-1.79,{ }^{*} \mathrm{p}=0.0477\right)($ Fig. 6.8).

$H 2 A F Y$, which encodes a replication-independent histone variant involved in transcription repression (Monteiro et al. 2014), displayed decreased expression in UWB1.289 (FC $\left.=-3.22,{ }^{*} \mathrm{p}=0.0467\right)$, OVCAR-3 $\left(\mathrm{FC}=-1.66,{ }^{*} \mathrm{p}=0.0398\right)$ and SKOV-3 $\left(\mathrm{FC}=-1.83,{ }^{*} \mathrm{p}=0.0352\right)$ cell lines (Fig. 6.8). TSPAN2, a trans-membrane 
protein involved in signal transduction related to cell development, activation, growth and motility (Otsubo et al. 2014), was up-regulated in all cell lines: UWB1.289 $\left(\mathrm{FC}=2.55,{ }^{*} \mathrm{p}=0.0264\right)$, OVCAR-3 $\left(\mathrm{FC}=1.94,{ }^{*} \mathrm{p}=0.0448\right)$ and SKOV-3 $\left(\mathrm{FC}=2.37,{ }^{*} \mathrm{p}=0.0396\right)$ (Fig. 6.8).

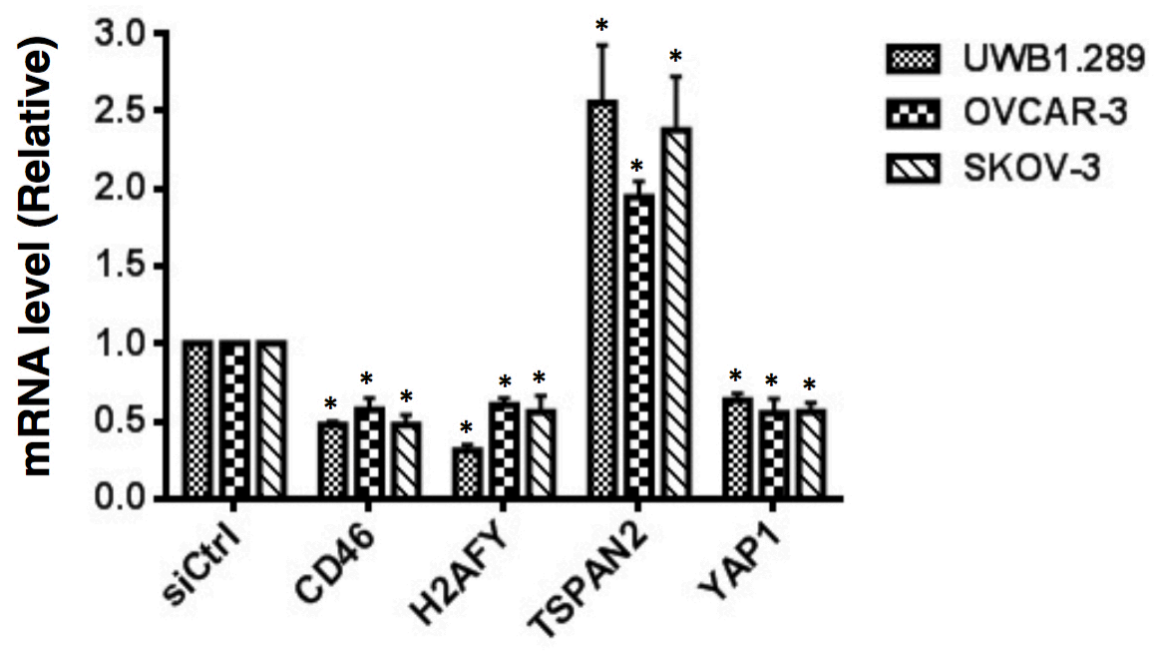

Figure 6. 8 Validation of common gene expression changes caused by HBO1 KD UWB1.289, OVCAR-3 and SKOV-3 cells were transfected with a pool of siRNAs targeting $\mathrm{HBOl}$ ('siHBO1') and non-targeting siRNAs ('siCtrl'). Cell lysates were extracted after $48 \mathrm{~h}$ whereupon RNA samples were prepared and analysed by qRTPCR to confirm the down-regulation of $C D 46, H 2 A F Y, Y A P 1$ and the up-regulation of TSPAN2. Relative levels of expression in cells treated with 'siHBO1' were compared to those of cells treated with 'siCtrl'. GAPDH expression was used as control. Statistical analysis consisted of one-way ANOVA and the Tukey's multiple comparison test. All values represent the mean \pm SD of three biological samples $\left(^{*} p\right.$ $<0.05)$.

These genes were subsequently considered as an 'HBO1 OC signature' gene set (Fig. 6.7 B), the expression of which are normally up-regulated by the acetyltransferase, suggesting that these genes could be direct targets of HBO1. To determine the extent to which the 'HBO1 OC signature' was present in other OC derived cells, the serous-like cell line UACC-1598 and the endometrioid TOV112D were investigated. These two cell lines were transfected with siRNAs targeting $\mathrm{HBOl}$, and qRT-PCR analyses were used to demonstrate the effective KD of HBO1 expression: UACC-1598 (FC $\left.=-2.18,{ }^{*} \mathrm{p}=0.0366\right)$, TOV-112D (FC $=-4.87, * * * * p<0.0001)($ Fig. 6.9). 


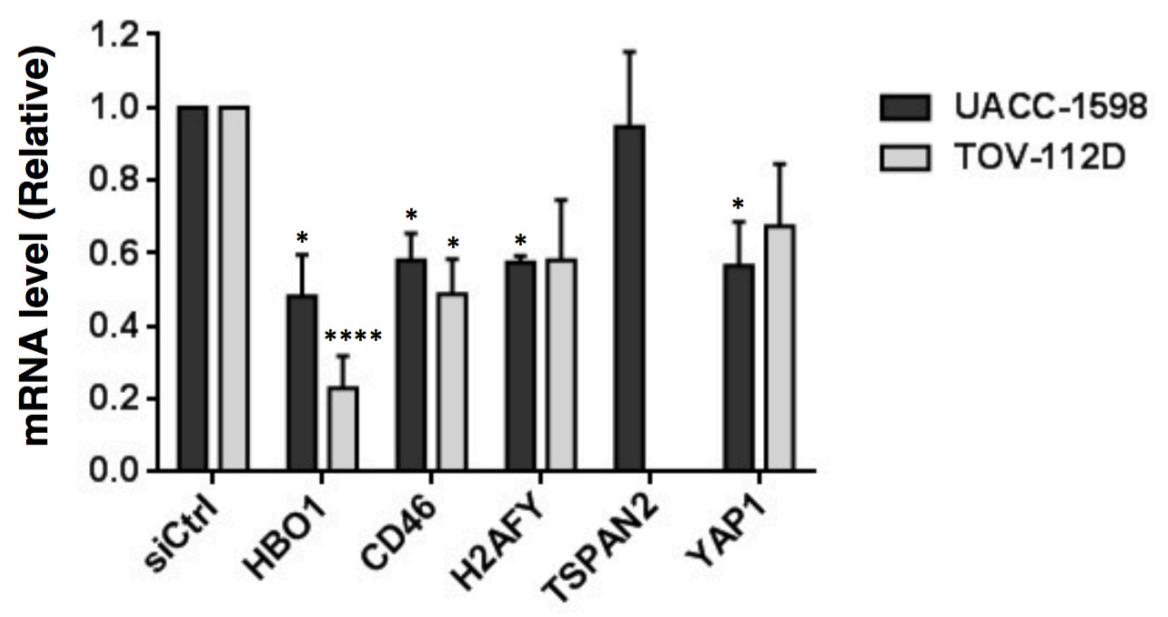

Figure 6. 9 Gene relative levels after HBO1 KD in UACC-1598 and TOV-112D UACC-1598 and TOV-112D cells were transfected with a pool of siRNAs targeting HBO1 ('siHBO1') and non-targeting siRNAs ('siCtrl'). Cell lysates were extracted after $48 \mathrm{~h}$ whereupon RNA samples were prepared and analysed by qRT-PCR to confirm the down-regulation of $H B O 1, C D 46, H 2 A F Y, Y A P 1$ and the up-regulation of TSPAN2. Relative levels of expression in cells treated with 'siHBO1' were compared to those of cells treated with 'siCtrl'. GAPDH expression was used as control. Statistical analysis consisted of one-way ANOVA and the Tukey's multiple comparison test. All values represent the mean \pm SD of three biological samples $(* p$ $\left.<0.05 ;{ }^{* * * *} \mathrm{p}<0.0001\right)$.

HBO1 KD in UACC-1598 resulted in the down-regulation of CD46 (FC $=-1.74,{ }^{*} \mathrm{p}$ $=0.0442), H 2 A F Y\left(\mathrm{FC}=-1.74,{ }^{*} \mathrm{p}=0.0418\right)$ and $Y A P 1\left(\mathrm{FC}=-1.81,{ }^{*} \mathrm{p}=0.0437\right)$ (Fig. 6.9). TSPAN2 (FC $=0.94 \pm 0.20, \mathrm{p}=0.6354$ ) levels were not increased, suggesting that the extension of the 'HBO1 OC signature' is limited to certain gene products or certain OC cell lines. HBO1 KD in the endometrioid cell line TOV$112 \mathrm{D}$ resulted in the down-regulation of $C D 46\left(\mathrm{FC}=-2.46,{ }^{*} \mathrm{p}=0.0284\right), H 2 A F Y$ $(\mathrm{FC}=-2.01, \mathrm{p}=0.0577)$ and YAP1 $(\mathrm{FC}=-1.34, \mathrm{p}=0.0522)$, whereas TSPAN2 gene transcripts could not be detected by qRT-PCR (Fig. 6.9).

These results suggest that tight regulation is exerted by HBO1 over a very specific subset of 'HBO1 OC signature' genes (CD46, H2AFY and YAPl) in all cell lines tested and could therefore represent key targets of HBO1 in OC development. 
Table 6. 6 Comparison of gene expression changes between OC cell lines

\begin{tabular}{|c|l|c|}
\hline Comparison & \multicolumn{1}{|c|}{ Common genes } & E $^{1}$ \\
\hline \multirow{2}{*}{$\begin{array}{c}\text { UWB1.289 and } \\
\text { OVCAR-3 (16) }\end{array}$} & OAS2, IFIT1, CXCL10, IFIT2, OAS1, MX1, IRF9, & \multirow{2}{|c|}{12} \\
\cline { 2 - 3 } & ABP5, GBP1, RASSF2, TLE4, MAP2 & \\
\hline \multirow{3}{*}{$\begin{array}{l}\text { UWB1.289 and } \\
\text { SKOV-3 (19) }\end{array}$} & ABTB1, CNTNAP1 & SAR2, LOC100133800, PGM2L1 \\
\cline { 2 - 3 } & NAPEPLD, LOC643668, PRMT6, LOC100133211, & 14 \\
\cline { 2 - 3 } & VPS36, SSR2, LOC345041, PRPF38A, F3 & \\
\hline OVCAR-3 and & SLNF5 & 1 \\
\cline { 2 - 3 } SKOV-3 (2) & LIFR & 1 \\
\hline
\end{tabular}

${ }^{1} \mathrm{E}$ : Expression. Red represents up-regulated genes in both microarrays. Blue represents down-regulated genes in both microarrays. Green represents common genes with opposing expression patterns between cell lines.

Two-way comparisons of each cell line with UWB1.289 revealed additional common gene sets of genes that were similar in size to the signature set (Table 6.6). Because of the limited size of all of the overlapping gene sets identified through functional mRNA analysis, it remained challenging to establish functional or regulatory relationships. Pathway analyses, which take into consideration all HBO1modulated transcripts for comparison, could provide a better perspective on the differences and similarities of the roles of HBO1 in OC cell lines and were therefore undertaken.

\subsection{Functional Analysis of Transient HBO1 Knockdown Profiles}

The large numbers of differentially expressed genes identified as a consequence of HBO1 depletion (Table 6.5) point to a potentially important regulatory role for HBO1, directly or indirectly suppressing and enhancing gene transcription in OC cells. Inspection of HBO1 UWB1.289 microarray data identified key target molecules involved in important regulatory functions, including genes associated with general cancer features (e.g. $C D K N 1 A / p 21$ or $M Y C$ ), particular OC characteristics (e.g. MUC16), histone acetylation (e.g. KAT2B) or HBO1 
functionality (e.g. $A R, I N G 4$ ). All six gene targets identified in the UWB1.289 microarray were validated via qRT-PCR: $\operatorname{KAT} 2 B(\mathrm{FC}=-2.39, * * * \mathrm{p}=0.0002)$ and MYC $\left(\mathrm{FC}=-1.65,{ }^{*} \mathrm{p}=0.0107\right)$ were similarly down-regulated, whereas CDKN1A $(\mathrm{FC}=2.32, * * * * \mathrm{p}<0.0001), \operatorname{MUC16}\left(\mathrm{FC}=2.68,{ }^{* * * *} \mathrm{p}=<0.0001\right), A R(\mathrm{FC}=2.32$, $\left.{ }^{* * *} \mathrm{p}=0.0001\right)$ and ING4 $\left(\mathrm{FC}=1.81,{ }^{* *} \mathrm{p}=0.0014\right)$ were also significantly upregulated (Fig. 6.10).

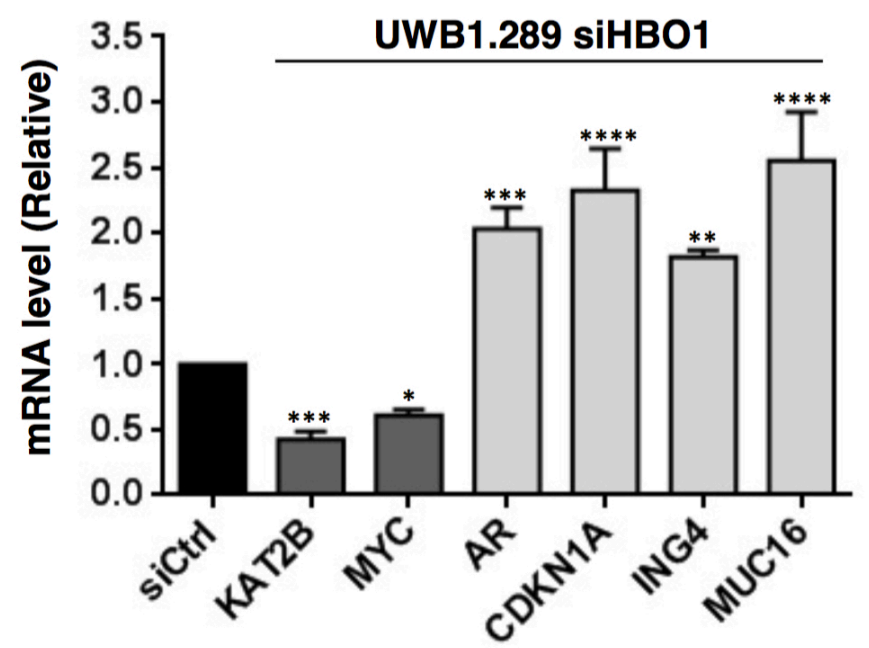

Figure 6. 10 Altered expression of key cancer genes caused by HBO1 KD UWB1.289 cells were transfected with a pool of siRNAs targeting $\mathrm{HBOl}$ ('siHBO1') and non-targeting siRNAs ('siCtrl'). Cell lysates were extracted after $48 \mathrm{~h}$ whereupon RNA samples were prepared and analysed by qRT-PCR to confirm the down-regulation of $K A T 2 B, M Y C$ and the up-regulation of $A R, C D K N 1 A, I N G 4$ and MUC16. Relative levels of expression in cells treated with 'siHBO1' were compared to those of cells treated with 'siCtrl'. GAPDH expression was used as control. Statistical analysis consisted of one-way ANOVA and the Tukey's multiple comparison test. All values represent the mean \pm SD of three biological samples $(* \mathrm{p}$ $\left.<0.05 ;{ }^{* *} \mathrm{p}<0.01 ;{ }^{* *} \mathrm{p}<0.001 ;{ }^{* * *} \mathrm{p}<0.0001\right)$.

In order to determine whether the modulatory activity of HBO1 over these genes was UWB1.289-cell type specific or a more wide regulatory phenomenon, their expression patterns were analysed by qRT-PCR in the rest of OC models. With the exception of $K A T 2 B$, a histone acetyltransferase that plays an important role in transcriptional regulation (Poux \& Marmorstein 2003), which was significantly down-regulated in all studied OC cell lines, none of the other gene targets were significantly altered in the serous-like cell lines OVCAR-3, SKOV-3, UACC-1598 or the endometrioid TOV-112D. These limited results suggest restricted implications 
of HBO1 in key cellular features. In order to elucidate which biological pathways are affected by HBO1 modulation, gene expression profiles were interrogated using two different pathway-prediction programmes: 'Ingenuity Pathway Analysis' (IPA) and 'Gene Set Enrichment Analysis' (GSEA), which is specifically designed for microarray profile analysis.

\subsubsection{Ingenuity Pathway Analysis of HBO1 knockdown profiles}

Firstly, the IPA 'Canonical pathways' feature predicted the significant activation/suppression of a number of pathways in UWB1.289 and OVCAR-3 cells (p-value $<0.05$ ) (Fig. 6.11). IPA did not detect any significantly altered canonical pathway or biological function in SKOV-3 cells, possibly due to the small number of differently expressed genes.

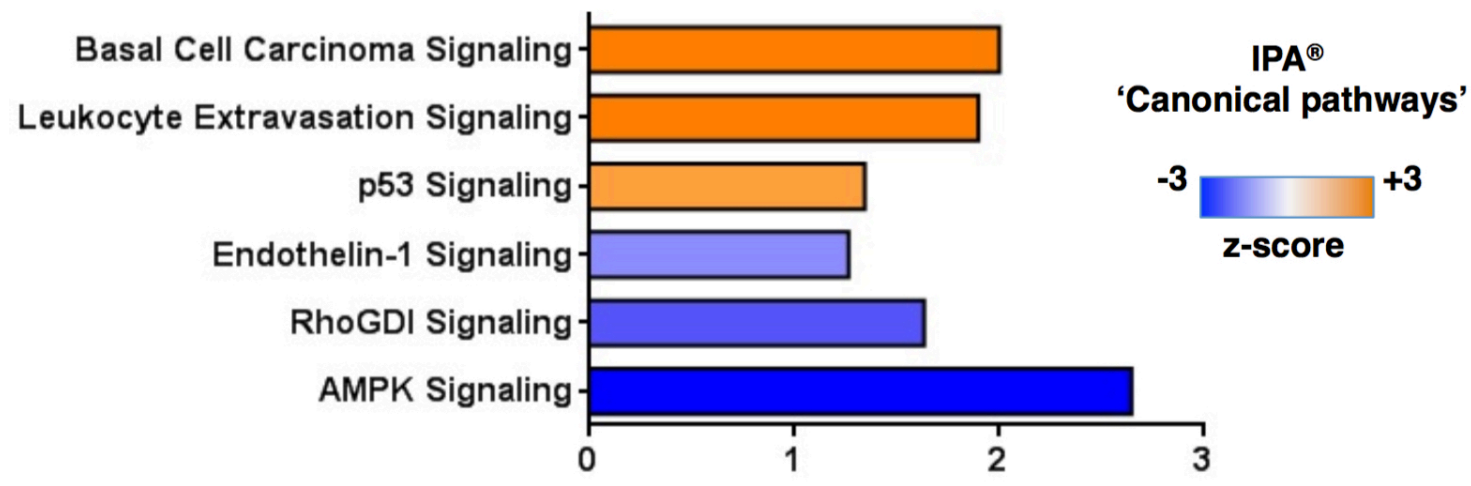

Figure 6. 11 IPA canonical pathways prediction after HBO1 KD in UWB1.289 The list of 889 differentially expressed genes (and their associated fold-change values) retrieved from the microarray analysis of HBO1 KD in UWB1.289 cells was uploaded into the IPA software. The IPA 'Canonical pathways' feature predicted pathway alterations following $\mathrm{HBO} 1 \mathrm{KD}$. Scale is shown to the right, where orange represents pathway activation and blue represents pathway inactivation.

The decrease of HBO1 levels in UWB1.289 cells was found to cause the significant activation of three canonical pathways: 'Basal cell carcinoma signalling' (Z-score $=$ $\left.2, \mathrm{p}=4.9 \times 10^{-02}\right)$, 'Leukocyte extravasation signalling' $\left(Z\right.$-score $=1.89, \mathrm{p}=2.2 \times 10^{-}$ ${ }^{02}$ ) and 'p53 signalling' $\left(Z\right.$-score $\left.=1.38, p=3.8 \times 10^{-02}\right)$ (Fig. 6.11). In addition, IPA predicted a decrease in activity of three signalling pathways, namely: Endothelin-1 $\left(\mathrm{Z}\right.$-score $\left.=1.26, \mathrm{p}=3.8 \times 10^{-02}\right)$, RhoGDI $\left(\mathrm{Z}\right.$-score $\left.=1.63, \mathrm{p}=4.8 \times 10^{-02}\right)$ and 'AMP- 
activated protein kinase' or AMPK $\left(Z\right.$-score $\left.=2.64, \mathrm{p}=4.9 \times 10^{-02}\right)$ (Fig. 6.11). Conversely, depletion of HBO1 in OVCAR-3 was found to cause the significant activation of 'Interferon Signalling' pathways $\left(\mathrm{Z}\right.$-score $\left.=3.46, \mathrm{p}=8.3 \times 10^{-07}\right)$.

Table 6. 7 List of common altered IPA functions after transient HBO1 KD

\begin{tabular}{|c|c|c|c|}
\hline \multirow{2}{*}{ Categories } & \multirow{2}{*}{ Biological function } & \multicolumn{2}{|c|}{ Z-score } \\
\cline { 3 - 4 } & & UWB1.289 & OVCAR-3 \\
\hline Organismal survival & Organismal death & -3.26 & -2.15 \\
Cellular movement & Migration of cells & 1.67 & 1.89 \\
Cellular movement & Invasion of cells & 2.01 & 2.14 \\
\hline
\end{tabular}

Next, the 'Downstream effects' feature of IPA was used to predict common biological functions affected by transient HBO1 KD in UWB1.289 and OVCAR-3 cells, which are summarized in Table 6.7 ( $p$-value $<0.05$ ). These data suggest prominent roles for $\mathrm{HBO} 1$ in $\mathrm{OC}$ cell viability (organismal death) and the suppression of cell movement (Table 6.7), a feature that had been already predicted (but not confirmed experimentally) in previous HBO1 KD studies (Fig. 5.6).
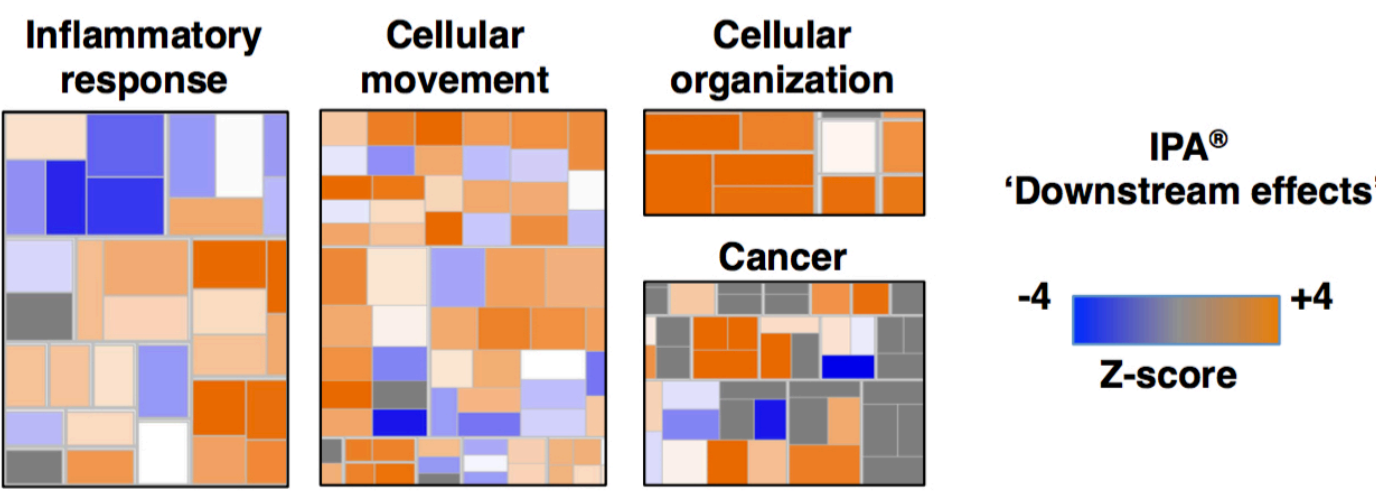

Figure 6. 12 IPA downstream effects prediction after HBO1 KD in UWB1.289

The list of 889 differentially expressed genes (and their associated fold-change values) retrieved from the microarray analysis of HBO1 KD in UWB1.289 cells was uploaded into the IPA software. The IPA 'Downstream effects' feature predicted alterations in biological functions, grouped according to IPA categories (e.g. Cellular movement), following HBO1 KD. Scale is shown next to the right panel, where orange represents pathway activation and blue represents pathway inactivation. Grey coloration represents non-affected biological functions. 
IPA 'Downstream effects' predictions in UWB1.289 cells alone infer the activation of biological processes including cellular movement and organization, cancer and inflammatory responses following transient $\mathrm{HBO} 1$ depletion (Fig. 6.12). Specifically, some of the activation predictions with the highest Z-score in UWB1.289 cells are associated with cellular motion, including the formation of filopodia $\left(\mathrm{p}=3.15 \times 10^{-04}\right)$, cell invasion $\left(\mathrm{p}=6.45 \times 10^{-04}\right)$ and metastasis $\left(\mathrm{p}=5.48 \times 10^{-}\right.$ ${ }^{04}$ ), suggesting a role for HBO1 in regulating the movement and spread of OC cells out of the ovary (Table 6.8). Furthermore, IPA predicted an augmentation in cytoplasm $\left(\mathrm{p}=4.85 \times 10^{-04}\right)$ and cytoskeleton $\left(\mathrm{p}=5.06 \times 10^{-05}\right)$ organization, pointing to HBO1-dependant re-arrangements of the cellular internal machinery (Table 6.8). A number of these characteristics had been previously predicted in the analyses of stable HBO1 KD in UWB1.289 cells (Table 5.2), where cytoskeleton alterations caused by the depletion of HBO1 were confirmed by AFM experiments (Fig. 5.7).

Table 6. 8 List of altered IPA functions after transient HBO1 KD

\begin{tabular}{|c|c|c|c|c|}
\hline & Categories & Biological function & Prediction & Z-score \\
\hline \multirow{4}{*}{ 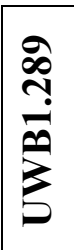 } & Cellular organization & Org. of cytoskeleton & Increased & 2.31 \\
\hline & Cellular organization & Org. of cytoplasm & Increased & 2.31 \\
\hline & Cancer & Metastasis of cells & Increased & 2.32 \\
\hline & Cellular movement & Formation of filopodia & Increased & 2.42 \\
\hline \multirow{4}{*}{$\frac{y}{3}$} & Infectious dise & \multirow{4}{*}{$\begin{array}{l}\text { Replication of viral RNA } \\
\text { Production of virus } \\
\text { Innate immune response } \\
\text { Leukocyte migration }\end{array}$} & Decre & -3.94 \\
\hline & Infectious diseases & & Decreased & -2.57 \\
\hline & Inflammatory response & & & 1.94 \\
\hline & Cellular movement & & Increased & 2.19 \\
\hline
\end{tabular}

In OVCAR-3 cells, IPA 'Downstream analysis' predictions pointed to a role for HBO1 in the activation of response against infectious diseases, since processes like 'replication of viral RNA' $\left(\mathrm{p}=1.02 \times 10^{-19}\right)$ and the 'production of viruses' $(\mathrm{p}=$ $1.97 \times 10^{-06}$ ) were predicted to decrease upon HBO1 depletion (Table 6.8). Conversely, a decrease in HBO1 levels was predicted to result in the activation of inflammatory responses, an effect also identified by IPA 'Canonical pathways'. An example of the inflammatory response is the activation of the innate immune response $\left(p=1.98 \times 10^{-09}\right)$ (Table 6.8). Another biological process identified was 
'Leukocyte migration' $\left(\mathrm{p}=3.77 \times 10^{-03}\right)$, which again links HBO1 with the regulation of cell motility (Table 6.8).

\subsubsection{Gene Set Enrichment Analysis of HBO1 knockdown profiles}

The GSEA software was used to predict which cellular functions were affected by the HBO1 KD loss-of-function phenotype in OC cells. To achieve this, each normalized microarray dataset was loaded into the GSEA programme, where it was converted to a ranked list of differentially expressed genes ('siHBO1' versus 'siCtrl'). Using the GSEA algorithm, this ranked gene list was compared sequentially with a collection of 'Hallmark Gene-Sets', representative of welldefined biological states or processes. If a large proportion of genes that belong to a 'Hallmark Gene-Set' are found at the top of the ranked list of differentially expressed genes, the GSEA prediction for this Gene-Set is activation. Conversely, if the genes were found at the bottom of the list, the Gene-Set prediction would be inactivation. Since the 'Hallmark Gene-Sets' represent biological states, processes or pathways, GSEA is therefore able to predict the activation/inactivation of biological processes or pathways.

Table 6. 9 List of common altered Gene-Sets following transient HBO1 KD

\begin{tabular}{|c|c|c|c|}
\hline \multirow{2}{*}{ Common Hallmark Gene-Sets } & \multicolumn{3}{|c|}{ Q-value } \\
\hline & OVCAR-3 & SKOV-3 & UWB1.289 \\
\hline IFN $\alpha$ response $^{1}$ & 0.0001 & 0.002 & 0.0001 \\
\hline IFN $\gamma$ response $^{1}$ & 0.0001 & 0.046 & 0.0001 \\
\hline k-RAS signalling ${ }^{2}$ & 0.0054 & 0.014 & 0.001 \\
\hline $\mathrm{EMT}^{3}$ & 0.0057 & 0.002 & 0.006 \\
\hline
\end{tabular}

${ }^{1}$ Genes up-regulated in response to IFN $\alpha / \gamma$

${ }^{2}$ Genes up-regulated by k-RAS activation

${ }^{3}$ Genes defining Epithelial-mesenchymal transition

GSEA analysis of OVCAR-3, SKOV-3 and UWB1.289 HBO1 KD profiles identified four common altered Gene-Sets, illustrated in Table 6.9. Amongst those, GSEA identified the up-regulation of genes associated to IFN $\alpha$ and IFN $\gamma$ responses, 
k-RAS signalling activation and epithelial-mesenchymal transition (EMT) (Table 6.9). The OVCAR-3 GSEA enrichment plot for the IFN $\alpha$ Gene-Set is illustrated in Figure 6.13 A, as well as the graphical depiction of some of the genes that integrate the corresponding Leading-edge Subset (57) (Fig. 6.13 B). GSEA did not predict common alterations of cellular pathways/processes linked to the differential downregulation of transcripts (bottom part of the ranked list), likely due to the small number of altered genes in some of the datasets and the diversity of associated functions.

A

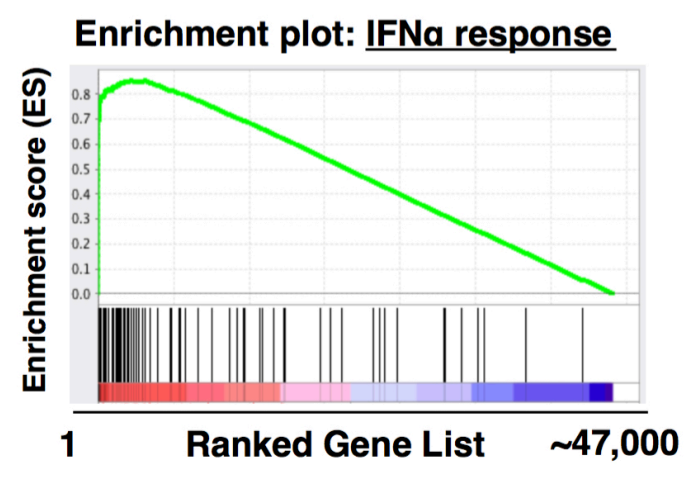

B

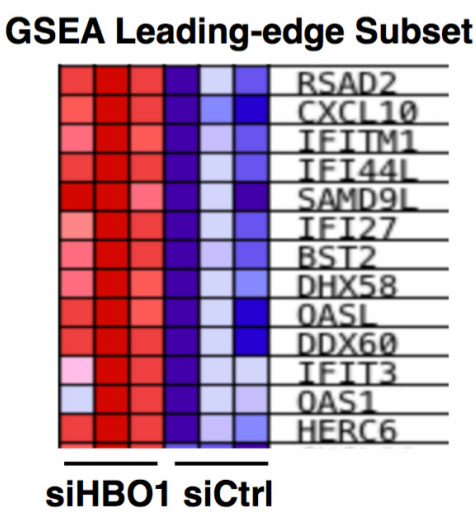

Figure 6. 13 Example of Gene-Set enrichment after HBO1 KD in OVCAR-3 Normalized microarray data obtained from the expression profiling of OVCAR-3 cells transfected with 'siHBO1' and 'siCtrl' was loaded into the GSEA programme. (A) The comparative analysis of this data with a series of 'Hallmarks Gene-Sets' resulted in GSEA predictions anticipating the significant activation of pathways/processes upon HBO1 depletion, including an IFNa response. (B) Example of the corresponding leading-edge subset. Out of the 96 genes that make up the IFN $\alpha$ response Gene-Set, 57 were grouped at the top of the ranked gene list. Red colour represents gene up-regulation, whereas blue colour represents downregulation.

Other GSEA predictions shared by at least two OC cell lines include the activation of 'tumour necrosis factor alpha' (TNF $\alpha)$ signalling, inflammatory responses and the TP53 network (Table 6.10), suggesting a general role of HBO1 in the inhibition of immune responses, inflammatory processes and 'TP53 pathways', mechanisms conventionally linked to OC progression. In addition, GSEA predicted the inactivation of the E2F pathway (Table 6.10), which would indicate a role of HBO1 in the regulation of E2F-related transcription. Interestingly, IPA had predicted the 
activation of some of these pathways, e.g. TP53 and inflammation (Table $6.10 \&$ Fig. 6.11). The fact that GSEA and IPA, which use different statistical methodology, predicted the stimulation of analogous pathways suggests a strong link between HBO1 and these processes in OC cells.

Table 6. 10 List of GSEA activated Gene-Sets after transient HBO1 KD

\begin{tabular}{|c|c|c|c|}
\hline \multirow{2}{*}{ Hallmark Gene-Sets } & \multicolumn{3}{|c|}{ Q-value } \\
\cline { 2 - 4 } & OVCAR-3 & SKOV-3 & UWB1.289 \\
\hline TNF $\alpha$ signalling via NF- $\kappa \beta^{1}$ & 0.002 & 0.002 & $-^{*}$ \\
\hline Inflammatory response $^{2}$ & 0.006 & - & 0.01 \\
\hline TP53 pathways ${ }^{3}$ & - & 0.004 & 0.026 \\
\hline E2F pathways $^{4}$ & - & 0.022 & 0.021 \\
\hline
\end{tabular}

* - Non identified Gene-Set

${ }^{1}$ Genes regulated by NF- $\kappa \beta$ in response to TNF $\alpha$ treatment

${ }^{2}$ Genes defining inflammatory response

${ }^{3}$ Genes involved in TP53 networks

${ }^{4}$ Genes encoding cell cycle related targets of E2F transcription factors

\subsubsection{Validation of HBO1 KD inflammatory responses}

The activation of immune and/or inflammatory responses (Tables 6.8, 6.9 \& 6.10) are often regarded as off-target effects of siRNA transfection (Marques \& Williams 2005; Robbins et al. 2009), and therefore the prediction analyses raised doubts about the specificity of potential HBO1 activities.

In order to dismiss the possibility that siRNAs caused off-target effects, the expression levels of ' $\mathrm{DExD} / \mathrm{H}-$ box helicase 60' (DDX60), 'interferon induced protein 44' (IFI44) and 'radical S-adenosyl methionine domain containing 2' (RSAD2), which have major roles in immune responses, were analysed in 'siHBO1', 'siCtrl' and 'Control' samples (Fig. 6.14). These genes are inducible via IFN signalling and were part of the GSEA leading-edge subset of genes predicted to activate the IFN $\alpha$ response (Fig. 6.13). 


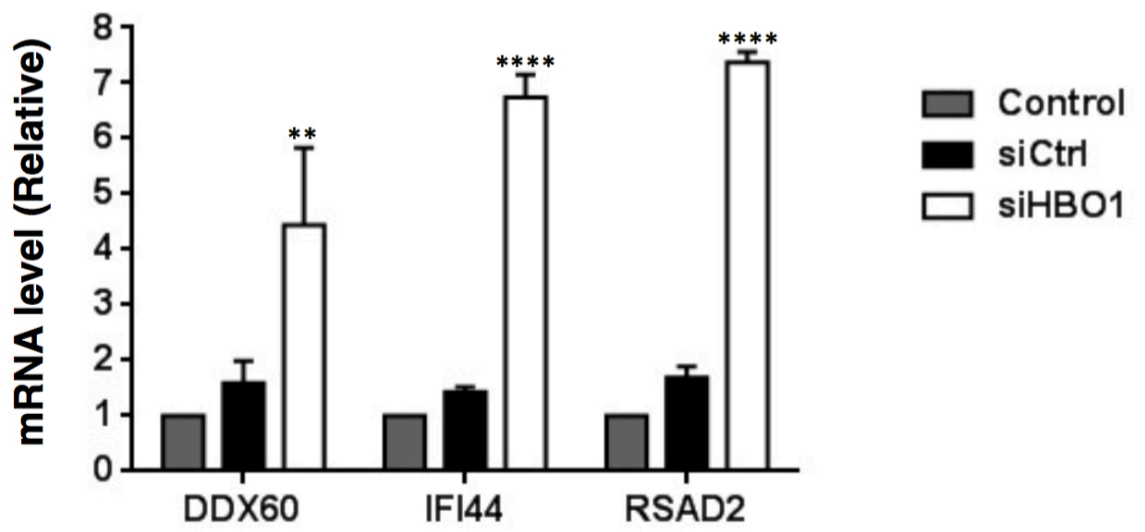

Figure 6. 14 Validation of IFN-related gene changes in OVCAR-3 cells OVCAR-3 cells were transfected with a pool of siRNAs targeting $\mathrm{HBOl}$ ('siHBO1'), as well as with non-targeting siRNAs ('siCtrl') and transfection reagent alone ('Control'). Cell lysates were extracted after $48 \mathrm{~h}$ whereupon RNA samples were prepared and analysed by qRT-PCR to confirm the up-regulation of $D D X 60$, IFI44 and RSAD2. Relative levels of expression in cells treated with 'siHBO1' were compared to those of cells treated with 'siCtrl' and 'Control'. RPL5 expression was used as control. Statistical analysis consisted of one-way ANOVA and the Tukey's multiple comparison test. All values represent the mean $\pm \mathrm{SD}$ of three biological samples $(* * \mathrm{p}<0.01 ; * * * * \mathrm{p}<0.0001)$.

The expression level of gene transcripts DDX60 (FC =1.59, $\mathrm{p}=0.8036)$, IFI44 (FC $=1.43, \mathrm{p}=0.9274)$ and RSAD2 $(\mathrm{FC}=1.69, \mathrm{p}=0.6609)$ was very similar in the 'siCtrl' and the transfection reagent alone samples ('Control') (Fig. 6.14). This largely suggests a role for $\mathrm{HBO} 1$ in this cellular response process, with $D D X 60$ (FC $\left.=4.44,{ }^{* *} \mathrm{p}=0.0017\right), \operatorname{IFI} 44\left(\mathrm{FC}=6.74,{ }^{* * * *} \mathrm{p}<0.0001\right)$ and RSAD2 $(\mathrm{FC}=7.37$, $* * * \mathrm{p}<0.0001)$ significantly up-regulated in the 'siHBO1' sample compared to the 'Control' (Fig. 6.14).

\subsection{In vitro Evaluation of HBO1-predicted Biological Functions}

Based on IPA and GSEA predictions on the activation and/or inactivation of similar biological processes and pathways, a number of in vitro assays were undertaken to assess the motility, invasiveness, viability and inflammatory responses of $\mathrm{HBO} 1 \mathrm{KD}$ cells. 


\subsubsection{Assessment of HBO1 suggested role in cell proliferation}

HBO1 has an essential role in DNA replication (Miotto \& Struhl 2010) suggesting the possibility that rates of cell proliferation may become altered as a result of HBO1 KD. To investigate this possibility, UWB1.289, OVCAR-3 and SKOV-3 cells were transfected with the correspondent siRNAs ('siHBO1' and 'siCtrl') for 48h, followed by the addition of $\mathrm{BrdU}$ for $4 \mathrm{~h}$ and $24 \mathrm{~h}$ and the measurement of the resultant absorbance. The proliferation assay is based on the use of the labelled nucleoside BrdU and its capability to incorporate into newly synthesized DNA of replicating cells.

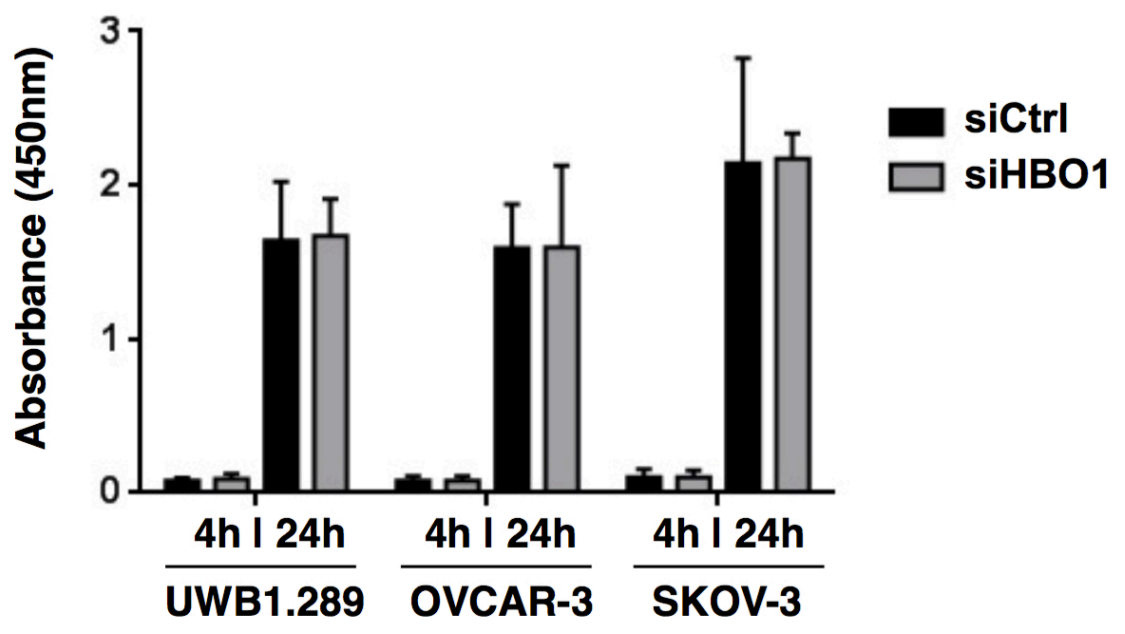

Figure 6. 15 Study of cell proliferation after HBO1 KD in OC cells

UWB1.289, OVCAR-3 and SKOV-3 cells were transfected with a pool of siRNAs targeting $\mathrm{HBOl}$ ('siHBO1') and non-targeting siRNAs ('siCtrl'). After 48h, BrdU was added to the cells for $4 \mathrm{~h}$ and $24 \mathrm{~h}$ to assess cell proliferation at $450 \mathrm{~nm}$. Absorbance was measured in duplicates.

The comparison of absorbance values between cells labelled with BrdU for 4 and 24 hours demonstrated the incorporation into active replicative cells (Fig. 6.15). However, the transient loss of HBO1 did not significantly affect the rates of cell proliferation of any of the studied cell lines (Fig. 6.15), a similar result to that obtained previously when using a stable HBO1 KD approach in UWB1.289 cells (Fig. 5.5). These findings support IPA and GSEA predictions, which did not identify cell proliferation changes as a consequence of $\mathrm{HBO} 1 \mathrm{KD}$. 
The results of cell proliferation studies further confirm that HBO1 is not essential for DNA replication in OC cells and point to a non-proliferative role for overexpressed $\mathrm{HBO} 1$ in $\mathrm{OC}$.

\subsubsection{Assessment of $\mathrm{HBO} 1$ predicted involvement in cell motility}

In order to evaluate the IPA predicted role for HBO1 in cellular movement (Table 6.7), phenotypical changes were measured using a scratch/wound-healing assay, which measures cell migration in vitro. First, UWB1.289, OVCAR-3 and SKOV-3 were transfected with the correspondent siRNAs ('siHBO1' and 'siCtrl') for $24 \mathrm{~h}$, whereupon cells were subjected to trypsin treatment for detachment and subsequent re-seeding aiming to obtain a confluent cell monolayer in the next $24 \mathrm{~h}$. After this (48h of siRNA transfection), a scratch was made and differences in cell migration were analysed (Fig. 6.16). In order to confirm the perpetuation of transient HBO1 KD post-trypsin treatment, samples of re-seeded cells were extracted and analysed using qRT-PCR; the transcript levels of HBO1 remained very low (not shown).

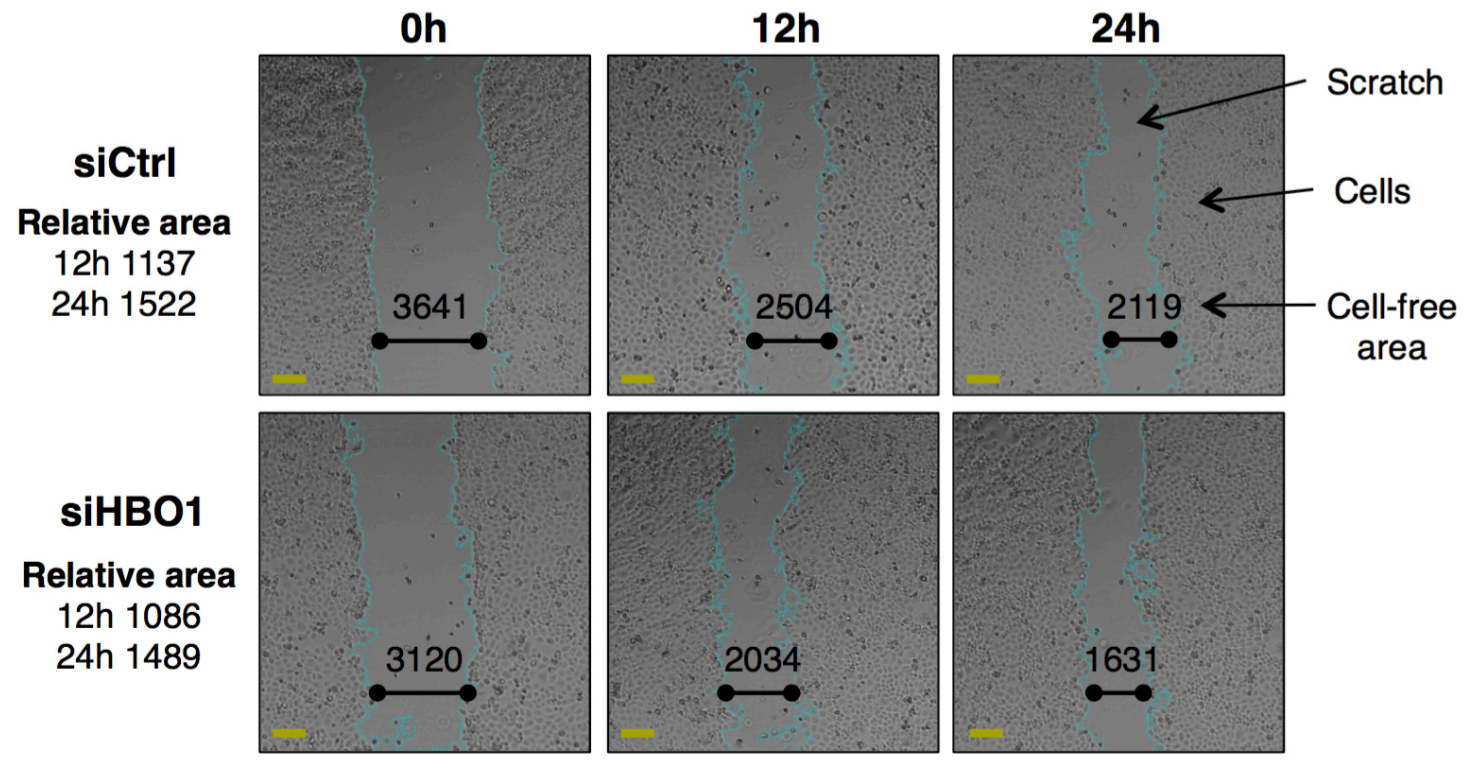

Figure 6. 16 Example of wound-healing assay after HBO1 KD in OVCAR-3

OVCAR-3 cells were transfected with a pool of siRNAs targeting $\mathrm{HBO1}$ ('siHBO1') and non-targeting siRNAs ('siCtrl'). After $48 \mathrm{~h}$, the confluent cell monolayer was partially removed by a pipette tip (scratch). Images were taken immediately after the scratch $(\mathrm{t}=0 \mathrm{~h})$ and after $12-24 \mathrm{~h}$; Image J software was used to calculate cell-free area values. The relative area was calculated subtracting cell-free area values at 12$24 \mathrm{~h}$ from cell-free area values at $\mathrm{t}=0 \mathrm{~h}$. Scales represent $100 \mu \mathrm{m}$. 
Analyses of wound-healing data did not reveal any differences in scratch closure as determined by the relative areas (RA) of 'siHBO1' and 'siCtrl' transfected cells 12 or 24 hours after the scratch in any of the studied cell lines (example of OVCAR-3 assay in Figure 6.16). The analyses of pathway dysregulation elicited by HBO1 KD in the SKOV-3 cell line did not include alterations in cell migratory properties (Table 6.7), which could explain for the absence of wound-healing assay differences in cell populations of SKOV-3. However, both OVCAR-3 and UWB1.289 KD profile analyses predicted an activation of gross cell migration (Table 6.7), but corresponding phenotypic assay failed to show the expected changes. This lack of significant discernible differences was also observed in previous scratch assays undertaken with shRNA-transduced UWB1.289 cells (Fig. 5.6).

Specifically, the differential expression of 25 (OVCAR-3) and 30 (UWB1.289) genes was associated with the activation of cell migration (Table 6.7). In OVCAR-3, some of these genes included: up-regulated BST2 $(\mathrm{FC}=2.79)$, the ' $\mathrm{C}-\mathrm{X}-\mathrm{C}$ motif chemokine 11' $(C X C L 11, \mathrm{FC}=1.8)$, the 'lymphoid enhancer binding factor 1' $(L E F 1, \mathrm{FC}=1.67)$ and STAT1 $(\mathrm{FC}=1.97)$, as well as down-regulated keratin 16 $(K R T 16, \mathrm{FC}=-1.5)$ and endothelin-2 $(E D N 2, \mathrm{FC}=-1.72)$. This group of genes displayed significant up/down-regulatory trends -some of which were corroborated using qRT-PCR - and have been previously linked to the promotion of cell migration (X. Gao et al. 2014). However, alterations of this set of genes alone may not have the ability to drive gross cell motility changes that can be observed in vitro. This suggestion is supported by the low z-score values obtained from the pathway analysis (Cell migration: OVCAR-3 1.89, UWB1.289 1.67; Table 6.7), which highlight the weakness of the prediction. Accordingly, HBO1 does not appear to be controlling this pathway in OC cells.

\subsubsection{Assessment of HBO1 predicted involvement in cellular invasion}

In order to evaluate the IPA predicted role for HBO1 in cellular invasion (Table 6.7), a CytoSelect ${ }^{\mathrm{TM}}$ cell invasion assay was undertaken. This assay utilises a basement membrane that serves as a barrier to discriminate invasive from noninvasive cells, depending on their ability to degrade proteins in the layer and pass through the generated pores. 
First, UWB1.289, OVCAR-3 and SKOV-3 were transfected with the correspondent siRNAs ('siHBO1' and 'siCtrl') for 24h, whereupon cells were subjected to trypsin treatment for detachment. Subsequently, cell suspensions $\left(7 \times 10^{5}\right.$ cells $\left./ \mathrm{mL}\right)$ were added to the basement membrane layer for $24 \mathrm{~h}$ or $48 \mathrm{~h}$ of incubation.

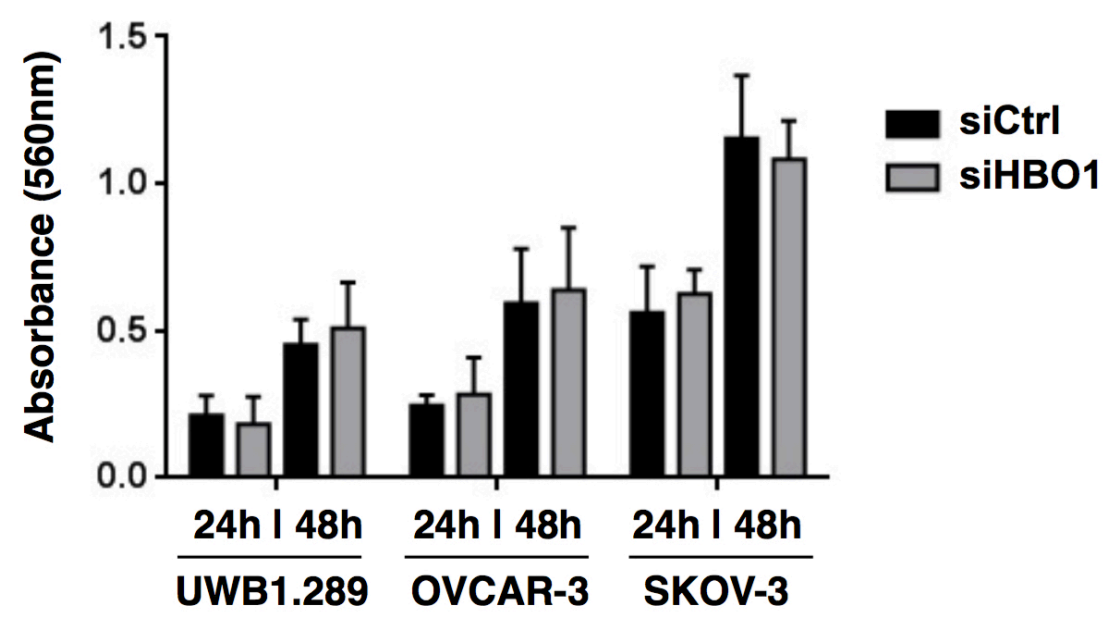

Figure 6. 17 Study of cell invasiveness after HBO1 KD in OC cells

UWB1.289, OVCAR-3 and SKOV-3 cells were transfected with a pool of siRNAs targeting $\mathrm{HBOl}$ ('siHBO1') and non-targeting siRNAs ('siCtrl'). After 24h, cells were transferred to CytoSelect ${ }^{\mathrm{TM}}$ wells containing a basement membrane layer and allowed to invade toward 10\% FBS for 24 and $48 \mathrm{~h}$. Invasive cells on the bottom of the invasion membrane were stained and quantified at $560 \mathrm{~nm}$. Absorbance of the final solution was measured in duplicates.

Measuring the absorbance of the solutions containing invasive cells that have migrated through the membrane did not reveal any differences between KD and control samples in any of the studied cell lines (Fig. 6.17). Similar to the previous situation with cell migration predictions, pathway analyses of the SKOV-3 KD profile did not anticipate alterations in the invasive properties of cells, suggesting that HBO1 is not involved in this pathway in SKOV-3 cells. On the other hand, OVCAR-3 and UWB1.289 KD profile analyses predicted an activation of gross cell invasion (Table 6.7), but corresponding phenotypic assay failed to show the expected changes (Fig. 6.17).

The differential expression of 22 (OVCAR-3) and 98 (UWB1.289) genes was associated with the activation of cell invasion (Table 6.7). In UWB1.289, some of 
these genes included: up-regulated matrix metallopeptidases 2/9 $(M M P 2, \mathrm{FC}=1.52$, $M M P 9 \mathrm{FC}=1.80), \operatorname{MUC16}(\mathrm{FC}=2.40)$ and E-cadherin $(C D H 1, \mathrm{FC}=1.67)$, as well as down-regulated $Y A P 1(\mathrm{FC}=-1.84)$ and the 'snail family transcriptional repressor 2' (SNAI2, FC = -1.65). In this case, both z-score values for predicted activation were significantly higher than those anticipating changes in cell migration (Cell invasion: OVCAR-3 2.14, UWB1.289 2.01; Table 6.7), and yet these findings suggest that HBO1 is not significantly linked to cell invasion in OC. However, since invasiveness requires several distinct cellular functions including adhesion, motility, detachment and extracellular matrix proteolysis, it is possible that HBO1 may be involved in the regulation of other processes, although it is not able to affect the process of cellular invasion in the studied in vitro conditions.

\subsubsection{Assessment of $\mathrm{HBO1}$ predicted involvement in cell viability}

IPA 'Downstream effects' analysis predicted that transient $\mathrm{KD}$ of $\mathrm{HBO}$ in UWB1.289 and OVCAR-3 lines might affect cell viability (Table 6.7: Biological function - Organismal survival). In order to evaluate this prediction in vitro, UWB1.289, OVCAR-3 and SKOV-3 cells were transfected with the correspondent siRNAs ('siHBO1' and 'siCtrl') for $48 \mathrm{~h}$, whereupon cells were subjected to the ApoTox-Glo ${ }^{\mathrm{TM}}$ triplex assay. This test combines three assay chemistries to easily assess cell viability, cytotoxicity and apoptosis events (caspase activity) in the same cell-based assay well.

The decrease in HBO1 transcript and protein levels led to a significant augmentation of cell viability in UWB1.289 (*p $=0.0238)$, OVCAR-3 $(* p=0.0144)$ and SKOV-3 $(* p=0.0482)($ Fig. 6.18 A). This increase in viability was accompanied by a significant reduction in cell cytotoxicity in the UWB1.289 cell line $\left({ }^{*} \mathrm{p}=0.0369\right)$, whereas both OVCAR-3 $(\mathrm{p}=0.7386)$ and SKOV-3 $(\mathrm{p}=0.8842)$ displayed substantial variability (Fig. 6.18 B). All three OC cell lines showed a decline of caspase activity, indicating a general reduction of apoptotic processes: UWB1.289 $\left({ }^{*} p=0.0314\right)$, OVCAR-3 (*p $\left.=0.0478\right), \operatorname{SKOV}-3(p=0.0537)$ (Fig. 6.18 C). 
A

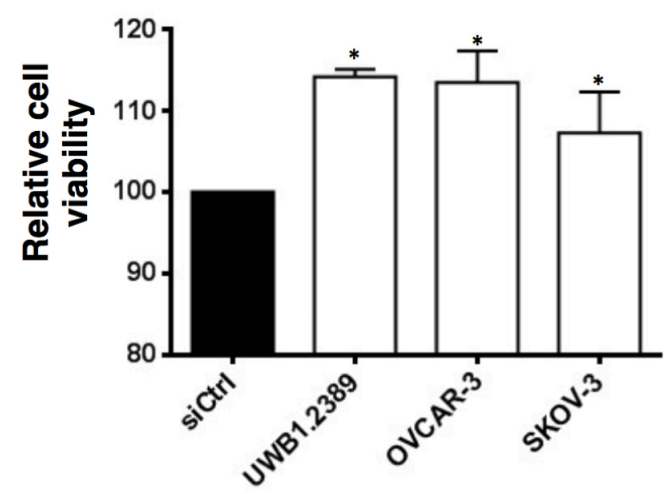

C

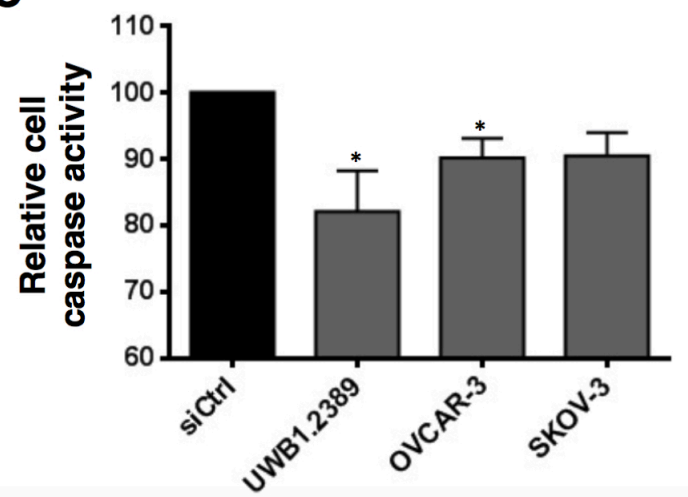

B

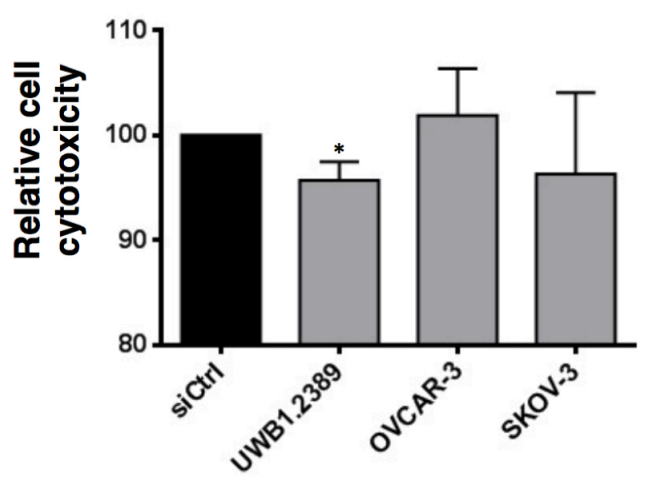

D

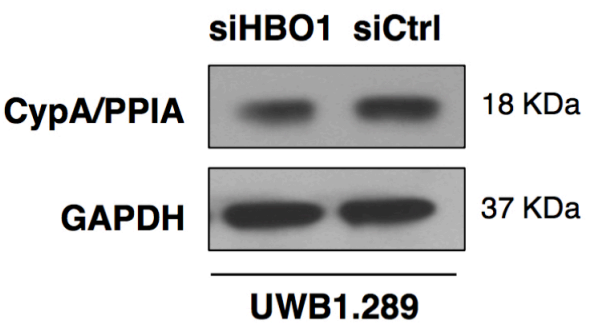

Figure 6. 18 Study of cell viability, cytotoxicity and apoptosis after HBO1 KD UWB1.289, OVCAR-3 and SKOV-3 cells were transfected with a pool of siRNAs targeting $\mathrm{HBOl}$ ('siHBO1') and non-targeting siRNAs ('siCtrl'). After 48h, changes in $(\mathbf{A})$ cell viability, $(\mathbf{B})$ cytotoxicity and $(\mathbf{C})$ caspase activity were measured using the ApoTox-Glo ${ }^{\mathrm{TM}}$ triplex assay. Relative cell values represent proportional differences between HBO1 KD and the correspondent 'siCtrl' raw measurements; only one control was plotted for simplification. (D) UWB1.289 protein extracts were analysed by western blot and probed with anti-CypA and anti-GAPDH antibodies. GAPDH was used as loading control. Statistical analyses consisted of one-way ANOVA and Tukey's multiple comparison tests. All values represent the mean \pm SD of three biological samples $\left({ }^{*} \mathrm{p}<0.05 ;{ }^{* *} \mathrm{p}<0.01\right)$.

To further investigate the reduction of cell death in HBO1 KD UWB1.289, protein lysates were analysed for Cyclophilin A (CyPA or PPIA). This protein is a biomarker of necrotic death or necroptosis, a caspase-independent form of programmed cell death linked to inflammatory processes (Christofferson \& Yuan 2010). HBO1 KD in UWB1.289 cells led to a significant reduction in the protein levels of CyPA/PPIA $\left(\mathrm{RD}=39.35 \pm 6.64 \%,{ }^{*} \mathrm{p}=0.04\right)$ (Fig. 6.18 D), supporting the ApoTox-Glo ${ }^{\mathrm{TM}}$ results that pointed to UWB1.289 cells being generally less apoptotic in the absence of HBO1. 
These findings support a general role for HBO1 in the regulation of cell viability, agreeing with in silico predictions that linked HBO1 KD with organismal death (assuming that the death of an organism implies the death of the different cells that compose it) in the three studied cell lines (Table 6.7).

\subsubsection{Assessment of $\mathrm{HBO} 1$ predicted involvement in inflammatory responses}

HBO1 KD in UWB1.289, OVCAR-3 and SKOV-3 cell lines led to the up-regulation of genes involved in the response to IFN $\alpha$ and IFN $\gamma$ (Table 6.9). Cellular membrane interactions of different cytokines are known to elicit the activation of IFN pathways, which trigger anti-viral, anti-tumour, anti-proliferative and immune-regulatory functions in cells, as well as the release of new IFN molecules (Haller et al. 2006; Kalliolias \& Ivashkiv 2010). In order to evaluate whether the response to IFN $\alpha$ and IFN $\gamma$ in OC cells triggers the secretion of newly synthesized IFNs, enzyme-linked immune-sorbent assays (ELISAs) were performed.

Prior to the ELISAs, IFNA, IFNG and IFNB (non-activated control) expression levels of untreated cells were determined by qRT-PCR in UWB1.289, OVCAR-3 and SKOV-3. IFNG was not present in any of the cell lines, suggesting very low expression levels that would make it difficult to detect via ELISA. These results are not uncommon, since the expression of type II IFNG is known to be limited to immune cells (e.g. T cells) (Schroder et al. 2003). In contrast, IFNB expression was detected in all OC cell lines, whereas IFNA expression was limited to OVCAR-3 and SKOV-3.

Following HBO1 KD in UWB1.289, OVCAR-3 and SKOV-3 cells, the culture medium was analysed for IFNs, and IFN cellular mRNA levels determined (Fig. 6.19). HBO1 KD led to a significant increase of IFNA transcripts in OVCAR-3 cells $\left(\mathrm{FC}=1.59,{ }^{*} \mathrm{p}=0.0478\right)($ Fig. 6.19 A). UWB1.289 cells did not express any detectable IFNA transcripts, while the expression levels in SKOV-3 cells appeared variable between experiments $(\mathrm{FC}=2.09, \mathrm{p}=0.7731)$. None of the $\mathrm{OC}$ cells lines displayed significant changes in IFNB expression: UWB1.289 $(\mathrm{FC}=0.962, \mathrm{p}=$ 0.7941), OVCAR-3 (FC = 1.12, p = 0.8566), SKOV-3 $(\mathrm{FC}=1.35, \mathrm{p}=0.7689)($ Fig. 6.19 A). 
A

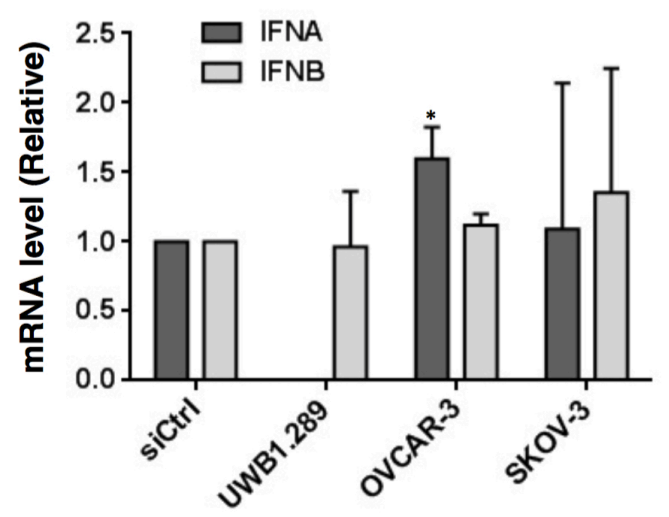

B

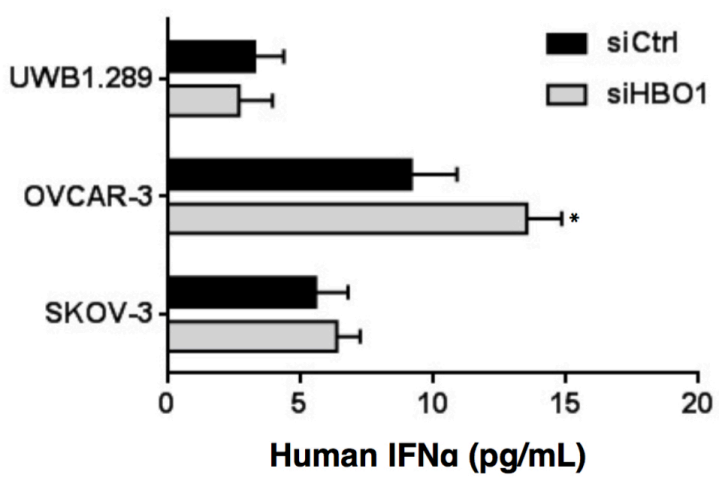

Figure 6. 19 IFN production and secretion after HBO1 KD in OC cells

UWB1.289, OVCAR-3 and SKOV-3 cells were transfected with a pool of siRNAs targeting HBOl ('siHBO1') and non-targeting siRNAs ('siCtrl'). (A) Cell lysates were extracted after $48 \mathrm{~h}$ whereupon RNA samples were prepared and analysed by qRT-PCR to assess the expression of $I F N A / B$. Relative levels of expression in cells treated with 'siHBO1' were compared to those of cells treated with 'siCtrl'. GAPDH expression was used as control. (B) Cell media was extracted after $48 \mathrm{~h}$ and analysed by ELISA. Sample measurements were compared to standard curves. Statistical analyses consisted of one-way ANOVA and Tukey's multiple comparison tests. All values represent the mean $\pm \mathrm{SD}$ of three biological samples $\left({ }^{*} \mathrm{p}<0.05\right)$.

The increase in IFNA expression levels in OVCAR-3 cells (Fig. 6.19 A) corresponded with the detection of higher levels of IFN $\alpha$ in the sample medium (*p $=0.0437$ ) (Fig. 6.19 B). For UWB1.289 and SKOV-3 cell lines, there were no significant differences in IFN $\alpha$ concentration in the media (Fig. 6.19 B). Similarly, IFN $\beta$-ELISA experiments revealed no differences and IFN $\gamma$ was undetectable by ELISA in all OC cell lines. These data support the predicted role of HBO1 modulating IFNa in OVCAR-3 cells but not in UWB1.289 and SKOV-3, demonstrating that there are limitations suggesting inaccuracies in the prediction software. 


\subsection{Discussion}

In order to further extend our knowledge about the roles of $\mathrm{HBO} 1$ in $\mathrm{OC}$, UWB1.289, OVCAR-3 and SKOV-3 cells were transfected with siRNAs targeting $\mathrm{HBO1}$ and subsequently monitored for gene and phenotypical alterations. First, nucleosome targets histone $\mathrm{H} 4$ and H3K14 were evaluated using acetylation-specific antibodies. Transient $\mathrm{HBO} 1 \mathrm{KD}$ resulted in the decrease of $\mathrm{H} 4$ acetylated residues in all OC cell lines (Fig. 6.1 \& Fig. 6.2), supporting a major role for HBO1 in the regulation of cellular $\mathrm{H} 4$ bulk acetylation. These results agree with previous findings observed in UWB1.289 cells expressing shRNAs targeting HBOI (Fig. 5.1 \& Fig. 5.2), as well as with a number of previous RNAi-based studies (Table 1.7). Conversely, and again in accordance with previous observations (Fig. 5.1 \& Fig. 5.2), HBO1 transient KD did not affect histone H3K14 acetylation (Fig. 6.1 \& Fig. 6.2).

Microarray analyses of gene expression changes caused by $\mathrm{HBO} 1$ transient depletion identified asymmetrical, yet compelling numbers of differentially expressed genes (Table 6.5). The largest number of HBO1 KD altered transcripts (889) was identified in the UWB1.289 cell line (Table 6.4), whereas a much smaller number of altered transcripts were identified in OVCAR-3 (155) and SKOV-3 (80). The number of genes identified was higher using this approach compared to using an shRNA approach (Table 5.1), which may be due to the differences in reduction of HBO1 expression levels, as well as the use of different cell lines. These differences could also reflect distinctive function for the HAT in diverse cancer stages/histotypes, which remains to be elucidated in primary tumour samples.

In order to analyse in depth the roles of $\mathrm{HBO} 1$ in OC, pathway analyses were implemented, followed by the in vitro confirmation of predicted phenotypes. IPA and GSEA analyses of the transient HBO1 KD expression profiles predicted differences and similarities on the functions of HBO1 in the three studied OC cell lines (Tables 6.7, 6.8, $6.9 \& 6.10$ ). Interestingly, some of these predictions (activated cell movement, reduction of cell death) were also anticipated by IPA analysis of HBO1 dependent genes in HeLa cells (Avvakumov et al. 2012), suggesting that certain HBO1-associated functions can be extrapolated to other cell lines with 
different origins. Notably, GSEA predicted common HBO1 dependent effects on (1) IFN responses, (2) k-RAS signalling and (3) the activation of epithelialmesenchymal transition (Table 6.9).

IFNs are a group of pleiotropic cytokines released in response to the presence of pathogens (e.g. viruses, bacteria and also tumour cells) with the consequence of causing nearby cells to enhance their anti-pathogenic defences (Haller et al. 2006). IFN signalling regulates the expression of many genes that could affect tumour cell growth, proliferation, differentiation, survival, migration or other specialized functions and interestingly, IFN signalling can either stimulate or suppress cellular immune response, depending on the targets of the IFN effects (e.g. cancerous, immune cells) and the nature of the IFN signalling pathway (Minn 2015). These data suggest that overexpressed HBO1 in OC could act as a negative regulator of: antitumorigenic immune-stimulatory signals or pro-tumorigenic immune-suppression. The negative regulation of IFN production (e.g. $I F N B$ ) was previously observed with other HATs such as GCN5 and PCAF in several cell types (Jin et al. 2014). Interestingly, $P C A F$ was down-regulated after $\mathrm{HBO} 1 \mathrm{KD}$ in all studied $\mathrm{OC}$ cell lines, suggesting a link between HBO1, PCAF and the inhibition of immune signalling. Nonetheless, the activation of IFN signalling was only confirmed in OVCAR-3 cells using IFN $\alpha$-ELISA (Fig. 6.19).

The activation of k-RAS signalling pathways involves the up-regulation of genes commonly associated with k-RAS activation, an oncogene involved in proliferation, differentiation, survival and other essential cellular mechanisms (Karnoub \& Weinberg 2008), suggesting a role for HBO1 in the inhibition of oncogenic transformation. Interestingly, the over-expression of HBO1 was shown to have a modest inhibitory effect on the h-RAS induced transformation of NIH-3T3 fibroblast cells (Johmura et al. 2008), highlighting the possible links of another member of the RAS signalling family with $\mathrm{HBO} 1$ and cancer. 
EMT is a biologic process that causes epithelial cells to lose their polarity and intercellular adhesion capabilities, subsequently driving their migratory and invasive properties as mesenchymal stem cells (Yeung \& Yang 2017). The up-regulation of genes that define epithelial-mesenchymal transition upon HBO1 KD suggests the involvement of $\mathrm{HBO} 1$ in the $\mathrm{OC}$-associated acquisition of invasive properties. Interestingly, previous analysis of stable $\mathrm{HBO} 1 \mathrm{KD}$ phenotypes also pinpointed this characteristic role (Fig. 5.7), highlighting the importance of HBO1 in cancer differentiation. In fact, the association of $\mathrm{HBO} 1$ with cancer differentiation has been previously shown in 76NE6-LMW-E breast cancer cells (Table 1.7), where HBO1 $\mathrm{KD}$ reduced cancer stem cell properties (e.g. mammosphere formation) and the expression of certain EMT gene markers, including CCNE1, E-cadherin ( $C D H 1), \mathrm{N}-$ cadherin $(\mathrm{CDH} 2)$, vimentin (VIM), the 'twist family BHLH transcription factor 1 ' (TWIST1) and the 'snail family transcriptional repressor 2' (SNAI2) (Duong et al. 2013).

Some of these effects had also been predicted using IPA in one or two OC cell lines. IPA predicted the significant activation of IFN signalling pathways in OVCAR-3 and the enhancement of cell migration/invasion in both OVCAR-3 and UWB1.289 (Table 6.7). However, and similar to the shRNA approach on UWB1.289 cells, the predicted increase in cellular migratory and invasive capacities was not confirmed by scratch/wound healing or invasion assays in UWB1.289, OVCAR-3 or SKOV-3 cells (Fig. 6.16 \& Fig. 6.17). Interestingly, the IPA 'Downstream effects' predicted HBO1-dependent changes in cell viability in OVCAR-3 and UWB1.289, a feature that was confirmed by viability, cytotoxicity and caspase activity assays in the three OC cell lines (Fig. 6.18). These results suggest a role for HBO1 in a live-dead balance and specifically point to repressive actions on the apoptotic-related death of cancerous cells. In this regard, it is worth highlighting the role of HBO1 as a regulator of $C D K N 1 A / p 21$, a TP53 transcription target implicated in cell cycle arrest and apoptosis inhibition (Jänicke et al. 2007; Abbas \& Dutta 2009). CDKN1A was up-regulated following transient $\mathrm{HBO} 1 \mathrm{KD}$ in UWB1.289 cells (Fig. 6.10), a phenomenon that agrees with the observed diminution of caspase activity (Fig 6.18). 
The considerable number of differentially expressed genes identified after HBO1 KD in UWB1.289 resulted in the highest number of predictions for the involvement of HBO1 in specific processes. For instance, IPA 'Canonical pathways' predicted the significant alteration of 6 biological pathways in UWB1.289 (Fig 6.11), of which the three potentially activated processes (basal cell carcinoma, leukocyte extravasation and p53 signalling) involve well-known cancer features and suggest a role for $\mathrm{HBO} 1$ in oncogenic transformation. The development of basal cell carcinoma, and therefore the activation of 'basal cell carcinoma signalling' pathways, is related to the deregulation of the sonic hedgehog pathway, whose activation is implicated in several cancers and entails the increase of stem-like characteristics of cancer cells (Epstein 2008). Interestingly, tumorigenic activation of the sonic hedgehog pathway has been reported in OC (Schmid et al. 2011), suggesting potential links between HBO1 and components of the pathway. Leukocyte extravasation is the movement of leukocytes out of the circulatory system and to the site of injury or infection, in order to trigger the subsequent immune response (Muller 2013). Accordingly, predicted HBO1associated suppression of this phenomenon in UWB1.289 cells agrees with previous observations that linked HBO1 with the inhibition of immune responses. The activation of 'TP53 signalling' pathways following HBO1 KD, also predicted by GSEA in SKOV-3 and UWB1.289 (Table 6.10), suggests a role for HBO1 in the regulation of the TP53-CDKN1A axis.

Predictions regarding process inhibition upon HBO1 KD (Endothelin-1, RhoGDI and AMPK signalling) are also involved in cancer progression, suggesting conflicting roles for HBO1 in OC. The endothelin-1 (EDN1) axis is involved in cancerous cell proliferation, survival and neovascularization, amongst other roles, and its aberrant activation is recognized as a common mechanism underlying the progression of OC (Rosanò et al. 2013). AMP-activated protein kinase (AMPK) is an important mediator in maintaining cellular metabolic/energy homeostasis, situated in the centre of a signalling network involving established TSGs (e.g. TP53) that can exert pro- or anti-tumorigenic roles in cancer (Faubert et al. 2015). Interestingly, activation of $A M P K$ suppresses the growth of OC malignant cells (Li et al. 2014; Yung et al. 2016), suggesting a pro-oncogenic role for HBO1 in the regulation of this network. RhoGDIs are signal transducers that control the location and activity of Rho guanosine triphosphate (GTP)-ases, and thus mediate several processes during 
tumorigenesis and cancer progression (Harding \& Theodorescu 2010). One member of the RhoGDI family (RhoGDI2) was found to antagonize OC growth, invasion and metastasis (Stevens et al. 2011), suggesting that the inhibition of 'RhoGDI signalling' may entail the promotion of cancerous properties and therefore an antioncogenic role for overexpressed HBO1.

Rho GTPases represent a family of small GTP-binding proteins involved in cell cytoskeleton organization, amongst other functions (Parri \& Chiarugi 2010). Notably, predicted HBO1 KD-associated inhibition of 'RhoGDI signalling' in UWB1.289 cells was accompanied by IPA predictions of increased cytoskeleton/cytoplasm organization (Table 6.8). These results are consistent with the promotion of higher levels of cellular organization (e.g. enhanced microtubule dynamics), a feature that was also observed in UWB1.289 cells expressing shRNAs targeting $\mathrm{HBOl}$ (Fig. 5.7), but this time phenotypical changes were not confirmed in vitro. One possibility for the negative AFM results using siRNAs could be the lack of treatment uniformity, given the absence of a marker indicating the cells in which HBO1 was in fact down-regulated (e.g. GFP) and that only a handful of cells were analysed at random due to the current limitations of AFM analysis.

Other potential inhibited processes identified in SKOV-3 and UWB1.289 (GSEA) are the 'E2F pathways' (Table 6.10). The E2F family encompasses a group of genes that codify transcription factors critical in cell cycle control (Ahlander \& Bosco 2009) and its inhibition points to a role for HBO1 in cell cycle regulation. However, these predictions could not be confirmed since cell growth rates remained unchanged following $\mathrm{HBO} 1 \mathrm{KD}$ in the three OC cell lines (Fig. 6.15).

Transcript levels of 13 genes, including $\mathrm{HBOl}$, were similarly altered in three OC cell lines, revealing a small 'HBO1 OC signature' (Fig. 6.7). These common transcripts likely represent the core functionality of HBO1 in specific cellular processes involving these genes. Three 'HBO1 OC signature' genes have been linked to $\mathrm{OC}$ and other cancerous malignancies: CD46, $H 2 A F Y$ and $Y A P 1$. Interestingly, these transcripts were also down-regulated following $\mathrm{HBO} 1 \mathrm{KD}$ in UACC-1598 and TOV-112D cell lines (Fig. 6.9), suggesting a strong association of these genes with HBO1 across OC cell-types. 
CD46 is a ubiquitously expressed membrane protein, whose main function is associated with the regulation of the complement system and plays other roles in immunity, autophagy and cell adhesion (Tang et al. 2016). This protein is commonly overexpressed in cancer tissues of the ovary, breast and lung, amongst others, with the aim to evade the immune system (Fishelson et al. 2003). In fact, CD46 expression is indicative of shorter revival-free survival for OC patients (Surowiak et al. 2006). $H 2 A F Y$ encodes a $\mathrm{H} 2 \mathrm{~A}$ histone variant (often called macroH2A1) that usually marks repressed autosomal chromatin and participates in stable $\mathrm{X}$ chromosome inactivation (Ladurner 2003). However, H2AFY also positively regulates transcription when located in the transcribed regions of certain gene subsets (Gamble et al. 2010). This protein has two different isoforms (macroH2A1.1 and macroH2A1.2) that are expressed with similar levels in normal adult cells, but differ in cancerous tissues (Monteiro et al. 2014). Notably, the percentage of macroH2A1.1 compared to total macroH2A1 was significantly reduced in OC samples compared to the percentage in the normal controls, a feature that has been commonly linked to tumours with poor prognosis (Novikov et al. 2011). YAP1 is a main downstream nuclear effector of the Hippo signalling pathway, which regulates tissue homeostasis, cell proliferation, organ size and regeneration (Moroishi et al. 2015). Dysregulation of the Hippo pathway is associated with cancer development and overexpression of YAP1 has been reported in OC (Steinhardt et al. 2008), where it is thought to act as an oncogene (X. Zhang et al. 2011).

Accordingly, the down-regulation of $C D 46, H 2 A F Y$ and $Y A P 1$ as a consequence of HBO1 KD suggests a role for HBO1 contributing to their pro-oncogenic overexpression. In addition, common gene regulation analyses also point to a tumorigenic role of HBO1 in OC cell lines, since 11 of the 12 targets were downregulated following HBO1 depletion (Fig. 6.7 B). However, the basal expression levels of CD46 and YAP1 did not display significant increasing trends, and only $H 2 A F Y$ was highly expressed in all OC cell lines compared to the control (H2AFY qRT-PCR primers target both macroH2A1.1 and macroH2A1.2 isoforms). Notably, none of the 'HBO1 OC signature' genes were identified as differentially expressed genes in HeLa, suggesting an OC-specific regulation. Further implications of these findings are examined in the final discussion. 
7. Discussion 
High-grade serous carcinoma is the most common and aggressive OC histo-type and its lethality is mainly due to the asymptomatic nature of early disease and the lack of effective treatment strategies for advanced conditions. With the exception of PARP inhibitors, and to some extent anti-angiogenic drugs like Bevacizumab, existing therapies ultimately yield disappointing results. Hence, the identification of druggable target molecules with key roles in HGSC phenotype and progression could facilitate the development of new therapies. In this scenario, the 'histone acetyltransferase binding to ORC-1' (HBO1, KAT7 or MYST2) was found overexpressed in a number of primary ovarian serous carcinomas (Iizuka et al. 2009). HBO1 is a nuclear protein, member of the MYST family of HATs (N Avvakumov \& Côté 2007), that functions within large multi-protein complexes and has roles in DNA replication and transcription (Doyon et al. 2006; Iizuka et al. 2006; Avvakumov et al. 2012). More importantly, HBO1 is an epigenetic regulator, specifically a 'writer', responsible for bulk H4K5, 8, 12, 16 and H3K14 acetylation (Iizuka et al. 2009; Kueh et al. 2011). Several lines of evidence suggest that HBO1 may play important roles in the OC phenotype and therefore could represent an interesting novel target that circumvents reliance on infrequent mutated genes.

The results of this study confirm the overexpression of HBO1, both at transcript and protein level, in representative OC cell lines compared to the HOSEpiC control (Fig. 3.1 \& Fig. 3.6). These findings are similar to overexpression of HBO1 in breast cancer cell lines (e.g. MCF-7, T47D) and bone osteosarcoma (Saos-2) cells (Iizuka et al. 2009; Duong et al. 2013), whereas conversely HBO1 expression was suppressed in acute myeloid leukaemia patients (Sauer et al. 2015), demonstrating the disease specific dysregulation of this protein. Additionally, other studies have documented overexpression of other HATs in a variety of carcinogenic malignancies, e.g. p300 in breast and prostate cancer (Debes et al. 2003; Xiao et al. 2011), suggesting that overexpression of 'writers' may play a significant role in cancer, possibly through the up-regulation of oncogenes via acetylation of the regulatory regions of these genes (Di Cerbo \& Schneider 2013). 
HBO1 transcript levels were highest in the group of cell lines representative of a serous subtype, suggesting possible links between HBO1 expression and the histotype. Interestingly, two of the cell lines that displayed lower levels of HBO1 expression, the mucinous COV644 and the clear-cell TOV-21G, were derived from un-treated patients, whereas OVCAR-3, UWB1.289 and SKOV-3 cell lines were derived from platinum-treated (OVCAR-3, UWB1.289) and non-platinum-treated (SKOV-3) patients diagnosed with HGSC. This suggests that HBO1 overexpression could, in some cases, be a side effect of the treatment with the potential outcome of increasing the resistance to chemotherapy (Koussounadis et al. 2014), although none of the cell lines display significant resistance according to previous studies (Beaufort et al. 2014). Furthermore, the endometrioid TOV-112D cell line, derived from an untreated patient, showed high levels of HBO1, suggesting that chemotherapeutic treatment may not be the only cause of elevated HBO1 in OC models.

HBO1 is expressed as a number of splice isoforms, the overexpression of which does not appear to be linked to cancer type or patient treatment (Fig. 3.3). In general terms, most transcript isoforms were overexpressed in every cell line compared to HOSEpiC, pointing to a cancer-related promoter deregulation phenomenon (e.g. DNA de-methylation or histone [auto-] acetylation). Certain isoforms, however, were not up-regulated in specific cell lines, for instance, isoforms 3 and 5 were unchanged in TOV-112D compared to HOSEpiC (Fig. 3.3 \& Appendix Fig. 1 C, E). These results suggest that the expression of specific isoforms of HBO1 transcription is cell line dependant.

All OC cell lines displayed overexpression of $\mathrm{HBOl}$ splice isoform 1 or 2 (Fig. 3.3 \& Appendix Fig. 1 A, B), which corresponded with the only protein isoforms detected by western blot, and therefore considered the most abundant variants of HBO1 (Fig. 3.4). While the RNA-seq analysis obtained from shRNA-transduced UWB1.289 cells pinpointed all $H B O 1$ variants amongst the 58,215 identified gene transcripts, isoforms 3, 4 and 5 displayed a very low effective count in both the control and $\mathrm{KD}$ samples, which translates to low basal transcript expression. Conversely, the effective count of $\mathrm{HBOl}$ isoforms 1 and 2 was high in both control and KD samples (yet significantly higher in the control compared to the KD due to effective $H B O 1$ silencing) (Table 5.1), further confirming the predominance of these 
isoforms in the global $\mathrm{HBOl}$ transcript composition. Microarray analyses did not identify HBO1 variants, as the human whole-genome expression Illumina ${ }^{\circledR}$ microarray incorporates bead probes corresponding only to HBO1 isoform 1.

HBO1 protein analysis revealed a molecular weight that was different to the predicted $70 \mathrm{KDa}$ for the unmodified protein (based on translation of the DNA sequence), as western blot exposed a smeared band that ranged from approximately $67 \mathrm{KDa}$ to $75 \mathrm{KDa}$ (Fig. 3.4 B). Moreover, UACC-1598 cells exhibited additional bands in the 90-100 KDa range. These observations suggest that HBO1 is very likely to undergo post-translational modifications, which would lead to altered HBO1 activity (Legube \& Trouche 2003). PTMs are essential mechanisms used by eukaryotic cells to modulate protein function, influencing enzyme activity, protein turnover, localization, protein-protein interactions and most importantly coordinating signalling networks and pathways (Karve \& Cheema 2011). Accordingly, cancerassociated PTM defects can trigger the functional modifications of key regulatory or structural proteins, resulting in aberrant cell physiology, structural integrity and gene expression, thus favouring cancer progression (Krueger \& Srivastava 2006).

The HBO1 protein encompasses a number of motifs that are known to undergo PTMs, including phosphorylation, acetylation and ubiquitination (Fig. 7.1), a number of which have been documented in vitro (Wu \& Liu 2008; Duong et al. 2013; Matsunuma et al. 2015). The serine-rich domain of HBO1 is thought to be hyper-phosphorylated under normal conditions (Miotto \& Struhl 2011), but few phosphorylation targets have been linked to changes in HBO1 activity. One of the sites that has been characterised is $\operatorname{Ser}^{57}$, the mutation of which eliminated its ability to be phosphorylated, resulting in decreased histone $\mathrm{H} 4$ acetylation activity of HBO1 in HeLa cells, suggesting that phosphorylation of $\mathrm{Ser}^{57}$ is involved in modulating the HAT activity of HBO1 (Wu \& Liu 2008). This observation was supported by others, as recombinant $\mathrm{HBO} 1$ protein expressed in E.coli had no detectable HAT activity (Iizuka \& Stillman 1999), possible due to the lack of eukaryotic PTMs. 


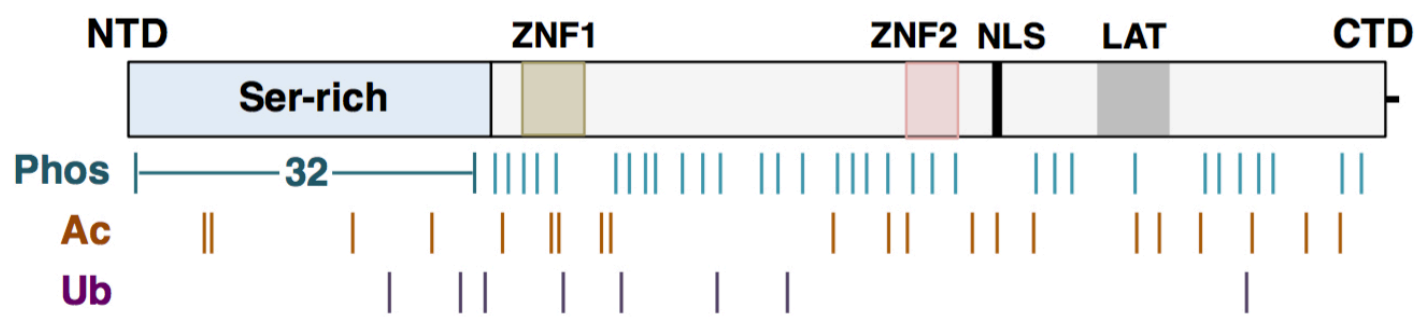

Figure 7. 1 Schematic depiction of potential HBO1 protein PTMs

The HBO1 protein encompasses 65 phosphorylation sites (including serine, threonine and tyrosine residues), 21 acetylation sites and 8 ubiquitination sites, according to PTM predictors NetPhos (Blom et al. 1999), PAIL (Li et al. 2006; Deng et al. 2016) and UbPred (Radivojac et al. 2010). Each vertical line represents a potential PTM site. The density of phosphorylation sites within the Ser-rich (serine rich) domain is so high that sites are represented by a numeral. Phos: Phosphorylation; Ac: Acetylation; Ub: Ubiquitination; NTD: Amino-terminus domain; ZNF1, 2: Zinc finger 1 and 2; NLS: Nuclear localization signal; LAT: Lysine acetyltransferase domain; CTD: Carboxyl-terminus domain.

Based on the literature and the specific composition of the HBO1 protein (Fig. 7.1), phosphorylation seems to be the most plausible source of protein size modifications. However, the addition of phosphate groups, which increase the weight of the target protein in approximately $0.08 \mathrm{KDa}$ (Parker et al. 2010), would not explain the existence of additional bands observed in UACC-1598 cells in the 90-100 KDa range (Fig. 3.4 A), similarly neither would the addition of acetyl- groups, which cause a mass shift of only $0.042 \mathrm{KDa}$ (Parker et al. 2010). In contrast, protein ubiquitination involves the attachment of $8.8 \mathrm{KDa}$ ubiquitin molecules, and can result in mono-ubiquitination, multi-ubiquitination or poly-ubiquitination (addition of a chain of ubiquitin molecules to one protein residue) (Parker et al. 2010; Sadowski et al. 2012), and have been previously reported to mark HBO1 proteins (Zou et al. 2013).

In order to assess the presence of ubiquitin molecules attached to HBO1, the protein was immune-precipitated and probed with an ubiquitin-specific antibody. The absence of ubiquitin-marked bands/smears in the gel suggests that the observed differences in the predicted molecular weight of HBO1 in UACC-1598 cells are not the result of protein ubiquitination (Fig. 3.5). Due to the presence of such an extensive array of putative phosphorylation sites within HBO1, the observed mass change may be due to phosphorylation of a number of these sites, and the size 
deviation could be attributed to a shift in the charge as well as mass, which may affect protein mobility in SDS-PAGE. This, however, remains to be tested.

It was confirmed that $\mathrm{HBO} 1$ is active in $\mathrm{OC}$ cells, since transient and constitutive HBO1 KD led to significant reductions in bulk histone $\mathrm{H} 4$ acetylation (Figs. 5.1, 5.2, 6.1 \& 6.2). These findings have been extensively discussed earlier and the main HBO1 acetylation targets in several KD studies are presented in Table $\mathbf{1 . 7}$ for comparison. Even though none of the KDs affected H3K14 acetylation levels, the evaluation of the capability of HBO1 to acetylate other lysine targets within the histone $\mathrm{H} 3$ tail might contribute to expand the current knowledge of its molecular range of action in cancer. Future objectives may include H3K9, an acetylation target that was found marginally regulated by $\mathrm{HBO} 1$ in a number of studies and has been linked to OC tumour progression (Zhen et al. 2010; Mishima et al. 2011; Yan et al. 2016), but could also involve different histone targets such as $\mathrm{H} 2 \mathrm{~A}$ and $\mathrm{H} 2 \mathrm{~B}$, which have never been linked to HBO1 activity before.

The manipulation of cells with the FLAG-tagged expression vector pCMV-Tag2BhHBOl increased HBO1 expression both at mRNA and protein level (Fig. $4.16 \mathrm{~A} \&$ Fig. 4.17), yet did not promote enhanced acetylation of histone H4 in shRNAtransduced UWB1.289 cells (Fig. 5.3). A similar phenomenon was reported by Foy et al. (2008), who observed that transfected HBO1 protein did not enhance H4 acetylation in human embryonic kidney (HEK) 293T cells, unless either JADE1L or JADE1S was also ectopically co-expressed (Foy et al. 2008). This suggests that assembly and stabilization of the HBO1 complex may require specific concentrations of HBO1 and JADE proteins, e.g. JADE1 ( $\mathrm{L}$ or S), in order to drive H4 acetylation (Foy et al. 2008). In support of this hypothesis, UWB1.289 cells showed relative low $J A D E 1$ and high $J A D E 2$ transcript levels compared to HOSEpiC (Fig. 3.20), suggesting that a presumable cell line specific shift in the HBO1 complex composition would retain enough JADE protein expression as to allow the successful acetylation of H4. In addition, the transfection of pCMVTag2B-hHBO1 in stable HBO1 KD UWB1.289 cells did not affect the levels of HDAC6, SNAP47, PCM1 or PMS2 gene transcripts (data not shown), considered $\mathrm{HBO} 1$ targets as their expression was altered following HBO1 depletion (Fig. 5.4). 
Several attempts were made to examine the localization of HBO1 in the OC genome, either via ChIP-qRT-PCR or ChIP-Seq, but none of these demonstrated any significant enrichment of HBO1 in the genome of OC cell models. CYR61 and $E R B B 2$, potential HBO1 targets derived from SQL correlations and tested for ChIP enrichment (Fig. 3.16) were identified as HBO1 targets in UWB1.289 cells through the analysis of microarray KD data. However, ChIP did not reveal HBO1 at the promoter region of these genes, highlighting the difficulties in 'ChIPing' endogenous HBO1. ChIP-Seq, if successful, would have provided insights into the way the HBO1 regulatory network performs its functions. For instance, it would have provided evidence of targets directly regulated by HBO1 via promoter binding, as oppose to protein-protein interactions, as well as association with known histone marks.

The potential reasons for this lack of success have been already extensively explored earlier, and point to the inability to immune-precipitate endogenous $\mathrm{HBO} 1$ protein in enough quantity to perform ChIP. A similar issue was reported by Hung et al. (2009), who were also unable to complete ChIP on HT1080 fibrosarcoma cells using an antibody targeting HBO1 (Santa Cruz ${ }^{\circledR}$ ), whereas they did manage to detect ING4 occupancy using an anti-Flag antibody targeting ectopically expressed Flag-ING4 (Hung et al. 2009). One possible explanation for the recurrent ChIP failure, which we favour, is the distinctive composition of the HBO1 multi-protein complex in UWB1.289 cells, with $J A D E 1 L / S$ transcripts present at very low levels and JADE2 present at very high levels (Fig. 3.20). The potential switch of complex scaffolding subunits may mask access to HBO1 by the antibody when recruited to the promoter of target genes, thus severely interfering with ChIP experiments.

Transient HBO1 depletion in OC cell lines was accompanied by cell-specific changes in gene expression (Table 6.5), suggesting that HBO1 may play subtly different roles in different $\mathrm{OC}$ sub-types. Amongst the different responses to the transient HBO1 KD identified in OVCAR-3, UWB1.289 and SKOV-3, the latter stands out due to (1) its low number of altered transcripts and (2) the change in regulatory trend, with three times more down-regulated genes than up-regulated counterparts, compared to the other cell lines. These differences might have their origin in the contrasting levels of HBO1 expression, but could also be dependent on 
the nature of the SKOV-3 cell line itself. SKOV-3 carries a null TP53 (due to a frameshift mutation) (Mullany et al. 2015), as opposed to the other serous-like OC cell lines that constitutively express TP53 due to missense point mutations (e.g. G743A in OVCAR-3), and has therefore recently been placed in the spotlight as to its suitability as a model for HGSC (Domcke et al. 2013; Coscia et al. 2016).

The transfection of siRNAs targeting $\mathrm{HBOl}$ in OC cell lines identified an 'HBO1 OC signature' gene list (Fig. 6.7), and whilst the basal expression level of these genes was similar to that of the HOSEpiC control cells, the majority of these genes (12/13; including $\mathrm{HBOl}$ itself) were down-regulated following HBO1 KD. Furthermore, the genome wide role of HBO1 may not have been fully revealed, as others have reported compensatory effects by certain HAT complexes after HBO1 KD. Notably, Avvakumov et al. (2012) observed increased levels of BRPF1 following $\mathrm{HBO} 1 \mathrm{KD}$ in HeLa cells, suggesting that the MOZ/MORF HAT complex may compensate for loss of HBO1 (Avvakumov et al. 2012).

The transient KD experiments pinpointed a group of pro-oncogenic genes, CD46, $H 2 A F Y$ and $Y A P 1$, that were down-regulated following HBO1 depletion in five OC cell lines (OVCAR-3, SKOV-3, UWB1.289, UACC-1598 and TOV-112D), suggesting an oncogenic potential of HBO1 in OC. Previous studies have reported overexpression of these genes in OC tissues (Fishelson et al. 2003; Steinhardt et al. 2008; Novikov et al. 2011) and this overexpression has been linked to shorter survival and poor prognosis (Surowiak et al. 2006; Novikov et al. 2011). CD46 is a complement inhibitor involved in the protection of tumour cells against the host immune system (Maciejczyk et al. 2011). HBO1 regulation of CD46 supports one pathway identified through in silico analysis of HBO1 function, namely the inhibition of IFN signalling pathways, confirmed in OVCAR-3 cells for IFNA/ $\alpha$ (Fig. 6.19). These findings suggest that $\mathrm{HBO} 1$ overexpression could be a mechanism deployed by cancerous cells, accomplished by the up-regulation of targets such as $C D 46$, in order to evade the immune system and their subsequent destruction.

The Hippo pathway transcriptional co-activator $Y A P 1$ is an $\mathrm{OC}$ oncogene whose expression has been shown to stimulate migration, invasion and anchorageindependent growth of OC cell lines (X. Zhang et al. 2011). HBO1 regulation of 
YAP1 may serve as a mechanism to enhance the oncogenic potential of OC cells, especially in terms of enhancing cell migration capacity. However, this was not conclusively demonstrated using scratch test assays (Fig. 5.6 \& Fig. 6.16).

Tumour suppressor genes were also altered following HBO1 depletion, e.g. ING4 was significantly up-regulated following HBO1 KD in UWB1.289 cells (Fig. 6.10). These findings suggest that HBO1 may not only act as an enhancer of pro-oncogenic gene expression in OC, but might also participate in the inhibition of certain TSGs. ING4, a member of the HBO1 multi-protein complex, has been shown to function in inhibitory activities including angiogenesis and cell migration (Garkavtsev et al. 2004; Shen et al. 2007), and is commonly down-regulated in OC (Liu et al. 2012). Accordingly, it seems likely that HBO1-driven repression of ING4 could contribute to a pro-oncogenic function in OC cells.

Another TSG up-regulated following HBO1 KD was the cyclin-dependant kinase inhibitor $C D K N 1 A / p 21$ (Fig. 6.10). CDKN1A has previously been identified as an HBO1 target following transient HBO1 KD in HeLa cells, however, conversely to the result obtained in OC cells, its expression was down-regulated in HeLa (Avvakumov et al. 2012). Although initially thought to suppress tumour growth by promoting cell cycle arrest, halting cell proliferation either as a p53-mediator or independently, evidence suggests that under certain conditions, CDKN1A can inhibit the apoptotic process thus promoting cellular proliferation and oncogenicity (Fischer 2017). The extent of CDKN1A expression in OC has been extensively studied, although the results obtained have not always been conclusive; while certain studies have found CDKNIA predominantly increased and associated with tumour progression (Ferrandina et al. 2000), others found significant links between low levels of $C D K N 1 A$ and high-grade tumours accompanied by poor survival rates (Anttila et al. 1999).

In UWB1.289 cells, the up-regulation of CDKN1A following HBO1 depletion coincided with a significant decrease in caspase activity (Fig. 6.18 C), suggesting a role in the inhibition of apoptosis and therefore a pro-oncogenic behaviour of $C D K N 1 A$. Thus, HBO1-mediated repression of $C D K N 1 A$ points to an anti-oncogenic role for HBO1 in OC cells, which is supported by the significant augmentation of 
cell viability rates following the KD (Fig. 6.18 A). Notably, and regardless of changes in cell caspase activity and cell viability, HBO1 KD did not elicit cell proliferation changes in OC cells (Fig 5.5 \& Fig. 6.15).

HBO1 KD using distinct silencing approaches in UWB1.289 cells revealed different gene expression signatures: whilst 889 genes where up/down-regulated in response to the transfection of siRNAs targeting $\mathrm{HBOl}$, only 68 genes displayed significant up/down-regulation in cells expressing shRNAs targeting HBO1. Although this may seem conflicting, given that both approaches are aimed at reducing the expression level of the same target protein, the different cell contexts in which this reduction occurs may account for the discrepancies in number and identity of gene targets.

The transfection of transient siRNAs involves that cells are in a situation in which, in less than one cycle of division ( $24 \mathrm{~h})$, protein levels of an important regulator have drastically declined, and therefore the concomitant expression of numerous associated-gene products may get altered (e.g. 889 genes in UWB1.289). On the other hand, the constitutive expression of shRNAs gives rise to a situation in which the cells, after going through different cycles of division in the absence of target protein, have been able to adapt and coexist with this circumstance, perhaps via modification of their transcriptome. Consequently, the genes that remained destabilised at the time of analysis (e.g. 68 genes in UWB1.289) could represent either transcripts that depend entirely on $\mathrm{HBO} 1$ regulation, and might therefore delineate the core functionality of $\mathrm{HBO}$, or a genomic response to counteract the lack of HBO1, although up-regulation of other HATs or HAT complex proteins was not observed.

Interestingly, the use of these two approaches to KD HBO1 (transient siRNAs and stable shRNAs) provides very valuable information about the possible effects that the silencing of this molecule would have at cellular level in different clinical contexts. The gene expression changes elicited by the transfection of siRNAs could be correlated to the genetic alterations that would take place at the beginning of a potential treatment against $\mathrm{HBO} 1$. As common therapeutic regimes are administered in cycles over time, it could also be assumed that after several cycles of KD treatment, cancer cells may adapt to a permanent shortage of HBO1, a situation that 
correlates with the expression of shRNAs targeting HBOl. Therefore, the development of the two silencing approaches provides a clear advantage in the study of $\mathrm{HBO} 1$ as a potential therapeutic target.

Table 7. 1 List of common altered IPA functions after HBO1 KD

\begin{tabular}{|l|c|c|c|}
\hline \multirow{2}{*}{\multicolumn{1}{|c|}{ Biological function }} & \multirow{2}{*}{ Prediction } & \multicolumn{2}{c|}{ Z-score } \\
\cline { 3 - 4 } & & Transient & Stable \\
\hline Microtubule dynamics & Increased & 1.67 & 2.24 \\
\hline Organization of cytoplasm & Increased & 2.31 & 2.24 \\
\hline Formation of cellular protrusions & Increased & 1.77 & 1.81 \\
\hline
\end{tabular}

As a whole, the genome wide analysis of HBO1 function revealed significant changes in the expression of more than 1000 genes, which when analysed for pathway/process activities in silico, resulted in a number of predictions, some of which were corroborated in vitro (e.g. activation of IFN signalling pathways in OVCAR-3). Most notably, in silico analyses of UWB1.289 gene signatures were successful in highlighting a role for HBO1 in microtubule dynamics and cytoplasm organization, pathways that were identified in both the individual analysis of transient and stable $\mathrm{HBO} 1 \mathrm{KD}$ datasets, thus suggesting a core function of HBO1 regulation (Table $\mathbf{7 . 1}$ ).

In line with these predictions, recent attention has been focused on extra factors that characterise cancer, including the study of mechanical changes undergone by tumour cells (Coceano et al. 2016). Such changes in the mechanical properties of single cells start early in tumorigenesis and involve several physiological processes that, by altering the membrane and cytoskeleton structure, convert a malignant cell into a metastatic one (Plodinec et al. 2012). It is known that cancer cells, regardless of cancer type, are softer/elastic and hence more deformable compared to normal, nonmalignant or even less differentiated cancer cells, which eventually leads to increased ability to infiltrate tissues and spread (Rother et al. 2014). For instance, the stiffness of metastatic cancer cells isolated from the pleural fluids of breast cancer patients was reported to be more than $70 \%$ lower than that of benign mesothelial 
cells (Cross et al. 2007). However, the underlying cause for this reduced stiffness, as well as its cause-effect relationship with other conformational changes observed during cancer growth and metastasis, remains not fully elucidated (Calzado-Martín et al. 2016).

AFM enables measuring cell stiffness, the resistance of a single cell to an externally induced deformation, helping to understand the correlation between cell structure, mechanics and functioning (Lekka 2016). Individual UWB1.289 cells expressing either 'shHBO1 1' (targeting HBOl) or 'shCtrl' (non-targeting control) were analysed using AFM in order to evaluate the state of internal cell mechanics upon shRNA expression. Analyses revealed that HBO1 KD cells presented higher stiffness in comparison with control cells (Fig. 5.7), a feature commonly linked to the development of significant internal cytoskeleton and cytoplasm rearrangements (Parsons et al. 2010). The reduced deformability of HBO1 KD cells probed by AFM suggests that they are likely to have reduced movement and migration, linked with decreased invasiveness (Lekka 2016), pointing to a pro-oncogenic role for HBO1 in OC cells. Specifically, the overexpression of HBO1 in OC cells is suggested to promote changes in chromatin architecture and cell mechanics towards more elastic, aggressive cancerous phenotypes.

The results presented in this work propose a new function for HBO1 in OC: the regulation of cytoplasm and cytoskeleton organization. The acetylation/deacetylation of tubulin proteins within microtubules is known to regulate microtubule function (Howes et al. 2014), suggesting a mechanism through which HBO1 can drive this novel function. For instance, the 'histone deacetylase 6' (HDAC6), which mediates tubulin deacetylation, has been shown to affect cell motility of cancer cells (Hubbert et al. 2002; Haggarty et al. 2003). Accordingly, one would expect that the overexpression of a HAT that may target tubulin for acetylation (e.g. HBO1) could elicit cytoskeleton re-arrangements aimed at acquiring higher degrees of cell elasticity. However, as it was highlighted by the in silico analysis (Table 7.1), HBO1 may also drive this function through the transcriptional regulation of genes involved in such processes. These findings may imply the opening of a completely new field of study, in which the activity of a known epigenetic chromatin modulator is also involved in the regulation of the mechanical properties of single cells. 
Even though HBO1 has never been directly linked with microtubule acetylation or with any other aspect related to cell organization, a number of studies have investigated its role in tumour cell differentiation towards cancerous phenotypes. For example, Kim et al. (2015) and Duong et al. (2013) reported that HBO1 is involved in the maintenance of murine embryonic stem cell self-renewal and pluripotency, as well as in the maintenance of breast cancer stem cell properties and expression of EMT markers. These studies point to a broad role for HBO1 in the establishment of aggressive cancer phenotypes, thus supporting the data obtained with AFM in OC cells.

HBO1 is known to frequently exert antagonistic roles, thus acting as an oncogene or a TSG depending on the specific background. This antagonistic functional duality has been observed for several genes, some of which have been discussed earlier (e.g. $C D K N 1 A$ ) and it is a common characteristic of HATs (e.g. PCAF) (Cohen et al. 2011; Stepanenko et al. 2013). The set of observations obtained in this study seem to support a dualistic role for HBO1 within the same cancer type, a kind of behaviour that has also been observed in HATs such as p300 in colorectal cancer cells (Gayther et al. 2000; Ishihama et al. 2007). HBO1 displayed certain pro-oncogenic features, which include (1) its overexpression in OC cells, (2) the positive regulation of OCassociated oncogenes (CD46, YAP1), and (3) the promotion of elastic tumour phenotypes. However, HBO1 was also associated with anti-oncogenic properties, mainly linked to its role as a regulator of cell viability, as it can be inferred from the KD profile displayed in viability/cytotoxicity assays, typical of TSGs.

While it may seem surprising that the overexpression of HBO1 in OC cells could be associated with anti-oncogenic activities, there are precedents for overexpression of proteins with anti-oncogenic activities in cancer cell types. The transcription factor nuclear receptor COUP-TFII $(N R 2 F 2)$ is overexpressed in breast cancer, yet $N R 2 F 2$ $\mathrm{KD}$ in MDAMB231 and MCF-7 breast cancer cells led to enhanced migration and invasion without changes in proliferation (Zhang et al. 2014). Human mammaglobin $(h M A M)$ is also overexpressed in breast cancer plasma and multiple breast cancer cell lines, yet $h M A M \mathrm{KD}$ enhances migration and invasiveness in MDA-MB231 cells (Koh et al. 2014). Finally, 'F-actin capping protein $\alpha 1$ subunit' (CAPZA1) is overexpressed in gastric carcinoma tissues and the MKN45 cell line, yet is 
associated with decreased cancer cell migration and invasion in this context (Lee et al. 2013).

Overall, the results of this study suggest conflicting activities for HBO1 in OC cell lines, which give an idea of the wide-ranging and dynamic roles of this important HAT. Based on the evidence contained herein, a potential therapeutic action reversing $\mathrm{HBO} 1$ overexpression in $\mathrm{OC}$ cells would have two major consequences: (1) it would decrease OC cell elasticity, interfering the progression towards more aggressive cancer phenotypes, and (2) it would increase the viability of OC cells, potentially benefiting the perpetuation of the disease. These responses may be considered as counterproductive since, while one seems to deter OC development, the other clearly favours its growth. Accordingly, more in depth studies and a better understanding of the roles of HBO1 in OC are needed in order to decide whether HBO1 would be a suitable target for OC treatment. In this regard, it would be particularly interesting to evaluate the behaviour of $\mathrm{HBO} 1 \mathrm{KD}$ OC cells in three dimensional (3D) culture models, closely related to the in vivo settings of OC growth. These in vitro 3D models may provide more relevant information about the actual effects of a potential therapeutic treatment targeting $\mathrm{HBO} 1$ in a population of OC cells. 


\section{Appendix}

Appendix 1

A

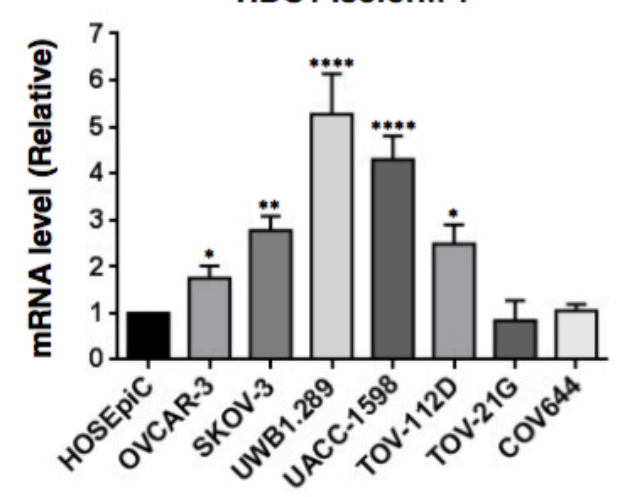

C

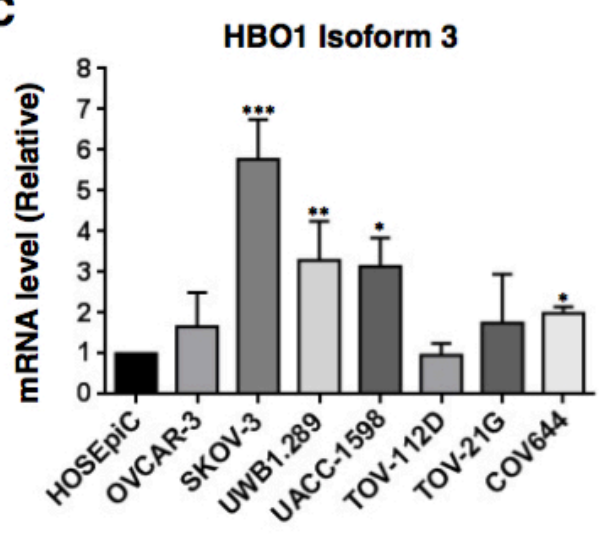

E

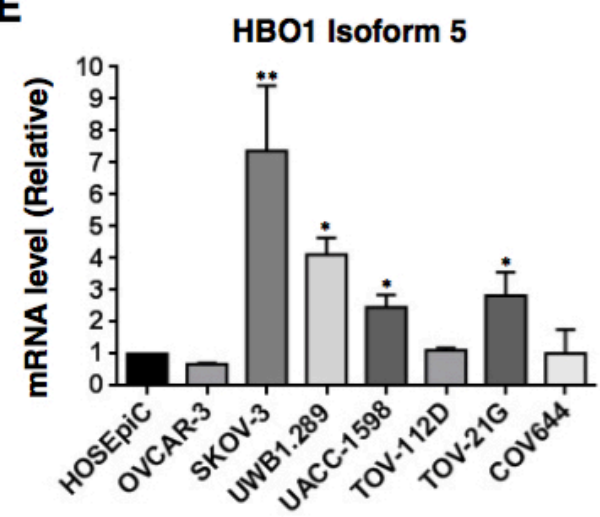

B
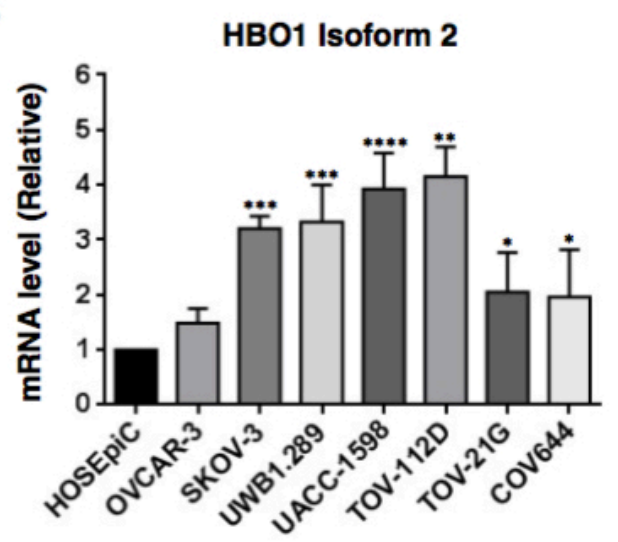

D

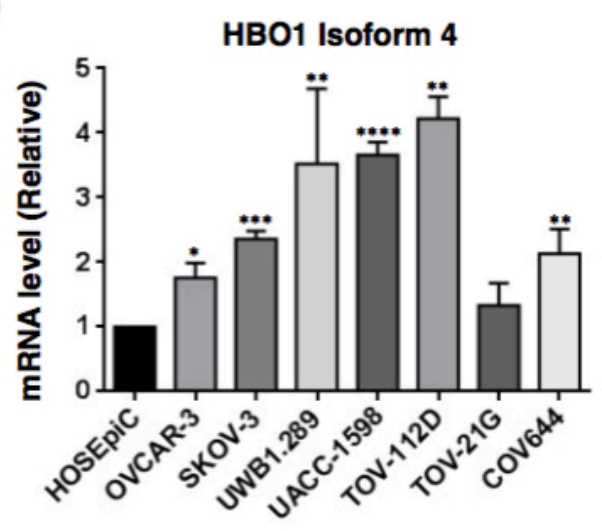

Appendix Figure 1 Comparative analysis of $\mathrm{HBO} 1$ expression in $\mathrm{OC}$ cell lines RNA lysates from HOSEpiC and 7 OC cell lines were prepared and analysed by qRT-PCR to evaluate the expression of $\mathrm{HBOl}$ isoforms 1, 2, 3, 4 and 5. Relative levels of $H B O 1$ expression were compared to those of the HOSEpiC non-cancerous control. GAPDH expression was used as control. Statistical analyses consisted of one-way ANOVA and Tukey's multiple comparison tests. All values represent the mean \pm SD of three biological samples $\left({ }^{*} \mathrm{p}<0.05 ;{ }^{* *} \mathrm{p}<0.01 ;{ }^{* * *} \mathrm{p}<0.001 ;{ }^{* * * *} \mathrm{p}<\right.$ $0.0001)$. 
Appendix 2

A

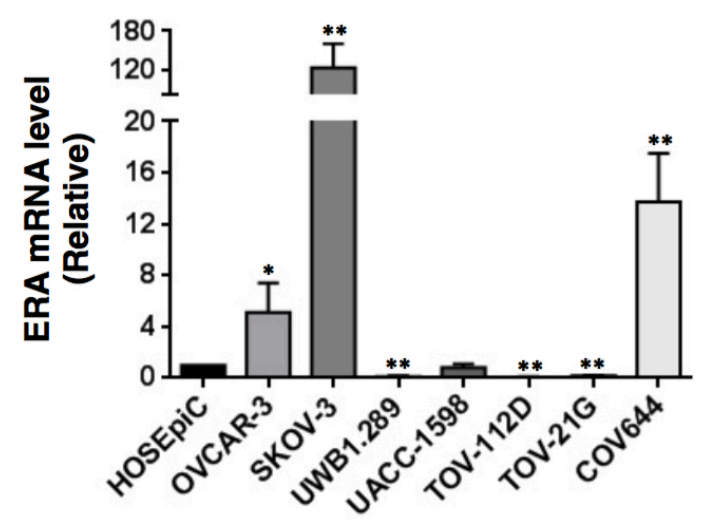

C

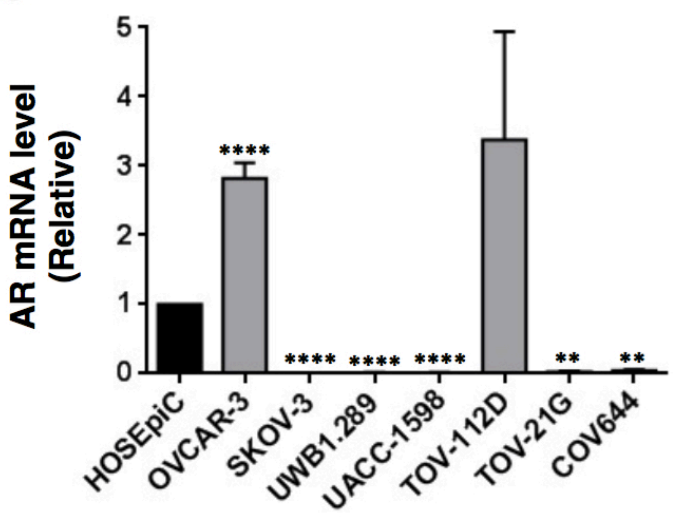

B

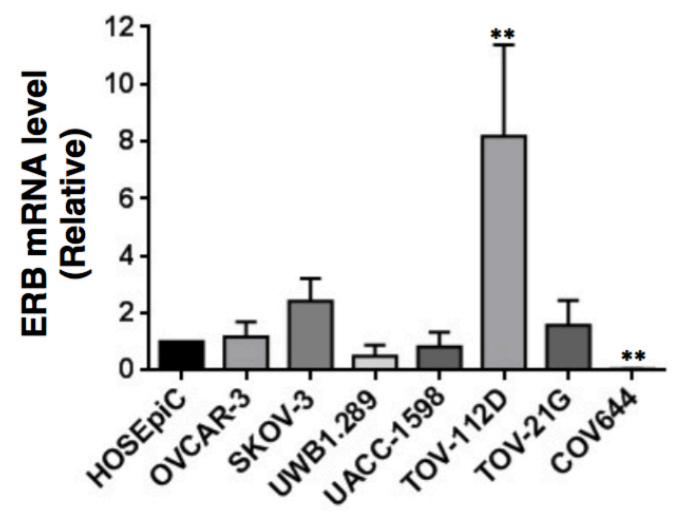

Appendix Figure 2 Comparative analysis of NR expression in OC cell lines RNA lysates from HOSEpiC and 7 OC cell lines were prepared and analysed by qRT-PCR to evaluate the expression of $E R A(\alpha), E R B(\beta)$ and $A R$. Relative levels of expression were compared to those of the HOSEpiC non-cancerous control. GAPDH expression was used as control. Statistical analyses consisted of one-way ANOVA and Tukey's multiple comparison tests. All values represent the mean $\pm \mathrm{SD}$ of three biological samples $\left({ }^{*} \mathrm{p}<0.05 ;{ }^{* *} \mathrm{p}<0.01 ;{ }^{* * *} \mathrm{p}<0.0001\right)$. 
Appendix 3

A

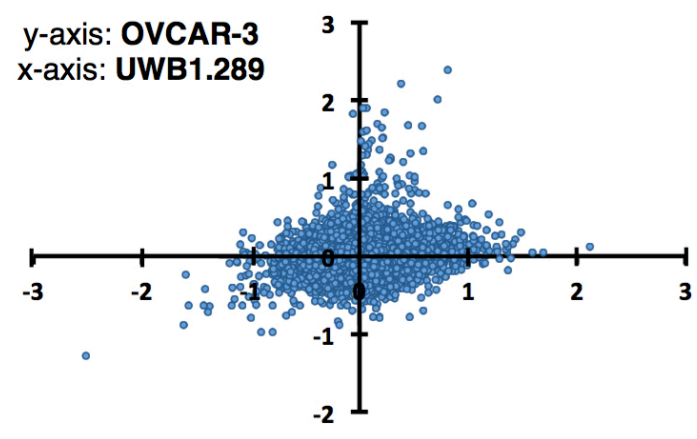

C

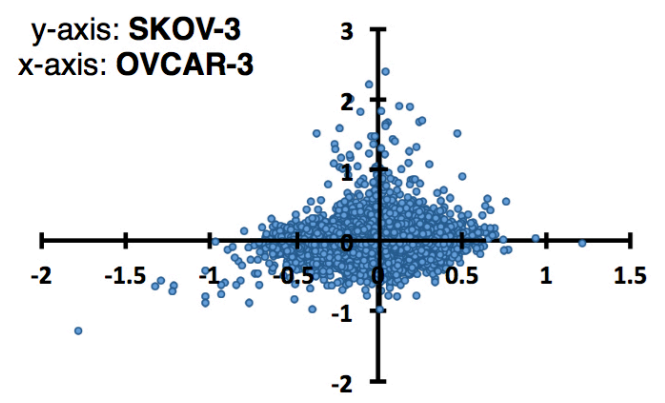

B

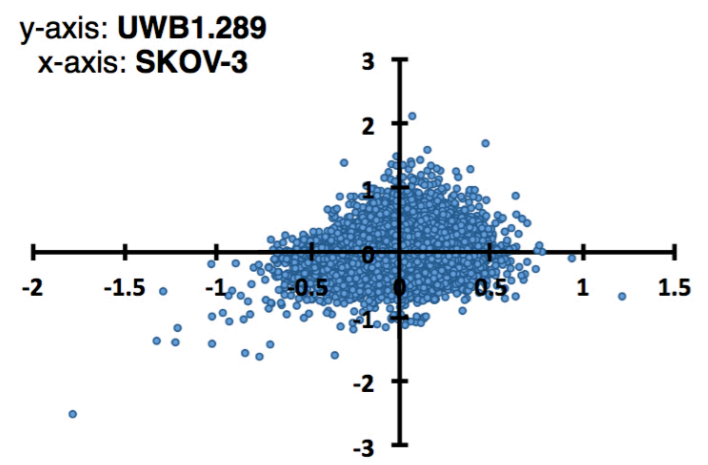

Appendix Figure 3 Comparative analysis of microarray KD profiles

Microarray-derived lists containing the FC expression value of more than 47,000 genes following HBO1 KD in UWB1.289, OVCAR-3 and SKOV-3 cell lines were plotted against each other for comparison. FC values were transformed into logarithms $(\log 2)$ prior to the analysis. Blue dots represent individual genes/probes. Final scatter-plots illustrate the genome-wide consistency of HBO1 KD amongst OC cell lines. 


\section{Bibliography}

Abbas, T. \& Dutta, A., 2009. p21 in cancer: intricate networks and multiple activities. Nature Reviews Cancer, 9(6), pp.400-414.

Abdulkareem, I. \& Zurmi, I., 2012. Review of hormonal treatment of breast cancer. Nigerian Journal of Clinical Practice, 15(1), p.9.

Adams, S.F. \& Benencia, F., 2015. Immunotherapy for ovarian cancer: what are the targets of the future? Future oncology (London, England), 11(9), pp.1293-6.

Aghajanian, C. et al., 2015. Final overall survival and safety analysis of OCEANS, a phase 3 trial of chemotherapy with or without bevacizumab in patients with platinum-sensitive recurrent ovarian cancer. Gynecologic Oncology, 139(1), pp.10-16.

Ahlander, J. \& Bosco, G., 2009. The RB/E2F pathway and regulation of RNA processing. Biochemical and biophysical research communications, 384(3), pp.280-3.

Ahmed, A.A. et al., 2010. Driver mutations in TP53 are ubiquitous in high grade serous carcinoma of the ovary. The Journal of pathology, 221(1), pp.49-56.

Ahmed, N. et al., 2016. Unique proteome signature of post-chemotherapy ovarian cancer ascites-derived tumor cells. Scientific Reports, 6(1), p.30061.

Al-Agha, O.M. \& Blake Gilks, C., 2011. High-Grade Serous Carcinoma Involving Fallopian Tube, Ovary and Peritoneum. Surgical Pathology Clinics, 4(1), pp.375-396.

Allis, C.D. et al., 2007. New Nomenclature for Chromatin-Modifying Enzymes. Cell, 131(4), pp.633-636.

Allis, C.D. \& Jenuwein, T., 2016. The molecular hallmarks of epigenetic control. Nature Reviews Genetics, 17(8), pp.487-500.

Alsop, K. et al., 2012. BRCA mutation frequency and patterns of treatment response in BRCA mutation-positive women with ovarian cancer: a report from the Australian Ovarian Cancer Study Group. Journal of clinical oncology : official journal of the American Society of Clinical Oncology, 30(21), pp.2654-63.

Alver, R.C., Chadha, G.S. \& Blow, J.J., 2014. The contribution of dormant origins to genome stability: from cell biology to human genetics. DNA repair, 19, pp.182-9.

Amadori, D. et al., 1997. Cell proliferation as a predictor of response to chemotherapy in metastatic breast cancer: a prospective study. Breast cancer research and treatment, 43(1), pp.7-14.

Amé, J.C. et al., 1999. PARP-2, A novel mammalian DNA damage-dependent poly(ADPribose) polymerase. The Journal of biological chemistry, 274(25), pp.17860-8.

Anttila, M.A. et al., 1999. p21/WAF1 expression as related to p53, cell proliferation and prognosis in epithelial ovarian cancer. British Journal of Cancer, 79(11-12), pp.18701878.

Argenta, P.A. et al., 2009. A phase II study of fulvestrant in the treatment of multiplyrecurrent epithelial ovarian cancer. Gynecologic oncology, 113(2), pp.205-9.

Arias, E.E. \& Walter, J.C., 2006. PCNA functions as a molecular platform to trigger Cdt1 destruction and prevent re-replication. Nature cell biology, 8(1), pp.84-90.

Armstrong, D.G. \& Webb, R., 1997. Ovarian follicular dominance: the role of intraovarian growth factors and novel proteins. Reviews of reproduction, 2(3), pp.139-46.

Ashworth, A., 2008. A Synthetic Lethal Therapeutic Approach: Poly(ADP) Ribose Polymerase Inhibitors for the Treatment of Cancers Deficient in DNA Double-Strand Break Repair. Journal of Clinical Oncology, 26(22), pp.3785-3790.

Aubert, G. \& Lansdorp, P.M., 2008. Telomeres and Aging. Physiological Reviews, 88(2), pp.557-579.

Auersperg, N. et al., 2001. Ovarian Surface Epithelium: Biology, Endocrinology, and Pathology 1. Endocrine Reviews, 22(2), pp.255-288.

Avvakumov, N. et al., 2012. Conserved molecular interactions within the HBO1 acetyltransferase complexes regulate cell proliferation. Molecular and cellular biology, 32(3), pp.689-703.

Avvakumov, N. \& Côté, J., 2007. Functions of myst family histone acetyltransferases and 
their link to disease. Sub-cellular biochemistry, 41, pp.295-317.

Avvakumov, N. \& Côté, J., 2007. The MYST family of histone acetyltransferases and their intimate links to cancer. Oncogene, 26(37), pp.5395-407.

Azad, N. et al., 2013. The future of epigenetic therapy in solid tumours--lessons from the past. Nature reviews. Clinical oncology, 10(5), pp.256-66.

Baba, A. \& Catoi, C., 2007. Carcinogenesis. In Comparative Oncology. The Publishing House of the Romanian Academy.

Baeriswyl, V. \& Christofori, G., 2009. The angiogenic switch in carcinogenesis. Seminars in Cancer Biology, 19(5), pp.329-337.

Balasubramanyam, K. et al., 2004. Curcumin, a Novel p300/CREB-binding Protein-specific Inhibitor of Acetyltransferase, Represses the Acetylation of Histone/Nonhistone Proteins and Histone Acetyltransferase-dependent Chromatin Transcription. Journal of Biological Chemistry, 279(49), pp.51163-51171.

Balch, C. et al., 2009. Minireview: epigenetic changes in ovarian cancer. Endocrinology, 150(9), pp.4003-11.

Baldwin, L.A. et al., 2012. Ten-Year Relative Survival for Epithelial Ovarian Cancer. Obstetrics \& Gynecology, 120(3), pp.612-618.

Baldwin, R.L. et al., 2000. BRCA1 Promoter Region Hypermethylation in Ovarian Carcinoma: A Population-based Study. Cancer Res., 60(19), pp.5329-5333.

Baldwin, R.L. et al., 2000. BRCA1 promoter region hypermethylation in ovarian carcinoma: a population-based study. Cancer research, 60(19), pp.5329-33.

Banerjee, S., Kaye, S.B. \& Ashworth, A., 2010. Making the best of PARP inhibitors in ovarian cancer. Nature Reviews Clinical Oncology, 7(9), pp.508-519.

Banerjee, S.K., 2011. Hormonal Carcinogenesis. In Encyclopedia of Cancer. Berlin, Heidelberg: Springer Berlin Heidelberg, pp. 1730-1733.

Bannister, A.J. et al., 2005. Spatial Distribution of Di- and Tri-methyl Lysine 36 of Histone H3 at Active Genes. Journal of Biological Chemistry, 280(18), pp.17732-17736.

Bansal, A., Singh, M.P. \& Rai, B., 2016. Human papillomavirus-associated cancers: A growing global problem. International journal of applied \& basic medical research, 6(2), pp.84-9.

Bapat, S.A. et al., 2005. Stem and progenitor-like cells contribute to the aggressive behavior of human epithelial ovarian cancer. Cancer research, 65(8), pp.3025-9.

Barbacid, M. et al., 2005. Cell cycle and cancer: genetic analysis of the role of cyclindependent kinases. Cold Spring Harbor symposia on quantitative biology, 70(0), pp.233-40.

Barlev, N.A. et al., 2001. Acetylation of p53 activates transcription through recruitment of coactivators/histone acetyltransferases. Molecular cell, 8(6), pp.1243-54.

Barnett, D.W. et al., 2011. BamTools: a C++ API and toolkit for analyzing and managing BAM files. Bioinformatics, 27(12), pp.1691-1692.

Bashashati, A. et al., 2013. Distinct evolutionary trajectories of primary high-grade serous ovarian cancers revealed through spatial mutational profiling. The Journal of pathology, 231(1), pp.21-34.

Bassi, S., 2007. A primer on python for life science researchers. PLoS computational biology, 3(11), p.e199.

Bast, R.C., 2010. CA 125 and the detection of recurrent ovarian cancer: a reasonably accurate biomarker for a difficult disease. Cancer, 116(12), pp.2850-3.

Bauerschlag, D.O. et al., 2010. The role of p53 as a surrogate marker for chemotherapeutical responsiveness in ovarian cancer. Journal of cancer research and clinical oncology, 136(1), pp.79-88.

Bavle, R.M., 2014. Mitosis at a glance. Journal of oral and maxillofacial pathology : JOMFP, 18(Suppl 1), pp.S2-5.

Baxevanis, C.N., Perez, S.A. \& Papamichail, M., 2009. Cancer immunotherapy. Critical Reviews in Clinical Laboratory Sciences, 46(4), pp.167-189.

Baxter, E. et al., 2014. Epigenetic regulation in cancer progression. Cell \& bioscience, 4, p.45. 
Beaufort, C.M. et al., 2014. Ovarian cancer cell line panel (OCCP): clinical importance of in vitro morphological subtypes. PloS one, 9(9), p.e103988.

Behnam-Motlagh, P. et al., 2010. Verotoxin-1 Treatment or Manipulation of its Receptor Globotriaosylceramide (Gb3) for Reversal of Multidrug Resistance to Cancer Chemotherapy. Toxins, 2(10), pp.2467-2477.

Bell, D.A., 2014. Low-grade serous tumors of ovary. International journal of gynecological pathology : official journal of the International Society of Gynecological Pathologists, 33(4), pp.348-56.

Bell, D.A., 2005. Origins and molecular pathology of ovarian cancer. Modern Pathology, 18, pp.S19-S32.

Bell, S.P. \& Dutta, A., 2002. DNA replication in eukaryotic cells. Annual review of biochemistry, 71, pp.333-74.

Bell, S.P. \& Stillman, B., 1992. ATP-dependent recognition of eukaryotic origins of DNA replication by a multiprotein complex. Nature, 357(6374), pp.128-134.

Berdasco, M. \& Esteller, M., 2013. Genetic syndromes caused by mutations in epigenetic genes. Human Genetics, 132(4), pp.359-383.

van den Berg-Bakker, C.A. et al., 1993. Establishment and characterization of 7 ovarian carcinoma cell lines and one granulosa tumor cell line: growth features and cytogenetics. International journal of cancer, 53(4), pp.613-20.

Berger, A.H., Knudson, A.G. \& Pandolfi, P.P., 2011. A continuum model for tumour suppression. Nature, 476(7359), pp.163-9.

Bergfeldt, K. et al., 2002. Risk of ovarian cancer in breast-cancer patients with a family history of breast or ovarian cancer: a population-based cohort study. The Lancet, 360(9337), pp.891-894.

Berns, E.M.J.J. \& Bowtell, D.D., 2012. The changing view of high-grade serous ovarian cancer. Cancer research, 72(11), pp.2701-4.

Bestor, T.H. \& Ingram, V.M., 1983. Two DNA methyltransferases from murine erythroleukemia cells: purification, sequence specificity, and mode of interaction with DNA. Proceedings of the National Academy of Sciences of the United States of America, 80(18), pp.5559-63.

Bi, F.-F., Li, D. \& Yang, Q., 2013. Promoter hypomethylation, especially around the E26 transformation-specific motif, and increased expression of poly (ADP-ribose) polymerase 1 in BRCA-mutated serous ovarian cancer. BMC Cancer, 13(1), p.90.

Bird, A. et al., 1985. A fraction of the mouse genome that is derived from islands of nonmethylated, CpG-rich DNA. Cell, 40(1), pp.91-9.

Blom, N., Gammeltoft, S. \& Brunak, S., 1999. Sequence and structure-based prediction of eukaryotic protein phosphorylation sites. Journal of Molecular Biology, 294(5), pp.1351-1362.

Boettcher, M. \& McManus, M.T., 2015. Choosing the Right Tool for the Job: RNAi, TALEN, or CRISPR. Molecular Cell, 58(4), pp.575-585.

Boffetta, P. et al., 2006. The burden of cancer attributable to alcohol drinking. International Journal of Cancer, 119(4), pp.884-887.

ten Bokkel Huinink, W. et al., 1997. Topotecan versus paclitaxel for the treatment of recurrent epithelial ovarian cancer. Journal of clinical oncology: official journal of the American Society of Clinical Oncology, 15(6), pp.2183-93.

BonDurant, A.E. et al., 2011. Quantitative detection of RASSF1A DNA promoter methylation in tumors and serum of patients with serous epithelial ovarian cancer. Gynecologic Oncology, 123(3), pp.581-587.

Bookman, M.A. et al., 2003. Evaluation of Monoclonal Humanized Anti-HER2 Antibody, Trastuzumab, in Patients With Recurrent or Refractory Ovarian or Primary Peritoneal Carcinoma With Overexpression of HER2: A Phase II Trial of the Gynecologic Oncology Group. Journal of Clinical Oncology, 21(2), pp.283-290.

Bottsford-Miller, J.N., Coleman, R.L. \& Sood, A.K., 2012. Resistance and escape from antiangiogenesis therapy: clinical implications and future strategies. Journal of clinical oncology : official journal of the American Society of Clinical Oncology, 30(32), 
pp.4026-34.

Boussios, S. et al., 2016. Non-epithelial Ovarian Cancer: Elucidating Uncommon Gynaecological Malignancies. Anticancer Research, 36(10), pp.5031-5042.

Bowen, N.J. et al., 2009. Gene expression profiling supports the hypothesis that human ovarian surface epithelia are multipotent and capable of serving as ovarian cancer initiating cells. BMC medical genomics, 2, p.71.

Bower, M. \& Waxman, J., 2015. Oncology - Lecture Notes 3rd ed., John Wiley \& Sons, Ltd.

Bowtell, D.D. et al., 2015. Rethinking ovarian cancer II: reducing mortality from high-grade serous ovarian cancer. Nature reviews. Cancer, 15(11), pp.668-79.

Bowtell, D.D.L., 2010. The genesis and evolution of high-grade serous ovarian cancer. Nature reviews. Cancer, 10(11), pp.803-8.

Braicu, E.-I. et al., 2011. Role of histological type on surgical outcome and survival following radical primary tumour debulking of epithelial ovarian, fallopian tube and peritoneal cancers. British journal of cancer, 105(12), pp.1818-24.

Breitling, R. et al., 2004. Rank products: a simple, yet powerful, new method to detect differentially regulated genes in replicated microarray experiments. FEBS letters, 573(1-3), pp.83-92.

Brien, G.L., Valerio, D.G. \& Armstrong, S.A., 2016. Exploiting the Epigenome to Control Cancer-Promoting Gene-Expression Programs. Cancer cell, 29(4), pp.464-76.

Bristow, R.E. et al., 2002. Survival effect of maximal cytoreductive surgery for advanced ovarian carcinoma during the platinum era: a meta-analysis. Journal of clinical oncology : official journal of the American Society of Clinical Oncology, 20(5), pp.1248-59.

Brose, K. \& Tessier-Lavigne, M., 2000. Slit proteins: key regulators of axon guidance, axonal branching, and cell migration. Current opinion in neurobiology, 10(1), pp.95102.

Brown, L.A. et al., 2006. Amplification of EMSY, a novel oncogene on 11q13, in high grade ovarian surface epithelial carcinomas. Gynecologic Oncology, 100(2), pp.264270.

Bryant, H.E. et al., 2009. PARP is activated at stalled forks to mediate Mre11-dependent replication restart and recombination. The EMBO journal, 28(17), pp.2601-15.

Burger, R.A. et al., 2011. Incorporation of bevacizumab in the primary treatment of ovarian cancer. The New England journal of medicine, 365(26), pp.2473-83.

Burke, T.W. et al., 2001. Replication factors MCM2 and ORC1 interact with the histone acetyltransferase HBO1. The Journal of biological chemistry, 276(18), pp.15397-408.

Bustin, S.A. et al., 2009. The MIQE guidelines: minimum information for publication of quantitative real-time PCR experiments. Clinical chemistry, 55(4), pp.611-22.

Callegaro-Filho, D. et al., 2016. Small cell carcinoma of the ovary-hypercalcemic type (SCCOHT): A review of 47 cases. Gynecologic oncology, 140(1), pp.53-7.

Calzado-Martín, A. et al., 2016. Effect of Actin Organization on the Stiffness of Living Breast Cancer Cells Revealed by Peak-Force Modulation Atomic Force Microscopy. ACS Nano, 10(3), pp.3365-3374.

Cambridge University Press, 1995. Gametes - The Oocyte First. J. . Grudzinskas \& J. . Yovich, eds., Press Syndicate of the University of Cambridge.

Cancer Research UK, 2010. Age-Standardised One-, Five-and Ten-Year Net Survival, Selected Cancers, Adults, England and Wales. Available at: http://www.cancerresearchuk.org/sites/default/files/cstreamnode/cs_surv_10common_male.pdf.

Cancer Statistics UK, C., 2014. CancerStats: Cancer Statistics for the UK. Available at: http://www.cancerresearchuk.org/health-professional/cancer-statistics-for-the-uk.

Cannistra, S.A., 2004. Cancer of the Ovary. New England Journal of Medicine, 351(24), pp.2519-2529.

Carbotti, G. et al., 2013. Activated leukocyte cell adhesion molecule soluble form: a potential biomarker of epithelial ovarian cancer is increased in type II tumors. International Journal of Cancer, 132(11), pp.2597-2605. 
Cardenas, C. et al., 2016. Redefining the origin and evolution of ovarian cancer: a hormonal connection. Endocrine-Related Cancer, 23(9), pp.R411-R422.

Carrozza, M.J. et al., 2003. The diverse functions of histone acetyltransferase complexes. Trends in genetics : TIG, 19(6), pp.321-9.

Cawley, S. et al., 2004. Unbiased mapping of transcription factor binding sites along human chromosomes 21 and 22 points to widespread regulation of noncoding RNAs. Cell, 116(4), pp.499-509.

Celojevic, D. et al., 2011. Effects of $17 \beta$-estradiol on proliferation, cell viability and intracellular redox status in native human lens epithelial cells. Molecular vision, 17, pp.1987-96.

Di Cerbo, V. \& Schneider, R., 2013. Cancers with wrong HATs: the impact of acetylation. Briefings in Functional Genomics, 12(3), pp.231-243.

Chadha, G.S. \& Blow, J.J., 2010. Histone acetylation by HBO1 tightens replication licensing. Molecular cell, 37(1), pp.5-6.

Champagne, K.S. et al., 2008. The crystal structure of the ING5 PHD finger in complex with an H3K4me3 histone peptide. Proteins, 72(4), pp.1371-6.

Chang, B.-D., Heidel, J.D. \& Primiano, T., 2014. Abstract 1946: Silencing of EVI1 with siRNA inhibits the growth of ovarian tumor cells. Cancer Research, 72(8 Supplement).

Chapman, J.R., Taylor, M.R.G. \& Boulton, S.J., 2012. Playing the End Game: DNA Double-Strand Break Repair Pathway Choice. Molecular Cell, 47(4), pp.497-510.

Chen, G. et al., 2012. Role of Tip60 in Human Melanoma Cell Migration, Metastasis, and Patient Survival. Journal of Investigative Dermatology, 132(11), pp.2632-2641.

Chen, V.W. et al., 2003. Pathology and classification of ovarian tumors. Cancer, 97(S10), pp.2631-2642.

Chen, Y., Zhang, L. \& Hao, Q., 2013. Candidate microRNA biomarkers in human epithelial ovarian cancer: systematic review profiling studies and experimental validation. Cancer cell international, 13(1), p.86.

Cheng, C.Y. \& Mruk, D.D., 2010. The biology of spermatogenesis: the past, present and future. Philosophical transactions of the Royal Society of London. Series B, Biological sciences, 365(1546), pp.1459-63.

Chhabra, N., 2013. Biochemistry for Medics. Available at:

$\mathrm{http} / / / \mathrm{www}$. namrata.co/oncogenes-lecture-3/conversion-of-proto-oncogenes-tooncogenes/.

Chi, Y. et al., 2014. Abnormal expression of CDK11p58 in prostate cancer. Cancer cell international, 14(1), p.2.

Chitalia, V.C. et al., 2008. Jade-1 inhibits Wnt signalling by ubiquitylating beta-catenin and mediates Wnt pathway inhibition by pVHL. Nature cell biology, 10(10), pp.1208-16.

Choudhary, C. et al., 2009. Lysine Acetylation Targets Protein Complexes and Co-Regulates Major Cellular Functions. Science, 325(5942).

Christofferson, D.E. \& Yuan, J., 2010. Cyclophilin A release as a biomarker of necrotic cell death. Cell death and differentiation, 17(12), pp.1942-3.

$\mathrm{Chu}$, V.T. et al., 2008. $\mathrm{MeV}+\mathrm{R}$ : using $\mathrm{MeV}$ as a graphical user interface for Bioconductor applications in microarray analysis. Genome biology, 9(7), p.R118.

Ciccia, A. \& Elledge, S.J., 2010. The DNA damage response: making it safe to play with knives. Molecular cell, 40(2), pp.179-204.

Clark, J. et al., 2002. Identification of amplified and expressed genes in breast cancer by comparative hybridization onto microarrays of randomly selected cDNA clones. Genes, chromosomes \& cancer, 34(1), pp.104-14.

Clark, P.R. et al., 1999. Polycations and cationic lipids enhance adenovirus transduction and transgene expression in tumor cells. Cancer Gene Therapy, 6(5), pp.437-446.

Clark, R. et al., 2013. Milky Spots Promote Ovarian Cancer Metastatic Colonization of Peritoneal Adipose in Experimental Models. The American Journal of Pathology, 183(2), pp.576-591.

Clark, W.H., 1991. Tumour progression and the nature of cancer. British journal of cancer, 64(4), pp.631-44. 
Coceano, G. et al., 2016. Investigation into local cell mechanics by atomic force microscopy mapping and optical tweezer vertical indentation. Nanotechnology, 27(6), p.65102.

Codd, E.F. \& F., E., 1970. A relational model of data for large shared data banks. Communications of the ACM, 13(6), pp.377-387.

Coffey, K. et al., 2012. Characterisation of a Tip60 specific inhibitor, NU9056, in prostate cancer. PloS one, 7(10), p.e45539.

Cohen, I. et al., 2011. Histone Modifiers in Cancer: Friends or Foes? Genes \& Cancer, 2(6), pp.631-647.

Cole, A.J. et al., 2016. Assessing mutant p53 in primary high-grade serous ovarian cancer using immunohistochemistry and massively parallel sequencing. Scientific Reports, 6(1), p.26191.

Coleman, R.L. et al., 2015. A phase II evaluation of the potent, highly selective PARP inhibitor veliparib in the treatment of persistent or recurrent epithelial ovarian, fallopian tube, or primary peritoneal cancer in patients who carry a germline BRCA1 or BRCA2 mutation. Gynecologic oncology, 137(3), pp.386-391.

Colland, F., 2010. The therapeutic potential of deubiquitinating enzyme inhibitors. Biochemical Society Transactions, 38(1), pp.137-143.

Contzler, R. et al., 2006. Histone acetyltransferase HBO1 inhibits NF-kappaB activity by coactivator sequestration. Biochemical and biophysical research communications, 350(1), pp.208-13.

Cooper, G., 2000. The Cell: A Molecular Approach Second., Sinauer Associates.

Coscia, F. et al., 2016. Integrative proteomic profiling of ovarian cancer cell lines reveals precursor cell associated proteins and functional status. Nature Communications, 7, p. 12645 .

Costello, J.F. et al., 2000. Aberrant CpG-island methylation has non-random and tumourtype-specific patterns. Nature Genetics, 24(2), pp.132-138.

Coukos, G., Tanyi, J. \& Kandalaft, L.E., 2016. Opportunities in immunotherapy of ovarian cancer. Annals of Oncology, 27(suppl 1), pp.i11-i15.

Courtnay, R. et al., 2015. Cancer metabolism and the Warburg effect: the role of HIF-1 and PI3K. Molecular Biology Reports, 42(4), pp.841-851.

Crawford, S., 2013. Is it time for a new paradigm for systemic cancer treatment? Lessons from a century of cancer chemotherapy. Frontiers in pharmacology, 4, p.68.

Cross, S.E. et al., 2007. Nanomechanical analysis of cells from cancer patients. Nature Nanotechnology, 2(12), pp.780-783.

Crow, J.F., 1994. Advantages of sexual reproduction. Developmental Genetics, 15(3), pp.205-213.

Cuatrecasas, M. et al., 2009. Transitional Cell Tumors of the Ovary. The American Journal of Surgical Pathology, 33(4), pp.556-567.

Cui, S. et al., 2015. The Emerging Role of Inhibitor of Growth 4 as a Tumor Suppressor in Multiple Human Cancers. Cellular Physiology and Biochemistry, 36(2), pp.409-422.

Curley, M.D. et al., 2009. CD133 expression defines a tumor initiating cell population in primary human ovarian cancer. Stem cells (Dayton, Ohio), 27(12), pp.2875-83.

Daniels, M.S. \& Lu, K.H., 2015. Clearer picture of PMS2-associated lynch syndrome is emerging. Journal of clinical oncology : official journal of the American Society of Clinical Oncology, 33(4), pp.299-300.

Dao, F. et al., 2016. Characteristics of 10-year survivors of high-grade serous ovarian carcinoma. Gynecologic Oncology, 141(2), pp.260-263.

Darb-Esfahani, S. et al., 2014. Interferon-stimulated Gene, 15 kDa (ISG15) in Ovarian Highgrade Serous Carcinoma. International Journal of Gynecological Pathology, 33(1), pp.16-22.

Davidowitz, R.A. et al., 2014. Mesenchymal gene program-expressing ovarian cancer spheroids exhibit enhanced mesothelial clearance. The Journal of clinical investigation, 124(6), pp.2611-25.

Dawson, M.A., Kouzarides, T. \& Huntly, B.J.P., 2012. Targeting Epigenetic Readers in Cancer. New England Journal of Medicine, 367(7), pp.647-657. 
Debes, J.D. et al., 2003. p300 in prostate cancer proliferation and progression. Cancer research, 63(22), pp.7638-40.

Decruze, S.B. et al., 2010. Paracentesis in Ovarian Cancer: A Study of the Physiology during Free Drainage of Ascites. Journal of Palliative Medicine, 13(3), pp.251-254.

Dellino, G.I. et al., 2013. Genome-wide mapping of human DNA-replication origins: levels of transcription at ORC1 sites regulate origin selection and replication timing. Genome research, 23(1), pp.1-11.

DelloRusso, C. et al., 2007. Functional characterization of a novel BRCA1-null ovarian cancer cell line in response to ionizing radiation. Molecular cancer research : MCR, 5(1), pp.35-45.

Deng, W. et al., 2016. GPS-PAIL: prediction of lysine acetyltransferase-specific modification sites from protein sequences. Scientific Reports, 6(1), p.39787.

DePamphilis, M.L., 2003. The "ORC cycle": a novel pathway for regulating eukaryotic DNA replication. Gene, 310, pp.1-15.

Dephoure, N. et al., 2008. A quantitative atlas of mitotic phosphorylation. Proceedings of the National Academy of Sciences of the United States of America, 105(31), pp.107627.

Desai, N. et al., 2013. Female and Male Gametogenesis. In Clinical Reproductive Medicine and Surgery. Springer New York, pp. 43-62.

Diaz, A., Nellore, A. \& Song, J.S., 2012. CHANCE: comprehensive software for quality control and validation of ChIP-seq data. Genome biology, 13(10), p.R98.

Dick, J.E., 2008. Stem cell concepts renew cancer research. Blood, 112(13), pp.4793-807.

Diederich, M.-H. et al., 2011. Anti-proliferative potential of curcumin in androgendependent prostate cancer cells occurs through modulation of the Wingless signaling pathway. International Journal of Oncology, 38(3), pp.603-11.

Dirat, B. et al., 2010. Unraveling the Obesity and Breast Cancer Links: A Role for CancerAssociated Adipocytes? In Adipose Tissue Development. Basel: KARGER, pp. 45-52.

Dizon, D.S., Blessing, J.A., et al., 2012. A phase II evaluation of belinostat and carboplatin in the treatment of recurrent or persistent platinum-resistant ovarian, fallopian tube, or primary peritoneal carcinoma: A gynecologic oncology group study. Gynecologic Oncology, 125(2), pp.367-371.

Dizon, D.S., Damstrup, L., et al., 2012. Phase II Activity of Belinostat (PXD-101), Carboplatin, and Paclitaxel in Women With Previously Treated Ovarian Cancer. International Journal of Gynecological Cancer, 22(6), pp.979-986.

Domchek, S.M. et al., 2016. Efficacy and safety of olaparib monotherapy in germline BRCA1/2 mutation carriers with advanced ovarian cancer and three or more lines of prior therapy. Gynecologic Oncology, 140(2), pp.199-203.

Domcke, S. et al., 2013. Evaluating cell lines as tumour models by comparison of genomic profiles. Nature communications, 4, p.2126.

Donnez, J. et al., 1985. Cyclic changes in ciliation, cell height, and mitotic activity in human tubal epithelium during reproductive life. Fertility and sterility, 43(4), pp.554-9.

Downward, J., 2004. RNA interference. BMJ (Clinical research ed.), 328(7450), pp.1245-8.

Doyon, Y. et al., 2006. ING tumor suppressor proteins are critical regulators of chromatin acetylation required for genome expression and perpetuation. Molecular cell, 21(1), pp.51-64.

Drazic, A. et al., 2016. The world of protein acetylation. Biochimica et Biophysica Acta (BBA) - Proteins and Proteomics, 1864(10), pp.1372-1401.

Drew, Y., 2015. The development of PARP inhibitors in ovarian cancer: from bench to bedside. British journal of cancer, 113 Suppl(S1), pp.S3-9.

Du, P., Kibbe, W.A. \& Lin, S.M., 2008. lumi: a pipeline for processing Illumina microarray. Bioinformatics (Oxford, England), 24(13), pp.1547-8.

Duda, K. et al., 2014. High-efficiency genome editing via 2A-coupled co-expression of fluorescent proteins and zinc finger nucleases or CRISPR/Cas9 nickase pairs. Nucleic Acids Research, 42(10), pp.e84-e84.

Duong, M.T. et al., 2013. Hbo1 is a cyclin E/CDK2 substrate that enriches breast cancer 
stem-like cells. Cancer research, 73(17), pp.5556-68.

Duraiswamy, J. et al., 2013. Dual Blockade of PD-1 and CTLA-4 Combined with Tumor Vaccine Effectively Restores T-Cell Rejection Function in Tumors. Cancer Research, 73(12), pp.3591-3603.

Dutta, A. \& Bell, S.P., 1997. Initiation of DNA replication in eukaryotic cells. Annual review of cell and developmental biology, 13, pp.293-332.

Easwaran, H. et al., 2012. A DNA hypermethylation module for the stem/progenitor cell signature of cancer. Genome Research, 22(5), pp.837-849.

Edlich, R.F., Winters, K.L. \& Lin, K.Y., 2005. Breast cancer and ovarian cancer genetics. Journal of long-term effects of medical implants, 15(5), pp.533-45.

Edwards, S.L. et al., 2008. Resistance to therapy caused by intragenic deletion in BRCA2. Nature, 451(7182), pp.1111-5.

Ehrenhofer-Murray, A.E., 2004. Chromatin dynamics at DNA replication, transcription and repair. European Journal of Biochemistry, 271(12), pp.2335-2349.

Elit, L. et al., 2007. Intraperitoneal chemotherapy in the first-line treatment of women with stage III epithelial ovarian cancer. Cancer, 109(4), pp.692-702.

Elmore, S., 2007. Apoptosis: a review of programmed cell death. Toxicologic pathology, 35(4), pp.495-516.

Elnakat, H. \& Ratnam, M., 2006. Role of folate receptor genes in reproduction and related cancers. Frontiers in bioscience : a journal and virtual library, 11, pp.506-19.

Epstein, E.H., 2008. Basal cell carcinomas: attack of the hedgehog. Nature reviews. Cancer, 8(10), pp.743-54.

Erzen, M. et al., 2001. Endometriosis-associated ovarian carcinoma (EAOC): an entity distinct from other ovarian carcinomas as suggested by a nested case-control study. Gynecologic oncology, 83(1), pp.100-8.

Esteller, M., 2008. Epigenetics in Cancer. New England Journal of Medicine, 358(11), pp.1148-1159.

Etemadmoghadam, D. et al., 2010. Amplicon-dependent CCNE1 expression is critical for clonogenic survival after cisplatin treatment and is correlated with $20 \mathrm{q} 11$ gain in ovarian cancer. N. Wong, ed. PloS one, 5(11), p.e15498.

Falcetta, F.S. et al., 2016. Laparoscopy versus laparotomy for FIGO stage I ovarian cancer. In D. D. Rosa, ed. Cochrane Database of Systematic Reviews. Chichester, UK: John Wiley \& Sons, Ltd.

Falchook, G.S. et al., 2013. Methylation and histone deacetylase inhibition in combination with platinum treatment in patients with advanced malignancies. Investigational New Drugs, 31(5), pp.1192-1200.

Fang, F. et al., 2010. A phase 1 and pharmacodynamic study of decitabine in combination with carboplatin in patients with recurrent, platinum-resistant, epithelial ovarian cancer. Cancer, 116(17), pp.4043-4053.

Farria, A., Li, W. \& Dent, S.Y.R., 2015. KATs in cancer: functions and therapies. Oncogene.

Fathalla, M.F., 1971. Incessant ovulation--a factor in ovarian neoplasia? Lancet (London, England), 2(7716), p.163.

Fathalla, M.F., 2013. Incessant ovulation and ovarian cancer - a hypothesis re-visited. Facts, views \& vision in ObGyn, 5(4), pp.292-7.

Fathalla, M.F., 2016. Non-hormonal interruption of incessant ovulation as a potential approach for ovarian cancer prevention. International Journal of Gynecology \& Obstetrics, 132(3), pp.356-358.

Fathalla, M.F., 2015. Women and the burden of human reproduction: An evolutionary perspective. Journal of Reproductive Health and Medicine, 1(2), pp.103-105.

Faubert, B. et al., 2015. The AMP-activated protein kinase (AMPK) and cancer: Many faces of a metabolic regulator. Cancer Letters, 356(2), pp.165-170.

Fay, D.S. \& Gerow, K., 2013. A biologist's guide to statistical thinking and analysis. WormBook: the online review of C. elegans biology, pp.1-54.

Feinberg, A.P. \& Tycko, B., 2004. The history of cancer epigenetics. Nature reviews. 
Cancer, 4(2), pp.143-53.

Feinberg, A.P. \& Vogelstein, B., 1983. Hypomethylation distinguishes genes of some human cancers from their normal counterparts. Nature, 301(5895), pp.89-92.

Feng, J. et al., 2012. Identifying ChIP-seq enrichment using MACS. Nature protocols, 7(9), pp.1728-40.

Feng, Y. et al., 2015. BRPF3-HBO1 regulates replication origin activation and histone H3K14 acetylation. The EMBO journal, 35(2), pp.176-192.

Ferrandina, G. et al., 2000. p21WAF1/CIP1 protein expression in primary ovarian cancer. International journal of oncology, 17(6), pp.1231-5.

Ferraris, D. V., 2010. Evolution of Poly(ADP-ribose) Polymerase-1 (PARP-1) Inhibitors. From Concept to Clinic. Journal of Medicinal Chemistry, 53(12), pp.4561-4584.

Fidler, I.J. \& Hart, I.R., 1982. Biological diversity in metastatic neoplasms: origins and implications. Science (New York, N.Y.), 217(4564), pp.998-1003.

Figueiredo, L.M., Cross, G.A.M. \& Janzen, C.J., 2009. Epigenetic regulation in African trypanosomes: a new kid on the block. Nature Reviews Microbiology, 7(7), pp.504513.

Finlayson, A., 2007. Endocrine and Reproductive Systems Third., Elsevier.

Fischer, M., 2017. Census and evaluation of p53 target genes. Oncogene, 36(28), pp.39433956.

Fishelson, Z. et al., 2003. Obstacles to cancer immunotherapy: expression of membrane complement regulatory proteins (mCRPs) in tumors. Molecular immunology, 40(2-4), pp.109-23.

Fletcher, D.A. \& Mullins, R.D., 2010. Cell mechanics and the cytoskeleton. Nature, 463(7280), pp.485-492.

Flotho, A. \& Melchior, F., 2013. Sumoylation: A Regulatory Protein Modification in Health and Disease. Annual Review of Biochemistry, 82(1), pp.357-385.

Fogh, J., Fogh, J.M. \& Orfeo, T., 1977. One hundred and twenty-seven cultured human tumor cell lines producing tumors in nude mice. Journal of the National Cancer Institute, 59(1), pp.221-6.

Fogh, J., Wright, W.C. \& Loveless, J.D., 1977. Absence of HeLa cell contamination in 169 cell lines derived from human tumors. Journal of the National Cancer Institute, 58(2), pp.209-14.

Fong, P.C. et al., 2009. Inhibition of poly(ADP-ribose) polymerase in tumors from BRCA mutation carriers. The New England journal of medicine, 361(2), pp.123-34.

Foster, K.A. et al., 1996. Somatic and germline mutations of the BRCA2 gene in sporadic ovarian cancer. Cancer research, 56(16), pp.3622-5.

Foy, R.L. et al., 2008. Role of Jade-1 in the histone acetyltransferase (HAT) HBO1 complex. The Journal of biological chemistry, 283(43), pp.28817-26.

Fradet-Turcotte, A. et al., 2016. BRCA2 functions: from DNA repair to replication fork stabilization. Endocrine-related cancer, 23(10), pp.T1-T17.

Fraga, M.F. et al., 2005. Loss of acetylation at Lys 16 and trimethylation at Lys 20 of histone H4 is a common hallmark of human cancer. Nature Genetics, 37(4), pp.391-400.

Freedman, R.S. et al., 2004. Peritoneal inflammation - A microenvironment for Epithelial Ovarian Cancer (EOC). Journal of Translational Medicine, 2(1), p.23.

Freeman, A. et al., 1999. Minichromosome Maintenance Proteins as Biological Markers of Dysplasia and Malignancy. Clinical Cancer Research, 5(8).

Friedberg, E.C., 2003. DNA damage and repair. Nature, 421(6921), pp.436-440.

$\mathrm{Fu}$, S. et al., 2011. Phase 1b-2a study to reverse platinum resistance through use of a hypomethylating agent, azacitidine, in patients with platinum-resistant or platinumrefractory epithelial ovarian cancer. Cancer, 117(8), pp.1661-1669.

Furey, T.S., 2012. ChIP-seq and beyond: new and improved methodologies to detect and characterize protein-DNA interactions. Nature reviews. Genetics, 13(12), pp.840-52.

Gade, P. \& Kalvakolanu, D. V, 2012. Chromatin immunoprecipitation assay as a tool for analyzing transcription factor activity. Methods in molecular biology (Clifton, N.J.), 809, pp.85-104. 
Gaitskell, K. et al., 2016. Tubal ligation and ovarian cancer risk in a large cohort: Substantial variation by histological type. International journal of cancer, 138(5), pp.1076-84.

Galtier-Dereure, F. et al., 1992. Estradiol stimulates cell growth and secretion of procathepsin D and a 120-kilodalton protein in the human ovarian cancer cell line BG1. The Journal of clinical endocrinology and metabolism, 75(6), pp.1497-502.

Gamble, M.J. et al., 2010. The histone variant macroH2A1 marks repressed autosomal chromatin, but protects a subset of its target genes from silencing. Genes \& Development, 24(1), pp.21-32.

Gao, C. et al., 2014. Rational design and validation of a Tip60 histone acetyltransferase inhibitor. Scientific reports, 4, p.5372.

Gao, X. et al., 2014. LEF1 regulates glioblastoma cell proliferation, migration, invasion, and cancer stem-like cell self-renewal. Tumor Biology, 35(11), pp.11505-11511.

Garkavtsev, I. et al., 2004. The candidate tumour suppressor protein ING4 regulates brain tumour growth and angiogenesis. Nature, 428(6980), pp.328-32.

Gavara, N., 2017. A beginner's guide to atomic force microscopy probing for cell mechanics. Microscopy research and technique, 80(1), pp.75-84.

Gayther, S.A. et al., 2000. Mutations truncating the EP300 acetylase in human cancers. Nature Genetics, 24(3), pp.300-303.

George, S.H.L. \& Shaw, P., 2014. BRCA and Early Events in the Development of Serous Ovarian Cancer. Frontiers in oncology, 4, p.5.

Georgiakaki, M. et al., 2006. Ligand-controlled interaction of histone acetyltransferase binding to ORC-1 (HBO1) with the $\mathrm{N}$-terminal transactivating domain of progesterone receptor induces steroid receptor coactivator 1-dependent coactivation of transcription. Molecular endocrinology (Baltimore, Md.), 20(9), pp.2122-40.

Geraghty, R.J. et al., 2014. Guidelines for the use of cell lines in biomedical research. British journal of cancer, 111(6), pp.1021-46.

Gershenson, D.M. et al., 2015. Impact of Age and Primary Disease Site on Outcome in Women With Low-Grade Serous Carcinoma of the Ovary or Peritoneum: Results of a Large Single-Institution Registry of a Rare Tumor. Journal of Clinical Oncology, 33(24), pp.2675-2682.

Gershenson, D.M., 2016. Ovarian germ cell tumors: Pathology, clinical manifestations, and diagnosis.

Gershey, E.L., Vidali, G. \& Allfrey, V.G., 1968. Chemical studies of histone acetylation. The occurrence of epsilon-N-acetyllysine in the f2al histone. The Journal of biological chemistry, 243(19), pp.5018-22.

Ghizzoni, M. et al., 2012. 6-alkylsalicylates are selective Tip60 inhibitors and target the acetyl-CoA binding site. European Journal of Medicinal Chemistry, 47(1), pp.337344.

Gilbert, S.F., 2000. Oogenesis. In Developmental Biology. Sinauer Associates.

Gilks, C.B. et al., 2008. Tumor cell type can be reproducibly diagnosed and is of independent prognostic significance in patients with maximally debulked ovarian carcinoma. Human Pathology, 39(8), pp.1239-1251.

Gilks, C.B. \& Prat, J., 2009. Ovarian carcinoma pathology and genetics: recent advances. Human pathology, 40(9), pp.1213-23.

Glasspool, R.M. et al., 2014. A randomised, phase II trial of the DNA-hypomethylating agent 5-aza-2'-deoxycytidine (decitabine) in combination with carboplatin vs carboplatin alone in patients with recurrent, partially platinum-sensitive ovarian cancer. British Journal of Cancer, 110(8), pp.1923-1929.

Goff, B.A. et al., 2004. Frequency of symptoms of ovarian cancer in women presenting to primary care clinics. JAMA, 291(22), pp.2705-12.

Goff, B.A. et al., 2000. Ovarian carcinoma diagnosis. Cancer, 89(10), pp.2068-2075.

Goh, A.M., Coffill, C.R. \& Lane, D.P., 2011. The role of mutant p53 in human cancer. The Journal of pathology, 223(2), pp.116-26.

Gong, H. et al., 2017. Method for Dual Viral Vector Mediated CRISPR-Cas9 Gene Disruption in Primary Human Endothelial Cells. Scientific Reports, 7, p.42127. 
Goren, A. et al., 2008. DNA replication timing of the human beta-globin domain is controlled by histone modification at the origin. Genes \& development, 22(10), pp.1319-24.

Gorringe, K.L. et al., 2007. High-Resolution Single Nucleotide Polymorphism Array Analysis of Epithelial Ovarian Cancer Reveals Numerous Microdeletions and Amplifications. Clinical Cancer Research, 13(16), pp.4731-4739.

Gourley, C. et al., 2014. Gynecologic Cancer InterGroup (GCIG) Consensus Review for Ovarian and Primary Peritoneal Low-Grade Serous Carcinomas. International Journal of Gynecological Cancer, 24, pp.S9-S13.

Gourley, C. et al., 2014. Molecular subgroup of high-grade serous ovarian cancer (HGSOC) as a predictor of outcome following bevacizumab. In: (Proceedings) 50th Annual Meeting of the American-Society-of-Clinical-Oncology (2014).

Graeber, T.G. et al., 1994. Hypoxia induces accumulation of $\mathrm{p} 53$ protein, but activation of a G1-phase checkpoint by low-oxygen conditions is independent of p53 status. Molecular and cellular biology, 14(9), pp.6264-77.

Greene, F.L. \& Sobin, L.H., 2008. The staging of cancer: a retrospective and prospective appraisal. CA: a cancer journal for clinicians, 58(3), pp.180-90.

Grienenberger, A. et al., 2002. The MYST Domain Acetyltransferase Chameau Functions in Epigenetic Mechanisms of Transcriptional Repression,

Grunewald, T. \& Ledermann, J.A., 2016. Targeted Therapies for Ovarian Cancer. Best Practice \& Research Clinical Obstetrics \& Gynaecology.

Guck, J. et al., 2005. Optical Deformability as an Inherent Cell Marker for Testing Malignant Transformation and Metastatic Competence. Biophysical Journal, 88(5), pp.3689-3698.

Gudmundsdottir, K. \& Ashworth, A., 2006. The roles of BRCA1 and BRCA2 and associated proteins in the maintenance of genomic stability. Oncogene, 25(43), pp.5864-5874.

Guglielmelli, P. et al., 2011. EZH2 mutational status predicts poor survival in myelofibrosis. Blood, 118(19), pp.5227-5234.

Gundelach, H., Braas, D. \& Klempnauer, K.-H., 2007. The promoter regions of the Mybregulated Adora2B and $\mathrm{Mcm} 4$ genes co-localize with origins of DNA replication. BMC Molecular Biology, 8(75).

Haeussler, M. et al., 2016. Evaluation of off-target and on-target scoring algorithms and integration into the guide RNA selection tool CRISPOR. Genome Biology, 17(1), p.148.

Haggarty, S.J. et al., 2003. Domain-selective small-molecule inhibitor of histone deacetylase 6 (HDAC6)-mediated tubulin deacetylation. Proceedings of the National Academy of Sciences of the United States of America, 100(8), pp.4389-94.

Halazonetis, T.D., Gorgoulis, V.G. \& Bartek, J., 2008. An Oncogene-Induced DNA Damage Model for Cancer Development. Science, 319(5868), pp.1352-1355.

Hall, J.E., 2016. Textbook of Medical Physiology 13th ed., Guyton and Hall.

Haller, O., Kochs, G. \& Weber, F., 2006. The interferon response circuit: Induction and suppression by pathogenic viruses. Virology, 344(1), pp.119-130.

Hamilton, T.C. et al., 1983. Characterization of a Human Ovarian Carcinoma Cell Line (NIH:OVCAR-3) with Androgen and Estrogen Receptors. Cancer Research, 43(11), pp.5379-5389.

Hanahan, D. et al., 2011. Hallmarks of cancer: the next generation. Cell, 144(5), pp.646-74.

Hanahan, D. \& Weinberg, R.A., 2000. The hallmarks of cancer. Cell, 100(1), pp.57-70.

Hanker, L.C. et al., 2012. The impact of second to sixth line therapy on survival of relapsed ovarian cancer after primary taxane/platinum-based therapy. Annals of Oncology, 23(10), pp.2605-2612.

Harding, M.A. \& Theodorescu, D., 2010. RhoGDI signaling provides targets for cancer therapy. European Journal of Cancer, 46(7), pp.1252-1259.

Harris, M.A. et al., 2003. Carcinosarcoma of the ovary. British journal of cancer, 88(5), pp.654-7. 
Harris, S.L. \& Levine, A.J., 2005. The p53 pathway: positive and negative feedback loops. Oncogene, 24(17), pp.2899-908.

Hauptmann, S. et al., 2002. Genetic alterations in epithelial ovarian tumors analyzed by comparative genomic hybridization. Human pathology, 33(6), pp.632-41.

Havasi, A. et al., 2013. Histone acetyl transferase (HAT) HBO1 and JADE1 in epithelial cell regeneration. The American journal of pathology, 182(1), pp.152-62.

Havrilesky, L.J. et al., 2013. Oral Contraceptive Pills as Primary Prevention for Ovarian Cancer. Obstetrics \& Gynecology, 122(1), pp.139-147.

He, G.H.Y. et al., 2005. Phylogenetic analysis of the ING family of PHD finger proteins. Molecular biology and evolution, 22(1), pp.104-16.

Heiskanen, M.A. et al., 2000. Detection of gene amplification by genomic hybridization to cDNA microarrays. Cancer research, 60(4), pp.799-802.

Helin, K. \& Dhanak, D., 2013. Chromatin proteins and modifications as drug targets. Nature, 502(7472), pp.480-488.

Helland, A. et al., 2011. Deregulation of MYCN, LIN28B and LET7 in a molecular subtype of aggressive high-grade serous ovarian cancers. PloS one, 6(4), p.e18064.

Henikoff, S. \& Smith, M.M., 2015. Histone Variants and Epigenetics. Cold Spring Harbor Perspectives in Biology, 7(1), p.a019364.

Hennessy, B.T., Coleman, R.L. \& Markman, M., 2009. Ovarian cancer. Lancet, 374(9698), pp.1371-82.

Hennessy, B.T. \& Mills, G.B., 2006. Ovarian cancer: homeobox genes, autocrine/paracrine growth, and kinase signaling. The international journal of biochemistry \& cell biology, 38(9), pp.1450-6.

Henry, R.A., Kuo, Y.-M. \& Andrews, A.J., 2013. Differences in Specificity and Selectivity Between CBP and p300 Acetylation of Histone H3 and H3/H4. Biochemistry, 52(34), pp.5746-5759.

Heppner, G.H., 1984. Tumor heterogeneity. Cancer research, 44(6), pp.2259-65.

Herman, J.G. \& Baylin, S.B., 2003. Gene Silencing in Cancer in Association with Promoter Hypermethylation. New England Journal of Medicine, 349(21), pp.2042-2054.

Herold, C.I. \& Cannistra, S.A., 2013. Gynecologic Cancer. Scientific American Medicine, Oncology, 40(2), pp.178-181.

Hess, V. et al., 2004. Mucinous epithelial ovarian cancer: a separate entity requiring specific treatment. Journal of clinical oncology : official journal of the American Society of Clinical Oncology, 22(6), pp.1040-4.

Hildebrand, J.S. et al., 2010. Postmenopausal hormone use and incident ovarian cancer: Associations differ by regimen. International journal of cancer, 127(12), pp.2928-35.

Hilton, J.L. et al., 2002. Inactivation of BRCA1 and BRCA2 in ovarian cancer. Journal of the National Cancer Institute, 94(18), pp.1396-406.

Hoeijmakers, J.H.J., 2001. Genome maintenance mechanisms for preventing cancer. Nature, 411(6835), pp.366-374.

Hoesel, B. \& Schmid, J.A., 2013. The complexity of NF-кB signaling in inflammation and cancer. Molecular cancer, 12, p.86.

Holliday, R. \& Pugh, J.E., 1975. DNA modification mechanisms and gene activity during development. Science (New York, N.Y.), 187(4173), pp.226-32.

Hon, G.C., Hawkins, R.D. \& Ren, B., 2009. Predictive chromatin signatures in the mammalian genome. Human Molecular Genetics, 18(R2), pp.R195-R201.

Housden, B.E. \& Perrimon, N., 2016. Comparing CRISPR and RNAi-based screening technologies. Nature Biotechnology, 34(6), pp.621-623.

Housman, G. et al., 2014. Drug resistance in cancer: an overview. Cancers, 6(3), pp.176992.

Howes, S.C. et al., 2014. Effects of tubulin acetylation and tubulin acetyltransferase binding on microtubule structure. Molecular biology of the cell, 25(2), pp.257-66.

Howitt, B.E. et al., 2015. Evidence for a dualistic model of high-grade serous carcinoma: BRCA mutation status, histology, and tubal intraepithelial carcinoma. The American journal of surgical pathology, 39(3), pp.287-93. 
$\mathrm{Hu}, \mathrm{X}$. et al., 2009. Genetic alterations and oncogenic pathways associated with breast cancer subtypes. Molecular cancer research : MCR, 7(4), pp.511-22.

Hua, W. et al., 1995. SKOV3 ovarian carcinoma cells have functional estrogen receptor but are growth-resistant to estrogen and antiestrogens. The Journal of Steroid Biochemistry and Molecular Biology, 55(3), pp.279-289.

Hubbert, C. et al., 2002. HDAC6 is a microtubule-associated deacetylase. Nature, 417(6887), pp.455-458.

Hulsen, T. et al., 2008. BioVenn - a web application for the comparison and visualization of biological lists using area-proportional Venn diagrams. BMC Genomics, 9(1), p.488.

Hung, T. et al., 2009. ING4 mediates crosstalk between histone H3 K4 trimethylation and H3 acetylation to attenuate cellular transformation. Molecular cell, 33(2), pp.248-56.

Hyman, E. et al., 2002. Impact of DNA amplification on gene expression patterns in breast cancer. Cancer research, 62(21), pp.6240-5.

Iizuka, M. et al., 2008. Hbo1 Links p53-dependent stress signaling to DNA replication licensing. Molecular and cellular biology, 28(1), pp.140-53.

Iizuka, M. et al., 2009. Histone acetyltransferase Hbo1: catalytic activity, cellular abundance, and links to primary cancers. Gene, 436(1-2), pp.108-14.

Iizuka, M. et al., 2013. Histone acetyltransferase Hbol destabilizes estrogen receptor $\alpha$ by ubiquitination and modulates proliferation of breast cancers. Cancer science, 104(12), pp.1647-55.

Iizuka, M. et al., 2006. Regulation of replication licensing by acetyltransferase Hbo1. Molecular and cellular biology, 26(3), pp.1098-108.

Iizuka, M. \& Stillman, B., 1999. Histone acetyltransferase HBO1 interacts with the ORC1 subunit of the human initiator protein. The Journal of biological chemistry, 274(33), pp.23027-34.

Impey, L. \& Child, T., 2012. Obstetrics \& Gynaecology Forth., Wiley-Blackwell.

Inoue, K. \& Fry, E., 2015. Aberrant Expression of Cyclin D1 in Cancer. Signal Transduction Insights, 4, p.1.

Ishihama, K. et al., 2007. Expression of HDAC1 and CBP/p300 in human colorectal carcinomas. Journal of Clinical Pathology, 60(11), pp.1205-1210.

Ishikawa, K. et al., 1998. Prediction of the coding sequences of unidentified human genes. $\mathrm{X}$. The complete sequences of 100 new cDNA clones from brain which can code for large proteins in vitro. DNA research : an international journal for rapid publication of reports on genes and genomes, 5(3), pp.169-76.

Jackson, A.L. \& Linsley, P.S., 2010. Recognizing and avoiding siRNA off-target effects for target identification and therapeutic application. Nature Reviews Drug Discovery, 9(1), pp.57-67.

Jacob, F. et al., 2014. Expression of GBGT1 is epigenetically regulated by DNA methylation in ovarian cancer cells. BMC Molecular Biology, 15(1), p.24.

Jacobs, I.J. et al., 1999. Screening for ovarian cancer: a pilot randomised controlled trial. The Lancet, 353(9160), pp.1207-1210.

Jain, S. et al., 2012. Auto-acetylation stabilizes p300 in cardiac myocytes during acute oxidative stress, promoting STAT3 accumulation and cell survival. Breast cancer research and treatment, 135(1), pp.103-14.

Jane, C., Louise, S. \& Hickey, M., 2012. Hormone replacement therapy. Hormone replacement therapy British Medical Journal, 344.

Jänicke, R.U. et al., 2007. The Multiple Battles Fought by Anti-Apoptotic p21. Cell Cycle, 6(4), pp.407-413.

Jasin, M. \& Rothstein, R., 2013. Repair of strand breaks by homologous recombination. Cold Spring Harbor perspectives in biology, 5(11), p.a012740.

Jeggo, P.A., Pearl, L.H. \& Carr, A.M., 2015. DNA repair, genome stability and cancer: a historical perspective. Nature Reviews Cancer, 16(1), pp.35-42.

Jenkins, G.W., Kemnitz, C.P. \& Tortora, G.J., 2008. Anatomy and Physiology: From Science to Life 2nd ed., Wiley.

Jenkins, M.A., 2009. Role of MSH6 and PMS2 in the DNA Mismatch Repair Process and 
Carcinogenesis. Surgical Oncology Clinics of North America, 18(4), pp.625-636.

Jin, Q. et al., 2014. Gen5 and PCAF negatively regulate interferon- $\beta$ production through

HAT-independent inhibition of TBK1. EMBO reports, 15(11), pp.1192-201.

Jinek, M. et al., 2012. A Programmable Dual-RNA-Guided DNA Endonuclease in Adaptive Bacterial Immunity. Science, 337(6096), pp.816-821.

Jinka, R. et al., 2012. Alterations in Cell-Extracellular Matrix Interactions during Progression of Cancers. International journal of cell biology, 2012, p.219196.

Johmura, Y., Suzuki, M., et al., 2008. FAD24, a regulator of adipogenesis and DNA replication, inhibits H-RAS-mediated transformation by repressing NF-kappaB activity. Biochemical and biophysical research communications, 369(2), pp.464-70.

Johmura, Y., Osada, S., et al., 2008. FAD24 acts in concert with histone acetyltransferase HBO1 to promote adipogenesis by controlling DNA replication. The Journal of biological chemistry, 283(4), pp.2265-74.

Johnson, J. et al., 2004. Germline stem cells and follicular renewal in the postnatal mammalian ovary. Nature, 428(6979), pp.145-150.

Johnson, M.H. \& Everitt, B.J., 2000. Essential Reproduction Fifth., Blackwell Publishing Inc.

Jones, P.A. \& Baylin, S.B., 2007. The Epigenomics of Cancer. Cell, 128(4), pp.683-692.

Jones, P.A. \& Baylin, S.B., 2002. The fundamental role of epigenetic events in cancer. Nature reviews. Genetics, 3(6), pp.415-28.

Jones, P.A., Issa, J.-P.J. \& Baylin, S., 2016. Targeting the cancer epigenome for therapy. Nature Reviews Genetics, 17(10), pp.630-641.

Jones, R.E. \& Lopez, K.H., 2013. Human Reproductive Biology,

Jordan, V.C., 1997. Tamoxifen: the herald of a new era of preventive therapeutics. Journal of the National Cancer Institute, 89(11), pp.747-9.

Kalkhoven, E., 2004. CBP and p300: HATs for different occasions. Biochemical Pharmacology, 68(6), pp.1145-1155.

Kalli, K.R. et al., 2008. Folate receptor alpha as a tumor target in epithelial ovarian cancer. Gynecologic Oncology, 108(3), pp.619-626.

Kalliolias, G.D. \& Ivashkiv, L.B., 2010. Overview of the biology of type I interferons. Arthritis Research \& Therapy, 12(Suppl 1), p.S1.

Kandalaft, L.E. et al., 2013. A Phase I vaccine trial using dendritic cells pulsed with autologous oxidized lysate for recurrent ovarian cancer. Journal of translational medicine, 11(1), p.149.

Karnani, N. et al., 2007. Pan-S replication patterns and chromosomal domains defined by genome-tiling arrays of ENCODE genomic areas. Genome research, 17(6), pp.865-76.

Karnezis, A.N. et al., 2016. The disparate origins of ovarian cancers: pathogenesis and prevention strategies. Nature Reviews Cancer, 17(1), pp.65-74.

Karnoub, A.E. \& Weinberg, R.A., 2008. Ras oncogenes: split personalities. Nature reviews. Molecular cell biology, 9(7), pp.517-31.

Karst, A.M., Levanon, K., Duraisamy, S., et al., 2011. Stathmin 1, a marker of PI3K pathway activation and regulator of microtubule dynamics, is expressed in early pelvic serous carcinomas. Gynecologic oncology, 123(1), pp.5-12.

Karst, A.M., Levanon, K. \& Drapkin, R., 2011. Modeling high-grade serous ovarian carcinogenesis from the fallopian tube. Proceedings of the National Academy of Sciences of the United States of America, 108(18), pp.7547-52.

Karve, T.M. \& Cheema, A.K., 2011. Small changes huge impact: the role of protein posttranslational modifications in cellular homeostasis and disease. Journal of amino acids, 2011, p.207691.

Kastan, M.B. et al., 1991. Participation of $\mathrm{p} 53$ protein in the cellular response to DNA damage. Cancer research, 51(23 Pt 1), pp.6304-11.

Kaur, G. \& Dufour, J.M., 2012. Cell lines: Valuable tools or useless artifacts. Spermatogenesis, 2(1), pp.1-5.

Kaverina, I. \& Straube, A., 2011. Regulation of cell migration by dynamic microtubules. Seminars in cell \& developmental biology, 22(9), pp.968-74. 
Kemp, M.G. et al., 2005. The histone deacetylase inhibitor trichostatin A alters the pattern of DNA replication origin activity in human cells. Nucleic acids research, 33(1), pp.32536.

Kenhub.com, 2017. Quiz / Test: Uterus and ovaries | Kenhub. Available at: https://www.kenhub.com/en/quizzes/uterus-and-ovaries [Accessed June 20, 2017].

Kenny, H.A. et al., 2014. Mesothelial cells promote early ovarian cancer metastasis through fibronectin secretion. The Journal of clinical investigation, 124(10), pp.4614-28.

Kent, W.J. et al., 2002. The human genome browser at UCSC. Genome research, 12(6), pp.996-1006.

Khandekar, M.J., Cohen, P. \& Spiegelman, B.M., 2011. Molecular mechanisms of cancer development in obesity. Nature reviews. Cancer, 11(12), pp.886-95.

Kim, J. et al., 2015. The ovary is an alternative site of origin for high-grade serous ovarian cancer in mice. Endocrinology, 156(6), pp.1975-81.

Kim, M.S. et al., 2015. The histone acetyltransferase Myst2 regulates Nanog expression, and is involved in maintaining pluripotency and self-renewal of embryonic stem cells. FEBS letters, 589(8), pp.941-50.

Kim, S. et al., 2004. A screen for genes that suppress loss of contact inhibition: identification of ING4 as a candidate tumor suppressor gene in human cancer. Proceedings of the National Academy of Sciences of the United States of America, 101(46), pp.16251-6.

Kindelberger, D.W. et al., 2007. Intraepithelial carcinoma of the fimbria and pelvic serous carcinoma: Evidence for a causal relationship. The American journal of surgical pathology, 31(2), pp.161-9.

Kishi, H. et al., 2001. Osmotic shock induces G1 arrest through p53 phosphorylation at Ser33 by activated p38MAPK without phosphorylation at Ser15 and Ser20. The Journal of biological chemistry, 276(42), pp.39115-22.

Kitawaki, J. et al., 1996. Immunohistochemical localisation of aromatase and its correlation with progesterone receptors in ovarian epithelial tumours. Anticancer research, 16(1), pp.91-7.

Kleff, S. et al., 1995. Identification of a gene encoding a yeast histone $\mathrm{H} 4$ acetyltransferase. The Journal of biological chemistry, 270(42), pp.24674-7.

Kleinsmith, L.J., 2006. Principles of Cancer Biology, Pearson Education Limited.

Klug, A., 2010. The Discovery of Zinc Fingers and Their Applications in Gene Regulation and Genome Manipulation. Annual Review of Biochemistry, 79(1), pp.213-231.

Knowles, M. \& Selby, P., 2005. Cellular and Molecular Biology of Cancer Fourth., Oxford University Press.

Kobayashi, H. et al., 2008. A randomized study of screening for ovarian cancer: a multicenter study in Japan. International journal of gynecological cancer : official journal of the International Gynecological Cancer Society, 18(3), pp.414-20.

Köbel, M. et al., 2009. A limited panel of immunomarkers can reliably distinguish between clear cell and high-grade serous carcinoma of the ovary. The American journal of surgical pathology, 33(1), pp.14-21.

Köbel, M. et al., 2008. Ovarian Carcinoma Subtypes Are Different Diseases: Implications for Biomarker Studies S. Narod, ed. PLoS Medicine, 5(12), p.e232.

Koh, E.-H. et al., 2014. Upregulation of Human Mammaglobin Reduces Migration and Invasion of Breast Cancer Cells. Cancer Investigation, 32(1), pp.22-29.

Korn, R.L. \& Crowley, J.J., 2013. Overview: progression-free survival as an endpoint in clinical trials with solid tumors. Clinical cancer research : an official journal of the American Association for Cancer Research, 19(10), pp.2607-12.

Kornberg, R.D., 1974. Chromatin structure: a repeating unit of histones and DNA. Science (New York, N.Y.), 184(4139), pp.868-71.

Koulouris, C.R. \& Penson, R.T., 2009. Ovarian Stromal and Germ Cell Tumors. Seminars in Oncology, 36(2), pp.126-136.

Koussounadis, A. et al., 2014. Chemotherapy-induced dynamic gene expression changes in vivo are prognostic in ovarian cancer. British Journal of Cancer, 110(12), pp.29752984. 
Kouzarides, T., 2007. Chromatin modifications and their function. Cell, 128(4), pp.693-705.

Koziol, J.A., 2010. Comments on the rank product method for analyzing replicated experiments. FEBS letters, 584(5), pp.941-4.

Krämer, A. et al., 2014. Causal analysis approaches in Ingenuity Pathway Analysis. Bioinformatics (Oxford, England), 30(4), pp.523-30.

Kreutz, C. et al., 2007. An error model for protein quantification. Bioinformatics (Oxford, England), 23(20), pp.2747-53.

Krueger, K.E. \& Srivastava, S., 2006. Posttranslational protein modifications: current implications for cancer detection, prevention, and therapeutics. Molecular \& cellular proteomics : $M C P, 5(10)$, pp.1799-810.

Kueh, A.J. et al., 2011. HBO1 is required for H3K14 acetylation and normal transcriptional activity during embryonic development. Molecular and cellular biology, 31(4), pp.845-60.

Kuhn, E. et al., 2010. Shortened Telomeres in Serous Tubal Intraepithelial Carcinoma: An Early Event in Ovarian High-grade Serous Carcinogenesis. The American Journal of Surgical Pathology, 34(6), pp.829-836.

Kuhn, E. et al., 2012. TP53 mutations in serous tubal intraepithelial carcinoma and concurrent pelvic high-grade serous carcinoma-evidence supporting the clonal relationship of the two lesions. The Journal of Pathology, 226(3), pp.421-426.

Kulkarni, A. \& Carley, H., 2016. Advances in the recognition and management of hereditary cancer. British Medical Bulletin, 120(1), pp.123-138.

Kundaje, A., 2013. A comprehensive collection of signal artifact blacklist regions in the human genome. ENCODE.

Kuo, Y.-M. \& Andrews, A.J., 2013. Quantitating the specificity and selectivity of Gcn5mediated acetylation of histone H3. PloS one, 8(2), p.e54896.

Kurdistani, S.K., 2011. Histone modifications in cancer biology and prognosis. Progress in drug research. Fortschritte der Arzneimittelforschung. Progres des recherches pharmaceutiques, 67, pp.91-106.

Kurman, R.J. \& Shih, I.-M., 2008. Pathogenesis of ovarian cancer: lessons from morphology and molecular biology and their clinical implications. International journal of gynecological pathology: official journal of the International Society of Gynecological Pathologists, 27(2), pp.151-60.

Kurman, R.J. \& Shih, I.-M., 2010. The origin and pathogenesis of epithelial ovarian cancer: a proposed unifying theory. The American journal of surgical pathology, 34(3), pp.433-43.

Kwong, J. et al., 2006. Candidate tumor-suppressor gene DLEC1 is frequently downregulated by promoter hypermethylation and histone hypoacetylation in human epithelial ovarian cancer. Neoplasia (New York, N.Y.), 8(4), pp.268-78.

Ladurner, A.G., 2003. Inactivating chromosomes: a macro domain that minimizes transcription. Molecular cell, 12(1), pp.1-3.

Laity, J.H., Lee, B.M. \& Wright, P.E., 2001. Zinc finger proteins: new insights into structural and functional diversity. Current opinion in structural biology, 11(1), pp.3946.

Lalonde, M.-E. et al., 2013. Exchange of associated factors directs a switch in HBO1 acetyltransferase histone tail specificity. Genes \& development, 27(18), pp.2009-24.

Lambrechts, D. et al., 2013. Markers of Response for the Antiangiogenic Agent Bevacizumab. Journal of Clinical Oncology, 31(9), pp.1219-1230.

Landt, S.G. et al., 2012. ChIP-seq guidelines and practices of the ENCODE and modENCODE consortia. Genome research, 22(9), pp.1813-31.

Langdon, S.P. et al., 1994. The regulation of growth and protein expression by estrogen in vitro: a study of 8 human ovarian carcinoma cell lines. The Journal of steroid biochemistry and molecular biology, 50(3-4), pp.131-5.

Langmead, B. et al., 2009. Ultrafast and memory-efficient alignment of short DNA sequences to the human genome. Genome biology, 10(3), p.R25.

Lataifeh, I. et al., 2005. Presenting symptoms of epithelial ovarian cancer. The Australian \& 
New Zealand journal of obstetrics \& gynaecology, 45(3), pp.211-4.

Lau, K.M., Mok, S.C. \& Ho, S.M., 1999. Expression of human estrogen receptor-alpha and beta, progesterone receptor, and androgen receptor mRNA in normal and malignant ovarian epithelial cells. Proceedings of the National Academy of Sciences of the United States of America, 96(10), pp.5722-7.

Ledermann, J. et al., 2014. Olaparib maintenance therapy in patients with platinum-sensitive relapsed serous ovarian cancer: a preplanned retrospective analysis of outcomes by BRCA status in a randomised phase 2 trial. The Lancet Oncology, 15(8), pp.852-861.

Ledermann, J.A. et al., 2016. Cediranib in patients with relapsed platinum-sensitive ovarian cancer (ICON6): a randomised, double-blind, placebo-controlled phase 3 trial. Lancet (London, England), 387(10023), pp.1066-74.

Lee, A.J.X. \& Swanton, C., 2012. Tumour heterogeneity and drug resistance: Personalising cancer medicine through functional genomics. Biochemical Pharmacology, 83(8), pp.1013-1020.

Lee, H. \& Romero, J., 2012. Origin of DNA replication at the human lamin B2 locus: OBR or ABR? Cell cycle (Georgetown, Tex.), 11(22), pp.4281-3.

Lee, K.K. \& Workman, J.L., 2007. Histone acetyltransferase complexes: one size doesn't fit all. Nature Reviews Molecular Cell Biology, 8(4), pp.284-295.

Lee, Y. et al., 2007. A candidate precursor to serous carcinoma that originates in the distal fallopian tube. The Journal of Pathology, 211(1), pp.26-35.

Lee, Y.-J. et al., 2013. Prognostic value of CAPZA1 overexpression in gastric cancer. International journal of oncology, 42(5), pp.1569-77.

Lee, Y.-Y. et al., 2011. Prognosis of ovarian clear cell carcinoma compared to other histological subtypes: A meta-analysis. Gynecologic Oncology, 122(3), pp.541-547.

Leggett, R.M. et al., 2013. Sequencing quality assessment tools to enable data-driven informatics for high throughput genomics. Frontiers in genetics, 4, p.288.

Legube, G. \& Trouche, D., 2003. Regulating histone acetyltransferases and deacetylases. EMBO reports, 4(10), pp.944-7.

Lei, Y. et al., 2015. Inhibition of ANKRD1 sensitizes human ovarian cancer cells to endoplasmic reticulum stress-induced apoptosis. Oncogene, 34(4), pp.485-495.

Lekka, M., 2016. Discrimination Between Normal and Cancerous Cells Using AFM. BioNanoScience, 6, pp.65-80.

Lemmon, M.A., Schlessinger, J. \& Ferguson, K.M., 2014. The EGFR Family: Not So Prototypical Receptor Tyrosine Kinases. Cold Spring Harbor Perspectives in Biology, 6(4), pp.a020768-a020768.

Lengyel, E., 2010. Ovarian cancer development and metastasis. The American journal of pathology, 177(3), pp.1053-64.

Lenhard, M. et al., 2012. Human chorionic gonadotropin and its relation to grade, stage and patient survival in ovarian cancer. BMC cancer, 12, p. 2 .

Ley, T.J. et al., 2010. DNMT3A Mutations in Acute Myeloid Leukemia. New England Journal of Medicine, 363(25), pp.2424-2433.

Li, A. et al., 2006. Prediction of Nepsilon-acetylation on internal lysines implemented in Bayesian Discriminant Method. Biochemical and biophysical research communications, 350(4), pp.818-24.

Li, C. et al., 2014. Reduced expression of AMPK- $\beta 1$ during tumor progression enhances the oncogenic capacity of advanced ovarian cancer. Molecular Cancer, 13(1), p.49.

Li, H. et al., 2009. The Sequence Alignment/Map format and SAMtools. Bioinformatics (Oxford, England), 25(16), pp.2078-9.

Liang, X. et al., 2015. Rapid and highly efficient mammalian cell engineering via Cas9 protein transfection. Journal of Biotechnology, 208, pp.44-53.

Lieber, M.R., 2010. The mechanism of double-strand DNA break repair by the nonhomologous DNA end-joining pathway. Annual review of biochemistry, 79, pp.181-211.

Lim, S.L. et al., 2008. Promoter hypermethylation of FANCF and outcome in advanced ovarian cancer. British Journal of Cancer, 98(8), pp.1452-1456. 
Liu, J.F. et al., 2014. Combination cediranib and olaparib versus olaparib alone for women with recurrent platinum-sensitive ovarian cancer: a randomised phase 2 study. The Lancet. Oncology, 15(11), pp.1207-14.

Liu, L. et al., 1999. p53 sites acetylated in vitro by PCAF and p300 are acetylated in vivo in response to DNA damage. Molecular and cellular biology, 19(2), pp.1202-9.

Liu, Y. et al., 2012. Expression of tumor suppressor gene ING4 in ovarian carcinoma is correlated with microvessel density. Journal of cancer research and clinical oncology, 138(4), pp.647-55.

Lodish, H. et al., 2000. Proto-Oncogenes and Tumor-Suppressor Genes. In Molecular Cell Biology. W. H. Freeman.

Loeb, L.A. \& Harris, C.C., 2008. Advances in chemical carcinogenesis: a historical review and prospective. Cancer research, 68(17), pp.6863-72.

London, R.E., 2015. The structural basis of XRCC1-mediated DNA repair. DNA Repair, 30, pp.90-103.

Lord, C.J. \& Ashworth, A., 2016. BRCAness revisited. Nature Reviews Cancer, 16(2), pp.110-120.

Love, M.I., Huber, W. \& Anders, S., 2014. Moderated estimation of fold change and dispersion for RNA-seq data with DESeq2. Genome biology, 15(12), p.550.

Lu, X. \& Lane, D.P., 1993. Differential induction of transcriptionally active p53 following UV or ionizing radiation: defects in chromosome instability syndromes? Cell, 75(4), pp.765-78.

Lucas, I. et al., 2007. High-throughput mapping of origins of replication in human cells. EMBO reports, 8(8), pp.770-7.

Luvero, D., Milani, A. \& Ledermann, J.A., 2014. Treatment options in recurrent ovarian cancer: latest evidence and clinical potential. Therapeutic advances in medical oncology, 6(5), pp.229-39.

Maciejczyk, A. et al., 2011. CD46 Expression is an Unfavorable Prognostic Factor in Breast Cancer Cases. Applied Immunohistochemistry \& Molecular Morphology, 19(6), pp.540-546.

Mackay, H.J. et al., 2010. Phase II trial of the histone deacetylase inhibitor belinostat in women with platinum resistant epithelial ovarian cancer and micropapillary (LMP) ovarian tumours. European Journal of Cancer, 46(9), pp.1573-1579.

Madhavan, H., 2007. Simple Laboratory methods to measure cell proliferation using DNA synthesis property. Journal of stem cells \& regenerative medicine, 3(1), pp.12-4.

Mahadevappa, A., Krishna, S.M. \& Vimala, M.G., 2017. Diagnostic and Prognostic Significance of Ki-67 Immunohistochemical Expression in Surface Epithelial Ovarian Carcinoma. Journal of clinical and diagnostic research: JCDR, 11(2), p.EC08-EC12.

Makris, G.M. et al., 2015. Ovarian carcinosarcoma: a case report, diagnosis, treatment and literature review. Hippokratia, 19(3), pp.256-9.

Maley, C.C. et al., 2004. Selectively advantageous mutations and hitchhikers in neoplasms: p16 lesions are selected in Barrett's esophagus. Cancer research, 64(10), pp.3414-27.

Malpica, A. et al., 2004. Grading ovarian serous carcinoma using a two-tier system. The American journal of surgical pathology, 28(4), pp.496-504.

Mantia-Smaldone, G.M., Edwards, R.P. \& Vlad, A.M., 2011. Targeted treatment of recurrent platinum-resistant ovarian cancer: current and emerging therapies. Cancer management and research, 3, pp.25-38.

Mao, Z. et al., 2008. Comparison of nonhomologous end joining and homologous recombination in human cells. DNA repair, 7(10), pp.1765-71.

Marieb, E.N. \& Hoehn, K., 2015. Human Anatomy \& Physiology 10th ed., Pearson Education Limited.

Markman, M. et al., 2004. Duration of response to second-line, platinum-based chemotherapy for ovarian cancer: implications for patient management and clinical trial design. Journal of clinical oncology : official journal of the American Society of Clinical Oncology, 22(15), pp.3120-5.

Marques, J.T. \& Williams, B.R.G., 2005. Activation of the mammalian immune system by 
siRNAs. Nature Biotechnology, 23(11), pp.1399-1405.

Marquez, R.T. et al., 2005. Patterns of Gene Expression in Different Histotypes of Epithelial Ovarian Cancer Correlate with Those in Normal Fallopian Tube, Endometrium, and Colon. Clinical Cancer Research, 11(17), pp.6116-6126.

Marraffini, L.A., 2016. The CRISPR-Cas system of Streptococcus pyogenes: function and applications, University of Oklahoma Health Sciences Center.

Marsh, D.J., Shah, J.S. \& Cole, A.J., 2014. Histones and their modifications in ovarian cancer - drivers of disease and therapeutic targets. Frontiers in oncology, 4, p.144.

Martini, F.H., Nath, J.L. \& Bartholomew, E.F., 2012. Fundamentals of Anatomy \& Physiology 9th ed., Pearson Education Limited.

Martins, F.C. et al., 2014. Combined image and genomic analysis of high-grade serous ovarian cancer reveals PTEN loss as a common driver event and prognostic classifier. Genome biology, 15(12), p.526.

Marusyk, A. \& Polyak, K., 2010. Tumor heterogeneity: causes and consequences. Biochimica et biophysica acta, 1805(1), pp.105-17.

Masai, H. et al., 2010. Eukaryotic Chromosome DNA Replication: Where, When, and How? Annual Review of Biochemistry, 79(1), pp.89-130.

Matei, D. et al., 2012. Epigenetic Resensitization to Platinum in Ovarian Cancer. Cancer Research, 72(9), pp.2197-2205.

Matsunuma, R. et al., 2016. UV Damage-Induced Phosphorylation of HBO1 Triggers CRL4DDB2-Mediated Degradation To Regulate Cell Proliferation. Molecular and cellular biology, 36(3), pp.394-406.

Matsunuma, R. et al., 2015. UV Damage-Induced Phosphorylation of HBO1 Triggers CRL4DDB2-Mediated Degradation To Regulate Cell Proliferation. Molecular and cellular biology, 36(3), pp.394-406.

Matulonis, U. et al., 2015. Phase I study of combination of vorinostat, carboplatin, and gemcitabine in women with recurrent, platinum-sensitive epithelial ovarian, fallopian tube, or peritoneal cancer. Cancer Chemotherapy and Pharmacology, 76(2), pp.417423.

Matulonis, U.A. et al., 2016. Ovarian cancer. Nature Reviews Disease Primers, 2, p.16061.

McCullagh, P. et al., 1999. The cloning, mapping and expression of a novel gene, BRL, related to the AF10 leukaemia gene. Oncogene, 18(52), pp.7442-7452.

McGuire, W.P., Markman, M. \& Markman, M., 2003. Primary ovarian cancer chemotherapy: current standards of care. British journal of cancer, 89 Suppl 3(Suppl 3), pp.S3-8.

McNulty, K. \& Gender, P., 2011. First Analysis of Tumor-Suppressor Interactions with Whole Genome in Normal Human Cells Reveals Key Differences with Cancer Cells. BNL Newsroom.

Méchali, M., 2010. Eukaryotic DNA replication origins: many choices for appropriate answers. Nature reviews. Molecular cell biology, 11(10), pp.728-38.

Mehra, K. et al., 2011. STICS, SCOUTs and p53 signatures; a new language for pelvic serous carcinogenesis. Frontiers in bioscience (Elite edition), 3, pp.625-34.

Meinhold-Heerlein, I. \& Hauptmann, S., 2014. The heterogeneity of ovarian cancer. Archives of gynecology and obstetrics, 289(2), pp.237-9.

Mendelsohn, J. et al., 2015. The Molecular Basis of Cancer 4th ed., Saunders, Elsevier, Inc.

Mendivil, A.A. et al., 2013. Increased Incidence of Severe Gastrointestinal Events With First-Line Paclitaxel, Carboplatin, and Vorinostat Chemotherapy for Advanced-Stage Epithelial Ovarian, Primary Peritoneal, and Fallopian Tube Cancer. International Journal of Gynecological Cancer, 23(3), pp.533-539.

Merlo, L.M.F. et al., 2006. Cancer as an evolutionary and ecological process. Nature Reviews Cancer, 6(12), pp.924-935.

Miller, L., 2010. Analyzing gels and western blots with ImageJ. Available at: http://www.lukemiller.org/ImageJ_gel_analysis.pdf.

Minn, A.J., 2015. Interferons and the Immunogenic Effects of Cancer Therapy. Trends in Immunology, 36(11), pp.725-737. 
Miotto, B. et al., 2006. Chameau HAT and DRpd3 HDAC function as antagonistic cofactors of JNK/AP-1-dependent transcription during Drosophila metamorphosis. Genes \& development, 20(1), pp.101-12.

Miotto, B. \& Struhl, K., 2006. Differential gene regulation by selective association of transcriptional coactivators and bZIP DNA-binding domains. Molecular and cellular biology, 26(16), pp.5969-82.

Miotto, B. \& Struhl, K., 2010. HBO1 histone acetylase activity is essential for DNA replication licensing and inhibited by Geminin. Molecular cell, 37(1), pp.57-66.

Miotto, B. \& Struhl, K., 2008. HBO1 histone acetylase is a coactivator of the replication licensing factor Cdt1. Genes \& development, 22(19), pp.2633-8.

Miotto, B. \& Struh1, K., 2011. JNK1 phosphorylation of Cdt1 inhibits recruitment of HBO1 histone acetylase and blocks replication licensing in response to stress. Molecular cell, 44(1), pp.62-71.

Mirza, M.R. et al., 2016. Niraparib Maintenance Therapy in Platinum-Sensitive, Recurrent Ovarian Cancer. New England Journal of Medicine, 375(22), pp.2154-2164.

Mishima, Y. et al., 2014. Histone acetylation mediated by Brd1 is crucial for Cd8 gene activation during early thymocyte development. Nature communications, 5, p.5872.

Mishima, Y., Miyagi, S., Saraya, A., Negishi, M., Endoh, M., et al., 2011. The Hbo1$\mathrm{Brd1/Brpf} 2$ complex is responsible for global acetylation of H3K14 and required for fetal liver erythropoiesis. Blood, 118(9), pp.2443-53.

Mishima, Y., Miyagi, S., Saraya, A., Negishi, M., Endoh, M., et al., 2011. The Hbo1$\mathrm{Brd} 1 / \mathrm{Brpf} 2$ complex is responsible for global acetylation of $\mathrm{H} 3 \mathrm{~K} 14$ and required for fetal liver erythropoiesis. Blood, 118(9), pp.2443-53.

Mitchison, T.J., 2012. The proliferation rate paradox in antimitotic chemotherapy. Molecular biology of the cell, 23(1), pp.1-6.

Mittal, D. et al., 2014. New insights into cancer immunoediting and its three component phases--elimination, equilibrium and escape. Current opinion in immunology, 27, pp.16-25.

Mittal, V., 2004. Improving the efficiency of RNA interference in mammals. Nature Reviews Genetics, 5(5), pp.355-365.

Miyamoto, N. et al., 2008. Tip60 Is Regulated by Circadian Transcription Factor Clock and Is Involved in Cisplatin Resistance. Journal of Biological Chemistry, 283(26), pp.18218-18226.

Mocellin, S. \& Provenzano, M., 2004. RNA interference: learning gene knock-down from cell physiology. Journal of translational medicine, 2(1), p.39.

Modesitt, S.C. et al., 2008. A phase II study of vorinostat in the treatment of persistent or recurrent epithelial ovarian or primary peritoneal carcinoma: A Gynecologic Oncology Group study. Gynecologic Oncology, 109(2), pp.182-186.

Moeendarbary, E. \& Harris, A.R., 2014. Cell mechanics: principles, practices, and prospects. Wiley interdisciplinary reviews. Systems biology and medicine, 6(5), pp.371-88.

Moll, U.M. et al., 2005. Transcription-independent pro-apoptotic functions of p53. Current opinion in cell biology, 17(6), pp.631-6.

Monk, B.J. et al., 2014. Anti-angiopoietin therapy with trebananib for recurrent ovarian cancer (TRINOVA-1): a randomised, multicentre, double-blind, placebo-controlled phase 3 trial. The Lancet. Oncology, 15(8), pp.799-808.

Monk, B.J. et al., 2010. Trabectedin Plus Pegylated Liposomal Doxorubicin in Recurrent Ovarian Cancer. Journal of Clinical Oncology, 28(19), pp.3107-3114.

Monneret, C., 2011. Platinum anticancer drugs. From serendipity to rational design. Annales pharmaceutiques francaises, 69(6), pp.286-95.

Monteiro, F.L. et al., 2014. Expression and functionality of histone H2A variants in cancer. Oncotarget, 5(11), pp.3428-43.

Moore, L.D., Le, T. \& Fan, G., 2013. DNA methylation and its basic function. Neuropsychopharmacology : official publication of the American College of Neuropsychopharmacology, 38(1), pp.23-38. 
Moore, P.S. \& Chang, Y., 2010. Why do viruses cause cancer? Highlights of the first century of human tumour virology. Nature Reviews Cancer, 10(12), pp.878-889.

Moroishi, T., Hansen, C.G. \& Guan, K.-L., 2015. The emerging roles of YAP and TAZ in cancer. Nature Reviews Cancer, 15(2), pp.73-79.

Mosashvilli, D. et al., 2010. Global histone acetylation levels: Prognostic relevance in patients with renal cell carcinoma. Cancer Science, 101(12), pp.2664-2669.

Mulier, S., 2009. HIPEC for ovarian cancer -patient information-. Available at: http://www.drmulier.com/3 en pat info ovarium.html [Accessed June 27, 2017].

Mullany, L.K. et al., 2015. Specific TP53 Mutants Overrepresented in Ovarian Cancer Impact CNV, TP53 Activity, Responses to Nutlin-3a, and Cell Survival. Neoplasia (New York, N.Y.), 17(10), pp.789-803.

Müller, A. \& Fishel, R., 2002. Mismatch repair and the hereditary non-polyposis colorectal cancer syndrome (HNPCC). Cancer investigation, 20(1), pp.102-9.

Muller, W.A., 2013. Getting Leukocytes to the Site of Inflammation. Veterinary pathology, $50(1)$, p.7.

Murdoch, W.J., 2005. Carcinogenic Potential of Ovulatory Genotoxicity. Biology of Reproduction, 73(4), pp.586-590.

Murdoch, W.J., 1995. Endothelial cell death in preovulatory ovine follicles: possible implication in the biomechanics of rupture. Journal of reproduction and fertility, 105(1), pp.161-4.

Murdoch, W.J. et al., 2010. Mechanisms and pathobiology of ovulation. Society of Reproduction and Fertility supplement, 67, pp.189-201.

Murdoch, W.J., Townsend, R.S. \& McDonnel, A.C., 2001. Ovulation-induced DNA damage in ovarian surface epithelial cells of ewes: prospective regulatory mechanisms of repair/survival and apoptosis. Biology of reproduction, 65(5), pp.1417-24.

Muroyama, A. \& Lechler, T., 2017. Microtubule organization, dynamics and functions in differentiated cells. Development (Cambridge, England), 144(17), pp.3012-3021.

Murphy, S.K., 2012. Targeting the epigenome in ovarian cancer. Future oncology (London, England), 8(2), pp.151-64.

Musselman, C.A. et al., 2012. Perceiving the epigenetic landscape through histone readers. Nature structural \& molecular biology, 19(12), pp.1218-27.

Mutch, D.G. et al., 2007. Randomized Phase III Trial of Gemcitabine Compared With Pegylated Liposomal Doxorubicin in Patients With Platinum-Resistant Ovarian Cancer. Journal of Clinical Oncology, 25(19), pp.2811-2818.

Nagaki, K. et al., 2003. Chromatin immunoprecipitation reveals that the 180-bp satellite repeat is the key functional DNA element of Arabidopsis thaliana centromeres. Genetics, 163(3), pp.1221-5.

National Academy of Medicine, 2016. Ovarian Cancers: Evolving Paradigms in Research and Care. The National Academies Press.

NCBI, N.C. for B.I., 2017. RefSeq: NCBI Reference Sequence Database. Available at: https://www.ncbi.nlm.nih.gov/refseq/.

NCBI Resource Coordinators, 2017. Database Resources of the National Center for Biotechnology Information. Nucleic Acids Research, 45(D1), pp.D12-D17.

Network, C.G.A.., 2011. Integrated genomic analyses of ovarian carcinoma. Nature, 474(7353), pp.609-15.

Nezhat, F.R. et al., 2015. New insights in the pathophysiology of ovarian cancer and implications for screening and prevention. American journal of obstetrics and gynecology, 213(3), pp.262-7.

$\mathrm{Ng}$, A. \& Barker, N., 2015. Ovary and fimbrial stem cells: biology, niche and cancer origins. Nature Reviews Molecular Cell Biology, 16(10), pp.625-638.

Nguyen, H.T., Tian, G. \& Murph, M.M., 2014. Molecular epigenetics in the management of ovarian cancer: are we investigating a rational clinical promise? Frontiers in oncology, 4, p.71.

Nicolson, G.L., 1984. Generation of phenotypic diversity and progression in metastatic tumor cells. Cancer metastasis reviews, 3(1), pp.25-42. 
Nieman, K.M. et al., 2011. Adipocytes promote ovarian cancer metastasis and provide energy for rapid tumor growth. Nature medicine, 17(11), pp.1498-503.

Nitiss, J.L., 2009. Targeting DNA topoisomerase II in cancer chemotherapy. Nature reviews. Cancer, 9(5), pp.338-50.

Noble, W.S., 2009. How does multiple testing correction work? Nature biotechnology, 27(12), pp.1135-7.

Norquist, B. et al., 2011. Secondary somatic mutations restoring BRCA1/2 predict chemotherapy resistance in hereditary ovarian carcinomas. Journal of clinical oncology : official journal of the American Society of Clinical Oncology, 29(22), pp.3008-15.

Norquist, B.M. et al., 2016. Inherited Mutations in Women With Ovarian Carcinoma. JAMA Oncology, 2(4), p.482.

Novikov, L. et al., 2011. QKI-Mediated Alternative Splicing of the Histone Variant MacroH2A1 Regulates Cancer Cell Proliferation. Molecular and Cellular Biology, 31(20), pp.4244-4255.

Nowell, P.C., 1976. The clonal evolution of tumor cell populations. Science (New York, N.Y.), 194(4260), pp.23-8.

O'Brien, C.A., Kreso, A. \& Jamieson, C.H.M., 2010. Cancer Stem Cells and Self-renewal. Clinical Cancer Research, 16(12), pp.3113-3120.

O'Donnell, A.J.M. et al., 2005. Estrogen receptor-alpha mediates gene expression changes and growth response in ovarian cancer cells exposed to estrogen. Endocrine-related cancer, 12(4), pp.851-66.

O'Driscoll, M. \& Jeggo, P.A., 2006. The role of double-strand break repair - insights from human genetics. Nature reviews. Genetics, 7(1), pp.45-54.

O'Leary, N.A. et al., 2016. Reference sequence (RefSeq) database at NCBI: current status, taxonomic expansion, and functional annotation. Nucleic Acids Research, 44(D1), pp.D733-D745.

Oats, J. \& Abraham, S., 2016. Fundamentals of Obstetrics and Gynaecology Tenth., Elsevier Limited.

Obenauf, A.C. \& Massagué, J., 2015. Surviving at a Distance: Organ-Specific Metastasis. Trends in Cancer, 1(1), pp.76-91.

Odunsi, K. et al., 2014. Epigenetic Potentiation of NY-ESO-1 Vaccine Therapy in Human Ovarian Cancer. Cancer Immunology Research, 2(1).

Oliver, K.E. \& McGuire, W.P., 2014. Ovarian cancer and antiangiogenic therapy: caveat emptor. Journal of clinical oncology : official journal of the American Society of Clinical Oncology, 32(30), pp.3353-6.

Olivier, R.I. et al., 2004. Clinical outcome of prophylactic oophorectomy in BRCA1/BRCA2 mutation carriers and events during follow-up. British Journal of Cancer, 90(8), pp.1492-1497.

Otsubo, C. et al., 2014. TSPAN2 Is Involved in Cell Invasion and Motility during Lung Cancer Progression. Cell Reports, 7(2), pp.527-538.

Oza, A.M. et al., 2015. A randomized double-blind phase III trial comparing vintafolide (EC145) and pegylated liposomal doxorubicin (PLD/Doxil®/Caelyx $\left.{ }^{\circledR}\right)$ in combination versus PLD in participants with platinum-resistant ovarian cancer (PROCEED) (NCT01170650). Gynecologic Oncology, 137, pp.5-6.

Ozer, A. \& Bruick, R.K., 2005. Regulation of HIF by prolyl hydroxylases: recruitment of the candidate tumor suppressor protein ING4. Cell cycle (Georgetown, Tex.), 4(9), pp.1153-6.

Pacchiarotti, J. et al., 2010. Differentiation potential of germ line stem cells derived from the postnatal mouse ovary 议. Differentiation, 79(3), pp.159-170.

Paik, D.Y. et al., 2012. Stem-like epithelial cells are concentrated in the distal end of the fallopian tube: a site for injury and serous cancer initiation. Stem cells (Dayton, Ohio), 30(11), pp.2487-97.

Paller, C.J. et al., 2014. Design of phase I combination trials: recommendations of the Clinical Trial Design Task Force of the NCI Investigational Drug Steering Committee. 
Clinical cancer research : an official journal of the American Association for Cancer Research, 20(16), pp.4210-7.

Panchenko, M. V., 2016. Structure, function and regulation of jade family PHD finger 1 (JADE1). Gene, 589(1), pp.1-11.

Panchenko, M. V, Zhou, M.I. \& Cohen, H.T., 2004. von Hippel-Lindau partner Jade-1 is a transcriptional co-activator associated with histone acetyltransferase activity. The Journal of biological chemistry, 279(53), pp.56032-41.

Park, C.C., Bissell, M.J. \& Barcellos-Hoff, M.H., 2000. The influence of the microenvironment on the malignant phenotype. Molecular medicine today, 6(8), pp.324-9.

Park, P.J., 2009. ChIP-seq: advantages and challenges of a maturing technology. Nature reviews. Genetics, 10(10), pp.669-80.

Park, S.-Y. et al., 2012. Globoside promotes activation of ERK by interaction with the epidermal growth factor receptor. Biochimica et Biophysica Acta (BBA) - General Subjects, 1820(7), pp.1141-1148.

Parker, C.E. et al., 2010. Mass Spectrometry for Post-Translational Modifications, CRC Press/Taylor \& Francis.

Parker, M.W., Botchan, M.R. \& Berger, J.M., 2017. Mechanisms and regulation of DNA replication initiation in eukaryotes. Critical Reviews in Biochemistry and Molecular Biology, pp.1-41.

Parker, W.B., 2009. Enzymology of purine and pyrimidine antimetabolites used in the treatment of cancer. Chemical reviews, 109(7), pp.2880-93.

Parri, M. \& Chiarugi, P., 2010. Rac and Rho GTPases in cancer cell motility control. Cell communication and signaling : CCS, 8, p.23.

Parsons, B.D. et al., 2009. A Direct Phenotypic Comparison of siRNA Pools and Multiple Individual Duplexes in a Functional Assay M. Freitag, ed. PLoS ONE, 4(12), p.e8471.

Parsons, J.T., Horwitz, A.R. \& Schwartz, M.A., 2010. Cell adhesion: integrating cytoskeletal dynamics and cellular tension. Nature reviews. Molecular cell biology, 11(9), pp.633-43.

Parthun, M.R., Widom, J. \& Gottschling, D.E., 1996. The major cytoplasmic histone acetyltransferase in yeast: links to chromatin replication and histone metabolism. Cell, 87(1), pp.85-94.

Patch, A.-M. et al., 2015. Whole-genome characterization of chemoresistant ovarian cancer. Nature, 521(7553), pp.489-494.

Patel, J.H. et al., 2004. The c-MYC Oncoprotein Is a Substrate of the Acetyltransferases hGCN5/PCAF and TIP60. Molecular and Cellular Biology, 24(24), pp.10826-10834.

Patrono, M.G. et al., 2013. Borderline tumours of the ovary, current controversies regarding their diagnosis and treatment. Ecancermedicalscience, 7, p.379.

Pattabiraman, D.R. \& Weinberg, R.A., 2014. Tackling the cancer stem cells - what challenges do they pose? Nature reviews. Drug discovery, 13(7), pp.497-512.

Pearl, L.H. et al., 2015. Therapeutic opportunities within the DNA damage response. Nature Reviews Cancer, 15(3), pp.166-180.

Pennington, K.P. \& Swisher, E.M., 2012. Hereditary ovarian cancer: beyond the usual suspects. Gynecologic oncology, 124(2), pp.347-53.

Perets, R. et al., 2013. Transformation of the Fallopian Tube Secretory Epithelium Leads to High-Grade Serous Ovarian Cancer in Brca;Tp53;Pten Models. Cancer Cell, 24(6), pp.751-765.

Perren, T.J. et al., 2011. A phase 3 trial of bevacizumab in ovarian cancer. The New England journal of medicine, 365(26), pp.2484-96.

Peschansky, V.J. \& Wahlestedt, C., 2014. Non-coding RNAs as direct and indirect modulators of epigenetic regulation. Epigenetics, 9(1), pp.3-12.

Pfisterer, J. et al., 2006. Gemcitabine plus carboplatin compared with carboplatin in patients with platinum-sensitive recurrent ovarian cancer: an intergroup trial of the AGOOVAR, the NCIC CTG, and the EORTC GCG. Journal of clinical oncology : official journal of the American Society of Clinical Oncology, 24(29), pp.4699-707. 
Phillips, D.M., 1963. The presence of acetyl groups of histones. The Biochemical journal, 87, pp.258-63.

Picard, F. et al., 2014. The spatiotemporal program of DNA replication is associated with specific combinations of chromatin marks in human cells. B. van Steensel, ed. PLoS genetics, 10(5), p.e1004282.

Piccart, M.J. et al., 2000. Randomized Intergroup Trial of Cisplatin-Paclitaxel Versus Cisplatin-Cyclophosphamide in Women With Advanced Epithelial Ovarian Cancer: Three-Year Results. Journal of the National Cancer Institute, 92(9), pp.699-708.

Piche, B. \& Li, G., 2010. Inhibitor of growth tumor suppressors in cancer progression. Cellular and molecular life sciences: CMLS, 67(12), pp.1987-99.

Pierotti, M.A., Sozzi, G. \& Croce, C.M., 2003. Mechanisms of oncogene activation. In Cancer Medicine. BC Decker.

Pignata, S. et al., 2011. Carboplatin plus paclitaxel versus carboplatin plus pegylated liposomal doxorubicin as first-line treatment for patients with ovarian cancer: the MITO-2 randomized phase III trial. Journal of clinical oncology : official journal of the American Society of Clinical Oncology, 29(27), pp.3628-35.

Piñon Jr, R., 2002. Biology of human reproduction First., University Science Books.

Plodinec, M. et al., 2012. The nanomechanical signature of breast cancer. Nature Nanotechnology, 7(11), pp.757-765.

Pollack, J.R. et al., 2002. Microarray analysis reveals a major direct role of DNA copy number alteration in the transcriptional program of human breast tumors. Proceedings of the National Academy of Sciences of the United States of America, 99(20), pp.12963-8.

Polyak, K., Haviv, I. \& Campbell, I.G., 2009. Co-evolution of tumor cells and their microenvironment. Trends in genetics : TIG, 25(1), pp.30-8.

Pop, C. \& Salvesen, G.S., 2009. Human caspases: activation, specificity, and regulation. The Journal of biological chemistry, 284(33), pp.21777-81.

Potter, H. \& Heller, R., 2003. Transfection by electroporation. Current protocols in molecular biology, Chapter 9, p.Unit 9.3.

Poux, A.N. \& Marmorstein, R., 2003. Molecular Basis for Gcn5/PCAF Histone Acetyltransferase Selectivity for Histone and Nonhistone Substrates $\dagger, \ddagger$. Biochemistry, 42(49), pp.14366-14374.

Pozo, P.N. \& Cook, J.G., 2016. Regulation and Function of Cdt1; A Key Factor in Cell Proliferation and Genome Stability. Genes, 8(1).

Pradeep, S. et al., 2014. Hematogenous Metastasis of Ovarian Cancer: Rethinking Mode of Spread. Cancer Cell, 26(1), pp.77-91.

Prat, J., 2012a. New insights into ovarian cancer pathology. Annals of oncology : official journal of the European Society for Medical Oncology / ESMO, 23 Suppl 1(suppl_10), pp.x111-7.

Prat, J., 2012b. Ovarian carcinomas: five distinct diseases with different origins, genetic alterations, and clinicopathological features. Virchows Archiv, 460(3), pp.237-249.

Prentice, L. et al., 2012. What is endosalpingiosis? Fertility and Sterility, 98(4), pp.942-947.

Prior, I.A., Lewis, P.D. \& Mattos, C., 2012. A Comprehensive Survey of Ras Mutations in Cancer. Cancer Research, 72(10).

Provencher, D.M. et al., 2000. Characterization of four novel epithelial ovarian cancer cell lines. In vitro cellular \& developmental biology. Animal, 36(6), pp.357-61.

Prowse, A.H. et al., 2006. Molecular genetic evidence that endometriosis is a precursor of ovarian cancer. International Journal of Cancer, 119(3), pp.556-562.

Pruitt, K.D. et al., 2009. The consensus coding sequence (CCDS) project: Identifying a common protein-coding gene set for the human and mouse genomes. Genome research, 19(7), pp.1316-23.

Przybycin, C.G. et al., 2010. Are All Pelvic (Nonuterine) Serous Carcinomas of Tubal Origin? The American Journal of Surgical Pathology, 34(10), pp.1407-1416.

Pujade-Lauraine, E. et al., 2014. Bevacizumab combined with chemotherapy for platinumresistant recurrent ovarian cancer: The AURELIA open-label randomized phase III 
trial. Journal of clinical oncology : official journal of the American Society of Clinical Oncology, 32(13), pp.1302-8.

Pujade-Lauraine, E. et al., 2010. Pegylated Liposomal Doxorubicin and Carboplatin Compared With Paclitaxel and Carboplatin for Patients With Platinum-Sensitive Ovarian Cancer in Late Relapse. Journal of Clinical Oncology, 28(20), pp.3323-3329.

Purdie, D.M. et al., 2003. Ovulation and risk of epithelial ovarian cancer. International Journal of Cancer, 104(2), pp.228-232.

Purnell, M.R. \& Whish, W.J., 1980. Novel inhibitors of poly(ADP-ribose) synthetase. The Biochemical journal, 185(3), pp.775-7.

Qi, L.S. et al., 2013. Repurposing CRISPR as an RNA-Guided Platform for SequenceSpecific Control of Gene Expression. Cell, 152(5), pp.1173-1183.

Qiu, H. et al., 2011. SLIT2 is epigenetically silenced in ovarian cancers and suppresses growth when activated. Asian Pacific journal of cancer prevention: APJCP, 12(3), pp.791-5.

Qiu, P. et al., 2004. Mutation detection using Surveyor nuclease. BioTechniques, 36(4), pp.702-7.

Quaresma, M., Coleman, M.P. \& Rachet, B., 2015. 40-year trends in an index of survival for all cancers combined and survival adjusted for age and sex for each cancer in England and Wales, 1971-2011: a population-based study. The Lancet, 385(9974), pp.12061218.

Quinlan, A.R., 2014. BEDTools: The Swiss-Army Tool for Genome Feature Analysis. In Current Protocols in Bioinformatics. Hoboken, NJ, USA: John Wiley \& Sons, Inc., p. 11.12.1-11.12.34.

Radivojac, P. et al., 2010. Identification, analysis, and prediction of protein ubiquitination sites. Proteins: Structure, Function, and Bioinformatics, 78(2), pp.365-380.

Raja, F.A. et al., 2013. Platinum versus platinum-combination chemotherapy in platinumsensitive recurrent ovarian cancer: a meta-analysis using individual patient data. Annals of oncology : official journal of the European Society for Medical Oncology, 24(12), pp.3028-34.

Rajan, P. et al., 2009. Alternative splicing and biological heterogeneity in prostate cancer. Nature Reviews Urology, 6(8), pp.454-460.

Ramirez, P.T. et al., 2008. Efficacy of letrozole in the treatment of recurrent platinum- and taxane-resistant high-grade cancer of the ovary or peritoneum. Gynecologic oncology, 110(1), pp.56-9.

Rao, B.R. \& Slotman, B.J., 1996. Endocrine role in ovarian cancer. Endocrine Related Cancer, 3(4), pp.309-326.

Rao, D.D. et al., 2009. siRNA vs. shRNA: Similarities and differences. Advanced Drug Delivery Reviews, 61(9), pp.746-759.

Rebbeck, T.R. et al., 2002. Prophylactic Oophorectomy in Carriers of BRCA1 or BRCA2 Mutations. New England Journal of Medicine, 346(21), pp.1616-1622.

Remus, D. \& Diffley, J.F.X., 2009. Eukaryotic DNA replication control: lock and load, then fire. Current opinion in cell biology, 21(6), pp.771-7.

Ribatti, D., 2011. From the discovery of Vascular Endothelial Growth Factor to the introduction of Avastin in clinical trials - an interview with Napoleone Ferrara. The International Journal of Developmental Biology, 55(4-5), pp.383-388.

Rice, M., Gladstone, W. \& Weir, M., 2004. Relational databases: a transparent framework for encouraging biology students to think informatically. Cell biology education, 3(4), pp.241-52.

Rice, M.S., Hankinson, S.E. \& Tworoger, S.S., 2014. Tubal ligation, hysterectomy, unilateral oophorectomy, and risk of ovarian cancer in the Nurses' Health Studies. Fertility and sterility, 102(1), p.192-198.e3.

Richards, J.S. et al., 2002. Ovulation: New Dimensions and New Regulators of the Inflammatory-Like Response. Annual Review of Physiology, 64(1), pp.69-92.

Richmond, T.J. et al., 1997. Crystal structure of the nucleosome core particle at $2.8 \mathrm{~A}$ resolution. Nature, 389(6648), pp.251-260. 
Rieu, I. \& Powers, S.J., 2009. Real-time quantitative RT-PCR: design, calculations, and statistics. The Plant cell, 21(4), pp.1031-3.

Rigakos, G. \& Razis, E., 2012. BRCAness: finding the Achilles heel in ovarian cancer. The oncologist, 17(7), pp.956-62.

Risch, H.A., 1998. Hormonal Etiology of Epithelial Ovarian Cancer, With a Hypothesis Concerning the Role of Androgens and Progesterone. JNCI Journal of the National Cancer Institute, 90(23), pp.1774-1786.

Robbins, M., Judge, A. \& MacLachlan, I., 2009. siRNA and Innate Immunity. Oligonucleotides, 19(2), pp.89-102.

Roberts, A. \& Pachter, L., 2013. Streaming fragment assignment for real-time analysis of sequencing experiments. Nature methods, 10(1), pp.71-3.

Robinson, J.T. et al., 2011. Integrative genomics viewer. Nature biotechnology, 29(1), pp.24-6.

Rosanò, L., Spinella, F. \& Bagnato, A., 2013. Endothelin 1 in cancer: biological implications and therapeutic opportunities. Nature Reviews Cancer, 13(9), pp.637-651.

Rosen, D.G. et al., 2009. Ovarian cancer: pathology, biology, and disease models. Frontiers in bioscience (Landmark edition), 14, pp.2089-102.

Rosenberg, P. et al., 2002. Randomized trial of single agent paclitaxel given weekly versus every three weeks and with peroral versus intravenous steroid premedication to patients with ovarian cancer previously treated with platinum. Acta oncologica (Stockholm, Sweden), 41(5), pp.418-24.

Rother, J. et al., 2014. Atomic force microscopy-based microrheology reveals significant differences in the viscoelastic response between malign and benign cell lines. Open biology, 4(5), p.140046.

Rouleau, M. et al., 2010. PARP inhibition: PARP1 and beyond. Nature reviews. Cancer, 10(4), pp.293-301.

Rundlett, S.E. et al., 1996. HDA1 and RPD3 are members of distinct yeast histone deacetylase complexes that regulate silencing and transcription. Proceedings of the National Academy of Sciences of the United States of America, 93(25), pp.14503-8.

Rustin, G. et al., 2011. A phase II trial evaluating two schedules of sagopilone (ZK-EPO), a novel epothilone, in patients with platinum-resistant ovarian cancer. Annals of oncology : official journal of the European Society for Medical Oncology, 22(11), pp.2411-6.

Rustin, G.J. et al., 2001. Use of CA-125 to define progression of ovarian cancer in patients with persistently elevated levels. Journal of clinical oncology : official journal of the American Society of Clinical Oncology, 19(20), pp.4054-7.

Sadoul, K. et al., 2008. Regulation of protein turnover by acetyltransferases and deacetylases. Biochimie, 90(2), pp.306-312.

Sadowski, M. et al., 2012. Protein Monoubiquitination and Polyubiquitination Generate Structural Diversity to Control Distinct Biological Processes. IUBMB Life, 64(2), pp.136-142.

Sakai, W. et al., 2008. Secondary mutations as a mechanism of cisplatin resistance in BRCA2-mutated cancers. Nature, 451(7182), pp.1116-20.

Saksouk, N. et al., 2009. HBO1 HAT complexes target chromatin throughout gene coding regions via multiple PHD finger interactions with histone $\mathrm{H} 3$ tail. Molecular cell, 33(2), pp.257-65.

Salani, R. et al., 2011. Posttreatment surveillance and diagnosis of recurrence in women with gynecologic malignancies: Society of Gynecologic Oncologists recommendations. American Journal of Obstetrics and Gynecology, 204(6), pp.466-478.

Sanchez, R. \& Zhou, M.-M., 2011. The PHD finger: a versatile epigenome reader. Trends in biochemical sciences, 36(7), pp.364-72.

Santoso, J.T. et al., 2014. Computed tomography adnexal mass score to estimate risk for ovarian cancer. Archives of Gynecology and Obstetrics, 289(3), pp.595-600.

Sauer, T. et al., 2015. MYST2 acetyltransferase expression and Histone H4 Lysine acetylation are suppressed in AML. Experimental Hematology, 43(9), p.794-802.e4. 
Scherer, W.F., Syverton, J.T. \& Gey, G.O., 1953. Studies on the propagation in vitro of poliomyelitis viruses. IV. Viral multiplication in a stable strain of human malignant epithelial cells (strain HeLa) derived from an epidermoid carcinoma of the cervix. The Journal of experimental medicine, 97(5), pp.695-710.

Schmid, S. et al., 2011. Wnt and Hedgehog Gene Pathway Expression in Serous Ovarian Cancer. International Journal of Gynecological Cancer, 21(6), pp.975-980.

Schmittgen, T.D. \& Livak, K.J., 2008. Analyzing real-time PCR data by the comparative CT method. Nature Protocols, 3(6), pp.1101-1108.

Schneider, C.A., Rasband, W.S. \& Eliceiri, K.W., 2012. NIH Image to ImageJ: 25 years of image analysis. Nature methods, 9(7), pp.671-5.

Schroder, K. et al., 2003. Interferon- : an overview of signals, mechanisms and functions. Journal of Leukocyte Biology, 75(2), pp.163-189.

Schwartz, P.E. et al., 1989. Tamoxifen in combination with cytotoxic chemotherapy in advanced epithelial ovarian cancer. A prospective randomized trial. Cancer, 63(6), pp.1074-8.

Seeber, L.M.S. \& van Diest, P.J., 2012. Epigenetics in ovarian cancer. Methods in molecular biology (Clifton, N.J.), 863, pp.253-69.

Sehdev, A.S. et al., 2010. Serous tubal intraepithelial carcinoma upregulates markers associated with high-grade serous carcinomas including Rsf-1 (HBXAP), cyclin E and fatty acid synthase. Modern Pathology, 23(6), pp.844-855.

Sehouli, J. et al., 2009. Intra-abdominal tumor dissemination pattern and surgical outcome in 214 patients with primary ovarian cancer. Journal of surgical oncology, 99(7), pp.4247.

Seidman, J.D. et al., 2004. The histologic type and stage distribution of ovarian carcinomas of surface epithelial origin. International journal of gynecological pathology : official journal of the International Society of Gynecological Pathologists, 23(1), pp.41-4.

Seidman, J.D., Kurman, R.J. \& Ronnett, B.M., 2003. Primary and metastatic mucinous adenocarcinomas in the ovaries: incidence in routine practice with a new approach to improve intraoperative diagnosis. The American journal of surgical pathology, 27(7), pp.985-93.

Seligson, D.B. et al., 2005. Global histone modification patterns predict risk of prostate cancer recurrence. Nature, 435(7046), pp.1262-1266.

Sellar, G.C. et al., 2003. OPCML at $11 \mathrm{q} 25$ is epigenetically inactivated and has tumorsuppressor function in epithelial ovarian cancer. Nature Genetics, 34(3), pp.337-343.

Sens, P. \& Plastino, J., 2015. Membrane tension and cytoskeleton organization in cell motility. Journal of Physics: Condensed Matter, 27(27), p.273103.

Seo, J.-A. et al., 2015. Curcumin induces apoptosis by inhibiting sarco/endoplasmic reticulum ca(2+) ATPase activity in ovarian cancer cells. Cancer letters, 371(1), pp.30-37.

Serrano-Olvera, A. et al., 2006. Prognostic, predictive and therapeutic implications of HER2 in invasive epithelial ovarian cancer. Cancer treatment reviews, 32(3), pp.180-90.

Shackleton, M. et al., 2009. Heterogeneity in cancer: cancer stem cells versus clonal evolution. Cell, 138(5), pp.822-9.

Shacter, E. \& Weitzman, S.A., 2002. Chronic inflammation and cancer. Oncology (Williston Park, N.Y.), 16(2), pp.217-26, 229-2.

Shah, D. et al., 2013. Doppler ultrasound: a good and reliable predictor of ovarian malignancy. Journal of obstetrics and gynaecology of India, 63(3), pp.186-9.

Shah, S., Prasad, S. \& Knudsen, K.E., 2012. Targeting Pioneering Factor and Hormone Receptor Cooperative Pathways to Suppress Tumor Progression. Cancer Research, 72(5), pp.1248-1259.

Shahbazian, M.D. \& Grunstein, M., 2007. Functions of site-specific histone acetylation and deacetylation. Annual review of biochemistry, 76, pp.75-100.

Shalem, O. et al., 2014. Genome-scale CRISPR-Cas9 knockout screening in human cells. Science (New York, N.Y.), 343(6166), pp.84-87.

Sharma, M. et al., 2000. Androgen receptor interacts with a novel MYST protein, HBO1. 
The Journal of biological chemistry, 275(45), pp.35200-8.

Sheikh, B.N., 2014. Crafting the Brain? Role of Histone Acetyltransferases in Neural Development and Disease. Cell and Tissue Research, 356(3), pp.553-573.

Shen, H. et al., 2014. CYR61 overexpression associated with the development and poor prognosis of ovarian carcinoma. Medical Oncology, 31(8), p.117.

Shen, J.-C. et al., 2007. Inhibitor of growth 4 suppresses cell spreading and cell migration by interacting with a novel binding partner, liprin alpha1. Cancer research, 67(6), pp.2552-8.

Sherr, C.J., 2004. Principles of tumor suppression. Cell, 116(2), pp.235-46.

Shi, L., Wu, Y. \& Li, C., 2015. Hormone therapy and risk of ovarian cancer in postmenopausal women. Menopause, 23(4), p.1.

Shih, I.-M. et al., 2010. Distinct DNA methylation profiles in ovarian serous neoplasms and their implications in ovarian carcinogenesis. American journal of obstetrics and gynecology, 203(6), p.584.e1-22.

Shih, I.-M. \& Kurman, R.J., 2005. Molecular Pathogenesis of Ovarian Borderline Tumors: New Insights and Old Challenges. Clinical Cancer Research, 11(20), pp.7273-7279.

Shih, I.-M. \& Kurman, R.J., 2004. Ovarian tumorigenesis: a proposed model based on morphological and molecular genetic analysis. The American journal of pathology, 164(5), pp.1511-8.

Shiseki, M. et al., 2003. p29ING4 and p28ING5 bind to p53 and p300, and enhance p53 activity. Cancer research, 63(10), pp.2373-8.

Shuang, T. et al., 2015. Over-expression of Sirt1 contributes to chemoresistance and indicates poor prognosis in serous epithelial ovarian cancer (EOC). Medical Oncology, 32(12), p.260.

Siegel, R.L., Miller, K.D. \& Jemal, A., 2016. Cancer statistics, 2016. CA: A Cancer Journal for Clinicians, 66(1), pp.7-30.

Sievers, F. et al., 2011. Fast, scalable generation of high-quality protein multiple sequence alignments using Clustal Omega. Molecular systems biology, 7(1), p.539.

Silva, J. et al., 2004. RNA-interference-based functional genomics in mammalian cells: reverse genetics coming of age. Oncogene, 23(51), pp.8401-8409.

Simon, A. \& Durrieu, M.-C., 2006. Strategies and results of atomic force microscopy in the study of cellular adhesion. Micron, 37(1), pp.1-13.

Simpkins, F., Garcia-Soto, A. \& Slingerland, J., 2013. New insights on the role of hormonal therapy in ovarian cancer. Steroids, 78(6), pp.530-7.

Singh, R.R. \& Kumar, R., 2005. Steroid hormone receptor signaling in tumorigenesis. Journal of Cellular Biochemistry, 96(3), pp.490-505.

Siriwardana, N.S., Meyer, R.D. \& Panchenko, M. V, 2015. The novel function of JADE1S in cytokinesis of epithelial cells. Cell cycle (Georgetown, Tex.), 14(17), pp.2821-34.

Sirmans, S.M. \& Pate, K.A., 2013. Epidemiology, diagnosis, and management of polycystic ovary syndrome. Clinical epidemiology, 6, pp.1-13.

Smale, S.T., 2010. Beta-Galactosidase Assay. Cold Spring Harbor Protocols, 2010(5), p.pdb.prot5423-prot5423.

Smith, H.J. et al., 2017. Epigenetic therapy for the treatment of epithelial ovarian cancer: A clinical review. Gynecologic oncology reports, 20, pp.81-86.

Smyth, G.K., 2004. Linear Models and Empirical Bayes Methods for Assessing Differential Expression in Microarray Experiments. Statistical Applications in Genetics and Molecular Biology, 3(1), pp.1-25.

Sohaib, S.A.A. \& Reznek, R.H., 2007. MR imaging in ovarian cancer. Cancer imaging : the official publication of the International Cancer Imaging Society, (Special issue A), pp.S119-29.

Sokalska, A. et al., 2009. Diagnostic accuracy of transvaginal ultrasound examination for assigning a specific diagnosis to adnexal masses. Ultrasound in obstetrics \& gynecology: the official journal of the International Society of Ultrasound in Obstetrics and Gynecology, 34(4), pp.462-70.

Sölétormos, G. et al., 2016. Clinical Use of Cancer Biomarkers in Epithelial Ovarian 
Cancer: Updated Guidelines From the European Group on Tumor Markers.

International journal of gynecological cancer : official journal of the International Gynecological Cancer Society, 26(1), pp.43-51.

Song, B. et al., 2013. Plk1 phosphorylation of orc2 and hbo1 contributes to gemcitabine resistance in pancreatic cancer. Molecular cancer therapeutics, 12(1), pp.58-68.

Soslow, R.A., 2008. Histologic Subtypes of Ovarian Carcinoma. International Journal of Gynecological Pathology, PAP(2), pp.161-74.

Southern, J.A. et al., 1991. Identification of an epitope on the $\mathrm{P}$ and $\mathrm{V}$ proteins of simian virus 5 that distinguishes between two isolates with different biological characteristics. Journal of General Virology, 72(7), pp.1551-1557.

SportsMD, 2015. Menstrual cycle.

Stadlmann, S. et al., 2005. Ovarian carcinoma cells and IL-1 $\beta$-activated human peritoneal mesothelial cells are possible sources of vascular endothelial growth factor in inflammatory and malignant peritoneal effusions. Gynecologic Oncology, 97(3), pp.784-789.

Staff, S.R.A.S., 2011. Using the SRA Toolkit to convert .sra files into other formats.

Stedman, W. et al., 2004. ORC, MCM, and histone hyperacetylation at the Kaposi's sarcoma-associated herpesvirus latent replication origin. Journal of virology, 78(22), pp.12566-75.

Steinhardt, A.A. et al., 2008. Expression of Yes-associated protein in common solid tumors. Human Pathology, 39(11), pp.1582-1589.

Stepanenko, A.A., Vassetzky, Y.S. \& Kavsan, V.M., 2013. Antagonistic functional duality of cancer genes. Gene, 529(2), pp.199-207.

Stevens, E. V et al., 2011. RhoGDI2 antagonizes ovarian carcinoma growth, invasion and metastasis. Small GTPases, 2(4), pp.202-210.

Stothard, P., 2000. The sequence manipulation suite: JavaScript programs for analyzing and formatting protein and DNA sequences. BioTechniques, 28(6), pp.1102, 1104.

Straatman, K., 2008. Wound healing assay. Available at: http://www.le.ac.uk/biochem/microscopy/pdf/Wound healing assay.pdf.

Stronach, E.A. et al., 2011. HDAC4-regulated STAT1 activation mediates platinum resistance in ovarian cancer. Cancer research, 71(13), pp.4412-22.

Subramanian, A. et al., 2005. Gene set enrichment analysis: a knowledge-based approach for interpreting genome-wide expression profiles. Proceedings of the National Academy of Sciences of the United States of America, 102(43), pp.15545-50.

Sugiyama, T. et al., 2000. Clinical characteristics of clear cell carcinoma of the ovary: a distinct histologic type with poor prognosis and resistance to platinum-based chemotherapy. Cancer, 88(11), pp.2584-9.

Surowiak, P. et al., 2006. CD46 expression is indicative of shorter revival-free survival for ovarian cancer patients. Anticancer research, 26(6C), pp.4943-8.

Suzuki, T. et al., 2002. New genes involved in cancer identified by retroviral tagging. Nature genetics, 32(1), pp.166-74.

Sveen, A. et al., 2016. Aberrant RNA splicing in cancer; expression changes and driver mutations of splicing factor genes. Oncogene, 35(19), pp.2413-2427.

Swiech, L. et al., 2014. In vivo interrogation of gene function in the mammalian brain using CRISPR-Cas9. Nature Biotechnology, 33(1), pp.102-106.

Sykes, S.M. et al., 2009. Acetylation of the DNA binding domain regulates transcriptionindependent apoptosis by p53. The Journal of biological chemistry, 284(30), pp.20197-205.

Sykes, S.M. et al., 2006. Acetylation of the p53 DNA-binding domain regulates apoptosis induction. Molecular cell, 24(6), pp.841-51.

Symeonides, S. \& Gourley, C., 2015. Ovarian Cancer Molecular Stratification and Tumor Heterogeneity: A Necessity and a Challenge. Frontiers in oncology, 5, p.229.

Szotek, P.P. et al., 2008. Normal ovarian surface epithelial label-retaining cells exhibit stem/progenitor cell characteristics. Proceedings of the National Academy of Sciences of the United States of America, 105(34), pp.12469-73. 
Tan, D.S., Agarwal, R. \& Kaye, S.B., 2006. Mechanisms of transcoelomic metastasis in ovarian cancer. The Lancet Oncology, 7(11), pp.925-934.

Tang, S.J. et al., 2016. Characterization of the Regulation of CD46 RNA Alternative Splicing. Journal of Biological Chemistry, 291(27), pp.14311-14323.

Tang, Y. et al., 2006. Tip60-dependent acetylation of p53 modulates the decision between cell-cycle arrest and apoptosis. Molecular cell, 24(6), pp.827-39.

Tavassoli, F. \& Deville, P., 2003. IARC Publications - Pathology and Genetics of Tumours of the Breast and Female Genital Organs, Available at: http://www.iarc.fr/en/publications/pdfs-online/pat-gen/bb4/.

Temkin, S.M. et al., 2016. Ovarian Cancer: The Fallopian Tube as the Site of Origin and Opportunities for Prevention., 6.

Teplinsky, E. \& Muggia, F., 2015. EGFR and HER2: is there a role in ovarian cancer? Translational Cancer Research, 4(1), pp.107-117.

Teplinsky, E. \& Muggia, F., 2014. Targeting HER2 in ovarian and uterine cancers: Challenges and future directions. Gynecologic Oncology, 135(2), pp.364-370.

Tessarz, P. \& Kouzarides, T., 2014. Histone core modifications regulating nucleosome structure and dynamics. Nature Reviews Molecular Cell Biology, 15(11), pp.703-708.

Tew, W.P. et al., 2014. Intravenous aflibercept in patients with platinum-resistant, advanced ovarian cancer: results of a randomized, double-blind, phase 2, parallel-arm study. Cancer, 120(3), pp.335-43.

Thibault, B. et al., 2014. Ovarian cancer microenvironment: implications for cancer dissemination and chemoresistance acquisition. Cancer and Metastasis Reviews, 33(1), pp.17-39.

Thigpen, J.T. et al., 1994. Phase II trial of paclitaxel in patients with progressive ovarian carcinoma after platinum-based chemotherapy: a Gynecologic Oncology Group study. Journal of clinical oncology: official journal of the American Society of Clinical Oncology, 12(9), pp.1748-53.

Thomas, T. \& Voss, A.K., 2007. The diverse biological roles of MYST histone acetyltransferase family proteins. Cell cycle (Georgetown, Tex.), 6(6), pp.696-704.

Thömmes, P. \& Blow, J.J., 1997. The DNA replication licensing system. Cancer surveys, 29, pp.75-90.

Thompson, K.A. et al., 1994. BR140, a novel zinc-finger protein with homology to the TAF250 subunit of TFIID. Biochemical and biophysical research communications, 198(3), pp.1143-52.

Thorvaldsdóttir, H., Robinson, J.T. \& Mesirov, J.P., 2013. Integrative Genomics Viewer (IGV): high-performance genomics data visualization and exploration. Briefings in bioinformatics, 14(2), pp.178-92.

Thun, M.J., Henley, S.J. \& Calle, E.E., 2002. Tobacco use and cancer: an epidemiologic perspective for geneticists. Oncogene, 21(48), pp.7307-25.

Todd, R. \& Wong, D.T., 1999. Oncogenes. Anticancer research, 19(6A), pp.4729-46.

Tothill, R.W. et al., 2008. Novel molecular subtypes of serous and endometrioid ovarian cancer linked to clinical outcome. Clinical cancer research : an official journal of the American Association for Cancer Research, 14(16), pp.5198-208.

Tourgeman, D.E. et al., 2002. Human chorionic gonadotropin suppresses ovarian epithelial neoplastic cell proliferation in vitro. Fertility and Sterility, 78(5), pp.1096-1099.

Tung, K.-H. et al., 2005. Effect of Anovulation Factors on Pre- and Postmenopausal Ovarian Cancer Risk: Revisiting the Incessant Ovulation Hypothesis. American Journal of Epidemiology, 161(4), pp.321-329.

Tzouanacou, E., Tweedie, S. \& Wilson, V., 2003. Identification of Jade1, a gene encoding a PHD zinc finger protein, in a gene trap mutagenesis screen for genes involved in anteroposterior axis development. Molecular and cellular biology, 23(23), pp.8553-2.

Ullah, M. et al., 2008. Molecular Architecture of Quartet MOZ/MORF Histone Acetyltransferase Complexes. Molecular and Cellular Biology, 28(22), pp.6828-6843.

UniProt Consortium, T.U., 2008. The universal protein resource (UniProt). Nucleic acids research, 36(Database issue), pp.D190-5. 
Unniyampurath, U., Pilankatta, R. \& Krishnan, M.N., 2016. RNA Interference in the Age of CRISPR: Will CRISPR Interfere with RNAi? International journal of molecular sciences, 17(3), p.291.

Untergasser, A. et al., 2012. Primer3--new capabilities and interfaces. Nucleic acids research, 40(15), p.e115.

Ushijima, T. \& Asada, K., 2010. Aberrant DNA methylation in contrast with mutations. Cancer science, 101(2), pp.300-5.

Utley, R.T. \& Côté, J., 2003. The MYST family of histone acetyltransferases. Current topics in microbiology and immunology, 274, pp.203-36.

Varras, M., 2004. Benefits and limitations of ultrasonographic evaluation of uterine adnexal lesions in early detection of ovarian cancer. Clinical and experimental obstetrics \& gynecology, 31(2), pp.85-98.

Vaughan, S. et al., 2011. Rethinking ovarian cancer: recommendations for improving outcomes. Nature reviews. Cancer, 11(10), pp.719-25.

Vergote, I. et al., 2009. A randomised, double-blind, phase II study of two doses of pemetrexed in the treatment of platinum-resistant, epithelial ovarian or primary peritoneal cancer. European Journal of Cancer, 45(8), pp.1415-1423.

Vergote, I. et al., 2016. A Randomized, Double-Blind, Placebo-Controlled, Phase III Study to Assess Efficacy and Safety of Weekly Farletuzumab in Combination With Carboplatin and Taxane in Patients With Ovarian Cancer in First Platinum-Sensitive Relapse. Journal of clinical oncology: official journal of the American Society of Clinical Oncology, 34(19), pp.2271-8.

Vezzoli, A. et al., 2010. Molecular basis of histone H3K36me3 recognition by the PWWP domain of Brpf1. Nature structural \& molecular biology, 17(5), pp.617-9.

Vignali, M. et al., 2000. ATP-dependent chromatin-remodeling complexes. Molecular and cellular biology, 20(6), pp.1899-910.

Vilming Elgaaen, B. et al., 2014. Global miRNA expression analysis of serous and clear cell ovarian carcinomas identifies differentially expressed miRNAs including miR-200c-3p as a prognostic marker. BMC Cancer, 14(1), p.80.

Vivas-Mejia, P.E. et al., 2011. Silencing survivin splice variant 2B leads to antitumor activity in taxane--resistant ovarian cancer. Clinical cancer research : an official journal of the American Association for Cancer Research, 17(11), pp.3716-26.

Vlad, A.M. et al., 2010. A phase II trial of intraperitoneal interleukin-2 in patients with platinum-resistant or platinum-refractory ovarian cancer. Cancer Immunology, Immunotherapy, 59(2), pp.293-301.

Vogelstein, B. \& Kinzler, K.W., 2004. Cancer genes and the pathways they control. Nature Medicine, 10(8), pp.789-799.

Vogelstein, B., Lane, D. \& Levine, A.J., 2000. Surfing the p53 network. Nature, 408(6810), pp.307-10.

Vousden, K.H. \& Lu, X., 2002. Live or let die: the cell's response to p53. Nature Reviews Cancer, 2(8), pp.594-604.

Walentowicz, P. et al., 2014. Serum Inhibin A and Inhibin B Levels in Epithelial Ovarian Cancer Patients S. K. Batra, ed. PLoS ONE, 9(3), p.e90575.

Walsh, T. et al., 2011. Mutations in 12 genes for inherited ovarian, fallopian tube, and peritoneal carcinoma identified by massively parallel sequencing. Proceedings of the National Academy of Sciences of the United States of America, 108(44), pp.18032-7.

Walter-Yohrling, J. et al., 2003. Identification of genes expressed in malignant cells that promote invasion. Cancer research, 63(24), pp.8939-47.

Wang, T. et al., 2015. Identification and characterization of essential genes in the human genome. Science, 350(6264), pp.1096-1101.

Wang, W. et al., 2010. Estrogen receptor $\alpha(E R \alpha)$ mediates 17 $\beta$-estradiol (E2)-activated expression of HBO1. Journal of experimental \& clinical cancer research : $C R, 29$, p.140.

Wang, Y. et al., 2014. Epigenetic Targeting of Ovarian Cancer Stem Cells. Cancer Research, 74(17), pp.4922-4936. 
Wang, Y. et al., 2014. Epigenetic targeting of ovarian cancer stem cells. Cancer research, 74(17), pp.4922-36.

Wang, Z. et al., 2008. Combinatorial patterns of histone acetylations and methylations in the human genome. Nature genetics, 40(7), pp.897-903.

Wani, M.C. \& Horwitz, S.B., 2014. Nature as a remarkable chemist: a personal story of the discovery and development of Taxol. Anti-cancer drugs, 25(5), pp.482-7.

Wapenaar, H. \& Dekker, F.J., 2016. Histone acetyltransferases: challenges in targeting bisubstrate enzymes. Clinical epigenetics, 8, p.59.

Weber, S. et al., 2011. Laparoscopic surgical staging of early ovarian cancer. Reviews in obstetrics \& gynecology, 4(3-4), pp.117-22.

Weiderpass, E. \& Labrèche, F., 2012. Malignant tumors of the female reproductive system. Safety and health at work, 3(3), pp.166-80.

Weinberg, R.A., 2014. The Biology of Cancer 2nd ed., Garland Science - Taylor\&Francis Group, LLC.

Weissman, S.M. et al., 2011. Genetic Counseling Considerations in the Evaluation of Families for Lynch Syndrome-A Review. Journal of Genetic Counseling, 20(1), pp.5-19.

Weston, A. \& Harris, C.C., 2003. Multistage Carcinogenesis. In D. Kufe, R. Pollock, \& R. Weichselbaum, eds. Cancer Medicine. BC Decker.

White, Y.A.R. et al., 2012. Oocyte formation by mitotically active germ cells purified from ovaries of reproductive-age women. Nature Medicine, 18(3), pp.413-421.

Widmaier, E.P., Raff, H. \& Strang, K.T., 2015. Vander's Human Physiology 14th ed., McGraw-Hill Education.

Wilson, R.C. \& Doudna, J.A., 2013. Molecular Mechanisms of RNA Interference. Annual Review of Biophysics, 42(1), pp.217-239.

Witschi, E., 1948. Migration of germ cells of human embryos from the yolk sac to the primitive gonadal folds. Contributions to Embryology of the Carnegie Institution of Washington, 32, pp.67-80.

Wong, P.G. et al., 2010. Chromatin unfolding by Cdt1 regulates MCM loading via opposing functions of HBO1 and HDAC11-geminin. Cell cycle (Georgetown, Tex.), 9(21), pp.4351-63.

Worley, M.J. et al., 2013. Endometriosis-associated ovarian cancer: a review of pathogenesis. International journal of molecular sciences, 14(3), pp.5367-79.

Wright, D.G. et al., 2016. Human T-cell leukemia virus type-1-encoded protein HBZ represses p53 function by inhibiting the acetyltransferase activity of p300/CBP and HBO1. Oncotarget, 7(2), pp.1687-706.

Wu, Z.-Q. \& Liu, X., 2008. Role for Plk1 phosphorylation of Hbol in regulation of replication licensing. Proceedings of the National Academy of Sciences of the United States of America, 105(6), pp.1919-24.

Xiao, X.-S. et al., 2011. High Expression of p300 in Human Breast Cancer Correlates with Tumor Recurrence and Predicts Adverse Prognosis. Chinese journal of cancer research $=$ Chung-kuo yen cheng yen chiu, 23(3), pp.201-7.

$\mathrm{Xu}, \mathrm{C}$. et al., 2013. miR-9 functions as a tumor suppressor in ovarian serous carcinoma by targeting TLN1. International Journal of Molecular Medicine, 32(2), pp.381-8.

$\mathrm{Xu}$, L. et al., 2000. Inhibition of malignant ascites and growth of human ovarian carcinoma by oral administration of a potent inhibitor of the vascular endothelial growth factor receptor tyrosine kinases. International journal of oncology, 16(3), pp.445-54.

$\mathrm{Xu}, \mathrm{W}$. et al., 2012. Cell Stiffness Is a Biomarker of the Metastatic Potential of Ovarian Cancer Cells S. K. Batra, ed. PLoS ONE, 7(10), p.e46609.

Xue, F., Janzen, D.M. \& Knecht, D.A., 2010. Contribution of Filopodia to Cell Migration: A Mechanical Link between Protrusion and Contraction. International journal of cell biology, 2010, p.507821.

Yan, K. et al., 2016. The Chromatin Regulator BRPF3 Preferentially Activates the HBO1 Acetyltransferase but Is Dispensable for Mouse Development and Survival. The Journal of biological chemistry, 291(6), pp.2647-63. 
Yan, Y. et al., 2000. Crystal structure of yeast Esa1 suggests a unified mechanism for catalysis and substrate binding by histone acetyltransferases. Molecular cell, 6(5), pp.1195-205.

Yang-Hartwich, Y. et al., 2014. Ovulation and extra-ovarian origin of ovarian cancer. Scientific Reports, 4, p.6116.

Yang, X.-J., 2004. The diverse superfamily of lysine acetyltransferases and their roles in leukemia and other diseases. Nucleic acids research, 32(3), pp.959-76.

Yang, X.-J. \& Seto, E., 2007. HATs and HDACs: from structure, function and regulation to novel strategies for therapy and prevention. Oncogene, 26(37), pp.5310-5318.

Yarden, Y. \& Pines, G., 2012. The ERBB network: at last, cancer therapy meets systems biology. Nature Reviews Cancer, 12(8), pp.553-563.

Ye, H., Karim, A.A. \& Loh, X.J., 2014. Current treatment options and drug delivery systems as potential therapeutic agents for ovarian cancer: A review. Materials Science and Engineering: C, 45, pp.609-619.

Ye, J. et al., 2012. Primer-BLAST: a tool to design target-specific primers for polymerase chain reaction. BMC bioinformatics, 13, p.134.

Yeung, K.T. \& Yang, J., 2017. Epithelial-mesenchymal transition in tumor metastasis. Molecular Oncology, 11(1), pp.28-39.

Yeung, T.-L. et al., 2015. Cellular and molecular processes in ovarian cancer metastasis. $\mathrm{Am}$ J Physiol Cell Physiol, 309, pp.444-456.

Yokoyama, Y., 2013. Redistribution of resistance and sensitivity to platinum during the observation period following treatment of epithelial ovarian cancer. Molecular and Clinical Oncology.

Yoshida, Y. et al., 2009. Positron emission tomography in ovarian cancer: 18F-deoxyglucose and 16alpha-18F-fluoro-17beta-estradiol PET. Journal of ovarian research, 2(1), p.7.

Yoshikawa, H. et al., 2000. Prevalence of endometriosis in ovarian cancer. Gynecologic and obstetric investigation, 50 Suppl 1, pp.11-7.

You, L. et al., 2014. Expression atlas of the multivalent epigenetic regulator Brpf1 and its requirement for survival of mouse embryos. Epigenetics, 9(6), pp.860-72.

You, L. et al., 2015. The chromatin regulator Brpf1 regulates embryo development and cell proliferation. The Journal of biological chemistry, 290(18), pp.11349-64.

Yuan, J.S. et al., 2006. Statistical analysis of real-time PCR data. BMC bioinformatics, 7(1), p.85.

Yung, M.M.H., Ngan, H.Y.S. \& Chan, D.W., 2016. Targeting AMPK signaling in combating ovarian cancers: opportunities and challenges. Acta Biochimica et Biophysica Sinica, 48(4), pp.301-317.

Yurkovetsky, Z. et al., 2010. Development of a multimarker assay for early detection of ovarian cancer. Journal of clinical oncology : official journal of the American Society of Clinical Oncology, 28(13), pp.2159-66.

Zaid, T.M. et al., 2013. Identification of FGFR4 as a Potential Therapeutic Target for Advanced-Stage, High-Grade Serous Ovarian Cancer. Clinical Cancer Research, 19(4), pp.809-820.

Zalel, Y. et al., 1996. Diagnosis and management of malignant germ cell ovarian tumors in young females. International journal of gynaecology and obstetrics: the official organ of the International Federation of Gynaecology and Obstetrics, 55(1), pp.1-10.

Zhang, C. et al., 2014. High NR2F2 transcript level is associated with increased survival and its expression inhibits TGF- $\beta$-dependent epithelial-mesenchymal transition in breast cancer. Breast Cancer Research and Treatment, 147(2), pp.265-281.

Zhang, F. et al., 2015. ING5 inhibits cancer aggressiveness via preventing EMT and is a potential prognostic biomarker for lung cancer. Oncotarget, 6(18), pp.16239-52.

Zhang, F. et al., 2011. The Inhibitor of Growth Protein 5 (ING5) Depends on INCA1 as a Co-Factor for Its Antiproliferative Effects A. Bergmann, ed. PLoS ONE, 6(7), p.e21505.

Zhang, H. et al., 2012. Experimental evidence showing that no mitotically active female 
germline progenitors exist in postnatal mouse ovaries. Proceedings of the National Academy of Sciences of the United States of America, 109(31), pp.12580-5.

Zhang, H. et al., 2008. Expression and promoter methylation status of mismatch repair gene hMLH1 and hMSH2 in epithelial ovarian cancer. The Australian \& New Zealand journal of obstetrics \& gynaecology, 48(5), pp.505-9.

Zhang, K. \& Dent, S.Y.R., 2005. Histone modifying enzymes and cancer: going beyond histones. Journal of cellular biochemistry, 96(6), pp.1137-48.

Zhang, S. et al., 2015. Clinically relevant microRNAs in ovarian cancer. Molecular cancer research : $M C R, 13(3)$, pp.393-401.

Zhang, S. et al., 2008. Identification and characterization of ovarian cancer-initiating cells from primary human tumors. Cancer research, 68(11), pp.4311-20.

Zhang, W. et al., 2016. PRAME expression and promoter hypomethylation in epithelial ovarian cancer. Oncotarget, 7(29), pp.45352-45369.

Zhang, X., George, J., Deb, S., Degoutin, J.L., Takano, E.A., Fox, S.B., Bowtell, D.D.L., et al., 2011. The Hippo pathway transcriptional co-activator, YAP, is an ovarian cancer oncogene. Oncogene, 30(25), pp.2810-2822.

Zhang, X., George, J., Deb, S., Degoutin, J.L., Takano, E.A., Fox, S.B., AOCS Study group, D.D.L., et al., 2011. The Hippo pathway transcriptional co-activator, YAP, is an ovarian cancer oncogene. Oncogene, 30(25), pp.2810-22.

Zhang, Y. et al., 2008. Model-based analysis of ChIP-Seq (MACS). Genome biology, 9(9), p.R137.

Zhen, L. et al., 2010. The Expression of H3K9Ac, H3K14Ac, and H4K20TriMe in Epithelial Ovarian Tumors and the Clinical Significance. International Journal of Gynecological Cancer, 20(1), pp.82-86.

Zheng, W. et al., 2000. Ovarian epithelial tumor growth promotion by follicle-stimulating hormone and inhibition of the effect by luteinizing hormone. Gynecologic oncology, 76(1), pp.80-8.

Zhou, J. et al., 2005. Epigenetic control of replication origins. Cell cycle (Georgetown, Tex.), 4(7), pp.889-92.

Zhou, M.I. et al., 2005. Jade-1, a candidate renal tumor suppressor that promotes apoptosis. Proceedings of the National Academy of Sciences of the United States of America, 102(31), pp.11035-40.

Zhou, M.I. et al., 2002. The von Hippel-Lindau tumor suppressor stabilizes novel plant homeodomain protein Jade-1. The Journal of biological chemistry, 277(42), pp.3988798.

Zhou, M.I. et al., 2004. Tumor suppressor von Hippel-Lindau (VHL) stabilization of Jade-1 protein occurs through plant homeodomains and is VHL mutation dependent. Cancer research, 64(4), pp.1278-86.

Zong, H. et al., 2005. Cyclin-dependent kinase 11p58 interacts with HBO1 and enhances its histone acetyltransferase activity. FEBS Letters, 579(17), pp.3579-3588.

Zong, H. et al., 2007. Cyclin D3/CDK11p58 complex is involved in the repression of androgen receptor. Molecular and cellular biology, 27(20), pp.7125-42.

Zou, C. et al., 2013. SCF(Fbxw15) mediates histone acetyltransferase binding to origin recognition complex (HBO1) ubiquitin-proteasomal degradation to regulate cell proliferation. The Journal of biological chemistry, 288(9), pp.6306-16.

Zou, K. et al., 2009. Production of offspring from a germline stem cell line derived from neonatal ovaries. Nature Cell Biology, 11(5), pp.631-636.

Zuckerman, S., 1951. The number of oocytes in the mature ovary. Rec Prog Horm Res, 6, pp.63-109. 